Pacific Northwest

National Laboratory

Operated by Battelle for the

U.S. Department of Energy

\title{
Hanford Site Near-Facility Environmental Monitoring Data Report for Calendar Year 2003
}

\author{
C. J. Perkins \\ R. M. Mitchell \\ R. T. Coffman \\ R. C. Roos \\ S. M. McKinney
}

Duratek Technical Services

Richland, Washington 99354

September 2004

Prepared for the U.S. Department of Energy

under Contract DE-AC06-76RL01830 


\title{
DISCLAIMER
}

This report was prepared as an account of work sponsored by an agency of the United States Government. Neither the United States Government nor any agency thereof, nor Battelle Memorial Institute, nor any of their employees, makes any warranty, express or implied, or assumes any legal liability or responsibility for the accuracy, completeness, or usefulness of any information, apparatus, product, or process disclosed, or represents that its use would not infringe privately owned rights. Reference herein to any specific commercial product, process, or service by trade name, trademark, manufacturer, or otherwise does not necessarily constitute or imply its endorsement, recommendation, or favoring by the United States Government or any agency thereof, or Battelle Memorial Institute. The views and opinions of authors expressed herein do not necessarily state or reflect those of the United States Government or any agency thereof.

\author{
PACIFIC NORTHWEST NATIONAL LABORATORY \\ operated by \\ BATTELLE \\ for the \\ UNITED STATES DEPARTMENT OF ENERGY
}

under Contract DE-AC06-76RL01830

Printed in the United States of America

Available to DOE and DOE contractors from the

Office of Scientific and Technical Information,

P.O. Box 62, Oak Ridge, TN 37831-0062;

ph: (865) 576-8401

fax: (865) 576-5728

email: reports@adonis.osti.gov

\begin{abstract}
Available to the public from the National Technical Information Service, U.S. Department of Commerce, 5285 Port Royal Rd., Springfield, VA 22161 ph: (800) 553-6847 fax: (703) 605-6900

email: orders@ntis.fedworld.gov

online ordering: http://www.ntis.gov/ordering.htm
\end{abstract}

This document was printed on recycled paper.

$(8 / 00)$ 
PNNL-14687, APP. 2

HANFORD SITE NEAR-FACILITY ENVIRONMENTAL MONITORING DATA REPORT FOR CALENDAR YEAR 2003

\author{
C. J. Perkins \\ R. T. Coffman \\ S. M. McKinney \\ R. M. Mitchell \\ R. C. Roos
}

Duratek Technical Services

Richland, Washington 99354

September 2004

Prepared for

the U.S. Department of Energy

under Contract DE-AC06-76RL01830

Submitted by

Pacific Northwest National Laboratory

Richland, Washington 99354 


\section{CONTENTS}

1.0 NEAR-FACILITY ENVIRONMENTAL MONITORING AT HANFORD ......1-1

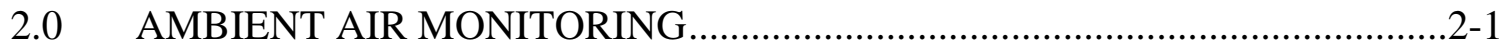

3.0 SOIL AND VEGETATION MONITORING ...................................................

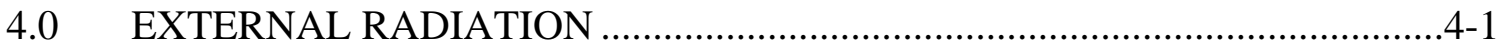

$5.0 \quad 100-N$ RIVERBANK SPRINGS MONITORING .........................................5-1

6.0 RADIOLOGICAL SURVEYS ..............................................................

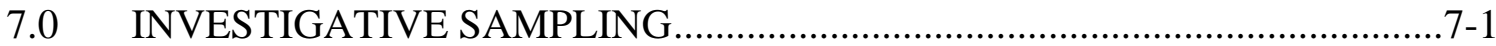

8.0 NOXIOUS WEED CONTROL PROGRAM …..............................................

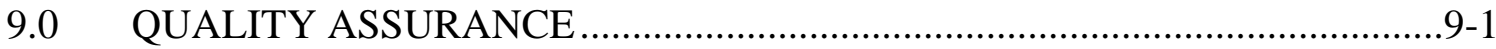

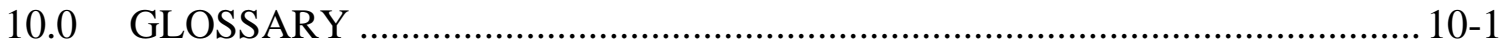

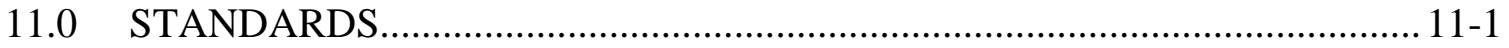

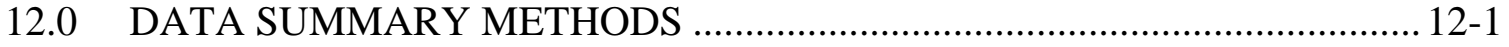

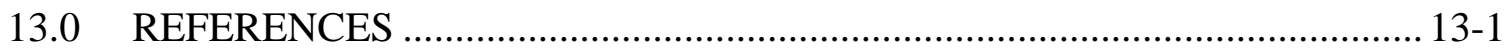




\section{LIST OF TERMS}

$\begin{array}{ll}\text { ALARA } & \text { As Low As Reasonably Achievable } \\ \text { CERCLA } & \text { Comprehensive Environmental Response, Compensation and Liability Act } \\ \text { CFR } & \text { Code of Federal Regulations } \\ \text { CSB } & \text { Canister Storage Building } \\ \text { DCG } & \text { derived concentration guide } \\ \text { DOE } & \text { U.S. Department of Energy } \\ \text { EDE } & \text { effective dose equivalent } \\ \text { ERC } & \text { Environmental Restoration Contractor } \\ \text { ERDF } & \text { Environmental Restoration Disposal Facility } \\ \text { ESD } & \text { environmental sites database } \\ \text { FH } & \text { Fluor Hanford, Inc. } \\ \text { GPS } & \text { global positioning system } \\ \text { HEPA } & \text { high-efficiency particulate air (filter) } \\ \text { HGIS } & \text { Hanford Geographical Information System } \\ \text { IDF } & \text { Integrated Disposal Facility } \\ \text { ILAW } & \text { Immobilized Low-Activity Waste Disposal } \\ \text { LWDF } & \text { Liquid Waste Disposal Facility } \\ \text { MCL } & \text { maximum contaminant level } \\ \text { PHMC } & \text { Project Hanford Management Contract } \\ \text { PNNL } & \text { Pacific Northwest National Laboratory } \\ \text { PFP } & \text { Plutonium Finishing Plant } \\ \text { PUREX } & \text { Plutonium-Uranium Extraction (Facility) } \\ \text { QA } & \text { quality assurance } \\ \text { RCRA } & \text { Resource Conservation and Recovery Act } \\ \text { RPP } & \text { River Protection Project } \\ \text { TLD } & \text { thermoluminescent dosimeters } \\ \text { WAC } & \text { Washington Administrative Code } \\ \text { WDOH } & \text { Washington State Department of Health } \\ \text { WSCF } & \text { Waste Sampling and Characterization Facility } \\ & \end{array}$




\subsection{NEAR-FACILITY ENVIRONMENTAL MONITORING AT HANFORD}

Near-facility environmental monitoring is defined as monitoring near facilities that have the potential to discharge or have discharged, stored, or disposed of radioactive or hazardous materials. Monitoring locations are associated with nuclear facilities such as the Plutonium Finishing Plant (PFP), Canister Storage Building (CSB), and the K Basins; inactive nuclear facilities such as N Reactor and the Plutonium-Uranium Extraction (PUREX) Facility; and waste storage or disposal facilities such as burial grounds, cribs, ditches, ponds, tank farms, and trenches.

Much of the monitoring consists of collecting and analyzing environmental samples and methodically surveying areas near facilities. The program is also designed to evaluate acquired analytical data, determine the effectiveness of facility effluent monitoring and controls, assess the adequacy of containment at waste disposal units, and detect and monitor unusual conditions. The program implements applicable portions of U.S. Department of Energy (DOE) Orders 435.1, 450.1 (replaced DOE Order 5400.1 in January 2003), and 5400.5 (DOE 1993); DOE Manual 231.1-1A, Environment, Safety, and Health Reporting Manual; Washington Administrative Code (WAC) 246-247; Title 40, Code of Federal Regulations (CFR) Part 61 (40 CFR 61), Subpart H; and 10 CFR 835.

Several types of environmental media are sampled near facilities to monitor waste management and restoration activities, and to evaluate the effectiveness of effluent treatment and control practices. Routine sampling and monitoring includes ambient air, water, external radiation, soil, and vegetation. The parameters typically monitored are radionuclide concentrations and radiation fields. Sampling methods are discussed in detail in the Duratek Technical Services, Operational Environmental Monitoring, DTS-OEM-001.

Samples are collected from known or expected effluent pathways. These pathways are generally downwind of potential or actual airborne releases and down gradient of liquid discharges. Table 1-1 shows the type, quantity, and location of routine near-facility monitoring samples collected in 2003.

Table 1-1. Near-Facility Routine Environmental Monitoring Samples and Locations, 2003.

\begin{tabular}{lcccccccccc} 
& $\begin{array}{c}\text { Number of sample } \\
\text { Sample Type }\end{array}$ & \multicolumn{10}{c}{ Operational area } \\
\cline { 3 - 11 } & locations & $100-\mathrm{B} / \mathrm{C}$ & $100-\mathrm{D} / \mathrm{DR}$ & $100-\mathrm{K}$ & $100-\mathrm{F}$ & $100-\mathrm{H}$ & $100-\mathrm{N}$ & ERDF $^{\mathrm{a}}$ & $200 / 600$ & $300 / 400$ \\
\hline Air & 82 & 6 & 3 & 11 & 6 & 2 & 5 & 3 & $41^{b}$ & 5 \\
Soil & 82 & 5 & 0 & 2 & 2 & 0 & 1 & 1 & 57 & 14 \\
Vegetation & 65 & 0 & 0 & 0 & 0 & 0 & 4 & 0 & 48 & 13 \\
External radiation & 134 & 4 & 0 & 20 & 5 & 0 & 14 & 3 & 67 & 21 \\
Water & 10 & 0 & 0 & 0 & 0 & 0 & 10 & 0 & 0 & 0
\end{tabular}

\footnotetext{
${ }^{\mathrm{a}}$ Environmental Restoration Disposal Facility in the 200 West Area.
}

b Includes 1 station at the Wye Barricade, 19 in the 200 East Area, and 21 in the 200 West Area. 
Strontium-90 results for this report period show overall lower values compared to historical trends. This was primarily due to changes in laboratory background correction calculations that were implemented in 2003. Both historical and current values are within accepted statistical ranges as evidenced by laboratory quality assurance (QA) and performance evaluation programs.

Waste disposal sites and the surrounding terrain are surveyed to detect and characterize radioactive surface contamination. Routine radiological surveys are conducted across the surfaces of underground radioactive material areas and along the perimeters of contamination areas. Locations include cribs, trenches, retention basins, ponds, ditches, solid waste disposal sites, unplanned release sites, tank farms, stabilized waste disposal sites, roads, and firebreaks in and around the Site operational areas.

Nonroutine, investigative samples are also collected as part of the Near-Facility Environmental Monitoring Program to confirm the absence or presence of radioactive and/or hazardous contaminants.

A Noxious Weed Control Program has been developed on the Hanford Site in response to Federal, State, and local laws requiring eradication or control of noxious weeds. A general discussion of the program and of control measures is provided in Section 8.0 of this Appendix.

This Appendix contains brief discussions, specific sampling location information, and complete analytical data results for the various near-facility environmental monitoring efforts for 2003. Detailed discussions and summarized analytical results are provided in Section 3.2 ("Near-Facility Environmental Monitoring") of the Hanford Site Environmental Report for Calendar Year 2003 (PNNL-14687).

\subsection{AIR MONITORING}

Near-facility air sampling monitors the effectiveness of waste management and environmental remediation controls, and effluent treatment systems in reducing effluents and emissions. These air samplers also monitor diffuse source emissions.

Ambient air monitoring is conducted to determine baseline concentrations of radionuclides in the operations areas, assess the impact of operations on the local environment, and monitor diffuse and fugitive emissions from sources located within the operations area. These measurements also provide an indication of the Project Hanford Management Contract (PHMC), River Protection Project (RPP), and Environmental Restoration Contractor (ERC) managed facilities' performance and are used to demonstrate compliance with environmental protection criteria.

In 2003, air radioactivity was sampled by a network of continuously operating samplers at 82 locations. Location-specific maps and monitoring results are provided in Section 2.0. 


\subsection{GROUNDWATER MONITORING}

The Near-Facility Environmental Monitoring Program did not conduct groundwater monitoring in 2002. Detailed discussion of groundwater monitoring management strategies and the 2003 monitoring results can be found in PNNL-14687 (Section 6.0, "Groundwater Monitoring") and in the Hanford Site Groundwater Monitoring for Fiscal Year 2003, PNNL-14548 (Hartman et al. 2004).

\subsection{SOIL AND VEGETATION SAMPLING}

Soil and vegetation samples were collected on or adjacent to waste disposal units, and from locations downwind and near or within the boundaries of the operating facilities. Samples were collected to detect potential migration and deposition of facility effluents. Migration of radionuclides can occur as the result of resuspension from radioactively contaminated surface areas, absorption by the roots of vegetation growing on or near underground and surface water disposal units, or intrusion by animals.

Radiological analyses of soil and vegetation samples included strontium-90, plutonium-239/240, isotopic uranium, and gamma-emitting radionuclides. Location-specific maps and the analytical results are presented in Section 3.0.

\subsection{EXTERNAL RADIATION}

External radiation levels were monitored near facilities and waste handling, storage, and disposal sites to measure, assess, and control the impacts of operations. Thermoluminescent dosimeters (TLDs) are used at numerous fixed locations to gather dose rate information over extended periods of time. TLD results can be used individually or averaged to determine dose rates in a given area for a particular sampling period.

Environmental dosimeters measure dose rates from all types of external radiation sources, including cosmic radiation, naturally occurring radioactivity in air and soil, and fallout from nuclear weapons testing, as well as any contribution from Hanford Site activities. During any year, changes in soil moisture and snow cover can cause external radiation levels to vary from $15 \%$ to $25 \%$ at any given location. The results are reported in units of millirems per year (mrem/yr). Individual TLD results and their locations are provided in Section 4.0.

\subsection{RIVERBANK SPRINGS MONITORING}

The springs along the 100-N Area Columbia River shoreline (N-Springs) were sampled in 2003 to assess the effectiveness of effluent and contamination controls. Ten water samples were collected. The radiological analyses were performed onsite at the Waste Sampling and Characterization Facility (WSCF), and the analyses included tritium, strontium-90, and 
gamma-emitting radionuclides. A location-specific map and the analytical results of the sampling are presented in Section 5.0.

\subsection{RADIOLOGICAL SURVEYS}

In 2003, the Hanford Site had approximately 3,651 ha (9,022 acres) of posted outdoor surface contamination, and 666 ha (1,646 acres) of posted underground radioactive material, not including the production facilities (e.g., PUREX, T-Plant, etc.). The total area of surface contamination was approximately six times larger than the area of underground radioactive material.

Since 1996, a global positioning system (GPS) has been utilized to accurately measure the surface area of these radiologically controlled sites. This collected information was entered into the Hanford Geographical Information System (HGIS), a computer database maintained by Fluor Hanford, Inc (FH). Survey location maps are provided in Section 6.0.

\subsection{INVESTIGATIVE SAMPLING}

Investigative sampling was conducted in the operations areas to confirm the absence or presence of radioactive and/or hazardous contaminants. Investigative sampling took place near facilities, such as storage and disposal sites, for at least one of the following reasons:

- $\quad$ To follow up radiological surface surveys that had indicated radioactive contamination was present.

- $\quad$ To conduct preoperational surveys to characterize the radiological/hazardous conditions at a site prior to facility construction, operation, or ultimate remediation.

- $\quad$ To determine if biotic intrusion (e.g., animal burrows or deep-rooted vegetation) has created a potential for contaminants to spread.

- $\quad$ To determine the integrity of waste containment systems.

Generally, the predominant radionuclides detected during these efforts were activation and fission products in the 100 Areas, fission products in the 200 Areas, and uranium in the 300 Area. Hazardous chemicals generally have not been identified above background levels in preoperational environmental monitoring samples. Special characterization samples collected in 2003 included soil and a cottontail rabbit from the 200 West Area, mice from the 100-K East Area, and a starling from the 300 Area. Complete results, including counting errors and field instrument and dose rate readings, where appropriate, are provided in Section 7.0. 


\subsection{NOXIOUS WEED CONTROL PROGRAM}

The Noxious Weed Control Program on the Hanford Site has been developed in response to Federal, State, and local laws requiring eradication or control of noxious weeds. A noxious weed is defined as "any plant which when established is highly destructive, competitive, or difficult to control by cultural or chemical practices." Typically, noxious weeds are non-native (alien) species that invade and displace native species, reduce habitat for fish and wildlife, and contribute to the extinction of sensitive species.

Ten plant species are on a high priority list for control at Hanford. These species are Yellow Starthistle (Centaurea solstitialis), Rush Skeletonweed (Chondrilla juncea), Babysbreath (Gypsophila paniculata), Medusa Head (Taeniatherum asperum), Dalmatian Toadflax (Linaria genistifolia ssp. Dalmatica), Spotted Knapweed (Centaurea maculosa), Diffuse Knapweed (Centaurea diffusa), Russian Knapweed (Acroptilon repens), Saltcedar (Tamarix spp.), and Purple Loosestrife (Lythrum salicaria).

Maps generally depicting the spatial distribution of these species across the Hanford Site can be found in Section 8.0. 
This page intentionally left blank. 


\subsection{AMBIENT AIR MONITORING}

Air samplers are located primarily at or near (within approximately $500 \mathrm{~m}[1,600 \mathrm{ft}]$ ) sites and/or facilities having the potential for, or history of, environmental releases, with emphasis on potential source terms as well as prevailing wind direction. Meteorological conditions are monitored continuously by the Pacific Northwest National Laboratory (PNNL) meteorology stations, which are strategically positioned in and around the Hanford Site.

For 2003, a network of continuously operating samplers at 82 locations (Table 2-1) sampled radioactivity in air. Location-specific maps are illustrated in Figures 2-1 through 2-11. Historical air sampling results for the 100-K, 100-N, 200 and 300 Areas are represented in graph form in Figures 2-12 through 2-23. A summary of ambient air sampling results for selected radionuclides collected during 2003 is presented in Table 2-2. The 2003 composited, sampler-specific monitoring results are provided in Table 2-3. Additional discussion of the 2003 results can be found in Section 3.2 of PNNL-14687.

Strontium-90 in air results for this report period show overall lower values compared to historical trends. This was primarily due to changes in laboratory background correction calculations that were implemented in 2003. Both historical and current values are within accepted statistical ranges as evidenced by laboratory QA and performance evaluation programs.

Several PNNL ambient air monitoring stations were utilized to provide additional information for several ERC remediation projects. The projects and the associated PNNL stations are the 100-B/C, 100-F, 100-KR-1 and 100-NR-1 remedial action projects (PNNL station "Yakima Barricade"), 300-FF-1\&2 remedial action project (PNNL stations "300NE," "300 Trench" and "300 Water Intake"), and the Environmental Restoration Disposal Facility (ERDF) project (PNNL station "200 West SE”). The 2003 air monitoring results for these locations can be found in Table 2-4.

Air monitoring within the 300 and 400 Areas was performed by PNNL as part of the Site Surface Environmental Surveillance Project. Data acquired are reviewed by Near-Facility Monitoring personnel. A more detailed discussion of these results is provided in PNNL-14687, Section 4.1, "Air Surveillance.”

Near-facility environmental air samplers operate at a flow rate of $0.057 \mathrm{~m}^{3} / \mathrm{min}$ $(2 \mathrm{ft} / \mathrm{min})$, drawing a sample through a $47 \mathrm{~mm}$ (2 in.), open-faced filter about $2 \mathrm{~m}(6 \mathrm{ft})$ aboveground. All sample filters are exchanged biweekly, held one week (to allow for decay of short-lived natural radioactivity), and then sent to the analytical laboratory for initial analysis of total alpha and total beta activity. These initial analyses serve as an indicator of potential environmental problems.

Depending on project/facility requirements, the filters were stored until the end of either a three- or six-month sample period, then segregated and composited by sample location for specific radionuclide analysis as shown in Table 2-1. Segregating and compositing air filters by 
site provides a larger sample size and, thus, a more sensitive and accurate measurement of the concentration of airborne radionuclides.

To help assess the impact of Site operations, monitoring results are compared to DOE derived concentration guides (DCGs), to the results obtained from the distant communities of Yakima and Sunnyside as reported by PNNL Site Environmental Surveillance Program, and to data acquired from collocated sampling locations managed by Near-Facility Monitoring, PNNL and the Washington State Department of Health (WDOH). Collocated sampling results are used for comparability and precision of data.

Table 2-1. Near-Facility Air Sampling Locations and Analyses, 2003.

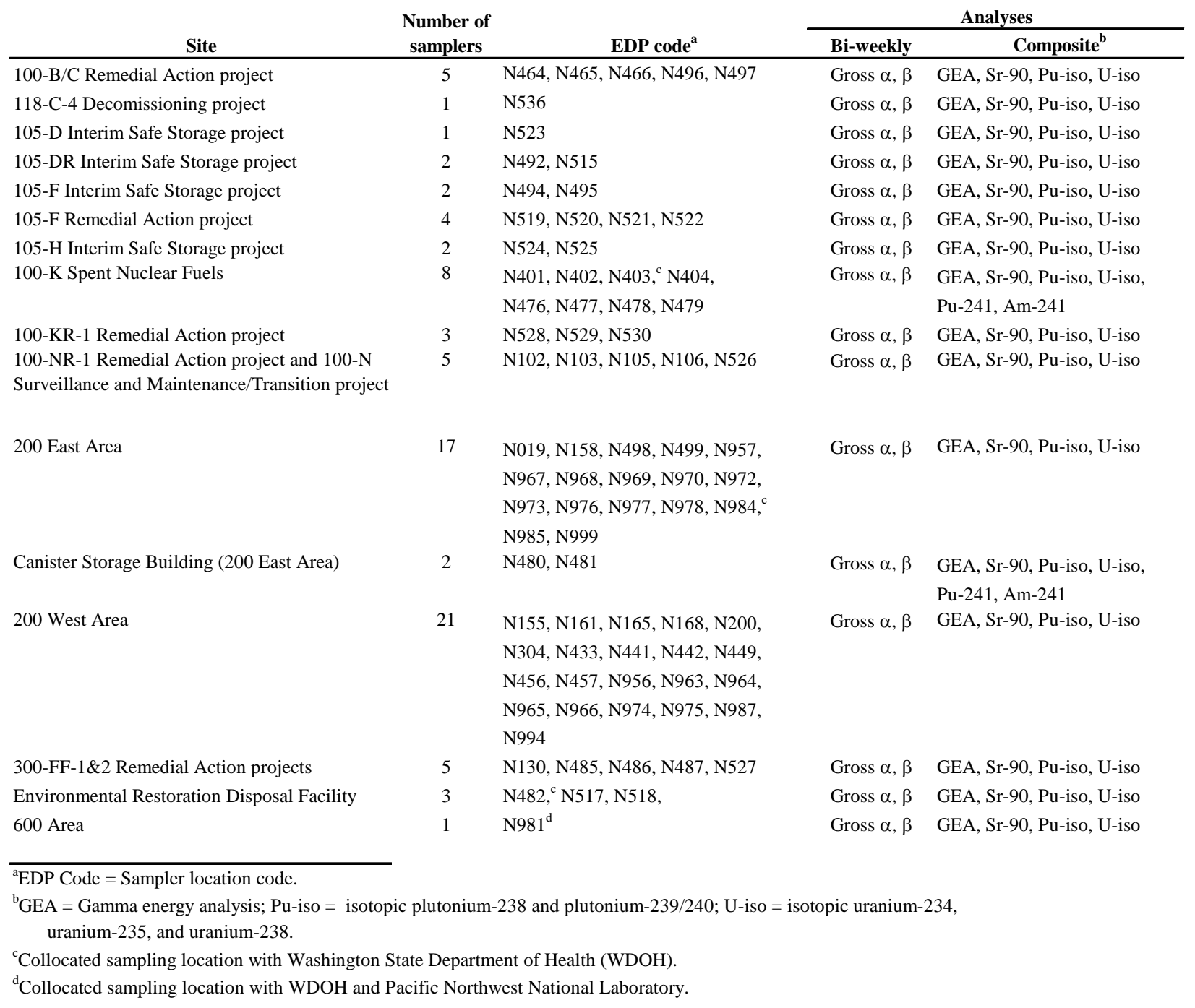




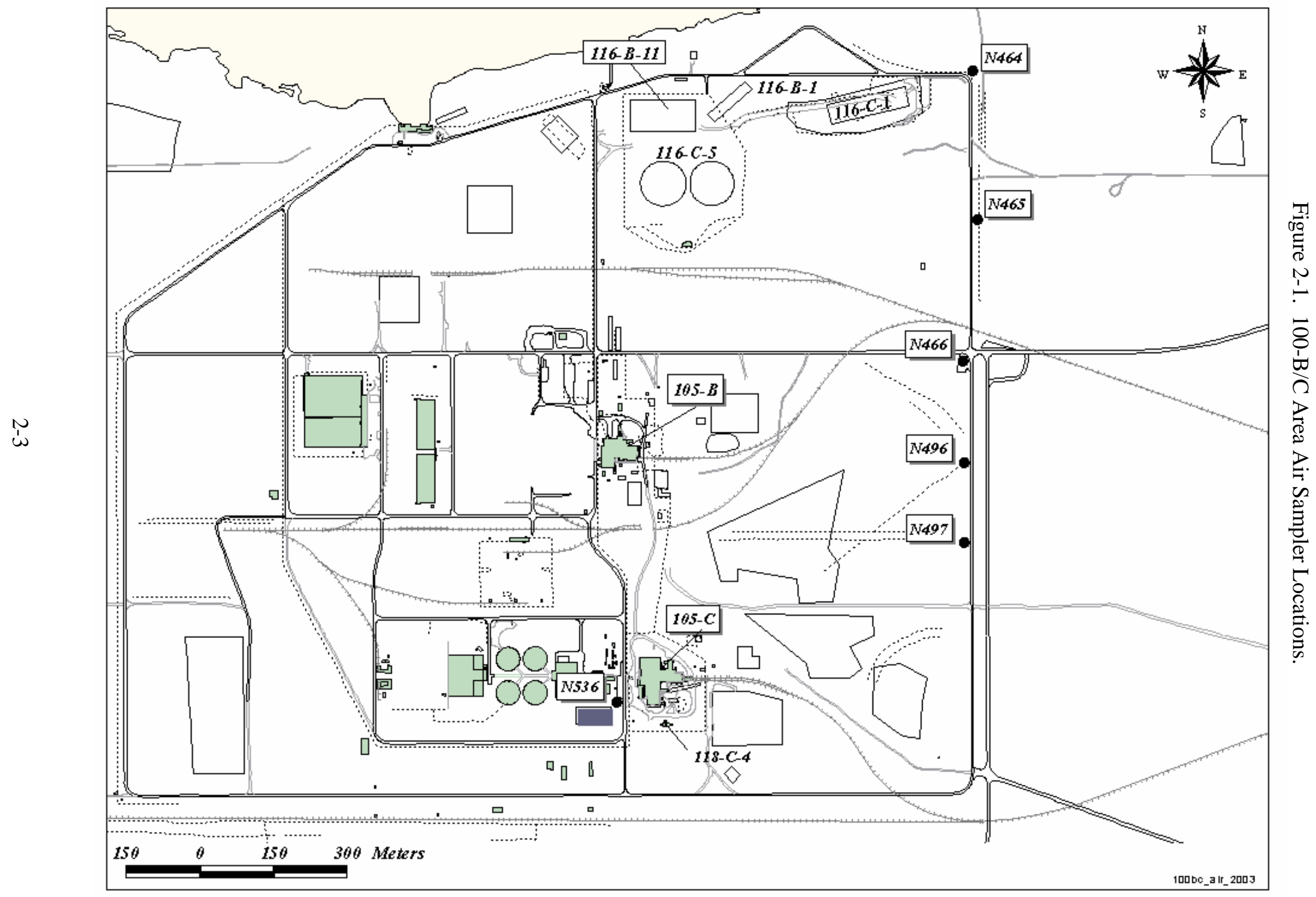




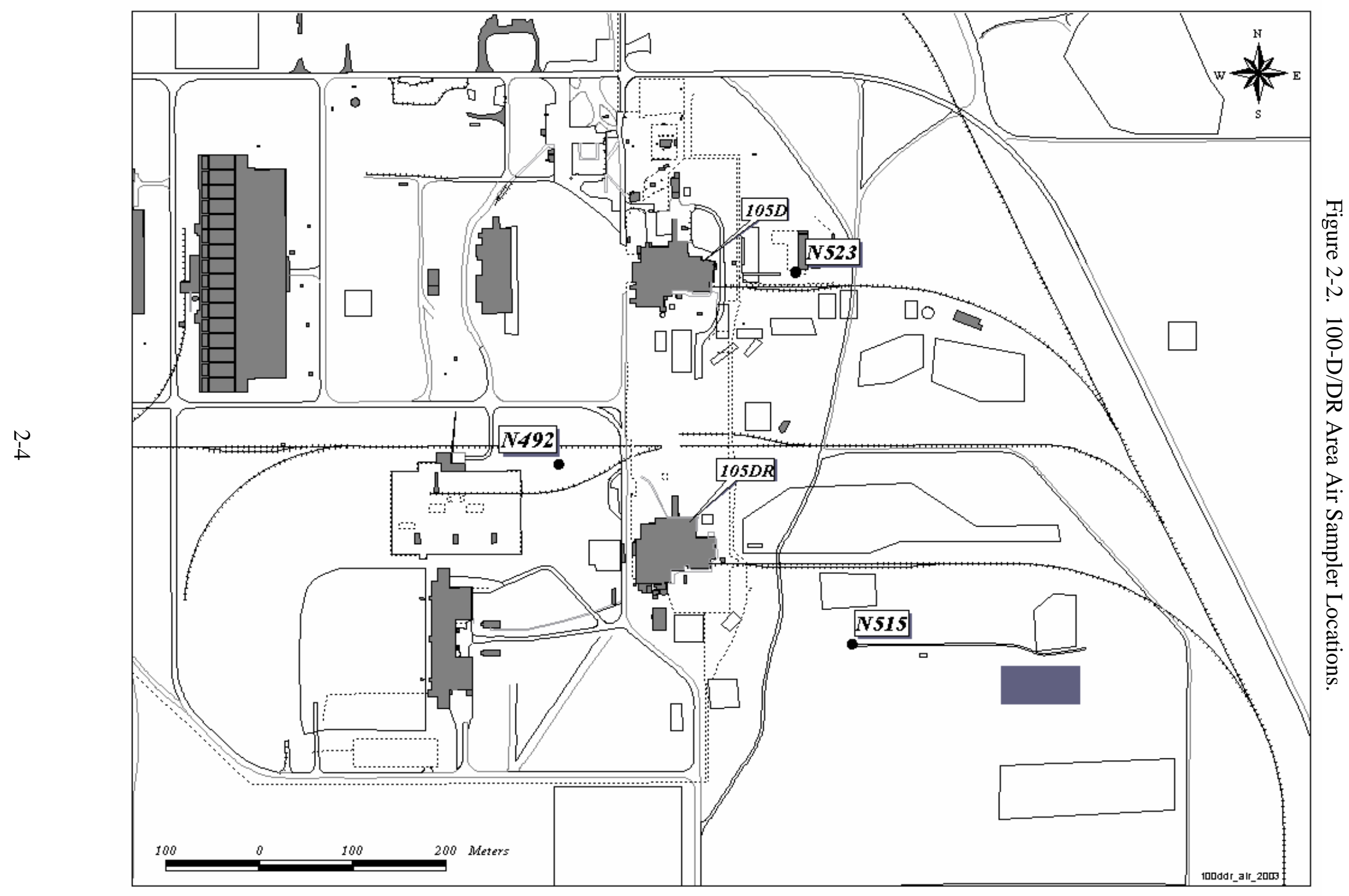




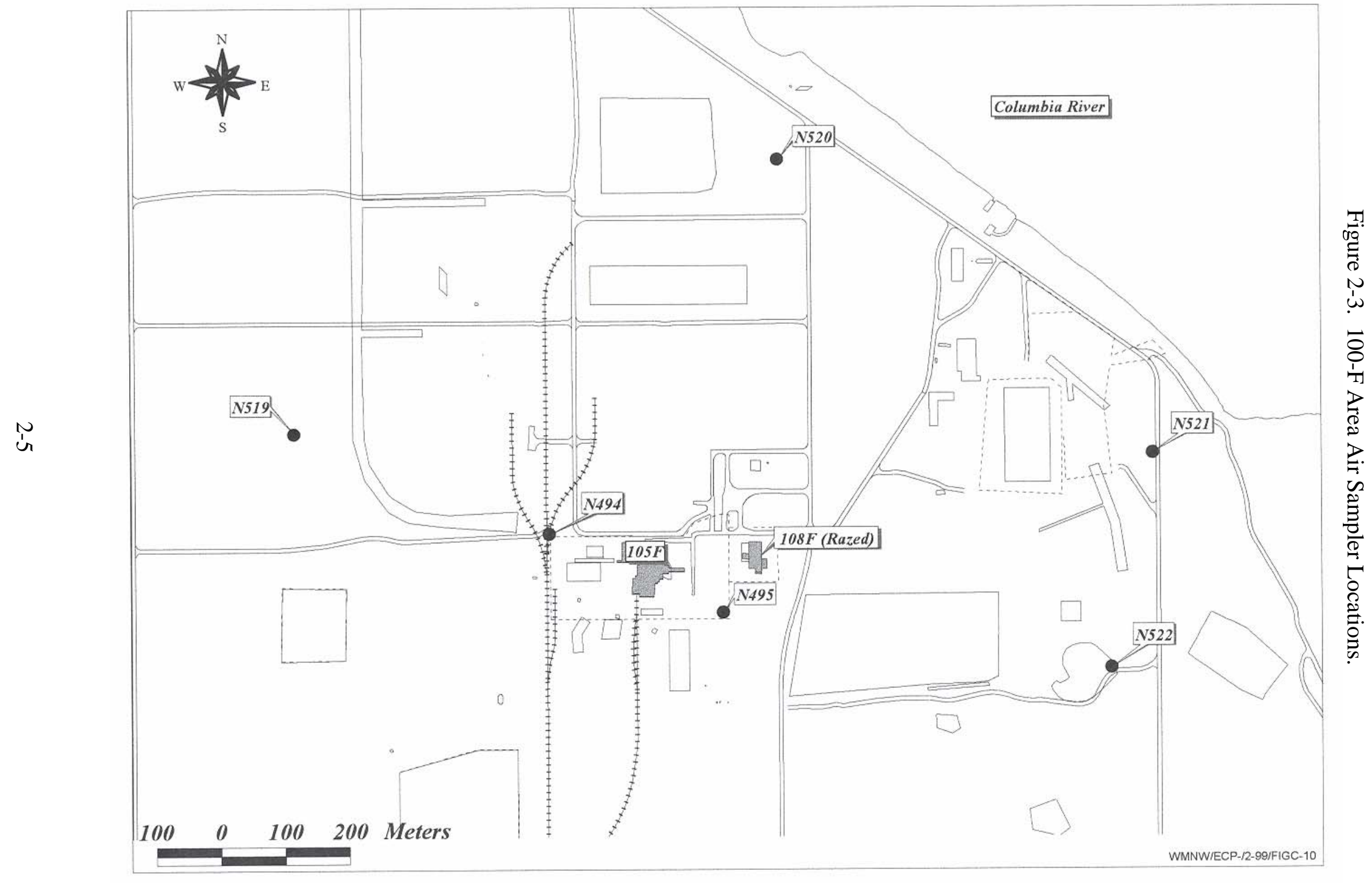




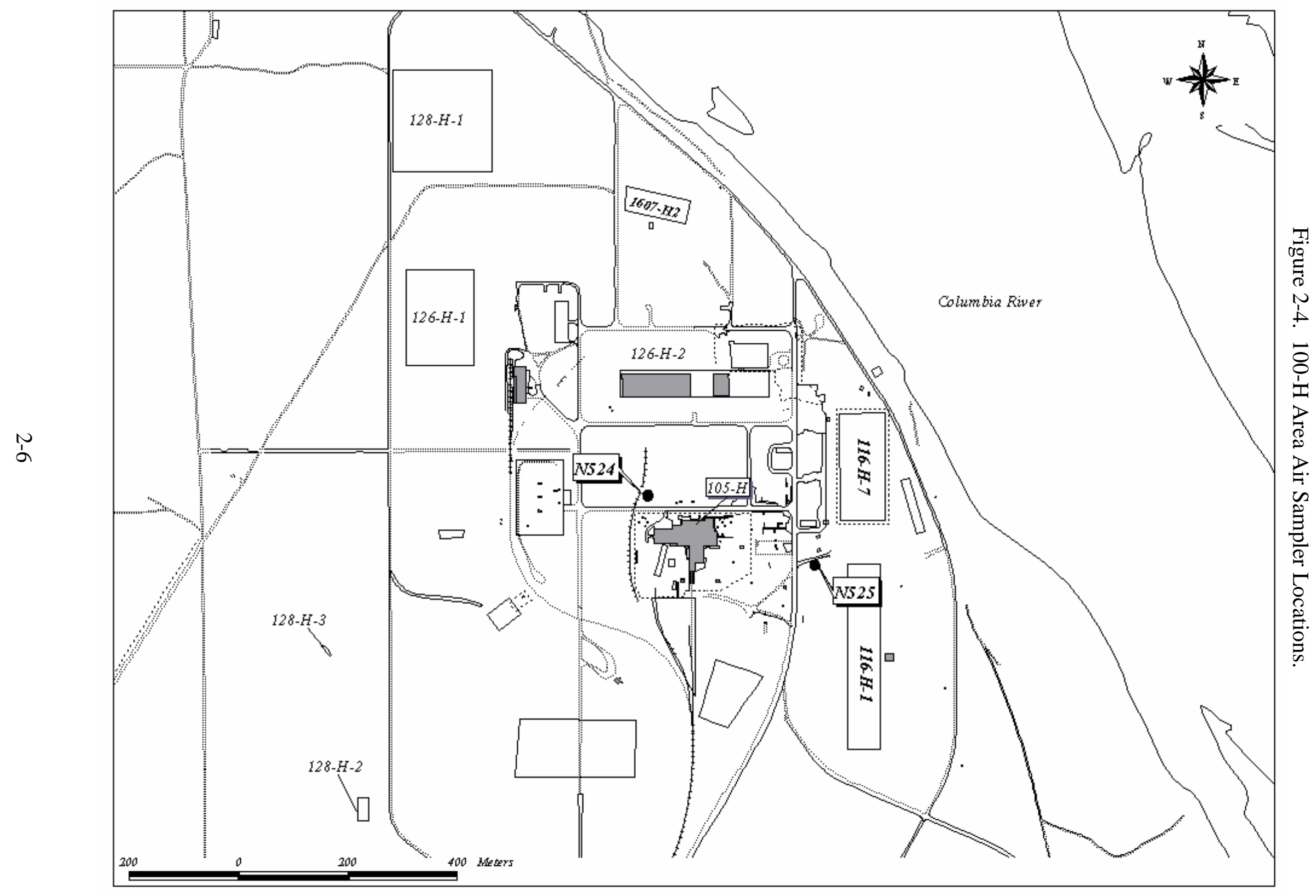




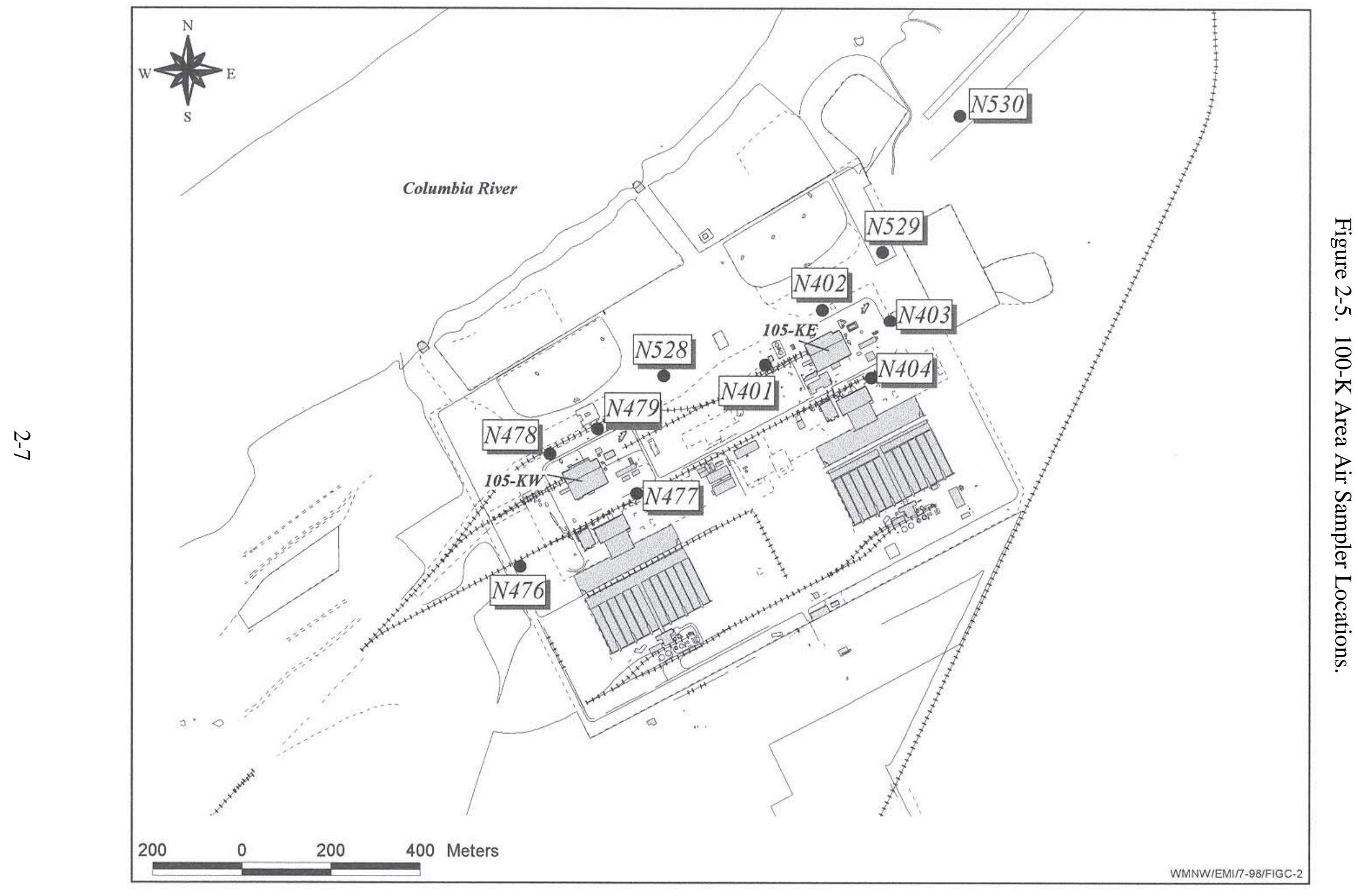




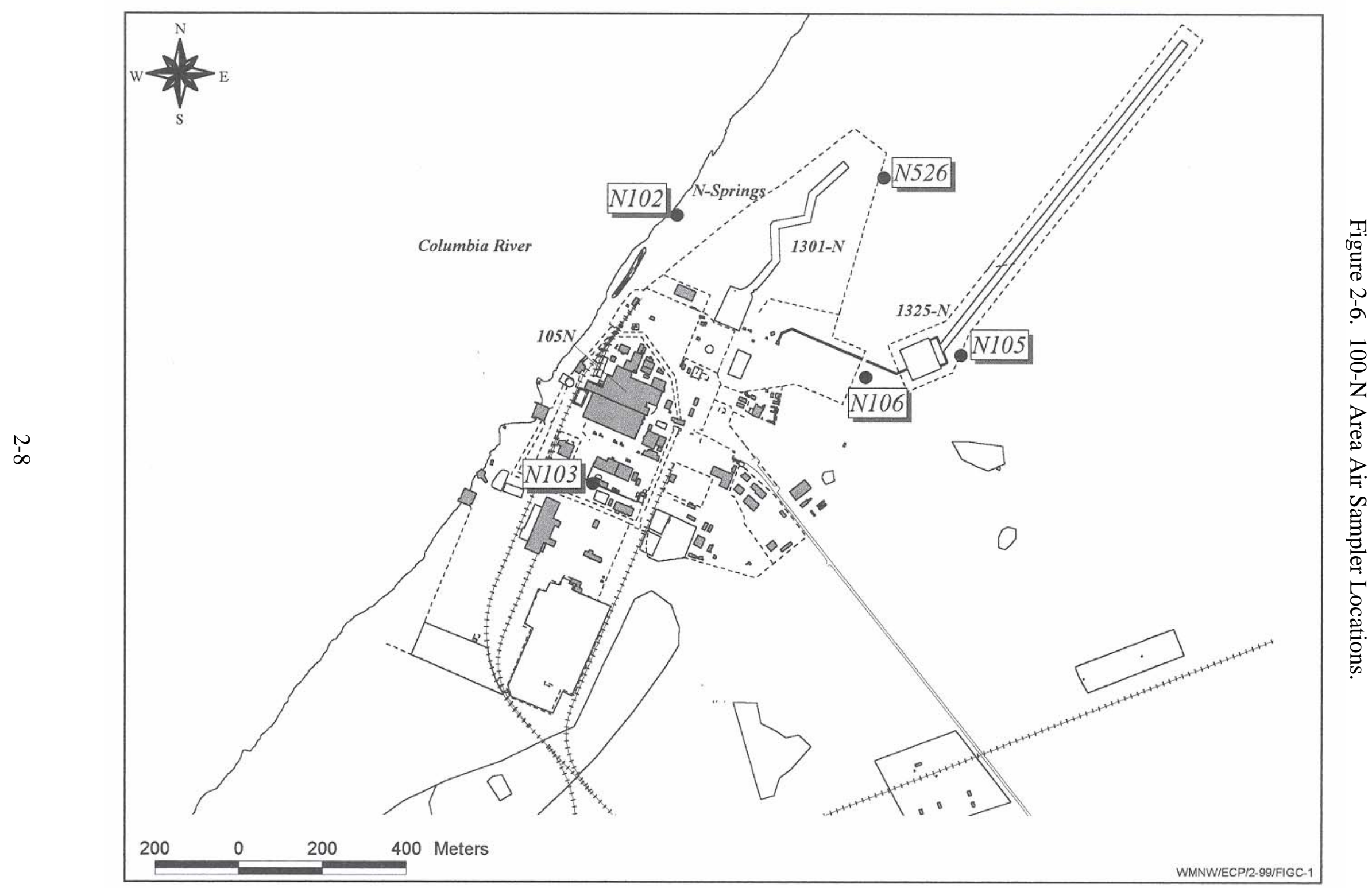




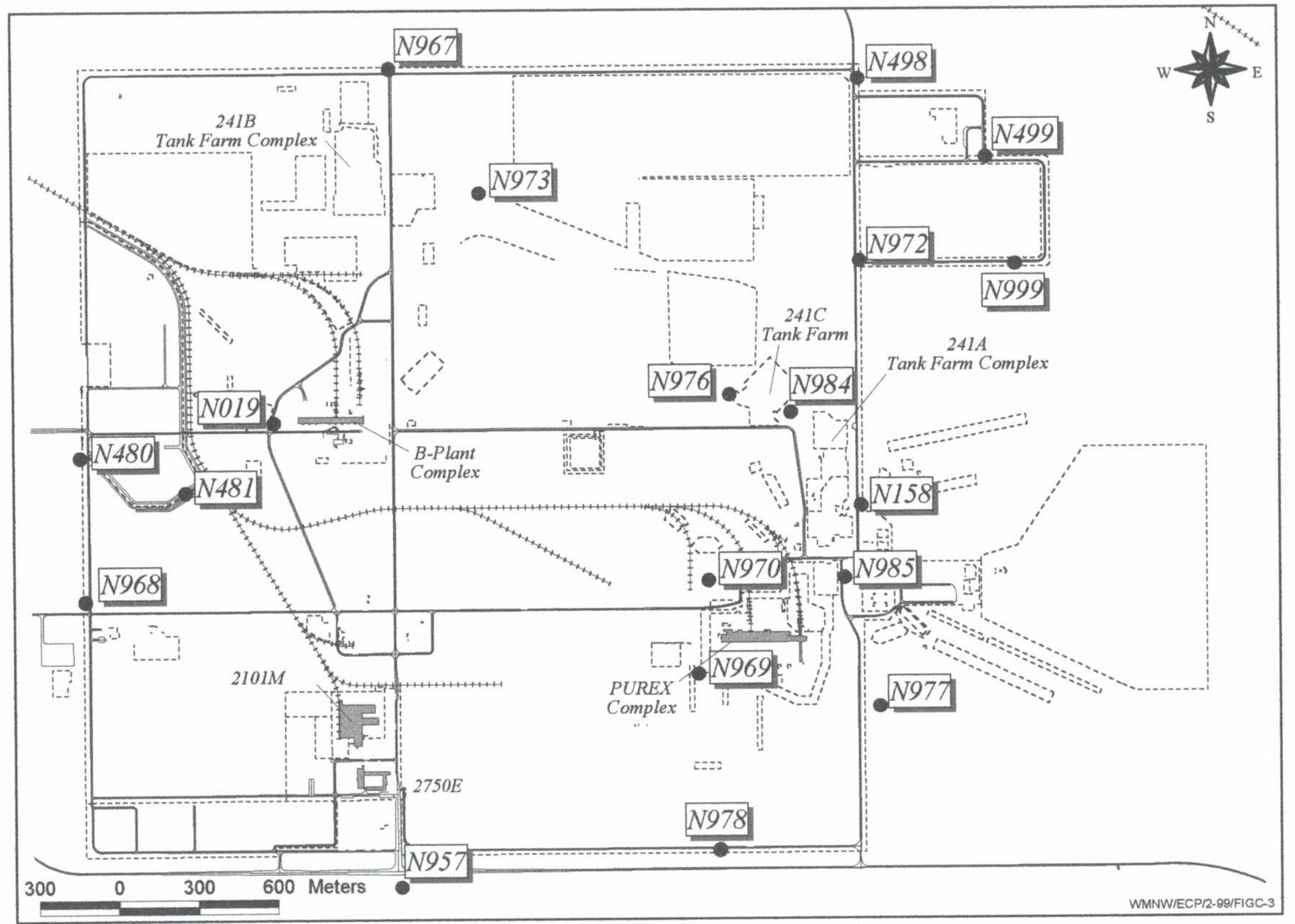

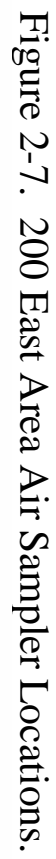




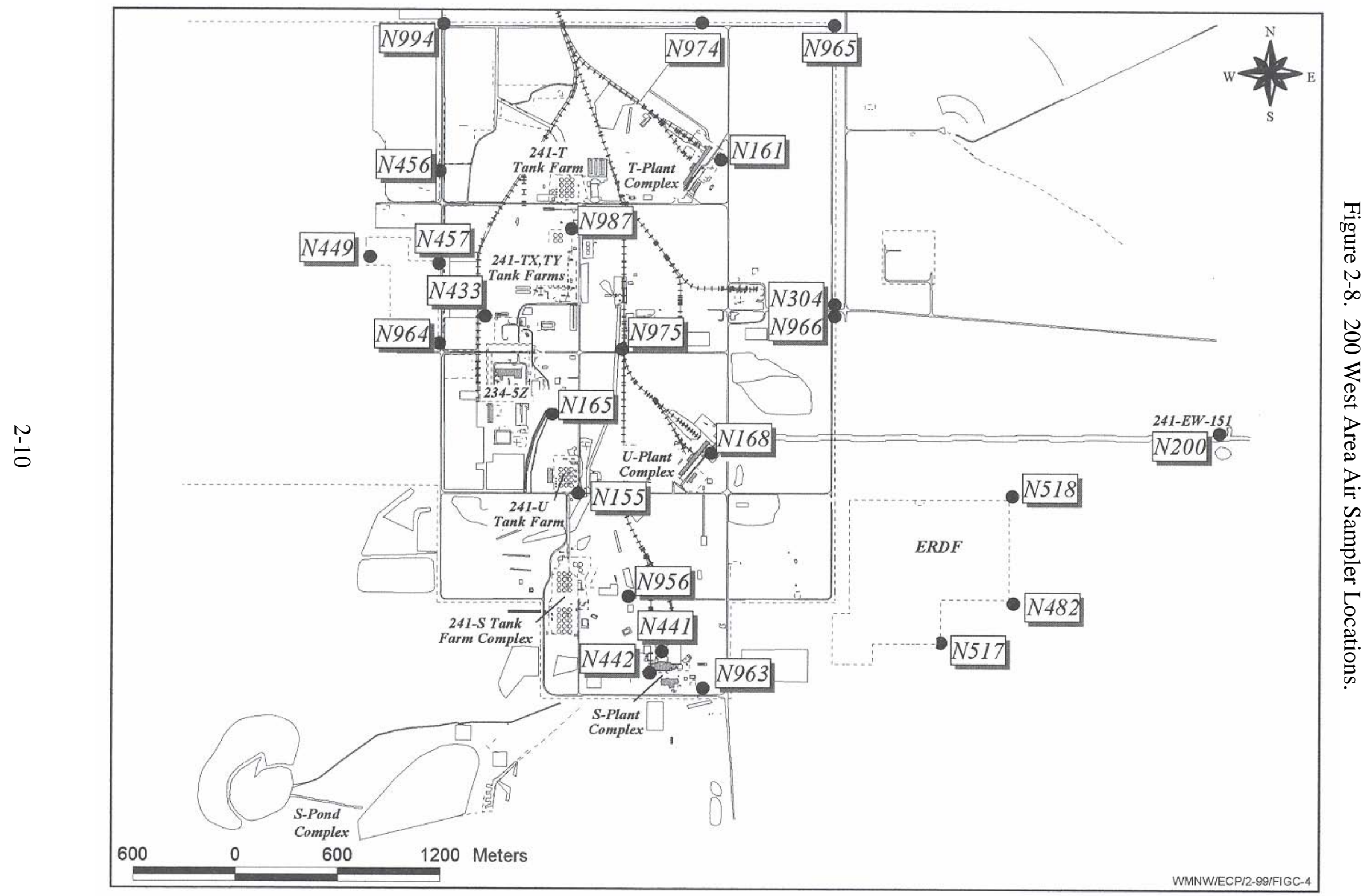


Figure 2-9. 300 Area Air Sampler Locations.

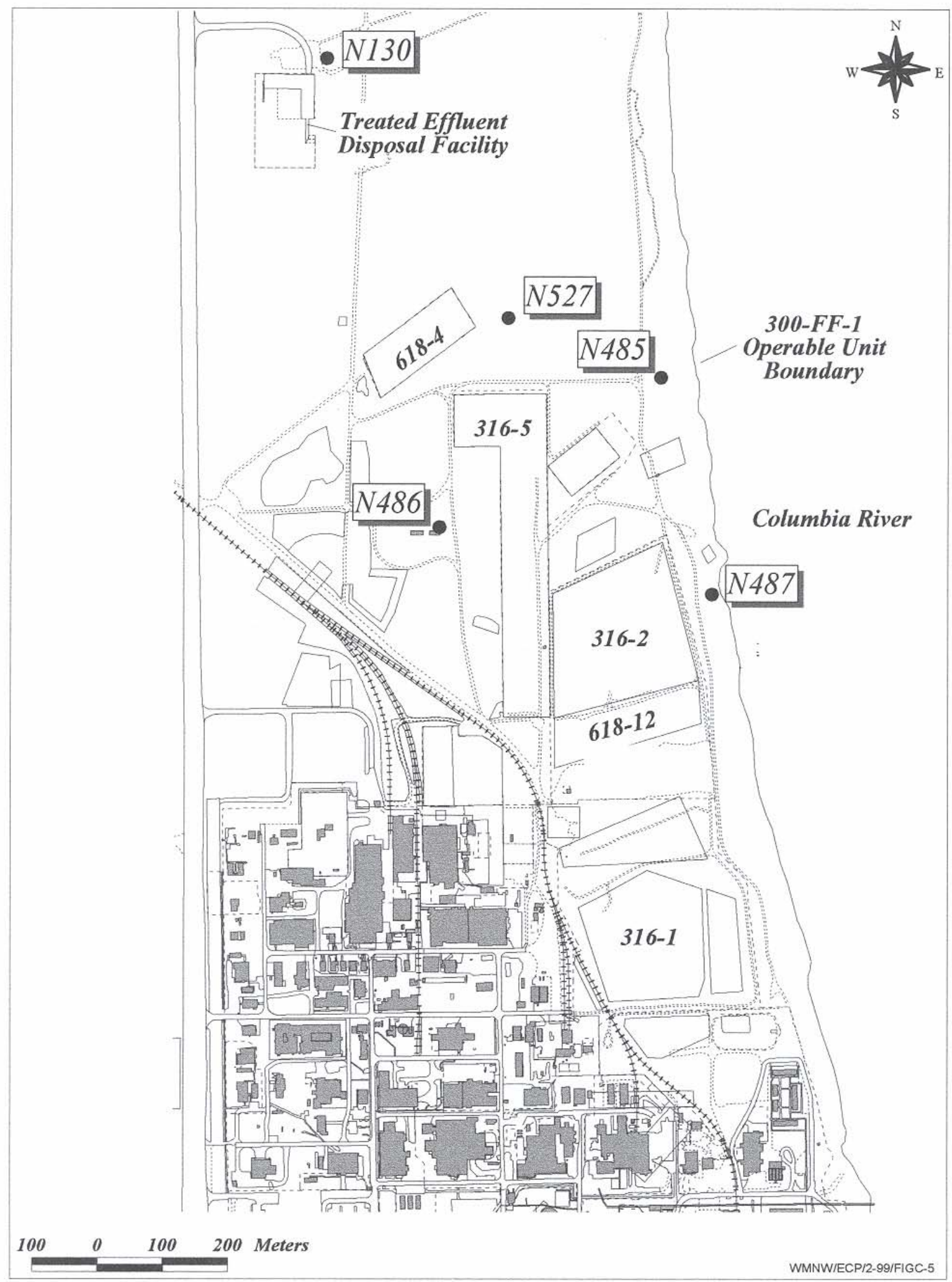




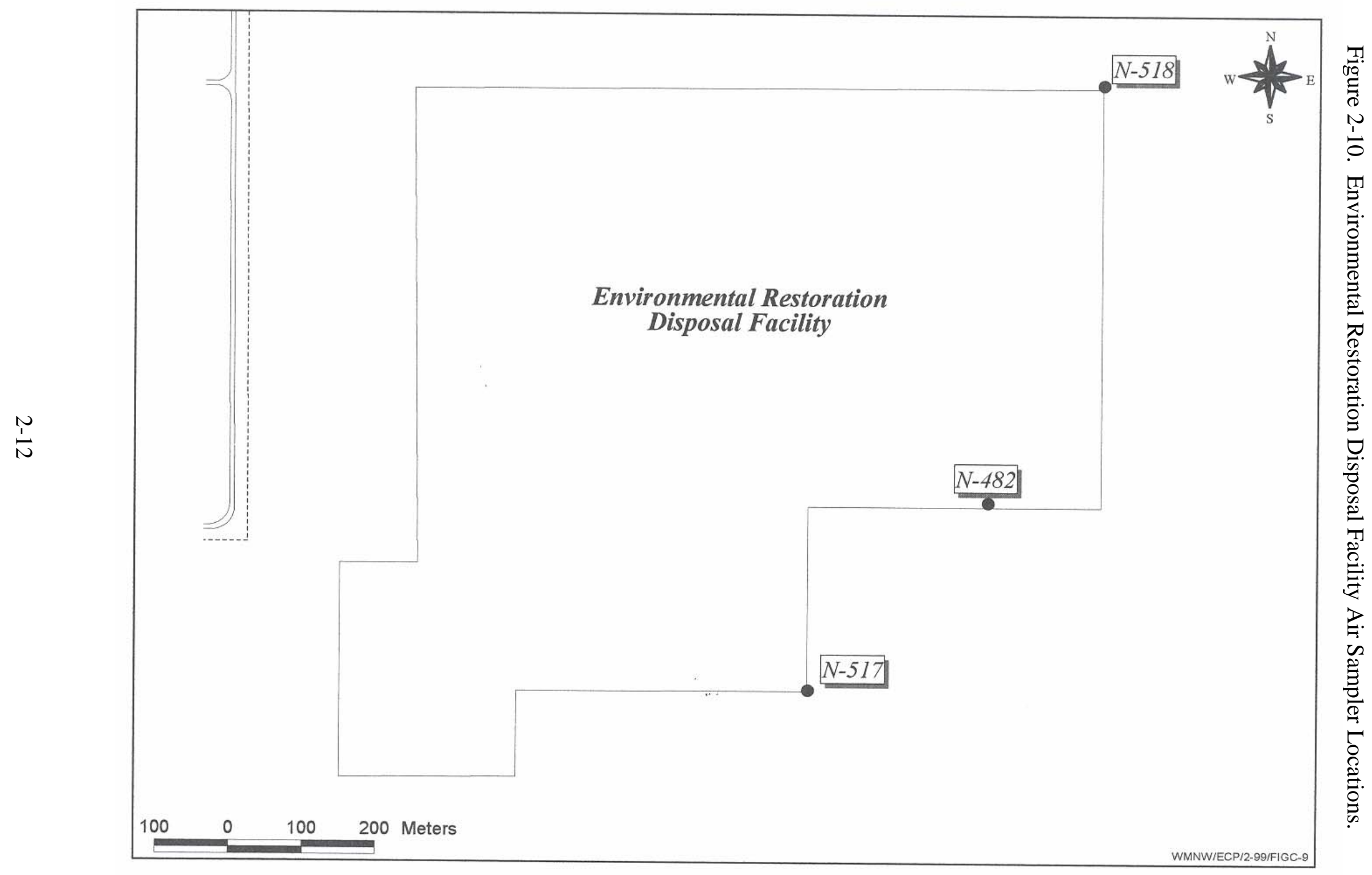


Figure 2-11. 600 Area Air Sampler Location.

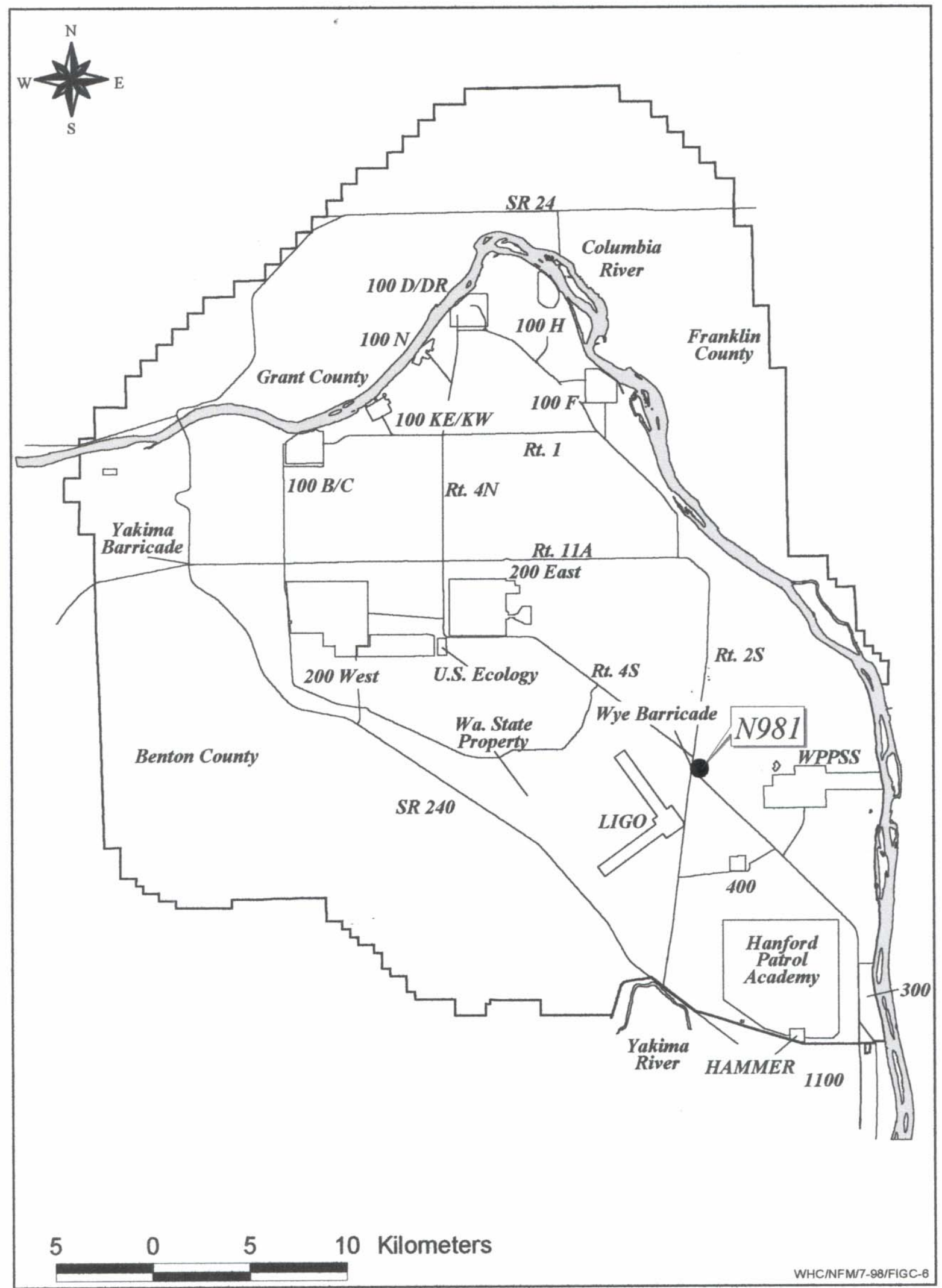


Figure 2-12. Annual Average Strontium-90 Concentrations in Air, 100-K Area.

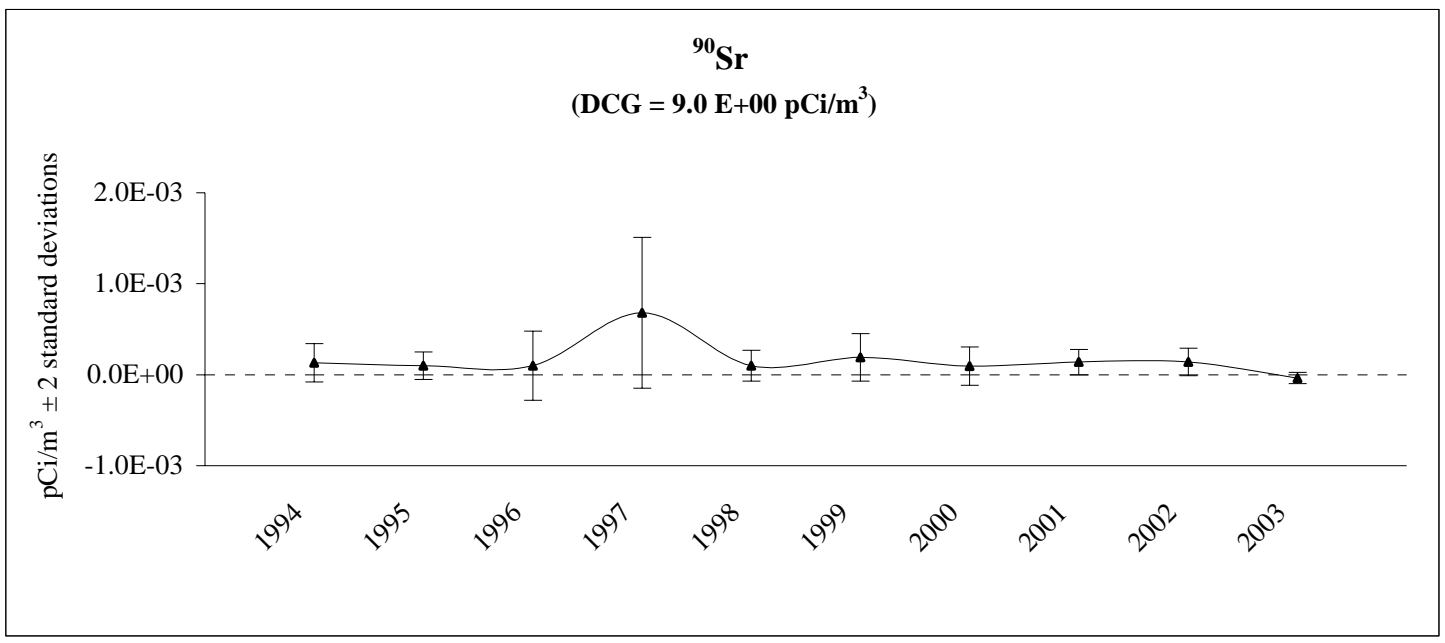

Figure 2-13. Annual Average Plutonium-239/240 Concentrations in Air, 100-K Area.

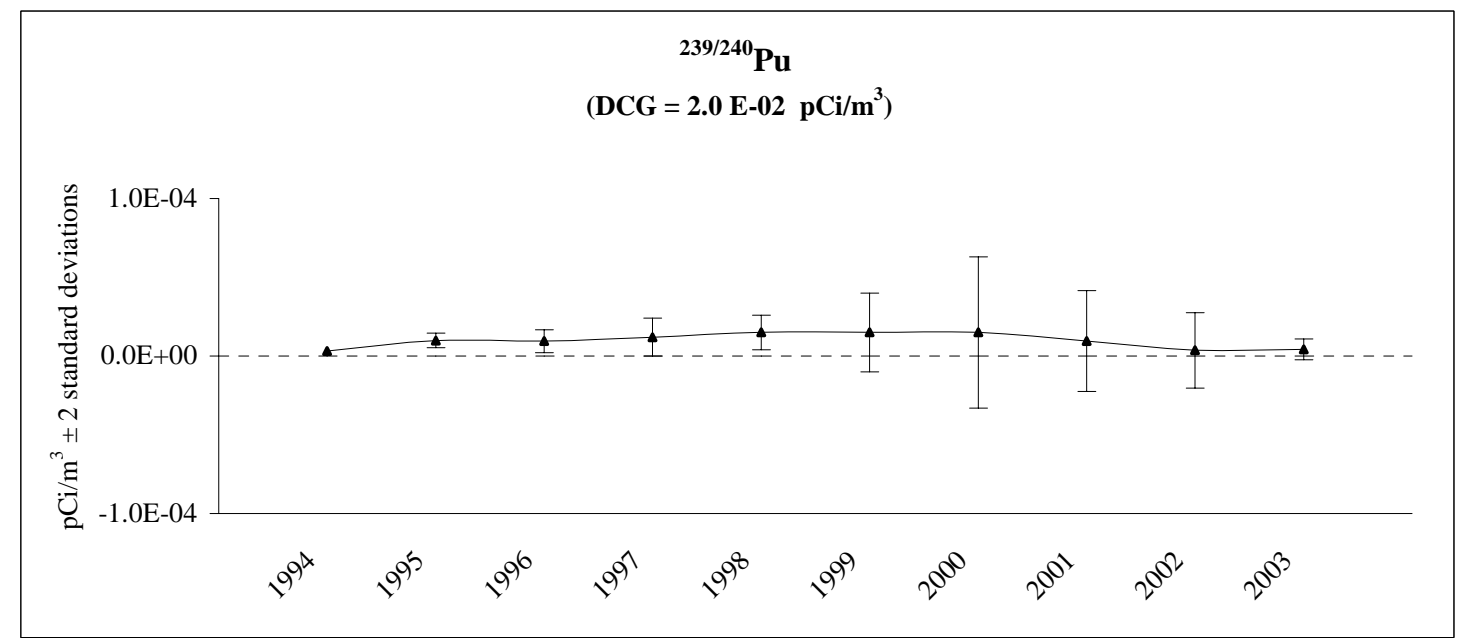

Figure 2-14. Annual Average Americium-241 Concentrations in Air, 100-K Area.

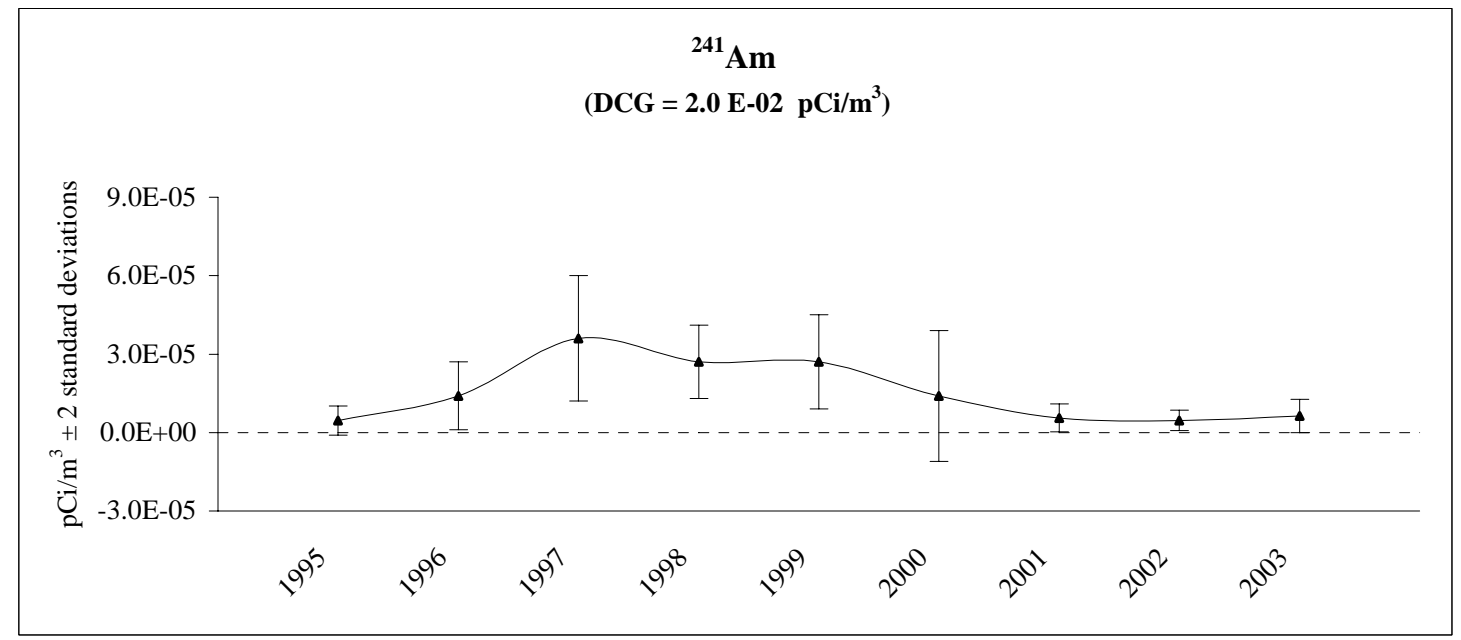


Figure 2-15. Annual Average Cobalt-60 Concentrations in Air, 100-N.

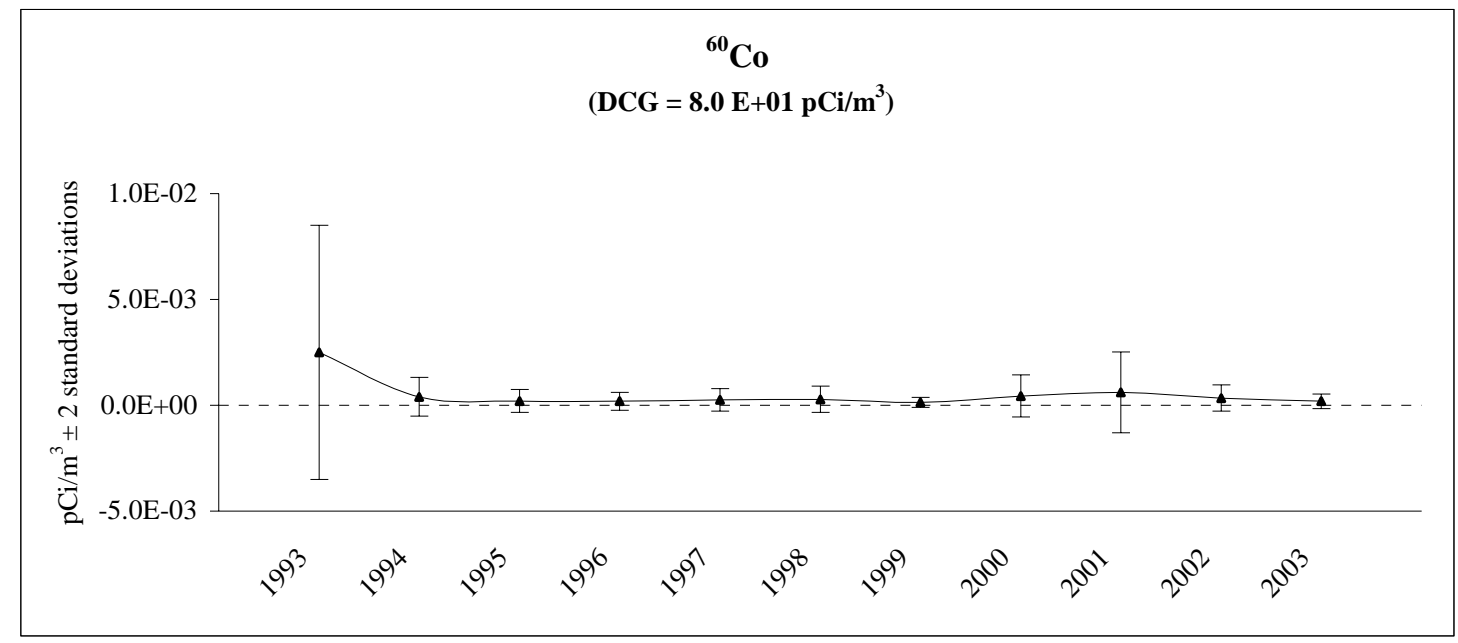

Figure 2-16. Annual Average Strontium-90 Concentrations in Air, 100-N.

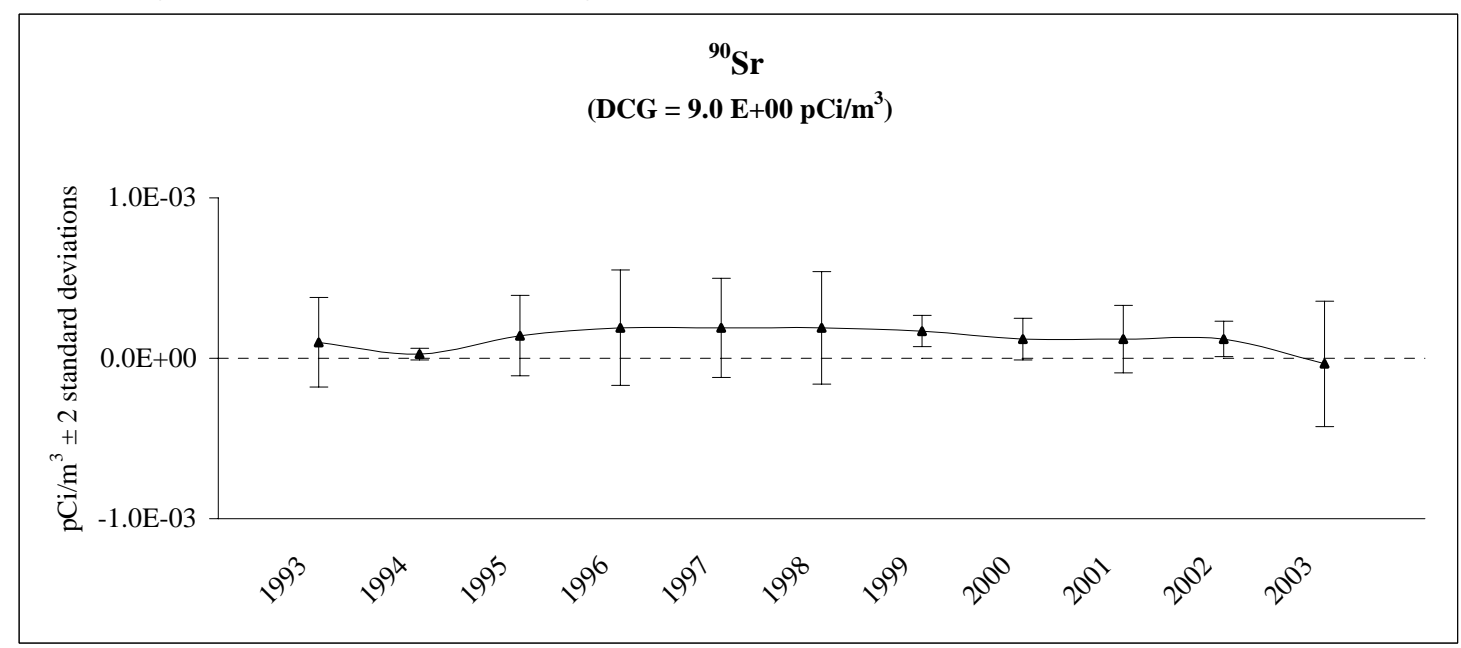

Figure 2-17. Annual Average Cesium-137 Concentrations in Air, 100-N.

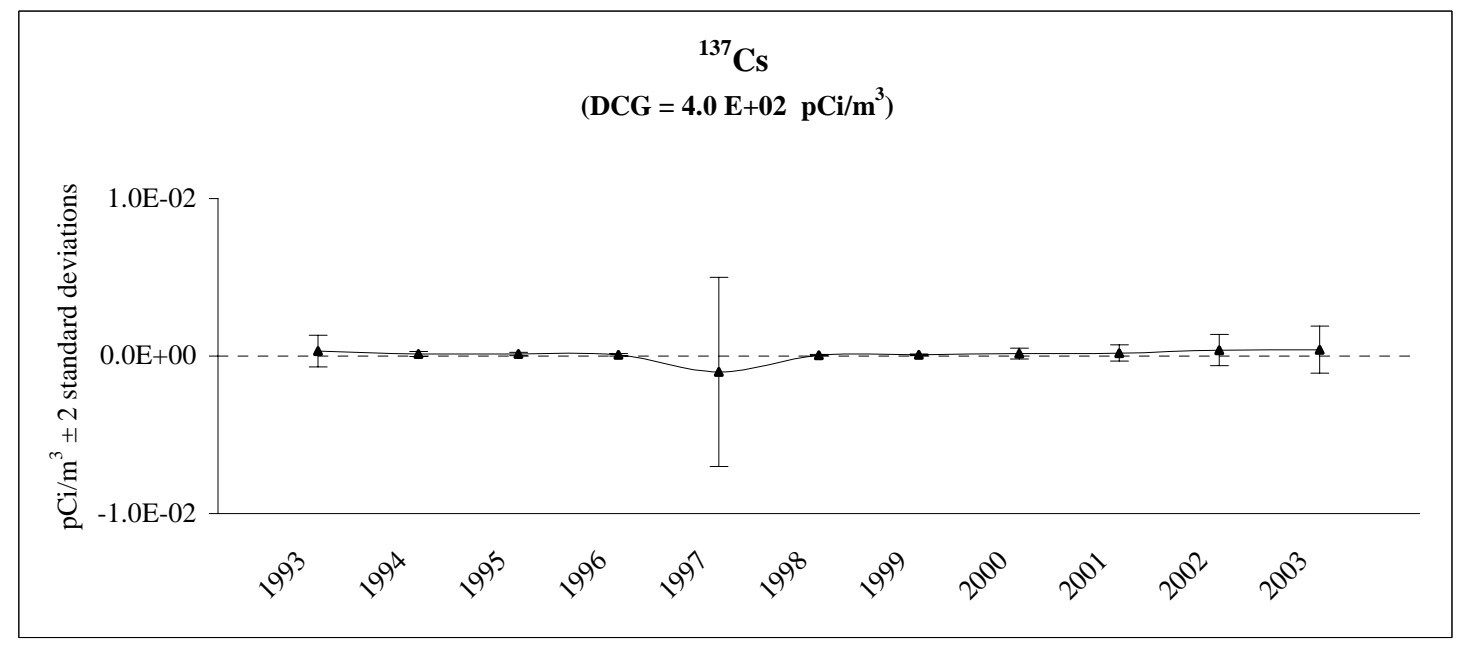


Figure 2-18. Annual Average Plutonium-239/240 Concentrations in Air, 100-N Area.

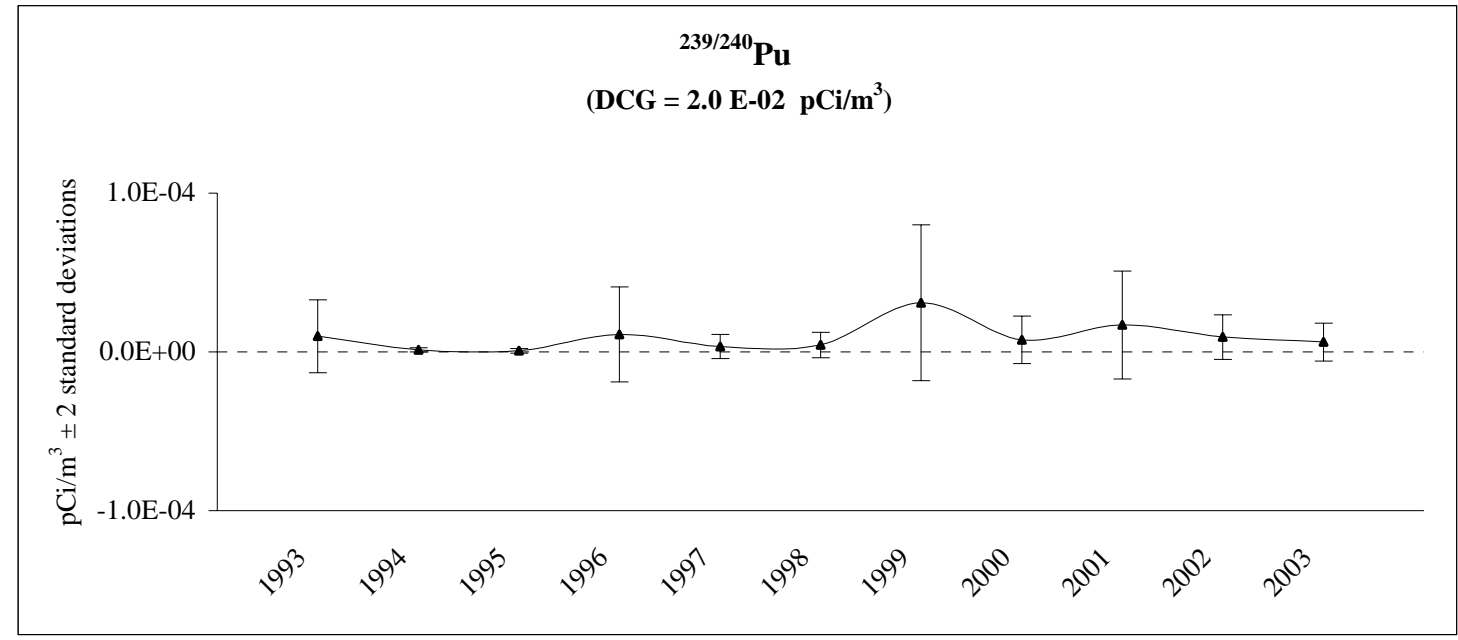

Figure 2-19. Annual Average Strontium-90 Concentrations in Air, 200 Areas.

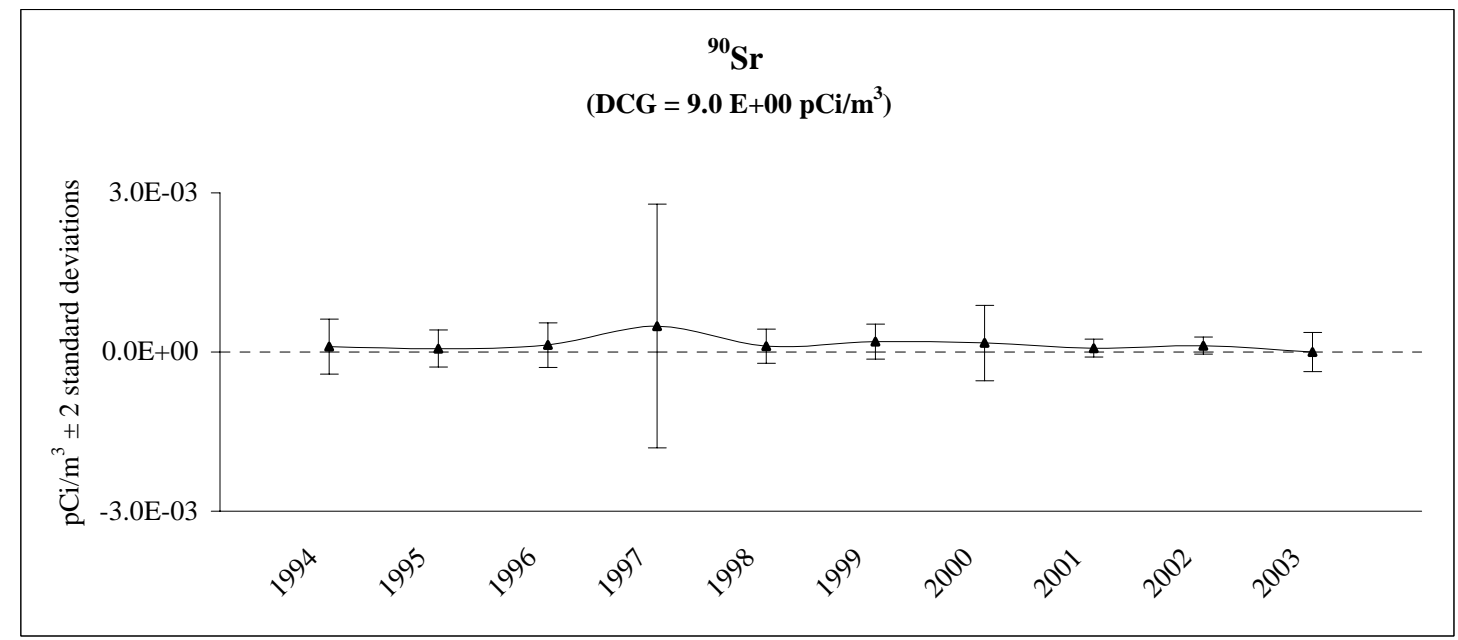

Figure 2-20. Annual Average Cesium-137 Concentrations in Air, 200 Areas.

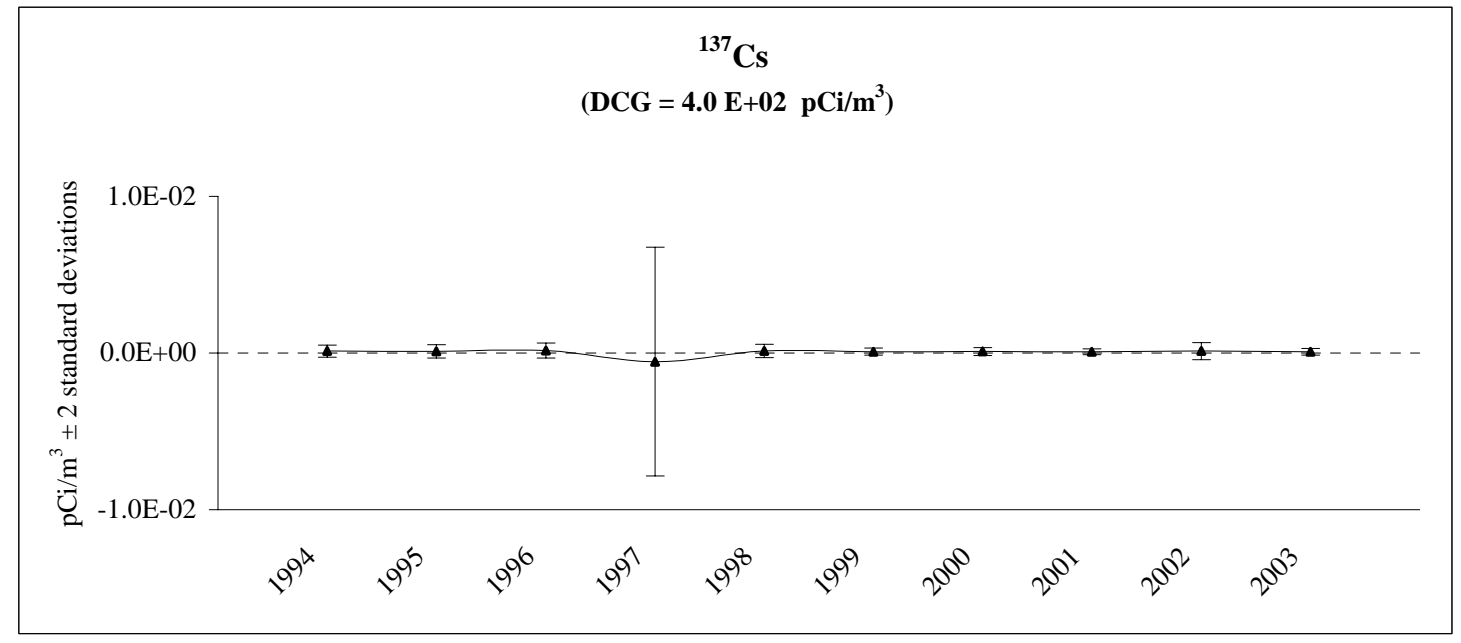


Figure 2-21. Annual Average Plutonium-239/240 Concentrations in Air, 200 Areas.

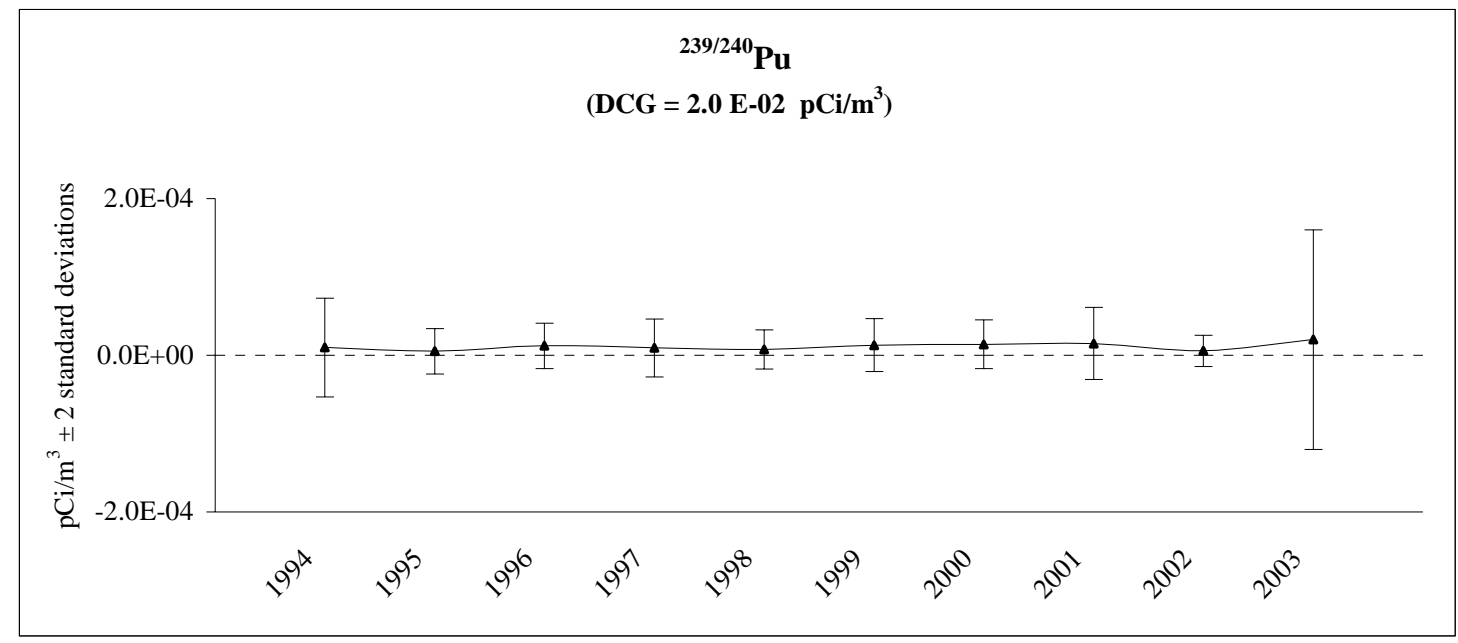

Figure 2-22. Annual Average Uranium-234 Concentrations in Air, 300 Area.

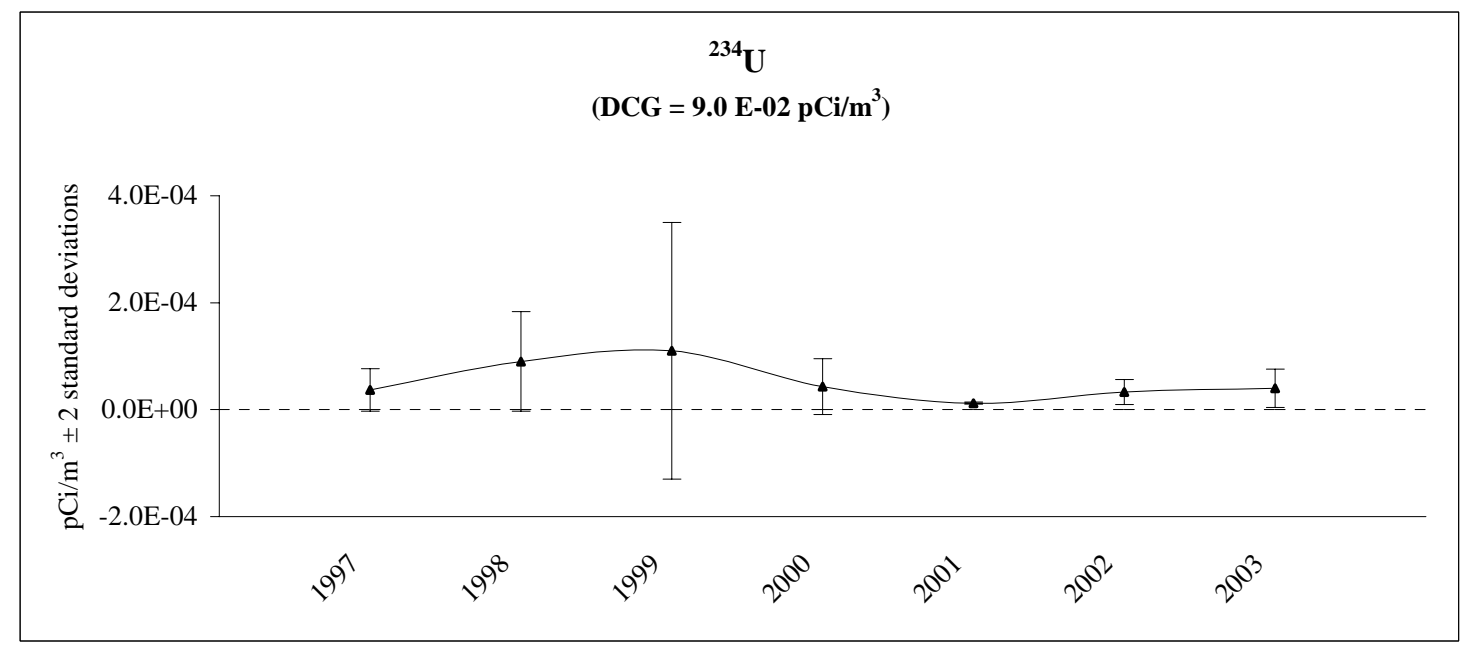

Figure 2-23. Annual Average Uranium-238 Concentrations in Air, 300 Area.

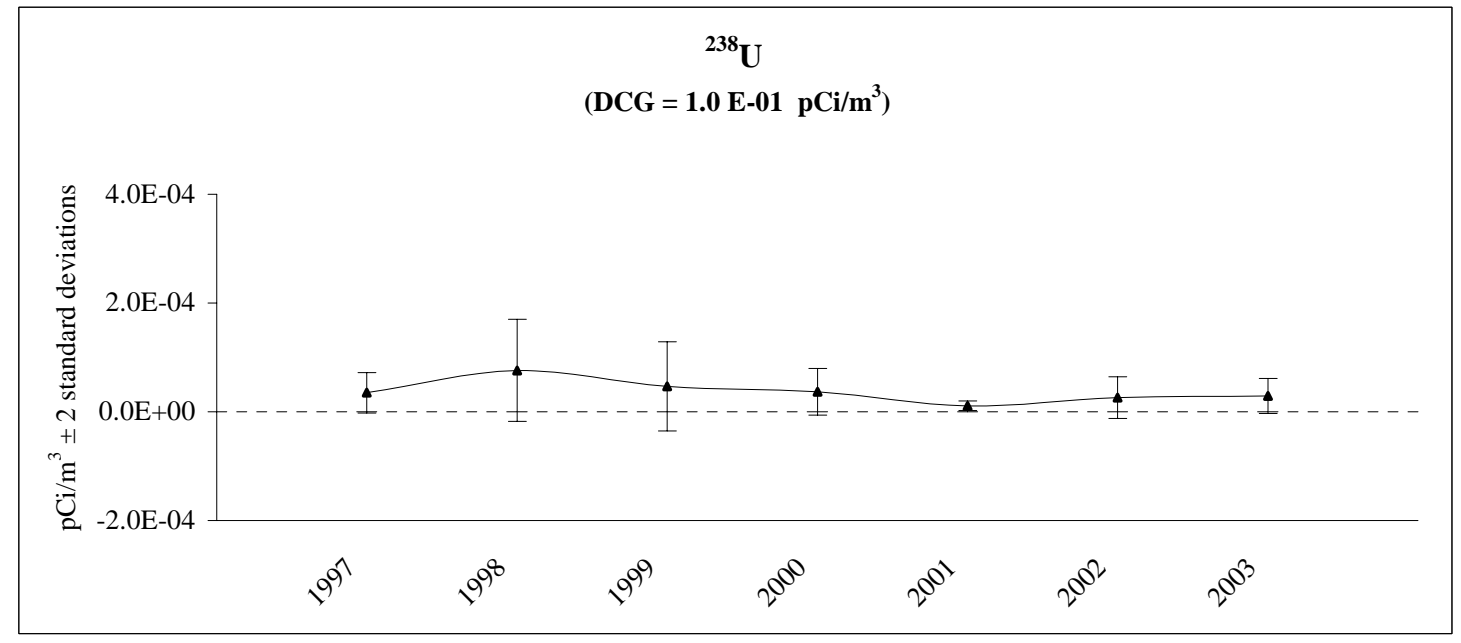


Table 2-2. Summary of Ambient Air Sampling Results $\left(\mathrm{pCi} / \mathrm{m}^{3}\right)$ for Selected Radionuclides, 2003.

\begin{tabular}{|c|c|c|c|c|c|c|}
\hline \multirow[b]{2}{*}{ Isotope } & \multicolumn{2}{|c|}{ Number of } & \multirow[b]{2}{*}{ Mean $^{a}$} & \multirow[b]{2}{*}{ Maximum $^{\mathbf{b}}$} & \multirow[b]{2}{*}{ Location } & \multirow[b]{2}{*}{ Sampler } \\
\hline & Detects & Samples & & & & \\
\hline${ }^{60} \mathrm{Co}$ & 2 & 158 & $8.1 \mathrm{E}-06 \pm 2.7 \mathrm{E}-04$ & $4.2 \mathrm{E}-04 \pm 1.8 \mathrm{E}-04$ & $100-\mathrm{N}$ & N526 \\
\hline${ }^{137} \mathrm{Cs}$ & 17 & 158 & $7.8 \mathrm{E}-05 \pm 5.3 \mathrm{E}-04$ & $2.5 \mathrm{E}-03 \pm 7.9 \mathrm{E}-04$ & $100-\mathrm{N}$ & N526 \\
\hline${ }^{239 / 240} \mathrm{Pu}$ & 38 & 151 & $1.6 \mathrm{E}-05 \pm 1.1 \mathrm{E}-04$ & $5.0 \mathrm{E}-04 \pm 1.9 \mathrm{E}-04$ & 200 West & N165 \\
\hline${ }^{90} \mathrm{Sr}$ & 15 & 151 & $-2.4 \mathrm{E}-05 \pm 4.6 \mathrm{E}-04$ & $1.0 \mathrm{E}-03 \pm 3.3 \mathrm{E}-04$ & 200 East & N984 \\
\hline${ }^{234} \mathrm{U}$ & 136 & 158 & $1.6 \mathrm{E}-05 \pm 2.1 \mathrm{E}-05$ & $6.9 \mathrm{E}-05 \pm 2.8 \mathrm{E}-05$ & 300-FF-1 & N487 \\
\hline${ }^{235} \mathrm{U}$ & 34 & 158 & $3.2 \mathrm{E}-06 \pm 7.0 \mathrm{E}-06$ & $2.2 \mathrm{E}-05 \pm 2.2 \mathrm{E}-05$ & 105-D & N523 \\
\hline${ }^{238} \mathrm{U}$ & 136 & 158 & $1.3 \mathrm{E}-05 \pm 1.9 \mathrm{E}-05$ & $6.4 \mathrm{E}-05 \pm 9.6 \mathrm{E}-05$ & $105-\mathrm{D}$ & N523 \\
\hline
\end{tabular}


Table 2-3. Near-Facility Air Sampling Results, 2003 (pCi/m³ \pm total analytical uncertainty).

\begin{tabular}{|c|c|c|c|c|c|c|c|}
\hline Location & Isotope & Result \pm Uncertainty & $\mathbf{R Q}^{*}$ & Location & Isotope & Result \pm Uncertainty & RQ* \\
\hline N464 (100-B/C) & ${ }^{144} \mathrm{Ce}$ & $-1.8 \mathrm{E}-04 \pm 7.8 \mathrm{E}-04$ & $\bar{U}$ & N464 (100-B/C) & ${ }^{144} \mathrm{Ce}$ & $5.2 \mathrm{E}-04 \pm 6.7 \mathrm{E}-04$ & $\mathrm{U}$ \\
\hline Composite Period & ${ }^{60} \mathrm{Co}$ & $3.0 \mathrm{E}-05 \pm 7.3 \mathrm{E}-05$ & $\mathrm{U}$ & Composite Period & ${ }^{60} \mathrm{Co}$ & $8.1 \mathrm{E}-05 \pm 9.0 \mathrm{E}-05$ & $\mathrm{U}$ \\
\hline \multirow[t]{16}{*}{$01 / 06 / 03$ to $06 / 23 / 03$} & ${ }^{134} \mathrm{Cs}$ & $2.9 \mathrm{E}-05 \pm 8.9 \mathrm{E}-05$ & $\mathrm{U}$ & $06 / 23 / 03$ to $12 / 15 / 03$ & ${ }^{134} \mathrm{Cs}$ & $-5.0 \mathrm{E}-05 \pm 7.1 \mathrm{E}-05$ & $\mathrm{U}$ \\
\hline & ${ }^{137} \mathrm{Cs}$ & $7.4 \mathrm{E}-05 \pm 7.8 \mathrm{E}-05$ & U & & ${ }^{137} \mathrm{Cs}$ & $-2.4 \mathrm{E}-05 \pm 6.9 \mathrm{E}-05$ & $\mathrm{U}$ \\
\hline & ${ }^{152} \mathrm{Eu}$ & $-1.9 \mathrm{E}-05 \pm 1.9 \mathrm{E}-04$ & $\mathrm{U}$ & & ${ }^{152} \mathrm{Eu}$ & $-1.1 \mathrm{E}-04 \pm 1.7 \mathrm{E}-04$ & $\mathrm{U}$ \\
\hline & ${ }^{154} \mathrm{Eu}$ & $8.7 \mathrm{E}-05 \pm 2.1 \mathrm{E}-04$ & U & & ${ }^{154} \mathrm{Eu}$ & $2.4 \mathrm{E}-05 \pm 2.4 \mathrm{E}-04$ & $\mathrm{U}$ \\
\hline & ${ }^{155} \mathrm{Eu}$ & $-6.9 \mathrm{E}-05 \pm 2.2 \mathrm{E}-04$ & $\mathrm{U}$ & & ${ }^{155} \mathrm{Eu}$ & $-2.0 \mathrm{E}-05 \pm 1.7 \mathrm{E}-04$ & $\mathrm{U}$ \\
\hline & ${ }^{238} \mathrm{Pu}$ & $1.9 \mathrm{E}-05 \pm 1.8 \mathrm{E}-05$ & $\mathrm{U}$ & & ${ }^{238} \mathrm{Pu}$ & $-2.0 \mathrm{E}-06 \pm 1.6 \mathrm{E}-05$ & $\mathrm{U}$ \\
\hline & ${ }^{239 / 240} \mathrm{Pu}$ & $9.3 \mathrm{E}-07 \pm 9.6 \mathrm{E}-07$ & $\mathrm{U}$ & & ${ }^{239 / 240} \mathrm{Pu}$ & $2.0 \mathrm{E}-06 \pm 4.1 \mathrm{E}-06$ & $\mathrm{U}$ \\
\hline & ${ }^{103} \mathrm{Ru}$ & $8.4 \mathrm{E}-05 \pm 8.6 \mathrm{E}-05$ & U & & ${ }^{103} \mathrm{Ru}$ & $-3.1 \mathrm{E}-06 \pm 3.1 \mathrm{E}-05$ & $\mathrm{U}$ \\
\hline & ${ }^{106} \mathrm{Ru}$ & $-4.4 \mathrm{E}-04 \pm 6.6 \mathrm{E}-04$ & $\mathrm{U}$ & & ${ }^{106} \mathrm{Ru}$ & $6.2 \mathrm{E}-05 \pm 6.0 \mathrm{E}-04$ & $\mathrm{U}$ \\
\hline & ${ }^{125} \mathrm{Sb}$ & $1.2 \mathrm{E}-04 \pm 1.9 \mathrm{E}-04$ & $\mathrm{U}$ & & ${ }^{125} \mathrm{Sb}$ & $-3.8 \mathrm{E}-05 \pm 1.4 \mathrm{E}-04$ & $\mathrm{U}$ \\
\hline & ${ }^{113} \mathrm{Sn}$ & $-2.0 \mathrm{E}-05 \pm 9.0 \mathrm{E}-05$ & $\mathrm{U}$ & & ${ }^{113} \mathrm{Sn}$ & $1.8 \mathrm{E}-05 \pm 8.0 \mathrm{E}-05$ & $\mathrm{U}$ \\
\hline & ${ }^{90} \mathrm{Sr}$ & $-7.0 \mathrm{E}-05 \pm 8.9 \mathrm{E}-05$ & $\mathrm{U}$ & & ${ }^{90} \mathrm{Sr}$ & $7.7 \mathrm{E}-06 \pm 8.0 \mathrm{E}-06$ & $\mathrm{U}$ \\
\hline & ${ }^{234} \mathrm{U}$ & $3.5 \mathrm{E}-06 \pm 3.6 \mathrm{E}-06$ & & & & $1.4 \mathrm{E}-05 \pm 8.5 \mathrm{E}-06$ & \\
\hline & ${ }^{235} \mathrm{U}$ & $9.3 \mathrm{E}-07 \pm 1.9 \mathrm{E}-06$ & $\mathrm{U}$ & & ${ }^{235} \mathrm{U}$ & $3.5 \mathrm{E}-06 \pm 4.3 \mathrm{E}-06$ & $\mathrm{U}$ \\
\hline & ${ }^{238} \mathrm{U}$ & $5.2 \mathrm{E}-06 \pm 4.7 \mathrm{E}-06$ & & & ${ }^{238} \mathrm{U}$ & $9.3 \mathrm{E}-06 \pm 6.7 \mathrm{E}-06$ & \\
\hline & ${ }^{65} \mathrm{Zn}$ & $-1.5 \mathrm{E}-04 \pm 1.7 \mathrm{E}-04$ & $\mathrm{U}$ & & ${ }^{65} \mathrm{Zn}$ & $1.2 \mathrm{E}-04 \pm 2.1 \mathrm{E}-04$ & $\mathrm{U}$ \\
\hline N465 (100-B/C) & ${ }^{144} \mathrm{Ce}$ & $-4.6 \mathrm{E}-05 \pm 4.6 \mathrm{E}-04$ & $\bar{U}$ & N465 (100-B/C) & ${ }^{144} \mathrm{Ce}$ & $-3.3 \mathrm{E}-04 \pm 8.3 \mathrm{E}-04$ & $\bar{U}$ \\
\hline Composite Period & ${ }^{60} \mathrm{Co}$ & $4.4 \mathrm{E}-06 \pm 4.4 \mathrm{E}-05$ & $\mathrm{U}$ & Composite Period & ${ }^{60} \mathrm{Co}$ & $-8.8 \mathrm{E}-06 \pm 8.8 \mathrm{E}-05$ & $\mathrm{U}$ \\
\hline \multirow[t]{16}{*}{ 01/06/03 to $06 / 23 / 03$} & ${ }^{134} \mathrm{Cs}$ & $-4.7 \mathrm{E}-05 \pm 8.8 \mathrm{E}-05$ & $\mathrm{U}$ & $06 / 23 / 03$ to $12 / 15 / 03$ & ${ }^{134} \mathrm{Cs}$ & $-8.0 \mathrm{E}-05 \pm 9.8 \mathrm{E}-05$ & $\mathrm{U}$ \\
\hline & ${ }^{137} \mathrm{Cs}$ & $5.7 \mathrm{E}-05 \pm 9.0 \mathrm{E}-05$ & $\mathrm{U}$ & & ${ }^{137} \mathrm{Cs}$ & $-1.6 \mathrm{E}-05 \pm 7.9 \mathrm{E}-05$ & $\mathrm{U}$ \\
\hline & ${ }^{152} \mathrm{Eu}$ & $5.5 \mathrm{E}-05 \pm 1.9 \mathrm{E}-04$ & $\mathrm{U}$ & & ${ }^{152} \mathrm{Eu}$ & $6.5 \mathrm{E}-05 \pm 1.8 \mathrm{E}-04$ & $\mathrm{U}$ \\
\hline & ${ }^{154} \mathrm{Eu}$ & $6.3 \mathrm{E}-05 \pm 2.8 \mathrm{E}-04$ & $\mathrm{U}$ & & ${ }^{154} \mathrm{Eu}$ & $2.7 \mathrm{E}-04 \pm 3.2 \mathrm{E}-04$ & $\mathrm{U}$ \\
\hline & ${ }^{155} \mathrm{Eu}$ & $-4.1 \mathrm{E}-05 \pm 1.9 \mathrm{E}-04$ & $\mathrm{U}$ & & ${ }^{155} \mathrm{Eu}$ & $-2.1 \mathrm{E}-04 \pm 2.1 \mathrm{E}-04$ & $\mathrm{U}$ \\
\hline & ${ }^{238} \mathrm{Pu}$ & $-1.1 \mathrm{E}-06 \pm 1.1 \mathrm{E}-05$ & $\mathrm{U}$ & & ${ }^{238} \mathrm{Pu}$ & $2.2 \mathrm{E}-05 \pm 1.9 \mathrm{E}-05$ & $\mathrm{U}$ \\
\hline & ${ }^{239 / 240} \mathrm{Pu}$ & $-1.1 \mathrm{E}-06 \pm 4.9 \mathrm{E}-06$ & $\mathrm{U}$ & & ${ }^{239 / 240} \mathrm{Pu}$ & $4.0 \mathrm{E}-06 \pm 4.1 \mathrm{E}-06$ & \\
\hline & ${ }^{103} \mathrm{Ru}$ & $2.6 \mathrm{E}-05 \pm 7.2 \mathrm{E}-05$ & $\mathrm{U}$ & & ${ }^{103} \mathrm{Ru}$ & $5.5 \mathrm{E}-05 \pm 1.0 \mathrm{E}-04$ & $\mathrm{U}$ \\
\hline & ${ }^{106} \mathrm{Ru}$ & $-2.1 \mathrm{E}-04 \pm 7.5 \mathrm{E}-04$ & $\mathrm{U}$ & & ${ }^{106} \mathrm{Ru}$ & $-2.7 \mathrm{E}-04 \pm 7.1 \mathrm{E}-04$ & $\mathrm{U}$ \\
\hline & ${ }^{125} \mathrm{Sb}$ & $-2.0 \mathrm{E}-04 \pm 2.1 \mathrm{E}-04$ & $\mathrm{U}$ & & ${ }^{125} \mathrm{Sb}$ & $-3.7 \mathrm{E}-05 \pm 1.7 \mathrm{E}-04$ & $\mathrm{U}$ \\
\hline & ${ }^{113} \mathrm{Sn}$ & $2.9 \mathrm{E}-05 \pm 7.9 \mathrm{E}-05$ & $\mathrm{U}$ & & ${ }^{113} \mathrm{Sn}$ & $-1.6 \mathrm{E}-05 \pm 9.1 \mathrm{E}-05$ & $\mathrm{U}$ \\
\hline & ${ }^{90} \mathrm{Sr}$ & $6.9 \mathrm{E}-05 \pm 9.9 \mathrm{E}-05$ & $\mathrm{U}$ & & ${ }^{90} \mathrm{Sr}$ & $-1.5 \mathrm{E}-05 \pm 7.8 \mathrm{E}-05$ & $\mathrm{U}$ \\
\hline & ${ }^{234} \mathrm{U}$ & $9.2 \mathrm{E}-06 \pm 6.3 \mathrm{E}-06$ & & & ${ }^{234} \mathrm{U}$ & $2.0 \mathrm{E}-05 \pm 1.1 \mathrm{E}-05$ & \\
\hline & ${ }^{235} \mathrm{U}$ & $2.5 \mathrm{E}-06 \pm 3.1 \mathrm{E}-06$ & & & ${ }^{235} \mathrm{U}$ & $2.5 \mathrm{E}-06 \pm 3.1 \mathrm{E}-06$ & \\
\hline & ${ }^{238} \mathrm{U}$ & $2.3 \mathrm{E}-06 \pm 2.8 \mathrm{E}-06$ & & & ${ }^{238} \mathrm{U}$ & $6.9 \mathrm{E}-06 \pm 5.2 \mathrm{E}-06$ & \\
\hline & ${ }^{65} \mathrm{Zn}$ & $-1.1 \mathrm{E}-04 \pm 1.8 \mathrm{E}-04$ & $\mathrm{U}$ & & ${ }^{65} \mathrm{Zn}$ & $-1.3 \mathrm{E}-04 \pm 2.7 \mathrm{E}-04$ & $\mathrm{U}$ \\
\hline
\end{tabular}

$\overline{\mathrm{RQ}}=$ Result Qualifier. $\mathrm{U}=$ The analyte was analyzed for but not detected. 
Table 2-3. Near-Facility Air Sampling Results, 2003 (pCi/m³ \pm total analytical uncertainty). (cont)

\begin{tabular}{ccrc}
\hline Location & Isotope & Result \pm Uncertainty & RQ* \\
\hline N466 (100-B/C) & ${ }^{144} \mathrm{Ce}$ & $-1.9 \mathrm{E}-04 \pm 5.6 \mathrm{E}-04$ & $\mathrm{U}$ \\
Composite Period & ${ }^{60} \mathrm{Co}$ & $4.9 \mathrm{E}-06 \pm 4.9 \mathrm{E}-05$ & $\mathrm{U}$ \\
01/06/03 to 06/23/03 & ${ }^{134} \mathrm{Cs}$ & $2.1 \mathrm{E}-05 \pm 7.7 \mathrm{E}-05$ & $\mathrm{U}$ \\
& ${ }^{137} \mathrm{Cs}$ & $9.3 \mathrm{E}-06 \pm 6.6 \mathrm{E}-05$ & $\mathrm{U}$ \\
& ${ }^{152} \mathrm{Eu}$ & $3.8 \mathrm{E}-06 \pm 3.8 \mathrm{E}-05$ & $\mathrm{U}$ \\
& ${ }^{154} \mathrm{Eu}$ & $-4.5 \mathrm{E}-05 \pm 2.1 \mathrm{E}-04$ & $\mathrm{U}$ \\
& ${ }^{155} \mathrm{Eu}$ & $-1.2 \mathrm{E}-05 \pm 1.2 \mathrm{E}-04$ & $\mathrm{U}$ \\
& ${ }^{238} \mathrm{Pu}$ & $-3.5 \mathrm{E}-06 \pm 1.5 \mathrm{E}-05$ & $\mathrm{U}$ \\
& ${ }^{239 / 240} \mathrm{Pu}$ & $9.1 \mathrm{E}-07 \pm 9.5 \mathrm{E}-07$ & $\mathrm{U}$ \\
& ${ }^{103} \mathrm{Ru}$ & $-2.4 \mathrm{E}-05 \pm 7.9 \mathrm{E}-05$ & $\mathrm{U}$ \\
& ${ }^{106} \mathrm{Ru}$ & $-1.6 \mathrm{E}-04 \pm 6.4 \mathrm{E}-04$ & $\mathrm{U}$ \\
& ${ }^{125} \mathrm{Sb}$ & $-8.6 \mathrm{E}-05 \pm 1.5 \mathrm{E}-04$ & $\mathrm{U}$ \\
& ${ }^{113} \mathrm{Sn}$ & $1.0 \mathrm{E}-04 \pm 8.3 \mathrm{E}-05$ & $\mathrm{U}$ \\
& ${ }^{90} \mathrm{Sr}$ & $7.6 \mathrm{E}-06 \pm 7.6 \mathrm{E}-05$ & $\mathrm{U}$ \\
& ${ }^{234} \mathrm{U}$ & $1.8 \mathrm{E}-05 \pm 1.0 \mathrm{E}-05$ & \\
& ${ }^{235} \mathrm{U}$ & $2.7 \mathrm{E}-06 \pm 4.1 \mathrm{E}-06$ & $\mathrm{U}$ \\
& ${ }^{238} \mathrm{U}$ & $8.4 \mathrm{E}-06 \pm 6.1 \mathrm{E}-06$ & \\
& ${ }^{63} \mathrm{Zn} \mathrm{U}$ & $-1.9 \mathrm{E}-04 \pm 2.0 \mathrm{E}-04$ & $\mathrm{U}$
\end{tabular}

\begin{tabular}{ccrc}
\hline Location & Isotope & Result \pm Uncertainty & RQ* \\
\hline N466 (100-B/C) & ${ }^{144} \mathrm{Ce}$ & $2.3 \mathrm{E}-05 \pm 2.3 \mathrm{E}-04$ & $\mathrm{U}$ \\
Composite Period & ${ }^{60} \mathrm{Co}$ & $1.6 \mathrm{E}-05 \pm 8.7 \mathrm{E}-05$ & $\mathrm{U}$ \\
06/23/03 to 12/22/03 & ${ }^{134} \mathrm{Cs}$ & $-6.9 \mathrm{E}-05 \pm 9.2 \mathrm{E}-05$ & $\mathrm{U}$ \\
& ${ }^{137} \mathrm{Cs}$ & $-4.7 \mathrm{E}-05 \pm 8.0 \mathrm{E}-05$ & $\mathrm{U}$ \\
& ${ }^{152} \mathrm{Eu}$ & $3.0 \mathrm{E}-05 \pm 1.9 \mathrm{E}-04$ & $\mathrm{U}$ \\
& ${ }^{154} \mathrm{Eu}$ & $-4.2 \mathrm{E}-05 \pm 3.0 \mathrm{E}-04$ & $\mathrm{U}$ \\
& ${ }^{155} \mathrm{Eu}$ & $-4.5 \mathrm{E}-05 \pm 1.8 \mathrm{E}-04$ & $\mathrm{U}$ \\
& ${ }^{238} \mathrm{Pu}$ & $-2.8 \mathrm{E}-06 \pm 1.6 \mathrm{E}-05$ & $\mathrm{U}$ \\
& ${ }^{239 / 240} \mathrm{Pu}$ & $9.2 \mathrm{E}-07 \pm 4.9 \mathrm{E}-06$ & $\mathrm{U}$ \\
& ${ }^{103} \mathrm{Ru}$ & $6.6 \mathrm{E}-06 \pm 6.6 \mathrm{E}-05$ & $\mathrm{U}$ \\
& ${ }^{106} \mathrm{Ru}$ & $-2.0 \mathrm{E}-04 \pm 6.9 \mathrm{E}-04$ & $\mathrm{U}$ \\
& ${ }^{125} \mathrm{Sb}$ & $3.6 \mathrm{E}-05 \pm 1.7 \mathrm{E}-04$ & $\mathrm{U}$ \\
& ${ }^{113} \mathrm{Sn}$ & $-2.1 \mathrm{E}-05 \pm 7.8 \mathrm{E}-05$ & $\mathrm{U}$ \\
& ${ }^{90} \mathrm{Sr}$ & $-8.5 \mathrm{E}-05 \pm 9.2 \mathrm{E}-05$ & $\mathrm{U}$ \\
& ${ }^{234} \mathrm{U}$ & $9.2 \mathrm{E}-06 \pm 6.5 \mathrm{E}-06$ & \\
& ${ }^{235} \mathrm{U}$ & $2.4 \mathrm{E}-06 \pm 3.0 \mathrm{E}-06$ & \\
& ${ }^{238} \mathrm{U}$ & $9.2 \mathrm{E}-06 \pm 6.2 \mathrm{E}-06$ & \\
& ${ }^{238} \mathrm{Z} \mathrm{Zn}$ & $1.1 \mathrm{E}-04 \pm 2.0 \mathrm{E}-04$ & $\mathrm{U}$
\end{tabular}

$\overline{\mathrm{RQ}}=$ Result Qualifier. $\mathrm{U}=$ The analyte was analyzed for but not detected. 
Table 2-3. Near-Facility Air Sampling Results, 2003 (pCi/m³ \pm total analytical uncertainty). (cont)

\begin{tabular}{|c|c|c|c|c|c|c|c|}
\hline Location & Isotope & Result \pm Uncertainty & $\mathbf{R Q}^{*}$ & Location & Isotope & Result \pm Uncertainty & $\mathbf{R Q}^{*}$ \\
\hline N497 (100-B/C) & ${ }^{144} \mathrm{Ce}$ & $-8.0 \mathrm{E}-05 \pm 7.6 \mathrm{E}-04$ & $\bar{U}$ & N497 (100-B/C) & ${ }^{144} \mathrm{Ce}$ & $-3.0 \mathrm{E}-04 \pm 7.9 \mathrm{E}-04$ & $\bar{U}$ \\
\hline Composite Period & ${ }^{60} \mathrm{Co}$ & $1.7 \mathrm{E}-06 \pm 1.7 \mathrm{E}-05$ & $\mathrm{U}$ & Composite Period & ${ }^{60} \mathrm{Co}$ & $6.3 \mathrm{E}-05 \pm 7.3 \mathrm{E}-05$ & $\mathrm{U}$ \\
\hline \multirow[t]{16}{*}{$01 / 06 / 03$ to $06 / 23 / 03$} & ${ }^{134} \mathrm{Cs}$ & $3.8 \mathrm{E}-05 \pm 8.1 \mathrm{E}-05$ & $\mathrm{U}$ & $06 / 23 / 03$ to $12 / 22 / 03$ & ${ }^{134} \mathrm{Cs}$ & $-1.3 \mathrm{E}-05 \pm 8.2 \mathrm{E}-05$ & $\mathrm{U}$ \\
\hline & ${ }^{137} \mathrm{Cs}$ & $3.0 \mathrm{E}-05 \pm 7.7 \mathrm{E}-05$ & $\mathrm{U}$ & & ${ }^{137} \mathrm{Cs}$ & $-7.6 \mathrm{E}-06 \pm 6.5 \mathrm{E}-05$ & $\mathrm{U}$ \\
\hline & ${ }^{152} \mathrm{Eu}$ & $5.3 \mathrm{E}-05 \pm 2.0 \mathrm{E}-04$ & $\mathrm{U}$ & & ${ }^{152} \mathrm{Eu}$ & $-3.0 \mathrm{E}-04 \pm 3.1 \mathrm{E}-04$ & $\mathrm{U}$ \\
\hline & ${ }^{154} \mathrm{Eu}$ & $-7.9 \mathrm{E}-05 \pm 2.6 \mathrm{E}-04$ & $\mathrm{U}$ & & ${ }^{154} \mathrm{Eu}$ & $-1.1 \mathrm{E}-04 \pm 2.1 \mathrm{E}-04$ & $\mathrm{U}$ \\
\hline & ${ }^{155} \mathrm{Eu}$ & $1.5 \mathrm{E}-04 \pm 1.9 \mathrm{E}-04$ & $\mathrm{U}$ & & ${ }^{155} \mathrm{Eu}$ & $3.4 \mathrm{E}-05 \pm 1.9 \mathrm{E}-04$ & $\mathrm{U}$ \\
\hline & ${ }^{238} \mathrm{Pu}$ & $-4.5 \mathrm{E}-06 \pm 1.0 \mathrm{E}-05$ & $\mathrm{U}$ & & ${ }^{238} \mathrm{Pu}$ & $3.8 \mathrm{E}-06 \pm 1.1 \mathrm{E}-05$ & $\mathrm{U}$ \\
\hline & ${ }^{239 / 240} \mathrm{Pu}$ & $9.1 \mathrm{E}-07 \pm 1.8 \mathrm{E}-06$ & $\mathrm{U}$ & & ${ }^{239 / 240} \mathrm{Pu}$ & $9.4 \mathrm{E}-07 \pm 5.6 \mathrm{E}-06$ & $\mathrm{U}$ \\
\hline & ${ }^{103} \mathrm{Ru}$ & $9.1 \mathrm{E}-05 \pm 9.1 \mathrm{E}-05$ & $\mathrm{U}$ & & ${ }^{103} \mathrm{Ru}$ & $1.1 \mathrm{E}-05 \pm 8.4 \mathrm{E}-05$ & $\mathrm{U}$ \\
\hline & ${ }^{106} \mathrm{Ru}$ & $-3.6 \mathrm{E}-04 \pm 6.9 \mathrm{E}-04$ & $\mathrm{U}$ & & ${ }^{106} \mathrm{Ru}$ & $1.7 \mathrm{E}-04 \pm 6.3 \mathrm{E}-04$ & $\mathrm{U}$ \\
\hline & ${ }^{125} \mathrm{Sb}$ & $8.5 \mathrm{E}-05 \pm 1.8 \mathrm{E}-04$ & $\mathrm{U}$ & & ${ }^{125} \mathrm{Sb}$ & $-3.4 \mathrm{E}-05 \pm 1.7 \mathrm{E}-04$ & $\mathrm{U}$ \\
\hline & ${ }^{113} \mathrm{Sn}$ & $-6.9 \mathrm{E}-05 \pm 9.3 \mathrm{E}-05$ & $\mathrm{U}$ & & ${ }^{113} \mathrm{Sn}$ & $-1.9 \mathrm{E}-05 \pm 8.5 \mathrm{E}-05$ & $\mathrm{U}$ \\
\hline & ${ }^{90} \mathrm{Sr}$ & $-7.6 \mathrm{E}-05 \pm 7.8 \mathrm{E}-05$ & $\mathrm{U}$ & & ${ }^{90} \mathrm{Sr}$ & $-5.1 \mathrm{E}-05 \pm 9.7 \mathrm{E}-05$ & $\mathrm{U}$ \\
\hline & ${ }^{234} \mathrm{U}$ & $9.1 \mathrm{E}-06 \pm 6.2 \mathrm{E}-06$ & & & ${ }^{234} \mathrm{U}$ & $9.4 \mathrm{E}-06 \pm 6.8 \mathrm{E}-06$ & \\
\hline & ${ }^{235} \mathrm{U}$ & $-8.3 \mathrm{E}-07 \pm 1.7 \mathrm{E}-06$ & $\mathrm{U}$ & & ${ }^{235} \mathrm{U}$ & $3.4 \mathrm{E}-06 \pm 3.5 \mathrm{E}-06$ & \\
\hline & ${ }^{238} \mathrm{U}$ & $6.9 \mathrm{E}-06 \pm 5.2 \mathrm{E}-06$ & & & ${ }^{238} \mathrm{U}$ & $8.0 \mathrm{E}-06 \pm 7.0 \mathrm{E}-06$ & $\mathrm{U}$ \\
\hline & ${ }^{65} \mathrm{Zn}$ & $-1.7 \mathrm{E}-04 \pm 2.0 \mathrm{E}-04$ & $\mathrm{U}$ & & ${ }^{65} \mathrm{Zn}$ & $-1.1 \mathrm{E}-04 \pm 2.0 \mathrm{E}-04$ & $\mathrm{U}$ \\
\hline N536 (100-B/C) & ${ }^{144} \mathrm{Ce}$ & $-5.8 \mathrm{E}-04 \pm 9.9 \mathrm{E}-04$ & $\mathrm{U}$ & N492 (100-D/DR) & ${ }^{144} \mathrm{Ce}$ & $-1.1 \mathrm{E}-03 \pm 1.7 \mathrm{E}-03$ & $\bar{U}$ \\
\hline Composite Period & ${ }^{60} \mathrm{Co}$ & $4.1 \mathrm{E}-05 \pm 1.2 \mathrm{E}-04$ & $\mathrm{U}$ & Composite Period & ${ }^{60} \mathrm{Co}$ & $1.5 \mathrm{E}-04 \pm 2.2 \mathrm{E}-04$ & $\mathrm{U}$ \\
\hline \multirow[t]{16}{*}{$02 / 26 / 03$ to $07 / 07 / 03$} & ${ }^{134} \mathrm{Cs}$ & $6.6 \mathrm{E}-05 \pm 1.3 \mathrm{E}-04$ & $\mathrm{U}$ & $01 / 07 / 03$ to $03 / 17 / 03$ & ${ }^{134} \mathrm{Cs}$ & $-2.7 \mathrm{E}-04 \pm 2.7 \mathrm{E}-04$ & $\mathrm{U}$ \\
\hline & ${ }^{137} \mathrm{Cs}$ & $-1.2 \mathrm{E}-05 \pm 1.2 \mathrm{E}-04$ & $\mathrm{U}$ & & ${ }^{137} \mathrm{Cs}$ & $-7.4 \mathrm{E}-05 \pm 2.0 \mathrm{E}-04$ & $\mathrm{U}$ \\
\hline & ${ }^{152} \mathrm{Eu}$ & $1.6 \mathrm{E}-06 \pm 1.6 \mathrm{E}-05$ & $\mathrm{U}$ & & ${ }^{152} \mathrm{Eu}$ & $2.7 \mathrm{E}-04 \pm 4.4 \mathrm{E}-04$ & $\mathrm{U}$ \\
\hline & ${ }^{154} \mathrm{Eu}$ & $2.4 \mathrm{E}-04 \pm 3.4 \mathrm{E}-04$ & $\mathrm{U}$ & & ${ }^{154} \mathrm{Eu}$ & $2.4 \mathrm{E}-04 \pm 6.1 \mathrm{E}-04$ & $\mathrm{U}$ \\
\hline & ${ }^{155} \mathrm{Eu}$ & $8.8 \mathrm{E}-05 \pm 2.5 \mathrm{E}-04$ & $\mathrm{U}$ & & ${ }^{155} \mathrm{Eu}$ & $1.3 \mathrm{E}-04 \pm 4.4 \mathrm{E}-04$ & $\mathrm{U}$ \\
\hline & ${ }^{238} \mathrm{Pu}$ & $2.1 \mathrm{E}-05 \pm 2.1 \mathrm{E}-05$ & $\mathrm{U}$ & & ${ }^{238} \mathrm{Pu}$ & $1.6 \mathrm{E}-05 \pm 1.8 \mathrm{E}-05$ & $\mathrm{U}$ \\
\hline & ${ }^{239 / 240} \mathrm{Pu}$ & $7.0 \mathrm{E}-06 \pm 7.9 \mathrm{E}-06$ & $\mathrm{U}$ & & ${ }^{239 / 240} \mathrm{Pu}$ & $1.8 \mathrm{E}-06 \pm 3.6 \mathrm{E}-06$ & $\mathrm{U}$ \\
\hline & ${ }^{103} \mathrm{Ru}$ & $-3.9 \mathrm{E}-05 \pm 1.2 \mathrm{E}-04$ & $\mathrm{U}$ & & ${ }^{103} \mathrm{Ru}$ & $-6.2 \mathrm{E}-05 \pm 1.9 \mathrm{E}-04$ & $\mathrm{U}$ \\
\hline & ${ }^{106} \mathrm{Ru}$ & $1.5 \mathrm{E}-04 \pm 1.0 \mathrm{E}-03$ & $\mathrm{U}$ & & ${ }^{106} \mathrm{Ru}$ & $-6.5 \mathrm{E}-05 \pm 6.5 \mathrm{E}-04$ & $\mathrm{U}$ \\
\hline & ${ }^{125} \mathrm{Sb}$ & $8.4 \mathrm{E}-05 \pm 2.6 \mathrm{E}-04$ & $\mathrm{U}$ & & ${ }^{125} \mathrm{Sb}$ & $-1.2 \mathrm{E}-04 \pm 4.8 \mathrm{E}-04$ & $\mathrm{U}$ \\
\hline & ${ }^{113} \mathrm{Sn}$ & $-2.3 \mathrm{E}-05 \pm 1.3 \mathrm{E}-04$ & $\mathrm{U}$ & & ${ }^{113} \mathrm{Sn}$ & $4.2 \mathrm{E}-05 \pm 2.1 \mathrm{E}-04$ & $\mathrm{U}$ \\
\hline & ${ }^{90} \mathrm{Sr}$ & $7.1 \mathrm{E}-05 \pm 1.4 \mathrm{E}-04$ & $\mathrm{U}$ & & ${ }^{90} \mathrm{Sr}$ & $1.7 \mathrm{E}-04 \pm 2.4 \mathrm{E}-04$ & $\mathrm{U}$ \\
\hline & ${ }^{234} \mathrm{U}$ & $1.0 \mathrm{E}-05 \pm 7.9 \mathrm{E}-06$ & & & ${ }^{234} \mathrm{U}$ & $2.4 \mathrm{E}-05 \pm 2.0 \mathrm{E}-05$ & $\mathrm{U}$ \\
\hline & ${ }^{235} \mathrm{U}$ & $4.4 \mathrm{E}-06 \pm 5.4 \mathrm{E}-06$ & $\mathrm{U}$ & & ${ }^{235} \mathrm{U}$ & $1.8 \mathrm{E}-06 \pm 1.9 \mathrm{E}-06$ & $\mathrm{U}$ \\
\hline & ${ }^{238} \mathrm{U}$ & $1.2 \mathrm{E}-05 \pm 9.1 \mathrm{E}-06$ & & & ${ }^{238} \mathrm{U}$ & $-1.8 \mathrm{E}-06 \pm 6.4 \mathrm{E}-06$ & $\mathrm{U}$ \\
\hline & ${ }^{65} \mathrm{Zn}$ & $8.0 \mathrm{E}-05 \pm 2.5 \mathrm{E}-04$ & $\mathrm{U}$ & & ${ }^{65} \mathrm{Zn}$ & $-1.4 \mathrm{E}-04 \pm 4.2 \mathrm{E}-04$ & $\mathrm{U}$ \\
\hline
\end{tabular}

$\overline{\mathrm{RQ}}=$ Result Qualifier. $\mathrm{U}=$ The analyte was analyzed for but not detected. 
Table 2-3. Near-Facility Air Sampling Results, 2003 (pCi/m³ \pm total analytical uncertainty). (cont)

\begin{tabular}{|c|c|c|c|c|c|c|c|}
\hline Location & Isotope & Result \pm Uncertainty & $\mathbf{R Q}^{*}$ & Location & Isotope & Result \pm Uncertainty & RQ* \\
\hline N492 (100-D/DR) & ${ }^{144} \mathrm{Ce}$ & $-4.8 \mathrm{E}-04 \pm 1.1 \mathrm{E}-03$ & $\mathrm{U}$ & N492 (100-D/DR) & ${ }^{144} \mathrm{Ce}$ & $8.9 \mathrm{E}-04 \pm 1.6 \mathrm{E}-03$ & $\mathrm{U}$ \\
\hline Composite Period & ${ }^{60} \mathrm{Co}$ & $-7.3 \mathrm{E}-05 \pm 1.3 \mathrm{E}-04$ & $\mathrm{U}$ & Composite Period & ${ }^{60} \mathrm{Co}$ & $-3.5 \mathrm{E}-05 \pm 2.1 \mathrm{E}-04$ & $\mathrm{U}$ \\
\hline \multirow[t]{16}{*}{$03 / 17 / 03$ to $06 / 23 / 03$} & ${ }^{134} \mathrm{Cs}$ & $-4.3 \mathrm{E}-05 \pm 1.2 \mathrm{E}-04$ & $\mathrm{U}$ & $06 / 23 / 03$ to $09 / 16 / 03$ & ${ }^{134} \mathrm{Cs}$ & $-3.5 \mathrm{E}-05 \pm 2.0 \mathrm{E}-04$ & $\mathrm{U}$ \\
\hline & ${ }^{137} \mathrm{Cs}$ & $-9.8 \mathrm{E}-06 \pm 9.8 \mathrm{E}-05$ & $\mathrm{U}$ & & ${ }^{137} \mathrm{Cs}$ & $-1.7 \mathrm{E}-04 \pm 2.0 \mathrm{E}-04$ & $\mathrm{U}$ \\
\hline & ${ }^{152} \mathrm{Eu}$ & $-1.7 \mathrm{E}-04 \pm 2.6 \mathrm{E}-04$ & $\mathrm{U}$ & & ${ }^{152} \mathrm{Eu}$ & $2.6 \mathrm{E}-04 \pm 4.2 \mathrm{E}-04$ & $\mathrm{U}$ \\
\hline & ${ }^{154} \mathrm{Eu}$ & $-2.7 \mathrm{E}-05 \pm 2.7 \mathrm{E}-04$ & $\mathrm{U}$ & & ${ }^{154} \mathrm{Eu}$ & $1.2 \mathrm{E}-04 \pm 6.0 \mathrm{E}-04$ & $\mathrm{U}$ \\
\hline & ${ }^{155} \mathrm{Eu}$ & $-2.1 \mathrm{E}-04 \pm 2.8 \mathrm{E}-04$ & $\mathrm{U}$ & & ${ }^{155} \mathrm{Eu}$ & $-3.5 \mathrm{E}-04 \pm 4.3 \mathrm{E}-04$ & $\mathrm{U}$ \\
\hline & ${ }^{238} \mathrm{Pu}$ & $6.8 \mathrm{E}-06 \pm 2.7 \mathrm{E}-05$ & $\mathrm{U}$ & & ${ }^{238} \mathrm{Pu}$ & $2.6 \mathrm{E}-05 \pm 3.0 \mathrm{E}-05$ & $\mathrm{U}$ \\
\hline & ${ }^{239 / 240} \mathrm{Pu}$ & $1.7 \mathrm{E}-06 \pm 7.7 \mathrm{E}-06$ & $\mathrm{U}$ & & ${ }^{239 / 240} \mathrm{Pu}$ & $1.3 \mathrm{E}-05 \pm 1.1 \mathrm{E}-05$ & \\
\hline & ${ }^{103} \mathrm{Ru}$ & $5.9 \mathrm{E}-05 \pm 9.4 \mathrm{E}-05$ & $\mathrm{U}$ & & ${ }^{103} \mathrm{Ru}$ & $-8.5 \mathrm{E}-06 \pm 8.5 \mathrm{E}-05$ & $\mathrm{U}$ \\
\hline & ${ }^{106} \mathrm{Ru}$ & $2.4 \mathrm{E}-04 \pm 1.0 \mathrm{E}-03$ & $\mathrm{U}$ & & ${ }^{106} \mathrm{Ru}$ & $-6.3 \mathrm{E}-04 \pm 1.9 \mathrm{E}-03$ & $\mathrm{U}$ \\
\hline & ${ }^{125} \mathrm{Sb}$ & 7.3E-05 $\pm 2.8 \mathrm{E}-04$ & $\mathrm{U}$ & & ${ }^{125} \mathrm{Sb}$ & $1.8 \mathrm{E}-04 \pm 4.1 \mathrm{E}-04$ & $\mathrm{U}$ \\
\hline & ${ }^{113} \mathrm{Sn}$ & $-1.3 \mathrm{E}-04 \pm 1.3 \mathrm{E}-04$ & $\mathrm{U}$ & & ${ }^{113} \mathrm{Sn}$ & $-1.2 \mathrm{E}-04 \pm 1.8 \mathrm{E}-04$ & $\mathrm{U}$ \\
\hline & ${ }^{90} \mathrm{Sr}$ & $-1.3 \mathrm{E}-05 \pm 1.2 \mathrm{E}-04$ & $\mathrm{U}$ & & ${ }^{90} \mathrm{Sr}$ & $2.1 \mathrm{E}-04 \pm 2.4 \mathrm{E}-04$ & \\
\hline & ${ }^{234} \mathrm{U}$ & $1.6 \mathrm{E}-05 \pm 1.1 \mathrm{E}-05$ & & & ${ }^{234} \mathrm{U}$ & $1.5 \mathrm{E}-05 \pm 1.3 \mathrm{E}-05$ & \\
\hline & ${ }^{235} \mathrm{U}$ & $2.6 \mathrm{E}-06 \pm 3.7 \mathrm{E}-06$ & $\mathrm{U}$ & & ${ }^{235} \mathrm{U}$ & $6.1 \mathrm{E}-06 \pm 7.4 \mathrm{E}-06$ & \\
\hline & ${ }^{238} \mathrm{U}$ & 7.3E-06 $\pm 7.4 \mathrm{E}-06$ & $\mathrm{U}$ & & ${ }^{238} \mathrm{U}$ & $7.4 \mathrm{E}-06 \pm 7.6 \mathrm{E}-06$ & \\
\hline & ${ }^{65} \mathrm{Zn}$ & $-1.9 \mathrm{E}-04 \pm 2.9 \mathrm{E}-04$ & $\mathrm{U}$ & & ${ }^{65} \mathrm{Zn}$ & 4.3E-05 $\pm 4.3 \mathrm{E}-04$ & $\mathrm{U}$ \\
\hline N492 (100-D/DR) & ${ }^{144} \mathrm{Ce}$ & $-1.5 \mathrm{E}-04 \pm 9.9 \mathrm{E}-04$ & $\bar{U}$ & N515 (100-D/DR) & ${ }^{144} \mathrm{Ce}$ & $1.9 \mathrm{E}-03 \pm 2.0 \mathrm{E}-03$ & $\mathrm{U}$ \\
\hline Composite Period & ${ }^{60} \mathrm{Co}$ & $3.6 \mathrm{E}-05 \pm 1.3 \mathrm{E}-04$ & $\mathrm{U}$ & Composite Period & ${ }^{60} \mathrm{Co}$ & $-1.5 \mathrm{E}-04 \pm 2.0 \mathrm{E}-04$ & $\mathrm{U}$ \\
\hline \multirow[t]{16}{*}{$09 / 16 / 03$ to $12 / 22 / 03$} & ${ }^{134} \mathrm{Cs}$ & $1.7 \mathrm{E}-05 \pm 1.3 \mathrm{E}-04$ & $\mathrm{U}$ & $01 / 07 / 03$ to $03 / 17 / 03$ & ${ }^{134} \mathrm{Cs}$ & $-1.6 \mathrm{E}-04 \pm 2.3 \mathrm{E}-04$ & $\mathrm{U}$ \\
\hline & ${ }^{137} \mathrm{Cs}$ & $-4.4 \mathrm{E}-05 \pm 1.1 \mathrm{E}-04$ & $\mathrm{U}$ & & ${ }^{137} \mathrm{Cs}$ & $7.6 \mathrm{E}-06 \pm 7.6 \mathrm{E}-05$ & $\mathrm{U}$ \\
\hline & ${ }^{152} \mathrm{Eu}$ & $-1.4 \mathrm{E}-05 \pm 1.4 \mathrm{E}-04$ & $\mathrm{U}$ & & ${ }^{152} \mathrm{Eu}$ & $-7.8 \mathrm{E}-05 \pm 4.9 \mathrm{E}-04$ & $\mathrm{U}$ \\
\hline & ${ }^{154} \mathrm{Eu}$ & $-2.4 \mathrm{E}-04 \pm 4.0 \mathrm{E}-04$ & $\mathrm{U}$ & & ${ }^{154} \mathrm{Eu}$ & $-3.8 \mathrm{E}-04 \pm 6.5 \mathrm{E}-04$ & $\mathrm{U}$ \\
\hline & ${ }^{155} \mathrm{Eu}$ & $-3.6 \mathrm{E}-04 \pm 3.7 \mathrm{E}-04$ & $\mathrm{U}$ & & ${ }^{155} \mathrm{Eu}$ & $-1.1 \mathrm{E}-04 \pm 5.6 \mathrm{E}-04$ & $\mathrm{U}$ \\
\hline & ${ }^{238} \mathrm{Pu}$ & $1.6 \mathrm{E}-05 \pm 2.1 \mathrm{E}-05$ & $\mathrm{U}$ & & ${ }^{238} \mathrm{Pu}$ & $-1.9 \mathrm{E}-06 \pm 1.6 \mathrm{E}-05$ & $\mathrm{U}$ \\
\hline & ${ }^{239 / 240} \mathrm{Pu}$ & $1.6 \mathrm{E}-06 \pm 1.7 \mathrm{E}-06$ & $\mathrm{U}$ & & ${ }^{239 / 240} \mathrm{Pu}$ & $3.7 \mathrm{E}-06 \pm 7.5 \mathrm{E}-06$ & $\mathrm{U}$ \\
\hline & ${ }^{103} \mathrm{Ru}$ & $-3.6 \mathrm{E}-05 \pm 1.0 \mathrm{E}-04$ & $\mathrm{U}$ & & ${ }^{103} \mathrm{Ru}$ & $-1.7 \mathrm{E}-04 \pm 2.2 \mathrm{E}-04$ & $\mathrm{U}$ \\
\hline & ${ }^{106} \mathrm{Ru}$ & $-3.0 \mathrm{E}-04 \pm 1.0 \mathrm{E}-03$ & $\mathrm{U}$ & & ${ }^{106} \mathrm{Ru}$ & $-1.2 \mathrm{E}-04 \pm 1.2 \mathrm{E}-03$ & $\mathrm{U}$ \\
\hline & ${ }^{125} \mathrm{Sb}$ & $9.1 \mathrm{E}-05 \pm 2.7 \mathrm{E}-04$ & $\mathrm{U}$ & & ${ }^{125} \mathrm{Sb}$ & $-1.6 \mathrm{E}-04 \pm 4.8 \mathrm{E}-04$ & $\mathrm{U}$ \\
\hline & ${ }^{113} \mathrm{Sn}$ & $-1.3 \mathrm{E}-04 \pm 1.3 \mathrm{E}-04$ & $\mathrm{U}$ & & ${ }^{113} \mathrm{Sn}$ & $-1.9 \mathrm{E}-04 \pm 2.4 \mathrm{E}-04$ & $\mathrm{U}$ \\
\hline & ${ }^{90} \mathrm{Sr}$ & $4.0 \mathrm{E}-05 \pm 2.4 \mathrm{E}-04$ & $\mathrm{U}$ & & ${ }^{90} \mathrm{Sr}$ & $-8.3 \mathrm{E}-05 \pm 2.1 \mathrm{E}-04$ & $\mathrm{U}$ \\
\hline & ${ }^{234} \mathrm{U}$ & $2.7 \mathrm{E}-05 \pm 1.5 \mathrm{E}-05$ & & & ${ }^{234} \mathrm{U}$ & $2.3 \mathrm{E}-05 \pm 1.6 \mathrm{E}-05$ & \\
\hline & ${ }^{235} \mathrm{U}$ & $1.6 \mathrm{E}-05 \pm 1.2 \mathrm{E}-05$ & & & ${ }^{235} \mathrm{U}$ & $5.2 \mathrm{E}-06 \pm 7.4 \mathrm{E}-06$ & $\mathrm{U}$ \\
\hline & ${ }^{238} \mathrm{U}$ & $1.6 \mathrm{E}-05 \pm 1.1 \mathrm{E}-05$ & & & ${ }^{238} \mathrm{U}$ & $7.1 \mathrm{E}-06 \pm 1.3 \mathrm{E}-05$ & $\mathrm{U}$ \\
\hline & ${ }^{65} \mathrm{Zn}$ & $-3.5 \mathrm{E}-04 \pm 3.6 \mathrm{E}-04$ & $\mathrm{U}$ & & ${ }^{65} \mathrm{Zn}$ & $-1.0 \mathrm{E}-04 \pm 4.3 \mathrm{E}-04$ & $\mathrm{U}$ \\
\hline
\end{tabular}

$\overline{\mathrm{RQ}}=$ Result Qualifier. $\mathrm{U}=$ The analyte was analyzed for but not detected. 
Table 2-3. Near-Facility Air Sampling Results, 2003 (pCi/m³ \pm total analytical uncertainty). (cont)

\begin{tabular}{|c|c|c|c|}
\hline Location & Isotope & Result \pm Uncertainty & $\mathbf{R Q}^{*}$ \\
\hline N515 (100-D/DR) & ${ }^{144} \mathrm{Ce}$ & $5.1 \mathrm{E}-04 \pm 9.1 \mathrm{E}-04$ & $\mathrm{U}$ \\
\hline Composite Period & ${ }^{60} \mathrm{Co}$ & $4.2 \mathrm{E}-05 \pm 9.5 \mathrm{E}-05$ & $\mathrm{U}$ \\
\hline \multirow[t]{16}{*}{$03 / 17 / 03$ to $06 / 23 / 03$} & ${ }^{134} \mathrm{Cs}$ & $-7.3 \mathrm{E}-05 \pm 9.5 \mathrm{E}-05$ & $\mathrm{U}$ \\
\hline & ${ }^{137} \mathrm{Cs}$ & $5.5 \mathrm{E}-05 \pm 8.0 \mathrm{E}-05$ & $\mathrm{U}$ \\
\hline & ${ }^{152} \mathrm{Eu}$ & $-1.5 \mathrm{E}-04 \pm 2.5 \mathrm{E}-04$ & $\mathrm{U}$ \\
\hline & ${ }^{154} \mathrm{Eu}$ & $2.6 \mathrm{E}-06 \pm 2.6 \mathrm{E}-05$ & $\mathrm{U}$ \\
\hline & ${ }^{155} \mathrm{Eu}$ & $-1.6 \mathrm{E}-04 \pm 2.5 \mathrm{E}-04$ & $\mathrm{U}$ \\
\hline & ${ }^{238} \mathrm{Pu}$ & $3.2 \mathrm{E}-06 \pm 1.6 \mathrm{E}-05$ & $\mathrm{U}$ \\
\hline & ${ }^{239 / 240} \mathrm{Pu}$ & $1.6 \mathrm{E}-06 \pm 7.3 \mathrm{E}-06$ & $\mathrm{U}$ \\
\hline & ${ }^{103} \mathrm{Ru}$ & $1.7 \mathrm{E}-06 \pm 1.7 \mathrm{E}-05$ & $\mathrm{U}$ \\
\hline & ${ }^{106} \mathrm{Ru}$ & $-3.6 \mathrm{E}-04 \pm 7.3 \mathrm{E}-04$ & $\mathrm{U}$ \\
\hline & ${ }^{125} \mathrm{Sb}$ & $-8.1 \mathrm{E}-05 \pm 2.0 \mathrm{E}-04$ & $\mathrm{U}$ \\
\hline & ${ }^{113} \mathrm{Sn}$ & $2.4 \mathrm{E}-05 \pm 9.4 \mathrm{E}-05$ & $\mathrm{U}$ \\
\hline & ${ }^{90} \mathrm{Sr}$ & $-2.9 \mathrm{E}-04 \pm 3.0 \mathrm{E}-04$ & $\mathrm{U}$ \\
\hline & ${ }^{234} \mathrm{U}$ & $1.3 \mathrm{E}-05 \pm 1.2 \mathrm{E}-05$ & $\mathrm{U}$ \\
\hline & ${ }^{235} \mathrm{U}$ & $1.4 \mathrm{E}-05 \pm 1.0 \mathrm{E}-05$ & \\
\hline & ${ }^{238} \mathrm{U}$ & $1.4 \mathrm{E}-05 \pm 1.0 \mathrm{E}-05$ & \\
\hline & ${ }^{65} \mathrm{Zn}$ & $-2.4 \mathrm{E}-05 \pm 2.3 \mathrm{E}-04$ & $\mathrm{U}$ \\
\hline N515 (100-D/DR) & ${ }^{144} \mathrm{Ce}$ & $2.2 \mathrm{E}-03 \pm 2.3 \mathrm{E}-03$ & \\
\hline Composite Period & ${ }^{60} \mathrm{Co}$ & $3.0 \mathrm{E}-05 \pm 1.8 \mathrm{E}-04$ & $\mathrm{U}$ \\
\hline \multirow[t]{16}{*}{$09 / 16 / 03$ to $12 / 22 / 03$} & ${ }^{134} \mathrm{Cs}$ & $-3.8 \mathrm{E}-05 \pm 1.5 \mathrm{E}-04$ & $\mathrm{U}$ \\
\hline & ${ }^{137} \mathrm{Cs}$ & $-1.5 \mathrm{E}-05 \pm 1.3 \mathrm{E}-04$ & $\mathrm{U}$ \\
\hline & ${ }^{152} \mathrm{Eu}$ & $-9.6 \mathrm{E}-05 \pm 3.8 \mathrm{E}-04$ & $\mathrm{U}$ \\
\hline & ${ }^{154} \mathrm{Eu}$ & $2.0 \mathrm{E}-05 \pm 2.0 \mathrm{E}-04$ & $\mathrm{U}$ \\
\hline & ${ }^{155} \mathrm{Eu}$ & $7.1 \mathrm{E}-05 \pm 3.3 \mathrm{E}-04$ & $\mathrm{U}$ \\
\hline & ${ }^{238} \mathrm{Pu}$ & $1.9 \mathrm{E}-05 \pm 2.4 \mathrm{E}-05$ & $\mathrm{U}$ \\
\hline & ${ }^{239 / 240} \mathrm{Pu}$ & $1.9 \mathrm{E}-05 \pm 1.3 \mathrm{E}-05$ & \\
\hline & ${ }^{103} \mathrm{Ru}$ & $7.4 \mathrm{E}-05 \pm 1.2 \mathrm{E}-04$ & $\mathrm{U}$ \\
\hline & ${ }^{106} \mathrm{Ru}$ & $1.2 \mathrm{E}-03 \pm 1.2 \mathrm{E}-03$ & $\mathrm{U}$ \\
\hline & ${ }^{125} \mathrm{Sb}$ & $-1.3 \mathrm{E}-04 \pm 3.2 \mathrm{E}-04$ & $\mathrm{U}$ \\
\hline & ${ }^{113} \mathrm{Sn}$ & $3.9 \mathrm{E}-05 \pm 1.5 \mathrm{E}-04$ & $\mathrm{U}$ \\
\hline & ${ }^{90} \mathrm{Sr}$ & $-3.9 \mathrm{E}-05 \pm 2.0 \mathrm{E}-04$ & $\mathrm{U}$ \\
\hline & ${ }^{234} \mathrm{U}$ & $2.2 \mathrm{E}-05 \pm 1.4 \mathrm{E}-05$ & \\
\hline & ${ }^{235} \mathrm{U}$ & $1.2 \mathrm{E}-06 \pm 1.2 \mathrm{E}-05$ & $\mathrm{U}$ \\
\hline & ${ }^{238} \mathrm{U}$ & $8.3 \mathrm{E}-06 \pm 6.9 \mathrm{E}-06$ & \\
\hline & ${ }^{65} \mathrm{Zn}$ & $2.2 \mathrm{E}-04 \pm 4.0 \mathrm{E}-04$ & $\mathrm{U}$ \\
\hline
\end{tabular}

\begin{tabular}{|c|c|c|c|}
\hline Location & Isotope & Result \pm Uncertainty & RQ* \\
\hline N515 (100-D/DR) & ${ }^{144} \mathrm{Ce}$ & $-7.5 \mathrm{E}-04 \pm 1.1 \mathrm{E}-03$ & $\bar{U}$ \\
\hline Composite Period & ${ }^{60} \mathrm{Co}$ & $-7.2 \mathrm{E}-05 \pm 1.7 \mathrm{E}-04$ & $\mathrm{U}$ \\
\hline \multirow[t]{16}{*}{$06 / 23 / 03$ to $09 / 16 / 03$} & ${ }^{134} \mathrm{Cs}$ & $-2.4 \mathrm{E}-05 \pm 1.9 \mathrm{E}-04$ & $\mathrm{U}$ \\
\hline & ${ }^{137} \mathrm{Cs}$ & $1.2 \mathrm{E}-05 \pm 1.2 \mathrm{E}-04$ & $\mathrm{U}$ \\
\hline & ${ }^{152} \mathrm{Eu}$ & $1.6 \mathrm{E}-05 \pm 1.6 \mathrm{E}-04$ & $\mathrm{U}$ \\
\hline & ${ }^{154} \mathrm{Eu}$ & 2.3E-04 $\pm 4.7 \mathrm{E}-04$ & $\mathrm{U}$ \\
\hline & ${ }^{155} \mathrm{Eu}$ & $-2.5 \mathrm{E}-04 \pm 3.7 \mathrm{E}-04$ & U \\
\hline & ${ }^{238} \mathrm{Pu}$ & $7.6 \mathrm{E}-06 \pm 1.8 \mathrm{E}-05$ & $\mathrm{U}$ \\
\hline & ${ }^{239 / 240} \mathrm{Pu}$ & $-3.9 \mathrm{E}-06 \pm 5.5 \mathrm{E}-06$ & $\mathrm{U}$ \\
\hline & ${ }^{103} \mathrm{Ru}$ & $-4.7 \mathrm{E}-05 \pm 1.3 \mathrm{E}-04$ & $\mathrm{U}$ \\
\hline & ${ }^{106} \mathrm{Ru}$ & $-8.0 \mathrm{E}-04 \pm 1.3 \mathrm{E}-03$ & $\mathrm{U}$ \\
\hline & ${ }^{125} \mathrm{Sb}$ & $-1.8 \mathrm{E}-04 \pm 3.6 \mathrm{E}-04$ & $\mathrm{U}$ \\
\hline & ${ }^{113} \mathrm{Sn}$ & $-3.3 \mathrm{E}-05 \pm 2.0 \mathrm{E}-04$ & U \\
\hline & ${ }^{90} \mathrm{Sr}$ & $2.5 \mathrm{E}-04 \pm 2.1 \mathrm{E}-04$ & \\
\hline & ${ }^{234} \mathrm{U}$ & $1.6 \mathrm{E}-05 \pm 1.3 \mathrm{E}-05$ & \\
\hline & ${ }^{235} \mathrm{U}$ & $1.8 \mathrm{E}-06 \pm 3.7 \mathrm{E}-06$ & $\mathrm{U}$ \\
\hline & ${ }^{238} \mathrm{U}$ & $2.0 \mathrm{E}-05 \pm 1.3 \mathrm{E}-05$ & \\
\hline & ${ }^{65} \mathrm{Zn}$ & $-2.2 \mathrm{E}-04 \pm 3.8 \mathrm{E}-04$ & $\mathrm{U}$ \\
\hline N523 (100-D/DR) & ${ }^{144} \mathrm{Ce}$ & $1.9 \mathrm{E}-03 \pm 1.1 \mathrm{E}-02$ & $\mathrm{U}$ \\
\hline Composite Period & ${ }^{60} \mathrm{Co}$ & $3.6 \mathrm{E}-04 \pm 1.2 \mathrm{E}-03$ & $\mathrm{U}$ \\
\hline \multirow[t]{16}{*}{$01 / 07 / 03$ to $03 / 17 / 03$} & ${ }^{134} \mathrm{Cs}$ & $3.1 \mathrm{E}-04 \pm 9.6 \mathrm{E}-04$ & $\mathrm{U}$ \\
\hline & ${ }^{137} \mathrm{Cs}$ & $-5.2 \mathrm{E}-04 \pm 1.1 \mathrm{E}-03$ & U \\
\hline & ${ }^{152} \mathrm{Eu}$ & $8.4 \mathrm{E}-04 \pm 2.8 \mathrm{E}-03$ & $\mathrm{U}$ \\
\hline & ${ }^{154} \mathrm{Eu}$ & $-1.9 \mathrm{E}-03 \pm 3.8 \mathrm{E}-03$ & $\mathrm{U}$ \\
\hline & ${ }^{155} \mathrm{Eu}$ & $1.1 \mathrm{E}-03 \pm 2.3 \mathrm{E}-03$ & $\mathrm{U}$ \\
\hline & ${ }^{238} \mathrm{Pu}$ & $9.7 \mathrm{E}-06 \pm 9.7 \mathrm{E}-06$ & $\mathrm{U}$ \\
\hline & ${ }^{239 / 240} \mathrm{Pu}$ & $9.7 \mathrm{E}-06 \pm 1.9 \mathrm{E}-05$ & $\mathrm{U}$ \\
\hline & ${ }^{103} \mathrm{Ru}$ & $6.9 \mathrm{E}-05 \pm 6.9 \mathrm{E}-04$ & $\mathrm{U}$ \\
\hline & ${ }^{106} \mathrm{Ru}$ & $3.2 \mathrm{E}-03 \pm 9.2 \mathrm{E}-03$ & $\mathrm{U}$ \\
\hline & ${ }^{125} \mathrm{Sb}$ & $2.6 \mathrm{E}-03 \pm 2.3 \mathrm{E}-03$ & $\mathrm{U}$ \\
\hline & ${ }^{113} \mathrm{Sn}$ & $3.5 \mathrm{E}-04 \pm 1.1 \mathrm{E}-03$ & $\mathrm{U}$ \\
\hline & ${ }^{90} \mathrm{Sr}$ & $-5.0 \mathrm{E}-04 \pm 1.3 \mathrm{E}-03$ & $\mathrm{U}$ \\
\hline & ${ }^{234} \mathrm{U}$ & $5.7 \mathrm{E}-05 \pm 6.3 \mathrm{E}-05$ & $\mathrm{U}$ \\
\hline & ${ }^{235} \mathrm{U}$ & $2.1 \mathrm{E}-05 \pm 4.2 \mathrm{E}-05$ & $\mathrm{U}$ \\
\hline & ${ }^{238} \mathrm{U}$ & $4.8 \mathrm{E}-05 \pm 5.3 \mathrm{E}-05$ & $\mathrm{U}$ \\
\hline & ${ }^{65} \mathrm{Zn}$ & $-8.8 \mathrm{E}-04 \pm 2.7 \mathrm{E}-03$ & $\mathrm{U}$ \\
\hline
\end{tabular}

$\overline{\mathrm{RQ}}=$ Result Qualifier. $\mathrm{U}=$ The analyte was analyzed for but not detected. 
Table 2-3. Near-Facility Air Sampling Results, 2003 (pCi/m³ \pm total analytical uncertainty). (cont)

\begin{tabular}{|c|c|c|c|}
\hline Location & Isotope & Result \pm Uncertainty & $\mathbf{R Q}^{*}$ \\
\hline N523 (100-D/DR) & ${ }^{144} \mathrm{Ce}$ & $-1.4 \mathrm{E}-03 \pm 5.6 \mathrm{E}-03$ & $\mathrm{U}$ \\
\hline Composite Period & ${ }^{60} \mathrm{Co}$ & $-2.1 \mathrm{E}-05 \pm 2.1 \mathrm{E}-04$ & $\mathrm{U}$ \\
\hline \multirow[t]{16}{*}{$03 / 17 / 03$ to $06 / 23 / 03$} & ${ }^{134} \mathrm{Cs}$ & $-2.8 \mathrm{E}-04 \pm 6.2 \mathrm{E}-04$ & $\mathrm{U}$ \\
\hline & ${ }^{137} \mathrm{Cs}$ & $2.6 \mathrm{E}-04 \pm 5.4 \mathrm{E}-04$ & $\mathrm{U}$ \\
\hline & ${ }^{152} \mathrm{Eu}$ & $-5.9 \mathrm{E}-04 \pm 1.7 \mathrm{E}-03$ & $\mathrm{U}$ \\
\hline & ${ }^{154} \mathrm{Eu}$ & $-1.3 \mathrm{E}-03 \pm 1.6 \mathrm{E}-03$ & $\mathrm{U}$ \\
\hline & ${ }^{155} \mathrm{Eu}$ & $8.4 \mathrm{E}-04 \pm 1.5 \mathrm{E}-03$ & $\mathrm{U}$ \\
\hline & ${ }^{238} \mathrm{Pu}$ & $-6.8 \mathrm{E}-05 \pm 9.6 \mathrm{E}-05$ & $\mathrm{U}$ \\
\hline & ${ }^{239 / 240} \mathrm{Pu}$ & $1.4 \mathrm{E}-05 \pm 3.4 \mathrm{E}-05$ & $\mathrm{U}$ \\
\hline & ${ }^{103} \mathrm{Ru}$ & $-4.0 \mathrm{E}-04 \pm 5.1 \mathrm{E}-04$ & $\mathrm{U}$ \\
\hline & ${ }^{106} \mathrm{Ru}$ & $1.6 \mathrm{E}-03 \pm 4.8 \mathrm{E}-03$ & $\mathrm{U}$ \\
\hline & ${ }^{125} \mathrm{Sb}$ & $7.3 \mathrm{E}-04 \pm 1.3 \mathrm{E}-03$ & $\mathrm{U}$ \\
\hline & ${ }^{113} \mathrm{Sn}$ & $1.4 \mathrm{E}-04 \pm 6.1 \mathrm{E}-04$ & $\mathrm{U}$ \\
\hline & ${ }^{90} \mathrm{Sr}$ & $-1.9 \mathrm{E}-04 \pm 5.7 \mathrm{E}-04$ & $\mathrm{U}$ \\
\hline & ${ }^{234} \mathrm{U}$ & $5.5 \mathrm{E}-05 \pm 4.1 \mathrm{E}-05$ & \\
\hline & ${ }^{235} \mathrm{U}$ & $-7.4 \mathrm{E}-06 \pm 1.5 \mathrm{E}-05$ & $\mathrm{U}$ \\
\hline & ${ }^{238} \mathrm{U}$ & $2.0 \mathrm{E}-05 \pm 2.5 \mathrm{E}-05$ & \\
\hline & ${ }^{65} \mathrm{Zn}$ & $-2.2 \mathrm{E}-04 \pm 1.5 \mathrm{E}-03$ & $\mathrm{U}$ \\
\hline N523 (100-D/DR) & ${ }^{144} \mathrm{Ce}$ & $5.8 \mathrm{E}-05 \pm 5.8 \mathrm{E}-04$ & $\mathrm{U}$ \\
\hline Composite Period & ${ }^{60} \mathrm{Co}$ & $1.9 \mathrm{E}-04 \pm 3.7 \mathrm{E}-04$ & $\mathrm{U}$ \\
\hline \multirow[t]{16}{*}{$12 / 09 / 03$ to $12 / 22 / 03$} & ${ }^{134} \mathrm{Cs}$ & $2.2 \mathrm{E}-04 \pm 4.0 \mathrm{E}-04$ & $\mathrm{U}$ \\
\hline & ${ }^{137} \mathrm{Cs}$ & $-6.6 \mathrm{E}-05 \pm 3.2 \mathrm{E}-04$ & $\mathrm{U}$ \\
\hline & ${ }^{152} \mathrm{Eu}$ & $-2.4 \mathrm{E}-04 \pm 1.0 \mathrm{E}-03$ & $\mathrm{U}$ \\
\hline & ${ }^{154} \mathrm{Eu}$ & $1.6 \mathrm{E}-05 \pm 1.6 \mathrm{E}-04$ & $\mathrm{U}$ \\
\hline & ${ }^{155} \mathrm{Eu}$ & $-3.3 \mathrm{E}-04 \pm 7.4 \mathrm{E}-04$ & $\mathrm{U}$ \\
\hline & ${ }^{238} \mathrm{Pu}$ & $5.2 \mathrm{E}-05 \pm 1.4 \mathrm{E}-04$ & $\mathrm{U}$ \\
\hline & ${ }^{239 / 240} \mathrm{Pu}$ & $2.5 \mathrm{E}-05 \pm 3.7 \mathrm{E}-05$ & $\mathrm{U}$ \\
\hline & ${ }^{103} \mathrm{Ru}$ & $-1.9 \mathrm{E}-04 \pm 0.0 \mathrm{E}+00$ & $\mathrm{U}$ \\
\hline & ${ }^{106} \mathrm{Ru}$ & $7.4 \mathrm{E}-04 \pm 2.9 \mathrm{E}-03$ & $\mathrm{U}$ \\
\hline & ${ }^{125} \mathrm{Sb}$ & $8.4 \mathrm{E}-05 \pm 7.0 \mathrm{E}-04$ & $\mathrm{U}$ \\
\hline & ${ }^{113} \mathrm{Sn}$ & $-2.4 \mathrm{E}-04 \pm 3.0 \mathrm{E}-04$ & $\mathrm{U}$ \\
\hline & ${ }^{90} \mathrm{Sr}$ & $-7.5 \mathrm{E}-05 \pm 1.3 \mathrm{E}-04$ & $\mathrm{U}$ \\
\hline & ${ }^{234} \mathrm{U}$ & $2.3 \mathrm{E}-05 \pm 2.3 \mathrm{E}-05$ & $\mathrm{U}$ \\
\hline & ${ }^{235} \mathrm{U}$ & $1.3 \mathrm{E}-05 \pm 1.6 \mathrm{E}-05$ & $\mathrm{U}$ \\
\hline & ${ }^{238} \mathrm{U}$ & $1.2 \mathrm{E}-05 \pm 1.4 \mathrm{E}-05$ & $\mathrm{U}$ \\
\hline & ${ }^{65} \mathrm{Zn}$ & $3.6 \mathrm{E}-04 \pm 9.7 \mathrm{E}-04$ & $\mathrm{U}$ \\
\hline
\end{tabular}

\begin{tabular}{|c|c|c|c|}
\hline Location & Isotope & Result \pm Uncertainty & $\mathbf{R Q}^{*}$ \\
\hline N523 (100-D/DR) & ${ }^{144} \mathrm{Ce}$ & $7.4 \mathrm{E}-03 \pm 1.8 \mathrm{E}-02$ & $\bar{U}$ \\
\hline Composite Period & ${ }^{60} \mathrm{Co}$ & $-1.4 \mathrm{E}-03 \pm 2.0 \mathrm{E}-03$ & $\mathrm{U}$ \\
\hline \multirow[t]{16}{*}{$06 / 23 / 03$ to $08 / 19 / 03$} & ${ }^{134} \mathrm{Cs}$ & $-1.1 \mathrm{E}-03 \pm 1.9 \mathrm{E}-03$ & $\mathrm{U}$ \\
\hline & ${ }^{137} \mathrm{Cs}$ & $1.3 \mathrm{E}-03 \pm 1.8 \mathrm{E}-03$ & $\mathrm{U}$ \\
\hline & ${ }^{152} \mathrm{Eu}$ & $-2.0 \mathrm{E}-03 \pm 4.7 \mathrm{E}-03$ & $\mathrm{U}$ \\
\hline & ${ }^{154} \mathrm{Eu}$ & $1.2 \mathrm{E}-03 \pm 6.2 \mathrm{E}-03$ & $\mathrm{U}$ \\
\hline & ${ }^{155} \mathrm{Eu}$ & $2.0 \mathrm{E}-03 \pm 5.4 \mathrm{E}-03$ & U \\
\hline & ${ }^{238} \mathrm{Pu}$ & $-2.8 \mathrm{E}-04 \pm 5.9 \mathrm{E}-04$ & $\mathrm{U}$ \\
\hline & ${ }^{239 / 240} \mathrm{Pu}$ & $3.0 \mathrm{E}-04 \pm 2.4 \mathrm{E}-04$ & \\
\hline & ${ }^{103} \mathrm{Ru}$ & $-7.9 \mathrm{E}-05 \pm 7.9 \mathrm{E}-04$ & $\mathrm{U}$ \\
\hline & ${ }^{106} \mathrm{Ru}$ & $-3.4 \mathrm{E}-03 \pm 1.7 \mathrm{E}-02$ & $\mathrm{U}$ \\
\hline & ${ }^{125} \mathrm{Sb}$ & $-2.6 \mathrm{E}-03 \pm 4.1 \mathrm{E}-03$ & $\mathrm{U}$ \\
\hline & ${ }^{113} \mathrm{Sn}$ & $-1.8 \mathrm{E}-04 \pm 1.8 \mathrm{E}-03$ & $\mathrm{U}$ \\
\hline & ${ }^{90} \mathrm{Sr}$ & $-1.8 \mathrm{E}-03 \pm 2.9 \mathrm{E}-03$ & $\mathrm{U}$ \\
\hline & ${ }^{234} \mathrm{U}$ & $4.4 \mathrm{E}-05 \pm 1.4 \mathrm{E}-04$ & $\mathrm{U}$ \\
\hline & ${ }^{235} \mathrm{U}$ & $2.2 \mathrm{E}-05 \pm 2.2 \mathrm{E}-05$ & $\mathrm{U}$ \\
\hline & ${ }^{238} \mathrm{U}$ & $6.4 \mathrm{E}-05 \pm 9.6 \mathrm{E}-05$ & $\mathrm{U}$ \\
\hline & ${ }^{65} \mathrm{Zn}$ & $-3.2 \mathrm{E}-03 \pm 3.7 \mathrm{E}-03$ & $\mathrm{U}$ \\
\hline N494 (100-F) & ${ }^{144} \mathrm{Ce}$ & $4.9 \mathrm{E}-04 \pm 1.7 \mathrm{E}-03$ & $\mathrm{U}$ \\
\hline Composite Period & ${ }^{60} \mathrm{Co}$ & $9.7 \mathrm{E}-05 \pm 1.7 \mathrm{E}-04$ & $\mathrm{U}$ \\
\hline \multirow[t]{16}{*}{$01 / 07 / 03$ to $03 / 18 / 03$} & ${ }^{134} \mathrm{Cs}$ & $-1.5 \mathrm{E}-04 \pm 1.8 \mathrm{E}-04$ & $\mathrm{U}$ \\
\hline & ${ }^{137} \mathrm{Cs}$ & $1.1 \mathrm{E}-05 \pm 1.1 \mathrm{E}-04$ & U \\
\hline & ${ }^{152} \mathrm{Eu}$ & $-1.8 \mathrm{E}-04 \pm 4.4 \mathrm{E}-04$ & $\mathrm{U}$ \\
\hline & ${ }^{154} \mathrm{Eu}$ & $4.3 \mathrm{E}-04 \pm 4.8 \mathrm{E}-04$ & $\mathrm{U}$ \\
\hline & ${ }^{155} \mathrm{Eu}$ & $-1.2 \mathrm{E}-04 \pm 4.9 \mathrm{E}-04$ & $\mathrm{U}$ \\
\hline & ${ }^{238} \mathrm{Pu}$ & $5.6 \mathrm{E}-06 \pm 1.6 \mathrm{E}-05$ & $\mathrm{U}$ \\
\hline & ${ }^{239 / 240} \mathrm{Pu}$ & $3.6 \mathrm{E}-06 \pm 7.3 \mathrm{E}-06$ & $\mathrm{U}$ \\
\hline & ${ }^{103} \mathrm{Ru}$ & $3.5 \mathrm{E}-05 \pm 1.9 \mathrm{E}-04$ & $\mathrm{U}$ \\
\hline & ${ }^{106} \mathrm{Ru}$ & $3.5 \mathrm{E}-04 \pm 1.6 \mathrm{E}-03$ & $\mathrm{U}$ \\
\hline & ${ }^{125} \mathrm{Sb}$ & $-8.2 \mathrm{E}-05 \pm 4.2 \mathrm{E}-04$ & $\mathrm{U}$ \\
\hline & ${ }^{113} \mathrm{Sn}$ & $-2.3 \mathrm{E}-05 \pm 2.0 \mathrm{E}-04$ & $\mathrm{U}$ \\
\hline & ${ }^{90} \mathrm{Sr}$ & $-2.5 \mathrm{E}-04 \pm 2.6 \mathrm{E}-04$ & $\mathrm{U}$ \\
\hline & ${ }^{234} \mathrm{U}$ & $4.0 \mathrm{E}-05 \pm 2.1 \mathrm{E}-05$ & \\
\hline & ${ }^{235} \mathrm{U}$ & $4.0 \mathrm{E}-06 \pm 8.0 \mathrm{E}-06$ & $\mathrm{U}$ \\
\hline & ${ }^{238} \mathrm{U}$ & $4.0 \mathrm{E}-05 \pm 2.0 \mathrm{E}-05$ & \\
\hline & ${ }^{65} \mathrm{Zn}$ & $-5.3 \mathrm{E}-04 \pm 5.4 \mathrm{E}-04$ & $\mathrm{U}$ \\
\hline
\end{tabular}

$\overline{\mathrm{RQ}}=$ Result Qualifier. $\mathrm{U}=$ The analyte was analyzed for but not detected. 
Table 2-3. Near-Facility Air Sampling Results, 2003 (pCi/m³ \pm total analytical uncertainty). (cont)

\begin{tabular}{|c|c|c|c|c|c|c|c|}
\hline Location & Isotope & Result \pm Uncertainty & $\mathbf{R Q}^{*}$ & Location & Isotope & Result \pm Uncertainty & RQ* \\
\hline N494 (100-F) & ${ }^{144} \mathrm{Ce}$ & $-6.4 \mathrm{E}-04 \pm 1.2 \mathrm{E}-03$ & $\mathrm{U}$ & N494 (100-F) & ${ }^{144} \mathrm{Ce}$ & $5.2 \mathrm{E}-04 \pm 1.1 \mathrm{E}-03$ & $\mathrm{U}$ \\
\hline Composite Period & ${ }^{60} \mathrm{Co}$ & $-1.4 \mathrm{E}-04 \pm 1.6 \mathrm{E}-04$ & $\mathrm{U}$ & Composite Period & ${ }^{60} \mathrm{Co}$ & $4.3 \mathrm{E}-05 \pm 1.7 \mathrm{E}-04$ & $\mathrm{U}$ \\
\hline \multirow[t]{16}{*}{$03 / 18 / 03$ to $06 / 24 / 03$} & ${ }^{134} \mathrm{Cs}$ & $-1.3 \mathrm{E}-04 \pm 1.5 \mathrm{E}-04$ & $\mathrm{U}$ & $06 / 24 / 03$ to $09 / 16 / 03$ & ${ }^{134} \mathrm{Cs}$ & $-1.3 \mathrm{E}-04 \pm 1.8 \mathrm{E}-04$ & $\mathrm{U}$ \\
\hline & ${ }^{137} \mathrm{Cs}$ & $6.4 \mathrm{E}-05 \pm 1.4 \mathrm{E}-04$ & $\mathrm{U}$ & & ${ }^{137} \mathrm{Cs}$ & $4.3 \mathrm{E}-05 \pm 1.5 \mathrm{E}-04$ & $\mathrm{U}$ \\
\hline & ${ }^{152} \mathrm{Eu}$ & $-6.4 \mathrm{E}-05 \pm 3.3 \mathrm{E}-04$ & $\mathrm{U}$ & & ${ }^{152} \mathrm{Eu}$ & $-1.9 \mathrm{E}-05 \pm 1.9 \mathrm{E}-04$ & $\mathrm{U}$ \\
\hline & ${ }^{154} \mathrm{Eu}$ & $-1.1 \mathrm{E}-04 \pm 4.9 \mathrm{E}-04$ & $\mathrm{U}$ & & ${ }^{154} \mathrm{Eu}$ & $8.0 \mathrm{E}-04 \pm 5.1 \mathrm{E}-04$ & $\mathrm{U}$ \\
\hline & ${ }^{155} \mathrm{Eu}$ & $-7.7 \mathrm{E}-05 \pm 3.1 \mathrm{E}-04$ & $\mathrm{U}$ & & ${ }^{155} \mathrm{Eu}$ & $1.0 \mathrm{E}-04 \pm 3.6 \mathrm{E}-04$ & $\mathrm{U}$ \\
\hline & ${ }^{238} \mathrm{Pu}$ & $-1.4 \mathrm{E}-06 \pm 1.4 \mathrm{E}-05$ & $\mathrm{U}$ & & ${ }^{238} \mathrm{Pu}$ & $2.4 \mathrm{E}-05 \pm 2.7 \mathrm{E}-05$ & $\mathrm{U}$ \\
\hline & ${ }^{239 / 240} \mathrm{Pu}$ & $7.3 \mathrm{E}-06 \pm 8.3 \mathrm{E}-06$ & $\mathrm{U}$ & & ${ }^{239 / 240} \mathrm{Pu}$ & $5.0 \mathrm{E}-06 \pm 9.1 \mathrm{E}-06$ & $\mathrm{U}$ \\
\hline & ${ }^{103} \mathrm{Ru}$ & $-4.6 \mathrm{E}-05 \pm 1.2 \mathrm{E}-04$ & $\mathrm{U}$ & & ${ }^{103} \mathrm{Ru}$ & $5.1 \mathrm{E}-05 \pm 1.3 \mathrm{E}-04$ & $\mathrm{U}$ \\
\hline & ${ }^{106} \mathrm{Ru}$ & $3.1 \mathrm{E}-04 \pm 1.2 \mathrm{E}-03$ & $\mathrm{U}$ & & ${ }^{106} \mathrm{Ru}$ & $-1.9 \mathrm{E}-04 \pm 1.3 \mathrm{E}-03$ & $\mathrm{U}$ \\
\hline & ${ }^{125} \mathrm{Sb}$ & $1.1 \mathrm{E}-04 \pm 3.0 \mathrm{E}-04$ & $\mathrm{U}$ & & ${ }^{125} \mathrm{Sb}$ & $-1.7 \mathrm{E}-04 \pm 3.4 \mathrm{E}-04$ & $\mathrm{U}$ \\
\hline & ${ }^{113} \mathrm{Sn}$ & $-8.5 \mathrm{E}-06 \pm 8.5 \mathrm{E}-05$ & $\mathrm{U}$ & & ${ }^{113} \mathrm{Sn}$ & $3.1 \mathrm{E}-05 \pm 1.9 \mathrm{E}-04$ & $\mathrm{U}$ \\
\hline & ${ }^{90} \mathrm{Sr}$ & $-3.9 \mathrm{E}-05 \pm 1.6 \mathrm{E}-04$ & $\mathrm{U}$ & & ${ }^{90} \mathrm{Sr}$ & $-3.1 \mathrm{E}-04 \pm 3.2 \mathrm{E}-04$ & $\mathrm{U}$ \\
\hline & ${ }^{234} \mathrm{U}$ & $1.1 \mathrm{E}-05 \pm 8.6 \mathrm{E}-06$ & & & ${ }^{234} \mathrm{U}$ & $2.3 \mathrm{E}-05 \pm 1.6 \mathrm{E}-05$ & \\
\hline & ${ }^{235} \mathrm{U}$ & $3.0 \mathrm{E}-06 \pm 6.1 \mathrm{E}-06$ & $\mathrm{U}$ & & ${ }^{235} \mathrm{U}$ & $1.8 \mathrm{E}-06 \pm 6.3 \mathrm{E}-06$ & $\mathrm{U}$ \\
\hline & ${ }^{238} \mathrm{U}$ & $1.1 \mathrm{E}-05 \pm 1.0 \mathrm{E}-05$ & $\mathrm{U}$ & & ${ }^{238} \mathrm{U}$ & $1.0 \mathrm{E}-05 \pm 1.0 \mathrm{E}-05$ & $\mathrm{U}$ \\
\hline & ${ }^{65} \mathrm{Zn}$ & $2.6 \mathrm{E}-04 \pm 3.5 \mathrm{E}-04$ & $\mathrm{U}$ & & ${ }^{65} \mathrm{Zn}$ & $-6.3 \mathrm{E}-05 \pm 3.9 \mathrm{E}-04$ & $\mathrm{U}$ \\
\hline N494 (100-F) & ${ }^{144} \mathrm{Ce}$ & $-5.8 \mathrm{E}-04 \pm 1.3 \mathrm{E}-03$ & $\bar{U}$ & N495 (100-F) & ${ }^{144} \mathrm{Ce}$ & $7.4 \mathrm{E}-04 \pm 1.6 \mathrm{E}-03$ & $\bar{U}$ \\
\hline Composite Period & ${ }^{60} \mathrm{Co}$ & $6.1 \mathrm{E}-05 \pm 1.2 \mathrm{E}-04$ & $\mathrm{U}$ & Composite Period & ${ }^{60} \mathrm{Co}$ & $2.7 \mathrm{E}-04 \pm 2.5 \mathrm{E}-04$ & $\mathrm{U}$ \\
\hline \multirow[t]{16}{*}{$09 / 16 / 03$ to $12 / 22 / 03$} & ${ }^{134} \mathrm{Cs}$ & $1.3 \mathrm{E}-04 \pm 1.7 \mathrm{E}-04$ & $\mathrm{U}$ & $01 / 07 / 03$ to $03 / 18 / 03$ & ${ }^{134} \mathrm{Cs}$ & $7.3 \mathrm{E}-05 \pm 2.3 \mathrm{E}-04$ & $\mathrm{U}$ \\
\hline & ${ }^{137} \mathrm{Cs}$ & $3.1 \mathrm{E}-05 \pm 1.2 \mathrm{E}-04$ & $\mathrm{U}$ & & ${ }^{137} \mathrm{Cs}$ & $-6.9 \mathrm{E}-05 \pm 2.0 \mathrm{E}-04$ & $\mathrm{U}$ \\
\hline & ${ }^{152} \mathrm{Eu}$ & $-1.8 \mathrm{E}-05 \pm 1.8 \mathrm{E}-04$ & $\mathrm{U}$ & & ${ }^{152} \mathrm{Eu}$ & $3.8 \mathrm{E}-04 \pm 4.4 \mathrm{E}-04$ & $\mathrm{U}$ \\
\hline & ${ }^{154} \mathrm{Eu}$ & $-2.9 \mathrm{E}-04 \pm 4.3 \mathrm{E}-04$ & $\mathrm{U}$ & & ${ }^{154} \mathrm{Eu}$ & $-6.4 \mathrm{E}-04 \pm 6.7 \mathrm{E}-04$ & $\mathrm{U}$ \\
\hline & ${ }^{155} \mathrm{Eu}$ & $-1.4 \mathrm{E}-04 \pm 3.4 \mathrm{E}-04$ & $\mathrm{U}$ & & ${ }^{155} \mathrm{Eu}$ & $-2.7 \mathrm{E}-04 \pm 4.7 \mathrm{E}-04$ & $\mathrm{U}$ \\
\hline & ${ }^{238} \mathrm{Pu}$ & $-4.4 \mathrm{E}-06 \pm 2.1 \mathrm{E}-05$ & $\mathrm{U}$ & & ${ }^{238} \mathrm{Pu}$ & $3.5 \mathrm{E}-06 \pm 1.4 \mathrm{E}-05$ & $\mathrm{U}$ \\
\hline & ${ }^{239 / 240} \mathrm{Pu}$ & $-1.5 \mathrm{E}-06 \pm 3.0 \mathrm{E}-06$ & U & & ${ }^{239 / 240} \mathrm{Pu}$ & $3.5 \mathrm{E}-06 \pm 7.1 \mathrm{E}-06$ & $\mathrm{U}$ \\
\hline & ${ }^{103} \mathrm{Ru}$ & $-1.4 \mathrm{E}-05 \pm 9.8 \mathrm{E}-05$ & $\mathrm{U}$ & & ${ }^{103} \mathrm{Ru}$ & $1.1 \mathrm{E}-04 \pm 2.1 \mathrm{E}-04$ & $\mathrm{U}$ \\
\hline & ${ }^{106} \mathrm{Ru}$ & $-3.3 \mathrm{E}-04 \pm 1.0 \mathrm{E}-03$ & $\mathrm{U}$ & & ${ }^{106} \mathrm{Ru}$ & $-2.8 \mathrm{E}-04 \pm 1.9 \mathrm{E}-03$ & $\mathrm{U}$ \\
\hline & ${ }^{125} \mathrm{Sb}$ & $3.9 \mathrm{E}-05 \pm 3.0 \mathrm{E}-04$ & $\mathrm{U}$ & & ${ }^{125} \mathrm{Sb}$ & $7.9 \mathrm{E}-05 \pm 4.6 \mathrm{E}-04$ & $\mathrm{U}$ \\
\hline & ${ }^{113} \mathrm{Sn}$ & $-5.5 \mathrm{E}-05 \pm 1.2 \mathrm{E}-04$ & $\mathrm{U}$ & & ${ }^{113} \mathrm{Sn}$ & $2.4 \mathrm{E}-04 \pm 2.5 \mathrm{E}-04$ & $\mathrm{U}$ \\
\hline & ${ }^{90} \mathrm{Sr}$ & $5.3 \mathrm{E}-05 \pm 2.2 \mathrm{E}-04$ & $\mathrm{U}$ & & ${ }^{90} \mathrm{Sr}$ & $7.4 \mathrm{E}-05 \pm 2.5 \mathrm{E}-04$ & $\mathrm{U}$ \\
\hline & ${ }^{234} \mathrm{U}$ & $1.5 \mathrm{E}-05 \pm 1.2 \mathrm{E}-05$ & & & ${ }^{234} \mathrm{U}$ & $2.2 \mathrm{E}-05 \pm 1.5 \mathrm{E}-05$ & \\
\hline & ${ }^{235} \mathrm{U}$ & $2.9 \mathrm{E}-06 \pm 4.2 \mathrm{E}-06$ & $\mathrm{U}$ & & ${ }^{235} \mathrm{U}$ & $2.2 \mathrm{E}-06 \pm 7.8 \mathrm{E}-06$ & $\mathrm{U}$ \\
\hline & ${ }^{238} \mathrm{U}$ & $1.3 \mathrm{E}-05 \pm 9.5 \mathrm{E}-06$ & & & ${ }^{238} \mathrm{U}$ & $4.1 \mathrm{E}-06 \pm 8.2 \mathrm{E}-06$ & $\mathrm{U}$ \\
\hline & ${ }^{65} \mathrm{Zn}$ & $-4.5 \mathrm{E}-04 \pm 4.6 \mathrm{E}-04$ & $\mathrm{U}$ & & ${ }^{65} \mathrm{Zn}$ & $-1.9 \mathrm{E}-04 \pm 5.1 \mathrm{E}-04$ & $\mathrm{U}$ \\
\hline
\end{tabular}

$\overline{\mathrm{RQ}}=$ Result Qualifier. $\mathrm{U}=$ The analyte was analyzed for but not detected. 
Table 2-3. Near-Facility Air Sampling Results, 2003 (pCi/m³ \pm total analytical uncertainty). (cont)

\begin{tabular}{ccrc}
\hline Location & Isotope & Result \pm Uncertainty & RQ* \\
\hline N495 (100-F) & ${ }^{144} \mathrm{Ce}$ & $-3.4 \mathrm{E}-04 \pm 1.0 \mathrm{E}-03$ & $\mathrm{U}$ \\
Composite Period & ${ }^{60} \mathrm{Co}$ & $5.5 \mathrm{E}-05 \pm 1.4 \mathrm{E}-04$ & $\mathrm{U}$ \\
03/18/03 to 06/24/03 & ${ }^{134} \mathrm{Cs}$ & $1.6 \mathrm{E}-04 \pm 1.4 \mathrm{E}-04$ & $\mathrm{U}$ \\
& ${ }^{137} \mathrm{Cs}$ & $1.3 \mathrm{E}-04 \pm 1.3 \mathrm{E}-04$ & $\mathrm{U}$ \\
& ${ }^{152} \mathrm{Eu}$ & $1.2 \mathrm{E}-04 \pm 2.6 \mathrm{E}-04$ & $\mathrm{U}$ \\
& ${ }^{154} \mathrm{Eu}$ & $-4.3 \mathrm{E}-04 \pm 4.5 \mathrm{E}-04$ & $\mathrm{U}$ \\
& ${ }^{155} \mathrm{Eu}$ & $2.0 \mathrm{E}-04 \pm 2.5 \mathrm{E}-04$ & $\mathrm{U}$ \\
& ${ }^{238} \mathrm{Pu}$ & $1.6 \mathrm{E}-05 \pm 2.4 \mathrm{E}-05$ & $\mathrm{U}$ \\
& ${ }^{239 / 240} \mathrm{Pu}$ & $1.8 \mathrm{E}-05 \pm 1.3 \mathrm{E}-05$ & \\
& ${ }^{103} \mathrm{Ru}$ & $5.3 \mathrm{E}-05 \pm 9.4 \mathrm{E}-05$ & $\mathrm{U}$ \\
& ${ }^{106} \mathrm{Ru}$ & $-4.1 \mathrm{E}-04 \pm 9.8 \mathrm{E}-04$ & $\mathrm{U}$ \\
& ${ }^{125} \mathrm{Sb}$ & $-2.7 \mathrm{E}-05 \pm 2.2 \mathrm{E}-04$ & $\mathrm{U}$ \\
& ${ }^{113} \mathrm{Sn}$ & $-1.6 \mathrm{E}-06 \pm 1.6 \mathrm{E}-05$ & $\mathrm{U}$ \\
& ${ }^{90} \mathrm{Sr}$ & $-1.4 \mathrm{E}-04 \pm 1.5 \mathrm{E}-04$ & $\mathrm{U}$ \\
& ${ }^{234} \mathrm{U}$ & $9.3 \mathrm{E}-06 \pm 9.4 \mathrm{E}-06$ & $\mathrm{U}$ \\
& ${ }^{235} \mathrm{U}$ & $8.8 \mathrm{E}-06 \pm 8.9 \mathrm{E}-06$ & $\mathrm{U}$ \\
& ${ }^{238} \mathrm{U}$ & $9.3 \mathrm{E}-06 \pm 1.0 \mathrm{E}-05$ & $\mathrm{U}$ \\
& ${ }^{63} \mathrm{Zn} \mathrm{U}$ & $-4.2 \mathrm{E}-04 \pm 4.3 \mathrm{E}-04$ & $\mathrm{U}$
\end{tabular}

\begin{tabular}{ccrc}
\hline Location & Isotope & Result \pm Uncertainty & RQ* \\
\hline N495 (100-F) & ${ }^{144} \mathrm{Ce}$ & $1.3 \mathrm{E}-03 \pm 1.7 \mathrm{E}-03$ & $\mathrm{U}$ \\
Composite Period & ${ }^{60} \mathrm{Co}$ & $8.3 \mathrm{E}-05 \pm 1.6 \mathrm{E}-04$ & $\mathrm{U}$ \\
06/24/03 to 09/16/03 & ${ }^{134} \mathrm{Cs}$ & $-1.7 \mathrm{E}-04 \pm 2.1 \mathrm{E}-04$ & $\mathrm{U}$ \\
& ${ }^{137} \mathrm{Cs}$ & $7.6 \mathrm{E}-05 \pm 1.5 \mathrm{E}-04$ & $\mathrm{U}$ \\
& ${ }^{152} \mathrm{Eu}$ & $-1.4 \mathrm{E}-04 \pm 4.6 \mathrm{E}-04$ & $\mathrm{U}$ \\
& ${ }^{154} \mathrm{Eu}$ & $1.3 \mathrm{E}-05 \pm 1.3 \mathrm{E}-04$ & $\mathrm{U}$ \\
& ${ }^{155} \mathrm{Eu}$ & $6.8 \mathrm{E}-07 \pm 6.8 \mathrm{E}-06$ & $\mathrm{U}$ \\
& ${ }^{238} \mathrm{Pu}$ & $1.3 \mathrm{E}-05 \pm 1.8 \mathrm{E}-05$ & $\mathrm{U}$ \\
& ${ }^{239 / 240} \mathrm{Pu}$ & $3.7 \mathrm{E}-06 \pm 9.4 \mathrm{E}-06$ & $\mathrm{U}$ \\
& ${ }^{103} \mathrm{Ru}$ & $2.5 \mathrm{E}-05 \pm 1.4 \mathrm{E}-04$ & $\mathrm{U}$ \\
& ${ }^{106} \mathrm{Ru}$ & $-7.9 \mathrm{E}-04 \pm 1.4 \mathrm{E}-03$ & $\mathrm{U}$ \\
& ${ }^{125} \mathrm{Sb}$ & $6.0 \mathrm{E}-05 \pm 3.7 \mathrm{E}-04$ & $\mathrm{U}$ \\
& ${ }^{113} \mathrm{Sn}$ & $8.4 \mathrm{E}-06 \pm 8.4 \mathrm{E}-05$ & $\mathrm{U}$ \\
& ${ }^{90} \mathrm{Sr}$ & $-3.9 \mathrm{E}-04 \pm 4.0 \mathrm{E}-04$ & $\mathrm{U}$ \\
& ${ }^{234} \mathrm{U}$ & $2.9 \mathrm{E}-05 \pm 1.7 \mathrm{E}-05$ & \\
& ${ }^{235} \mathrm{U}$ & $2.0 \mathrm{E}-06 \pm 6.9 \mathrm{E}-06$ & $\mathrm{U}$ \\
& ${ }^{238} \mathrm{U}$ & $2.1 \mathrm{E}-05 \pm 1.4 \mathrm{E}-05$ & \\
& ${ }^{65} \mathrm{Zn}$ & $9.8 \mathrm{E}-05 \pm 2.6 \mathrm{E}-04$ & $\mathrm{U}$
\end{tabular}

$\overline{\mathrm{RQ}}=$ Result Qualifier. $\mathrm{U}=$ The analyte was analyzed for but not detected. 
Table 2-3. Near-Facility Air Sampling Results, 2003 (pCi/m³ \pm total analytical uncertainty). (cont)

\begin{tabular}{|c|c|c|c|c|c|c|c|}
\hline Location & Isotope & Result \pm Uncertainty & RQ* & Location & Isotope & Result \pm Uncertainty & RQ* \\
\hline N520 (100-F) & ${ }^{144} \mathrm{Ce}$ & $2.2 \mathrm{E}-04 \pm 1.3 \mathrm{E}-03$ & $\mathrm{U}$ & N521 (100-F) & ${ }^{144} \mathrm{Ce}$ & $9.8 \mathrm{E}-04 \pm 1.2 \mathrm{E}-03$ & $\mathrm{U}$ \\
\hline Composite Period & ${ }^{60} \mathrm{Co}$ & $-3.8 \mathrm{E}-05 \pm 1.6 \mathrm{E}-04$ & $\mathrm{U}$ & Composite Period & ${ }^{60} \mathrm{Co}$ & $4.1 \mathrm{E}-05 \pm 1.2 \mathrm{E}-04$ & $\mathrm{U}$ \\
\hline \multirow[t]{16}{*}{$01 / 07 / 03$ to $04 / 30 / 03$} & ${ }^{134} \mathrm{Cs}$ & $5.3 \mathrm{E}-05 \pm 1.3 \mathrm{E}-04$ & $\mathrm{U}$ & $01 / 07 / 03$ to $04 / 30 / 03$ & ${ }^{134} \mathrm{Cs}$ & $-4.0 \mathrm{E}-05 \pm 1.3 \mathrm{E}-04$ & $\mathrm{U}$ \\
\hline & ${ }^{137} \mathrm{Cs}$ & $1.5 \mathrm{E}-04 \pm 1.4 \mathrm{E}-04$ & $\mathrm{U}$ & & ${ }^{137} \mathrm{Cs}$ & $5.3 \mathrm{E}-06 \pm 5.4 \mathrm{E}-05$ & $\mathrm{U}$ \\
\hline & ${ }^{152} \mathrm{Eu}$ & $-6.3 \mathrm{E}-05 \pm 3.1 \mathrm{E}-04$ & $\mathrm{U}$ & & ${ }^{152} \mathrm{Eu}$ & $-2.3 \mathrm{E}-04 \pm 3.4 \mathrm{E}-04$ & $\mathrm{U}$ \\
\hline & ${ }^{154} \mathrm{Eu}$ & $3.2 \mathrm{E}-04 \pm 4.3 \mathrm{E}-04$ & $\mathrm{U}$ & & ${ }^{154} \mathrm{Eu}$ & $1.9 \mathrm{E}-04 \pm 3.9 \mathrm{E}-04$ & $\mathrm{U}$ \\
\hline & ${ }^{155} \mathrm{Eu}$ & $-1.2 \mathrm{E}-04 \pm 3.5 \mathrm{E}-04$ & $\mathrm{U}$ & & ${ }^{155} \mathrm{Eu}$ & $-4.8 \mathrm{E}-04 \pm 5.0 \mathrm{E}-04$ & $\mathrm{U}$ \\
\hline & ${ }^{238} \mathrm{Pu}$ & $-5.1 \mathrm{E}-06 \pm 2.1 \mathrm{E}-05$ & $\mathrm{U}$ & & ${ }^{238} \mathrm{Pu}$ & $1.5 \mathrm{E}-06 \pm 1.5 \mathrm{E}-05$ & $\mathrm{U}$ \\
\hline & ${ }^{239 / 240} \mathrm{Pu}$ & $-3.4 \mathrm{E}-06 \pm 6.8 \mathrm{E}-06$ & $\mathrm{U}$ & & ${ }^{239 / 240} \mathrm{Pu}$ & $5.4 \mathrm{E}-05 \pm 2.7 \mathrm{E}-05$ & \\
\hline & ${ }^{103} \mathrm{Ru}$ & $-9.0 \mathrm{E}-05 \pm 1.5 \mathrm{E}-04$ & $\mathrm{U}$ & & ${ }^{103} \mathrm{Ru}$ & $-4.9 \mathrm{E}-05 \pm 1.5 \mathrm{E}-04$ & $\mathrm{U}$ \\
\hline & ${ }^{106} \mathrm{Ru}$ & $1.0 \mathrm{E}-03 \pm 1.2 \mathrm{E}-03$ & $\mathrm{U}$ & & ${ }^{106} \mathrm{Ru}$ & $-6.7 \mathrm{E}-04 \pm 1.2 \mathrm{E}-03$ & $\mathrm{U}$ \\
\hline & ${ }^{125} \mathrm{Sb}$ & $-1.4 \mathrm{E}-04 \pm 3.0 \mathrm{E}-04$ & $\mathrm{U}$ & & ${ }^{125} \mathrm{Sb}$ & $9.0 \mathrm{E}-05 \pm 2.8 \mathrm{E}-04$ & $\mathrm{U}$ \\
\hline & ${ }^{113} \mathrm{Sn}$ & $8.6 \mathrm{E}-05 \pm 1.6 \mathrm{E}-04$ & $\mathrm{U}$ & & ${ }^{113} \mathrm{Sn}$ & $2.1 \mathrm{E}-05 \pm 1.6 \mathrm{E}-04$ & $\mathrm{U}$ \\
\hline & ${ }^{90} \mathrm{Sr}$ & $-2.0 \mathrm{E}-04 \pm 2.0 \mathrm{E}-04$ & $\mathrm{U}$ & & ${ }^{90} \mathrm{Sr}$ & $-1.1 \mathrm{E}-04 \pm 1.3 \mathrm{E}-04$ & $\mathrm{U}$ \\
\hline & ${ }^{234} \mathrm{U}$ & $6.1 \mathrm{E}-06 \pm 7.5 \mathrm{E}-06$ & $\mathrm{U}$ & & ${ }^{234} \mathrm{U}$ & $2.5 \mathrm{E}-05 \pm 1.4 \mathrm{E}-05$ & \\
\hline & ${ }^{235} \mathrm{U}$ & $5.3 \mathrm{E}-06 \pm 5.5 \mathrm{E}-06$ & & & ${ }^{235} \mathrm{U}$ & $7.6 \mathrm{E}-06 \pm 6.8 \mathrm{E}-06$ & \\
\hline & ${ }^{238} \mathrm{U}$ & $4.9 \mathrm{E}-06 \pm 6.0 \mathrm{E}-06$ & $\mathrm{U}$ & & ${ }^{238} \mathrm{U}$ & $5.8 \mathrm{E}-06 \pm 7.1 \mathrm{E}-06$ & $\mathrm{U}$ \\
\hline & ${ }^{65} \mathrm{Zn}$ & $-2.0 \mathrm{E}-04 \pm 3.4 \mathrm{E}-04$ & $\mathrm{U}$ & & ${ }^{65} \mathrm{Zn}$ & $-1.9 \mathrm{E}-04 \pm 2.7 \mathrm{E}-04$ & $\mathrm{U}$ \\
\hline N522 (100-F) & ${ }^{144} \mathrm{Ce}$ & $-7.7 \mathrm{E}-04 \pm 1.3 \mathrm{E}-03$ & $\bar{U}$ & N524 (100-H) & ${ }^{144} \mathrm{Ce}$ & $8.6 \mathrm{E}-04 \pm 1.8 \mathrm{E}-03$ & $\mathrm{U}$ \\
\hline Composite Period & ${ }^{60} \mathrm{Co}$ & $7.8 \mathrm{E}-06 \pm 7.8 \mathrm{E}-05$ & $\mathrm{U}$ & Composite Period & ${ }^{60} \mathrm{Co}$ & $5.2 \mathrm{E}-05 \pm 2.3 \mathrm{E}-04$ & $\mathrm{U}$ \\
\hline \multirow[t]{16}{*}{$01 / 07 / 03$ to $04 / 30 / 03$} & ${ }^{134} \mathrm{Cs}$ & $-4.8 \mathrm{E}-05 \pm 1.2 \mathrm{E}-04$ & $\mathrm{U}$ & $01 / 07 / 03$ to $03 / 17 / 03$ & ${ }^{134} \mathrm{Cs}$ & $4.9 \mathrm{E}-05 \pm 2.5 \mathrm{E}-04$ & $\mathrm{U}$ \\
\hline & ${ }^{137} \mathrm{Cs}$ & $3.5 \mathrm{E}-05 \pm 1.2 \mathrm{E}-04$ & $\mathrm{U}$ & & ${ }^{137} \mathrm{Cs}$ & $4.7 \mathrm{E}-05 \pm 2.1 \mathrm{E}-04$ & $\mathrm{U}$ \\
\hline & ${ }^{152} \mathrm{Eu}$ & $1.3 \mathrm{E}-04 \pm 3.2 \mathrm{E}-04$ & $\mathrm{U}$ & & ${ }^{152} \mathrm{Eu}$ & $-2.2 \mathrm{E}-04 \pm 4.7 \mathrm{E}-04$ & $\mathrm{U}$ \\
\hline & ${ }^{154} \mathrm{Eu}$ & $1.3 \mathrm{E}-04 \pm 3.1 \mathrm{E}-04$ & $\mathrm{U}$ & & ${ }^{154} \mathrm{Eu}$ & $4.0 \mathrm{E}-04 \pm 6.7 \mathrm{E}-04$ & $\mathrm{U}$ \\
\hline & ${ }^{155} \mathrm{Eu}$ & $7.5 \mathrm{E}-06 \pm 7.5 \mathrm{E}-05$ & $\mathrm{U}$ & & ${ }^{155} \mathrm{Eu}$ & $-1.8 \mathrm{E}-04 \pm 4.5 \mathrm{E}-04$ & $\mathrm{U}$ \\
\hline & ${ }^{238} \mathrm{Pu}$ & $-3.4 \mathrm{E}-06 \pm 2.6 \mathrm{E}-05$ & $\mathrm{U}$ & & ${ }^{238} \mathrm{Pu}$ & $1.8 \mathrm{E}-06 \pm 1.1 \mathrm{E}-05$ & $\mathrm{U}$ \\
\hline & ${ }^{239 / 240} \mathrm{Pu}$ & $1.8 \mathrm{E}-06 \pm 1.8 \mathrm{E}-06$ & $\mathrm{U}$ & & ${ }^{239 / 240} \mathrm{Pu}$ & $-3.9 \mathrm{E}-06 \pm 5.5 \mathrm{E}-06$ & $\mathrm{U}$ \\
\hline & ${ }^{103} \mathrm{Ru}$ & $8.8 \mathrm{E}-06 \pm 8.8 \mathrm{E}-05$ & $\mathrm{U}$ & & ${ }^{103} \mathrm{Ru}$ & $1.3 \mathrm{E}-04 \pm 2.1 \mathrm{E}-04$ & $\mathrm{U}$ \\
\hline & ${ }^{106} \mathrm{Ru}$ & $9.7 \mathrm{E}-04 \pm 1.2 \mathrm{E}-03$ & $\mathrm{U}$ & & ${ }^{106} \mathrm{Ru}$ & $2.9 \mathrm{E}-03 \pm 2.0 \mathrm{E}-03$ & $\mathrm{U}$ \\
\hline & ${ }^{125} \mathrm{Sb}$ & $4.8 \mathrm{E}-05 \pm 2.7 \mathrm{E}-04$ & $\mathrm{U}$ & & ${ }^{125} \mathrm{Sb}$ & $1.3 \mathrm{E}-05 \pm 1.3 \mathrm{E}-04$ & $\mathrm{U}$ \\
\hline & ${ }^{113} \mathrm{Sn}$ & $-6.8 \mathrm{E}-07 \pm 6.9 \mathrm{E}-06$ & $\mathrm{U}$ & & ${ }^{113} \mathrm{Sn}$ & $3.7 \mathrm{E}-05 \pm 2.1 \mathrm{E}-04$ & $\mathrm{U}$ \\
\hline & ${ }^{90} \mathrm{Sr}$ & $3.8 \mathrm{E}-05 \pm 1.4 \mathrm{E}-04$ & $\mathrm{U}$ & & ${ }^{90} \mathrm{Sr}$ & $-2.8 \mathrm{E}-04 \pm 2.8 \mathrm{E}-04$ & $\mathrm{U}$ \\
\hline & ${ }^{234} \mathrm{U}$ & $1.2 \mathrm{E}-05 \pm 8.4 \mathrm{E}-06$ & & & ${ }^{234} \mathrm{U}$ & $8.0 \mathrm{E}-06 \pm 9.7 \mathrm{E}-06$ & $\mathrm{U}$ \\
\hline & ${ }^{235} \mathrm{U}$ & $3.5 \mathrm{E}-06 \pm 4.3 \mathrm{E}-06$ & & & ${ }^{235} \mathrm{U}$ & $2.0 \mathrm{E}-06 \pm 2.1 \mathrm{E}-06$ & $\mathrm{U}$ \\
\hline & ${ }^{238} \mathrm{U}$ & $7.6 \mathrm{E}-06 \pm 6.4 \mathrm{E}-06$ & & & ${ }^{238} \mathrm{U}$ & $3.9 \mathrm{E}-06 \pm 7.8 \mathrm{E}-06$ & $\mathrm{U}$ \\
\hline & ${ }^{65} \mathrm{Zn}$ & $-2.1 \mathrm{E}-04 \pm 2.7 \mathrm{E}-04$ & $\mathrm{U}$ & & ${ }^{65} \mathrm{Zn}$ & $-1.6 \mathrm{E}-04 \pm 5.3 \mathrm{E}-04$ & $\mathrm{U}$ \\
\hline
\end{tabular}

$\overline{\mathrm{RQ}}=$ Result Qualifier. $\mathrm{U}=$ The analyte was analyzed for but not detected. 
Table 2-3. Near-Facility Air Sampling Results, 2003 (pCi/m³ \pm total analytical uncertainty). (cont)

\begin{tabular}{|c|c|c|c|c|c|c|c|}
\hline Location & Isotope & Result \pm Uncertainty & $\mathbf{R Q}^{*}$ & Location & Isotope & Result \pm Uncertainty & $\mathbf{R Q}^{*}$ \\
\hline N524 (100-H) & ${ }^{144} \mathrm{Ce}$ & $4.6 \mathrm{E}-04 \pm 1.0 \mathrm{E}-03$ & $\mathrm{U}$ & N524 (100-H) & ${ }^{144} \mathrm{Ce}$ & $-8.8 \mathrm{E}-04 \pm 1.6 \mathrm{E}-03$ & $\bar{U}$ \\
\hline Composite Period & ${ }^{60} \mathrm{Co}$ & $2.4 \mathrm{E}-05 \pm 1.3 \mathrm{E}-04$ & $\mathrm{U}$ & Composite Period & ${ }^{60} \mathrm{Co}$ & $6.8 \mathrm{E}-05 \pm 1.7 \mathrm{E}-04$ & $\mathrm{U}$ \\
\hline \multirow[t]{16}{*}{$03 / 17 / 03$ to $06 / 24 / 03$} & ${ }^{134} \mathrm{Cs}$ & $-3.3 \mathrm{E}-06 \pm 3.3 \mathrm{E}-05$ & $\mathrm{U}$ & $06 / 24 / 03$ to $09 / 16 / 03$ & ${ }^{134} \mathrm{Cs}$ & $-2.8 \mathrm{E}-05 \pm 1.5 \mathrm{E}-04$ & $\mathrm{U}$ \\
\hline & ${ }^{137} \mathrm{Cs}$ & $8.6 \mathrm{E}-06 \pm 8.6 \mathrm{E}-05$ & $\mathrm{U}$ & & ${ }^{137} \mathrm{Cs}$ & $1.1 \mathrm{E}-03 \pm 4.0 \mathrm{E}-04$ & \\
\hline & ${ }^{152} \mathrm{Eu}$ & $6.8 \mathrm{E}-05 \pm 2.5 \mathrm{E}-04$ & $\mathrm{U}$ & & ${ }^{152} \mathrm{Eu}$ & $8.8 \mathrm{E}-05 \pm 4.1 \mathrm{E}-04$ & $\mathrm{U}$ \\
\hline & ${ }^{154} \mathrm{Eu}$ & $-2.0 \mathrm{E}-04 \pm 3.9 \mathrm{E}-04$ & $\mathrm{U}$ & & ${ }^{154} \mathrm{Eu}$ & $2.0 \mathrm{E}-04 \pm 4.1 \mathrm{E}-04$ & $\mathrm{U}$ \\
\hline & ${ }^{155} \mathrm{Eu}$ & $5.9 \mathrm{E}-05 \pm 2.7 \mathrm{E}-04$ & $\mathrm{U}$ & & ${ }^{155} \mathrm{Eu}$ & $-1.4 \mathrm{E}-04 \pm 4.6 \mathrm{E}-04$ & $\mathrm{U}$ \\
\hline & ${ }^{238} \mathrm{Pu}$ & $-6.6 \mathrm{E}-06 \pm 2.3 \mathrm{E}-05$ & $\mathrm{U}$ & & ${ }^{238} \mathrm{Pu}$ & $2.7 \mathrm{E}-05 \pm 3.5 \mathrm{E}-05$ & $\mathrm{U}$ \\
\hline & ${ }^{239 / 240} \mathrm{Pu}$ & $3.1 \mathrm{E}-05 \pm 1.9 \mathrm{E}-05$ & & & ${ }^{239 / 240} \mathrm{Pu}$ & $1.6 \mathrm{E}-04 \pm 6.4 \mathrm{E}-05$ & \\
\hline & ${ }^{103} \mathrm{Ru}$ & $-7.3 \mathrm{E}-05 \pm 9.8 \mathrm{E}-05$ & $\mathrm{U}$ & & ${ }^{103} \mathrm{Ru}$ & $-9.8 \mathrm{E}-06 \pm 9.8 \mathrm{E}-05$ & $\mathrm{U}$ \\
\hline & ${ }^{106} \mathrm{Ru}$ & $-4.0 \mathrm{E}-04 \pm 9.2 \mathrm{E}-04$ & $\mathrm{U}$ & & ${ }^{106} \mathrm{Ru}$ & $5.4 \mathrm{E}-04 \pm 1.4 \mathrm{E}-03$ & $\mathrm{U}$ \\
\hline & ${ }^{125} \mathrm{Sb}$ & $1.4 \mathrm{E}-04 \pm 3.0 \mathrm{E}-04$ & $\mathrm{U}$ & & ${ }^{125} \mathrm{Sb}$ & $-7.8 \mathrm{E}-05 \pm 4.1 \mathrm{E}-04$ & $\mathrm{U}$ \\
\hline & ${ }^{113} \mathrm{Sn}$ & $-3.2 \mathrm{E}-05 \pm 1.1 \mathrm{E}-04$ & $\mathrm{U}$ & & ${ }^{113} \mathrm{Sn}$ & $7.8 \mathrm{E}-05 \pm 1.8 \mathrm{E}-04$ & $\mathrm{U}$ \\
\hline & ${ }^{90} \mathrm{Sr}$ & $3.4 \mathrm{E}-04 \pm 1.9 \mathrm{E}-04$ & & & ${ }^{90} \mathrm{Sr}$ & $6.7 \mathrm{E}-04 \pm 2.6 \mathrm{E}-04$ & \\
\hline & ${ }^{234} \mathrm{U}$ & $1.6 \mathrm{E}-05 \pm 1.1 \mathrm{E}-05$ & & & ${ }^{234} \mathrm{U}$ & $3.0 \mathrm{E}-05 \pm 2.0 \mathrm{E}-05$ & \\
\hline & ${ }^{235} \mathrm{U}$ & $8.8 \mathrm{E}-06 \pm 7.9 \mathrm{E}-06$ & & & ${ }^{235} \mathrm{U}$ & $1.8 \mathrm{E}-06 \pm 1.9 \mathrm{E}-06$ & $\mathrm{U}$ \\
\hline & ${ }^{238} \mathrm{U}$ & $1.1 \mathrm{E}-05 \pm 8.5 \mathrm{E}-06$ & & & ${ }^{238} \mathrm{U}$ & $2.0 \mathrm{E}-05 \pm 1.4 \mathrm{E}-05$ & \\
\hline & ${ }^{65} \mathrm{Zn}$ & $-2.9 \mathrm{E}-06 \pm 2.9 \mathrm{E}-05$ & $\mathrm{U}$ & & ${ }^{65} \mathrm{Zn}$ & $1.7 \mathrm{E}-04 \pm 3.2 \mathrm{E}-04$ & $\mathrm{U}$ \\
\hline N524 $(100-\mathrm{H})$ & ${ }^{144} \mathrm{Ce}$ & $-9.8 \mathrm{E}-04 \pm 1.3 \mathrm{E}-03$ & $\mathrm{U}$ & N525 $(100-\mathrm{H})$ & ${ }^{144} \mathrm{Ce}$ & $-4.4 \mathrm{E}-04 \pm 1.8 \mathrm{E}-03$ & $\bar{U}$ \\
\hline Composite Period & ${ }^{60} \mathrm{Co}$ & $-1.6 \mathrm{E}-04 \pm 1.6 \mathrm{E}-04$ & $\mathrm{U}$ & Composite Period & ${ }^{60} \mathrm{Co}$ & $5.2 \mathrm{E}-05 \pm 1.9 \mathrm{E}-04$ & $\mathrm{U}$ \\
\hline \multirow[t]{16}{*}{$09 / 16 / 03$ to $12 / 22 / 03$} & ${ }^{134} \mathrm{Cs}$ & $-3.3 \mathrm{E}-05 \pm 1.4 \mathrm{E}-04$ & $\mathrm{U}$ & $01 / 07 / 03$ to $03 / 17 / 03$ & ${ }^{134} \mathrm{Cs}$ & $1.5 \mathrm{E}-04 \pm 2.0 \mathrm{E}-04$ & $\mathrm{U}$ \\
\hline & ${ }^{137} \mathrm{Cs}$ & $6.2 \mathrm{E}-04 \pm 3.1 \mathrm{E}-04$ & & & ${ }^{137} \mathrm{Cs}$ & $-2.1 \mathrm{E}-04 \pm 2.2 \mathrm{E}-04$ & $\mathrm{U}$ \\
\hline & ${ }^{152} \mathrm{Eu}$ & $-1.3 \mathrm{E}-04 \pm 4.0 \mathrm{E}-04$ & $\mathrm{U}$ & & ${ }^{152} \mathrm{Eu}$ & $-6.9 \mathrm{E}-04 \pm 7.1 \mathrm{E}-04$ & $\mathrm{U}$ \\
\hline & ${ }^{154} \mathrm{Eu}$ & $-2.1 \mathrm{E}-04 \pm 4.2 \mathrm{E}-04$ & $\mathrm{U}$ & & ${ }^{154} \mathrm{Eu}$ & $-3.0 \mathrm{E}-05 \pm 3.0 \mathrm{E}-04$ & $\mathrm{U}$ \\
\hline & ${ }^{155} \mathrm{Eu}$ & $3.2 \mathrm{E}-05 \pm 3.2 \mathrm{E}-04$ & $\mathrm{U}$ & & ${ }^{155} \mathrm{Eu}$ & $2.3 \mathrm{E}-05 \pm 2.3 \mathrm{E}-04$ & $\mathrm{U}$ \\
\hline & ${ }^{238} \mathrm{Pu}$ & $-6.2 \mathrm{E}-06 \pm 2.1 \mathrm{E}-05$ & $\mathrm{U}$ & & ${ }^{238} \mathrm{Pu}$ & $2.0 \mathrm{E}-06 \pm 2.1 \mathrm{E}-06$ & $\mathrm{U}$ \\
\hline & ${ }^{239 / 240} \mathrm{Pu}$ & $4.9 \mathrm{E}-05 \pm 2.5 \mathrm{E}-05$ & & & ${ }^{239 / 240} \mathrm{Pu}$ & $-5.9 \mathrm{E}-06 \pm 1.1 \mathrm{E}-05$ & $\mathrm{U}$ \\
\hline & ${ }^{103} \mathrm{Ru}$ & $1.8 \mathrm{E}-06 \pm 1.8 \mathrm{E}-05$ & $\mathrm{U}$ & & ${ }^{103} \mathrm{Ru}$ & $2.5 \mathrm{E}-05 \pm 2.3 \mathrm{E}-04$ & $\mathrm{U}$ \\
\hline & ${ }^{106} \mathrm{Ru}$ & $-1.1 \mathrm{E}-03 \pm 1.1 \mathrm{E}-03$ & $\mathrm{U}$ & & ${ }^{106} \mathrm{Ru}$ & $-8.2 \mathrm{E}-04 \pm 1.5 \mathrm{E}-03$ & $\mathrm{U}$ \\
\hline & ${ }^{125} \mathrm{Sb}$ & $-2.3 \mathrm{E}-05 \pm 2.3 \mathrm{E}-04$ & $\mathrm{U}$ & & ${ }^{125} \mathrm{Sb}$ & $-1.4 \mathrm{E}-04 \pm 4.3 \mathrm{E}-04$ & $\mathrm{U}$ \\
\hline & ${ }^{113} \mathrm{Sn}$ & $3.6 \mathrm{E}-10 \pm 3.6 \mathrm{E}-09$ & $\mathrm{U}$ & & ${ }^{113} \mathrm{Sn}$ & $-1.3 \mathrm{E}-04 \pm 2.0 \mathrm{E}-04$ & $\mathrm{U}$ \\
\hline & ${ }^{90} \mathrm{Sr}$ & $2.3 \mathrm{E}-04 \pm 2.2 \mathrm{E}-04$ & & & ${ }^{90} \mathrm{Sr}$ & $-2.2 \mathrm{E}-04 \pm 2.3 \mathrm{E}-04$ & $\mathrm{U}$ \\
\hline & ${ }^{234} \mathrm{U}$ & $2.8 \mathrm{E}-05 \pm 1.6 \mathrm{E}-05$ & & & ${ }^{234} \mathrm{U}$ & $1.4 \mathrm{E}-05 \pm 1.2 \mathrm{E}-05$ & \\
\hline & ${ }^{235} \mathrm{U}$ & $4.2 \mathrm{E}-06 \pm 6.4 \mathrm{E}-06$ & $\mathrm{U}$ & & ${ }^{235} \mathrm{U}$ & $1.8 \mathrm{E}-06 \pm 1.9 \mathrm{E}-06$ & $\mathrm{U}$ \\
\hline & ${ }^{238} \mathrm{U}$ & $2.1 \mathrm{E}-05 \pm 1.3 \mathrm{E}-05$ & & & ${ }^{238} \mathrm{U}$ & $9.1 \mathrm{E}-06 \pm 1.0 \mathrm{E}-05$ & $\mathrm{U}$ \\
\hline & ${ }^{65} \mathrm{Zn}$ & $2.0 \mathrm{E}-04 \pm 3.4 \mathrm{E}-04$ & $\mathrm{U}$ & & ${ }^{65} \mathrm{Zn}$ & $-2.0 \mathrm{E}-05 \pm 2.0 \mathrm{E}-04$ & $\mathrm{U}$ \\
\hline
\end{tabular}

$\overline{\mathrm{RQ}}=$ Result Qualifier. $\mathrm{U}=$ The analyte was analyzed for but not detected. 
Table 2-3. Near-Facility Air Sampling Results, 2003 (pCi/m³ \pm total analytical uncertainty). (cont)

\begin{tabular}{|c|c|c|c|}
\hline Location & Isotope & Result \pm Uncertainty & $\mathbf{R Q}^{*}$ \\
\hline N525 $(100-\mathrm{H})$ & ${ }^{144} \mathrm{Ce}$ & $2.3 \mathrm{E}-04 \pm 7.5 \mathrm{E}-04$ & $\mathrm{U}$ \\
\hline Composite Period & ${ }^{60} \mathrm{Co}$ & $3.9 \mathrm{E}-05 \pm 8.9 \mathrm{E}-05$ & $\mathrm{U}$ \\
\hline \multirow[t]{16}{*}{$03 / 17 / 03$ to $06 / 24 / 03$} & ${ }^{134} \mathrm{Cs}$ & $-1.3 \mathrm{E}-05 \pm 7.2 \mathrm{E}-05$ & $\mathrm{U}$ \\
\hline & ${ }^{137} \mathrm{Cs}$ & $-5.7 \mathrm{E}-06 \pm 5.7 \mathrm{E}-05$ & $\mathrm{U}$ \\
\hline & ${ }^{152} \mathrm{Eu}$ & $-6.5 \mathrm{E}-05 \pm 1.6 \mathrm{E}-04$ & $\mathrm{U}$ \\
\hline & ${ }^{154} \mathrm{Eu}$ & $-5.0 \mathrm{E}-05 \pm 2.4 \mathrm{E}-04$ & $\mathrm{U}$ \\
\hline & ${ }^{155} \mathrm{Eu}$ & $8.6 \mathrm{E}-05 \pm 1.6 \mathrm{E}-04$ & $\mathrm{U}$ \\
\hline & ${ }^{238} \mathrm{Pu}$ & $-1.0 \mathrm{E}-05 \pm 1.9 \mathrm{E}-05$ & $\mathrm{U}$ \\
\hline & ${ }^{239 / 240} \mathrm{Pu}$ & $-1.4 \mathrm{E}-06 \pm 5.0 \mathrm{E}-06$ & $\mathrm{U}$ \\
\hline & ${ }^{103} \mathrm{Ru}$ & $3.8 \mathrm{E}-05 \pm 6.2 \mathrm{E}-05$ & $\mathrm{U}$ \\
\hline & ${ }^{106} \mathrm{Ru}$ & $-7.4 \mathrm{E}-04 \pm 7.7 \mathrm{E}-04$ & $\mathrm{U}$ \\
\hline & ${ }^{125} \mathrm{Sb}$ & $-4.8 \mathrm{E}-05 \pm 1.6 \mathrm{E}-04$ & $\mathrm{U}$ \\
\hline & ${ }^{113} \mathrm{Sn}$ & $-2.7 \mathrm{E}-05 \pm 7.3 \mathrm{E}-05$ & $\mathrm{U}$ \\
\hline & ${ }^{90} \mathrm{Sr}$ & $-1.2 \mathrm{E}-04 \pm 1.6 \mathrm{E}-04$ & $\mathrm{U}$ \\
\hline & ${ }^{234} \mathrm{U}$ & $9.6 \mathrm{E}-06 \pm 9.0 \mathrm{E}-06$ & $\mathrm{U}$ \\
\hline & ${ }^{235} \mathrm{U}$ & $1.3 \mathrm{E}-06 \pm 4.5 \mathrm{E}-06$ & $\mathrm{U}$ \\
\hline & ${ }^{238} \mathrm{U}$ & $1.1 \mathrm{E}-05 \pm 8.2 \mathrm{E}-06$ & \\
\hline & ${ }^{65} \mathrm{Zn}$ & $1.2 \mathrm{E}-05 \pm 1.2 \mathrm{E}-04$ & $\mathrm{U}$ \\
\hline N525 $(100-H)$ & ${ }^{144} \mathrm{Ce}$ & $-4.9 \mathrm{E}-04 \pm 1.1 \mathrm{E}-03$ & $\mathrm{U}$ \\
\hline Composite Period & ${ }^{60} \mathrm{Co}$ & $1.2 \mathrm{E}-04 \pm 1.5 \mathrm{E}-04$ & $\mathrm{U}$ \\
\hline \multirow[t]{16}{*}{$09 / 16 / 03$ to $12 / 22 / 03$} & ${ }^{134} \mathrm{Cs}$ & $1.0 \mathrm{E}-04 \pm 1.2 \mathrm{E}-04$ & $\mathrm{U}$ \\
\hline & ${ }^{137} \mathrm{Cs}$ & $4.0 \mathrm{E}-05 \pm 1.2 \mathrm{E}-04$ & $\mathrm{U}$ \\
\hline & ${ }^{152} \mathrm{Eu}$ & $-6.9 \mathrm{E}-05 \pm 2.7 \mathrm{E}-04$ & $\mathrm{U}$ \\
\hline & ${ }^{154} \mathrm{Eu}$ & $-8.5 \mathrm{E}-05 \pm 3.9 \mathrm{E}-04$ & $\mathrm{U}$ \\
\hline & ${ }^{155} \mathrm{Eu}$ & $1.0 \mathrm{E}-04 \pm 2.9 \mathrm{E}-04$ & $\mathrm{U}$ \\
\hline & ${ }^{238} \mathrm{Pu}$ & $4.9 \mathrm{E}-06 \pm 2.7 \mathrm{E}-05$ & $\mathrm{U}$ \\
\hline & ${ }^{239 / 240} \mathrm{Pu}$ & $3.2 \mathrm{E}-06 \pm 1.0 \mathrm{E}-05$ & $\mathrm{U}$ \\
\hline & ${ }^{103} \mathrm{Ru}$ & $1.2 \mathrm{E}-05 \pm 1.0 \mathrm{E}-04$ & $\mathrm{U}$ \\
\hline & ${ }^{106} \mathrm{Ru}$ & $-3.3 \mathrm{E}-04 \pm 9.3 \mathrm{E}-04$ & $\mathrm{U}$ \\
\hline & ${ }^{125} \mathrm{Sb}$ & $7.0 \mathrm{E}-05 \pm 2.7 \mathrm{E}-04$ & $\mathrm{U}$ \\
\hline & ${ }^{113} \mathrm{Sn}$ & $3.7 \mathrm{E}-05 \pm 1.1 \mathrm{E}-04$ & $\mathrm{U}$ \\
\hline & ${ }^{90} \mathrm{Sr}$ & $-2.7 \mathrm{E}-04 \pm 2.8 \mathrm{E}-04$ & $\mathrm{U}$ \\
\hline & ${ }^{234} \mathrm{U}$ & $3.1 \mathrm{E}-05 \pm 1.7 \mathrm{E}-05$ & \\
\hline & ${ }^{235} \mathrm{U}$ & $4.0 \mathrm{E}-06 \pm 5.0 \mathrm{E}-06$ & \\
\hline & ${ }^{238} \mathrm{U}$ & $8.7 \mathrm{E}-06 \pm 7.2 \mathrm{E}-06$ & \\
\hline & ${ }^{65} \mathrm{Zn}$ & $7.6 \mathrm{E}-05 \pm 2.9 \mathrm{E}-04$ & $\mathrm{U}$ \\
\hline
\end{tabular}

\begin{tabular}{|c|c|c|c|}
\hline Location & Isotope & Result \pm Uncertainty & RQ* \\
\hline N525 $(100-\mathrm{H})$ & ${ }^{144} \mathrm{Ce}$ & $-3.9 \mathrm{E}-04 \pm 1.7 \mathrm{E}-03$ & $\bar{U}$ \\
\hline Composite Period & ${ }^{60} \mathrm{Co}$ & $-8.5 \mathrm{E}-05 \pm 1.6 \mathrm{E}-04$ & $\mathrm{U}$ \\
\hline \multirow[t]{16}{*}{$06 / 24 / 03$ to $09 / 16 / 03$} & ${ }^{134} \mathrm{Cs}$ & $-8.6 \mathrm{E}-05 \pm 1.7 \mathrm{E}-04$ & $\mathrm{U}$ \\
\hline & ${ }^{137} \mathrm{Cs}$ & $8.1 \mathrm{E}-05 \pm 1.6 \mathrm{E}-04$ & $\mathrm{U}$ \\
\hline & ${ }^{152} \mathrm{Eu}$ & $-8.9 \mathrm{E}-05 \pm 4.2 \mathrm{E}-04$ & $\mathrm{U}$ \\
\hline & ${ }^{154} \mathrm{Eu}$ & $-3.3 \mathrm{E}-05 \pm 3.3 \mathrm{E}-04$ & $\mathrm{U}$ \\
\hline & ${ }^{155} \mathrm{Eu}$ & $-1.2 \mathrm{E}-04 \pm 4.6 \mathrm{E}-04$ & U \\
\hline & ${ }^{238} \mathrm{Pu}$ & $-7.2 \mathrm{E}-06 \pm 2.7 \mathrm{E}-05$ & $\mathrm{U}$ \\
\hline & ${ }^{239 / 240} \mathrm{Pu}$ & $1.8 \mathrm{E}-06 \pm 1.8 \mathrm{E}-06$ & $\mathrm{U}$ \\
\hline & ${ }^{103} \mathrm{Ru}$ & $2.3 \mathrm{E}-05 \pm 1.5 \mathrm{E}-04$ & $\mathrm{U}$ \\
\hline & ${ }^{106} \mathrm{Ru}$ & $-1.8 \mathrm{E}-04 \pm 1.3 \mathrm{E}-03$ & $\mathrm{U}$ \\
\hline & ${ }^{125} \mathrm{Sb}$ & $-7.8 \mathrm{E}-05 \pm 3.9 \mathrm{E}-04$ & $\mathrm{U}$ \\
\hline & ${ }^{113} \mathrm{Sn}$ & $6.9 \mathrm{E}-05 \pm 1.8 \mathrm{E}-04$ & U \\
\hline & ${ }^{90} \mathrm{Sr}$ & $-3.3 \mathrm{E}-05 \pm 2.6 \mathrm{E}-04$ & $\mathrm{U}$ \\
\hline & ${ }^{234} \mathrm{U}$ & $1.9 \mathrm{E}-05 \pm 1.4 \mathrm{E}-05$ & \\
\hline & ${ }^{235} \mathrm{U}$ & $3.9 \mathrm{E}-06 \pm 7.9 \mathrm{E}-06$ & $\mathrm{U}$ \\
\hline & ${ }^{238} \mathrm{U}$ & $1.1 \mathrm{E}-05 \pm 1.1 \mathrm{E}-05$ & $\mathrm{U}$ \\
\hline & ${ }^{65} \mathrm{Zn}$ & $3.5 \mathrm{E}-04 \pm 3.6 \mathrm{E}-04$ & $\mathrm{U}$ \\
\hline N401 (100-K) & ${ }^{241} \mathrm{Am}$ & $1.2 \mathrm{E}-05 \pm 1.7 \mathrm{E}-05$ & $\mathrm{U}$ \\
\hline Composite Period & ${ }^{144} \mathrm{Ce}$ & $5.9 \mathrm{E}-05 \pm 5.9 \mathrm{E}-04$ & $\mathrm{U}$ \\
\hline \multirow[t]{18}{*}{ 01/06/03 to $06 / 23 / 03$} & ${ }^{60} \mathrm{Co}$ & $7.5 \mathrm{E}-05 \pm 8.8 \mathrm{E}-05$ & $\mathrm{U}$ \\
\hline & ${ }^{134} \mathrm{Cs}$ & $-1.2 \mathrm{E}-05 \pm 7.9 \mathrm{E}-05$ & U \\
\hline & ${ }^{137} \mathrm{Cs}$ & $-8.5 \mathrm{E}-05 \pm 8.8 \mathrm{E}-05$ & $\mathrm{U}$ \\
\hline & ${ }^{152} \mathrm{Eu}$ & $6.0 \mathrm{E}-05 \pm 1.9 \mathrm{E}-04$ & $\mathrm{U}$ \\
\hline & ${ }^{154} \mathrm{Eu}$ & $1.1 \mathrm{E}-04 \pm 2.7 \mathrm{E}-04$ & $\mathrm{U}$ \\
\hline & ${ }^{155} \mathrm{Eu}$ & $1.5 \mathrm{E}-05 \pm 1.5 \mathrm{E}-04$ & $\mathrm{U}$ \\
\hline & ${ }^{238} \mathrm{Pu}$ & $1.1 \mathrm{E}-05 \pm 3.4 \mathrm{E}-05$ & $\mathrm{U}$ \\
\hline & ${ }^{239 / 240} \mathrm{Pu}$ & $3.8 \mathrm{E}-06 \pm 7.8 \mathrm{E}-06$ & $\mathrm{U}$ \\
\hline & ${ }^{241} \mathrm{Pu}$ & $1.7 \mathrm{E}-04 \pm 1.2 \mathrm{E}-03$ & $\mathrm{U}$ \\
\hline & ${ }^{103} \mathrm{Ru}$ & $3.7 \mathrm{E}-05 \pm 6.8 \mathrm{E}-05$ & $\mathrm{U}$ \\
\hline & ${ }^{106} \mathrm{Ru}$ & $4.4 \mathrm{E}-05 \pm 4.4 \mathrm{E}-04$ & $\mathrm{U}$ \\
\hline & ${ }^{125} \mathrm{Sb}$ & $1.2 \mathrm{E}-04 \pm 1.7 \mathrm{E}-04$ & $\mathrm{U}$ \\
\hline & ${ }^{113} \mathrm{Sn}$ & $7.6 \mathrm{E}-05 \pm 8.9 \mathrm{E}-05$ & $\mathrm{U}$ \\
\hline & ${ }^{90} \mathrm{Sr}$ & $4.5 \mathrm{E}-05 \pm 9.6 \mathrm{E}-05$ & $\mathrm{U}$ \\
\hline & ${ }^{234} \mathrm{U}$ & $1.1 \mathrm{E}-05 \pm 7.0 \mathrm{E}-06$ & \\
\hline & ${ }^{235} \mathrm{U}$ & $5.5 \mathrm{E}-06 \pm 4.7 \mathrm{E}-06$ & \\
\hline & ${ }^{238} \mathrm{U}$ & $9.8 \mathrm{E}-06 \pm 6.4 \mathrm{E}-06$ & \\
\hline & 6 & $6.1 F-05+19 F-04$ & \\
\hline
\end{tabular}

$\overline{\mathrm{RQ}}=$ Result Qualifier. $\mathrm{U}=$ The analyte was analyzed for but not detected. 
Table 2-3. Near-Facility Air Sampling Results, 2003 (pCi/m³ \pm total analytical uncertainty). (cont)

\begin{tabular}{|c|c|c|c|c|c|c|c|}
\hline Location & Isotope & Result \pm Uncertainty & RQ* & Location & Isotope & Result \pm Uncertainty & $\mathbf{R Q}^{*}$ \\
\hline N401 $(100-K)$ & ${ }^{241} \mathrm{Am}$ & $1.1 \mathrm{E}-05 \pm 1.6 \mathrm{E}-05$ & $\bar{U}$ & N402 (100-K) & ${ }^{241} \mathrm{Am}$ & $7.1 \mathrm{E}-06 \pm 1.4 \mathrm{E}-05$ & $\mathrm{U}$ \\
\hline Composite Period & ${ }^{144} \mathrm{Ce}$ & $8.2 \mathrm{E}-05 \pm 6.8 \mathrm{E}-04$ & $\mathrm{U}$ & Composite Period & ${ }^{144} \mathrm{Ce}$ & $-1.3 \mathrm{E}-04 \pm 7.8 \mathrm{E}-04$ & $\mathrm{U}$ \\
\hline \multirow[t]{18}{*}{$06 / 23 / 03$ to $12 / 22 / 03$} & ${ }^{60} \mathrm{Co}$ & $4.2 \mathrm{E}-05 \pm 6.9 \mathrm{E}-05$ & $\mathrm{U}$ & $01 / 06 / 03$ to $06 / 23 / 03$ & ${ }^{60} \mathrm{Co}$ & $-4.8 \mathrm{E}-05 \pm 1.0 \mathrm{E}-04$ & $\mathrm{U}$ \\
\hline & ${ }^{134} \mathrm{Cs}$ & $-3.2 \mathrm{E}-05 \pm 6.6 \mathrm{E}-05$ & $\mathrm{U}$ & & ${ }^{134} \mathrm{Cs}$ & $-2.5 \mathrm{E}-05 \pm 9.8 \mathrm{E}-05$ & $\mathrm{U}$ \\
\hline & ${ }^{137} \mathrm{Cs}$ & $-2.8 \mathrm{E}-05 \pm 6.8 \mathrm{E}-05$ & $\mathrm{U}$ & & ${ }^{137} \mathrm{Cs}$ & $1.0 \mathrm{E}-04 \pm 9.2 \mathrm{E}-05$ & $\mathrm{U}$ \\
\hline & ${ }^{152} \mathrm{Eu}$ & $-1.0 \mathrm{E}-04 \pm 2.1 \mathrm{E}-04$ & $\mathrm{U}$ & & ${ }^{152} \mathrm{Eu}$ & $2.5 \mathrm{E}-05 \pm 2.0 \mathrm{E}-04$ & $\mathrm{U}$ \\
\hline & ${ }^{154} \mathrm{Eu}$ & $-1.8 \mathrm{E}-04 \pm 2.3 \mathrm{E}-04$ & $\mathrm{U}$ & & ${ }^{154} \mathrm{Eu}$ & $2.3 \mathrm{E}-04 \pm 2.9 \mathrm{E}-04$ & $\mathrm{U}$ \\
\hline & ${ }^{155} \mathrm{Eu}$ & $-3.1 \mathrm{E}-05 \pm 1.9 \mathrm{E}-04$ & $\mathrm{U}$ & & ${ }^{155} \mathrm{Eu}$ & $2.2 \mathrm{E}-05 \pm 2.0 \mathrm{E}-04$ & $\mathrm{U}$ \\
\hline & ${ }^{238} \mathrm{Pu}$ & $-1.3 \mathrm{E}-05 \pm 3.0 \mathrm{E}-05$ & $\mathrm{U}$ & & ${ }^{238} \mathrm{Pu}$ & $-9.0 \mathrm{E}-06 \pm 2.9 \mathrm{E}-05$ & $\mathrm{U}$ \\
\hline & ${ }^{239 / 240} \mathrm{Pu}$ & $1.2 \mathrm{E}-05 \pm 9.8 \mathrm{E}-06$ & & & ${ }^{239 / 240} \mathrm{Pu}$ & $1.1 \mathrm{E}-05 \pm 1.1 \mathrm{E}-05$ & $\mathrm{U}$ \\
\hline & ${ }^{241} \mathrm{Pu}$ & $-6.4 \mathrm{E}-04 \pm 6.6 \mathrm{E}-04$ & $\mathrm{U}$ & & ${ }^{241} \mathrm{Pu}$ & $1.1 \mathrm{E}-05 \pm 1.1 \mathrm{E}-04$ & $\mathrm{U}$ \\
\hline & ${ }^{103} \mathrm{Ru}$ & $-1.9 \mathrm{E}-05 \pm 5.9 \mathrm{E}-05$ & $\mathrm{U}$ & & ${ }^{103} \mathrm{Ru}$ & $7.1 \mathrm{E}-05 \pm 8.9 \mathrm{E}-05$ & $\mathrm{U}$ \\
\hline & ${ }^{106} \mathrm{Ru}$ & $-9.8 \mathrm{E}-05 \pm 5.5 \mathrm{E}-04$ & $\mathrm{U}$ & & ${ }^{106} \mathrm{Ru}$ & $2.8 \mathrm{E}-04 \pm 7.8 \mathrm{E}-04$ & $\mathrm{U}$ \\
\hline & ${ }^{125} \mathrm{Sb}$ & $1.4 \mathrm{E}-04 \pm 1.7 \mathrm{E}-04$ & $\mathrm{U}$ & & ${ }^{125} \mathrm{Sb}$ & $1.1 \mathrm{E}-04 \pm 1.9 \mathrm{E}-04$ & $\mathrm{U}$ \\
\hline & ${ }^{113} \mathrm{Sn}$ & $-1.4 \mathrm{E}-05 \pm 6.9 \mathrm{E}-05$ & $\mathrm{U}$ & & ${ }^{113} \mathrm{Sn}$ & $2.4 \mathrm{E}-05 \pm 9.2 \mathrm{E}-05$ & $\mathrm{U}$ \\
\hline & ${ }^{90} \mathrm{Sr}$ & $-2.9 \mathrm{E}-05 \pm 8.6 \mathrm{E}-05$ & $\mathrm{U}$ & & ${ }^{90} \mathrm{Sr}$ & $-5.7 \mathrm{E}-05 \pm 8.7 \mathrm{E}-05$ & $\mathrm{U}$ \\
\hline & ${ }^{234} \mathrm{U}$ & $1.5 \mathrm{E}-05 \pm 8.5 \mathrm{E}-06$ & & & ${ }^{234} \mathrm{U}$ & $9.0 \mathrm{E}-06 \pm 6.0 \mathrm{E}-06$ & \\
\hline & ${ }^{235} \mathrm{U}$ & $8.0 \mathrm{E}-07 \pm 1.6 \mathrm{E}-06$ & $\mathrm{U}$ & & ${ }^{235} \mathrm{U}$ & $2.9 \mathrm{E}-06 \pm 3.0 \mathrm{E}-06$ & \\
\hline & ${ }^{238} \mathrm{U}$ & $1.5 \mathrm{E}-05 \pm 8.9 \mathrm{E}-06$ & & & ${ }^{238} \mathrm{U}$ & $8.0 \mathrm{E}-06 \pm 5.5 \mathrm{E}-06$ & \\
\hline & ${ }^{65} \mathrm{Zn}$ & $2.3 \mathrm{E}-04 \pm 1.9 \mathrm{E}-04$ & $\mathrm{U}$ & & ${ }^{65} \mathrm{Zn}$ & $5.3 \mathrm{E}-05 \pm 2.2 \mathrm{E}-04$ & $\mathrm{U}$ \\
\hline N402 (100-K) & ${ }^{241} \mathrm{Am}$ & $-9.5 E-07 \pm 9.5 E-06$ & $\bar{U}$ & N403 (100-K) & ${ }^{241} \mathrm{Am}$ & $1.9 \mathrm{E}-06 \pm 1.5 \mathrm{E}-05$ & $\bar{U}$ \\
\hline Composite Period & ${ }^{144} \mathrm{Ce}$ & $-6.5 \mathrm{E}-04 \pm 6.7 \mathrm{E}-04$ & $\mathrm{U}$ & Composite Period & ${ }^{144} \mathrm{Ce}$ & $-4.3 \mathrm{E}-05 \pm 4.3 \mathrm{E}-04$ & $\mathrm{U}$ \\
\hline \multirow[t]{18}{*}{$06 / 23 / 03$ to $12 / 22 / 03$} & ${ }^{60} \mathrm{Co}$ & $-6.6 \mathrm{E}-05 \pm 8.1 \mathrm{E}-05$ & $\mathrm{U}$ & $01 / 06 / 03$ to $06 / 23 / 03$ & ${ }^{60} \mathrm{Co}$ & $1.7 \mathrm{E}-05 \pm 8.3 \mathrm{E}-05$ & $\mathrm{U}$ \\
\hline & ${ }^{134} \mathrm{Cs}$ & $-1.7 \mathrm{E}-05 \pm 6.9 \mathrm{E}-05$ & U & & ${ }^{134} \mathrm{Cs}$ & $2.8 \mathrm{E}-05 \pm 7.3 \mathrm{E}-05$ & $\mathrm{U}$ \\
\hline & ${ }^{137} \mathrm{Cs}$ & 7.7E-06 $\pm 6.7 \mathrm{E}-05$ & $\mathrm{U}$ & & ${ }^{137} \mathrm{Cs}$ & $5.4 \mathrm{E}-05 \pm 6.5 \mathrm{E}-05$ & $\mathrm{U}$ \\
\hline & ${ }^{152} \mathrm{Eu}$ & $1.4 \mathrm{E}-04 \pm 1.5 \mathrm{E}-04$ & $\mathrm{U}$ & & ${ }^{152} \mathrm{Eu}$ & $-2.0 \mathrm{E}-04 \pm 2.1 \mathrm{E}-04$ & $\mathrm{U}$ \\
\hline & ${ }^{154} \mathrm{Eu}$ & $-1.4 \mathrm{E}-04 \pm 2.3 \mathrm{E}-04$ & $\mathrm{U}$ & & ${ }^{154} \mathrm{Eu}$ & $5.6 \mathrm{E}-07 \pm 5.6 \mathrm{E}-06$ & U \\
\hline & ${ }^{155} \mathrm{Eu}$ & $-2.9 \mathrm{E}-05 \pm 1.5 \mathrm{E}-04$ & $\mathrm{U}$ & & ${ }^{155} \mathrm{Eu}$ & $1.2 \mathrm{E}-04 \pm 1.5 \mathrm{E}-04$ & $\mathrm{U}$ \\
\hline & ${ }^{238} \mathrm{Pu}$ & $1.2 \mathrm{E}-05 \pm 2.6 \mathrm{E}-05$ & $\mathrm{U}$ & & ${ }^{238} \mathrm{Pu}$ & $-1.3 \mathrm{E}-05 \pm 2.0 \mathrm{E}-05$ & $\mathrm{U}$ \\
\hline & ${ }^{239 / 240} \mathrm{Pu}$ & $3.5 \mathrm{E}-06 \pm 7.1 \mathrm{E}-06$ & $\mathrm{U}$ & & ${ }^{239 / 240} \mathrm{Pu}$ & $5.9 \mathrm{E}-06 \pm 7.2 \mathrm{E}-06$ & \\
\hline & ${ }^{241} \mathrm{Pu}$ & $4.7 \mathrm{E}-04 \pm 7.4 \mathrm{E}-04$ & $\mathrm{U}$ & & ${ }^{241} \mathrm{Pu}$ & $8.9 \mathrm{E}-04 \pm 1.1 \mathrm{E}-03$ & $\mathrm{U}$ \\
\hline & ${ }^{103} \mathrm{Ru}$ & $-1.8 \mathrm{E}-06 \pm 1.8 \mathrm{E}-05$ & $\mathrm{U}$ & & ${ }^{103} \mathrm{Ru}$ & $3.8 \mathrm{E}-05 \pm 6.1 \mathrm{E}-05$ & $\mathrm{U}$ \\
\hline & ${ }^{106} \mathrm{Ru}$ & $-6.2 \mathrm{E}-04 \pm 6.4 \mathrm{E}-04$ & $\mathrm{U}$ & & ${ }^{106} \mathrm{Ru}$ & $2.2 \mathrm{E}-04 \pm 6.1 \mathrm{E}-04$ & $\mathrm{U}$ \\
\hline & ${ }^{125} \mathrm{Sb}$ & $3.0 \mathrm{E}-05 \pm 1.3 \mathrm{E}-04$ & $\mathrm{U}$ & & ${ }^{125} \mathrm{Sb}$ & $-9.0 \mathrm{E}-05 \pm 1.5 \mathrm{E}-04$ & $\mathrm{U}$ \\
\hline & ${ }^{113} \mathrm{Sn}$ & $-2.4 \mathrm{E}-05 \pm 6.3 \mathrm{E}-05$ & $\mathrm{U}$ & & ${ }^{113} \mathrm{Sn}$ & $3.7 \mathrm{E}-05 \pm 7.1 \mathrm{E}-05$ & $\mathrm{U}$ \\
\hline & ${ }^{90} \mathrm{Sr}$ & $-1.2 \mathrm{E}-04 \pm 1.2 \mathrm{E}-04$ & $\mathrm{U}$ & & ${ }^{90} \mathrm{Sr}$ & $-3.7 \mathrm{E}-05 \pm 8.2 \mathrm{E}-05$ & $\mathrm{U}$ \\
\hline & ${ }^{234} \mathrm{U}$ & $1.1 \mathrm{E}-05 \pm 8.5 \mathrm{E}-06$ & & & ${ }^{234} \mathrm{U}$ & $7.1 \mathrm{E}-06 \pm 5.9 \mathrm{E}-06$ & \\
\hline & ${ }^{235} \mathrm{U}$ & $8.1 \mathrm{E}-07 \pm 8.4 \mathrm{E}-07$ & $\mathrm{U}$ & & ${ }^{235} \mathrm{U}$ & $2.3 \mathrm{E}-06 \pm 2.8 \mathrm{E}-06$ & \\
\hline & ${ }^{238} \mathrm{U}$ & $1.8 \mathrm{E}-05 \pm 1.0 \mathrm{E}-05$ & & & ${ }^{238} \mathrm{U}$ & $1.1 \mathrm{E}-05 \pm 6.9 \mathrm{E}-06$ & \\
\hline & ${ }^{65} \mathrm{Zn}$ & $-7.4 \mathrm{E}-06 \pm 7.4 \mathrm{E}-05$ & $\mathrm{U}$ & & ${ }^{65} \mathrm{Zn}$ & $-5.3 \mathrm{E}-05 \pm 1.5 \mathrm{E}-04$ & $\mathrm{U}$ \\
\hline
\end{tabular}

$\overline{\mathrm{RQ}}=$ Result Qualifier. $\mathrm{U}=$ The analyte was analyzed for but not detected. 
Table 2-3. Near-Facility Air Sampling Results, 2003 (pCi/m³ \pm total analytical uncertainty). (cont)

\begin{tabular}{|c|c|c|c|c|c|c|c|}
\hline Location & Isotope & Result \pm Uncertainty & $\mathbf{R Q}^{*}$ & Location & Isotope & Result \pm Uncertainty & $\mathbf{R Q}^{*}$ \\
\hline N403 (100-K) & ${ }^{241} \mathrm{Am}$ & $7.9 \mathrm{E}-06 \pm 1.5 \mathrm{E}-05$ & $\bar{U}$ & N404 (100-K) & ${ }^{241} \mathrm{Am}$ & $1.3 \mathrm{E}-05 \pm 1.6 \mathrm{E}-05$ & $\bar{U}$ \\
\hline Composite Period & ${ }^{144} \mathrm{Ce}$ & $2.7 \mathrm{E}-04 \pm 6.8 \mathrm{E}-04$ & $\mathrm{U}$ & Composite Period & ${ }^{144} \mathrm{Ce}$ & $-3.6 \mathrm{E}-05 \pm 3.6 \mathrm{E}-04$ & U \\
\hline \multirow[t]{18}{*}{$06 / 23 / 03$ to $12 / 22 / 03$} & ${ }^{60} \mathrm{Co}$ & $8.1 \mathrm{E}-05 \pm 9.3 \mathrm{E}-05$ & $\mathrm{U}$ & $01 / 06 / 03$ to $06 / 23 / 03$ & ${ }^{60} \mathrm{Co}$ & $-3.2 \mathrm{E}-05 \pm 9.3 \mathrm{E}-05$ & $\mathrm{U}$ \\
\hline & ${ }^{134} \mathrm{Cs}$ & $3.2 \mathrm{E}-05 \pm 8.3 \mathrm{E}-05$ & $\mathrm{U}$ & & ${ }^{134} \mathrm{Cs}$ & $2.2 \mathrm{E}-05 \pm 8.5 \mathrm{E}-05$ & $\mathrm{U}$ \\
\hline & ${ }^{137} \mathrm{Cs}$ & $3.1 \mathrm{E}-05 \pm 7.5 \mathrm{E}-05$ & $\mathrm{U}$ & & ${ }^{137} \mathrm{Cs}$ & $-1.2 \mathrm{E}-04 \pm 1.2 \mathrm{E}-04$ & $\mathrm{U}$ \\
\hline & ${ }^{152} \mathrm{Eu}$ & $1.2 \mathrm{E}-04 \pm 1.8 \mathrm{E}-04$ & $\mathrm{U}$ & & ${ }^{152} \mathrm{Eu}$ & $1.6 \mathrm{E}-04 \pm 2.1 \mathrm{E}-04$ & $\mathrm{U}$ \\
\hline & ${ }^{154} \mathrm{Eu}$ & $1.1 \mathrm{E}-04 \pm 2.6 \mathrm{E}-04$ & $\mathrm{U}$ & & ${ }^{154} \mathrm{Eu}$ & $3.8 \mathrm{E}-04 \pm 3.1 \mathrm{E}-04$ & $\mathrm{U}$ \\
\hline & ${ }^{155} \mathrm{Eu}$ & $6.4 \mathrm{E}-05 \pm 1.7 \mathrm{E}-04$ & $\mathrm{U}$ & & ${ }^{155} \mathrm{Eu}$ & $9.2 \mathrm{E}-05 \pm 2.1 \mathrm{E}-04$ & $\mathrm{U}$ \\
\hline & ${ }^{238} \mathrm{Pu}$ & $-1.6 \mathrm{E}-06 \pm 1.6 \mathrm{E}-05$ & $\mathrm{U}$ & & ${ }^{238} \mathrm{Pu}$ & $2.4 \mathrm{E}-05 \pm 2.9 \mathrm{E}-05$ & $\mathrm{U}$ \\
\hline & ${ }^{239 / 240} \mathrm{Pu}$ & $4.6 \mathrm{E}-06 \pm 7.0 \mathrm{E}-06$ & $\mathrm{U}$ & & ${ }^{239 / 240} \mathrm{Pu}$ & $9.7 \mathrm{E}-06 \pm 1.2 \mathrm{E}-05$ & $\mathrm{U}$ \\
\hline & ${ }^{241} \mathrm{Pu}$ & $-4.2 \mathrm{E}-04 \pm 4.4 \mathrm{E}-04$ & $\mathrm{U}$ & & ${ }^{241} \mathrm{Pu}$ & $2.8 \mathrm{E}-04 \pm 1.2 \mathrm{E}-03$ & $\mathrm{U}$ \\
\hline & ${ }^{103} \mathrm{Ru}$ & $-5.5 \mathrm{E}-05 \pm 7.1 \mathrm{E}-05$ & $\mathrm{U}$ & & ${ }^{103} \mathrm{Ru}$ & $-1.5 \mathrm{E}-05 \pm 6.9 \mathrm{E}-05$ & $\mathrm{U}$ \\
\hline & ${ }^{106} \mathrm{Ru}$ & $1.9 \mathrm{E}-04 \pm 6.2 \mathrm{E}-04$ & $\mathrm{U}$ & & ${ }^{106} \mathrm{Ru}$ & $-4.8 \mathrm{E}-04 \pm 7.2 \mathrm{E}-04$ & $\mathrm{U}$ \\
\hline & ${ }^{125} \mathrm{Sb}$ & $-8.1 \mathrm{E}-05 \pm 1.7 \mathrm{E}-04$ & $\mathrm{U}$ & & ${ }^{125} \mathrm{Sb}$ & $-3.7 \mathrm{E}-05 \pm 1.9 \mathrm{E}-04$ & $\mathrm{U}$ \\
\hline & ${ }^{113} \mathrm{Sn}$ & $2.3 \mathrm{E}-05 \pm 7.8 \mathrm{E}-05$ & $\mathrm{U}$ & & ${ }^{113} \mathrm{Sn}$ & $4.4 \mathrm{E}-05 \pm 8.6 \mathrm{E}-05$ & $\mathrm{U}$ \\
\hline & ${ }^{90} \mathrm{Sr}$ & $1.0 \mathrm{E}-04 \pm 9.9 \mathrm{E}-05$ & & & ${ }^{90} \mathrm{Sr}$ & $1.6 \mathrm{E}-05 \pm 9.9 \mathrm{E}-05$ & $\mathrm{U}$ \\
\hline & ${ }^{234} \mathrm{U}$ & $1.6 \mathrm{E}-05 \pm 9.7 \mathrm{E}-06$ & & & ${ }^{234} \mathrm{U}$ & $1.3 \mathrm{E}-05 \pm 8.2 \mathrm{E}-06$ & \\
\hline & ${ }^{235} \mathrm{U}$ & $5.2 \mathrm{E}-06 \pm 5.3 \mathrm{E}-06$ & $\mathrm{U}$ & & ${ }^{235} \mathrm{U}$ & $1.7 \mathrm{E}-06 \pm 2.4 \mathrm{E}-06$ & $\mathrm{U}$ \\
\hline & ${ }^{238} \mathrm{U}$ & $1.5 \mathrm{E}-05 \pm 9.2 \mathrm{E}-06$ & & & ${ }^{238} \mathrm{U}$ & $4.6 \mathrm{E}-06 \pm 4.2 \mathrm{E}-06$ & \\
\hline & ${ }^{65} \mathrm{Zn}$ & $5.2 \mathrm{E}-05 \pm 2.2 \mathrm{E}-04$ & $\mathrm{U}$ & & ${ }^{65} \mathrm{Zn}$ & $-3.1 \mathrm{E}-05 \pm 1.9 \mathrm{E}-04$ & $\mathrm{U}$ \\
\hline N404 (100-K) & ${ }^{241} \mathrm{Am}$ & $-5.5 \mathrm{E}-06 \pm 1.3 \mathrm{E}-05$ & $\mathrm{U}$ & N476 (100-K) & ${ }^{241} \mathrm{Am}$ & $1.1 \mathrm{E}-05 \pm 1.5 \mathrm{E}-05$ & $\mathrm{U}$ \\
\hline Composite Period & ${ }^{144} \mathrm{Ce}$ & $1.2 \mathrm{E}-04 \pm 5.2 \mathrm{E}-04$ & $\mathrm{U}$ & Composite Period & ${ }^{144} \mathrm{Ce}$ & $7.6 \mathrm{E}-04 \pm 7.9 \mathrm{E}-04$ & $\mathrm{U}$ \\
\hline \multirow[t]{18}{*}{ 06/23/03 to $12 / 22 / 03$} & ${ }^{60} \mathrm{Co}$ & $1.8 \mathrm{E}-05 \pm 8.7 \mathrm{E}-05$ & $\mathrm{U}$ & $01 / 06 / 03$ to $06 / 23 / 03$ & ${ }^{60} \mathrm{Co}$ & $4.8 \mathrm{E}-05 \pm 9.9 \mathrm{E}-05$ & $\mathrm{U}$ \\
\hline & ${ }^{134} \mathrm{Cs}$ & $-3.3 \mathrm{E}-05 \pm 7.0 \mathrm{E}-05$ & $\mathrm{U}$ & & ${ }^{134} \mathrm{Cs}$ & $-2.9 \mathrm{E}-05 \pm 8.7 \mathrm{E}-05$ & $\mathrm{U}$ \\
\hline & ${ }^{137} \mathrm{Cs}$ & $2.8 \mathrm{E}-05 \pm 5.7 \mathrm{E}-05$ & $\mathrm{U}$ & & ${ }^{137} \mathrm{Cs}$ & $-2.1 \mathrm{E}-05 \pm 7.8 \mathrm{E}-05$ & $\mathrm{U}$ \\
\hline & ${ }^{152} \mathrm{Eu}$ & $-6.1 \mathrm{E}-05 \pm 1.4 \mathrm{E}-04$ & $\mathrm{U}$ & & ${ }^{152} \mathrm{Eu}$ & $9.0 \mathrm{E}-05 \pm 2.0 \mathrm{E}-04$ & $\mathrm{U}$ \\
\hline & ${ }^{154} \mathrm{Eu}$ & $-1.5 \mathrm{E}-04 \pm 2.2 \mathrm{E}-04$ & $\mathrm{U}$ & & ${ }^{154} \mathrm{Eu}$ & $-6.7 \mathrm{E}-05 \pm 2.6 \mathrm{E}-04$ & $\mathrm{U}$ \\
\hline & ${ }^{155} \mathrm{Eu}$ & $1.5 \mathrm{E}-05 \pm 1.3 \mathrm{E}-04$ & $\mathrm{U}$ & & ${ }^{155} \mathrm{Eu}$ & $-1.3 \mathrm{E}-05 \pm 1.3 \mathrm{E}-04$ & $\mathrm{U}$ \\
\hline & ${ }^{238} \mathrm{Pu}$ & $-3.2 \mathrm{E}-06 \pm 2.5 \mathrm{E}-05$ & $\mathrm{U}$ & & ${ }^{238} \mathrm{Pu}$ & $3.8 \mathrm{E}-06 \pm 3.0 \mathrm{E}-05$ & $\mathrm{U}$ \\
\hline & ${ }^{239 / 240} \mathrm{Pu}$ & $-6.4 \mathrm{E}-06 \pm 7.8 \mathrm{E}-06$ & $\mathrm{U}$ & & ${ }^{239 / 240} \mathrm{Pu}$ & $1.9 \mathrm{E}-06 \pm 3.8 \mathrm{E}-06$ & $\mathrm{U}$ \\
\hline & ${ }^{241} \mathrm{Pu}$ & $-4.4 \mathrm{E}-04 \pm 4.6 \mathrm{E}-04$ & U & & ${ }^{241} \mathrm{Pu}$ & $3.6 \mathrm{E}-04 \pm 1.1 \mathrm{E}-03$ & $\mathrm{U}$ \\
\hline & ${ }^{103} \mathrm{Ru}$ & $9.2 \mathrm{E}-06 \pm 5.2 \mathrm{E}-05$ & $\mathrm{U}$ & & ${ }^{103} \mathrm{Ru}$ & $-7.0 \mathrm{E}-05 \pm 8.9 \mathrm{E}-05$ & $\mathrm{U}$ \\
\hline & ${ }^{106} \mathrm{Ru}$ & $4.0 \mathrm{E}-05 \pm 4.0 \mathrm{E}-04$ & $\mathrm{U}$ & & ${ }^{106} \mathrm{Ru}$ & $-2.0 \mathrm{E}-05 \pm 2.0 \mathrm{E}-04$ & $\mathrm{U}$ \\
\hline & ${ }^{125} \mathrm{Sb}$ & $4.6 \mathrm{E}-05 \pm 1.3 \mathrm{E}-04$ & $\mathrm{U}$ & & ${ }^{125} \mathrm{Sb}$ & $2.3 \mathrm{E}-05 \pm 1.9 \mathrm{E}-04$ & $\mathrm{U}$ \\
\hline & ${ }^{113} \mathrm{Sn}$ & $6.2 \mathrm{E}-06 \pm 6.2 \mathrm{E}-05$ & $\mathrm{U}$ & & ${ }^{113} \mathrm{Sn}$ & $-3.3 \mathrm{E}-05 \pm 9.2 \mathrm{E}-05$ & $\mathrm{U}$ \\
\hline & ${ }^{90} \mathrm{Sr}$ & $-9.1 \mathrm{E}-05 \pm 9.4 \mathrm{E}-05$ & $\mathrm{U}$ & & ${ }^{90} \mathrm{Sr}$ & $-5.3 \mathrm{E}-05 \pm 7.7 \mathrm{E}-05$ & $\mathrm{U}$ \\
\hline & ${ }^{234} \mathrm{U}$ & $1.8 \mathrm{E}-05 \pm 9.8 \mathrm{E}-06$ & & & ${ }^{234} \mathrm{U}$ & $1.1 \mathrm{E}-05 \pm 7.6 \mathrm{E}-06$ & \\
\hline & ${ }^{235} \mathrm{U}$ & $-7.0 \mathrm{E}-07 \pm 1.4 \mathrm{E}-06$ & $\mathrm{U}$ & & ${ }^{235} \mathrm{U}$ & $1.6 \mathrm{E}-06 \pm 4.0 \mathrm{E}-06$ & $\mathrm{U}$ \\
\hline & ${ }^{238} \mathrm{U}$ & $6.7 \mathrm{E}-06 \pm 5.3 \mathrm{E}-06$ & & & ${ }^{238} \mathrm{U}$ & $5.9 \mathrm{E}-06 \pm 5.5 \mathrm{E}-06$ & $\mathrm{U}$ \\
\hline & ${ }^{65} \mathrm{Zn}$ & $1.2 \mathrm{E}-05 \pm 1.3 \mathrm{E}-04$ & $\mathrm{U}$ & & ${ }^{65} \mathrm{Zn}$ & $-2.3 \mathrm{E}-04 \pm 2.4 \mathrm{E}-04$ & $\mathrm{U}$ \\
\hline
\end{tabular}

$\overline{\mathrm{RQ}}=$ Result Qualifier. $\mathrm{U}=$ The analyte was analyzed for but not detected. 
Table 2-3. Near-Facility Air Sampling Results, 2003 (pCi/m³ \pm total analytical uncertainty). (cont)

\begin{tabular}{|c|c|c|c|c|c|c|c|}
\hline Location & Isotope & Result \pm Uncertainty & $\mathbf{R Q}^{*}$ & Location & Isotope & Result \pm Uncertainty & RQ* \\
\hline N476 (100-K) & ${ }^{241} \mathrm{Am}$ & $8.3 \mathrm{E}-06 \pm 1.4 \mathrm{E}-05$ & $\bar{U}$ & N477 (100-K) & ${ }^{241} \mathrm{Am}$ & $1.1 \mathrm{E}-06 \pm 1.1 \mathrm{E}-05$ & $\bar{U}$ \\
\hline Composite Period & ${ }^{144} \mathrm{Ce}$ & $2.1 \mathrm{E}-04 \pm 7.9 \mathrm{E}-04$ & $\mathrm{U}$ & Composite Period & ${ }^{144} \mathrm{Ce}$ & $2.5 \mathrm{E}-04 \pm 7.7 \mathrm{E}-04$ & $\mathrm{U}$ \\
\hline \multirow[t]{18}{*}{$06 / 23 / 03$ to $12 / 22 / 03$} & ${ }^{60} \mathrm{Co}$ & $5.9 \mathrm{E}-05 \pm 9.4 \mathrm{E}-05$ & $\mathrm{U}$ & $01 / 06 / 03$ to $06 / 23 / 03$ & ${ }^{60} \mathrm{Co}$ & $5.0 \mathrm{E}-05 \pm 7.9 \mathrm{E}-05$ & $\mathrm{U}$ \\
\hline & ${ }^{134} \mathrm{Cs}$ & $-3.3 \mathrm{E}-05 \pm 9.6 \mathrm{E}-05$ & $\mathrm{U}$ & & ${ }^{134} \mathrm{Cs}$ & $-8.6 \mathrm{E}-06 \pm 7.7 \mathrm{E}-05$ & $\mathrm{U}$ \\
\hline & ${ }^{137} \mathrm{Cs}$ & $9.1 \mathrm{E}-05 \pm 8.6 \mathrm{E}-05$ & $\mathrm{U}$ & & ${ }^{137} \mathrm{Cs}$ & $-2.5 \mathrm{E}-05 \pm 7.5 \mathrm{E}-05$ & $\mathrm{U}$ \\
\hline & ${ }^{152} \mathrm{Eu}$ & $7.1 \mathrm{E}-05 \pm 2.1 \mathrm{E}-04$ & $\mathrm{U}$ & & ${ }^{152} \mathrm{Eu}$ & $2.3 \mathrm{E}-05 \pm 2.2 \mathrm{E}-04$ & $\mathrm{U}$ \\
\hline & ${ }^{154} \mathrm{Eu}$ & $-2.4 \mathrm{E}-04 \pm 2.7 \mathrm{E}-04$ & $\mathrm{U}$ & & ${ }^{154} \mathrm{Eu}$ & $-2.5 \mathrm{E}-04 \pm 2.5 \mathrm{E}-04$ & $\mathrm{U}$ \\
\hline & ${ }^{155} \mathrm{Eu}$ & $1.3 \mathrm{E}-04 \pm 1.9 \mathrm{E}-04$ & $\mathrm{U}$ & & ${ }^{155} \mathrm{Eu}$ & $-8.6 \mathrm{E}-05 \pm 2.2 \mathrm{E}-04$ & $\mathrm{U}$ \\
\hline & ${ }^{238} \mathrm{Pu}$ & $1.1 \mathrm{E}-05 \pm 2.1 \mathrm{E}-05$ & $\mathrm{U}$ & & ${ }^{238} \mathrm{Pu}$ & $-6.1 \mathrm{E}-06 \pm 3.3 \mathrm{E}-05$ & $\mathrm{U}$ \\
\hline & ${ }^{239 / 240} \mathrm{Pu}$ & $-3.2 \mathrm{E}-06 \pm 6.6 \mathrm{E}-06$ & $\mathrm{U}$ & & ${ }^{239 / 240} \mathrm{Pu}$ & $-4.0 \mathrm{E}-06 \pm 1.0 \mathrm{E}-05$ & $\mathrm{U}$ \\
\hline & ${ }^{241} \mathrm{Pu}$ & $3.8 \mathrm{E}-05 \pm 3.8 \mathrm{E}-04$ & $\mathrm{U}$ & & ${ }^{241} \mathrm{Pu}$ & $3.1 \mathrm{E}-04 \pm 1.1 \mathrm{E}-03$ & $\mathrm{U}$ \\
\hline & ${ }^{103} \mathrm{Ru}$ & $2.6 \mathrm{E}-05 \pm 9.9 \mathrm{E}-05$ & $\mathrm{U}$ & & ${ }^{103} \mathrm{Ru}$ & $-2.7 \mathrm{E}-05 \pm 9.0 \mathrm{E}-05$ & $\mathrm{U}$ \\
\hline & ${ }^{106} \mathrm{Ru}$ & $-1.3 \mathrm{E}-04 \pm 7.1 \mathrm{E}-04$ & $\mathrm{U}$ & & ${ }^{106} \mathrm{Ru}$ & $-2.8 \mathrm{E}-04 \pm 7.9 \mathrm{E}-04$ & $\mathrm{U}$ \\
\hline & ${ }^{125} \mathrm{Sb}$ & $8.8 \mathrm{E}-05 \pm 1.8 \mathrm{E}-04$ & $\mathrm{U}$ & & ${ }^{125} \mathrm{Sb}$ & $-7.6 \mathrm{E}-05 \pm 1.8 \mathrm{E}-04$ & $\mathrm{U}$ \\
\hline & ${ }^{113} \mathrm{Sn}$ & $1.3 \mathrm{E}-05 \pm 9.2 \mathrm{E}-05$ & $\mathrm{U}$ & & ${ }^{113} \mathrm{Sn}$ & $-2.0 \mathrm{E}-05 \pm 8.8 \mathrm{E}-05$ & $\mathrm{U}$ \\
\hline & ${ }^{90} \mathrm{Sr}$ & $-1.1 \mathrm{E}-04 \pm 1.2 \mathrm{E}-04$ & $\mathrm{U}$ & & ${ }^{90} \mathrm{Sr}$ & $-6.7 \mathrm{E}-05 \pm 7.6 \mathrm{E}-05$ & $\mathrm{U}$ \\
\hline & ${ }^{234} \mathrm{U}$ & $1.7 \mathrm{E}-05 \pm 9.9 \mathrm{E}-06$ & & & ${ }^{234} \mathrm{U}$ & $9.0 \mathrm{E}-06 \pm 6.5 \mathrm{E}-06$ & \\
\hline & ${ }^{235} \mathrm{U}$ & $1.7 \mathrm{E}-06 \pm 3.4 \mathrm{E}-06$ & $\mathrm{U}$ & & ${ }^{235} \mathrm{U}$ & $3.2 \mathrm{E}-06 \pm 4.0 \mathrm{E}-06$ & $\mathrm{U}$ \\
\hline & ${ }^{238} \mathrm{U}$ & $8.3 \mathrm{E}-06 \pm 5.9 \mathrm{E}-06$ & & & ${ }^{238} \mathrm{U}$ & $9.7 \mathrm{E}-06 \pm 6.5 \mathrm{E}-06$ & \\
\hline & ${ }^{65} \mathrm{Zn}$ & $-1.7 \mathrm{E}-04 \pm 2.3 \mathrm{E}-04$ & $\mathrm{U}$ & & ${ }^{65} \mathrm{Zn}$ & $-1.2 \mathrm{E}-04 \pm 1.7 \mathrm{E}-04$ & $\mathrm{U}$ \\
\hline N477 (100-K) & ${ }^{241} \mathrm{Am}$ & $5.3 \mathrm{E}-06 \pm 4.9 \mathrm{E}-06$ & $\bar{U}$ & N478 (100-K) & ${ }^{241} \mathrm{Am}$ & $1.7 \mathrm{E}-06 \pm 1.1 \mathrm{E}-05$ & $\mathrm{U}$ \\
\hline Composite Period & ${ }^{144} \mathrm{Ce}$ & $2.9 \mathrm{E}-05 \pm 2.9 \mathrm{E}-04$ & $\mathrm{U}$ & Composite Period & ${ }^{144} \mathrm{Ce}$ & $5.6 \mathrm{E}-04 \pm 7.6 \mathrm{E}-04$ & $\mathrm{U}$ \\
\hline \multirow[t]{18}{*}{$06 / 23 / 03$ to $12 / 22 / 03$} & ${ }^{60} \mathrm{Co}$ & $-3.0 \mathrm{E}-05 \pm 9.3 \mathrm{E}-05$ & $\mathrm{U}$ & $01 / 06 / 03$ to $06 / 23 / 03$ & ${ }^{60} \mathrm{Co}$ & $-6.0 \mathrm{E}-06 \pm 6.0 \mathrm{E}-05$ & $\mathrm{U}$ \\
\hline & ${ }^{134} \mathrm{Cs}$ & $-2.1 \mathrm{E}-05 \pm 6.7 \mathrm{E}-05$ & U & & ${ }^{134} \mathrm{Cs}$ & $1.7 \mathrm{E}-05 \pm 8.7 \mathrm{E}-05$ & $\mathrm{U}$ \\
\hline & ${ }^{137} \mathrm{Cs}$ & $2.0 \mathrm{E}-05 \pm 6.0 \mathrm{E}-05$ & $\mathrm{U}$ & & ${ }^{137} \mathrm{Cs}$ & $-8.7 \mathrm{E}-06 \pm 7.9 \mathrm{E}-05$ & $\mathrm{U}$ \\
\hline & ${ }^{152} \mathrm{Eu}$ & $2.6 \mathrm{E}-04 \pm 1.8 \mathrm{E}-04$ & $\mathrm{U}$ & & ${ }^{152} \mathrm{Eu}$ & $5.1 \mathrm{E}-05 \pm 2.1 \mathrm{E}-04$ & $\mathrm{U}$ \\
\hline & ${ }^{154} \mathrm{Eu}$ & $2.0 \mathrm{E}-04 \pm 2.3 \mathrm{E}-04$ & $\mathrm{U}$ & & ${ }^{154} \mathrm{Eu}$ & $9.1 \mathrm{E}-05 \pm 2.9 \mathrm{E}-04$ & $\mathrm{U}$ \\
\hline & ${ }^{155} \mathrm{Eu}$ & $1.2 \mathrm{E}-05 \pm 1.3 \mathrm{E}-04$ & $\mathrm{U}$ & & ${ }^{155} \mathrm{Eu}$ & $-3.2 \mathrm{E}-05 \pm 2.0 \mathrm{E}-04$ & $\mathrm{U}$ \\
\hline & ${ }^{238} \mathrm{Pu}$ & $-2.8 \mathrm{E}-06 \pm 1.6 \mathrm{E}-05$ & $\mathrm{U}$ & & ${ }^{238} \mathrm{Pu}$ & $2.0 \mathrm{E}-05 \pm 3.0 \mathrm{E}-05$ & $\mathrm{U}$ \\
\hline & ${ }^{239 / 240} \mathrm{Pu}$ & $4.2 \mathrm{E}-06 \pm 7.7 \mathrm{E}-06$ & $\mathrm{U}$ & & ${ }^{239 / 240} \mathrm{Pu}$ & $5.8 \mathrm{E}-06 \pm 1.1 \mathrm{E}-05$ & $\mathrm{U}$ \\
\hline & ${ }^{241} \mathrm{Pu}$ & $-3.3 \mathrm{E}-04 \pm 3.4 \mathrm{E}-04$ & $\mathrm{U}$ & & ${ }^{241} \mathrm{Pu}$ & $2.8 \mathrm{E}-04 \pm 1.0 \mathrm{E}-03$ & $\mathrm{U}$ \\
\hline & ${ }^{103} \mathrm{Ru}$ & $-6.8 \mathrm{E}-07 \pm 6.8 \mathrm{E}-06$ & $\mathrm{U}$ & & ${ }^{103} \mathrm{Ru}$ & $1.7 \mathrm{E}-05 \pm 9.3 \mathrm{E}-05$ & $\mathrm{U}$ \\
\hline & ${ }^{106} \mathrm{Ru}$ & $-7.2 \mathrm{E}-05 \pm 5.7 \mathrm{E}-04$ & $\mathrm{U}$ & & ${ }^{106} \mathrm{Ru}$ & $-3.4 \mathrm{E}-04 \pm 7.1 \mathrm{E}-04$ & $\mathrm{U}$ \\
\hline & ${ }^{125} \mathrm{Sb}$ & $-6.9 \mathrm{E}-05 \pm 1.4 \mathrm{E}-04$ & $\mathrm{U}$ & & ${ }^{125} \mathrm{Sb}$ & $5.5 \mathrm{E}-05 \pm 1.8 \mathrm{E}-04$ & $\mathrm{U}$ \\
\hline & ${ }^{113} \mathrm{Sn}$ & $1.6 \mathrm{E}-05 \pm 6.7 \mathrm{E}-05$ & $\mathrm{U}$ & & ${ }^{113} \mathrm{Sn}$ & $-2.2 \mathrm{E}-05 \pm 8.6 \mathrm{E}-05$ & $\mathrm{U}$ \\
\hline & ${ }^{90} \mathrm{Sr}$ & $7.0 \mathrm{E}-06 \pm 7.0 \mathrm{E}-05$ & $\mathrm{U}$ & & ${ }^{90} \mathrm{Sr}$ & $7.6 \mathrm{E}-06 \pm 7.6 \mathrm{E}-05$ & $\mathrm{U}$ \\
\hline & ${ }^{234} \mathrm{U}$ & $1.6 \mathrm{E}-05 \pm 9.7 \mathrm{E}-06$ & & & ${ }^{234} \mathrm{U}$ & $9.9 \mathrm{E}-06 \pm 7.6 \mathrm{E}-06$ & \\
\hline & ${ }^{235} \mathrm{U}$ & $7.7 \mathrm{E}-07 \pm 1.6 \mathrm{E}-06$ & $\mathrm{U}$ & & ${ }^{235} \mathrm{U}$ & $2.3 \mathrm{E}-06 \pm 3.6 \mathrm{E}-06$ & $\mathrm{U}$ \\
\hline & ${ }^{238} \mathrm{U}$ & $1.2 \mathrm{E}-05 \pm 7.8 \mathrm{E}-06$ & & & ${ }^{238} \mathrm{U}$ & $6.4 \mathrm{E}-06 \pm 5.3 \mathrm{E}-06$ & \\
\hline & ${ }^{65} \mathrm{Zn}$ & $-2.6 \mathrm{E}-04 \pm 2.7 \mathrm{E}-04$ & $\mathrm{U}$ & & ${ }^{65} \mathrm{Zn}$ & $8.0 \mathrm{E}-05 \pm 2.1 \mathrm{E}-04$ & $\mathrm{U}$ \\
\hline
\end{tabular}

$\overline{\mathrm{RQ}}=$ Result Qualifier. $\mathrm{U}=$ The analyte was analyzed for but not detected. 
Table 2-3. Near-Facility Air Sampling Results, 2003 (pCi/m³ \pm total analytical uncertainty). (cont)

\begin{tabular}{ccrc}
\hline Location & Isotope & Result \pm Uncertainty & RQ* \\
\hline N478 (100-K) & ${ }^{241} \mathrm{Am}$ & $1.9 \mathrm{E}-05 \pm 1.5 \mathrm{E}-05$ & $\mathrm{U}$ \\
Composite Period & ${ }^{144} \mathrm{Ce}$ & $1.6 \mathrm{E}-04 \pm 1.1 \mathrm{E}-03$ & $\mathrm{U}$ \\
06/23/03 to 12/22/03 & ${ }^{60} \mathrm{Co}$ & $-1.2 \mathrm{E}-04 \pm 1.3 \mathrm{E}-04$ & $\mathrm{U}$ \\
& ${ }^{134} \mathrm{Cs}$ & $3.0 \mathrm{E}-05 \pm 1.2 \mathrm{E}-04$ & $\mathrm{U}$ \\
& ${ }^{137} \mathrm{Cs}$ & $-1.2 \mathrm{E}-06 \pm 1.2 \mathrm{E}-05$ & $\mathrm{U}$ \\
& ${ }^{152} \mathrm{Eu}$ & $3.0 \mathrm{E}-04 \pm 3.0 \mathrm{E}-04$ & $\mathrm{U}$ \\
& ${ }^{154} \mathrm{Eu}$ & $1.9 \mathrm{E}-04 \pm 3.6 \mathrm{E}-04$ & $\mathrm{U}$ \\
& ${ }^{155} \mathrm{Eu}$ & $4.3 \mathrm{E}-06 \pm 4.3 \mathrm{E}-05$ & $\mathrm{U}$ \\
& ${ }^{238} \mathrm{Pu}$ & $1.8 \mathrm{E}-06 \pm 1.9 \mathrm{E}-06$ & $\mathrm{U}$ \\
& ${ }^{239 / 240} \mathrm{Pu}$ & $5.3 \mathrm{E}-06 \pm 6.5 \mathrm{E}-06$ & \\
& ${ }^{241} \mathrm{Pu}$ & $-3.7 \mathrm{E}-05 \pm 3.9 \mathrm{E}-05$ & $\mathrm{U}$ \\
& ${ }^{103} \mathrm{Ru}$ & $-2.3 \mathrm{E}-05 \pm 1.3 \mathrm{E}-04$ & $\mathrm{U}$ \\
& ${ }^{106} \mathrm{Ru}$ & $9.7 \mathrm{E}-05 \pm 9.7 \mathrm{E}-04$ & $\mathrm{U}$ \\
& ${ }^{125} \mathrm{Sb}$ & $-1.3 \mathrm{E}-04 \pm 2.0 \mathrm{E}-04$ & $\mathrm{U}$ \\
& ${ }^{113} \mathrm{Sn}$ & $5.9 \mathrm{E}-05 \pm 1.2 \mathrm{E}-04$ & $\mathrm{U}$ \\
& ${ }^{90} \mathrm{Sr}$ & $-8.2 \mathrm{E}-05 \pm 9.3 \mathrm{E}-05$ & $\mathrm{U}$ \\
& ${ }^{23} \mathrm{Zn} \mathrm{U}$ & $-8.9 \mathrm{E}-05 \pm 2.4 \mathrm{E}-04$ & $\mathrm{U}$
\end{tabular}

\begin{tabular}{|c|c|c|c|}
\hline Location & Isotope & Result \pm Uncertainty & $\mathbf{R Q}^{*}$ \\
\hline N479 (100-K) & ${ }^{241} \mathrm{Am}$ & $9.2 \mathrm{E}-06 \pm 1.4 \mathrm{E}-05$ & $\bar{U}$ \\
\hline Composite Period & ${ }^{144} \mathrm{Ce}$ & $-2.2 \mathrm{E}-04 \pm 8.2 \mathrm{E}-04$ & $\mathrm{U}$ \\
\hline \multirow[t]{18}{*}{$01 / 06 / 03$ to $06 / 23 / 03$} & ${ }^{60} \mathrm{Co}$ & $-7.6 \mathrm{E}-05 \pm 8.1 \mathrm{E}-05$ & U \\
\hline & ${ }^{134} \mathrm{Cs}$ & $-6.2 \mathrm{E}-05 \pm 8.4 \mathrm{E}-05$ & $\mathrm{U}$ \\
\hline & ${ }^{137} \mathrm{Cs}$ & $1.7 \mathrm{E}-05 \pm 7.5 \mathrm{E}-05$ & $\mathrm{U}$ \\
\hline & ${ }^{152} \mathrm{Eu}$ & $-1.3 \mathrm{E}-05 \pm 1.3 \mathrm{E}-04$ & $\mathrm{U}$ \\
\hline & ${ }^{154} \mathrm{Eu}$ & $6.4 \mathrm{E}-05 \pm 2.3 \mathrm{E}-04$ & $\mathrm{U}$ \\
\hline & ${ }^{155} \mathrm{Eu}$ & $5.7 \mathrm{E}-05 \pm 2.2 \mathrm{E}-04$ & $\mathrm{U}$ \\
\hline & ${ }^{238} \mathrm{Pu}$ & $9.2 \mathrm{E}-06 \pm 3.0 \mathrm{E}-05$ & $\mathrm{U}$ \\
\hline & ${ }^{239 / 240} \mathrm{Pu}$ & $6.7 \mathrm{E}-06 \pm 1.0 \mathrm{E}-05$ & $\mathrm{U}$ \\
\hline & ${ }^{241} \mathrm{Pu}$ & $3.1 \mathrm{E}-04 \pm 1.2 \mathrm{E}-03$ & $\mathrm{U}$ \\
\hline & ${ }^{103} \mathrm{Ru}$ & $-6.8 \mathrm{E}-05 \pm 9.3 \mathrm{E}-05$ & $\mathrm{U}$ \\
\hline & ${ }^{106} \mathrm{Ru}$ & $-4.2 \mathrm{E}-04 \pm 7.0 \mathrm{E}-04$ & $\mathrm{U}$ \\
\hline & ${ }^{125} \mathrm{Sb}$ & $5.5 \mathrm{E}-05 \pm 1.8 \mathrm{E}-04$ & $\mathrm{U}$ \\
\hline & ${ }^{113} \mathrm{Sn}$ & $-7.5 \mathrm{E}-05 \pm 8.9 \mathrm{E}-05$ & $\mathrm{U}$ \\
\hline & ${ }^{90} \mathrm{Sr}$ & $7.7 \mathrm{E}-06 \pm 7.7 \mathrm{E}-05$ & $\mathrm{U}$ \\
\hline & ${ }^{234} \mathrm{U}$ & $9.2 \mathrm{E}-06 \pm 7.9 \mathrm{E}-06$ & $\mathrm{U}$ \\
\hline & ${ }^{235} \mathrm{U}$ & $4.2 \mathrm{E}-06 \pm 6.4 \mathrm{E}-06$ & $\mathrm{U}$ \\
\hline & ${ }^{238} \mathrm{U}$ & $7.7 \mathrm{E}-06 \pm 5.6 \mathrm{E}-06$ & \\
\hline & ${ }^{65} \mathrm{Zn}$ & $-9.7 \mathrm{E}-05 \pm 1.8 \mathrm{E}-04$ & $\mathrm{U}$ \\
\hline N528 $(100-K)$ & ${ }^{144} \mathrm{Ce}$ & $-3.2 \mathrm{E}-04 \pm 8.6 \mathrm{E}-04$ & $\mathrm{U}$ \\
\hline Composite Period & ${ }^{60} \mathrm{Co}$ & $-5.5 \mathrm{E}-05 \pm 8.1 \mathrm{E}-05$ & $\mathrm{U}$ \\
\hline \multirow[t]{16}{*}{$01 / 07 / 03$ to $06 / 23 / 03$} & ${ }^{134} \mathrm{Cs}$ & $6.1 \mathrm{E}-05 \pm 8.5 \mathrm{E}-05$ & $\mathrm{U}$ \\
\hline & ${ }^{137} \mathrm{Cs}$ & $7.2 \mathrm{E}-05 \pm 7.7 \mathrm{E}-05$ & $\mathrm{U}$ \\
\hline & ${ }^{152} \mathrm{Eu}$ & $-5.4 \mathrm{E}-05 \pm 2.3 \mathrm{E}-04$ & $\mathrm{U}$ \\
\hline & ${ }^{154} \mathrm{Eu}$ & $-1.3 \mathrm{E}-04 \pm 2.2 \mathrm{E}-04$ & $\mathrm{U}$ \\
\hline & ${ }^{155} \mathrm{Eu}$ & $-9.2 \mathrm{E}-05 \pm 2.2 \mathrm{E}-04$ & $\mathrm{U}$ \\
\hline & ${ }^{238} \mathrm{Pu}$ & $-9.4 \mathrm{E}-07 \pm 9.4 \mathrm{E}-06$ & U \\
\hline & ${ }^{239 / 240} \mathrm{Pu}$ & $2.9 \mathrm{E}-06 \pm 4.4 \mathrm{E}-06$ & $\mathrm{U}$ \\
\hline & ${ }^{103} \mathrm{Ru}$ & $4.5 \mathrm{E}-06 \pm 4.5 \mathrm{E}-05$ & $\mathrm{U}$ \\
\hline & ${ }^{106} \mathrm{Ru}$ & $-1.6 \mathrm{E}-04 \pm 7.1 \mathrm{E}-04$ & $\mathrm{U}$ \\
\hline & ${ }^{125} \mathrm{Sb}$ & $1.1 \mathrm{E}-04 \pm 2.0 \mathrm{E}-04$ & $\mathrm{U}$ \\
\hline & ${ }^{113} \mathrm{Sn}$ & $-6.9 \mathrm{E}-05 \pm 9.8 \mathrm{E}-05$ & $\mathrm{U}$ \\
\hline & ${ }^{90} \mathrm{Sr}$ & $-3.6 \mathrm{E}-07 \pm 3.6 \mathrm{E}-06$ & $\mathrm{U}$ \\
\hline & ${ }^{234} \mathrm{U}$ & $1.1 \mathrm{E}-05 \pm 7.1 \mathrm{E}-06$ & \\
\hline & ${ }^{235} \mathrm{U}$ & $3.3 \mathrm{E}-06 \pm 3.4 \mathrm{E}-06$ & \\
\hline & ${ }^{238} \mathrm{U}$ & $9.4 \mathrm{E}-06 \pm 6.8 \mathrm{E}-06$ & \\
\hline & ${ }^{65} \mathrm{Zn}$ & $-3.2 \mathrm{E}-04 \pm 3.4 \mathrm{E}-04$ & $\mathrm{U}$ \\
\hline
\end{tabular}

$\overline{\mathrm{RQ}}=$ Result Qualifier. $\mathrm{U}=$ The analyte was analyzed for but not detected. 
Table 2-3. Near-Facility Air Sampling Results, 2003 (pCi/m³ \pm total analytical uncertainty). (cont)

\begin{tabular}{|c|c|c|c|c|c|c|c|}
\hline Location & Isotope & Result \pm Uncertainty & $\mathbf{R Q}^{*}$ & Location & Isotope & Result \pm Uncertainty & RQ* \\
\hline N528 $(100-K)$ & ${ }^{144} \mathrm{Ce}$ & $-5.2 \mathrm{E}-04 \pm 6.7 \mathrm{E}-04$ & $\bar{U}$ & N529 $(100-K)$ & ${ }^{144} \mathrm{Ce}$ & $3.8 \mathrm{E}-04 \pm 8.0 \mathrm{E}-04$ & $\mathrm{U}$ \\
\hline Composite Period & ${ }^{60} \mathrm{Co}$ & $-7.0 \mathrm{E}-05 \pm 1.0 \mathrm{E}-04$ & $\mathrm{U}$ & Composite Period & ${ }^{60} \mathrm{Co}$ & $-4.8 \mathrm{E}-05 \pm 1.0 \mathrm{E}-04$ & $\mathrm{U}$ \\
\hline \multirow[t]{16}{*}{$06 / 23 / 03$ to $12 / 22 / 03$} & ${ }^{134} \mathrm{Cs}$ & $-1.1 \mathrm{E}-05 \pm 7.7 \mathrm{E}-05$ & $\mathrm{U}$ & $01 / 07 / 03$ to $06 / 23 / 03$ & ${ }^{134} \mathrm{Cs}$ & $-2.5 \mathrm{E}-06 \pm 2.5 \mathrm{E}-05$ & $\mathrm{U}$ \\
\hline & ${ }^{137} \mathrm{Cs}$ & $-5.8 \mathrm{E}-06 \pm 5.8 \mathrm{E}-05$ & $\mathrm{U}$ & & ${ }^{137} \mathrm{Cs}$ & $2.3 \mathrm{E}-04 \pm 1.7 \mathrm{E}-04$ & \\
\hline & ${ }^{152} \mathrm{Eu}$ & $-1.8 \mathrm{E}-05 \pm 1.6 \mathrm{E}-04$ & $\mathrm{U}$ & & ${ }^{152} \mathrm{Eu}$ & $4.8 \mathrm{E}-05 \pm 2.0 \mathrm{E}-04$ & $\mathrm{U}$ \\
\hline & ${ }^{154} \mathrm{Eu}$ & $-1.3 \mathrm{E}-04 \pm 2.5 \mathrm{E}-04$ & $\mathrm{U}$ & & ${ }^{154} \mathrm{Eu}$ & $5.6 \mathrm{E}-05 \pm 2.5 \mathrm{E}-04$ & $\mathrm{U}$ \\
\hline & ${ }^{155} \mathrm{Eu}$ & $-1.3 \mathrm{E}-04 \pm 1.7 \mathrm{E}-04$ & $\mathrm{U}$ & & ${ }^{155} \mathrm{Eu}$ & $1.3 \mathrm{E}-04 \pm 2.0 \mathrm{E}-04$ & $\mathrm{U}$ \\
\hline & ${ }^{238} \mathrm{Pu}$ & $-7.9 \mathrm{E}-06 \pm 1.5 \mathrm{E}-05$ & $\mathrm{U}$ & & ${ }^{238} \mathrm{Pu}$ & $1.4 \mathrm{E}-05 \pm 1.5 \mathrm{E}-05$ & $\mathrm{U}$ \\
\hline & ${ }^{239 / 240} \mathrm{Pu}$ & $7.1 \mathrm{E}-06 \pm 6.7 \mathrm{E}-06$ & $\mathrm{U}$ & & ${ }^{239 / 240} \mathrm{Pu}$ & $8.3 \mathrm{E}-07 \pm 8.6 \mathrm{E}-07$ & $\mathrm{U}$ \\
\hline & ${ }^{103} \mathrm{Ru}$ & $-1.1 \mathrm{E}-05 \pm 8.6 \mathrm{E}-05$ & $\mathrm{U}$ & & ${ }^{103} \mathrm{Ru}$ & $-4.0 \mathrm{E}-06 \pm 4.0 \mathrm{E}-05$ & $\mathrm{U}$ \\
\hline & ${ }^{106} \mathrm{Ru}$ & $-4.0 \mathrm{E}-04 \pm 6.0 \mathrm{E}-04$ & $\mathrm{U}$ & & ${ }^{106} \mathrm{Ru}$ & $-2.8 \mathrm{E}-04 \pm 7.4 \mathrm{E}-04$ & $\mathrm{U}$ \\
\hline & ${ }^{125} \mathrm{Sb}$ & $3.1 \mathrm{E}-05 \pm 1.6 \mathrm{E}-04$ & $\mathrm{U}$ & & ${ }^{125} \mathrm{Sb}$ & $4.7 \mathrm{E}-05 \pm 1.9 \mathrm{E}-04$ & $\mathrm{U}$ \\
\hline & ${ }^{113} \mathrm{Sn}$ & $-3.9 \mathrm{E}-06 \pm 3.9 \mathrm{E}-05$ & $\mathrm{U}$ & & ${ }^{113} \mathrm{Sn}$ & $2.8 \mathrm{E}-05 \pm 8.8 \mathrm{E}-05$ & $\mathrm{U}$ \\
\hline & ${ }^{90} \mathrm{Sr}$ & $-2.0 \mathrm{E}-04 \pm 2.0 \mathrm{E}-04$ & $\mathrm{U}$ & & ${ }^{90} \mathrm{Sr}$ & $-2.6 \mathrm{E}-06 \pm 2.6 \mathrm{E}-05$ & $\mathrm{U}$ \\
\hline & ${ }^{234} \mathrm{U}$ & $2.2 \mathrm{E}-05 \pm 1.2 \mathrm{E}-05$ & & & ${ }^{234} \mathrm{U}$ & $7.1 \mathrm{E}-06 \pm 6.3 \mathrm{E}-06$ & $\mathrm{U}$ \\
\hline & ${ }^{235} \mathrm{U}$ & $2.5 \mathrm{E}-06 \pm 3.8 \mathrm{E}-06$ & $\mathrm{U}$ & & ${ }^{235} \mathrm{U}$ & $7.6 \mathrm{E}-07 \pm 2.7 \mathrm{E}-06$ & $\mathrm{U}$ \\
\hline & ${ }^{238} \mathrm{U}$ & $9.5 \mathrm{E}-06 \pm 6.8 \mathrm{E}-06$ & & & ${ }^{238} \mathrm{U}$ & $9.1 \mathrm{E}-06 \pm 6.1 \mathrm{E}-06$ & \\
\hline & ${ }^{65} \mathrm{Zn}$ & $2.6 \mathrm{E}-05 \pm 2.0 \mathrm{E}-04$ & $\mathrm{U}$ & & ${ }^{65} \mathrm{Zn}$ & $-5.3 \mathrm{E}-05 \pm 2.2 \mathrm{E}-04$ & $\mathrm{U}$ \\
\hline N529 (100-K) & ${ }^{144} \mathrm{Ce}$ & $7.8 \mathrm{E}-05 \pm 7.1 \mathrm{E}-04$ & $\bar{U}$ & N530 (100-K) & ${ }^{144} \mathrm{Ce}$ & $2.5 \mathrm{E}-04 \pm 7.7 \mathrm{E}-04$ & $\mathrm{U}$ \\
\hline Composite Period & ${ }^{60} \mathrm{Co}$ & $1.1 \mathrm{E}-04 \pm 8.4 \mathrm{E}-05$ & $\mathrm{U}$ & Composite Period & ${ }^{60} \mathrm{Co}$ & $5.1 \mathrm{E}-05 \pm 8.1 \mathrm{E}-05$ & $\mathrm{U}$ \\
\hline \multirow[t]{16}{*}{$06 / 23 / 03$ to $12 / 22 / 03$} & ${ }^{134} \mathrm{Cs}$ & $-4.1 \mathrm{E}-05 \pm 6.6 \mathrm{E}-05$ & $\mathrm{U}$ & $01 / 07 / 03$ to $06 / 23 / 03$ & ${ }^{134} \mathrm{Cs}$ & 4.3E-05 $\pm 7.9 \mathrm{E}-05$ & $\mathrm{U}$ \\
\hline & ${ }^{137} \mathrm{Cs}$ & $1.7 \mathrm{E}-05 \pm 6.1 \mathrm{E}-05$ & $\mathrm{U}$ & & ${ }^{137} \mathrm{Cs}$ & $1.2 \mathrm{E}-07 \pm 1.2 \mathrm{E}-06$ & $\mathrm{U}$ \\
\hline & ${ }^{152} \mathrm{Eu}$ & $2.6 \mathrm{E}-04 \pm 2.2 \mathrm{E}-04$ & $\mathrm{U}$ & & ${ }^{152} \mathrm{Eu}$ & $-6.8 \mathrm{E}-05 \pm 1.9 \mathrm{E}-04$ & $\mathrm{U}$ \\
\hline & ${ }^{154} \mathrm{Eu}$ & $-4.5 \mathrm{E}-05 \pm 1.9 \mathrm{E}-04$ & $\mathrm{U}$ & & ${ }^{154} \mathrm{Eu}$ & $1.0 \mathrm{E}-04 \pm 2.2 \mathrm{E}-04$ & $\mathrm{U}$ \\
\hline & ${ }^{155} \mathrm{Eu}$ & $3.2 \mathrm{E}-05 \pm 1.9 \mathrm{E}-04$ & $\mathrm{U}$ & & ${ }^{155} \mathrm{Eu}$ & $1.2 \mathrm{E}-04 \pm 2.2 \mathrm{E}-04$ & $\mathrm{U}$ \\
\hline & ${ }^{238} \mathrm{Pu}$ & $-1.3 \mathrm{E}-05 \pm 1.4 \mathrm{E}-05$ & $\mathrm{U}$ & & ${ }^{238} \mathrm{Pu}$ & $2.9 \mathrm{E}-06 \pm 1.7 \mathrm{E}-05$ & $\mathrm{U}$ \\
\hline & ${ }^{239 / 240} \mathrm{Pu}$ & $9.9 \mathrm{E}-06 \pm 8.6 \mathrm{E}-06$ & $\mathrm{U}$ & & ${ }^{239 / 240} \mathrm{Pu}$ & $4.9 \mathrm{E}-06 \pm 5.5 \mathrm{E}-06$ & $\mathrm{U}$ \\
\hline & ${ }^{103} \mathrm{Ru}$ & $-9.2 \mathrm{E}-06 \pm 8.0 \mathrm{E}-05$ & $\mathrm{U}$ & & ${ }^{103} \mathrm{Ru}$ & $-5.1 \mathrm{E}-08 \pm 5.1 \mathrm{E}-07$ & $\mathrm{U}$ \\
\hline & ${ }^{106} \mathrm{Ru}$ & $1.9 \mathrm{E}-04 \pm 5.6 \mathrm{E}-04$ & $\mathrm{U}$ & & ${ }^{106} \mathrm{Ru}$ & $-3.4 \mathrm{E}-06 \pm 3.4 \mathrm{E}-05$ & $\mathrm{U}$ \\
\hline & ${ }^{125} \mathrm{Sb}$ & $-9.3 \mathrm{E}-05 \pm 1.6 \mathrm{E}-04$ & $\mathrm{U}$ & & ${ }^{125} \mathrm{Sb}$ & $6.3 \mathrm{E}-05 \pm 2.1 \mathrm{E}-04$ & $\mathrm{U}$ \\
\hline & ${ }^{113} \mathrm{Sn}$ & $2.9 \mathrm{E}-05 \pm 9.5 \mathrm{E}-05$ & $\mathrm{U}$ & & ${ }^{113} \mathrm{Sn}$ & $-2.7 \mathrm{E}-05 \pm 8.3 \mathrm{E}-05$ & $\mathrm{U}$ \\
\hline & ${ }^{90} \mathrm{Sr}$ & $-2.7 \mathrm{E}-05 \pm 9.4 \mathrm{E}-05$ & $\mathrm{U}$ & & ${ }^{90} \mathrm{Sr}$ & $-1.5 \mathrm{E}-05 \pm 9.0 \mathrm{E}-05$ & $\mathrm{U}$ \\
\hline & ${ }^{234} \mathrm{U}$ & $2.0 \mathrm{E}-05 \pm 1.0 \mathrm{E}-05$ & & & ${ }^{234} \mathrm{U}$ & $6.7 \mathrm{E}-06 \pm 5.9 \mathrm{E}-06$ & $\mathrm{U}$ \\
\hline & ${ }^{235} \mathrm{U}$ & $2.8 \mathrm{E}-06 \pm 3.5 \mathrm{E}-06$ & $\mathrm{U}$ & & ${ }^{235} \mathrm{U}$ & $2.4 \mathrm{E}-06 \pm 3.0 \mathrm{E}-06$ & \\
\hline & ${ }^{238} \mathrm{U}$ & $1.5 \mathrm{E}-05 \pm 8.5 \mathrm{E}-06$ & & & ${ }^{238} \mathrm{U}$ & $8.2 \mathrm{E}-06 \pm 5.9 \mathrm{E}-06$ & \\
\hline & ${ }^{65} \mathrm{Zn}$ & $-4.8 \mathrm{E}-05 \pm 1.8 \mathrm{E}-04$ & $\mathrm{U}$ & & ${ }^{65} \mathrm{Zn}$ & $-3.5 \mathrm{E}-07 \pm 3.5 \mathrm{E}-06$ & $\mathrm{U}$ \\
\hline
\end{tabular}

$\overline{\mathrm{RQ}}=$ Result Qualifier. $\mathrm{U}=$ The analyte was analyzed for but not detected. 
Table 2-3. Near-Facility Air Sampling Results, 2003 (pCi/m³ \pm total analytical uncertainty). (cont)

\begin{tabular}{ccrc}
\hline Location & Isotope & Result \pm Uncertainty & RQ* \\
\hline N530 (100-K) & ${ }^{144} \mathrm{Ce}$ & $-7.7 \mathrm{E}-05 \pm 6.2 \mathrm{E}-04$ & $\mathrm{U}$ \\
Composite Period & ${ }^{60} \mathrm{Co}$ & $-2.2 \mathrm{E}-05 \pm 7.8 \mathrm{E}-05$ & $\mathrm{U}$ \\
06/23/03 to 12/22/03 & ${ }^{134} \mathrm{Cs}$ & $-1.4 \mathrm{E}-05 \pm 6.5 \mathrm{E}-05$ & $\mathrm{U}$ \\
& ${ }^{137} \mathrm{Cs}$ & $-4.3 \mathrm{E}-06 \pm 4.3 \mathrm{E}-05$ & $\mathrm{U}$ \\
& ${ }^{152} \mathrm{Eu}$ & $2.6 \mathrm{E}-05 \pm 1.5 \mathrm{E}-04$ & $\mathrm{U}$ \\
& ${ }^{154} \mathrm{Eu}$ & $-7.2 \mathrm{E}-06 \pm 7.2 \mathrm{E}-05$ & $\mathrm{U}$ \\
& ${ }^{155} \mathrm{Eu}$ & $8.4 \mathrm{E}-05 \pm 1.8 \mathrm{E}-04$ & $\mathrm{U}$ \\
& ${ }^{238} \mathrm{Pu}$ & $1.7 \mathrm{E}-06 \pm 1.3 \mathrm{E}-05$ & $\mathrm{U}$ \\
& ${ }^{239 / 240} \mathrm{Pu}$ & $2.5 \mathrm{E}-06 \pm 3.1 \mathrm{E}-06$ & \\
& ${ }^{103} \mathrm{Ru}$ & $-1.6 \mathrm{E}-05 \pm 7.3 \mathrm{E}-05$ & $\mathrm{U}$ \\
& ${ }^{106} \mathrm{Ru}$ & $-2.8 \mathrm{E}-04 \pm 5.5 \mathrm{E}-04$ & $\mathrm{U}$ \\
& ${ }^{125} \mathrm{Sb}$ & $5.5 \mathrm{E}-06 \pm 5.6 \mathrm{E}-05$ & $\mathrm{U}$ \\
& ${ }^{113} \mathrm{Sn}$ & $4.4 \mathrm{E}-05 \pm 6.8 \mathrm{E}-05$ & $\mathrm{U}$ \\
& ${ }^{90} \mathrm{Sr}$ & $-1.2 \mathrm{E}-04 \pm 1.3 \mathrm{E}-04$ & $\mathrm{U}$ \\
& ${ }^{234} \mathrm{U}$ & $1.6 \mathrm{E}-05 \pm 9.4 \mathrm{E}-06$ & \\
& ${ }^{235} \mathrm{U}$ & $1.5 \mathrm{E}-06 \pm 3.1 \mathrm{E}-06$ & $\mathrm{U}$ \\
& ${ }^{238} \mathrm{U}$ & $1.0 \mathrm{E}-05 \pm 6.6 \mathrm{E}-06$ & \\
& ${ }^{65} \mathrm{Zn}$ & $-8.3 \mathrm{E}-06 \pm 8.3 \mathrm{E}-05$ & $\mathrm{U}$
\end{tabular}

\begin{tabular}{ccrc}
\hline Location & Isotope & Result \pm Uncertainty & RQ* \\
\hline N102 (100-N) & ${ }^{144} \mathrm{Ce}$ & $-1.0 \mathrm{E}-04 \pm 8.2 \mathrm{E}-04$ & $\mathrm{U}$ \\
Composite Period & ${ }^{60} \mathrm{Co}$ & $1.1 \mathrm{E}-05 \pm 7.9 \mathrm{E}-05$ & $\mathrm{U}$ \\
01/07/03 to 06/23/03 & ${ }^{134} \mathrm{Cs}$ & $2.5 \mathrm{E}-05 \pm 8.3 \mathrm{E}-05$ & $\mathrm{U}$ \\
& ${ }^{137} \mathrm{Cs}$ & $3.7 \mathrm{E}-05 \pm 7.6 \mathrm{E}-05$ & $\mathrm{U}$ \\
& ${ }^{152} \mathrm{Eu}$ & $-4.1 \mathrm{E}-05 \pm 2.3 \mathrm{E}-04$ & $\mathrm{U}$ \\
& ${ }^{154} \mathrm{Eu}$ & $-5.9 \mathrm{E}-05 \pm 2.3 \mathrm{E}-04$ & $\mathrm{U}$ \\
& ${ }^{155} \mathrm{Eu}$ & $-1.7 \mathrm{E}-04 \pm 2.3 \mathrm{E}-04$ & $\mathrm{U}$ \\
& ${ }^{238} \mathrm{Pu}$ & $-3.9 \mathrm{E}-06 \pm 1.2 \mathrm{E}-05$ & $\mathrm{U}$ \\
& ${ }^{239 / 240} \mathrm{Pu}$ & $1.2 \mathrm{E}-05 \pm 8.0 \mathrm{E}-06$ & \\
& ${ }^{103} \mathrm{Ru}$ & $-4.6 \mathrm{E}-05 \pm 8.7 \mathrm{E}-05$ & $\mathrm{U}$ \\
& ${ }^{106} \mathrm{Ru}$ & $3.7 \mathrm{E}-04 \pm 6.8 \mathrm{E}-04$ & $\mathrm{U}$ \\
& ${ }^{125} \mathrm{Sb}$ & $-4.0 \mathrm{E}-05 \pm 1.9 \mathrm{E}-04$ & $\mathrm{U}$ \\
& ${ }^{113} \mathrm{Sn}$ & $2.0 \mathrm{E}-05 \pm 9.4 \mathrm{E}-05$ & $\mathrm{U}$ \\
& ${ }^{90} \mathrm{Sr}$ & $-9.3 \mathrm{E}-05 \pm 9.6 \mathrm{E}-05$ & $\mathrm{U}$ \\
& ${ }^{234} \mathrm{U}$ & $9.3 \mathrm{E}-06 \pm 6.5 \mathrm{E}-06$ & \\
& ${ }^{235} \mathrm{U}$ & $7.6 \mathrm{E}-07 \pm 2.7 \mathrm{E}-06$ & $\mathrm{U}$ \\
& ${ }^{238} \mathrm{U}$ & $6.2 \mathrm{E}-06 \pm 4.7 \mathrm{E}-06$ & \\
& ${ }^{65} \mathrm{Zn} \mathrm{Zn}$ & $3.0 \mathrm{E}-05 \pm 2.0 \mathrm{E}-04$ & $\mathrm{U}$ \\
\hline${ }^{638} \mathrm{U}$ & $8.5 \mathrm{E}-06 \pm 5.8 \mathrm{E}-06$ & \\
& ${ }^{235} \mathrm{U}$ & $2.1 \mathrm{E}-04 \pm 2.3 \mathrm{E}-04$ & $\mathrm{U}$
\end{tabular}

$\overline{\mathrm{RQ}}=$ Result Qualifier. $\mathrm{U}=$ The analyte was analyzed for but not detected. 
Table 2-3. Near-Facility Air Sampling Results, 2003 (pCi/m³ \pm total analytical uncertainty). (cont)

\begin{tabular}{|c|c|c|c|c|c|c|c|}
\hline Location & Isotope & Result \pm Uncertainty & $\mathbf{R Q}^{*}$ & Location & Isotope & Result \pm Uncertainty & $\mathbf{R Q}^{*}$ \\
\hline N103 $(100-N)$ & ${ }^{144} \mathrm{Ce}$ & $-3.1 \mathrm{E}-05 \pm 3.1 \mathrm{E}-04$ & $\bar{U}$ & N105 $(100-N)$ & ${ }^{144} \mathrm{Ce}$ & $-1.5 \mathrm{E}-04 \pm 7.8 \mathrm{E}-04$ & $\mathrm{U}$ \\
\hline Composite Period & ${ }^{60} \mathrm{Co}$ & $4.7 \mathrm{E}-05 \pm 8.0 \mathrm{E}-05$ & $\mathrm{U}$ & Composite Period & ${ }^{60} \mathrm{Co}$ & $2.2 \mathrm{E}-04 \pm 9.8 \mathrm{E}-05$ & \\
\hline \multirow[t]{16}{*}{$06 / 23 / 03$ to $12 / 22 / 03$} & ${ }^{134} \mathrm{Cs}$ & $3.7 \mathrm{E}-05 \pm 7.0 \mathrm{E}-05$ & $\mathrm{U}$ & $01 / 07 / 03$ to $06 / 23 / 03$ & ${ }^{134} \mathrm{Cs}$ & $-5.5 \mathrm{E}-05 \pm 8.4 \mathrm{E}-05$ & $\mathrm{U}$ \\
\hline & ${ }^{137} \mathrm{Cs}$ & $-5.6 \mathrm{E}-06 \pm 5.7 \mathrm{E}-05$ & $\mathrm{U}$ & & ${ }^{137} \mathrm{Cs}$ & $3.6 \mathrm{E}-04 \pm 1.7 \mathrm{E}-04$ & \\
\hline & ${ }^{152} \mathrm{Eu}$ & $-2.0 \mathrm{E}-04 \pm 2.1 \mathrm{E}-04$ & $\mathrm{U}$ & & ${ }^{152} \mathrm{Eu}$ & $-2.0 \mathrm{E}-04 \pm 2.1 \mathrm{E}-04$ & $\mathrm{U}$ \\
\hline & ${ }^{154} \mathrm{Eu}$ & $2.2 \mathrm{E}-05 \pm 1.9 \mathrm{E}-04$ & $\mathrm{U}$ & & ${ }^{154} \mathrm{Eu}$ & $7.2 \mathrm{E}-05 \pm 2.0 \mathrm{E}-04$ & $\mathrm{U}$ \\
\hline & ${ }^{155} \mathrm{Eu}$ & $-9.5 \mathrm{E}-05 \pm 2.0 \mathrm{E}-04$ & $\mathrm{U}$ & & ${ }^{155} \mathrm{Eu}$ & $4.6 \mathrm{E}-05 \pm 2.2 \mathrm{E}-04$ & $\mathrm{U}$ \\
\hline & ${ }^{238} \mathrm{Pu}$ & $-9.0 \mathrm{E}-06 \pm 1.5 \mathrm{E}-05$ & $\mathrm{U}$ & & ${ }^{238} \mathrm{Pu}$ & $-1.9 \mathrm{E}-06 \pm 1.1 \mathrm{E}-05$ & $\mathrm{U}$ \\
\hline & ${ }^{239 / 240} \mathrm{Pu}$ & $5.2 \mathrm{E}-06 \pm 5.9 \mathrm{E}-06$ & $\mathrm{U}$ & & ${ }^{239 / 240} \mathrm{Pu}$ & $6.8 \mathrm{E}-06 \pm 6.4 \mathrm{E}-06$ & $\mathrm{U}$ \\
\hline & ${ }^{103} \mathrm{Ru}$ & $-2.4 \mathrm{E}-05 \pm 7.2 \mathrm{E}-05$ & $\mathrm{U}$ & & ${ }^{103} \mathrm{Ru}$ & $1.5 \mathrm{E}-05 \pm 8.5 \mathrm{E}-05$ & $\mathrm{U}$ \\
\hline & ${ }^{106} \mathrm{Ru}$ & $-1.9 \mathrm{E}-04 \pm 5.6 \mathrm{E}-04$ & $\mathrm{U}$ & & ${ }^{106} \mathrm{Ru}$ & $5.0 \mathrm{E}-04 \pm 7.2 \mathrm{E}-04$ & $\mathrm{U}$ \\
\hline & ${ }^{125} \mathrm{Sb}$ & $7.7 \mathrm{E}-05 \pm 1.5 \mathrm{E}-04$ & $\mathrm{U}$ & & ${ }^{125} \mathrm{Sb}$ & $3.9 \mathrm{E}-05 \pm 1.9 \mathrm{E}-04$ & $\mathrm{U}$ \\
\hline & ${ }^{113} \mathrm{Sn}$ & $3.0 \mathrm{E}-06 \pm 3.0 \mathrm{E}-05$ & $\mathrm{U}$ & & ${ }^{113} \mathrm{Sn}$ & $3.9 \mathrm{E}-05 \pm 9.5 \mathrm{E}-05$ & $\mathrm{U}$ \\
\hline & ${ }^{90} \mathrm{Sr}$ & $-1.4 \mathrm{E}-04 \pm 1.5 \mathrm{E}-04$ & $\mathrm{U}$ & & ${ }^{90} \mathrm{Sr}$ & $-5.4 \mathrm{E}-05 \pm 5.6 \mathrm{E}-05$ & $\mathrm{U}$ \\
\hline & ${ }^{234} \mathrm{U}$ & $1.7 \mathrm{E}-05 \pm 1.0 \mathrm{E}-05$ & & & ${ }^{234} \mathrm{U}$ & $1.1 \mathrm{E}-05 \pm 7.4 \mathrm{E}-06$ & \\
\hline & ${ }^{235} \mathrm{U}$ & $7.5 \mathrm{E}-07 \pm 1.5 \mathrm{E}-06$ & $\mathrm{U}$ & & ${ }^{235} \mathrm{U}$ & $1.6 \mathrm{E}-06 \pm 3.2 \mathrm{E}-06$ & $\mathrm{U}$ \\
\hline & ${ }^{238} \mathrm{U}$ & $1.2 \mathrm{E}-05 \pm 7.6 \mathrm{E}-06$ & & & ${ }^{238} \mathrm{U}$ & $2.2 \mathrm{E}-06 \pm 2.7 \mathrm{E}-06$ & \\
\hline & ${ }^{65} \mathrm{Zn}$ & $-1.6 \mathrm{E}-04 \pm 1.7 \mathrm{E}-04$ & $\mathrm{U}$ & & ${ }^{65} \mathrm{Zn}$ & $1.1 \mathrm{E}-04 \pm 1.7 \mathrm{E}-04$ & $\mathrm{U}$ \\
\hline N105 $(100-N)$ & ${ }^{144} \mathrm{Ce}$ & $4.7 \mathrm{E}-04 \pm 1.1 \mathrm{E}-03$ & $\mathrm{U}$ & N106 $(100-N)$ & ${ }^{144} \mathrm{Ce}$ & $-1.4 \mathrm{E}-05 \pm 1.4 \mathrm{E}-04$ & $\bar{U}$ \\
\hline Composite Period & ${ }^{60} \mathrm{Co}$ & $5.4 \mathrm{E}-04 \pm 2.3 \mathrm{E}-04$ & & Composite Period & ${ }^{60} \mathrm{Co}$ & $5.1 \mathrm{E}-05 \pm 8.2 \mathrm{E}-05$ & $\mathrm{U}$ \\
\hline \multirow[t]{16}{*}{$06 / 23 / 03$ to $09 / 30 / 03$} & ${ }^{134} \mathrm{Cs}$ & $3.5 \mathrm{E}-05 \pm 1.2 \mathrm{E}-04$ & $\mathrm{U}$ & $01 / 07 / 03$ to $06 / 23 / 03$ & ${ }^{134} \mathrm{Cs}$ & $3.0 \mathrm{E}-05 \pm 6.9 \mathrm{E}-05$ & $\mathrm{U}$ \\
\hline & ${ }^{137} \mathrm{Cs}$ & $2.8 \mathrm{E}-04 \pm 2.1 \mathrm{E}-04$ & & & ${ }^{137} \mathrm{Cs}$ & $-3.7 \mathrm{E}-06 \pm 3.7 \mathrm{E}-05$ & $\mathrm{U}$ \\
\hline & ${ }^{152} \mathrm{Eu}$ & $-1.4 \mathrm{E}-04 \pm 2.7 \mathrm{E}-04$ & $\mathrm{U}$ & & ${ }^{152} \mathrm{Eu}$ & $8.4 \mathrm{E}-05 \pm 1.7 \mathrm{E}-04$ & $\mathrm{U}$ \\
\hline & ${ }^{154} \mathrm{Eu}$ & $2.1 \mathrm{E}-04 \pm 4.0 \mathrm{E}-04$ & $\mathrm{U}$ & & ${ }^{154} \mathrm{Eu}$ & $7.2 \mathrm{E}-06 \pm 7.2 \mathrm{E}-05$ & $\mathrm{U}$ \\
\hline & ${ }^{155} \mathrm{Eu}$ & $2.1 \mathrm{E}-05 \pm 2.1 \mathrm{E}-04$ & $\mathrm{U}$ & & ${ }^{155} \mathrm{Eu}$ & $8.4 \mathrm{E}-05 \pm 1.5 \mathrm{E}-04$ & $\mathrm{U}$ \\
\hline & ${ }^{238} \mathrm{Pu}$ & $-1.2 \mathrm{E}-05 \pm 2.7 \mathrm{E}-05$ & $\mathrm{U}$ & & ${ }^{238} \mathrm{Pu}$ & $7.3 \mathrm{E}-06 \pm 1.4 \mathrm{E}-05$ & $\mathrm{U}$ \\
\hline & ${ }^{239 / 240} \mathrm{Pu}$ & $5.0 \mathrm{E}-06 \pm 1.0 \mathrm{E}-05$ & $\mathrm{U}$ & & ${ }^{239 / 240} \mathrm{Pu}$ & $-3.7 \mathrm{E}-06 \pm 4.5 \mathrm{E}-06$ & $\mathrm{U}$ \\
\hline & ${ }^{103} \mathrm{Ru}$ & $-8.3 \mathrm{E}-05 \pm 1.2 \mathrm{E}-04$ & $\mathrm{U}$ & & ${ }^{103} \mathrm{Ru}$ & $-6.4 \mathrm{E}-05 \pm 7.6 \mathrm{E}-05$ & $\mathrm{U}$ \\
\hline & ${ }^{106} \mathrm{Ru}$ & $-3.3 \mathrm{E}-04 \pm 1.0 \mathrm{E}-03$ & $\mathrm{U}$ & & ${ }^{106} \mathrm{Ru}$ & $6.2 \mathrm{E}-04 \pm 7.0 \mathrm{E}-04$ & $\mathrm{U}$ \\
\hline & ${ }^{125} \mathrm{Sb}$ & $9.8 \mathrm{E}-05 \pm 2.6 \mathrm{E}-04$ & $\mathrm{U}$ & & ${ }^{125} \mathrm{Sb}$ & $-7.2 \mathrm{E}-05 \pm 1.5 \mathrm{E}-04$ & $\mathrm{U}$ \\
\hline & ${ }^{113} \mathrm{Sn}$ & $-2.3 \mathrm{E}-05 \pm 1.2 \mathrm{E}-04$ & $\mathrm{U}$ & & ${ }^{113} \mathrm{Sn}$ & $1.8 \mathrm{E}-05 \pm 7.6 \mathrm{E}-05$ & $\mathrm{U}$ \\
\hline & ${ }^{90} \mathrm{Sr}$ & $-1.3 \mathrm{E}-04 \pm 1.7 \mathrm{E}-04$ & $\mathrm{U}$ & & ${ }^{90} \mathrm{Sr}$ & $-8.3 \mathrm{E}-05 \pm 8.6 \mathrm{E}-05$ & $\mathrm{U}$ \\
\hline & ${ }^{234} \mathrm{U}$ & $2.4 \mathrm{E}-05 \pm 1.4 \mathrm{E}-05$ & & & ${ }^{234} \mathrm{U}$ & $8.3 \mathrm{E}-06 \pm 6.3 \mathrm{E}-06$ & \\
\hline & ${ }^{235} \mathrm{U}$ & $3.2 \mathrm{E}-06 \pm 4.5 \mathrm{E}-06$ & $\mathrm{U}$ & & ${ }^{235} \mathrm{U}$ & $2.3 \mathrm{E}-06 \pm 3.4 \mathrm{E}-06$ & $\mathrm{U}$ \\
\hline & ${ }^{238} \mathrm{U}$ & $1.4 \mathrm{E}-05 \pm 1.1 \mathrm{E}-05$ & & & ${ }^{238} \mathrm{U}$ & $6.3 \mathrm{E}-06 \pm 4.8 \mathrm{E}-06$ & \\
\hline & ${ }^{65} \mathrm{Zn}$ & $-1.9 \mathrm{E}-04 \pm 3.4 \mathrm{E}-04$ & $\mathrm{U}$ & & ${ }^{65} \mathrm{Zn}$ & $6.3 \mathrm{E}-05 \pm 1.9 \mathrm{E}-04$ & $\mathrm{U}$ \\
\hline
\end{tabular}

$\overline{\mathrm{RQ}}=$ Result Qualifier. $\mathrm{U}=$ The analyte was analyzed for but not detected. 
Table 2-3. Near-Facility Air Sampling Results, 2003 (pCi/m³ \pm total analytical uncertainty). (cont)

\begin{tabular}{ccrc}
\hline Location & Isotope & Result \pm Uncertainty & RQ* \\
\hline N106 (100-N) & ${ }^{144} \mathrm{Ce}$ & $-5.8 \mathrm{E}-04 \pm 8.4 \mathrm{E}-04$ & $\mathrm{U}$ \\
Composite Period & ${ }^{60} \mathrm{Co}$ & $1.1 \mathrm{E}-04 \pm 1.0 \mathrm{E}-04$ & $\mathrm{U}$ \\
06/23/03 to 12/22/03 & ${ }^{134} \mathrm{Cs}$ & $-7.8 \mathrm{E}-06 \pm 7.8 \mathrm{E}-05$ & $\mathrm{U}$ \\
& ${ }^{137} \mathrm{Cs}$ & $1.1 \mathrm{E}-05 \pm 8.5 \mathrm{E}-05$ & $\mathrm{U}$ \\
& ${ }^{152} \mathrm{Eu}$ & $2.8 \mathrm{E}-05 \pm 1.9 \mathrm{E}-04$ & $\mathrm{U}$ \\
& ${ }^{154} \mathrm{Eu}$ & $5.6 \mathrm{E}-06 \pm 5.6 \mathrm{E}-05$ & $\mathrm{U}$ \\
& ${ }^{155} \mathrm{Eu}$ & $-1.8 \mathrm{E}-04 \pm 2.1 \mathrm{E}-04$ & $\mathrm{U}$ \\
& ${ }^{238} \mathrm{Pu}$ & $4.0 \mathrm{E}-06 \pm 1.2 \mathrm{E}-05$ & $\mathrm{U}$ \\
& ${ }^{239 / 240} \mathrm{Pu}$ & $4.8 \mathrm{E}-06 \pm 6.4 \mathrm{E}-06$ & $\mathrm{U}$ \\
& ${ }^{103} \mathrm{Ru}$ & $3.3 \mathrm{E}-05 \pm 1.1 \mathrm{E}-04$ & $\mathrm{U}$ \\
& ${ }^{106} \mathrm{Ru}$ & $-8.9 \mathrm{E}-05 \pm 8.5 \mathrm{E}-04$ & $\mathrm{U}$ \\
& ${ }^{125} \mathrm{Sb}$ & $1.3 \mathrm{E}-04 \pm 2.0 \mathrm{E}-04$ & $\mathrm{U}$ \\
& ${ }^{113} \mathrm{Sn}$ & $-1.7 \mathrm{E}-05 \pm 9.3 \mathrm{E}-05$ & $\mathrm{U}$ \\
& ${ }^{90} \mathrm{Sr}$ & $-1.8 \mathrm{E}-04 \pm 1.8 \mathrm{E}-04$ & $\mathrm{U}$ \\
& ${ }^{234} \mathrm{U}$ & $1.3 \mathrm{E}-05 \pm 8.7 \mathrm{E}-06$ & \\
& ${ }^{235} \mathrm{U}$ & $-1.5 \mathrm{E}-06 \pm 3.9 \mathrm{E}-06$ & $\mathrm{U}$ \\
& ${ }^{238} \mathrm{U}$ & $1.0 \mathrm{E}-05 \pm 7.4 \mathrm{E}-06$ & \\
& ${ }^{63} \mathrm{Zn} \mathrm{U}$ & $-2.2 \mathrm{E}-04 \pm 2.2 \mathrm{E}-04$ & $\mathrm{U}$
\end{tabular}

\begin{tabular}{ccrc}
\hline Location & Isotope & Result \pm Uncertainty & RQ* \\
\hline N526 (100-N) & ${ }^{144} \mathrm{Ce}$ & $-3.7 \mathrm{E}-04 \pm 8.4 \mathrm{E}-04$ & $\mathrm{U}$ \\
Composite Period & ${ }^{60} \mathrm{Co}$ & $4.2 \mathrm{E}-04 \pm 1.8 \mathrm{E}-04$ & \\
$01 / 07 / 03$ to 06/23/03 & ${ }^{134} \mathrm{Cs}$ & $3.1 \mathrm{E}-05 \pm 9.0 \mathrm{E}-05$ & $\mathrm{U}$ \\
& ${ }^{137} \mathrm{Cs}$ & $2.5 \mathrm{E}-03 \pm 7.9 \mathrm{E}-04$ & \\
& ${ }^{152} \mathrm{Eu}$ & $9.0 \mathrm{E}-05 \pm 2.2 \mathrm{E}-04$ & $\mathrm{U}$ \\
& ${ }^{154} \mathrm{Eu}$ & $1.3 \mathrm{E}-04 \pm 2.1 \mathrm{E}-04$ & $\mathrm{U}$ \\
& ${ }^{155} \mathrm{Eu}$ & $-1.6 \mathrm{E}-04 \pm 2.3 \mathrm{E}-04$ & $\mathrm{U}$ \\
& ${ }^{238} \mathrm{Pu}$ & $-5.1 \mathrm{E}-06 \pm 1.8 \mathrm{E}-05$ & $\mathrm{U}$ \\
& ${ }^{239 / 240} \mathrm{Pu}$ & $2.0 \mathrm{E}-05 \pm 1.2 \mathrm{E}-05$ & \\
& ${ }^{103} \mathrm{Ru}$ & $2.6 \mathrm{E}-05 \pm 1.0 \mathrm{E}-04$ & $\mathrm{U}$ \\
& ${ }^{106} \mathrm{Ru}$ & $-1.4 \mathrm{E}-04 \pm 7.0 \mathrm{E}-04$ & $\mathrm{U}$ \\
& ${ }^{125} \mathrm{Sb}$ & $6.2 \mathrm{E}-05 \pm 2.0 \mathrm{E}-04$ & $\mathrm{U}$ \\
& ${ }^{113} \mathrm{Sn}$ & $3.5 \mathrm{E}-05 \pm 9.9 \mathrm{E}-05$ & $\mathrm{U}$ \\
& ${ }^{90} \mathrm{Sr}$ & $7.7 \mathrm{E}-05 \pm 1.0 \mathrm{E}-04$ & $\mathrm{U}$ \\
& ${ }^{234} \mathrm{U}$ & $6.4 \mathrm{E}-06 \pm 5.3 \mathrm{E}-06$ & \\
& ${ }^{235} \mathrm{U}$ & $1.5 \mathrm{E}-06 \pm 3.1 \mathrm{E}-06$ & $\mathrm{U}$ \\
& ${ }^{238} \mathrm{U}$ & $8.4 \mathrm{E}-06 \pm 6.1 \mathrm{E}-06$ & \\
& ${ }^{238} \mathrm{U} \mathrm{Zn}$ & $-1.9 \mathrm{E}-04 \pm 2.2 \mathrm{E}-04$ & $\mathrm{U}$
\end{tabular}

$\overline{\mathrm{RQ}}=$ Result Qualifier. $\mathrm{U}=$ The analyte was analyzed for but not detected. 
Table 2-3. Near-Facility Air Sampling Results, 2003 (pCi/m³ \pm total analytical uncertainty). (cont)

\begin{tabular}{|c|c|c|c|c|c|c|c|}
\hline Location & Isotope & Result \pm Uncertainty & $\mathbf{R Q}^{*}$ & Location & Isotope & Result \pm Uncertainty & RQ* \\
\hline N482 (ERDF) & ${ }^{144} \mathrm{Ce}$ & $1.4 \mathrm{E}-05 \pm 1.4 \mathrm{E}-04$ & $\bar{U}$ & N517 (ERDF) & ${ }^{144} \mathrm{Ce}$ & $6.3 \mathrm{E}-04 \pm 6.1 \mathrm{E}-04$ & $\bar{U}$ \\
\hline Composite Period & ${ }^{60} \mathrm{Co}$ & $-5.8 \mathrm{E}-05 \pm 8.0 \mathrm{E}-05$ & U & Composite Period & ${ }^{60} \mathrm{Co}$ & $-6.8 \mathrm{E}-05 \pm 8.8 \mathrm{E}-05$ & $\mathrm{U}$ \\
\hline \multirow[t]{16}{*}{$06 / 24 / 03$ to $12 / 22 / 03$} & ${ }^{134} \mathrm{Cs}$ & $1.3 \mathrm{E}-06 \pm 1.3 \mathrm{E}-05$ & $\mathrm{U}$ & $01 / 08 / 03$ to $06 / 24 / 03$ & ${ }^{134} \mathrm{Cs}$ & $-1.7 \mathrm{E}-06 \pm 1.7 \mathrm{E}-05$ & $\mathrm{U}$ \\
\hline & ${ }^{137} \mathrm{Cs}$ & $2.8 \mathrm{E}-05 \pm 6.4 \mathrm{E}-05$ & $\mathrm{U}$ & & ${ }^{137} \mathrm{Cs}$ & $2.4 \mathrm{E}-05 \pm 7.8 \mathrm{E}-05$ & U \\
\hline & ${ }^{152} \mathrm{Eu}$ & $-3.7 \mathrm{E}-05 \pm 1.6 \mathrm{E}-04$ & $\mathrm{U}$ & & ${ }^{152} \mathrm{Eu}$ & $-5.7 \mathrm{E}-05 \pm 1.6 \mathrm{E}-04$ & $\mathrm{U}$ \\
\hline & ${ }^{154} \mathrm{Eu}$ & $-1.0 \mathrm{E}-04 \pm 2.1 \mathrm{E}-04$ & $\mathrm{U}$ & & ${ }^{154} \mathrm{Eu}$ & $7.2 \mathrm{E}-05 \pm 2.5 \mathrm{E}-04$ & $\mathrm{U}$ \\
\hline & ${ }^{155} \mathrm{Eu}$ & $1.5 \mathrm{E}-07 \pm 1.5 \mathrm{E}-06$ & U & & ${ }^{155} \mathrm{Eu}$ & $-1.6 \mathrm{E}-05 \pm 1.5 \mathrm{E}-04$ & $\mathrm{U}$ \\
\hline & ${ }^{238} \mathrm{Pu}$ & $1.1 \mathrm{E}-05 \pm 1.3 \mathrm{E}-05$ & $\mathrm{U}$ & & ${ }^{238} \mathrm{Pu}$ & $-5.5 \mathrm{E}-06 \pm 1.7 \mathrm{E}-05$ & $\mathrm{U}$ \\
\hline & ${ }^{239 / 240} \mathrm{Pu}$ & $3.2 \mathrm{E}-06 \pm 4.5 \mathrm{E}-06$ & $\mathrm{U}$ & & ${ }^{239 / 240} \mathrm{Pu}$ & $2.2 \mathrm{E}-06 \pm 3.2 \mathrm{E}-06$ & $\mathrm{U}$ \\
\hline & ${ }^{103} \mathrm{Ru}$ & $1.2 \mathrm{E}-06 \pm 1.2 \mathrm{E}-05$ & $\mathrm{U}$ & & ${ }^{103} \mathrm{Ru}$ & $-2.4 \mathrm{E}-05 \pm 7.9 \mathrm{E}-05$ & $\mathrm{U}$ \\
\hline & ${ }^{106} \mathrm{Ru}$ & $8.7 \mathrm{E}-05 \pm 6.4 \mathrm{E}-04$ & U & & ${ }^{106} \mathrm{Ru}$ & $-8.2 \mathrm{E}-05 \pm 6.3 \mathrm{E}-04$ & $\mathrm{U}$ \\
\hline & ${ }^{125} \mathrm{Sb}$ & $1.2 \mathrm{E}-04 \pm 1.4 \mathrm{E}-04$ & $\mathrm{U}$ & & ${ }^{125} \mathrm{Sb}$ & $-2.2 \mathrm{E}-05 \pm 1.6 \mathrm{E}-04$ & $\mathrm{U}$ \\
\hline & ${ }^{113} \mathrm{Sn}$ & $3.5 \mathrm{E}-05 \pm 6.9 \mathrm{E}-05$ & $\mathrm{U}$ & & ${ }^{113} \mathrm{Sn}$ & $4.4 \mathrm{E}-05 \pm 8.7 \mathrm{E}-05$ & $\mathrm{U}$ \\
\hline & ${ }^{90} \mathrm{Sr}$ & $1.0 \mathrm{E}-04 \pm 1.1 \mathrm{E}-04$ & & & ${ }^{90} \mathrm{Sr}$ & $-1.4 \mathrm{E}-04 \pm 1.4 \mathrm{E}-04$ & $\mathrm{U}$ \\
\hline & ${ }^{234} \mathrm{U}$ & $2.2 \mathrm{E}-05 \pm 1.2 \mathrm{E}-05$ & & & ${ }^{234} \mathrm{U}$ & $2.1 \mathrm{E}-05 \pm 1.2 \mathrm{E}-05$ & \\
\hline & ${ }^{235} \mathrm{U}$ & $2.4 \mathrm{E}-06 \pm 3.7 \mathrm{E}-06$ & $\mathrm{U}$ & & ${ }^{235} \mathrm{U}$ & $3.8 \mathrm{E}-06 \pm 4.7 \mathrm{E}-06$ & U \\
\hline & ${ }^{238} \mathrm{U}$ & $2.7 \mathrm{E}-05 \pm 1.4 \mathrm{E}-05$ & & & ${ }^{238} \mathrm{U}$ & $1.2 \mathrm{E}-05 \pm 8.1 \mathrm{E}-06$ & \\
\hline & ${ }^{65} \mathrm{Zn}$ & $7.9 \mathrm{E}-05 \pm 1.6 \mathrm{E}-04$ & $\mathrm{U}$ & & ${ }^{65} \mathrm{Zn}$ & $4.6 \mathrm{E}-06 \pm 4.6 \mathrm{E}-05$ & $\mathrm{U}$ \\
\hline N517 (ERDF) & ${ }^{144} \mathrm{Ce}$ & $3.3 \mathrm{E}-04 \pm 6.5 \mathrm{E}-04$ & $\mathrm{U}$ & N518 (ERDF) & ${ }^{144} \mathrm{Ce}$ & $-1.5 \mathrm{E}-04 \pm 8.0 \mathrm{E}-04$ & $\mathrm{U}$ \\
\hline Composite Period & ${ }^{60} \mathrm{Co}$ & $1.5 \mathrm{E}-05 \pm 7.7 \mathrm{E}-05$ & $\mathrm{U}$ & Composite Period & ${ }^{60} \mathrm{Co}$ & $5.4 \mathrm{E}-05 \pm 9.4 \mathrm{E}-05$ & $\mathrm{U}$ \\
\hline \multirow[t]{16}{*}{$06 / 24 / 03$ to $12 / 22 / 03$} & ${ }^{134} \mathrm{Cs}$ & $5.6 \mathrm{E}-05 \pm 7.1 \mathrm{E}-05$ & $\mathrm{U}$ & $01 / 08 / 03$ to $06 / 24 / 03$ & ${ }^{134} \mathrm{Cs}$ & $3.3 \mathrm{E}-05 \pm 8.8 \mathrm{E}-05$ & $\mathrm{U}$ \\
\hline & ${ }^{137} \mathrm{Cs}$ & $7.6 \mathrm{E}-05 \pm 5.9 \mathrm{E}-05$ & $\mathrm{U}$ & & ${ }^{137} \mathrm{Cs}$ & $1.8 \mathrm{E}-05 \pm 8.0 \mathrm{E}-05$ & $\mathrm{U}$ \\
\hline & ${ }^{152} \mathrm{Eu}$ & $1.7 \mathrm{E}-05 \pm 1.6 \mathrm{E}-04$ & $\mathrm{U}$ & & ${ }^{152} \mathrm{Eu}$ & $-5.0 \mathrm{E}-05 \pm 2.0 \mathrm{E}-04$ & $\mathrm{U}$ \\
\hline & ${ }^{154} \mathrm{Eu}$ & $7.2 \mathrm{E}-05 \pm 2.3 \mathrm{E}-04$ & $\mathrm{U}$ & & ${ }^{154} \mathrm{Eu}$ & $7.8 \mathrm{E}-06 \pm 7.8 \mathrm{E}-05$ & $\mathrm{U}$ \\
\hline & ${ }^{155} \mathrm{Eu}$ & $-5.3 \mathrm{E}-05 \pm 1.5 \mathrm{E}-04$ & $\mathrm{U}$ & & ${ }^{155} \mathrm{Eu}$ & $-7.1 \mathrm{E}-06 \pm 7.1 \mathrm{E}-05$ & $\mathrm{U}$ \\
\hline & ${ }^{238} \mathrm{Pu}$ & $3.2 \mathrm{E}-06 \pm 1.3 \mathrm{E}-05$ & $\mathrm{U}$ & & ${ }^{238} \mathrm{Pu}$ & $-9.3 \mathrm{E}-07 \pm 9.3 \mathrm{E}-06$ & $\mathrm{U}$ \\
\hline & ${ }^{239 / 240} \mathrm{Pu}$ & $4.8 \mathrm{E}-06 \pm 5.4 \mathrm{E}-06$ & $\mathrm{U}$ & & ${ }^{239 / 240} \mathrm{Pu}$ & $-9.3 \mathrm{E}-07 \pm 5.0 \mathrm{E}-06$ & $\mathrm{U}$ \\
\hline & ${ }^{103} \mathrm{Ru}$ & $1.8 \mathrm{E}-05 \pm 6.6 \mathrm{E}-05$ & $\mathrm{U}$ & & ${ }^{103} \mathrm{Ru}$ & $-9.3 \mathrm{E}-06 \pm 9.3 \mathrm{E}-05$ & $\mathrm{U}$ \\
\hline & ${ }^{106} \mathrm{Ru}$ & $-2.2 \mathrm{E}-04 \pm 5.8 \mathrm{E}-04$ & $\mathrm{U}$ & & ${ }^{106} \mathrm{Ru}$ & $-1.8 \mathrm{E}-05 \pm 1.8 \mathrm{E}-04$ & $\mathrm{U}$ \\
\hline & ${ }^{125} \mathrm{Sb}$ & $-1.8 \mathrm{E}-05 \pm 1.3 \mathrm{E}-04$ & $\mathrm{U}$ & & ${ }^{125} \mathrm{Sb}$ & $-1.2 \mathrm{E}-05 \pm 1.2 \mathrm{E}-04$ & $\mathrm{U}$ \\
\hline & ${ }^{113} \mathrm{Sn}$ & $1.7 \mathrm{E}-05 \pm 7.1 \mathrm{E}-05$ & $\mathrm{U}$ & & ${ }^{113} \mathrm{Sn}$ & $8.5 \mathrm{E}-05 \pm 1.0 \mathrm{E}-04$ & $\mathrm{U}$ \\
\hline & ${ }^{90} \mathrm{Sr}$ & $6.8 \mathrm{E}-05 \pm 1.1 \mathrm{E}-04$ & $\mathrm{U}$ & & ${ }^{90} \mathrm{Sr}$ & $-1.2 \mathrm{E}-04 \pm 1.2 \mathrm{E}-04$ & $\mathrm{U}$ \\
\hline & ${ }^{234} \mathrm{U}$ & $2.6 \mathrm{E}-05 \pm 1.3 \mathrm{E}-05$ & & & ${ }^{234} \mathrm{U}$ & $2.7 \mathrm{E}-05 \pm 1.4 \mathrm{E}-05$ & \\
\hline & ${ }^{235} \mathrm{U}$ & $3.8 \mathrm{E}-06 \pm 4.3 \mathrm{E}-06$ & $\mathrm{U}$ & & ${ }^{235} \mathrm{U}$ & $5.8 \mathrm{E}-06 \pm 5.2 \mathrm{E}-06$ & \\
\hline & ${ }^{238} \mathrm{U}$ & $1.8 \mathrm{E}-05 \pm 9.8 \mathrm{E}-06$ & & & ${ }^{238} \mathrm{U}$ & $1.9 \mathrm{E}-05 \pm 1.1 \mathrm{E}-05$ & \\
\hline & ${ }^{65} \mathrm{Zn}$ & $1.6 \mathrm{E}-04 \pm 1.6 \mathrm{E}-04$ & $\mathrm{U}$ & & ${ }^{65} \mathrm{Zn}$ & $-2.0 \mathrm{E}-04 \pm 2.1 \mathrm{E}-04$ & $\mathrm{U}$ \\
\hline
\end{tabular}

$\overline{\mathrm{RQ}}=$ Result Qualifier. $\mathrm{U}=$ The analyte was analyzed for but not detected. 
Table 2-3. Near-Facility Air Sampling Results, 2003 (pCi/m³ \pm total analytical uncertainty). (cont)

\begin{tabular}{|c|c|c|c|c|c|c|c|}
\hline Location & Isotope & Result \pm Uncertainty & $\mathbf{R Q}^{*}$ & Location & Isotope & Result \pm Uncertainty & $\mathbf{R Q}^{*}$ \\
\hline N518 (ERDF) & ${ }^{144} \mathrm{Ce}$ & $2.3 \mathrm{E}-04 \pm 7.1 \mathrm{E}-04$ & $\bar{U}$ & N019 (200-East) & ${ }^{144} \mathrm{Ce}$ & $-1.0 \mathrm{E}-05 \pm 1.1 \mathrm{E}-04$ & $\bar{U}$ \\
\hline Composite Period & ${ }^{60} \mathrm{Co}$ & $-3.1 \mathrm{E}-05 \pm 9.3 \mathrm{E}-05$ & U & Composite Period & ${ }^{60} \mathrm{Co}$ & $-8.5 \mathrm{E}-06 \pm 8.6 \mathrm{E}-05$ & $\mathrm{U}$ \\
\hline \multirow[t]{16}{*}{$06 / 24 / 03$ to $12 / 22 / 03$} & ${ }^{134} \mathrm{Cs}$ & $3.3 \mathrm{E}-05 \pm 9.7 \mathrm{E}-05$ & $\mathrm{U}$ & $01 / 06 / 03$ to $06 / 23 / 03$ & ${ }^{134} \mathrm{Cs}$ & $1.3 \mathrm{E}-05 \pm 7.3 \mathrm{E}-05$ & $\mathrm{U}$ \\
\hline & ${ }^{137} \mathrm{Cs}$ & $7.1 \mathrm{E}-05 \pm 8.5 \mathrm{E}-05$ & $\mathrm{U}$ & & ${ }^{137} \mathrm{Cs}$ & $-1.6 \mathrm{E}-05 \pm 9.2 \mathrm{E}-05$ & U \\
\hline & ${ }^{152} \mathrm{Eu}$ & $1.4 \mathrm{E}-04 \pm 2.2 \mathrm{E}-04$ & $\mathrm{U}$ & & ${ }^{152} \mathrm{Eu}$ & $-4.7 \mathrm{E}-06 \pm 4.7 \mathrm{E}-05$ & $\mathrm{U}$ \\
\hline & ${ }^{154} \mathrm{Eu}$ & $-1.8 \mathrm{E}-04 \pm 2.8 \mathrm{E}-04$ & $\mathrm{U}$ & & ${ }^{154} \mathrm{Eu}$ & $9.9 \mathrm{E}-06 \pm 9.9 \mathrm{E}-05$ & $\mathrm{U}$ \\
\hline & ${ }^{155} \mathrm{Eu}$ & $-1.2 \mathrm{E}-04 \pm 1.9 \mathrm{E}-04$ & U & & ${ }^{155} \mathrm{Eu}$ & $1.3 \mathrm{E}-05 \pm 1.3 \mathrm{E}-04$ & $\mathrm{U}$ \\
\hline & ${ }^{238} \mathrm{Pu}$ & $-1.2 \mathrm{E}-05 \pm 1.6 \mathrm{E}-05$ & $\mathrm{U}$ & & ${ }^{238} \mathrm{Pu}$ & $1.0 \mathrm{E}-05 \pm 1.5 \mathrm{E}-05$ & $\mathrm{U}$ \\
\hline & ${ }^{239 / 240} \mathrm{Pu}$ & $4.6 \mathrm{E}-06 \pm 5.6 \mathrm{E}-06$ & $\mathrm{U}$ & & ${ }^{239 / 240} \mathrm{Pu}$ & $9.2 \mathrm{E}-07 \pm 9.6 \mathrm{E}-07$ & $\mathrm{U}$ \\
\hline & ${ }^{103} \mathrm{Ru}$ & $-7.7 \mathrm{E}-05 \pm 8.1 \mathrm{E}-05$ & $\mathrm{U}$ & & ${ }^{103} \mathrm{Ru}$ & $9.6 \mathrm{E}-06 \pm 6.4 \mathrm{E}-05$ & $\mathrm{U}$ \\
\hline & ${ }^{106} \mathrm{Ru}$ & $-8.3 \mathrm{E}-07 \pm 8.3 \mathrm{E}-06$ & U & & ${ }^{106} \mathrm{Ru}$ & $1.3 \mathrm{E}-04 \pm 7.0 \mathrm{E}-04$ & $\mathrm{U}$ \\
\hline & ${ }^{125} \mathrm{Sb}$ & $-1.7 \mathrm{E}-04 \pm 1.9 \mathrm{E}-04$ & $\mathrm{U}$ & & ${ }^{125} \mathrm{Sb}$ & $-2.1 \mathrm{E}-04 \pm 2.1 \mathrm{E}-04$ & $\mathrm{U}$ \\
\hline & ${ }^{113} \mathrm{Sn}$ & $-3.4 \mathrm{E}-05 \pm 8.6 \mathrm{E}-05$ & $\mathrm{U}$ & & ${ }^{113} \mathrm{Sn}$ & $-9.7 \mathrm{E}-06 \pm 9.1 \mathrm{E}-05$ & $\mathrm{U}$ \\
\hline & ${ }^{90} \mathrm{Sr}$ & $3.9 \mathrm{E}-05 \pm 1.0 \mathrm{E}-04$ & $\mathrm{U}$ & & ${ }^{90} \mathrm{Sr}$ & $1.5 \mathrm{E}-05 \pm 1.2 \mathrm{E}-04$ & $\mathrm{U}$ \\
\hline & ${ }^{234} \mathrm{U}$ & $1.3 \mathrm{E}-05 \pm 8.1 \mathrm{E}-06$ & & & ${ }^{234} \mathrm{U}$ & $6.2 \mathrm{E}-06 \pm 5.9 \mathrm{E}-06$ & $\mathrm{U}$ \\
\hline & ${ }^{235} \mathrm{U}$ & $4.3 \mathrm{E}-06 \pm 4.2 \mathrm{E}-06$ & & & ${ }^{235} \mathrm{U}$ & $2.5 \mathrm{E}-06 \pm 3.1 \mathrm{E}-06$ & \\
\hline & ${ }^{238} \mathrm{U}$ & $1.7 \mathrm{E}-05 \pm 9.8 \mathrm{E}-06$ & & & ${ }^{238} \mathrm{U}$ & $3.2 \mathrm{E}-06 \pm 3.9 \mathrm{E}-06$ & $\mathrm{U}$ \\
\hline & ${ }^{65} \mathrm{Zn}$ & $-1.5 \mathrm{E}-05 \pm 1.5 \mathrm{E}-04$ & $\mathrm{U}$ & & ${ }^{65} \mathrm{Zn}$ & $-1.4 \mathrm{E}-04 \pm 2.2 \mathrm{E}-04$ & $\mathrm{U}$ \\
\hline N019 (200-East) & ${ }^{144} \mathrm{Ce}$ & $1.7 \mathrm{E}-04 \pm 6.0 \mathrm{E}-04$ & $\mathrm{U}$ & N158 (200-East) & ${ }^{144} \mathrm{Ce}$ & $-4.3 \mathrm{E}-04 \pm 7.1 \mathrm{E}-04$ & $\mathrm{U}$ \\
\hline Composite Period & ${ }^{60} \mathrm{Co}$ & $1.1 \mathrm{E}-05 \pm 7.9 \mathrm{E}-05$ & $\mathrm{U}$ & Composite Period & ${ }^{60} \mathrm{Co}$ & $-2.3 \mathrm{E}-05 \pm 8.9 \mathrm{E}-05$ & $\mathrm{U}$ \\
\hline \multirow[t]{16}{*}{$06 / 23 / 03$ to $12 / 22 / 03$} & ${ }^{134} \mathrm{Cs}$ & $6.0 \mathrm{E}-05 \pm 6.9 \mathrm{E}-05$ & $\mathrm{U}$ & $01 / 06 / 03$ to $06 / 23 / 03$ & ${ }^{134} \mathrm{Cs}$ & $1.1 \mathrm{E}-04 \pm 1.1 \mathrm{E}-04$ & $\mathrm{U}$ \\
\hline & ${ }^{137} \mathrm{Cs}$ & $2.0 \mathrm{E}-05 \pm 6.8 \mathrm{E}-05$ & $\mathrm{U}$ & & ${ }^{137} \mathrm{Cs}$ & $1.4 \mathrm{E}-04 \pm 1.3 \mathrm{E}-04$ & \\
\hline & ${ }^{152} \mathrm{Eu}$ & $2.5 \mathrm{E}-05 \pm 1.5 \mathrm{E}-04$ & $\mathrm{U}$ & & ${ }^{152} \mathrm{Eu}$ & $3.7 \mathrm{E}-05 \pm 1.8 \mathrm{E}-04$ & $\mathrm{U}$ \\
\hline & ${ }^{154} \mathrm{Eu}$ & $2.2 \mathrm{E}-05 \pm 2.1 \mathrm{E}-04$ & $\mathrm{U}$ & & ${ }^{154} \mathrm{Eu}$ & $5.2 \mathrm{E}-05 \pm 2.7 \mathrm{E}-04$ & U \\
\hline & ${ }^{155} \mathrm{Eu}$ & $-8.4 \mathrm{E}-05 \pm 1.6 \mathrm{E}-04$ & $\mathrm{U}$ & & ${ }^{155} \mathrm{Eu}$ & $-7.1 \mathrm{E}-05 \pm 1.9 \mathrm{E}-04$ & $\mathrm{U}$ \\
\hline & ${ }^{238} \mathrm{Pu}$ & $-1.1 \mathrm{E}-05 \pm 1.5 \mathrm{E}-05$ & $\mathrm{U}$ & & ${ }^{238} \mathrm{Pu}$ & $6.6 \mathrm{E}-06 \pm 1.4 \mathrm{E}-05$ & $\mathrm{U}$ \\
\hline & ${ }^{239 / 240} \mathrm{Pu}$ & 4.3E-06 \pm 6.1E-06 & $\mathrm{U}$ & & ${ }^{239 / 240} \mathrm{Pu}$ & $9.1 \mathrm{E}-07 \pm 9.4 \mathrm{E}-07$ & $\mathrm{U}$ \\
\hline & ${ }^{103} \mathrm{Ru}$ & $8.2 \mathrm{E}-05 \pm 6.0 \mathrm{E}-05$ & $\mathrm{U}$ & & ${ }^{103} \mathrm{Ru}$ & $1.0 \mathrm{E}-05 \pm 6.7 \mathrm{E}-05$ & $\mathrm{U}$ \\
\hline & ${ }^{106} \mathrm{Ru}$ & $3.1 \mathrm{E}-04 \pm 5.2 \mathrm{E}-04$ & $\mathrm{U}$ & & ${ }^{106} \mathrm{Ru}$ & $1.1 \mathrm{E}-04 \pm 6.2 \mathrm{E}-04$ & $\mathrm{U}$ \\
\hline & ${ }^{125} \mathrm{Sb}$ & $-2.7 \mathrm{E}-05 \pm 1.4 \mathrm{E}-04$ & $\mathrm{U}$ & & ${ }^{125} \mathrm{Sb}$ & $-6.5 \mathrm{E}-05 \pm 1.9 \mathrm{E}-04$ & $\mathrm{U}$ \\
\hline & ${ }^{113} \mathrm{Sn}$ & $-2.2 \mathrm{E}-05 \pm 6.5 \mathrm{E}-05$ & $\mathrm{U}$ & & ${ }^{113} \mathrm{Sn}$ & $-1.2 \mathrm{E}-05 \pm 8.1 \mathrm{E}-05$ & $\mathrm{U}$ \\
\hline & ${ }^{90} \mathrm{Sr}$ & $-2.3 \mathrm{E}-04 \pm 1.1 \mathrm{E}-04$ & $\mathrm{U}$ & & ${ }^{90} \mathrm{Sr}$ & $-1.2 \mathrm{E}-04 \pm 1.3 \mathrm{E}-04$ & $\mathrm{U}$ \\
\hline & ${ }^{234} \mathrm{U}$ & $1.8 \mathrm{E}-05 \pm 1.0 \mathrm{E}-05$ & & & ${ }^{234} \mathrm{U}$ & $1.2 \mathrm{E}-05 \pm 7.6 \mathrm{E}-06$ & \\
\hline & ${ }^{235} \mathrm{U}$ & $1.7 \mathrm{E}-06 \pm 4.2 \mathrm{E}-06$ & $\mathrm{U}$ & & ${ }^{235} \mathrm{U}$ & $7.6 \mathrm{E}-07 \pm 2.7 \mathrm{E}-06$ & $\mathrm{U}$ \\
\hline & ${ }^{238} \mathrm{U}$ & $1.7 \mathrm{E}-05 \pm 9.5 \mathrm{E}-06$ & & & ${ }^{238} \mathrm{U}$ & $5.0 \mathrm{E}-06 \pm 4.7 \mathrm{E}-06$ & $\mathrm{U}$ \\
\hline & ${ }^{65} \mathrm{Zn}$ & $-2.3 \mathrm{E}-04 \pm 2.4 \mathrm{E}-04$ & $\mathrm{U}$ & & ${ }^{65} \mathrm{Zn}$ & $-1.0 \mathrm{E}-04 \pm 1.9 \mathrm{E}-04$ & $\mathrm{U}$ \\
\hline
\end{tabular}

$\overline{\mathrm{RQ}}=$ Result Qualifier. $\mathrm{U}=$ The analyte was analyzed for but not detected. 
Table 2-3. Near-Facility Air Sampling Results, 2003 (pCi/m³ \pm total analytical uncertainty). (cont)

\begin{tabular}{ccrc}
\hline Location & Isotope & Result \pm Uncertainty & RQ* \\
\hline N158 (200-East) & ${ }^{144} \mathrm{Ce}$ & $-1.3 \mathrm{E}-04 \pm 7.9 \mathrm{E}-04$ & $\mathrm{U}$ \\
Composite Period & ${ }^{60} \mathrm{Co}$ & $7.6 \mathrm{E}-05 \pm 8.5 \mathrm{E}-05$ & $\mathrm{U}$ \\
$06 / 23 / 03$ to 12/22/03 & ${ }^{134} \mathrm{Cs}$ & $3.0 \mathrm{E}-05 \pm 8.1 \mathrm{E}-05$ & $\mathrm{U}$ \\
& ${ }^{137} \mathrm{Cs}$ & $1.7 \mathrm{E}-04 \pm 1.7 \mathrm{E}-04$ & \\
& ${ }^{152} \mathrm{Eu}$ & $-1.2 \mathrm{E}-04 \pm 1.9 \mathrm{E}-04$ & $\mathrm{U}$ \\
& ${ }^{154} \mathrm{Eu}$ & $-3.6 \mathrm{E}-06 \pm 3.6 \mathrm{E}-05$ & $\mathrm{U}$ \\
& ${ }^{155} \mathrm{Eu}$ & $-1.5 \mathrm{E}-04 \pm 2.2 \mathrm{E}-04$ & $\mathrm{U}$ \\
& ${ }^{238} \mathrm{Pu}$ & $-1.1 \mathrm{E}-06 \pm 1.1 \mathrm{E}-05$ & $\mathrm{U}$ \\
& ${ }^{239 / 240} \mathrm{Pu}$ & $1.1 \mathrm{E}-06 \pm 1.1 \mathrm{E}-06$ & $\mathrm{U}$ \\
& ${ }^{103} \mathrm{Ru}$ & $-2.3 \mathrm{E}-05 \pm 6.6 \mathrm{E}-05$ & $\mathrm{U}$ \\
& ${ }^{106} \mathrm{Ru}$ & $-6.7 \mathrm{E}-04 \pm 7.0 \mathrm{E}-04$ & $\mathrm{U}$ \\
& ${ }^{125} \mathrm{Sb}$ & $1.3 \mathrm{E}-04 \pm 1.7 \mathrm{E}-04$ & $\mathrm{U}$ \\
& ${ }^{113} \mathrm{Sn}$ & $2.3 \mathrm{E}-05 \pm 7.8 \mathrm{E}-05$ & $\mathrm{U}$ \\
& ${ }^{90} \mathrm{Sr}$ & $-1.3 \mathrm{E}-04 \pm 1.3 \mathrm{E}-04$ & $\mathrm{U}$ \\
& ${ }^{234} \mathrm{U}$ & $1.8 \mathrm{E}-05 \pm 1.0 \mathrm{E}-05$ & \\
& ${ }^{235} \mathrm{U}$ & $8.1 \mathrm{E}-07 \pm 8.4 \mathrm{E}-07$ & $\mathrm{U}$ \\
& ${ }^{238} \mathrm{U}$ & $9.7 \mathrm{E}-06 \pm 7.0 \mathrm{E}-06$ & \\
& ${ }^{65} \mathrm{Zn}$ & $6.5 \mathrm{E}-05 \pm 1.9 \mathrm{E}-04$ & $\mathrm{U}$
\end{tabular}

\begin{tabular}{|c|c|c|c|}
\hline Location & Isotope & Result \pm Uncertainty & $\mathbf{R Q}^{*}$ \\
\hline N480 (200-East) & ${ }^{241} \mathrm{Am}$ & $4.0 \mathrm{E}-06 \pm 1.2 \mathrm{E}-05$ & $\bar{U}$ \\
\hline Composite Period & ${ }^{144} \mathrm{Ce}$ & $3.8 \mathrm{E}-05 \pm 3.8 \mathrm{E}-04$ & $\mathrm{U}$ \\
\hline \multirow[t]{18}{*}{$01 / 06 / 03$ to $06 / 23 / 03$} & ${ }^{60} \mathrm{Co}$ & $-8.1 \mathrm{E}-05 \pm 1.0 \mathrm{E}-04$ & $\mathrm{U}$ \\
\hline & ${ }^{134} \mathrm{Cs}$ & $-2.8 \mathrm{E}-05 \pm 8.2 \mathrm{E}-05$ & $\mathrm{U}$ \\
\hline & ${ }^{137} \mathrm{Cs}$ & $3.7 \mathrm{E}-05 \pm 8.1 \mathrm{E}-05$ & $\mathrm{U}$ \\
\hline & ${ }^{152} \mathrm{Eu}$ & $3.0 \mathrm{E}-05 \pm 2.0 \mathrm{E}-04$ & $\mathrm{U}$ \\
\hline & ${ }^{154} \mathrm{Eu}$ & $-5.6 \mathrm{E}-05 \pm 3.4 \mathrm{E}-04$ & $\mathrm{U}$ \\
\hline & ${ }^{155} \mathrm{Eu}$ & $6.7 \mathrm{E}-05 \pm 1.9 \mathrm{E}-04$ & $\mathrm{U}$ \\
\hline & ${ }^{238} \mathrm{Pu}$ & $3.7 \mathrm{E}-05 \pm 2.8 \mathrm{E}-05$ & \\
\hline & ${ }^{239 / 240} \mathrm{Pu}$ & $2.0 \mathrm{E}-06 \pm 4.0 \mathrm{E}-06$ & $\mathrm{U}$ \\
\hline & ${ }^{241} \mathrm{Pu}$ & $1.2 \mathrm{E}-04 \pm 1.2 \mathrm{E}-03$ & $\mathrm{U}$ \\
\hline & ${ }^{103} \mathrm{Ru}$ & $3.3 \mathrm{E}-05 \pm 8.8 \mathrm{E}-05$ & $\mathrm{U}$ \\
\hline & ${ }^{106} \mathrm{Ru}$ & $-1.9 \mathrm{E}-05 \pm 1.9 \mathrm{E}-04$ & $\mathrm{U}$ \\
\hline & ${ }^{125} \mathrm{Sb}$ & $2.7 \mathrm{E}-05 \pm 1.9 \mathrm{E}-04$ & U \\
\hline & ${ }^{113} \mathrm{Sn}$ & $4.3 \mathrm{E}-05 \pm 9.3 \mathrm{E}-05$ & $\mathrm{U}$ \\
\hline & ${ }^{90} \mathrm{Sr}$ & $-1.5 \mathrm{E}-05 \pm 8.2 \mathrm{E}-05$ & $\mathrm{U}$ \\
\hline & ${ }^{234} \mathrm{II}$ & $1.3 \mathrm{E}-05 \pm 8.3 \mathrm{E}-06$ & \\
\hline & ${ }^{235} \mathrm{U}$ & $4.9 \mathrm{E}-06 \pm 4.4 \mathrm{E}-06$ & \\
\hline & ${ }^{238} \mathrm{U}$ & $1.2 \mathrm{E}-05 \pm 7.5 \mathrm{E}-06$ & \\
\hline & ${ }^{65} \mathrm{Zn}$ & $-1.6 \mathrm{E}-04 \pm 2.0 \mathrm{E}-04$ & $\mathrm{U}$ \\
\hline N481 (200-East) & ${ }^{241} \mathrm{Am}$ & $6.7 \mathrm{E}-06 \pm 1.0 \mathrm{E}-05$ & $\bar{U}$ \\
\hline Composite Period & ${ }^{144} \mathrm{Ce}$ & $-1.7 \mathrm{E}-04 \pm 7.8 \mathrm{E}-04$ & $\mathrm{U}$ \\
\hline \multirow[t]{18}{*}{$01 / 06 / 03$ to $06 / 23 / 03$} & ${ }^{60} \mathrm{Co}$ & $2.6 \mathrm{E}-05 \pm 7.2 \mathrm{E}-05$ & $\mathrm{U}$ \\
\hline & ${ }^{134}$ & $-5.7 \mathrm{E}-05 \pm 7.9 \mathrm{E}-05$ & $\mathrm{U}$ \\
\hline & ${ }^{137} \mathrm{Cs}$ & $6.8 \mathrm{E}-06 \pm 6.8 \mathrm{E}-05$ & $\mathrm{U}$ \\
\hline & ${ }^{152} \mathrm{Eu}$ & $-5.3 \mathrm{E}-05 \pm 1.9 \mathrm{E}-04$ & U \\
\hline & ${ }^{154} \mathrm{Eu}$ & $1.8 \mathrm{E}-05 \pm 1.8 \mathrm{E}-04$ & $\mathrm{U}$ \\
\hline & ${ }^{155} \mathrm{Eu}$ & $3.4 \mathrm{E}-05 \pm 2.2 \mathrm{E}-04$ & $\mathrm{U}$ \\
\hline & ${ }^{238} \mathrm{Pu}$ & $1.2 \mathrm{E}-05 \pm 3.1 \mathrm{E}-05$ & U \\
\hline & ${ }^{239 / 240} \mathrm{Pu}$ & $2.1 \mathrm{E}-05 \pm 1.6 \mathrm{E}-05$ & \\
\hline & ${ }^{241} \mathrm{Pu}$ & $-8.4 \mathrm{E}-04 \pm 8.4 \mathrm{E}-03$ & U \\
\hline & ${ }^{103} \mathrm{Ru}$ & $-1.9 \mathrm{E}-05 \pm 9.8 \mathrm{E}-05$ & U \\
\hline & ${ }^{106} \mathrm{Ru}$ & $-1.0 \mathrm{E}-04 \pm 6.8 \mathrm{E}-04$ & $\mathrm{U}$ \\
\hline & ${ }^{125} \mathrm{Sb}$ & $-1.4 \mathrm{E}-04 \pm 1.9 \mathrm{E}-04$ & $\mathrm{U}$ \\
\hline & ${ }^{113} \mathrm{Sn}$ & $-1.0 \mathrm{E}-05 \pm 8.9 \mathrm{E}-05$ & $\mathrm{U}$ \\
\hline & ${ }^{90} \mathrm{Sr}$ & $-7.6 \mathrm{E}-06 \pm 7.6 \mathrm{E}-05$ & $\mathrm{U}$ \\
\hline & ${ }^{234} \mathrm{U}$ & $8.4 \mathrm{E}-06 \pm 6.6 \mathrm{E}-06$ & \\
\hline & ${ }^{235} \mathrm{U}$ & $8.4 \mathrm{E}-06 \pm 6.9 \mathrm{E}-06$ & \\
\hline & ${ }^{238} \mathrm{U}$ & $9.2 \mathrm{E}-06 \pm 6.9 \mathrm{E}-06$ & \\
\hline & ${ }^{65} \mathrm{Zn}$ & $8.7 \mathrm{E}-05 \pm 1.8 \mathrm{E}-04$ & U \\
\hline
\end{tabular}

\begin{tabular}{ccrc}
\hline N480 (200-East) & ${ }^{241} \mathrm{Am}$ & $5.0 \mathrm{E}-06 \pm 7.6 \mathrm{E}-06$ & $\mathrm{U}$ \\
Composite Period & ${ }^{144} \mathrm{Ce}$ & $-3.0 \mathrm{E}-04 \pm 5.6 \mathrm{E}-04$ & $\mathrm{U}$ \\
$06 / 23 / 03$ to 12/22/03 & ${ }^{60} \mathrm{Co}$ & $-8.1 \mathrm{E}-06 \pm 8.1 \mathrm{E}-05$ & $\mathrm{U}$ \\
& ${ }^{134} \mathrm{Cs}$ & $-6.7 \mathrm{E}-06 \pm 6.7 \mathrm{E}-05$ & $\mathrm{U}$ \\
& ${ }^{137} \mathrm{Cs}$ & $-5.2 \mathrm{E}-05 \pm 6.3 \mathrm{E}-05$ & $\mathrm{U}$ \\
& ${ }^{152} \mathrm{Eu}$ & $-5.9 \mathrm{E}-05 \pm 1.5 \mathrm{E}-04$ & $\mathrm{U}$ \\
& ${ }^{154} \mathrm{Eu}$ & $-8.8 \mathrm{E}-05 \pm 2.4 \mathrm{E}-04$ & $\mathrm{U}$ \\
& ${ }^{155} \mathrm{Eu}$ & $1.2 \mathrm{E}-04 \pm 1.5 \mathrm{E}-04$ & $\mathrm{U}$ \\
& ${ }^{238} \mathrm{Pu}$ & $-1.5 \mathrm{E}-05 \pm 2.4 \mathrm{E}-05$ & $\mathrm{U}$ \\
& ${ }^{239 / 240} \mathrm{Pu}$ & $3.6 \mathrm{E}-06 \pm 9.0 \mathrm{E}-06$ & $\mathrm{U}$ \\
& ${ }^{241} \mathrm{Pu}$ & $-2.2 \mathrm{E}-04 \pm 2.3 \mathrm{E}-04$ & $\mathrm{U}$ \\
& ${ }^{103} \mathrm{Ru}$ & $-8.3 \mathrm{E}-06 \pm 6.7 \mathrm{E}-05$ & $\mathrm{U}$ \\
& ${ }^{106} \mathrm{Ru}$ & $-2.1 \mathrm{E}-04 \pm 5.7 \mathrm{E}-04$ & $\mathrm{U}$ \\
& ${ }^{125} \mathrm{Sb}$ & $-2.7 \mathrm{E}-05 \pm 1.3 \mathrm{E}-04$ & $\mathrm{U}$ \\
& ${ }^{113} \mathrm{Sn}$ & $-8.1 \mathrm{E}-06 \pm 6.9 \mathrm{E}-05$ & $\mathrm{U}$ \\
& ${ }^{90} \mathrm{Sr}$ & $-1.2 \mathrm{E}-04 \pm 1.2 \mathrm{E}-04$ & $\mathrm{U}$ \\
& ${ }^{234} \mathrm{U}$ & $1.2 \mathrm{E}-05 \pm 7.2 \mathrm{E}-06$ & \\
${ }^{235} \mathrm{U}$ & $1.5 \mathrm{E}-06 \pm 3.1 \mathrm{E}-06$ & $\mathrm{U}$ \\
${ }^{238} \mathrm{U}$ & $5.6 \mathrm{E}-06 \pm 4.4 \mathrm{E}-06$ & \\
${ }^{65} \mathrm{Zn}$ & $-4.4 \mathrm{E}-05 \pm 1.8 \mathrm{E}-04$ & $\mathrm{U}$
\end{tabular}

$\overline{\mathrm{RQ}}=$ Result Qualifier. $\mathrm{U}=$ The analyte was analyzed for but not detected. 
Table 2-3. Near-Facility Air Sampling Results, 2003 (pCi/m³ \pm total analytical uncertainty). (cont)

\begin{tabular}{|c|c|c|c|c|c|c|c|}
\hline Location & Isotope & Result \pm Uncertainty & $\mathbf{R Q}^{*}$ & Location & Isotope & Result \pm Uncertainty & RQ* \\
\hline N481 (200-East) & ${ }^{241} \mathrm{Am}$ & $-2.9 \mathrm{E}-06 \pm 1.0 \mathrm{E}-05$ & $\bar{U}$ & N498 (200-East) & ${ }^{144} \mathrm{Ce}$ & $-2.5 \mathrm{E}-04 \pm 7.4 \mathrm{E}-04$ & $\bar{U}$ \\
\hline Composite Period & ${ }^{144} \mathrm{Ce}$ & $6.6 \mathrm{E}-05 \pm 6.7 \mathrm{E}-04$ & $\mathrm{U}$ & Composite Period & ${ }^{60} \mathrm{Co}$ & $3.8 \mathrm{E}-05 \pm 9.1 \mathrm{E}-05$ & $\mathrm{U}$ \\
\hline \multirow[t]{18}{*}{$06 / 23 / 03$ to $12 / 22 / 03$} & ${ }^{60} \mathrm{Co}$ & $1.9 \mathrm{E}-05 \pm 7.3 \mathrm{E}-05$ & $\mathrm{U}$ & $01 / 06 / 03$ to $06 / 23 / 03$ & ${ }^{134} \mathrm{Cs}$ & $3.5 \mathrm{E}-05 \pm 8.1 \mathrm{E}-05$ & $\mathrm{U}$ \\
\hline & ${ }^{134} \mathrm{Cs}$ & $-4.4 \mathrm{E}-05 \pm 7.2 \mathrm{E}-05$ & $\mathrm{U}$ & & ${ }^{137} \mathrm{Cs}$ & 3.3E-05 $\pm 8.3 \mathrm{E}-05$ & $\mathrm{U}$ \\
\hline & ${ }^{137} \mathrm{Cs}$ & $-8.4 \mathrm{E}-06 \pm 6.6 \mathrm{E}-05$ & $\mathrm{U}$ & & ${ }^{152} \mathrm{Eu}$ & $7.6 \mathrm{E}-05 \pm 1.9 \mathrm{E}-04$ & $\mathrm{U}$ \\
\hline & ${ }^{152} \mathrm{Eu}$ & $-1.1 \mathrm{E}-04 \pm 1.9 \mathrm{E}-04$ & $\mathrm{U}$ & & ${ }^{154} \mathrm{Eu}$ & $-3.2 \mathrm{E}-04 \pm 3.3 \mathrm{E}-04$ & $\mathrm{U}$ \\
\hline & ${ }^{154} \mathrm{Eu}$ & $-1.5 \mathrm{E}-04 \pm 2.3 \mathrm{E}-04$ & $\mathrm{U}$ & & ${ }^{155} \mathrm{Eu}$ & $-1.4 \mathrm{E}-04 \pm 2.0 \mathrm{E}-04$ & $\mathrm{U}$ \\
\hline & ${ }^{155} \mathrm{Eu}$ & $3.6 \mathrm{E}-05 \pm 2.2 \mathrm{E}-04$ & $\mathrm{U}$ & & ${ }^{238} \mathrm{Pu}$ & $-9.1 \mathrm{E}-06 \pm 1.5 \mathrm{E}-05$ & $\mathrm{U}$ \\
\hline & ${ }^{238} \mathrm{Pu}$ & $6.7 \mathrm{E}-06 \pm 2.3 \mathrm{E}-05$ & $\mathrm{U}$ & & ${ }^{239 / 240} \mathrm{Pu}$ & $9.9 \mathrm{E}-07 \pm 5.2 \mathrm{E}-06$ & $\mathrm{U}$ \\
\hline & ${ }^{239 / 240} \mathrm{Pu}$ & $3.3 \mathrm{E}-06 \pm 8.3 \mathrm{E}-06$ & $\mathrm{U}$ & & ${ }^{103} \mathrm{Ru}$ & $-5.1 \mathrm{E}-06 \pm 5.1 \mathrm{E}-05$ & $\mathrm{U}$ \\
\hline & ${ }^{241} \mathrm{Pu}$ & $3.6 \mathrm{E}-04 \pm 6.7 \mathrm{E}-04$ & $\mathrm{U}$ & & ${ }^{106} \mathrm{Ru}$ & $-5.4 \mathrm{E}-04 \pm 7.1 \mathrm{E}-04$ & $\mathrm{U}$ \\
\hline & ${ }^{103} \mathrm{Ru}$ & $4.9 \mathrm{E}-06 \pm 4.9 \mathrm{E}-05$ & $\mathrm{U}$ & & ${ }^{125} \mathrm{Sb}$ & $-1.6 \mathrm{E}-05 \pm 1.6 \mathrm{E}-04$ & $\mathrm{U}$ \\
\hline & ${ }^{106} \mathrm{Ru}$ & $2.3 \mathrm{E}-04 \pm 6.1 \mathrm{E}-04$ & $\mathrm{U}$ & & ${ }^{113} \mathrm{Sn}$ & $-1.1 \mathrm{E}-05 \pm 8.0 \mathrm{E}-05$ & $\mathrm{U}$ \\
\hline & ${ }^{125} \mathrm{Sb}$ & $3.2 \mathrm{E}-06 \pm 3.2 \mathrm{E}-05$ & $\mathrm{U}$ & & ${ }^{90} \mathrm{Sr}$ & $4.5 \mathrm{E}-04 \pm 1.8 \mathrm{E}-04$ & \\
\hline & ${ }^{113} \mathrm{Sn}$ & $1.9 \mathrm{E}-05 \pm 7.8 \mathrm{E}-05$ & $\mathrm{U}$ & & ${ }^{234} \mathrm{U}$ & $1.6 \mathrm{E}-05 \pm 9.1 \mathrm{E}-06$ & \\
\hline & ${ }^{90} \mathrm{Sr}$ & $3.0 \mathrm{E}-05 \pm 1.2 \mathrm{E}-04$ & $\mathrm{U}$ & & ${ }^{235} \mathrm{U}$ & $2.5 \mathrm{E}-06 \pm 3.1 \mathrm{E}-06$ & \\
\hline & ${ }^{234} \mathrm{U}$ & $7.3 \mathrm{E}-06 \pm 5.8 \mathrm{E}-06$ & & & ${ }^{238} \mathrm{U}$ & $7.6 \mathrm{E}-06 \pm 6.3 \mathrm{E}-06$ & \\
\hline & ${ }^{235} \mathrm{U}$ & $5.0 \mathrm{E}-06 \pm 4.2 \mathrm{E}-06$ & & & ${ }^{65} \mathrm{Zn}$ & $1.7 \mathrm{E}-05 \pm 1.7 \mathrm{E}-04$ & $\mathrm{U}$ \\
\hline & ${ }^{238} \mathrm{U}$ & $9.0 \mathrm{E}-06 \pm 6.3 \mathrm{E}-06$ & & & & & \\
\hline & ${ }^{65} \mathrm{Zn}$ & $2.9 \mathrm{E}-05 \pm 1.7 \mathrm{E}-04$ & $\mathrm{U}$ & & & & \\
\hline N498 (200-East) & ${ }^{144} \mathrm{Ce}$ & $-2.6 \mathrm{E}-04 \pm 7.0 \mathrm{E}-04$ & $\mathrm{U}$ & N499 (200-East) & ${ }^{144} \mathrm{Ce}$ & $-1.5 \mathrm{E}-04 \pm 7.5 \mathrm{E}-04$ & $\mathrm{U}$ \\
\hline Composite Period & ${ }^{60} \mathrm{Co}$ & $-1.5 \mathrm{E}-05 \pm 7.2 \mathrm{E}-05$ & $\mathrm{U}$ & Composite Period & ${ }^{60} \mathrm{Co}$ & $1.4 \mathrm{E}-05 \pm 9.8 \mathrm{E}-05$ & $\mathrm{U}$ \\
\hline \multirow[t]{16}{*}{$06 / 23 / 03$ to $12 / 22 / 03$} & ${ }^{134} \mathrm{Cs}$ & $-2.5 \mathrm{E}-05 \pm 6.9 \mathrm{E}-05$ & $\mathrm{U}$ & $01 / 06 / 03$ to $06 / 23 / 03$ & ${ }^{134} \mathrm{Cs}$ & $-1.0 \mathrm{E}-04 \pm 1.1 \mathrm{E}-04$ & $\mathrm{U}$ \\
\hline & ${ }^{137} \mathrm{Cs}$ & $-5.1 \mathrm{E}-05 \pm 6.6 \mathrm{E}-05$ & $\mathrm{U}$ & & ${ }^{137} \mathrm{Cs}$ & $-2.0 \mathrm{E}-06 \pm 2.0 \mathrm{E}-05$ & $\mathrm{U}$ \\
\hline & ${ }^{152} \mathrm{Eu}$ & $-1.2 \mathrm{E}-04 \pm 1.8 \mathrm{E}-04$ & $\mathrm{U}$ & & ${ }^{152} \mathrm{Eu}$ & $7.3 \mathrm{E}-05 \pm 2.0 \mathrm{E}-04$ & $\mathrm{U}$ \\
\hline & ${ }^{154} \mathrm{Eu}$ & $-2.2 \mathrm{E}-05 \pm 2.1 \mathrm{E}-04$ & $\mathrm{U}$ & & ${ }^{154} \mathrm{Eu}$ & $1.3 \mathrm{E}-04 \pm 2.8 \mathrm{E}-04$ & $\mathrm{U}$ \\
\hline & ${ }^{155} \mathrm{Eu}$ & $3.1 \mathrm{E}-05 \pm 1.9 \mathrm{E}-04$ & $\mathrm{U}$ & & ${ }^{155} \mathrm{Eu}$ & $-8.4 \mathrm{E}-05 \pm 1.9 \mathrm{E}-04$ & $\mathrm{U}$ \\
\hline & ${ }^{238} \mathrm{Pu}$ & $-7.0 \mathrm{E}-06 \pm 1.1 \mathrm{E}-05$ & $\mathrm{U}$ & & ${ }^{238} \mathrm{Pu}$ & $-6.9 \mathrm{E}-06 \pm 1.2 \mathrm{E}-05$ & $\mathrm{U}$ \\
\hline & ${ }^{239 / 240} \mathrm{Pu}$ & $1.4 \mathrm{E}-05 \pm 9.0 \mathrm{E}-06$ & & & ${ }^{239 / 240} \mathrm{Pu}$ & $8.6 \mathrm{E}-07 \pm 3.0 \mathrm{E}-06$ & $\mathrm{U}$ \\
\hline & ${ }^{103} \mathrm{Ru}$ & $-3.7 \mathrm{E}-05 \pm 7.1 \mathrm{E}-05$ & U & & ${ }^{103} \mathrm{Ru}$ & $-3.1 \mathrm{E}-05 \pm 8.5 \mathrm{E}-05$ & $\mathrm{U}$ \\
\hline & ${ }^{106} \mathrm{Ru}$ & $-4.4 \mathrm{E}-05 \pm 4.4 \mathrm{E}-04$ & $\mathrm{U}$ & & ${ }^{106} \mathrm{Ru}$ & $-3.4 \mathrm{E}-04 \pm 8.3 \mathrm{E}-04$ & $\mathrm{U}$ \\
\hline & ${ }^{125} \mathrm{Sb}$ & $5.4 \mathrm{E}-05 \pm 1.6 \mathrm{E}-04$ & $\mathrm{U}$ & & ${ }^{125} \mathrm{Sb}$ & $-5.5 \mathrm{E}-05 \pm 1.8 \mathrm{E}-04$ & $\mathrm{U}$ \\
\hline & ${ }^{113} \mathrm{Sn}$ & $-8.4 \mathrm{E}-05 \pm 8.7 \mathrm{E}-05$ & $\mathrm{U}$ & & ${ }^{113} \mathrm{Sn}$ & $5.2 \mathrm{E}-05 \pm 9.0 \mathrm{E}-05$ & $\mathrm{U}$ \\
\hline & ${ }^{90} \mathrm{Sr}$ & $5.8 \mathrm{E}-05 \pm 1.0 \mathrm{E}-04$ & $\mathrm{U}$ & & ${ }^{90} \mathrm{Sr}$ & $6.3 \mathrm{E}-05 \pm 9.3 \mathrm{E}-05$ & $\mathrm{U}$ \\
\hline & ${ }^{234} \mathrm{U}$ & 1.7E-05 $\pm 9.3 \mathrm{E}-06$ & & & ${ }^{234} \mathrm{U}$ & $1.0 \mathrm{E}-05 \pm 6.9 \mathrm{E}-06$ & \\
\hline & ${ }^{235} \mathrm{U}$ & $1.4 \mathrm{E}-06 \pm 2.9 \mathrm{E}-06$ & $\mathrm{U}$ & & ${ }^{235} \mathrm{U}$ & $4.8 \mathrm{E}-06 \pm 4.3 \mathrm{E}-06$ & \\
\hline & ${ }^{238} \mathrm{U}$ & $1.1 \mathrm{E}-05 \pm 7.0 \mathrm{E}-06$ & & & ${ }^{238} \mathrm{U}$ & $8.6 \mathrm{E}-06 \pm 5.9 \mathrm{E}-06$ & \\
\hline & ${ }^{65} \mathrm{Zn}$ & $-3.9 \mathrm{E}-05 \pm 1.8 \mathrm{E}-04$ & $\mathrm{U}$ & & ${ }^{65} \mathrm{Zn}$ & $7.6 \mathrm{E}-05 \pm 2.2 \mathrm{E}-04$ & $\mathrm{U}$ \\
\hline
\end{tabular}

$\overline{\mathrm{RQ}}=$ Result Qualifier. $\mathrm{U}=$ The analyte was analyzed for but not detected. 
Table 2-3. Near-Facility Air Sampling Results, 2003 (pCi/m³ \pm total analytical uncertainty). (cont)

\begin{tabular}{|c|c|c|c|c|c|c|c|}
\hline Location & Isotope & Result \pm Uncertainty & RQ* & Location & Isotope & Result \pm Uncertainty & RQ* \\
\hline N499 (200-East) & ${ }^{144} \mathrm{Ce}$ & $-1.7 \mathrm{E}-04 \pm 6.4 \mathrm{E}-04$ & $\mathrm{U}$ & N957 (200-East) & ${ }^{144} \mathrm{Ce}$ & $-7.3 \mathrm{E}-04 \pm 8.3 \mathrm{E}-04$ & $\mathrm{U}$ \\
\hline Composite Period & ${ }^{60} \mathrm{Co}$ & $1.9 \mathrm{E}-06 \pm 1.9 \mathrm{E}-05$ & $\mathrm{U}$ & Composite Period & ${ }^{60} \mathrm{Co}$ & $-4.1 \mathrm{E}-05 \pm 7.6 \mathrm{E}-05$ & $\mathrm{U}$ \\
\hline \multirow[t]{16}{*}{$06 / 23 / 03$ to $12 / 22 / 03$} & ${ }^{134} \mathrm{Cs}$ & $-4.6 \mathrm{E}-05 \pm 7.4 \mathrm{E}-05$ & $\mathrm{U}$ & $01 / 06 / 03$ to $06 / 23 / 03$ & ${ }^{134} \mathrm{Cs}$ & $-4.5 \mathrm{E}-05 \pm 7.4 \mathrm{E}-05$ & $\mathrm{U}$ \\
\hline & ${ }^{137} \mathrm{Cs}$ & $1.8 \mathrm{E}-05 \pm 5.9 \mathrm{E}-05$ & $\mathrm{U}$ & & ${ }^{137} \mathrm{Cs}$ & $7.2 \mathrm{E}-05 \pm 7.6 \mathrm{E}-05$ & $\mathrm{U}$ \\
\hline & ${ }^{152} \mathrm{Eu}$ & $-2.2 \mathrm{E}-04 \pm 2.3 \mathrm{E}-04$ & $\mathrm{U}$ & & ${ }^{152} \mathrm{Eu}$ & $-1.0 \mathrm{E}-04 \pm 1.9 \mathrm{E}-04$ & $\mathrm{U}$ \\
\hline & ${ }^{154} \mathrm{Eu}$ & $-7.8 \mathrm{E}-05 \pm 2.2 \mathrm{E}-04$ & $\mathrm{U}$ & & ${ }^{154} \mathrm{Eu}$ & $1.2 \mathrm{E}-05 \pm 1.2 \mathrm{E}-04$ & $\mathrm{U}$ \\
\hline & ${ }^{155} \mathrm{Eu}$ & $1.6 \mathrm{E}-05 \pm 1.6 \mathrm{E}-04$ & $\mathrm{U}$ & & ${ }^{155} \mathrm{Eu}$ & $-1.2 \mathrm{E}-04 \pm 2.2 \mathrm{E}-04$ & $\mathrm{U}$ \\
\hline & ${ }^{238} \mathrm{Pu}$ & $-6.5 \mathrm{E}-06 \pm 1.4 \mathrm{E}-05$ & $\mathrm{U}$ & & ${ }^{238} \mathrm{Pu}$ & $-7.2 \mathrm{E}-06 \pm 1.2 \mathrm{E}-05$ & $\mathrm{U}$ \\
\hline & ${ }^{239 / 240} \mathrm{Pu}$ & $3.2 \mathrm{E}-06 \pm 3.9 \mathrm{E}-06$ & & & ${ }^{239 / 240} \mathrm{Pu}$ & $8.4 \mathrm{E}-06 \pm 7.0 \mathrm{E}-06$ & $\mathrm{U}$ \\
\hline & ${ }^{103} \mathrm{Ru}$ & $5.1 \mathrm{E}-06 \pm 5.1 \mathrm{E}-05$ & $\mathrm{U}$ & & ${ }^{103} \mathrm{Ru}$ & $8.9 \mathrm{E}-06 \pm 7.7 \mathrm{E}-05$ & $\mathrm{U}$ \\
\hline & ${ }^{106} \mathrm{Ru}$ & $8.9 \mathrm{E}-05 \pm 5.9 \mathrm{E}-04$ & $\mathrm{U}$ & & ${ }^{106} \mathrm{Ru}$ & $1.8 \mathrm{E}-05 \pm 1.8 \mathrm{E}-04$ & $\mathrm{U}$ \\
\hline & ${ }^{125} \mathrm{Sb}$ & $4.1 \mathrm{E}-05 \pm 1.5 \mathrm{E}-04$ & $\mathrm{U}$ & & ${ }^{125} \mathrm{Sb}$ & $-2.7 \mathrm{E}-05 \pm 1.9 \mathrm{E}-04$ & $\mathrm{U}$ \\
\hline & ${ }^{113} \mathrm{Sn}$ & $8.8 \mathrm{E}-05 \pm 9.0 \mathrm{E}-05$ & $\mathrm{U}$ & & ${ }^{113} \mathrm{Sn}$ & $-4.5 \mathrm{E}-05 \pm 9.1 \mathrm{E}-05$ & $\mathrm{U}$ \\
\hline & ${ }^{90} \mathrm{Sr}$ & $-1.3 \mathrm{E}-04 \pm 1.4 \mathrm{E}-04$ & $\mathrm{U}$ & & ${ }^{90} \mathrm{Sr}$ & $2.1 \mathrm{E}-04 \pm 1.2 \mathrm{E}-04$ & \\
\hline & ${ }^{234} \mathrm{U}$ & $2.6 \mathrm{E}-05 \pm 1.4 \mathrm{E}-05$ & & & ${ }^{234} \mathrm{U}$ & $8.4 \mathrm{E}-06 \pm 7.6 \mathrm{E}-06$ & $\mathrm{U}$ \\
\hline & ${ }^{235} \mathrm{U}$ & $7.8 \mathrm{E}-06 \pm 5.9 \mathrm{E}-06$ & & & ${ }^{235} \mathrm{U}$ & $2.2 \mathrm{E}-06 \pm 4.9 \mathrm{E}-06$ & $\mathrm{U}$ \\
\hline & ${ }^{238} \mathrm{U}$ & $1.6 \mathrm{E}-05 \pm 9.0 \mathrm{E}-06$ & & & ${ }^{238} \mathrm{U}$ & $3.4 \mathrm{E}-06 \pm 3.9 \mathrm{E}-06$ & $\mathrm{U}$ \\
\hline & ${ }^{65} \mathrm{Zn}$ & $-2.1 \mathrm{E}-05 \pm 2.0 \mathrm{E}-04$ & $\mathrm{U}$ & & ${ }^{65} \mathrm{Zn}$ & $4.9 \mathrm{E}-05 \pm 1.7 \mathrm{E}-04$ & $\mathrm{U}$ \\
\hline N957 (200-East) & ${ }^{144} \mathrm{Ce}$ & $-2.6 \mathrm{E}-05 \pm 2.6 \mathrm{E}-04$ & $\mathrm{U}$ & N967 (200-East) & ${ }^{144} \mathrm{Ce}$ & $-1.0 \mathrm{E}-04 \pm 8.0 \mathrm{E}-04$ & $\mathrm{U}$ \\
\hline Composite Period & ${ }^{60} \mathrm{Co}$ & $-2.3 \mathrm{E}-05 \pm 6.8 \mathrm{E}-05$ & $\mathrm{U}$ & Composite Period & ${ }^{60} \mathrm{Co}$ & $6.9 \mathrm{E}-05 \pm 7.7 \mathrm{E}-05$ & $\mathrm{U}$ \\
\hline \multirow[t]{16}{*}{$06 / 23 / 03$ to $12 / 23 / 03$} & ${ }^{134} \mathrm{Cs}$ & $-6.4 \mathrm{E}-06 \pm 6.4 \mathrm{E}-05$ & $\mathrm{U}$ & $01 / 06 / 03$ to $06 / 23 / 03$ & ${ }^{134} \mathrm{Cs}$ & $3.9 \mathrm{E}-05 \pm 8.2 \mathrm{E}-05$ & $\mathrm{U}$ \\
\hline & ${ }^{137} \mathrm{Cs}$ & $5.8 \mathrm{E}-05 \pm 6.4 \mathrm{E}-05$ & $\mathrm{U}$ & & ${ }^{137} \mathrm{Cs}$ & $2.1 \mathrm{E}-04 \pm 1.4 \mathrm{E}-04$ & \\
\hline & ${ }^{152} \mathrm{Eu}$ & $-2.3 \mathrm{E}-04 \pm 2.4 \mathrm{E}-04$ & $\mathrm{U}$ & & ${ }^{152} \mathrm{Eu}$ & $-2.2 \mathrm{E}-04 \pm 2.3 \mathrm{E}-04$ & $\mathrm{U}$ \\
\hline & ${ }^{154} \mathrm{Eu}$ & $4.7 \mathrm{E}-05 \pm 2.1 \mathrm{E}-04$ & $\mathrm{U}$ & & ${ }^{154} \mathrm{Eu}$ & $-3.2 \mathrm{E}-05 \pm 2.2 \mathrm{E}-04$ & $\mathrm{U}$ \\
\hline & ${ }^{155} \mathrm{Eu}$ & $1.9 \mathrm{E}-04 \pm 2.2 \mathrm{E}-04$ & $\mathrm{U}$ & & ${ }^{155} \mathrm{Eu}$ & $-2.3 \mathrm{E}-04 \pm 2.4 \mathrm{E}-04$ & $\mathrm{U}$ \\
\hline & ${ }^{238} \mathrm{Pu}$ & $7.4 \mathrm{E}-06 \pm 9.1 \mathrm{E}-06$ & $\mathrm{U}$ & & ${ }^{238} \mathrm{Pu}$ & $-2.8 \mathrm{E}-06 \pm 1.2 \mathrm{E}-05$ & $\mathrm{U}$ \\
\hline & ${ }^{239 / 240} \mathrm{Pu}$ & $3.7 \mathrm{E}-06 \pm 3.8 \mathrm{E}-06$ & & & ${ }^{239 / 240} \mathrm{Pu}$ & $4.6 \mathrm{E}-06 \pm 6.5 \mathrm{E}-06$ & $\mathrm{U}$ \\
\hline & ${ }^{103} \mathrm{Ru}$ & $-7.0 \mathrm{E}-06 \pm 6.7 \mathrm{E}-05$ & $\mathrm{U}$ & & ${ }^{103} \mathrm{Ru}$ & 8.3E-06 \pm 7.7E-05 & $\mathrm{U}$ \\
\hline & ${ }^{106} \mathrm{Ru}$ & $3.6 \mathrm{E}-04 \pm 5.9 \mathrm{E}-04$ & $\mathrm{U}$ & & ${ }^{106} \mathrm{Ru}$ & $-1.1 \mathrm{E}-04 \pm 6.9 \mathrm{E}-04$ & $\mathrm{U}$ \\
\hline & ${ }^{125} \mathrm{Sb}$ & $-1.8 \mathrm{E}-06 \pm 1.8 \mathrm{E}-05$ & $\mathrm{U}$ & & ${ }^{125} \mathrm{Sb}$ & $2.6 \mathrm{E}-07 \pm 2.6 \mathrm{E}-06$ & $\mathrm{U}$ \\
\hline & ${ }^{113} \mathrm{Sn}$ & $1.6 \mathrm{E}-05 \pm 7.3 \mathrm{E}-05$ & $\mathrm{U}$ & & ${ }^{113} \mathrm{Sn}$ & $-1.6 \mathrm{E}-06 \pm 1.6 \mathrm{E}-05$ & $\mathrm{U}$ \\
\hline & ${ }^{90} \mathrm{Sr}$ & $-7.4 \mathrm{E}-05 \pm 9.5 \mathrm{E}-05$ & $\mathrm{U}$ & & ${ }^{90} \mathrm{Sr}$ & $-9.8 \mathrm{E}-05 \pm 1.0 \mathrm{E}-04$ & $\mathrm{U}$ \\
\hline & ${ }^{234} \mathrm{U}$ & $1.5 \mathrm{E}-05 \pm 8.7 \mathrm{E}-06$ & & & ${ }^{234} \mathrm{U}$ & $6.5 \mathrm{E}-06 \pm 6.1 \mathrm{E}-06$ & $\mathrm{U}$ \\
\hline & ${ }^{235} \mathrm{U}$ & $4.2 \mathrm{E}-06 \pm 4.7 \mathrm{E}-06$ & $\mathrm{U}$ & & ${ }^{235} \mathrm{U}$ & $4.7 \mathrm{E}-06 \pm 4.3 \mathrm{E}-06$ & \\
\hline & ${ }^{238} \mathrm{U}$ & $1.2 \mathrm{E}-05 \pm 7.4 \mathrm{E}-06$ & & & ${ }^{238} \mathrm{U}$ & 4.3E-06 \pm 4.4E-06 & $\mathrm{U}$ \\
\hline & ${ }^{65} \mathrm{Zn}$ & $1.6 \mathrm{E}-04 \pm 1.8 \mathrm{E}-04$ & $\mathrm{U}$ & & ${ }^{65} \mathrm{Zn}$ & $-2.5 \mathrm{E}-04 \pm 2.6 \mathrm{E}-04$ & $\mathrm{U}$ \\
\hline
\end{tabular}

$\overline{\mathrm{RQ}}=$ Result Qualifier. $\mathrm{U}=$ The analyte was analyzed for but not detected. 
Table 2-3. Near-Facility Air Sampling Results, 2003 (pCi/m³ \pm total analytical uncertainty). (cont)

\begin{tabular}{|c|c|c|c|c|c|c|c|}
\hline Location & Isotope & Result \pm Uncertainty & $\mathbf{R Q}^{*}$ & Location & Isotope & Result \pm Uncertainty & $\mathbf{R Q}^{*}$ \\
\hline N967 (200-East) & ${ }^{144} \mathrm{Ce}$ & $6.3 \mathrm{E}-05 \pm 6.0 \mathrm{E}-04$ & $\mathrm{U}$ & N968 (200-East) & ${ }^{144} \mathrm{Ce}$ & $8.9 \mathrm{E}-05 \pm 7.8 \mathrm{E}-04$ & $\mathrm{U}$ \\
\hline Composite Period & ${ }^{60} \mathrm{Co}$ & $5.5 \mathrm{E}-05 \pm 7.9 \mathrm{E}-05$ & $\mathrm{U}$ & Composite Period & ${ }^{60} \mathrm{Co}$ & $5.1 \mathrm{E}-05 \pm 7.9 \mathrm{E}-05$ & $\mathrm{U}$ \\
\hline \multirow[t]{16}{*}{$06 / 23 / 03$ to $12 / 22 / 03$} & ${ }^{134} \mathrm{Cs}$ & $-2.0 \mathrm{E}-05 \pm 7.5 \mathrm{E}-05$ & $\mathrm{U}$ & $01 / 06 / 03$ to $06 / 23 / 03$ & ${ }^{134} \mathrm{Cs}$ & $-5.8 \mathrm{E}-05 \pm 1.1 \mathrm{E}-04$ & $\mathrm{U}$ \\
\hline & ${ }^{137} \mathrm{Cs}$ & $1.1 \mathrm{E}-04 \pm 8.3 \mathrm{E}-05$ & $\mathrm{U}$ & & ${ }^{137} \mathrm{Cs}$ & $4.4 \mathrm{E}-05 \pm 7.8 \mathrm{E}-05$ & $\mathrm{U}$ \\
\hline & ${ }^{152} \mathrm{Eu}$ & $-1.1 \mathrm{E}-04 \pm 1.4 \mathrm{E}-04$ & U & & ${ }^{152} \mathrm{Eu}$ & $6.9 \mathrm{E}-07 \pm 6.9 \mathrm{E}-06$ & $\mathrm{U}$ \\
\hline & ${ }^{154} \mathrm{Eu}$ & $-1.2 \mathrm{E}-04 \pm 2.0 \mathrm{E}-04$ & $\mathrm{U}$ & & ${ }^{154} \mathrm{Eu}$ & $-4.6 \mathrm{E}-05 \pm 2.1 \mathrm{E}-04$ & $\mathrm{U}$ \\
\hline & ${ }^{155} \mathrm{Eu}$ & $7.9 \mathrm{E}-05 \pm 1.4 \mathrm{E}-04$ & $\mathrm{U}$ & & ${ }^{155} \mathrm{Eu}$ & $-6.7 \mathrm{E}-05 \pm 2.2 \mathrm{E}-04$ & $\mathrm{U}$ \\
\hline & ${ }^{238} \mathrm{Pu}$ & $1.1 \mathrm{E}-05 \pm 1.6 \mathrm{E}-05$ & $\mathrm{U}$ & & ${ }^{238} \mathrm{Pu}$ & $-6.8 \mathrm{E}-06 \pm 1.4 \mathrm{E}-05$ & $\mathrm{U}$ \\
\hline & ${ }^{239 / 240} \mathrm{Pu}$ & $2.6 \mathrm{E}-05 \pm 1.4 \mathrm{E}-05$ & & & ${ }^{239 / 240} \mathrm{Pu}$ & $1.7 \mathrm{E}-06 \pm 3.5 \mathrm{E}-06$ & $\mathrm{U}$ \\
\hline & ${ }^{103} \mathrm{Ru}$ & $-9.2 \mathrm{E}-06 \pm 5.9 \mathrm{E}-05$ & $\mathrm{U}$ & & ${ }^{103} \mathrm{Ru}$ & $-7.8 \mathrm{E}-05 \pm 1.0 \mathrm{E}-04$ & $\mathrm{U}$ \\
\hline & ${ }^{106} \mathrm{Ru}$ & $-1.8 \mathrm{E}-04 \pm 5.1 \mathrm{E}-04$ & $\mathrm{U}$ & & ${ }^{106} \mathrm{Ru}$ & $-4.6 \mathrm{E}-04 \pm 6.9 \mathrm{E}-04$ & $\mathrm{U}$ \\
\hline & ${ }^{125} \mathrm{Sb}$ & $-8.0 \mathrm{E}-05 \pm 1.3 \mathrm{E}-04$ & $\mathrm{U}$ & & ${ }^{125} \mathrm{Sb}$ & $1.3 \mathrm{E}-05 \pm 1.3 \mathrm{E}-04$ & $\mathrm{U}$ \\
\hline & ${ }^{113} \mathrm{Sn}$ & $3.8 \mathrm{E}-05 \pm 6.5 \mathrm{E}-05$ & $\mathrm{U}$ & & ${ }^{113} \mathrm{Sn}$ & $1.7 \mathrm{E}-05 \pm 1.0 \mathrm{E}-04$ & $\mathrm{U}$ \\
\hline & ${ }^{90} \mathrm{Sr}$ & $7.1 \mathrm{E}-04 \pm 2.4 \mathrm{E}-04$ & & & ${ }^{90} \mathrm{Sr}$ & $-8.6 \mathrm{E}-05 \pm 8.9 \mathrm{E}-05$ & $\mathrm{U}$ \\
\hline & ${ }^{234} \mathrm{U}$ & $5.8 \mathrm{E}-06 \pm 8.8 \mathrm{E}-06$ & $\mathrm{U}$ & & ${ }^{234} \mathrm{U}$ & $1.2 \mathrm{E}-05 \pm 8.3 \mathrm{E}-06$ & \\
\hline & ${ }^{235} \mathrm{U}$ & $-3.2 \mathrm{E}-06 \pm 5.7 \mathrm{E}-06$ & $\mathrm{U}$ & & ${ }^{235} \mathrm{U}$ & $7.8 \mathrm{E}-07 \pm 2.7 \mathrm{E}-06$ & $\mathrm{U}$ \\
\hline & ${ }^{238} \mathrm{U}$ & $6.5 \mathrm{E}-06 \pm 6.8 \mathrm{E}-06$ & $\mathrm{U}$ & & ${ }^{238} \mathrm{U}$ & $8.6 \mathrm{E}-06 \pm 6.4 \mathrm{E}-06$ & \\
\hline & ${ }^{65} \mathrm{Zn}$ & $-6.4 \mathrm{E}-05 \pm 1.7 \mathrm{E}-04$ & $\mathrm{U}$ & & ${ }^{65} \mathrm{Zn}$ & $5.2 \mathrm{E}-06 \pm 5.2 \mathrm{E}-05$ & $\mathrm{U}$ \\
\hline N968 (200-East) & ${ }^{144} \mathrm{Ce}$ & $-2.3 \mathrm{E}-04 \pm 6.8 \mathrm{E}-04$ & $\bar{U}$ & N969 (200-East) & ${ }^{144} \mathrm{Ce}$ & $-6.6 \mathrm{E}-04 \pm 7.9 \mathrm{E}-04$ & $\mathrm{U}$ \\
\hline Composite Period & ${ }^{60} \mathrm{Co}$ & $3.7 \mathrm{E}-05 \pm 6.6 \mathrm{E}-05$ & $\mathrm{U}$ & Composite Period & ${ }^{60} \mathrm{Co}$ & $1.6 \mathrm{E}-05 \pm 9.6 \mathrm{E}-05$ & $\mathrm{U}$ \\
\hline \multirow[t]{16}{*}{$06 / 23 / 03$ to $12 / 22 / 03$} & ${ }^{134} \mathrm{Cs}$ & $-8.6 \mathrm{E}-06 \pm 7.2 \mathrm{E}-05$ & $\mathrm{U}$ & $01 / 06 / 03$ to $06 / 23 / 03$ & ${ }^{134} \mathrm{Cs}$ & $8.8 \mathrm{E}-05 \pm 1.0 \mathrm{E}-04$ & $\mathrm{U}$ \\
\hline & ${ }^{137} \mathrm{Cs}$ & $4.8 \mathrm{E}-06 \pm 4.8 \mathrm{E}-05$ & $\mathrm{U}$ & & ${ }^{137} \mathrm{Cs}$ & $3.3 \mathrm{E}-05 \pm 9.1 \mathrm{E}-05$ & $\mathrm{U}$ \\
\hline & ${ }^{152} \mathrm{Eu}$ & $2.1 \mathrm{E}-05 \pm 1.7 \mathrm{E}-04$ & $\mathrm{U}$ & & ${ }^{152} \mathrm{Eu}$ & $-2.4 \mathrm{E}-05 \pm 2.0 \mathrm{E}-04$ & $\mathrm{U}$ \\
\hline & ${ }^{154} \mathrm{Eu}$ & $-6.9 \mathrm{E}-05 \pm 2.0 \mathrm{E}-04$ & $\mathrm{U}$ & & ${ }^{154} \mathrm{Eu}$ & $-1.5 \mathrm{E}-06 \pm 1.5 \mathrm{E}-05$ & $\mathrm{U}$ \\
\hline & ${ }^{155} \mathrm{Eu}$ & $2.6 \mathrm{E}-06 \pm 2.6 \mathrm{E}-05$ & $\mathrm{U}$ & & ${ }^{155} \mathrm{Eu}$ & $-5.4 \mathrm{E}-05 \pm 2.0 \mathrm{E}-04$ & $\mathrm{U}$ \\
\hline & ${ }^{238} \mathrm{Pu}$ & $9.4 \mathrm{E}-06 \pm 1.3 \mathrm{E}-05$ & $\mathrm{U}$ & & ${ }^{238} \mathrm{Pu}$ & $8.3 \mathrm{E}-06 \pm 1.8 \mathrm{E}-05$ & $\mathrm{U}$ \\
\hline & ${ }^{239 / 240} \mathrm{Pu}$ & $2.3 \mathrm{E}-06 \pm 3.5 \mathrm{E}-06$ & $\mathrm{U}$ & & ${ }^{239 / 240} \mathrm{Pu}$ & $3.2 \mathrm{E}-06 \pm 3.9 \mathrm{E}-06$ & \\
\hline & ${ }^{103} \mathrm{Ru}$ & $1.8 \mathrm{E}-05 \pm 7.0 \mathrm{E}-05$ & $\mathrm{U}$ & & ${ }^{103} \mathrm{Ru}$ & $2.0 \mathrm{E}-05 \pm 9.1 \mathrm{E}-05$ & $\mathrm{U}$ \\
\hline & ${ }^{106} \mathrm{Ru}$ & $-3.9 \mathrm{E}-04 \pm 5.6 \mathrm{E}-04$ & $\mathrm{U}$ & & ${ }^{106} \mathrm{Ru}$ & $-5.4 \mathrm{E}-04 \pm 8.7 \mathrm{E}-04$ & $\mathrm{U}$ \\
\hline & ${ }^{125} \mathrm{Sb}$ & $3.6 \mathrm{E}-05 \pm 1.5 \mathrm{E}-04$ & $\mathrm{U}$ & & ${ }^{125} \mathrm{Sb}$ & $-1.1 \mathrm{E}-04 \pm 2.0 \mathrm{E}-04$ & $\mathrm{U}$ \\
\hline & ${ }^{113} \mathrm{Sn}$ & $-1.1 \mathrm{E}-05 \pm 7.1 \mathrm{E}-05$ & $\mathrm{U}$ & & ${ }^{113} \mathrm{Sn}$ & $-5.5 \mathrm{E}-05 \pm 9.6 \mathrm{E}-05$ & $\mathrm{U}$ \\
\hline & ${ }^{90} \mathrm{Sr}$ & $-4.4 \mathrm{E}-05 \pm 1.1 \mathrm{E}-04$ & $\mathrm{U}$ & & ${ }^{90} \mathrm{Sr}$ & $-3.3 \mathrm{E}-05 \pm 1.1 \mathrm{E}-04$ & $\mathrm{U}$ \\
\hline & ${ }^{234} \mathrm{U}$ & $2.0 \mathrm{E}-05 \pm 1.1 \mathrm{E}-05$ & & & ${ }^{234} \mathrm{U}$ & $1.2 \mathrm{E}-05 \pm 8.7 \mathrm{E}-06$ & \\
\hline & ${ }^{235} \mathrm{U}$ & $-7.3 \mathrm{E}-07 \pm 3.3 \mathrm{E}-06$ & $\mathrm{U}$ & & ${ }^{235} \mathrm{U}$ & $7.1 \mathrm{E}-06 \pm 6.2 \mathrm{E}-06$ & \\
\hline & ${ }^{238} \mathrm{U}$ & $1.3 \mathrm{E}-05 \pm 7.9 \mathrm{E}-06$ & & & ${ }^{238} \mathrm{U}$ & $1.2 \mathrm{E}-05 \pm 7.9 \mathrm{E}-06$ & \\
\hline & ${ }^{65} \mathrm{Zn}$ & $-9.2 \mathrm{E}-05 \pm 1.5 \mathrm{E}-04$ & $\mathrm{U}$ & & ${ }^{65} \mathrm{Zn}$ & $-1.6 \mathrm{E}-06 \pm 1.6 \mathrm{E}-05$ & $\mathrm{U}$ \\
\hline
\end{tabular}

$\overline{\mathrm{RQ}}=$ Result Qualifier. $\mathrm{U}=$ The analyte was analyzed for but not detected. 
Table 2-3. Near-Facility Air Sampling Results, 2003 (pCi/m³ \pm total analytical uncertainty). (cont)

\begin{tabular}{|c|c|c|c|c|c|c|c|}
\hline Location & Isotope & Result \pm Uncertainty & $\mathbf{R Q}^{*}$ & Location & Isotope & Result \pm Uncertainty & $\mathbf{R Q}^{*}$ \\
\hline N969 (200-East) & ${ }^{144} \mathrm{Ce}$ & $-4.7 \mathrm{E}-04 \pm 6.1 \mathrm{E}-04$ & $\bar{U}$ & N970 (200-East) & ${ }^{144} \mathrm{Ce}$ & $-1.4 \mathrm{E}-04 \pm 5.3 \mathrm{E}-04$ & $\mathrm{U}$ \\
\hline Composite Period & ${ }^{60} \mathrm{Co}$ & $4.1 \mathrm{E}-05 \pm 6.6 \mathrm{E}-05$ & $\mathrm{U}$ & Composite Period & ${ }^{60} \mathrm{Co}$ & $9.3 \mathrm{E}-05 \pm 9.1 \mathrm{E}-05$ & $\mathrm{U}$ \\
\hline \multirow[t]{16}{*}{$06 / 23 / 03$ to $12 / 23 / 03$} & ${ }^{134} \mathrm{Cs}$ & $-6.9 \mathrm{E}-05 \pm 7.2 \mathrm{E}-05$ & $\mathrm{U}$ & $01 / 06 / 03$ to $06 / 23 / 03$ & ${ }^{134} \mathrm{Cs}$ & $-1.3 \mathrm{E}-05 \pm 7.0 \mathrm{E}-05$ & $\mathrm{U}$ \\
\hline & ${ }^{137} \mathrm{Cs}$ & $-3.0 \mathrm{E}-05 \pm 6.3 \mathrm{E}-05$ & $\mathrm{U}$ & & ${ }^{137} \mathrm{Cs}$ & $-6.9 \mathrm{E}-06 \pm 6.7 \mathrm{E}-05$ & $\mathrm{U}$ \\
\hline & ${ }^{152} \mathrm{Eu}$ & $-7.4 \mathrm{E}-06 \pm 7.4 \mathrm{E}-05$ & U & & ${ }^{152} \mathrm{Eu}$ & $4.2 \mathrm{E}-05 \pm 1.6 \mathrm{E}-04$ & U \\
\hline & ${ }^{154} \mathrm{Eu}$ & $-1.2 \mathrm{E}-05 \pm 1.2 \mathrm{E}-04$ & $\mathrm{U}$ & & ${ }^{154} \mathrm{Eu}$ & $-9.8 \mathrm{E}-05 \pm 2.1 \mathrm{E}-04$ & $\mathrm{U}$ \\
\hline & ${ }^{155} \mathrm{Eu}$ & $3.7 \mathrm{E}-05 \pm 1.5 \mathrm{E}-04$ & $\mathrm{U}$ & & ${ }^{155} \mathrm{Eu}$ & $5.8 \mathrm{E}-05 \pm 1.5 \mathrm{E}-04$ & $\mathrm{U}$ \\
\hline & ${ }^{238} \mathrm{Pu}$ & $4.0 \mathrm{E}-06 \pm 1.0 \mathrm{E}-05$ & $\mathrm{U}$ & & ${ }^{238} \mathrm{Pu}$ & $-7.5 \mathrm{E}-07 \pm 7.3 \mathrm{E}-06$ & $\mathrm{U}$ \\
\hline & ${ }^{239 / 240} \mathrm{Pu}$ & $1.2 \mathrm{E}-05 \pm 8.5 \mathrm{E}-06$ & & & ${ }^{239 / 240} \mathrm{Pu}$ & $2.3 \mathrm{E}-06 \pm 3.6 \mathrm{E}-06$ & $\mathrm{U}$ \\
\hline & ${ }^{103} \mathrm{Ru}$ & $-4.5 \mathrm{E}-05 \pm 6.3 \mathrm{E}-05$ & $\mathrm{U}$ & & ${ }^{103} \mathrm{Ru}$ & $9.1 \mathrm{E}-06 \pm 7.1 \mathrm{E}-05$ & $\mathrm{U}$ \\
\hline & ${ }^{106} \mathrm{Ru}$ & $-8.9 \mathrm{E}-05 \pm 5.2 \mathrm{E}-04$ & $\mathrm{U}$ & & ${ }^{106} \mathrm{Ru}$ & $-2.1 \mathrm{E}-04 \pm 6.5 \mathrm{E}-04$ & $\mathrm{U}$ \\
\hline & ${ }^{125} \mathrm{Sb}$ & $8.9 \mathrm{E}-05 \pm 1.4 \mathrm{E}-04$ & $\mathrm{U}$ & & ${ }^{125} \mathrm{Sb}$ & $1.4 \mathrm{E}-04 \pm 1.6 \mathrm{E}-04$ & $\mathrm{U}$ \\
\hline & ${ }^{113} \mathrm{Sn}$ & $-2.8 \mathrm{E}-05 \pm 6.2 \mathrm{E}-05$ & $\mathrm{U}$ & & ${ }^{113} \mathrm{Sn}$ & $2.0 \mathrm{E}-05 \pm 7.6 \mathrm{E}-05$ & $\mathrm{U}$ \\
\hline & ${ }^{90} \mathrm{Sr}$ & $-5.1 \mathrm{E}-05 \pm 1.0 \mathrm{E}-04$ & $\mathrm{U}$ & & ${ }^{90} \mathrm{Sr}$ & $-6.8 \mathrm{E}-05 \pm 1.0 \mathrm{E}-04$ & $\mathrm{U}$ \\
\hline & ${ }^{234} \mathrm{U}$ & $1.5 \mathrm{E}-05 \pm 8.8 \mathrm{E}-06$ & & & ${ }^{234} \mathrm{U}$ & $6.5 \mathrm{E}-06 \pm 5.1 \mathrm{E}-06$ & \\
\hline & ${ }^{235} \mathrm{U}$ & $6.8 \mathrm{E}-07 \pm 7.0 \mathrm{E}-07$ & $\mathrm{U}$ & & ${ }^{235} \mathrm{U}$ & $2.1 \mathrm{E}-06 \pm 3.9 \mathrm{E}-06$ & $\mathrm{U}$ \\
\hline & ${ }^{238} \mathrm{U}$ & $6.8 \mathrm{E}-06 \pm 5.7 \mathrm{E}-06$ & & & ${ }^{238} \mathrm{U}$ & $6.5 \mathrm{E}-06 \pm 4.8 \mathrm{E}-06$ & \\
\hline & ${ }^{65} \mathrm{Zn}$ & $-1.1 \mathrm{E}-04 \pm 1.7 \mathrm{E}-04$ & $\mathrm{U}$ & & ${ }^{65} \mathrm{Zn}$ & $-1.3 \mathrm{E}-05 \pm 1.3 \mathrm{E}-04$ & $\mathrm{U}$ \\
\hline N970 (200-East) & ${ }^{144} \mathrm{Ce}$ & $-3.8 \mathrm{E}-04 \pm 6.8 \mathrm{E}-04$ & $\bar{U}$ & N972 (200-East) & ${ }^{144} \mathrm{Ce}$ & $4.2 \mathrm{E}-04 \pm 7.8 \mathrm{E}-04$ & $\mathrm{U}$ \\
\hline Composite Period & ${ }^{60} \mathrm{Co}$ & $7.2 \mathrm{E}-05 \pm 8.1 \mathrm{E}-05$ & $\mathrm{U}$ & Composite Period & ${ }^{60} \mathrm{Co}$ & $-6.1 \mathrm{E}-05 \pm 7.6 \mathrm{E}-05$ & $\mathrm{U}$ \\
\hline \multirow[t]{16}{*}{$06 / 23 / 03$ to $12 / 22 / 03$} & ${ }^{134} \mathrm{Cs}$ & $-7.4 \mathrm{E}-05 \pm 9.0 \mathrm{E}-05$ & $\mathrm{U}$ & $01 / 06 / 03$ to $06 / 23 / 03$ & ${ }^{134} \mathrm{Cs}$ & $-3.8 \mathrm{E}-05 \pm 7.8 \mathrm{E}-05$ & $\mathrm{U}$ \\
\hline & ${ }^{137} \mathrm{Cs}$ & $-1.9 \mathrm{E}-05 \pm 6.9 \mathrm{E}-05$ & $\mathrm{U}$ & & ${ }^{137} \mathrm{Cs}$ & $7.8 \mathrm{E}-05 \pm 8.4 \mathrm{E}-05$ & $\mathrm{U}$ \\
\hline & ${ }^{152} \mathrm{Eu}$ & $-1.0 \mathrm{E}-04 \pm 1.8 \mathrm{E}-04$ & $\mathrm{U}$ & & ${ }^{152} \mathrm{Eu}$ & $-9.6 \mathrm{E}-05 \pm 1.9 \mathrm{E}-04$ & $\mathrm{U}$ \\
\hline & ${ }^{154} \mathrm{Eu}$ & $1.0 \mathrm{E}-04 \pm 2.5 \mathrm{E}-04$ & $\mathrm{U}$ & & ${ }^{154} \mathrm{Eu}$ & $2.8 \mathrm{E}-04 \pm 2.3 \mathrm{E}-04$ & $\mathrm{U}$ \\
\hline & ${ }^{155} \mathrm{Eu}$ & $-1.6 \mathrm{E}-04 \pm 1.8 \mathrm{E}-04$ & $\mathrm{U}$ & & ${ }^{155} \mathrm{Eu}$ & $-2.4 \mathrm{E}-04 \pm 2.5 \mathrm{E}-04$ & $\mathrm{U}$ \\
\hline & ${ }^{238} \mathrm{Pu}$ & $-1.8 \mathrm{E}-06 \pm 9.6 \mathrm{E}-06$ & $\mathrm{U}$ & & ${ }^{238} \mathrm{Pu}$ & $8.3 \mathrm{E}-07 \pm 7.6 \mathrm{E}-06$ & $\mathrm{U}$ \\
\hline & ${ }^{239 / 240} \mathrm{Pu}$ & $8.6 \mathrm{E}-07 \pm 3.9 \mathrm{E}-06$ & $\mathrm{U}$ & & ${ }^{239 / 240} \mathrm{Pu}$ & $1.7 \mathrm{E}-06 \pm 3.4 \mathrm{E}-06$ & $\mathrm{U}$ \\
\hline & ${ }^{103} \mathrm{Ru}$ & $2.5 \mathrm{E}-07 \pm 2.5 \mathrm{E}-06$ & $\mathrm{U}$ & & ${ }^{103} \mathrm{Ru}$ & $1.5 \mathrm{E}-05 \pm 7.8 \mathrm{E}-05$ & $\mathrm{U}$ \\
\hline & ${ }^{106} \mathrm{Ru}$ & $-4.4 \mathrm{E}-04 \pm 6.4 \mathrm{E}-04$ & $\mathrm{U}$ & & ${ }^{106} \mathrm{Ru}$ & $3.0 \mathrm{E}-04 \pm 6.9 \mathrm{E}-04$ & $\mathrm{U}$ \\
\hline & ${ }^{125} \mathrm{Sb}$ & $-1.8 \mathrm{E}-05 \pm 1.7 \mathrm{E}-04$ & $\mathrm{U}$ & & ${ }^{125} \mathrm{Sb}$ & $6.9 \mathrm{E}-05 \pm 1.8 \mathrm{E}-04$ & $\mathrm{U}$ \\
\hline & ${ }^{113} \mathrm{Sn}$ & $-3.3 \mathrm{E}-05 \pm 8.2 \mathrm{E}-05$ & $\mathrm{U}$ & & ${ }^{113} \mathrm{Sn}$ & $3.0 \mathrm{E}-05 \pm 8.6 \mathrm{E}-05$ & $\mathrm{U}$ \\
\hline & ${ }^{90} \mathrm{Sr}$ & $-5.8 \mathrm{E}-05 \pm 9.6 \mathrm{E}-05$ & $\mathrm{U}$ & & ${ }^{90} \mathrm{Sr}$ & $1.5 \mathrm{E}-07 \pm 1.5 \mathrm{E}-06$ & $\mathrm{U}$ \\
\hline & ${ }^{234} \mathrm{U}$ & $1.6 \mathrm{E}-05 \pm 9.3 \mathrm{E}-06$ & & & ${ }^{234} \mathrm{U}$ & $8.3 \mathrm{E}-06 \pm 6.3 \mathrm{E}-06$ & \\
\hline & ${ }^{235} \mathrm{U}$ & $4.5 \mathrm{E}-06 \pm 4.1 \mathrm{E}-06$ & & & ${ }^{235} \mathrm{U}$ & $6.7 \mathrm{E}-07 \pm 7.0 \mathrm{E}-07$ & $\mathrm{U}$ \\
\hline & ${ }^{238} \mathrm{U}$ & $1.2 \mathrm{E}-05 \pm 7.6 \mathrm{E}-06$ & & & ${ }^{238} \mathrm{U}$ & 4.7E-06 $\pm 3.9 \mathrm{E}-06$ & \\
\hline & ${ }^{65} \mathrm{Zn}$ & $1.8 \mathrm{E}-04 \pm 2.2 \mathrm{E}-04$ & $\mathrm{U}$ & & ${ }^{65} \mathrm{Zn}$ & $-3.5 \mathrm{E}-05 \pm 1.5 \mathrm{E}-04$ & $\mathrm{U}$ \\
\hline
\end{tabular}

$\overline{\mathrm{RQ}}=$ Result Qualifier. $\mathrm{U}=$ The analyte was analyzed for but not detected. 
Table 2-3. Near-Facility Air Sampling Results, 2003 (pCi/m³ \pm total analytical uncertainty). (cont)

\begin{tabular}{ccrc}
\hline Location & Isotope & Result \pm Uncertainty & RQ* \\
\hline N972 (200-East) & ${ }^{144} \mathrm{Ce}$ & $2.7 \mathrm{E}-05 \pm 2.7 \mathrm{E}-04$ & $\mathrm{U}$ \\
Composite Period & ${ }^{60} \mathrm{Co}$ & $7.1 \mathrm{E}-06 \pm 7.1 \mathrm{E}-05$ & $\mathrm{U}$ \\
06/23/03 to 12/22/03 & ${ }^{134} \mathrm{Cs}$ & $-3.6 \mathrm{E}-06 \pm 3.6 \mathrm{E}-05$ & $\mathrm{U}$ \\
& ${ }^{137} \mathrm{Cs}$ & $-1.3 \mathrm{E}-05 \pm 6.4 \mathrm{E}-05$ & $\mathrm{U}$ \\
& ${ }^{152} \mathrm{Eu}$ & $-5.4 \mathrm{E}-05 \pm 1.4 \mathrm{E}-04$ & $\mathrm{U}$ \\
& ${ }^{154} \mathrm{Eu}$ & $2.7 \mathrm{E}-07 \pm 2.7 \mathrm{E}-06$ & $\mathrm{U}$ \\
& ${ }^{155} \mathrm{Eu}$ & $8.8 \mathrm{E}-05 \pm 1.5 \mathrm{E}-04$ & $\mathrm{U}$ \\
& ${ }^{238} \mathrm{Pu}$ & $-3.7 \mathrm{E}-06 \pm 1.2 \mathrm{E}-05$ & $\mathrm{U}$ \\
& ${ }^{239 / 240} \mathrm{Pu}$ & $1.2 \mathrm{E}-06 \pm 2.4 \mathrm{E}-06$ & $\mathrm{U}$ \\
& ${ }^{103} \mathrm{Ru}$ & $-6.0 \mathrm{E}-05 \pm 6.3 \mathrm{E}-05$ & $\mathrm{U}$ \\
& ${ }^{106} \mathrm{Ru}$ & $-4.9 \mathrm{E}-04 \pm 5.6 \mathrm{E}-04$ & $\mathrm{U}$ \\
& ${ }^{125} \mathrm{Sb}$ & $-3.7 \mathrm{E}-05 \pm 1.4 \mathrm{E}-04$ & $\mathrm{U}$ \\
& ${ }^{113} \mathrm{Sn}$ & $-1.1 \mathrm{E}-05 \pm 6.8 \mathrm{E}-05$ & $\mathrm{U}$ \\
& ${ }^{90} \mathrm{Sr}$ & $7.1 \mathrm{E}-05 \pm 9.4 \mathrm{E}-05$ & $\mathrm{U}$ \\
& ${ }^{234} \mathrm{U}$ & $8.5 \mathrm{E}-06 \pm 6.1 \mathrm{E}-06$ & \\
& ${ }^{235} \mathrm{U}$ & $1.6 \mathrm{E}-06 \pm 3.1 \mathrm{E}-06$ & $\mathrm{U}$ \\
& ${ }^{238} \mathrm{U}$ & $9.2 \mathrm{E}-06 \pm 6.5 \mathrm{E}-06$ & \\
& ${ }^{65} \mathrm{Zn} \mathrm{U}$ & $1.2 \mathrm{E}-05 \pm 1.2 \mathrm{E}-04$ & $\mathrm{U}$
\end{tabular}

\begin{tabular}{|c|c|c|c|}
\hline Location & Isotope & Result \pm Uncertainty & RQ* \\
\hline N973 (200-East) & ${ }^{144} \mathrm{Ce}$ & $1.3 \mathrm{E}-04 \pm 8.1 \mathrm{E}-04$ & $\bar{U}$ \\
\hline Composite Period & ${ }^{60} \mathrm{Co}$ & $3.6 \mathrm{E}-05 \pm 9.9 \mathrm{E}-05$ & $\mathrm{U}$ \\
\hline \multirow[t]{16}{*}{$01 / 06 / 03$ to $06 / 23 / 03$} & ${ }^{134} \mathrm{Cs}$ & $-2.6 \mathrm{E}-05 \pm 8.7 \mathrm{E}-05$ & $\mathrm{U}$ \\
\hline & ${ }^{137} \mathrm{Cs}$ & $3.0 \mathrm{E}-04 \pm 1.5 \mathrm{E}-04$ & \\
\hline & ${ }^{152} \mathrm{Eu}$ & $-1.1 \mathrm{E}-04 \pm 2.0 \mathrm{E}-04$ & $\mathrm{U}$ \\
\hline & ${ }^{154} \mathrm{Eu}$ & $-9.0 \mathrm{E}-05 \pm 3.3 \mathrm{E}-04$ & $\mathrm{U}$ \\
\hline & ${ }^{155} \mathrm{Eu}$ & $-1.3 \mathrm{E}-04 \pm 1.9 \mathrm{E}-04$ & U \\
\hline & ${ }^{238} \mathrm{Pu}$ & $-3.1 \mathrm{E}-06 \pm 1.6 \mathrm{E}-05$ & $\mathrm{U}$ \\
\hline & ${ }^{239 / 240} \mathrm{Pu}$ & $1.1 \mathrm{E}-06 \pm 3.7 \mathrm{E}-06$ & $\mathrm{U}$ \\
\hline & ${ }^{103} \mathrm{Ru}$ & $6.5 \mathrm{E}-05 \pm 8.9 \mathrm{E}-05$ & $\mathrm{U}$ \\
\hline & ${ }^{106} \mathrm{Ru}$ & $1.3 \mathrm{E}-04 \pm 7.6 \mathrm{E}-04$ & $\mathrm{U}$ \\
\hline & ${ }^{125} \mathrm{Sb}$ & $6.6 \mathrm{E}-05 \pm 1.7 \mathrm{E}-04$ & $\mathrm{U}$ \\
\hline & ${ }^{113} \mathrm{Sn}$ & $-2.3 \mathrm{E}-05 \pm 9.6 \mathrm{E}-05$ & $\mathrm{U}$ \\
\hline & ${ }^{90} \mathrm{Sr}$ & $4.0 \mathrm{E}-05 \pm 1.1 \mathrm{E}-04$ & $\mathrm{U}$ \\
\hline & ${ }^{234} \mathrm{U}$ & $1.3 \mathrm{E}-05 \pm 7.9 \mathrm{E}-06$ & \\
\hline & ${ }^{235} \mathrm{U}$ & $3.3 \mathrm{E}-06 \pm 3.4 \mathrm{E}-06$ & \\
\hline & ${ }^{238} \mathrm{U}$ & $1.5 \mathrm{E}-05 \pm 8.5 \mathrm{E}-06$ & \\
\hline & ${ }^{65} \mathrm{Zn}$ & $-1.9 \mathrm{E}-04 \pm 2.0 \mathrm{E}-04$ & $\mathrm{U}$ \\
\hline N976 (200-East) & ${ }^{144} \mathrm{Ce}$ & $-1.4 \mathrm{E}-05 \pm 1.4 \mathrm{E}-04$ & $\bar{U}$ \\
\hline Composite Period & ${ }^{60} \mathrm{Co}$ & $-1.4 \mathrm{E}-05 \pm 7.6 \mathrm{E}-05$ & $\mathrm{U}$ \\
\hline \multirow[t]{16}{*}{$01 / 06 / 03$ to $06 / 23 / 03$} & ${ }^{134} \mathrm{Cs}$ & $-6.2 \mathrm{E}-05 \pm 7.7 \mathrm{E}-05$ & $\mathrm{U}$ \\
\hline & ${ }^{137} \mathrm{Cs}$ & $6.4 \mathrm{E}-05 \pm 7.9 \mathrm{E}-05$ & $\mathrm{U}$ \\
\hline & ${ }^{152} \mathrm{Eu}$ & $-2.5 \mathrm{E}-05 \pm 1.8 \mathrm{E}-04$ & $\mathrm{U}$ \\
\hline & ${ }^{154} \mathrm{Eu}$ & $1.1 \mathrm{E}-04 \pm 2.3 \mathrm{E}-04$ & $\mathrm{U}$ \\
\hline & ${ }^{155} \mathrm{Eu}$ & $-2.0 \mathrm{E}-05 \pm 2.0 \mathrm{E}-04$ & $\mathrm{U}$ \\
\hline & ${ }^{238} \mathrm{Pu}$ & $4.6 \mathrm{E}-06 \pm 1.2 \mathrm{E}-05$ & $\mathrm{U}$ \\
\hline & ${ }^{239 / 240} \mathrm{Pu}$ & $-2.3 \mathrm{E}-06 \pm 4.2 \mathrm{E}-06$ & $\mathrm{U}$ \\
\hline & ${ }^{103} \mathrm{Ru}$ & $-8.7 \mathrm{E}-05 \pm 9.0 \mathrm{E}-05$ & $\mathrm{U}$ \\
\hline & ${ }^{106} \mathrm{Ru}$ & $5.1 \mathrm{E}-04 \pm 7.1 \mathrm{E}-04$ & $\mathrm{U}$ \\
\hline & ${ }^{125} \mathrm{Sb}$ & $2.3 \mathrm{E}-05 \pm 1.8 \mathrm{E}-04$ & $\mathrm{U}$ \\
\hline & ${ }^{113} \mathrm{Sn}$ & $-5.2 \mathrm{E}-05 \pm 8.5 \mathrm{E}-05$ & $\mathrm{U}$ \\
\hline & ${ }^{90} \mathrm{Sr}$ & $-2.3 \mathrm{E}-05 \pm 8.7 \mathrm{E}-05$ & $\mathrm{U}$ \\
\hline & ${ }^{234} \mathrm{U}$ & $1.5 \mathrm{E}-05 \pm 9.0 \mathrm{E}-06$ & \\
\hline & ${ }^{235} \mathrm{U}$ & $5.1 \mathrm{E}-06 \pm 4.8 \mathrm{E}-06$ & $\mathrm{U}$ \\
\hline & ${ }^{238} \mathrm{U}$ & $2.0 \mathrm{E}-05 \pm 1.1 \mathrm{E}-05$ & \\
\hline & ${ }^{65} \mathrm{Zn}$ & $1.3 \mathrm{E}-04 \pm 1.7 \mathrm{E}-04$ & $\mathrm{U}$ \\
\hline
\end{tabular}

$\overline{\mathrm{RQ}}=$ Result Qualifier. $\mathrm{U}=$ The analyte was analyzed for but not detected. 
Table 2-3. Near-Facility Air Sampling Results, 2003 (pCi/m³ \pm total analytical uncertainty). (cont)

\begin{tabular}{|c|c|c|c|c|c|c|c|}
\hline Location & Isotope & Result \pm Uncertainty & $\mathbf{R Q}^{*}$ & Location & Isotope & Result \pm Uncertainty & $\mathbf{R Q}^{*}$ \\
\hline N976 (200-East) & ${ }^{144} \mathrm{Ce}$ & $5.2 \mathrm{E}-04 \pm 6.3 \mathrm{E}-04$ & $\bar{U}$ & N977 (200-East) & ${ }^{144} \mathrm{Ce}$ & $-2.7 \mathrm{E}-04 \pm 7.6 \mathrm{E}-04$ & $\bar{U}$ \\
\hline Composite Period & ${ }^{60} \mathrm{Co}$ & $5.6 \mathrm{E}-05 \pm 8.9 \mathrm{E}-05$ & U & Composite Period & ${ }^{60} \mathrm{Co}$ & $-2.5 \mathrm{E}-05 \pm 1.0 \mathrm{E}-04$ & $\mathrm{U}$ \\
\hline \multirow[t]{16}{*}{ 06/23/03 to $12 / 22 / 03$} & ${ }^{134} \mathrm{Cs}$ & $1.3 \mathrm{E}-05 \pm 7.0 \mathrm{E}-05$ & $\mathrm{U}$ & $01 / 06 / 03$ to $06 / 23 / 03$ & ${ }^{134} \mathrm{Cs}$ & $-1.6 \mathrm{E}-05 \pm 8.4 \mathrm{E}-05$ & $\mathrm{U}$ \\
\hline & ${ }^{137} \mathrm{Cs}$ & $1.4 \mathrm{E}-04 \pm 1.3 \mathrm{E}-04$ & & & ${ }^{137} \mathrm{Cs}$ & $-2.2 \mathrm{E}-05 \pm 8.0 \mathrm{E}-05$ & $\mathrm{U}$ \\
\hline & ${ }^{152} \mathrm{Eu}$ & $3.0 \mathrm{E}-05 \pm 1.4 \mathrm{E}-04$ & $\mathrm{U}$ & & ${ }^{152} \mathrm{Eu}$ & $1.8 \mathrm{E}-04 \pm 2.0 \mathrm{E}-04$ & $\mathrm{U}$ \\
\hline & ${ }^{154} \mathrm{Eu}$ & $5.2 \mathrm{E}-04 \pm 3.5 \mathrm{E}-04$ & & & ${ }^{154} \mathrm{Eu}$ & $9.7 \mathrm{E}-06 \pm 9.7 \mathrm{E}-05$ & $\mathrm{U}$ \\
\hline & ${ }^{155} \mathrm{Eu}$ & $4.4 \mathrm{E}-05 \pm 1.5 \mathrm{E}-04$ & U & & ${ }^{155} \mathrm{Eu}$ & $1.7 \mathrm{E}-04 \pm 1.9 \mathrm{E}-04$ & $\mathrm{U}$ \\
\hline & ${ }^{238} \mathrm{Pu}$ & $6.1 \mathrm{E}-06 \pm 1.1 \mathrm{E}-05$ & $\mathrm{U}$ & & ${ }^{238} \mathrm{Pu}$ & $-2.5 \mathrm{E}-06 \pm 1.2 \mathrm{E}-05$ & $\mathrm{U}$ \\
\hline & ${ }^{239 / 240} \mathrm{Pu}$ & $4.4 \mathrm{E}-06 \pm 4.2 \mathrm{E}-06$ & & & ${ }^{239 / 240} \mathrm{Pu}$ & $4.1 \mathrm{E}-06 \pm 4.6 \mathrm{E}-06$ & $\mathrm{U}$ \\
\hline & ${ }^{103} \mathrm{Ru}$ & $4.0 \mathrm{E}-05 \pm 6.2 \mathrm{E}-05$ & $\mathrm{U}$ & & ${ }^{103} \mathrm{Ru}$ & $-6.2 \mathrm{E}-07 \pm 6.2 \mathrm{E}-06$ & $\mathrm{U}$ \\
\hline & ${ }^{106} \mathrm{Ru}$ & $3.1 \mathrm{E}-04 \pm 5.4 \mathrm{E}-04$ & $\mathrm{U}$ & & ${ }^{106} \mathrm{Ru}$ & $-2.0 \mathrm{E}-05 \pm 2.0 \mathrm{E}-04$ & $\mathrm{U}$ \\
\hline & ${ }^{125} \mathrm{Sb}$ & $9.3 \mathrm{E}-05 \pm 1.4 \mathrm{E}-04$ & $\mathrm{U}$ & & ${ }^{125} \mathrm{Sb}$ & $1.2 \mathrm{E}-04 \pm 1.8 \mathrm{E}-04$ & $\mathrm{U}$ \\
\hline & ${ }^{113} \mathrm{Sn}$ & $1.2 \mathrm{E}-05 \pm 6.9 \mathrm{E}-05$ & $\mathrm{U}$ & & ${ }^{113} \mathrm{Sn}$ & $-1.9 \mathrm{E}-05 \pm 8.9 \mathrm{E}-05$ & $\mathrm{U}$ \\
\hline & ${ }^{90} \mathrm{Sr}$ & $1.6 \mathrm{E}-04 \pm 1.1 \mathrm{E}-04$ & & & ${ }^{90} \mathrm{Sr}$ & $-1.8 \mathrm{E}-04 \pm 1.9 \mathrm{E}-04$ & $\mathrm{U}$ \\
\hline & ${ }^{234} \mathrm{U}$ & $2.9 \mathrm{E}-05 \pm 1.4 \mathrm{E}-05$ & & & ${ }^{234} \mathrm{U}$ & $5.9 \mathrm{E}-06 \pm 5.5 \mathrm{E}-06$ & $\mathrm{U}$ \\
\hline & ${ }^{235} \mathrm{U}$ & $4.2 \mathrm{E}-06 \pm 4.8 \mathrm{E}-06$ & $\mathrm{U}$ & & ${ }^{235} \mathrm{U}$ & $1.6 \mathrm{E}-06 \pm 3.3 \mathrm{E}-06$ & $\mathrm{U}$ \\
\hline & ${ }^{238} \mathrm{U}$ & $4.0 \mathrm{E}-05 \pm 1.9 \mathrm{E}-05$ & & & ${ }^{238} \mathrm{U}$ & $5.9 \mathrm{E}-06 \pm 5.5 \mathrm{E}-06$ & $\mathrm{U}$ \\
\hline & ${ }^{65} \mathrm{Zn}$ & $1.2 \mathrm{E}-04 \pm 1.6 \mathrm{E}-04$ & $\mathrm{U}$ & & ${ }^{65} \mathrm{Zn}$ & $-1.0 \mathrm{E}-04 \pm 2.0 \mathrm{E}-04$ & $\mathrm{U}$ \\
\hline N977 (200-East) & ${ }^{144} \mathrm{Ce}$ & $7.2 \mathrm{E}-05 \pm 6.9 \mathrm{E}-04$ & $\mathrm{U}$ & N978 (200-East) & ${ }^{144} \mathrm{Ce}$ & $3.4 \mathrm{E}-04 \pm 7.1 \mathrm{E}-04$ & $\mathrm{U}$ \\
\hline Composite Period & ${ }^{60} \mathrm{Co}$ & $-3.3 \mathrm{E}-05 \pm 7.0 \mathrm{E}-05$ & $\mathrm{U}$ & Composite Period & ${ }^{60} \mathrm{Co}$ & $1.5 \mathrm{E}-06 \pm 1.5 \mathrm{E}-05$ & $\mathrm{U}$ \\
\hline \multirow[t]{16}{*}{$06 / 23 / 03$ to $12 / 22 / 03$} & ${ }^{134} \mathrm{Cs}$ & $2.7 \mathrm{E}-05 \pm 6.3 \mathrm{E}-05$ & U & $01 / 06 / 03$ to $06 / 23 / 03$ & ${ }^{134} \mathrm{Cs}$ & $-4.3 \mathrm{E}-05 \pm 9.1 \mathrm{E}-05$ & $\mathrm{U}$ \\
\hline & ${ }^{137} \mathrm{Cs}$ & $-8.5 \mathrm{E}-05 \pm 8.9 \mathrm{E}-05$ & $\mathrm{U}$ & & ${ }^{137} \mathrm{Cs}$ & $-4.9 \mathrm{E}-06 \pm 4.9 \mathrm{E}-05$ & $\mathrm{U}$ \\
\hline & ${ }^{152} \mathrm{Eu}$ & $6.6 \mathrm{E}-05 \pm 1.5 \mathrm{E}-04$ & $\mathrm{U}$ & & ${ }^{152} \mathrm{Eu}$ & $4.3 \mathrm{E}-05 \pm 1.8 \mathrm{E}-04$ & $\mathrm{U}$ \\
\hline & ${ }^{154} \mathrm{Eu}$ & $-1.2 \mathrm{E}-04 \pm 2.4 \mathrm{E}-04$ & $\mathrm{U}$ & & ${ }^{154} \mathrm{Eu}$ & $2.5 \mathrm{E}-05 \pm 2.5 \mathrm{E}-04$ & $\mathrm{U}$ \\
\hline & ${ }^{155} \mathrm{Eu}$ & $-2.7 \mathrm{E}-05 \pm 1.5 \mathrm{E}-04$ & $\mathrm{U}$ & & ${ }^{155} \mathrm{Eu}$ & $1.4 \mathrm{E}-04 \pm 1.9 \mathrm{E}-04$ & $\mathrm{U}$ \\
\hline & ${ }^{238} \mathrm{Pu}$ & $4.8 \mathrm{E}-06 \pm 9.2 \mathrm{E}-06$ & $\mathrm{U}$ & & ${ }^{238} \mathrm{Pu}$ & $6.1 \mathrm{E}-06 \pm 1.4 \mathrm{E}-05$ & $\mathrm{U}$ \\
\hline & ${ }^{239 / 240} \mathrm{Pu}$ & $-4.8 \mathrm{E}-06 \pm 5.0 \mathrm{E}-06$ & $\mathrm{U}$ & & ${ }^{239 / 240} \mathrm{Pu}$ & $1.8 \mathrm{E}-05 \pm 1.1 \mathrm{E}-05$ & \\
\hline & ${ }^{103} \mathrm{Ru}$ & $1.2 \mathrm{E}-05 \pm 6.2 \mathrm{E}-05$ & $\mathrm{U}$ & & ${ }^{103} \mathrm{Ru}$ & $1.9 \mathrm{E}-05 \pm 8.2 \mathrm{E}-05$ & $\mathrm{U}$ \\
\hline & ${ }^{106} \mathrm{Ru}$ & $-4.3 \mathrm{E}-05 \pm 4.3 \mathrm{E}-04$ & $\mathrm{U}$ & & ${ }^{106} \mathrm{Ru}$ & $-2.8 \mathrm{E}-04 \pm 7.6 \mathrm{E}-04$ & $\mathrm{U}$ \\
\hline & ${ }^{125} \mathrm{Sb}$ & $-9.8 \mathrm{E}-05 \pm 1.4 \mathrm{E}-04$ & $\mathrm{U}$ & & ${ }^{125} \mathrm{Sb}$ & $2.2 \mathrm{E}-05 \pm 1.7 \mathrm{E}-04$ & $\mathrm{U}$ \\
\hline & ${ }^{113} \mathrm{Sn}$ & $3.2 \mathrm{E}-05 \pm 6.4 \mathrm{E}-05$ & $\mathrm{U}$ & & ${ }^{113} \mathrm{Sn}$ & $-3.2 \mathrm{E}-05 \pm 8.7 \mathrm{E}-05$ & $\mathrm{U}$ \\
\hline & ${ }^{90} \mathrm{Sr}$ & $1.5 \mathrm{E}-05 \pm 8.2 \mathrm{E}-05$ & $\mathrm{U}$ & & ${ }^{90} \mathrm{Sr}$ & $7.5 \mathrm{E}-05 \pm 9.6 \mathrm{E}-05$ & $\mathrm{U}$ \\
\hline & ${ }^{234} \mathrm{U}$ & $1.2 \mathrm{E}-05 \pm 7.9 \mathrm{E}-06$ & & & ${ }^{234} \mathrm{U}$ & $1.3 \mathrm{E}-05 \pm 7.5 \mathrm{E}-06$ & \\
\hline & ${ }^{235} \mathrm{U}$ & $3.5 \mathrm{E}-06 \pm 3.7 \mathrm{E}-06$ & & & ${ }^{235} \mathrm{U}$ & $3.8 \mathrm{E}-06 \pm 4.6 \mathrm{E}-06$ & $\mathrm{U}$ \\
\hline & ${ }^{238} \mathrm{U}$ & $1.5 \mathrm{E}-05 \pm 8.9 \mathrm{E}-06$ & & & ${ }^{238} \mathrm{U}$ & $1.0 \mathrm{E}-05 \pm 6.7 \mathrm{E}-06$ & \\
\hline & ${ }^{65} \mathrm{Zn}$ & $-6.0 \mathrm{E}-05 \pm 1.8 \mathrm{E}-04$ & $\mathrm{U}$ & & ${ }^{65} \mathrm{Zn}$ & $2.3 \mathrm{E}-05 \pm 2.1 \mathrm{E}-04$ & $\mathrm{U}$ \\
\hline
\end{tabular}

$\overline{\mathrm{RQ}}=$ Result Qualifier. $\mathrm{U}=$ The analyte was analyzed for but not detected. 
Table 2-3. Near-Facility Air Sampling Results, 2003 (pCi/m³ \pm total analytical uncertainty). (cont)

\begin{tabular}{|c|c|c|c|}
\hline Location & Isotope & Result \pm Uncertainty & $\mathbf{R Q}^{*}$ \\
\hline N978 (200-East) & ${ }^{144} \mathrm{Ce}$ & $1.3 \mathrm{E}-05 \pm 1.3 \mathrm{E}-04$ & $\mathrm{U}$ \\
\hline Composite Period & ${ }^{60} \mathrm{Co}$ & $4.5 \mathrm{E}-05 \pm 9.4 \mathrm{E}-05$ & $\mathrm{U}$ \\
\hline \multirow[t]{16}{*}{$06 / 23 / 03$ to $12 / 23 / 03$} & ${ }^{134} \mathrm{Cs}$ & $-5.7 \mathrm{E}-05 \pm 9.2 \mathrm{E}-05$ & $\mathrm{U}$ \\
\hline & ${ }^{137} \mathrm{Cs}$ & $1.3 \mathrm{E}-05 \pm 7.8 \mathrm{E}-05$ & $\mathrm{U}$ \\
\hline & ${ }^{152} \mathrm{Eu}$ & $-8.7 \mathrm{E}-05 \pm 2.1 \mathrm{E}-04$ & $\mathrm{U}$ \\
\hline & ${ }^{154} \mathrm{Eu}$ & $8.9 \mathrm{E}-05 \pm 2.8 \mathrm{E}-04$ & $\mathrm{U}$ \\
\hline & ${ }^{155} \mathrm{Eu}$ & $-6.8 \mathrm{E}-05 \pm 1.7 \mathrm{E}-04$ & $\mathrm{U}$ \\
\hline & ${ }^{238} \mathrm{Pu}$ & $-2.4 \mathrm{E}-06 \pm 1.0 \mathrm{E}-05$ & $\mathrm{U}$ \\
\hline & ${ }^{239 / 240} \mathrm{Pu}$ & $2.4 \mathrm{E}-06 \pm 4.8 \mathrm{E}-06$ & $\mathrm{U}$ \\
\hline & ${ }^{103} \mathrm{Ru}$ & $1.0 \mathrm{E}-05 \pm 7.8 \mathrm{E}-05$ & $\mathrm{U}$ \\
\hline & ${ }^{106} \mathrm{Ru}$ & $-6.0 \mathrm{E}-04 \pm 7.0 \mathrm{E}-04$ & $\mathrm{U}$ \\
\hline & ${ }^{125} \mathrm{Sb}$ & $-4.6 \mathrm{E}-05 \pm 1.7 \mathrm{E}-04$ & $\mathrm{U}$ \\
\hline & ${ }^{113} \mathrm{Sn}$ & $3.3 \mathrm{E}-05 \pm 8.2 \mathrm{E}-05$ & $\mathrm{U}$ \\
\hline & ${ }^{90} \mathrm{Sr}$ & $7.5 \mathrm{E}-06 \pm 7.5 \mathrm{E}-05$ & \\
\hline & ${ }^{234} \mathrm{U}$ & $1.4 \mathrm{E}-05 \pm 8.9 \mathrm{E}-06$ & \\
\hline & ${ }^{235} \mathrm{U}$ & $5.1 \mathrm{E}-06 \pm 5.2 \mathrm{E}-06$ & $\mathrm{U}$ \\
\hline & ${ }^{238} \mathrm{U}$ & $1.9 \mathrm{E}-05 \pm 1.1 \mathrm{E}-05$ & \\
\hline & ${ }^{65} \mathrm{Zn}$ & $6.0 \mathrm{E}-05 \pm 2.2 \mathrm{E}-04$ & $\mathrm{U}$ \\
\hline N984 (200-East) & ${ }^{144} \mathrm{Ce}$ & $-1.8 \mathrm{E}-04 \pm 7.5 \mathrm{E}-04$ & $\mathrm{U}$ \\
\hline Composite Period & ${ }^{60} \mathrm{Co}$ & $-8.8 \mathrm{E}-05 \pm 9.1 \mathrm{E}-05$ & $\mathrm{U}$ \\
\hline \multirow[t]{16}{*}{$06 / 23 / 03$ to $12 / 22 / 03$} & ${ }^{134} \mathrm{Cs}$ & $-1.7 \mathrm{E}-05 \pm 6.9 \mathrm{E}-05$ & $\mathrm{U}$ \\
\hline & ${ }^{137} \mathrm{Cs}$ & $8.5 \mathrm{E}-05 \pm 8.3 \mathrm{E}-05$ & $\mathrm{U}$ \\
\hline & ${ }^{152} \mathrm{Eu}$ & $-2.3 \mathrm{E}-04 \pm 2.4 \mathrm{E}-04$ & $\mathrm{U}$ \\
\hline & ${ }^{154} \mathrm{Eu}$ & $5.9 \mathrm{E}-06 \pm 5.9 \mathrm{E}-05$ & $\mathrm{U}$ \\
\hline & ${ }^{155} \mathrm{Eu}$ & $-1.2 \mathrm{E}-04 \pm 2.1 \mathrm{E}-04$ & $\mathrm{U}$ \\
\hline & ${ }^{238} \mathrm{Pu}$ & $1.8 \mathrm{E}-06 \pm 9.4 \mathrm{E}-06$ & $\mathrm{U}$ \\
\hline & ${ }^{239 / 240} \mathrm{Pu}$ & $2.2 \mathrm{E}-05 \pm 1.2 \mathrm{E}-05$ & \\
\hline & ${ }^{103} \mathrm{Ru}$ & $-4.6 \mathrm{E}-06 \pm 4.6 \mathrm{E}-05$ & $\mathrm{U}$ \\
\hline & ${ }^{106} \mathrm{Ru}$ & $-3.2 \mathrm{E}-04 \pm 6.3 \mathrm{E}-04$ & $\mathrm{U}$ \\
\hline & ${ }^{125} \mathrm{Sb}$ & $-5.4 \mathrm{E}-05 \pm 1.5 \mathrm{E}-04$ & $\mathrm{U}$ \\
\hline & ${ }^{113} \mathrm{Sn}$ & $-6.5 \mathrm{E}-06 \pm 6.5 \mathrm{E}-05$ & $\mathrm{U}$ \\
\hline & ${ }^{90} \mathrm{Sr}$ & $1.0 \mathrm{E}-03 \pm 3.3 \mathrm{E}-04$ & \\
\hline & ${ }^{234} \mathrm{U}$ & $1.3 \mathrm{E}-05 \pm 8.1 \mathrm{E}-06$ & \\
\hline & ${ }^{235} \mathrm{U}$ & $3.6 \mathrm{E}-06 \pm 3.8 \mathrm{E}-06$ & \\
\hline & ${ }^{238} \mathrm{U}$ & $1.0 \mathrm{E}-05 \pm 7.2 \mathrm{E}-06$ & \\
\hline & ${ }^{65} \mathrm{Zn}$ & $4.8 \mathrm{E}-05 \pm 1.5 \mathrm{E}-04$ & $\mathrm{U}$ \\
\hline
\end{tabular}

\begin{tabular}{|c|c|c|c|}
\hline Location & Isotope & Result \pm Uncertainty & RQ* \\
\hline N984 (200-East) & ${ }^{144} \mathrm{Ce}$ & $-2.2 \mathrm{E}-05 \pm 2.2 \mathrm{E}-04$ & $\bar{U}$ \\
\hline Composite Period & ${ }^{60} \mathrm{Co}$ & $6.3 \mathrm{E}-05 \pm 9.4 \mathrm{E}-05$ & $\mathrm{U}$ \\
\hline \multirow[t]{16}{*}{$01 / 06 / 03$ to $06 / 23 / 03$} & ${ }^{134} \mathrm{Cs}$ & $6.4 \mathrm{E}-05 \pm 9.3 \mathrm{E}-05$ & $\mathrm{U}$ \\
\hline & ${ }^{137} \mathrm{Cs}$ & $2.8 \mathrm{E}-04 \pm 1.6 \mathrm{E}-04$ & \\
\hline & ${ }^{152} \mathrm{Eu}$ & $1.3 \mathrm{E}-04 \pm 1.9 \mathrm{E}-04$ & $\mathrm{U}$ \\
\hline & ${ }^{154} \mathrm{Eu}$ & $-3.4 \mathrm{E}-05 \pm 2.8 \mathrm{E}-04$ & $\mathrm{U}$ \\
\hline & ${ }^{155} \mathrm{Eu}$ & $4.4 \mathrm{E}-05 \pm 2.0 \mathrm{E}-04$ & U \\
\hline & ${ }^{238} \mathrm{Pu}$ & $2.0 \mathrm{E}-06 \pm 1.5 \mathrm{E}-05$ & $\mathrm{U}$ \\
\hline & ${ }^{239 / 240} \mathrm{Pu}$ & $2.9 \mathrm{E}-06 \pm 3.6 \mathrm{E}-06$ & \\
\hline & ${ }^{103} \mathrm{Ru}$ & $-1.9 \mathrm{E}-05 \pm 8.8 \mathrm{E}-05$ & $\mathrm{U}$ \\
\hline & ${ }^{106} \mathrm{Ru}$ & $-7.1 \mathrm{E}-06 \pm 7.1 \mathrm{E}-05$ & $\mathrm{U}$ \\
\hline & ${ }^{125} \mathrm{Sb}$ & $2.5 \mathrm{E}-05 \pm 2.0 \mathrm{E}-04$ & $\mathrm{U}$ \\
\hline & ${ }^{113} \mathrm{Sn}$ & $1.2 \mathrm{E}-05 \pm 8.9 \mathrm{E}-05$ & $\mathrm{U}$ \\
\hline & ${ }^{90} \mathrm{Sr}$ & $6.5 \mathrm{E}-04 \pm 2.3 \mathrm{E}-04$ & \\
\hline & ${ }^{234} \mathrm{U}$ & $1.0 \mathrm{E}-05 \pm 6.6 \mathrm{E}-06$ & \\
\hline & ${ }^{235} \mathrm{U}$ & $8.4 \mathrm{E}-07 \pm 1.7 \mathrm{E}-06$ & $\mathrm{U}$ \\
\hline & ${ }^{238} \mathrm{U}$ & $1.2 \mathrm{E}-05 \pm 7.6 \mathrm{E}-06$ & \\
\hline & ${ }^{65} \mathrm{Zn}$ & $1.6 \mathrm{E}-05 \pm 1.6 \mathrm{E}-04$ & $\mathrm{U}$ \\
\hline N985 (200-East) & ${ }^{144} \mathrm{Ce}$ & $-2.7 \mathrm{E}-05 \pm 2.7 \mathrm{E}-04$ & $\bar{U}$ \\
\hline Composite Period & ${ }^{60} \mathrm{Co}$ & $-8.7 \mathrm{E}-05 \pm 9.3 \mathrm{E}-05$ & $\mathrm{U}$ \\
\hline \multirow[t]{16}{*}{$01 / 06 / 03$ to $06 / 23 / 03$} & ${ }^{134} \mathrm{Cs}$ & $-4.2 \mathrm{E}-05 \pm 8.0 \mathrm{E}-05$ & $\mathrm{U}$ \\
\hline & ${ }^{137} \mathrm{Cs}$ & $2.0 \mathrm{E}-04 \pm 1.3 \mathrm{E}-04$ & \\
\hline & ${ }^{152} \mathrm{Eu}$ & $-2.4 \mathrm{E}-05 \pm 1.6 \mathrm{E}-04$ & $\mathrm{U}$ \\
\hline & ${ }^{154} \mathrm{Eu}$ & $-7.5 \mathrm{E}-05 \pm 2.2 \mathrm{E}-04$ & $\mathrm{U}$ \\
\hline & ${ }^{155} \mathrm{Eu}$ & $2.0 \mathrm{E}-05 \pm 1.6 \mathrm{E}-04$ & $\mathrm{U}$ \\
\hline & ${ }^{238} \mathrm{Pu}$ & $5.3 \mathrm{E}-06 \pm 1.3 \mathrm{E}-05$ & $\mathrm{U}$ \\
\hline & ${ }^{239 / 240} \mathrm{Pu}$ & $9.2 \mathrm{E}-07 \pm 9.5 \mathrm{E}-07$ & $\mathrm{U}$ \\
\hline & ${ }^{103} \mathrm{Ru}$ & $-4.8 \mathrm{E}-05 \pm 8.6 \mathrm{E}-05$ & $\mathrm{U}$ \\
\hline & ${ }^{106} \mathrm{Ru}$ & $2.1 \mathrm{E}-04 \pm 6.5 \mathrm{E}-04$ & $\mathrm{U}$ \\
\hline & ${ }^{125} \mathrm{Sb}$ & $1.3 \mathrm{E}-04 \pm 1.7 \mathrm{E}-04$ & $\mathrm{U}$ \\
\hline & ${ }^{113} \mathrm{Sn}$ & $-1.4 \mathrm{E}-05 \pm 7.8 \mathrm{E}-05$ & $\mathrm{U}$ \\
\hline & ${ }^{90} \mathrm{Sr}$ & $3.0 \mathrm{E}-05 \pm 8.9 \mathrm{E}-05$ & $\mathrm{U}$ \\
\hline & ${ }^{234} \mathrm{U}$ & $9.2 \mathrm{E}-06 \pm 7.1 \mathrm{E}-06$ & \\
\hline & ${ }^{235} \mathrm{U}$ & $7.6 \mathrm{E}-07 \pm 3.4 \mathrm{E}-06$ & $\mathrm{U}$ \\
\hline & ${ }^{238} \mathrm{U}$ & $1.2 \mathrm{E}-05 \pm 7.7 \mathrm{E}-06$ & \\
\hline & ${ }^{65} \mathrm{Zn}$ & $1.2 \mathrm{E}-04 \pm 1.9 \mathrm{E}-04$ & $\mathrm{U}$ \\
\hline
\end{tabular}

$\overline{\mathrm{RQ}}=$ Result Qualifier. $\mathrm{U}=$ The analyte was analyzed for but not detected. 
Table 2-3. Near-Facility Air Sampling Results, 2003 (pCi/m³ \pm total analytical uncertainty). (cont)

\begin{tabular}{|c|c|c|c|c|c|c|c|}
\hline Location & Isotope & Result \pm Uncertainty & $\mathbf{R Q}^{*}$ & Location & Isotope & Result \pm Uncertainty & RQ* \\
\hline N985 (200-East) & ${ }^{144} \mathrm{Ce}$ & $5.4 \mathrm{E}-05 \pm 5.4 \mathrm{E}-04$ & $\bar{U}$ & N999 (200-East) & ${ }^{144} \mathrm{Ce}$ & $5.7 \mathrm{E}-04 \pm 7.7 \mathrm{E}-04$ & $\bar{U}$ \\
\hline Composite Period & ${ }^{60} \mathrm{Co}$ & $-4.7 \mathrm{E}-05 \pm 7.8 \mathrm{E}-05$ & U & Composite Period & ${ }^{60} \mathrm{Co}$ & $2.8 \mathrm{E}-05 \pm 8.7 \mathrm{E}-05$ & $\mathrm{U}$ \\
\hline \multirow[t]{16}{*}{$06 / 23 / 03$ to $12 / 22 / 03$} & ${ }^{134} \mathrm{Cs}$ & $4.0 \mathrm{E}-05 \pm 6.9 \mathrm{E}-05$ & $\mathrm{U}$ & $01 / 06 / 03$ to $06 / 23 / 03$ & ${ }^{134} \mathrm{Cs}$ & $6.1 \mathrm{E}-06 \pm 6.2 \mathrm{E}-05$ & $\mathrm{U}$ \\
\hline & ${ }^{137} \mathrm{Cs}$ & $1.3 \mathrm{E}-04 \pm 9.8 \mathrm{E}-05$ & & & ${ }^{137} \mathrm{Cs}$ & $4.5 \mathrm{E}-05 \pm 8.4 \mathrm{E}-05$ & U \\
\hline & ${ }^{152} \mathrm{Eu}$ & $-7.4 \mathrm{E}-05 \pm 1.5 \mathrm{E}-04$ & $\mathrm{U}$ & & ${ }^{152} \mathrm{Eu}$ & $-1.5 \mathrm{E}-04 \pm 1.8 \mathrm{E}-04$ & $\mathrm{U}$ \\
\hline & ${ }^{154} \mathrm{Eu}$ & $1.0 \mathrm{E}-04 \pm 2.2 \mathrm{E}-04$ & $\mathrm{U}$ & & ${ }^{154} \mathrm{Eu}$ & $7.5 \mathrm{E}-05 \pm 2.3 \mathrm{E}-04$ & $\mathrm{U}$ \\
\hline & ${ }^{155} \mathrm{Eu}$ & $-1.7 \mathrm{E}-05 \pm 1.6 \mathrm{E}-04$ & U & & ${ }^{155} \mathrm{Eu}$ & $7.3 \mathrm{E}-05 \pm 2.0 \mathrm{E}-04$ & $\mathrm{U}$ \\
\hline & ${ }^{238} \mathrm{Pu}$ & $5.6 \mathrm{E}-06 \pm 1.0 \mathrm{E}-05$ & $\mathrm{U}$ & & ${ }^{238} \mathrm{Pu}$ & $9.0 \mathrm{E}-07 \pm 9.0 \mathrm{E}-06$ & U \\
\hline & ${ }^{239 / 240} \mathrm{Pu}$ & $5.6 \mathrm{E}-06 \pm 6.3 \mathrm{E}-06$ & $\mathrm{U}$ & & ${ }^{239 / 240} \mathrm{Pu}$ & $1.7 \mathrm{E}-06 \pm 2.5 \mathrm{E}-06$ & $\mathrm{U}$ \\
\hline & ${ }^{103} \mathrm{Ru}$ & $1.7 \mathrm{E}-05 \pm 6.3 \mathrm{E}-05$ & $\mathrm{U}$ & & ${ }^{103} \mathrm{Ru}$ & $-6.6 \mathrm{E}-05 \pm 8.6 \mathrm{E}-05$ & $\mathrm{U}$ \\
\hline & ${ }^{106} \mathrm{Ru}$ & $-1.5 \mathrm{E}-04 \pm 5.2 \mathrm{E}-04$ & $\mathrm{U}$ & & ${ }^{106} \mathrm{Ru}$ & $-5.7 \mathrm{E}-04 \pm 7.7 \mathrm{E}-04$ & $\mathrm{U}$ \\
\hline & ${ }^{125} \mathrm{Sb}$ & $-4.6 \mathrm{E}-06 \pm 4.6 \mathrm{E}-05$ & $\mathrm{U}$ & & ${ }^{125} \mathrm{Sb}$ & $-4.8 \mathrm{E}-05 \pm 2.0 \mathrm{E}-04$ & $\mathrm{U}$ \\
\hline & ${ }^{113} \mathrm{Sn}$ & $1.6 \mathrm{E}-06 \pm 1.6 \mathrm{E}-05$ & $\mathrm{U}$ & & ${ }^{113} \mathrm{Sn}$ & $-2.8 \mathrm{E}-05 \pm 8.4 \mathrm{E}-05$ & $\mathrm{U}$ \\
\hline & ${ }^{90} \mathrm{Sr}$ & $1.5 \mathrm{E}-04 \pm 9.7 \mathrm{E}-05$ & & & ${ }^{90} \mathrm{Sr}$ & $-2.2 \mathrm{E}-05 \pm 1.1 \mathrm{E}-04$ & $\mathrm{U}$ \\
\hline & ${ }^{234} \mathrm{U}$ & $2.4 \mathrm{E}-05 \pm 1.2 \mathrm{E}-05$ & & & ${ }^{234} \mathrm{U}$ & $6.4 \mathrm{E}-06 \pm 6.1 \mathrm{E}-06$ & $\mathrm{U}$ \\
\hline & ${ }^{235} \mathrm{U}$ & $3.3 \mathrm{E}-06 \pm 4.7 \mathrm{E}-06$ & $\mathrm{U}$ & & ${ }^{235} \mathrm{U}$ & $7.0 \mathrm{E}-06 \pm 5.8 \mathrm{E}-06$ & \\
\hline & ${ }^{238} \mathrm{U}$ & $1.5 \mathrm{E}-05 \pm 8.9 \mathrm{E}-06$ & & & ${ }^{238} \mathrm{U}$ & $9.7 \mathrm{E}-06 \pm 6.3 \mathrm{E}-06$ & \\
\hline & ${ }^{65} \mathrm{Zn}$ & $-1.2 \mathrm{E}-04 \pm 1.6 \mathrm{E}-04$ & $\mathrm{U}$ & & ${ }^{65} \mathrm{Zn}$ & $1.2 \mathrm{E}-04 \pm 2.0 \mathrm{E}-04$ & $\mathrm{U}$ \\
\hline N999 (200-East) & ${ }^{144} \mathrm{Ce}$ & $-5.5 \mathrm{E}-04 \pm 8.0 \mathrm{E}-04$ & $\mathrm{U}$ & N155 (200-West) & ${ }^{144} \mathrm{Ce}$ & $-2.5 \mathrm{E}-04 \pm 7.2 \mathrm{E}-04$ & $\mathrm{U}$ \\
\hline Composite Period & ${ }^{60} \mathrm{Co}$ & $8.8 \mathrm{E}-05 \pm 7.9 \mathrm{E}-05$ & $\mathrm{U}$ & Composite Period & ${ }^{60} \mathrm{Co}$ & $-4.7 \mathrm{E}-06 \pm 4.7 \mathrm{E}-05$ & $\mathrm{U}$ \\
\hline \multirow[t]{16}{*}{$06 / 23 / 03$ to $12 / 22 / 03$} & ${ }^{134} \mathrm{Cs}$ & $-2.4 \mathrm{E}-05 \pm 7.1 \mathrm{E}-05$ & $\mathrm{U}$ & $01 / 06 / 03$ to $06 / 23 / 03$ & ${ }^{134} \mathrm{Cs}$ & $2.7 \mathrm{E}-05 \pm 7.9 \mathrm{E}-05$ & $\mathrm{U}$ \\
\hline & ${ }^{137} \mathrm{Cs}$ & $8.4 \mathrm{E}-05 \pm 7.5 \mathrm{E}-05$ & $\mathrm{U}$ & & ${ }^{137} \mathrm{Cs}$ & $5.1 \mathrm{E}-04 \pm 2.1 \mathrm{E}-04$ & \\
\hline & ${ }^{152} \mathrm{Eu}$ & $1.5 \mathrm{E}-05 \pm 1.5 \mathrm{E}-04$ & $\mathrm{U}$ & & ${ }^{152} \mathrm{Eu}$ & $-1.4 \mathrm{E}-04 \pm 2.0 \mathrm{E}-04$ & $\mathrm{U}$ \\
\hline & ${ }^{154} \mathrm{Eu}$ & $-7.9 \mathrm{E}-05 \pm 2.1 \mathrm{E}-04$ & $\mathrm{U}$ & & ${ }^{154} \mathrm{Eu}$ & $1.0 \mathrm{E}-04 \pm 1.9 \mathrm{E}-04$ & $\mathrm{U}$ \\
\hline & ${ }^{155} \mathrm{Eu}$ & $2.4 \mathrm{E}-05 \pm 1.8 \mathrm{E}-04$ & $\mathrm{U}$ & & ${ }^{155} \mathrm{Eu}$ & $-1.3 \mathrm{E}-04 \pm 2.1 \mathrm{E}-04$ & $\mathrm{U}$ \\
\hline & ${ }^{238} \mathrm{Pu}$ & $-5.9 \mathrm{E}-06 \pm 1.2 \mathrm{E}-05$ & $\mathrm{U}$ & & ${ }^{238} \mathrm{Pu}$ & $-9.0 \mathrm{E}-07 \pm 9.0 \mathrm{E}-06$ & $\mathrm{U}$ \\
\hline & ${ }^{239 / 240} \mathrm{Pu}$ & $8.5 \mathrm{E}-07 \pm 8.8 \mathrm{E}-07$ & $\mathrm{U}$ & & ${ }^{239 / 240} \mathrm{Pu}$ & $9.7 \mathrm{E}-06 \pm 7.3 \mathrm{E}-06$ & \\
\hline & ${ }^{103} \mathrm{Ru}$ & $3.7 \mathrm{E}-06 \pm 3.7 \mathrm{E}-05$ & $\mathrm{U}$ & & ${ }^{103} \mathrm{Ru}$ & $-5.8 \mathrm{E}-05 \pm 7.2 \mathrm{E}-05$ & $\mathrm{U}$ \\
\hline & ${ }^{106} \mathrm{Ru}$ & $1.2 \mathrm{E}-05 \pm 1.2 \mathrm{E}-04$ & $\mathrm{U}$ & & ${ }^{106} \mathrm{Ru}$ & $-6.2 \mathrm{E}-05 \pm 6.2 \mathrm{E}-04$ & $\mathrm{U}$ \\
\hline & ${ }^{125} \mathrm{Sb}$ & $1.7 \mathrm{E}-04 \pm 1.5 \mathrm{E}-04$ & $\mathrm{U}$ & & ${ }^{125} \mathrm{Sb}$ & $1.1 \mathrm{E}-04 \pm 1.9 \mathrm{E}-04$ & $\mathrm{U}$ \\
\hline & ${ }^{113} \mathrm{Sn}$ & $3.7 \mathrm{E}-05 \pm 7.6 \mathrm{E}-05$ & $\mathrm{U}$ & & ${ }^{113} \mathrm{Sn}$ & $3.1 \mathrm{E}-05 \pm 8.7 \mathrm{E}-05$ & $\mathrm{U}$ \\
\hline & ${ }^{90} \mathrm{Sr}$ & $8.5 \mathrm{E}-05 \pm 9.7 \mathrm{E}-05$ & $\mathrm{U}$ & & ${ }^{90} \mathrm{Sr}$ & $-3.0 \mathrm{E}-05 \pm 9.8 \mathrm{E}-05$ & $\mathrm{U}$ \\
\hline & ${ }^{234} \mathrm{U}$ & $1.6 \mathrm{E}-05 \pm 9.3 \mathrm{E}-06$ & & & ${ }^{234} \mathrm{U}$ & $2.7 \mathrm{E}-06 \pm 4.4 \mathrm{E}-06$ & $\mathrm{U}$ \\
\hline & ${ }^{235} \mathrm{U}$ & $2.6 \mathrm{E}-06 \pm 3.1 \mathrm{E}-06$ & & & ${ }^{235} \mathrm{U}$ & $2.2 \mathrm{E}-06 \pm 3.4 \mathrm{E}-06$ & $\mathrm{U}$ \\
\hline & ${ }^{238} \mathrm{U}$ & $1.5 \mathrm{E}-05 \pm 9.0 \mathrm{E}-06$ & & & ${ }^{238} \mathrm{U}$ & $6.1 \mathrm{E}-06 \pm 4.6 \mathrm{E}-06$ & \\
\hline & ${ }^{65} \mathrm{Zn}$ & $2.0 \mathrm{E}-04 \pm 2.5 \mathrm{E}-04$ & $\mathrm{U}$ & & ${ }^{65} \mathrm{Zn}$ & $-1.2 \mathrm{E}-04 \pm 1.7 \mathrm{E}-04$ & $\mathrm{U}$ \\
\hline
\end{tabular}

$\overline{\mathrm{RQ}}=$ Result Qualifier. $\mathrm{U}=$ The analyte was analyzed for but not detected. 
Table 2-3. Near-Facility Air Sampling Results, 2003 (pCi/m³ \pm total analytical uncertainty). (cont)

\begin{tabular}{ccrc}
\hline Location & Isotope & Result \pm Uncertainty & RQ* \\
\hline N155 (200-West) & ${ }^{144} \mathrm{Ce}$ & $9.0 \mathrm{E}-05 \pm 5.7 \mathrm{E}-04$ & $\mathrm{U}$ \\
Composite Period & ${ }^{60} \mathrm{Co}$ & $-2.8 \mathrm{E}-05 \pm 7.7 \mathrm{E}-05$ & $\mathrm{U}$ \\
06/23/03 to 12/22/03 & ${ }^{134} \mathrm{Cs}$ & $3.0 \mathrm{E}-05 \pm 7.3 \mathrm{E}-05$ & $\mathrm{U}$ \\
& ${ }^{137} \mathrm{Cs}$ & $4.5 \mathrm{E}-04 \pm 1.9 \mathrm{E}-04$ & \\
& ${ }^{152} \mathrm{Eu}$ & $8.7 \mathrm{E}-05 \pm 1.5 \mathrm{E}-04$ & $\mathrm{U}$ \\
& ${ }^{154} \mathrm{Eu}$ & $-2.6 \mathrm{E}-05 \pm 2.5 \mathrm{E}-04$ & $\mathrm{U}$ \\
& ${ }^{155} \mathrm{Eu}$ & $-1.2 \mathrm{E}-05 \pm 1.2 \mathrm{E}-04$ & $\mathrm{U}$ \\
& ${ }^{238} \mathrm{Pu}$ & $-1.0 \mathrm{E}-05 \pm 2.3 \mathrm{E}-05$ & $\mathrm{U}$ \\
& ${ }^{239 / 240} \mathrm{Pu}$ & $2.0 \mathrm{E}-05 \pm 1.3 \mathrm{E}-05$ & \\
& ${ }^{103} \mathrm{Ru}$ & $-1.3 \mathrm{E}-06 \pm 1.3 \mathrm{E}-05$ & $\mathrm{U}$ \\
& ${ }^{106} \mathrm{Ru}$ & $-2.0 \mathrm{E}-04 \pm 5.3 \mathrm{E}-04$ & $\mathrm{U}$ \\
& ${ }^{125} \mathrm{Sb}$ & $6.6 \mathrm{E}-06 \pm 6.6 \mathrm{E}-05$ & $\mathrm{U}$ \\
& ${ }^{113} \mathrm{Sn}$ & $-9.9 \mathrm{E}-06 \pm 6.6 \mathrm{E}-05$ & $\mathrm{U}$ \\
& ${ }^{90} \mathrm{Sr}$ & $-1.5 \mathrm{E}-05 \pm 8.7 \mathrm{E}-05$ & $\mathrm{U}$ \\
& ${ }^{234} \mathrm{U}$ & $1.3 \mathrm{E}-05 \pm 8.1 \mathrm{E}-06$ & \\
& ${ }^{235} \mathrm{U}$ & $2.2 \mathrm{E}-06 \pm 3.4 \mathrm{E}-06$ & $\mathrm{U}$ \\
& ${ }^{238} \mathrm{U}$ & $1.0 \mathrm{E}-05 \pm 6.4 \mathrm{E}-06$ & \\
& ${ }^{65} \mathrm{Zn} \mathrm{U}$ & $1.1 \mathrm{E}-05 \pm 7.2 \mathrm{E}-06$ & \\
& ${ }^{65} \mathrm{Zn}$ & $1.6 \mathrm{E}-05 \pm 1.6 \mathrm{E}-04$ & $\mathrm{U}$ \\
\hline${ }^{235} \mathrm{U}$ & $2.2 \mathrm{E}-04 \pm 2.2 \mathrm{E}-04$ & $\mathrm{U}$
\end{tabular}

\begin{tabular}{ccrc}
\hline Location & Isotope & Result \pm Uncertainty & RQ* \\
\hline N161 (200-West) & ${ }^{144} \mathrm{Ce}$ & $3.4 \mathrm{E}-04 \pm 5.9 \mathrm{E}-04$ & $\mathrm{U}$ \\
Composite Period & ${ }^{60} \mathrm{Co}$ & $5.2 \mathrm{E}-05 \pm 8.7 \mathrm{E}-05$ & $\mathrm{U}$ \\
01/06/03 to 06/23/03 & ${ }^{134} \mathrm{Cs}$ & $-2.9 \mathrm{E}-06 \pm 2.9 \mathrm{E}-05$ & $\mathrm{U}$ \\
& ${ }^{137} \mathrm{Cs}$ & $4.9 \mathrm{E}-05 \pm 7.7 \mathrm{E}-05$ & $\mathrm{U}$ \\
& ${ }^{152} \mathrm{Eu}$ & $-3.4 \mathrm{E}-05 \pm 1.6 \mathrm{E}-04$ & $\mathrm{U}$ \\
& ${ }^{154} \mathrm{Eu}$ & $1.1 \mathrm{E}-04 \pm 1.9 \mathrm{E}-04$ & $\mathrm{U}$ \\
& ${ }^{155} \mathrm{Eu}$ & $4.8 \mathrm{E}-05 \pm 1.5 \mathrm{E}-04$ & $\mathrm{U}$ \\
& ${ }^{238} \mathrm{Pu}$ & $-2.6 \mathrm{E}-06 \pm 6.8 \mathrm{E}-06$ & $\mathrm{U}$ \\
& ${ }^{239 / 240} \mathrm{Pu}$ & $6.2 \mathrm{E}-06 \pm 5.2 \mathrm{E}-06$ & \\
& ${ }^{103} \mathrm{Ru}$ & $-1.2 \mathrm{E}-05 \pm 6.3 \mathrm{E}-05$ & $\mathrm{U}$ \\
& ${ }^{106} \mathrm{Ru}$ & $-2.7 \mathrm{E}-04 \pm 6.2 \mathrm{E}-04$ & $\mathrm{U}$ \\
& ${ }^{125} \mathrm{Sb}$ & $-7.0 \mathrm{E}-05 \pm 1.6 \mathrm{E}-04$ & $\mathrm{U}$ \\
& ${ }^{113} \mathrm{Sn}$ & $3.0 \mathrm{E}-05 \pm 7.0 \mathrm{E}-05$ & $\mathrm{U}$ \\
& ${ }^{90} \mathrm{Sr}$ & $-1.5 \mathrm{E}-05 \pm 1.4 \mathrm{E}-04$ & $\mathrm{U}$ \\
& ${ }^{234} \mathrm{U}$ & $1.7 \mathrm{E}-05 \pm 9.5 \mathrm{E}-06$ & \\
& ${ }^{235} \mathrm{U}$ & $3.9 \mathrm{E}-06 \pm 4.5 \mathrm{E}-06$ & $\mathrm{U}$ \\
& ${ }^{238} \mathrm{U}$ & $8.5 \mathrm{E}-06 \pm 6.1 \mathrm{E}-06$ & \\
& ${ }^{65} \mathrm{Zn}$ & $-1.2 \mathrm{E}-04 \pm 1.5 \mathrm{E}-04$ & $\mathrm{U}$
\end{tabular}

$\overline{\mathrm{RQ}}=$ Result Qualifier. $\mathrm{U}=$ The analyte was analyzed for but not detected. 
Table 2-3. Near-Facility Air Sampling Results, 2003 (pCi/m³ \pm total analytical uncertainty). (cont)

\begin{tabular}{|c|c|c|c|c|c|c|c|}
\hline Location & Isotope & Result \pm Uncertainty & RQ* & Location & Isotope & Result \pm Uncertainty & RQ* \\
\hline N165 (200-West) & ${ }^{144} \mathrm{Ce}$ & $3.2 \mathrm{E}-04 \pm 5.3 \mathrm{E}-04$ & $\mathrm{U}$ & N168 (200-West) & ${ }^{144} \mathrm{Ce}$ & $-3.4 \mathrm{E}-04 \pm 7.2 \mathrm{E}-04$ & $\mathrm{U}$ \\
\hline Composite Period & ${ }^{60} \mathrm{Co}$ & $3.0 \mathrm{E}-05 \pm 7.4 \mathrm{E}-05$ & $\mathrm{U}$ & Composite Period & ${ }^{60} \mathrm{Co}$ & $-1.5 \mathrm{E}-05 \pm 9.3 \mathrm{E}-05$ & $\mathrm{U}$ \\
\hline \multirow[t]{16}{*}{$06 / 23 / 03$ to $12 / 22 / 03$} & ${ }^{134} \mathrm{Cs}$ & $7.4 \mathrm{E}-05 \pm 6.1 \mathrm{E}-05$ & $\mathrm{U}$ & $01 / 06 / 03$ to $06 / 23 / 03$ & ${ }^{134} \mathrm{Cs}$ & $3.8 \mathrm{E}-06 \pm 3.8 \mathrm{E}-05$ & $\mathrm{U}$ \\
\hline & ${ }^{137} \mathrm{Cs}$ & $7.6 \mathrm{E}-05 \pm 7.3 \mathrm{E}-05$ & $\mathrm{U}$ & & ${ }^{137} \mathrm{Cs}$ & $6.4 \mathrm{E}-05 \pm 9.4 \mathrm{E}-05$ & $\mathrm{U}$ \\
\hline & ${ }^{152} \mathrm{Eu}$ & $-4.6 \mathrm{E}-05 \pm 1.4 \mathrm{E}-04$ & $\mathrm{U}$ & & ${ }^{152} \mathrm{Eu}$ & $5.1 \mathrm{E}-05 \pm 1.9 \mathrm{E}-04$ & $\mathrm{U}$ \\
\hline & ${ }^{154} \mathrm{Eu}$ & $-1.4 \mathrm{E}-04 \pm 2.2 \mathrm{E}-04$ & $\mathrm{U}$ & & ${ }^{154} \mathrm{Eu}$ & $-2.1 \mathrm{E}-05 \pm 2.1 \mathrm{E}-04$ & $\mathrm{U}$ \\
\hline & ${ }^{155} \mathrm{Eu}$ & $-4.7 \mathrm{E}-05 \pm 1.4 \mathrm{E}-04$ & $\mathrm{U}$ & & ${ }^{155} \mathrm{Eu}$ & $2.1 \mathrm{E}-04 \pm 2.0 \mathrm{E}-04$ & $\mathrm{U}$ \\
\hline & ${ }^{238} \mathrm{Pu}$ & $-1.1 \mathrm{E}-06 \pm 1.1 \mathrm{E}-05$ & $\mathrm{U}$ & & ${ }^{238} \mathrm{Pu}$ & $-3.4 \mathrm{E}-06 \pm 1.4 \mathrm{E}-05$ & U \\
\hline & ${ }^{239 / 240} \mathrm{Pu}$ & $5.0 \mathrm{E}-04 \pm 1.9 \mathrm{E}-04$ & & & ${ }^{239 / 240} \mathrm{Pu}$ & $5.1 \mathrm{E}-06 \pm 4.6 \mathrm{E}-06$ & \\
\hline & ${ }^{103} \mathrm{Ru}$ & $3.1 \mathrm{E}-05 \pm 5.6 \mathrm{E}-05$ & $\mathrm{U}$ & & ${ }^{103} \mathrm{Ru}$ & $3.2 \mathrm{E}-05 \pm 7.6 \mathrm{E}-05$ & $\mathrm{U}$ \\
\hline & ${ }^{106} \mathrm{Ru}$ & $-5.9 \mathrm{E}-04 \pm 6.2 \mathrm{E}-04$ & $\mathrm{U}$ & & ${ }^{106} \mathrm{Ru}$ & $4.5 \mathrm{E}-04 \pm 7.4 \mathrm{E}-04$ & $\mathrm{U}$ \\
\hline & ${ }^{125} \mathrm{Sb}$ & $-1.0 \mathrm{E}-05 \pm 1.1 \mathrm{E}-04$ & $\mathrm{U}$ & & ${ }^{125} \mathrm{Sb}$ & $-1.2 \mathrm{E}-05 \pm 1.2 \mathrm{E}-04$ & $\mathrm{U}$ \\
\hline & ${ }^{113} \mathrm{Sn}$ & $-9.2 \mathrm{E}-07 \pm 9.2 \mathrm{E}-06$ & $\mathrm{U}$ & & ${ }^{113} \mathrm{Sn}$ & $1.4 \mathrm{E}-05 \pm 8.3 \mathrm{E}-05$ & $\mathrm{U}$ \\
\hline & ${ }^{90} \mathrm{Sr}$ & $-5.7 \mathrm{E}-05 \pm 8.9 \mathrm{E}-05$ & $\mathrm{U}$ & & ${ }^{90} \mathrm{Sr}$ & $-6.9 \mathrm{E}-05 \pm 1.1 \mathrm{E}-04$ & $\mathrm{U}$ \\
\hline & ${ }^{234} \mathrm{U}$ & $1.2 \mathrm{E}-05 \pm 7.3 \mathrm{E}-06$ & & & ${ }^{234} \mathrm{U}$ & $2.0 \mathrm{E}-05 \pm 1.1 \mathrm{E}-05$ & \\
\hline & ${ }^{235} \mathrm{U}$ & $2.1 \mathrm{E}-06 \pm 3.9 \mathrm{E}-06$ & $\mathrm{U}$ & & ${ }^{235} \mathrm{U}$ & $5.6 \mathrm{E}-06 \pm 4.7 \mathrm{E}-06$ & \\
\hline & ${ }^{238} \mathrm{U}$ & $1.6 \mathrm{E}-05 \pm 8.9 \mathrm{E}-06$ & & & ${ }^{238} \mathrm{U}$ & $1.2 \mathrm{E}-05 \pm 7.7 \mathrm{E}-06$ & \\
\hline & ${ }^{65} \mathrm{Zn}$ & $4.5 \mathrm{E}-06 \pm 4.5 \mathrm{E}-05$ & $\mathrm{U}$ & & ${ }^{65} \mathrm{Zn}$ & $-1.0 \mathrm{E}-04 \pm 2.2 \mathrm{E}-04$ & $\mathrm{U}$ \\
\hline N168 (200-West) & ${ }^{144} \mathrm{Ce}$ & $-7.2 \mathrm{E}-04 \pm 8.0 \mathrm{E}-04$ & $\mathrm{U}$ & N200 (200-West) & ${ }^{144} \mathrm{Ce}$ & $-2.1 \mathrm{E}-04 \pm 8.5 \mathrm{E}-04$ & $\mathrm{U}$ \\
\hline Composite Period & ${ }^{60} \mathrm{Co}$ & $4.4 \mathrm{E}-06 \pm 4.4 \mathrm{E}-05$ & $\mathrm{U}$ & Composite Period & ${ }^{60} \mathrm{Co}$ & $-2.8 \mathrm{E}-05 \pm 1.2 \mathrm{E}-04$ & $\mathrm{U}$ \\
\hline \multirow[t]{16}{*}{$06 / 23 / 03$ to $12 / 22 / 03$} & ${ }^{134} \mathrm{Cs}$ & $-1.5 \mathrm{E}-05 \pm 7.3 \mathrm{E}-05$ & $\mathrm{U}$ & $02 / 25 / 03$ to $06 / 24 / 03$ & ${ }^{134} \mathrm{Cs}$ & $-1.2 \mathrm{E}-04 \pm 1.2 \mathrm{E}-04$ & $\mathrm{U}$ \\
\hline & ${ }^{137} \mathrm{Cs}$ & $1.9 \mathrm{E}-05 \pm 7.2 \mathrm{E}-05$ & $\mathrm{U}$ & & ${ }^{137} \mathrm{Cs}$ & $8.2 \mathrm{E}-05 \pm 1.1 \mathrm{E}-04$ & $\mathrm{U}$ \\
\hline & ${ }^{152} \mathrm{Eu}$ & $-7.9 \mathrm{E}-05 \pm 2.0 \mathrm{E}-04$ & $\mathrm{U}$ & & ${ }^{152} \mathrm{Eu}$ & $1.8 \mathrm{E}-04 \pm 2.7 \mathrm{E}-04$ & $\mathrm{U}$ \\
\hline & ${ }^{154} \mathrm{Eu}$ & $9.9 \mathrm{E}-06 \pm 9.9 \mathrm{E}-05$ & $\mathrm{U}$ & & ${ }^{154} \mathrm{Eu}$ & $2.3 \mathrm{E}-04 \pm 3.0 \mathrm{E}-04$ & $\mathrm{U}$ \\
\hline & ${ }^{155} \mathrm{Eu}$ & $-1.1 \mathrm{E}-05 \pm 1.1 \mathrm{E}-04$ & $\mathrm{U}$ & & ${ }^{155} \mathrm{Eu}$ & $-1.8 \mathrm{E}-04 \pm 2.5 \mathrm{E}-04$ & $\mathrm{U}$ \\
\hline & ${ }^{238} \mathrm{Pu}$ & $-8.6 \mathrm{E}-06 \pm 1.2 \mathrm{E}-05$ & $\mathrm{U}$ & & ${ }^{238} \mathrm{Pu}$ & $1.3 \mathrm{E}-05 \pm 2.6 \mathrm{E}-05$ & $\mathrm{U}$ \\
\hline & ${ }^{239 / 240} \mathrm{Pu}$ & $4.1 \mathrm{E}-06 \pm 6.2 \mathrm{E}-06$ & U & & ${ }^{239 / 240} \mathrm{Pu}$ & $1.5 \mathrm{E}-06 \pm 3.1 \mathrm{E}-06$ & $\mathrm{U}$ \\
\hline & ${ }^{103} \mathrm{Ru}$ & $2.3 \mathrm{E}-05 \pm 6.5 \mathrm{E}-05$ & $\mathrm{U}$ & & ${ }^{103} \mathrm{Ru}$ & $4.8 \mathrm{E}-05 \pm 9.4 \mathrm{E}-05$ & $\mathrm{U}$ \\
\hline & ${ }^{106} \mathrm{Ru}$ & $-2.4 \mathrm{E}-05 \pm 2.4 \mathrm{E}-04$ & $\mathrm{U}$ & & ${ }^{106} \mathrm{Ru}$ & $2.7 \mathrm{E}-04 \pm 9.5 \mathrm{E}-04$ & $\mathrm{U}$ \\
\hline & ${ }^{125} \mathrm{Sb}$ & $9.3 \mathrm{E}-05 \pm 1.7 \mathrm{E}-04$ & $\mathrm{U}$ & & ${ }^{125} \mathrm{Sb}$ & $-1.1 \mathrm{E}-05 \pm 1.1 \mathrm{E}-04$ & $\mathrm{U}$ \\
\hline & ${ }^{113} \mathrm{Sn}$ & $1.7 \mathrm{E}-05 \pm 7.8 \mathrm{E}-05$ & $\mathrm{U}$ & & ${ }^{113} \mathrm{Sn}$ & $-3.6 \mathrm{E}-05 \pm 1.2 \mathrm{E}-04$ & $\mathrm{U}$ \\
\hline & ${ }^{90} \mathrm{Sr}$ & $8.6 \mathrm{E}-06 \pm 8.6 \mathrm{E}-05$ & $\mathrm{U}$ & & ${ }^{90} \mathrm{Sr}$ & $-1.9 \mathrm{E}-04 \pm 1.9 \mathrm{E}-04$ & $\mathrm{U}$ \\
\hline & ${ }^{234} \mathrm{U}$ & $1.9 \mathrm{E}-05 \pm 1.1 \mathrm{E}-05$ & & & ${ }^{234} \mathrm{U}$ & $9.6 \mathrm{E}-06 \pm 7.2 \mathrm{E}-06$ & \\
\hline & ${ }^{235} \mathrm{U}$ & $3.3 \mathrm{E}-06 \pm 3.4 \mathrm{E}-06$ & & & ${ }^{235} \mathrm{U}$ & $5.9 \mathrm{E}-06 \pm 5.6 \mathrm{E}-06$ & \\
\hline & ${ }^{238} \mathrm{U}$ & $1.9 \mathrm{E}-05 \pm 1.0 \mathrm{E}-05$ & & & ${ }^{238} \mathrm{U}$ & $5.4 \mathrm{E}-06 \pm 6.6 \mathrm{E}-06$ & $\mathrm{U}$ \\
\hline & ${ }^{65} \mathrm{Zn}$ & $6.3 \mathrm{E}-05 \pm 1.9 \mathrm{E}-04$ & $\mathrm{U}$ & & ${ }^{65} \mathrm{Zn}$ & $-2.8 \mathrm{E}-04 \pm 3.0 \mathrm{E}-04$ & $\mathrm{U}$ \\
\hline
\end{tabular}

$\overline{\mathrm{RQ}}=$ Result Qualifier. $\mathrm{U}=$ The analyte was analyzed for but not detected. 
Table 2-3. Near-Facility Air Sampling Results, 2003 (pCi/m³ \pm total analytical uncertainty). (cont)

\begin{tabular}{ccrc}
\hline Location & Isotope & Result \pm Uncertainty & RQ* \\
\hline N200 (200-West) & ${ }^{144} \mathrm{Ce}$ & $1.9 \mathrm{E}-04 \pm 6.9 \mathrm{E}-04$ & $\mathrm{U}$ \\
Composite Period & ${ }^{60} \mathrm{Co}$ & $7.3 \mathrm{E}-05 \pm 9.1 \mathrm{E}-05$ & $\mathrm{U}$ \\
06/24/03 to 12/22/03 & ${ }^{134} \mathrm{Cs}$ & $-1.6 \mathrm{E}-05 \pm 9.3 \mathrm{E}-05$ & $\mathrm{U}$ \\
& ${ }^{137} \mathrm{Cs}$ & $2.0 \mathrm{E}-05 \pm 8.9 \mathrm{E}-05$ & $\mathrm{U}$ \\
& ${ }^{152} \mathrm{Eu}$ & $-3.4 \mathrm{E}-05 \pm 1.8 \mathrm{E}-04$ & $\mathrm{U}$ \\
& ${ }^{154} \mathrm{Eu}$ & $5.4 \mathrm{E}-05 \pm 2.6 \mathrm{E}-04$ & $\mathrm{U}$ \\
& ${ }^{155} \mathrm{Eu}$ & $-1.2 \mathrm{E}-04 \pm 2.0 \mathrm{E}-04$ & $\mathrm{U}$ \\
& ${ }^{238} \mathrm{Pu}$ & $1.6 \mathrm{E}-06 \pm 1.1 \mathrm{E}-05$ & $\mathrm{U}$ \\
& ${ }^{239 / 240} \mathrm{Pu}$ & $4.0 \mathrm{E}-06 \pm 3.9 \mathrm{E}-06$ & \\
& ${ }^{103} \mathrm{Ru}$ & $4.3 \mathrm{E}-05 \pm 7.3 \mathrm{E}-05$ & $\mathrm{U}$ \\
& ${ }^{106} \mathrm{Ru}$ & $-2.6 \mathrm{E}-04 \pm 6.9 \mathrm{E}-04$ & $\mathrm{U}$ \\
& ${ }^{125} \mathrm{Sb}$ & $-1.1 \mathrm{E}-04 \pm 1.8 \mathrm{E}-04$ & $\mathrm{U}$ \\
& ${ }^{113} \mathrm{Sn}$ & $2.2 \mathrm{E}-05 \pm 7.9 \mathrm{E}-05$ & $\mathrm{U}$ \\
& ${ }^{90} \mathrm{Sr}$ & $-7.4 \mathrm{E}-05 \pm 1.2 \mathrm{E}-04$ & $\mathrm{U}$ \\
& ${ }^{234} \mathrm{U}$ & $1.6 \mathrm{E}-05 \pm 9.4 \mathrm{E}-06$ & \\
& ${ }^{235} \mathrm{U}$ & $8.2 \mathrm{E}-07 \pm 1.6 \mathrm{E}-06$ & $\mathrm{U}$ \\
& ${ }^{238} \mathrm{U}$ & $1.8 \mathrm{E}-05 \pm 9.8 \mathrm{E}-06$ & \\
& ${ }^{65} \mathrm{Zn} \mathrm{U}$ & $1.7 \mathrm{E}-05 \pm 9.4 \mathrm{E}-06$ & \\
& ${ }^{65} \mathrm{Zn}$ & $2.4 \mathrm{E}-05 \pm 1.9 \mathrm{E}-04$ & $\mathrm{U}$ \\
\hline${ }^{235} \mathrm{U}$ & ${ }^{144} \mathrm{Ce}$ & $5.3 \mathrm{E}-05 \pm 5.3 \mathrm{E}-04$ & $\mathrm{U}$ \\
& ${ }^{155} \mathrm{Eu}$ & $4.4 \mathrm{E}-05 \pm 1.8 \mathrm{E}-04$ & $\mathrm{U}$
\end{tabular}

\begin{tabular}{|c|c|c|c|}
\hline Location & Isotope & Result \pm Uncertainty & RQ* \\
\hline N304 (200-West) & ${ }^{144} \mathrm{Ce}$ & $-1.4 \mathrm{E}-04 \pm 7.6 \mathrm{E}-04$ & $\bar{U}$ \\
\hline Composite Period & ${ }^{60} \mathrm{Co}$ & $-1.5 \mathrm{E}-05 \pm 7.0 \mathrm{E}-05$ & $\mathrm{U}$ \\
\hline \multirow[t]{16}{*}{$01 / 06 / 03$ to $06 / 23 / 03$} & ${ }^{134} \mathrm{Cs}$ & $1.9 \mathrm{E}-05 \pm 7.8 \mathrm{E}-05$ & $\mathrm{U}$ \\
\hline & ${ }^{137} \mathrm{Cs}$ & $-6.4 \mathrm{E}-06 \pm 6.4 \mathrm{E}-05$ & $\mathrm{U}$ \\
\hline & ${ }^{152} \mathrm{Eu}$ & $1.3 \mathrm{E}-04 \pm 2.1 \mathrm{E}-04$ & $\mathrm{U}$ \\
\hline & ${ }^{154} \mathrm{Eu}$ & $-1.6 \mathrm{E}-05 \pm 1.6 \mathrm{E}-04$ & $\mathrm{U}$ \\
\hline & ${ }^{155} \mathrm{Eu}$ & $-6.6 \mathrm{E}-05 \pm 2.1 \mathrm{E}-04$ & U \\
\hline & ${ }^{238} \mathrm{Pu}$ & $-1.1 \mathrm{E}-06 \pm 1.1 \mathrm{E}-05$ & $\mathrm{U}$ \\
\hline & ${ }^{239 / 240} \mathrm{Pu}$ & $7.0 \mathrm{E}-06 \pm 6.3 \mathrm{E}-06$ & \\
\hline & ${ }^{103} \mathrm{Ru}$ & $-1.0 \mathrm{E}-05 \pm 7.3 \mathrm{E}-05$ & $\mathrm{U}$ \\
\hline & ${ }^{106} \mathrm{Ru}$ & $-4.7 \mathrm{E}-04 \pm 6.7 \mathrm{E}-04$ & $\mathrm{U}$ \\
\hline & ${ }^{125} \mathrm{Sb}$ & $1.9 \mathrm{E}-05 \pm 1.9 \mathrm{E}-04$ & $\mathrm{U}$ \\
\hline & ${ }^{113} \mathrm{Sn}$ & $-6.3 \mathrm{E}-06 \pm 6.3 \mathrm{E}-05$ & $\mathrm{U}$ \\
\hline & ${ }^{90} \mathrm{Sr}$ & $-2.3 \mathrm{E}-05 \pm 9.6 \mathrm{E}-05$ & $\mathrm{U}$ \\
\hline & ${ }^{234} \mathrm{U}$ & $2.7 \mathrm{E}-05 \pm 1.8 \mathrm{E}-05$ & \\
\hline & ${ }^{235} \mathrm{U}$ & $1.3 \mathrm{E}-05 \pm 1.5 \mathrm{E}-05$ & $\mathrm{U}$ \\
\hline & ${ }^{238} \mathrm{U}$ & $1.5 \mathrm{E}-05 \pm 1.3 \mathrm{E}-05$ & \\
\hline & ${ }^{65} \mathrm{Zn}$ & $-1.5 \mathrm{E}-06 \pm 1.5 \mathrm{E}-05$ & $\mathrm{U}$ \\
\hline N433 (200-West) & ${ }^{144} \mathrm{Ce}$ & $5.8 \mathrm{E}-04 \pm 7.2 \mathrm{E}-04$ & $\mathrm{U}$ \\
\hline Composite Period & ${ }^{60} \mathrm{Co}$ & $6.6 \mathrm{E}-06 \pm 6.6 \mathrm{E}-05$ & $\mathrm{U}$ \\
\hline \multirow[t]{16}{*}{$01 / 06 / 03$ to $06 / 23 / 03$} & ${ }^{134} \mathrm{Cs}$ & $-1.6 \mathrm{E}-05 \pm 9.2 \mathrm{E}-05$ & $\mathrm{U}$ \\
\hline & ${ }^{137} \mathrm{Cs}$ & $7.1 \mathrm{E}-05 \pm 8.8 \mathrm{E}-05$ & $\mathrm{U}$ \\
\hline & ${ }^{152} \mathrm{Eu}$ & $-5.2 \mathrm{E}-06 \pm 5.2 \mathrm{E}-05$ & $\mathrm{U}$ \\
\hline & ${ }^{154} \mathrm{Eu}$ & $-8.4 \mathrm{E}-06 \pm 8.4 \mathrm{E}-05$ & $\mathrm{U}$ \\
\hline & ${ }^{155} \mathrm{Eu}$ & $1.2 \mathrm{E}-04 \pm 1.9 \mathrm{E}-04$ & $\mathrm{U}$ \\
\hline & ${ }^{238} \mathrm{Pu}$ & $-2.0 \mathrm{E}-05 \pm 2.1 \mathrm{E}-05$ & $\mathrm{U}$ \\
\hline & ${ }^{239 / 240} \mathrm{Pu}$ & $2.3 \mathrm{E}-04 \pm 9.4 \mathrm{E}-05$ & \\
\hline & ${ }^{103} \mathrm{Ru}$ & $4.8 \mathrm{E}-05 \pm 7.6 \mathrm{E}-05$ & $\mathrm{U}$ \\
\hline & ${ }^{106} \mathrm{Ru}$ & $-3.4 \mathrm{E}-04 \pm 9.0 \mathrm{E}-04$ & $\mathrm{U}$ \\
\hline & ${ }^{125} \mathrm{Sb}$ & $3.2 \mathrm{E}-05 \pm 1.7 \mathrm{E}-04$ & $\mathrm{U}$ \\
\hline & ${ }^{113} \mathrm{Sn}$ & $1.8 \mathrm{E}-05 \pm 8.5 \mathrm{E}-05$ & $\mathrm{U}$ \\
\hline & ${ }^{90} \mathrm{Sr}$ & $-1.3 \mathrm{E}-04 \pm 1.4 \mathrm{E}-04$ & $\mathrm{U}$ \\
\hline & ${ }^{234} \mathrm{U}$ & $2.2 \mathrm{E}-05 \pm 1.1 \mathrm{E}-05$ & \\
\hline & ${ }^{235} \mathrm{U}$ & $5.0 \mathrm{E}-06 \pm 4.5 \mathrm{E}-06$ & \\
\hline & ${ }^{238} \mathrm{U}$ & $1.9 \mathrm{E}-05 \pm 1.0 \mathrm{E}-05$ & \\
\hline & ${ }^{65} \mathrm{Zn}$ & $-1.3 \mathrm{E}-04 \pm 2.0 \mathrm{E}-04$ & $\mathrm{U}$ \\
\hline
\end{tabular}

$\overline{\mathrm{RQ}}=$ Result Qualifier. $\mathrm{U}=$ The analyte was analyzed for but not detected. 
Table 2-3. Near-Facility Air Sampling Results, 2003 (pCi/m³ \pm total analytical uncertainty). (cont)

\begin{tabular}{|c|c|c|c|}
\hline Location & Isotope & Result \pm Uncertainty & $\mathbf{R Q}^{*}$ \\
\hline N433 (200-West) & ${ }^{144} \mathrm{Ce}$ & $4.1 \mathrm{E}-04 \pm 8.7 \mathrm{E}-04$ & $\mathrm{U}$ \\
\hline Composite Period & ${ }^{60} \mathrm{Co}$ & $3.1 \mathrm{E}-06 \pm 3.1 \mathrm{E}-05$ & $\mathrm{U}$ \\
\hline \multirow[t]{16}{*}{$06 / 23 / 03$ to $12 / 22 / 03$} & ${ }^{134} \mathrm{Cs}$ & $2.3 \mathrm{E}-05 \pm 8.3 \mathrm{E}-05$ & $\mathrm{U}$ \\
\hline & ${ }^{137} \mathrm{Cs}$ & $4.6 \mathrm{E}-05 \pm 7.5 \mathrm{E}-05$ & $\mathrm{U}$ \\
\hline & ${ }^{152} \mathrm{Eu}$ & $-3.7 \mathrm{E}-05 \pm 2.3 \mathrm{E}-04$ & $\mathrm{U}$ \\
\hline & ${ }^{154} \mathrm{Eu}$ & $-2.1 \mathrm{E}-04 \pm 3.1 \mathrm{E}-04$ & $\mathrm{U}$ \\
\hline & ${ }^{155} \mathrm{Eu}$ & $-6.2 \mathrm{E}-05 \pm 2.3 \mathrm{E}-04$ & $\mathrm{U}$ \\
\hline & ${ }^{238} \mathrm{Pu}$ & $-3.9 \mathrm{E}-06 \pm 1.5 \mathrm{E}-05$ & $\mathrm{U}$ \\
\hline & ${ }^{239 / 240} \mathrm{Pu}$ & $2.5 \mathrm{E}-05 \pm 1.4 \mathrm{E}-05$ & \\
\hline & ${ }^{103} \mathrm{Ru}$ & $-1.1 \mathrm{E}-05 \pm 7.6 \mathrm{E}-05$ & $\mathrm{U}$ \\
\hline & ${ }^{106} \mathrm{Ru}$ & $-1.3 \mathrm{E}-04 \pm 6.5 \mathrm{E}-04$ & $\mathrm{U}$ \\
\hline & ${ }^{125} \mathrm{Sb}$ & $-1.5 \mathrm{E}-04 \pm 1.9 \mathrm{E}-04$ & $\mathrm{U}$ \\
\hline & ${ }^{113} \mathrm{Sn}$ & $-3.4 \mathrm{E}-05 \pm 8.6 \mathrm{E}-05$ & $\mathrm{U}$ \\
\hline & ${ }^{90} \mathrm{Sr}$ & $-4.9 \mathrm{E}-05 \pm 1.0 \mathrm{E}-04$ & $\mathrm{U}$ \\
\hline & ${ }^{234} \mathrm{U}$ & $2.1 \mathrm{E}-05 \pm 1.2 \mathrm{E}-05$ & \\
\hline & ${ }^{235} \mathrm{U}$ & $3.6 \mathrm{E}-06 \pm 4.4 \mathrm{E}-06$ & $\mathrm{U}$ \\
\hline & ${ }^{238} \mathrm{U}$ & $2.7 \mathrm{E}-05 \pm 1.4 \mathrm{E}-05$ & \\
\hline & ${ }^{65} \mathrm{Zn}$ & $3.0 \mathrm{E}-04 \pm 2.3 \mathrm{E}-04$ & $\mathrm{U}$ \\
\hline N441 (200-West) & ${ }^{144} \mathrm{Ce}$ & $9.8 \mathrm{E}-04 \pm 9.5 \mathrm{E}-04$ & $\mathrm{U}$ \\
\hline Composite Period & ${ }^{60} \mathrm{Co}$ & $-4.7 \mathrm{E}-05 \pm 8.5 \mathrm{E}-05$ & $\mathrm{U}$ \\
\hline \multirow[t]{16}{*}{$06 / 23 / 03$ to $12 / 22 / 03$} & ${ }^{134} \mathrm{Cs}$ & $-3.8 \mathrm{E}-05 \pm 9.0 \mathrm{E}-05$ & $\mathrm{U}$ \\
\hline & ${ }^{137} \mathrm{Cs}$ & $1.0 \mathrm{E}-04 \pm 8.2 \mathrm{E}-05$ & $\mathrm{U}$ \\
\hline & ${ }^{152} \mathrm{Eu}$ & $9.0 \mathrm{E}-05 \pm 1.6 \mathrm{E}-04$ & $\mathrm{U}$ \\
\hline & ${ }^{154} \mathrm{Eu}$ & $1.6 \mathrm{E}-04 \pm 2.5 \mathrm{E}-04$ & $\mathrm{U}$ \\
\hline & ${ }^{155} \mathrm{Eu}$ & $2.7 \mathrm{E}-05 \pm 1.6 \mathrm{E}-04$ & $\mathrm{U}$ \\
\hline & ${ }^{238} \mathrm{Pu}$ & $5.3 \mathrm{E}-06 \pm 1.3 \mathrm{E}-05$ & $\mathrm{U}$ \\
\hline & ${ }^{239 / 240} \mathrm{Pu}$ & $1.9 \mathrm{E}-05 \pm 1.0 \mathrm{E}-05$ & \\
\hline & ${ }^{103} \mathrm{Ru}$ & $4.6 \mathrm{E}-05 \pm 6.4 \mathrm{E}-05$ & $\mathrm{U}$ \\
\hline & ${ }^{106} \mathrm{Ru}$ & $-5.3 \mathrm{E}-04 \pm 7.6 \mathrm{E}-04$ & $\mathrm{U}$ \\
\hline & ${ }^{125} \mathrm{Sb}$ & $-7.0 \mathrm{E}-05 \pm 1.6 \mathrm{E}-04$ & $\mathrm{U}$ \\
\hline & ${ }^{113} \mathrm{Sn}$ & $2.3 \mathrm{E}-05 \pm 7.6 \mathrm{E}-05$ & $\mathrm{U}$ \\
\hline & ${ }^{90} \mathrm{Sr}$ & $1.4 \mathrm{E}-04 \pm 1.1 \mathrm{E}-04$ & \\
\hline & ${ }^{234} \mathrm{U}$ & $2.4 \mathrm{E}-05 \pm 1.3 \mathrm{E}-05$ & \\
\hline & ${ }^{235} \mathrm{U}$ & $4.8 \mathrm{E}-06 \pm 4.3 \mathrm{E}-06$ & \\
\hline & ${ }^{238} \mathrm{U}$ & $1.6 \mathrm{E}-05 \pm 9.2 \mathrm{E}-06$ & \\
\hline & ${ }^{65} \mathrm{Zn}$ & 7.3E-05 $\pm 1.7 \mathrm{E}-04$ & $\mathrm{U}$ \\
\hline
\end{tabular}

\begin{tabular}{|c|c|c|c|}
\hline Location & Isotope & Result \pm Uncertainty & RQ* \\
\hline N441 (200-West) & ${ }^{144} \mathrm{Ce}$ & $-1.7 \mathrm{E}-04 \pm 5.5 \mathrm{E}-04$ & $\bar{U}$ \\
\hline Composite Period & ${ }^{60} \mathrm{Co}$ & $-1.4 \mathrm{E}-05 \pm 7.4 \mathrm{E}-05$ & $\mathrm{U}$ \\
\hline \multirow[t]{16}{*}{$01 / 06 / 03$ to $06 / 23 / 03$} & ${ }^{134} \mathrm{Cs}$ & $3.5 \mathrm{E}-05 \pm 7.8 \mathrm{E}-05$ & $\mathrm{U}$ \\
\hline & ${ }^{137} \mathrm{Cs}$ & $1.2 \mathrm{E}-04 \pm 8.3 \mathrm{E}-05$ & $\mathrm{U}$ \\
\hline & ${ }^{152} \mathrm{Eu}$ & $-9.4 \mathrm{E}-05 \pm 1.6 \mathrm{E}-04$ & $\mathrm{U}$ \\
\hline & ${ }^{154} \mathrm{Eu}$ & $2.6 \mathrm{E}-05 \pm 2.1 \mathrm{E}-04$ & $\mathrm{U}$ \\
\hline & ${ }^{155} \mathrm{Eu}$ & $-1.8 \mathrm{E}-05 \pm 1.4 \mathrm{E}-04$ & U \\
\hline & ${ }^{238} \mathrm{Pu}$ & $5.4 \mathrm{E}-06 \pm 1.5 \mathrm{E}-05$ & $\mathrm{U}$ \\
\hline & ${ }^{239 / 240} \mathrm{Pu}$ & $4.5 \mathrm{E}-06 \pm 6.4 \mathrm{E}-06$ & $\mathrm{U}$ \\
\hline & ${ }^{103} \mathrm{Ru}$ & $-2.9 \mathrm{E}-05 \pm 5.9 \mathrm{E}-05$ & $\mathrm{U}$ \\
\hline & ${ }^{106} \mathrm{Ru}$ & $-3.5 \mathrm{E}-04 \pm 6.1 \mathrm{E}-04$ & $\mathrm{U}$ \\
\hline & ${ }^{125} \mathrm{Sb}$ & $-2.3 \mathrm{E}-06 \pm 2.3 \mathrm{E}-05$ & $\mathrm{U}$ \\
\hline & ${ }^{113} \mathrm{Sn}$ & $-2.1 \mathrm{E}-06 \pm 2.1 \mathrm{E}-05$ & $\mathrm{U}$ \\
\hline & ${ }^{90} \mathrm{Sr}$ & $-7.5 \mathrm{E}-05 \pm 1.1 \mathrm{E}-04$ & $\mathrm{U}$ \\
\hline & ${ }^{234} \mathrm{U}$ & $1.4 \mathrm{E}-05 \pm 7.9 \mathrm{E}-06$ & \\
\hline & ${ }^{235} \mathrm{U}$ & $3.8 \mathrm{E}-06 \pm 3.7 \mathrm{E}-06$ & \\
\hline & ${ }^{238} \mathrm{U}$ & $9.0 \mathrm{E}-06 \pm 6.0 \mathrm{E}-06$ & \\
\hline & ${ }^{65} \mathrm{Zn}$ & $-1.4 \mathrm{E}-04 \pm 1.5 \mathrm{E}-04$ & $\mathrm{U}$ \\
\hline N442 (200-West) & ${ }^{144} \mathrm{Ce}$ & $-2.6 \mathrm{E}-05 \pm 2.6 \mathrm{E}-04$ & $\bar{U}$ \\
\hline Composite Period & ${ }^{60} \mathrm{Co}$ & $2.9 \mathrm{E}-06 \pm 2.9 \mathrm{E}-05$ & $\mathrm{U}$ \\
\hline \multirow[t]{16}{*}{$01 / 06 / 03$ to $06 / 23 / 03$} & ${ }^{134} \mathrm{Cs}$ & $-1.4 \mathrm{E}-05 \pm 7.7 \mathrm{E}-05$ & $\mathrm{U}$ \\
\hline & ${ }^{137} \mathrm{Cs}$ & $1.5 \mathrm{E}-04 \pm 9.1 \mathrm{E}-05$ & U \\
\hline & ${ }^{152} \mathrm{Eu}$ & $-3.3 \mathrm{E}-05 \pm 1.8 \mathrm{E}-04$ & $\mathrm{U}$ \\
\hline & ${ }^{154} \mathrm{Eu}$ & $-1.8 \mathrm{E}-05 \pm 1.8 \mathrm{E}-04$ & $\mathrm{U}$ \\
\hline & ${ }^{155} \mathrm{Eu}$ & $-2.4 \mathrm{E}-04 \pm 2.5 \mathrm{E}-04$ & $\mathrm{U}$ \\
\hline & ${ }^{238} \mathrm{Pu}$ & $1.1 \mathrm{E}-06 \pm 1.1 \mathrm{E}-06$ & $\mathrm{U}$ \\
\hline & ${ }^{239 / 240} \mathrm{Pu}$ & $5.4 \mathrm{E}-06 \pm 6.7 \mathrm{E}-06$ & $\mathrm{U}$ \\
\hline & ${ }^{103} \mathrm{Ru}$ & $2.8 \mathrm{E}-06 \pm 2.8 \mathrm{E}-05$ & $\mathrm{U}$ \\
\hline & ${ }^{106} \mathrm{Ru}$ & $-8.3 \mathrm{E}-04 \pm 8.6 \mathrm{E}-04$ & $\mathrm{U}$ \\
\hline & ${ }^{125} \mathrm{Sb}$ & $8.2 \mathrm{E}-05 \pm 1.8 \mathrm{E}-04$ & $\mathrm{U}$ \\
\hline & ${ }^{113} \mathrm{Sn}$ & $-3.4 \mathrm{E}-05 \pm 7.6 \mathrm{E}-05$ & $\mathrm{U}$ \\
\hline & ${ }^{90} \mathrm{Sr}$ & $6.8 \mathrm{E}-05 \pm 1.0 \mathrm{E}-04$ & $\mathrm{U}$ \\
\hline & ${ }^{234} \mathrm{U}$ & $4.9 \mathrm{E}-06 \pm 4.1 \mathrm{E}-06$ & \\
\hline & ${ }^{235} \mathrm{U}$ & $1.5 \mathrm{E}-06 \pm 2.2 \mathrm{E}-06$ & $\mathrm{U}$ \\
\hline & ${ }^{238} \mathrm{U}$ & $7.5 \mathrm{E}-06 \pm 5.3 \mathrm{E}-06$ & \\
\hline & ${ }^{65} \mathrm{Zn}$ & $-1.2 \mathrm{E}-04 \pm 1.8 \mathrm{E}-04$ & $\mathrm{U}$ \\
\hline
\end{tabular}

$\overline{\mathrm{RQ}}=$ Result Qualifier. $\mathrm{U}=$ The analyte was analyzed for but not detected. 
Table 2-3. Near-Facility Air Sampling Results, 2003 (pCi/m³ \pm total analytical uncertainty). (cont)

\begin{tabular}{|c|c|c|c|}
\hline Location & Isotope & Result \pm Uncertainty & $\mathbf{R Q}^{*}$ \\
\hline N442 (200-West) & ${ }^{144} \mathrm{Ce}$ & $7.3 \mathrm{E}-05 \pm 5.1 \mathrm{E}-04$ & $\mathrm{U}$ \\
\hline Composite Period & ${ }^{60} \mathrm{Co}$ & $9.7 \mathrm{E}-05 \pm 8.5 \mathrm{E}-05$ & $\mathrm{U}$ \\
\hline \multirow[t]{16}{*}{$06 / 23 / 03$ to $12 / 22 / 03$} & ${ }^{134} \mathrm{Cs}$ & $3.7 \mathrm{E}-05 \pm 7.1 \mathrm{E}-05$ & $\mathrm{U}$ \\
\hline & ${ }^{137} \mathrm{Cs}$ & $8.3 \mathrm{E}-05 \pm 6.5 \mathrm{E}-05$ & $\mathrm{U}$ \\
\hline & ${ }^{152} \mathrm{Eu}$ & $-7.0 \mathrm{E}-06 \pm 7.0 \mathrm{E}-05$ & $\mathrm{U}$ \\
\hline & ${ }^{154} \mathrm{Eu}$ & $2.6 \mathrm{E}-04 \pm 2.2 \mathrm{E}-04$ & $\mathrm{U}$ \\
\hline & ${ }^{155} \mathrm{Eu}$ & $4.7 \mathrm{E}-05 \pm 1.4 \mathrm{E}-04$ & $\mathrm{U}$ \\
\hline & ${ }^{238} \mathrm{Pu}$ & $9.2 \mathrm{E}-06 \pm 1.3 \mathrm{E}-05$ & $\mathrm{U}$ \\
\hline & ${ }^{239 / 240} \mathrm{Pu}$ & $1.1 \mathrm{E}-04 \pm 4.6 \mathrm{E}-05$ & \\
\hline & ${ }^{103} \mathrm{Ru}$ & $-3.8 \mathrm{E}-05 \pm 5.3 \mathrm{E}-05$ & $\mathrm{U}$ \\
\hline & ${ }^{106} \mathrm{Ru}$ & $5.7 \mathrm{E}-05 \pm 5.4 \mathrm{E}-04$ & $\mathrm{U}$ \\
\hline & ${ }^{125} \mathrm{Sb}$ & $3.2 \mathrm{E}-04 \pm 2.6 \mathrm{E}-04$ & \\
\hline & ${ }^{113} \mathrm{Sn}$ & $1.4 \mathrm{E}-05 \pm 6.0 \mathrm{E}-05$ & $\mathrm{U}$ \\
\hline & ${ }^{90} \mathrm{Sr}$ & $4.9 \mathrm{E}-05 \pm 1.0 \mathrm{E}-04$ & $\mathrm{U}$ \\
\hline & ${ }^{234} \mathrm{U}$ & $1.2 \mathrm{E}-05 \pm 9.6 \mathrm{E}-06$ & $\mathrm{U}$ \\
\hline & ${ }^{235} \mathrm{U}$ & $-2.7 \mathrm{E}-06 \pm 5.4 \mathrm{E}-06$ & $\mathrm{U}$ \\
\hline & ${ }^{238} \mathrm{U}$ & $1.1 \mathrm{E}-05 \pm 7.6 \mathrm{E}-06$ & \\
\hline & ${ }^{65} \mathrm{Zn}$ & $2.9 \mathrm{E}-05 \pm 1.5 \mathrm{E}-04$ & $\mathrm{U}$ \\
\hline N449 (200-West) & ${ }^{144} \mathrm{Ce}$ & $3.5 \mathrm{E}-04 \pm 8.3 \mathrm{E}-04$ & $\mathrm{U}$ \\
\hline Composite Period & ${ }^{60} \mathrm{Co}$ & $1.6 \mathrm{E}-05 \pm 1.3 \mathrm{E}-04$ & $\mathrm{U}$ \\
\hline \multirow[t]{16}{*}{$06 / 23 / 03$ to $12 / 22 / 03$} & ${ }^{134} \mathrm{Cs}$ & $2.0 \mathrm{E}-05 \pm 8.9 \mathrm{E}-05$ & $\mathrm{U}$ \\
\hline & ${ }^{137} \mathrm{Cs}$ & $7.6 \mathrm{E}-05 \pm 8.0 \mathrm{E}-05$ & $\mathrm{U}$ \\
\hline & ${ }^{152} \mathrm{Eu}$ & $1.7 \mathrm{E}-04 \pm 2.0 \mathrm{E}-04$ & $\mathrm{U}$ \\
\hline & ${ }^{154} \mathrm{Eu}$ & $-5.3 \mathrm{E}-05 \pm 2.8 \mathrm{E}-04$ & $\mathrm{U}$ \\
\hline & ${ }^{155} \mathrm{Eu}$ & $-4.3 \mathrm{E}-05 \pm 2.1 \mathrm{E}-04$ & $\mathrm{U}$ \\
\hline & ${ }^{238} \mathrm{Pu}$ & $2.1 \mathrm{E}-05 \pm 2.0 \mathrm{E}-05$ & $\mathrm{U}$ \\
\hline & ${ }^{239 / 240} \mathrm{Pu}$ & $8.7 \mathrm{E}-06 \pm 7.2 \mathrm{E}-06$ & \\
\hline & ${ }^{103} \mathrm{Ru}$ & $-6.7 \mathrm{E}-05 \pm 7.8 \mathrm{E}-05$ & $\mathrm{U}$ \\
\hline & ${ }^{106} \mathrm{Ru}$ & $4.5 \mathrm{E}-04 \pm 7.7 \mathrm{E}-04$ & $\mathrm{U}$ \\
\hline & ${ }^{125} \mathrm{Sb}$ & $5.2 \mathrm{E}-05 \pm 1.8 \mathrm{E}-04$ & $\mathrm{U}$ \\
\hline & ${ }^{113} \mathrm{Sn}$ & $-1.5 \mathrm{E}-05 \pm 9.2 \mathrm{E}-05$ & $\mathrm{U}$ \\
\hline & ${ }^{90} \mathrm{Sr}$ & $-2.4 \mathrm{E}-04 \pm 2.5 \mathrm{E}-04$ & $\mathrm{U}$ \\
\hline & ${ }^{234} \mathrm{U}$ & $1.5 \mathrm{E}-05 \pm 1.0 \mathrm{E}-05$ & \\
\hline & ${ }^{235} \mathrm{U}$ & $1.0 \mathrm{E}-06 \pm 5.4 \mathrm{E}-06$ & $\mathrm{U}$ \\
\hline & ${ }^{238} \mathrm{U}$ & $1.5 \mathrm{E}-05 \pm 9.4 \mathrm{E}-06$ & \\
\hline & ${ }^{65} \mathrm{Zn}$ & $-1.5 \mathrm{E}-04 \pm 2.1 \mathrm{E}-04$ & $\mathrm{U}$ \\
\hline
\end{tabular}

\begin{tabular}{ccrc}
\hline Location & Isotope & Result \pm Uncertainty & RQ* \\
\hline N449 (200-West) & ${ }^{144} \mathrm{Ce}$ & $-1.0 \mathrm{E}-03 \pm 1.1 \mathrm{E}-03$ & $\mathrm{U}$ \\
Composite Period & ${ }^{60} \mathrm{Co}$ & $9.7 \mathrm{E}-06 \pm 9.7 \mathrm{E}-05$ & $\mathrm{U}$ \\
01/06/03 to 06/23/03 & ${ }^{134} \mathrm{Cs}$ & $9.0 \mathrm{E}-05 \pm 8.4 \mathrm{E}-05$ & $\mathrm{U}$ \\
& ${ }^{137} \mathrm{Cs}$ & $-3.4 \mathrm{E}-05 \pm 9.2 \mathrm{E}-05$ & $\mathrm{U}$ \\
& ${ }^{152} \mathrm{Eu}$ & $1.3 \mathrm{E}-04 \pm 2.0 \mathrm{E}-04$ & $\mathrm{U}$ \\
& ${ }^{154} \mathrm{Eu}$ & $5.0 \mathrm{E}-05 \pm 2.6 \mathrm{E}-04$ & $\mathrm{U}$ \\
& ${ }^{155} \mathrm{Eu}$ & $7.1 \mathrm{E}-05 \pm 1.9 \mathrm{E}-04$ & $\mathrm{U}$ \\
& ${ }^{238} \mathrm{Pu}$ & $-1.3 \mathrm{E}-05 \pm 1.6 \mathrm{E}-05$ & $\mathrm{U}$ \\
& ${ }^{239 / 240} \mathrm{Pu}$ & $3.5 \mathrm{E}-06 \pm 4.3 \mathrm{E}-06$ & \\
& ${ }^{103} \mathrm{Ru}$ & $-4.1 \mathrm{E}-05 \pm 7.3 \mathrm{E}-05$ & $\mathrm{U}$ \\
& ${ }^{106} \mathrm{Ru}$ & $-2.8 \mathrm{E}-04 \pm 7.6 \mathrm{E}-04$ & $\mathrm{U}$ \\
& ${ }^{125} \mathrm{Sb}$ & $7.7 \mathrm{E}-05 \pm 1.7 \mathrm{E}-04$ & $\mathrm{U}$ \\
& ${ }^{113} \mathrm{Sn}$ & $4.7 \mathrm{E}-05 \pm 9.1 \mathrm{E}-05$ & $\mathrm{U}$ \\
& ${ }^{90} \mathrm{Sr}$ & $-4.8 \mathrm{E}-05 \pm 1.0 \mathrm{E}-04$ & $\mathrm{U}$ \\
& ${ }^{234} \mathrm{U}$ & $1.4 \mathrm{E}-05 \pm 8.5 \mathrm{E}-06$ & \\
& ${ }^{235} \mathrm{U}$ & $3.4 \mathrm{E}-06 \pm 4.2 \mathrm{E}-06$ & $\mathrm{U}$ \\
& ${ }^{238} \mathrm{U}$ & $9.5 \mathrm{E}-06 \pm 7.2 \mathrm{E}-06$ & \\
& ${ }^{65} \mathrm{Zn} \mathrm{Zn}$ & $-9.9 \mathrm{E}-05 \pm 2.5 \mathrm{E}-04$ & $\mathrm{U}$ \\
\hline${ }^{65} \mathrm{Zn} \mathrm{U}$ & $-9.5 \mathrm{E}-05 \pm 2.1 \mathrm{E}-04$ & $\mathrm{U}$
\end{tabular}

$\overline{\mathrm{RQ}}=$ Result Qualifier. $\mathrm{U}=$ The analyte was analyzed for but not detected. 
Table 2-3. Near-Facility Air Sampling Results, 2003 (pCi/m³ \pm total analytical uncertainty). (cont)

\begin{tabular}{|c|c|c|c|c|c|c|c|}
\hline Location & Isotope & Result \pm Uncertainty & $\mathbf{R Q}^{*}$ & Location & Isotope & Result \pm Uncertainty & RQ* \\
\hline N456 (200-West) & ${ }^{144} \mathrm{Ce}$ & $3.3 \mathrm{E}-04 \pm 6.1 \mathrm{E}-04$ & $\mathrm{U}$ & N457 (200-West) & ${ }^{144} \mathrm{Ce}$ & $-3.8 \mathrm{E}-04 \pm 7.8 \mathrm{E}-04$ & $\mathrm{U}$ \\
\hline Composite Period & ${ }^{60} \mathrm{Co}$ & $1.3 \mathrm{E}-05 \pm 7.8 \mathrm{E}-05$ & $\mathrm{U}$ & Composite Period & ${ }^{60} \mathrm{Co}$ & $-4.1 \mathrm{E}-05 \pm 7.8 \mathrm{E}-05$ & $\mathrm{U}$ \\
\hline \multirow[t]{16}{*}{$06 / 23 / 03$ to $12 / 22 / 03$} & ${ }^{134} \mathrm{Cs}$ & $-3.1 \mathrm{E}-05 \pm 6.1 \mathrm{E}-05$ & $\mathrm{U}$ & $01 / 06 / 03$ to $06 / 23 / 03$ & ${ }^{134} \mathrm{Cs}$ & $-7.1 \mathrm{E}-05 \pm 8.1 \mathrm{E}-05$ & $\mathrm{U}$ \\
\hline & ${ }^{137} \mathrm{Cs}$ & $5.5 \mathrm{E}-05 \pm 6.6 \mathrm{E}-05$ & $\mathrm{U}$ & & ${ }^{137} \mathrm{Cs}$ & $7.9 \mathrm{E}-05 \pm 7.9 \mathrm{E}-05$ & $\mathrm{U}$ \\
\hline & ${ }^{152} \mathrm{Eu}$ & $5.8 \mathrm{E}-05 \pm 1.4 \mathrm{E}-04$ & U & & ${ }^{152} \mathrm{Eu}$ & $-1.9 \mathrm{E}-05 \pm 1.9 \mathrm{E}-04$ & U \\
\hline & ${ }^{154} \mathrm{Eu}$ & $-3.6 \mathrm{E}-05 \pm 2.2 \mathrm{E}-04$ & $\mathrm{U}$ & & ${ }^{154} \mathrm{Eu}$ & $-4.0 \mathrm{E}-05 \pm 2.1 \mathrm{E}-04$ & $\mathrm{U}$ \\
\hline & ${ }^{155} \mathrm{Eu}$ & $1.1 \mathrm{E}-04 \pm 1.6 \mathrm{E}-04$ & $\mathrm{U}$ & & ${ }^{155} \mathrm{Eu}$ & $-8.4 \mathrm{E}-05 \pm 2.1 \mathrm{E}-04$ & $\mathrm{U}$ \\
\hline & ${ }^{238} \mathrm{Pu}$ & $4.0 \mathrm{E}-06 \pm 1.5 \mathrm{E}-05$ & $\mathrm{U}$ & & ${ }^{238} \mathrm{Pu}$ & $8.5 \mathrm{E}-06 \pm 1.8 \mathrm{E}-05$ & $\mathrm{U}$ \\
\hline & ${ }^{239 / 240} \mathrm{Pu}$ & $2.0 \mathrm{E}-06 \pm 5.0 \mathrm{E}-06$ & $\mathrm{U}$ & & ${ }^{239 / 240} \mathrm{Pu}$ & $7.2 \mathrm{E}-06 \pm 6.8 \mathrm{E}-06$ & $\mathrm{U}$ \\
\hline & ${ }^{103} \mathrm{Ru}$ & $1.2 \mathrm{E}-06 \pm 1.2 \mathrm{E}-05$ & $\mathrm{U}$ & & ${ }^{103} \mathrm{Ru}$ & $-6.7 \mathrm{E}-05 \pm 8.4 \mathrm{E}-05$ & $\mathrm{U}$ \\
\hline & ${ }^{106} \mathrm{Ru}$ & $-1.8 \mathrm{E}-04 \pm 5.0 \mathrm{E}-04$ & $\mathrm{U}$ & & ${ }^{106} \mathrm{Ru}$ & $-1.4 \mathrm{E}-04 \pm 7.0 \mathrm{E}-04$ & $\mathrm{U}$ \\
\hline & ${ }^{125} \mathrm{Sb}$ & $3.8 \mathrm{E}-05 \pm 1.4 \mathrm{E}-04$ & $\mathrm{U}$ & & ${ }^{125} \mathrm{Sb}$ & $-7.4 \mathrm{E}-05 \pm 1.9 \mathrm{E}-04$ & $\mathrm{U}$ \\
\hline & ${ }^{113} \mathrm{Sn}$ & $-2.6 \mathrm{E}-05 \pm 6.5 \mathrm{E}-05$ & $\mathrm{U}$ & & ${ }^{113} \mathrm{Sn}$ & $3.9 \mathrm{E}-05 \pm 8.6 \mathrm{E}-05$ & $\mathrm{U}$ \\
\hline & ${ }^{90} \mathrm{Sr}$ & $-1.1 \mathrm{E}-04 \pm 1.2 \mathrm{E}-04$ & $\mathrm{U}$ & & ${ }^{90} \mathrm{Sr}$ & $7.7 \mathrm{E}-06 \pm 7.7 \mathrm{E}-05$ & $\mathrm{U}$ \\
\hline & ${ }^{234} \mathrm{U}$ & $1.7 \mathrm{E}-05 \pm 1.0 \mathrm{E}-05$ & & & ${ }^{234} \mathrm{U}$ & $7.2 \mathrm{E}-06 \pm 5.7 \mathrm{E}-06$ & \\
\hline & ${ }^{235} \mathrm{U}$ & $1.8 \mathrm{E}-06 \pm 2.5 \mathrm{E}-06$ & $\mathrm{U}$ & & ${ }^{235} \mathrm{U}$ & $3.9 \mathrm{E}-06 \pm 3.8 \mathrm{E}-06$ & \\
\hline & ${ }^{238} \mathrm{U}$ & $1.8 \mathrm{E}-05 \pm 1.1 \mathrm{E}-05$ & & & ${ }^{238} \mathrm{U}$ & $5.7 \mathrm{E}-06 \pm 4.6 \mathrm{E}-06$ & \\
\hline & ${ }^{65} \mathrm{Zn}$ & $1.3 \mathrm{E}-04 \pm 1.7 \mathrm{E}-04$ & $\mathrm{U}$ & & ${ }^{65} \mathrm{Zn}$ & $-1.5 \mathrm{E}-04 \pm 1.7 \mathrm{E}-04$ & $\mathrm{U}$ \\
\hline N457 (200-West) & ${ }^{144} \mathrm{Ce}$ & $-6.8 \mathrm{E}-05 \pm 6.1 \mathrm{E}-04$ & $\bar{U}$ & N956 (200-West) & ${ }^{144} \mathrm{Ce}$ & $-6.0 \mathrm{E}-04 \pm 7.9 \mathrm{E}-04$ & $\mathrm{U}$ \\
\hline Composite Period & ${ }^{60} \mathrm{Co}$ & $-1.7 \mathrm{E}-05 \pm 7.9 \mathrm{E}-05$ & $\mathrm{U}$ & Composite Period & ${ }^{60} \mathrm{Co}$ & $2.9 \mathrm{E}-05 \pm 9.9 \mathrm{E}-05$ & $\mathrm{U}$ \\
\hline \multirow[t]{16}{*}{$06 / 23 / 03$ to $12 / 22 / 03$} & ${ }^{134} \mathrm{Cs}$ & $4.8 \mathrm{E}-05 \pm 6.9 \mathrm{E}-05$ & $\mathrm{U}$ & $01 / 06 / 03$ to $06 / 23 / 03$ & ${ }^{134} \mathrm{Cs}$ & $2.4 \mathrm{E}-05 \pm 8.5 \mathrm{E}-05$ & $\mathrm{U}$ \\
\hline & ${ }^{137} \mathrm{Cs}$ & $9.3 \mathrm{E}-05 \pm 7.1 \mathrm{E}-05$ & $\mathrm{U}$ & & ${ }^{137} \mathrm{Cs}$ & $4.2 \mathrm{E}-04 \pm 1.9 \mathrm{E}-04$ & \\
\hline & ${ }^{152} \mathrm{Eu}$ & $3.0 \mathrm{E}-06 \pm 3.0 \mathrm{E}-05$ & $\mathrm{U}$ & & ${ }^{152} \mathrm{Eu}$ & $-1.1 \mathrm{E}-04 \pm 1.9 \mathrm{E}-04$ & $\mathrm{U}$ \\
\hline & ${ }^{154} \mathrm{Eu}$ & $-3.6 \mathrm{E}-05 \pm 2.5 \mathrm{E}-04$ & $\mathrm{U}$ & & ${ }^{154} \mathrm{Eu}$ & $-7.5 \mathrm{E}-06 \pm 7.5 \mathrm{E}-05$ & $\mathrm{U}$ \\
\hline & ${ }^{155} \mathrm{Eu}$ & $-5.6 \mathrm{E}-05 \pm 1.6 \mathrm{E}-04$ & $\mathrm{U}$ & & ${ }^{155} \mathrm{Eu}$ & $-2.4 \mathrm{E}-05 \pm 1.9 \mathrm{E}-04$ & $\mathrm{U}$ \\
\hline & ${ }^{238} \mathrm{Pu}$ & $3.7 \mathrm{E}-06 \pm$ 5.3E-06 & $\mathrm{U}$ & & ${ }^{238} \mathrm{Pu}$ & $3.3 \mathrm{E}-06 \pm 1.4 \mathrm{E}-05$ & $\mathrm{U}$ \\
\hline & ${ }^{239 / 240} \mathrm{Pu}$ & $2.8 \mathrm{E}-06 \pm 3.4 \mathrm{E}-06$ & & & ${ }^{239 / 240} \mathrm{Pu}$ & $6.7 \mathrm{E}-06 \pm 5.8 \mathrm{E}-06$ & \\
\hline & ${ }^{103} \mathrm{Ru}$ & $-1.3 \mathrm{E}-05 \pm 6.2 \mathrm{E}-05$ & $\mathrm{U}$ & & ${ }^{103} \mathrm{Ru}$ & $6.3 \mathrm{E}-06 \pm 6.3 \mathrm{E}-05$ & $\mathrm{U}$ \\
\hline & ${ }^{106} \mathrm{Ru}$ & $1.2 \mathrm{E}-04 \pm 5.6 \mathrm{E}-04$ & $\mathrm{U}$ & & ${ }^{106} \mathrm{Ru}$ & $-8.6 \mathrm{E}-04 \pm 8.9 \mathrm{E}-04$ & $\mathrm{U}$ \\
\hline & ${ }^{125} \mathrm{Sb}$ & $-2.4 \mathrm{E}-06 \pm 2.4 \mathrm{E}-05$ & $\mathrm{U}$ & & ${ }^{125} \mathrm{Sb}$ & $-5.3 \mathrm{E}-05 \pm 1.8 \mathrm{E}-04$ & $\mathrm{U}$ \\
\hline & ${ }^{113} \mathrm{Sn}$ & $3.0 \mathrm{E}-05 \pm 6.7 \mathrm{E}-05$ & $\mathrm{U}$ & & ${ }^{113} \mathrm{Sn}$ & $5.9 \mathrm{E}-05 \pm 9.6 \mathrm{E}-05$ & $\mathrm{U}$ \\
\hline & ${ }^{90} \mathrm{Sr}$ & $-1.4 \mathrm{E}-05 \pm 9.4 \mathrm{E}-05$ & $\mathrm{U}$ & & ${ }^{90} \mathrm{Sr}$ & $-1.1 \mathrm{E}-04 \pm 1.1 \mathrm{E}-04$ & $\mathrm{U}$ \\
\hline & ${ }^{234} \mathrm{U}$ & $1.3 \mathrm{E}-05 \pm 8.0 \mathrm{E}-06$ & & & ${ }^{234} \mathrm{U}$ & $2.0 \mathrm{E}-05 \pm 1.1 \mathrm{E}-05$ & \\
\hline & ${ }^{235} \mathrm{U}$ & $2.2 \mathrm{E}-06 \pm 2.7 \mathrm{E}-06$ & & & ${ }^{235} \mathrm{U}$ & 7.3E-07 $\pm 7.3 \mathrm{E}-06$ & $\mathrm{U}$ \\
\hline & ${ }^{238} \mathrm{U}$ & $1.5 \mathrm{E}-05 \pm 8.6 \mathrm{E}-06$ & & & ${ }^{238} \mathrm{U}$ & $1.2 \mathrm{E}-05 \pm 7.5 \mathrm{E}-06$ & \\
\hline & ${ }^{65} \mathrm{Zn}$ & $1.3 \mathrm{E}-04 \pm 1.8 \mathrm{E}-04$ & $\mathrm{U}$ & & ${ }^{65} \mathrm{Zn}$ & $1.5 \mathrm{E}-05 \pm 1.5 \mathrm{E}-04$ & $\mathrm{U}$ \\
\hline
\end{tabular}

$\overline{\mathrm{RQ}}=$ Result Qualifier. $\mathrm{U}=$ The analyte was analyzed for but not detected. 
Table 2-3. Near-Facility Air Sampling Results, 2003 (pCi/m³ \pm total analytical uncertainty). (cont)

\begin{tabular}{|c|c|c|c|c|c|c|c|}
\hline Location & Isotope & Result \pm Uncertainty & $\mathbf{R Q}^{*}$ & Location & Isotope & Result \pm Uncertainty & RQ* \\
\hline N956 (200-West) & ${ }^{144} \mathrm{Ce}$ & $-3.7 \mathrm{E}-04 \pm 5.7 \mathrm{E}-04$ & $\bar{U}$ & N963 (200-West) & ${ }^{144} \mathrm{Ce}$ & $-1.1 \mathrm{E}-05 \pm 1.1 \mathrm{E}-04$ & $\mathrm{U}$ \\
\hline Composite Period & ${ }^{60} \mathrm{Co}$ & $3.8 \mathrm{E}-05 \pm 7.7 \mathrm{E}-05$ & $\mathrm{U}$ & Composite Period & ${ }^{60} \mathrm{Co}$ & $6.2 \mathrm{E}-05 \pm 9.2 \mathrm{E}-05$ & $\mathrm{U}$ \\
\hline \multirow[t]{16}{*}{$06 / 23 / 03$ to $12 / 22 / 03$} & ${ }^{134} \mathrm{Cs}$ & $5.1 \mathrm{E}-06 \pm 5.1 \mathrm{E}-05$ & $\mathrm{U}$ & $01 / 06 / 03$ to $06 / 23 / 03$ & ${ }^{134} \mathrm{Cs}$ & $1.3 \mathrm{E}-05 \pm 8.2 \mathrm{E}-05$ & $\mathrm{U}$ \\
\hline & ${ }^{137} \mathrm{Cs}$ & $2.3 \mathrm{E}-04 \pm 1.4 \mathrm{E}-04$ & & & ${ }^{137} \mathrm{Cs}$ & $5.9 \mathrm{E}-05 \pm 7.9 \mathrm{E}-05$ & $\mathrm{U}$ \\
\hline & ${ }^{152} \mathrm{Eu}$ & $3.3 \mathrm{E}-05 \pm 1.5 \mathrm{E}-04$ & $\mathrm{U}$ & & ${ }^{152} \mathrm{Eu}$ & $-7.6 \mathrm{E}-06 \pm 7.6 \mathrm{E}-05$ & $\mathrm{U}$ \\
\hline & ${ }^{154} \mathrm{Eu}$ & $2.1 \mathrm{E}-04 \pm 2.4 \mathrm{E}-04$ & $\mathrm{U}$ & & ${ }^{154} \mathrm{Eu}$ & $-1.9 \mathrm{E}-05 \pm 1.9 \mathrm{E}-04$ & $\mathrm{U}$ \\
\hline & ${ }^{155} \mathrm{Eu}$ & $-5.0 \mathrm{E}-05 \pm 1.5 \mathrm{E}-04$ & $\mathrm{U}$ & & ${ }^{155} \mathrm{Eu}$ & $3.1 \mathrm{E}-05 \pm 1.9 \mathrm{E}-04$ & $\mathrm{U}$ \\
\hline & ${ }^{238} \mathrm{Pu}$ & $8.1 \mathrm{E}-06 \pm 1.1 \mathrm{E}-05$ & $\mathrm{U}$ & & ${ }^{238} \mathrm{Pu}$ & $8.4 \mathrm{E}-07 \pm 8.7 \mathrm{E}-07$ & $\mathrm{U}$ \\
\hline & ${ }^{239 / 240} \mathrm{Pu}$ & $4.6 \mathrm{E}-06 \pm 5.3 \mathrm{E}-06$ & $\mathrm{U}$ & & ${ }^{239 / 240} \mathrm{Pu}$ & $1.6 \mathrm{E}-05 \pm 9.6 \mathrm{E}-06$ & \\
\hline & ${ }^{103} \mathrm{Ru}$ & $4.0 \mathrm{E}-05 \pm 6.8 \mathrm{E}-05$ & $\mathrm{U}$ & & ${ }^{103} \mathrm{Ru}$ & $4.6 \mathrm{E}-05 \pm 7.7 \mathrm{E}-05$ & $\mathrm{U}$ \\
\hline & ${ }^{106} \mathrm{Ru}$ & $-1.8 \mathrm{E}-04 \pm 5.5 \mathrm{E}-04$ & $\mathrm{U}$ & & ${ }^{106} \mathrm{Ru}$ & $-4.2 \mathrm{E}-04 \pm 7.6 \mathrm{E}-04$ & $\mathrm{U}$ \\
\hline & ${ }^{125} \mathrm{Sb}$ & $-7.7 \mathrm{E}-05 \pm 1.5 \mathrm{E}-04$ & $\mathrm{U}$ & & ${ }^{125} \mathrm{Sb}$ & $9.8 \mathrm{E}-05 \pm 1.8 \mathrm{E}-04$ & $\mathrm{U}$ \\
\hline & ${ }^{113} \mathrm{Sn}$ & $2.3 \mathrm{E}-05 \pm 6.7 \mathrm{E}-05$ & $\mathrm{U}$ & & ${ }^{113} \mathrm{Sn}$ & $-4.4 \mathrm{E}-05 \pm 8.9 \mathrm{E}-05$ & $\mathrm{U}$ \\
\hline & ${ }^{90} \mathrm{Sr}$ & $-7.4 \mathrm{E}-06 \pm 7.4 \mathrm{E}-05$ & $\mathrm{U}$ & & ${ }^{90} \mathrm{Sr}$ & $-1.5 \mathrm{E}-05 \pm 7.7 \mathrm{E}-05$ & $\mathrm{U}$ \\
\hline & ${ }^{234} \mathrm{U}$ & $1.7 \mathrm{E}-05 \pm 9.9 \mathrm{E}-06$ & & & ${ }^{234} \mathrm{U}$ & $1.4 \mathrm{E}-05 \pm 8.0 \mathrm{E}-06$ & \\
\hline & ${ }^{235} \mathrm{U}$ & $4.6 \mathrm{E}-06 \pm 4.1 \mathrm{E}-06$ & & & ${ }^{235} \mathrm{U}$ & $1.5 \mathrm{E}-06 \pm 2.1 \mathrm{E}-06$ & $\mathrm{U}$ \\
\hline & ${ }^{238} \mathrm{U}$ & $2.2 \mathrm{E}-05 \pm 1.2 \mathrm{E}-05$ & & & ${ }^{238} \mathrm{U}$ & $4.1 \mathrm{E}-06 \pm 3.7 \mathrm{E}-06$ & \\
\hline & ${ }^{65} \mathrm{Zn}$ & $1.5 \mathrm{E}-04 \pm 1.9 \mathrm{E}-04$ & $\mathrm{U}$ & & ${ }^{65} \mathrm{Zn}$ & $-3.9 \mathrm{E}-05 \pm 1.8 \mathrm{E}-04$ & $\mathrm{U}$ \\
\hline N963 (200-West) & ${ }^{144} \mathrm{Ce}$ & $8.1 \mathrm{E}-05 \pm 5.8 \mathrm{E}-04$ & $\bar{U}$ & N964 (200-West) & ${ }^{144} \mathrm{Ce}$ & $2.3 \mathrm{E}-04 \pm 7.2 \mathrm{E}-04$ & $\mathrm{U}$ \\
\hline Composite Period & ${ }^{60} \mathrm{Co}$ & $8.8 \mathrm{E}-05 \pm 8.9 \mathrm{E}-05$ & $\mathrm{U}$ & Composite Period & ${ }^{60} \mathrm{Co}$ & $1.2 \mathrm{E}-05 \pm 9.2 \mathrm{E}-05$ & $\mathrm{U}$ \\
\hline \multirow[t]{16}{*}{$06 / 23 / 03$ to $12 / 22 / 03$} & ${ }^{134} \mathrm{Cs}$ & $-4.0 \mathrm{E}-05 \pm 7.7 \mathrm{E}-05$ & $\mathrm{U}$ & $01 / 06 / 03$ to $06 / 23 / 03$ & ${ }^{134} \mathrm{Cs}$ & $5.4 \mathrm{E}-05 \pm 9.6 \mathrm{E}-05$ & $\mathrm{U}$ \\
\hline & ${ }^{137} \mathrm{Cs}$ & $2.4 \mathrm{E}-05 \pm 6.6 \mathrm{E}-05$ & $\mathrm{U}$ & & ${ }^{137} \mathrm{Cs}$ & $1.1 \mathrm{E}-05 \pm 8.5 \mathrm{E}-05$ & $\mathrm{U}$ \\
\hline & ${ }^{152} \mathrm{Eu}$ & $-2.1 \mathrm{E}-05 \pm 1.4 \mathrm{E}-04$ & $\mathrm{U}$ & & ${ }^{152} \mathrm{Eu}$ & $3.6 \mathrm{E}-06 \pm 3.6 \mathrm{E}-05$ & $\mathrm{U}$ \\
\hline & ${ }^{154} \mathrm{Eu}$ & $-8.6 \mathrm{E}-05 \pm 2.1 \mathrm{E}-04$ & $\mathrm{U}$ & & ${ }^{154} \mathrm{Eu}$ & $1.0 \mathrm{E}-04 \pm 2.9 \mathrm{E}-04$ & $\mathrm{U}$ \\
\hline & ${ }^{155} \mathrm{Eu}$ & $1.3 \mathrm{E}-05 \pm 1.3 \mathrm{E}-04$ & $\mathrm{U}$ & & ${ }^{155} \mathrm{Eu}$ & $9.2 \mathrm{E}-06 \pm 9.2 \mathrm{E}-05$ & $\mathrm{U}$ \\
\hline & ${ }^{238} \mathrm{Pu}$ & $8.8 \mathrm{E}-07 \pm 5.0 \mathrm{E}-07$ & $\mathrm{U}$ & & ${ }^{238} \mathrm{Pu}$ & $1.4 \mathrm{E}-05 \pm 1.3 \mathrm{E}-05$ & $\mathrm{U}$ \\
\hline & ${ }^{239 / 240} \mathrm{Pu}$ & $6.4 \mathrm{E}-05 \pm 2.8 \mathrm{E}-05$ & & & ${ }^{239 / 240} \mathrm{Pu}$ & $1.6 \mathrm{E}-06 \pm 4.0 \mathrm{E}-06$ & $\mathrm{U}$ \\
\hline & ${ }^{103} \mathrm{Ru}$ & $-2.6 \mathrm{E}-05 \pm 6.3 \mathrm{E}-05$ & $\mathrm{U}$ & & ${ }^{103} \mathrm{Ru}$ & $3.4 \mathrm{E}-05 \pm 7.6 \mathrm{E}-05$ & $\mathrm{U}$ \\
\hline & ${ }^{106} \mathrm{Ru}$ & $3.2 \mathrm{E}-04 \pm 5.6 \mathrm{E}-04$ & $\mathrm{U}$ & & ${ }^{106} \mathrm{Ru}$ & $2.2 \mathrm{E}-04 \pm 7.8 \mathrm{E}-04$ & $\mathrm{U}$ \\
\hline & ${ }^{125} \mathrm{Sb}$ & $3.9 \mathrm{E}-05 \pm 1.4 \mathrm{E}-04$ & $\mathrm{U}$ & & ${ }^{125} \mathrm{Sb}$ & $1.6 \mathrm{E}-04 \pm 2.0 \mathrm{E}-04$ & $\mathrm{U}$ \\
\hline & ${ }^{113} \mathrm{Sn}$ & $8.8 \mathrm{E}-06 \pm 6.8 \mathrm{E}-05$ & $\mathrm{U}$ & & ${ }^{113} \mathrm{Sn}$ & $-3.6 \mathrm{E}-05 \pm 9.0 \mathrm{E}-05$ & $\mathrm{U}$ \\
\hline & ${ }^{90} \mathrm{Sr}$ & $-1.2 \mathrm{E}-04 \pm 1.2 \mathrm{E}-04$ & $\mathrm{U}$ & & ${ }^{90} \mathrm{Sr}$ & $7.7 \mathrm{E}-06 \pm 7.7 \mathrm{E}-05$ & $\mathrm{U}$ \\
\hline & ${ }^{234} \mathrm{U}$ & $1.2 \mathrm{E}-05 \pm 7.9 \mathrm{E}-06$ & & & ${ }^{234} \mathrm{U}$ & $1.3 \mathrm{E}-05 \pm 7.6 \mathrm{E}-06$ & \\
\hline & ${ }^{235} \mathrm{U}$ & $1.6 \mathrm{E}-06 \pm 2.3 \mathrm{E}-06$ & $\mathrm{U}$ & & ${ }^{235} \mathrm{U}$ & $1.5 \mathrm{E}-06 \pm 2.2 \mathrm{E}-06$ & $\mathrm{U}$ \\
\hline & ${ }^{238} \mathrm{U}$ & $1.1 \mathrm{E}-05 \pm 7.0 \mathrm{E}-06$ & & & ${ }^{238} \mathrm{U}$ & $5.6 \mathrm{E}-06 \pm 4.5 \mathrm{E}-06$ & \\
\hline & ${ }^{65} \mathrm{Zn}$ & $-4.9 \mathrm{E}-07 \pm 4.9 \mathrm{E}-06$ & $\mathrm{U}$ & & ${ }^{65} \mathrm{Zn}$ & $3.1 \mathrm{E}-05 \pm 2.0 \mathrm{E}-04$ & $\mathrm{U}$ \\
\hline
\end{tabular}

$\overline{\mathrm{RQ}}=$ Result Qualifier. $\mathrm{U}=$ The analyte was analyzed for but not detected. 
Table 2-3. Near-Facility Air Sampling Results, 2003 (pCi/m³ \pm total analytical uncertainty). (cont)

\begin{tabular}{|c|c|c|c|c|c|c|c|}
\hline Location & Isotope & Result \pm Uncertainty & $\mathbf{R Q}^{*}$ & Location & Isotope & Result \pm Uncertainty & RQ* \\
\hline N964 (200-West) & ${ }^{144} \mathrm{Ce}$ & $2.4 \mathrm{E}-04 \pm 8.5 \mathrm{E}-04$ & $\bar{U}$ & N965 (200-West) & ${ }^{144} \mathrm{Ce}$ & $-6.2 \mathrm{E}-04 \pm 8.0 \mathrm{E}-04$ & $\bar{U}$ \\
\hline Composite Period & ${ }^{60} \mathrm{Co}$ & $-2.2 \mathrm{E}-05 \pm 7.2 \mathrm{E}-05$ & U & Composite Period & ${ }^{60} \mathrm{Co}$ & $2.1 \mathrm{E}-05 \pm 7.6 \mathrm{E}-05$ & $\mathrm{U}$ \\
\hline \multirow[t]{16}{*}{$06 / 23 / 03$ to $12 / 22 / 03$} & ${ }^{134} \mathrm{Cs}$ & $-4.4 \mathrm{E}-05 \pm 7.2 \mathrm{E}-05$ & $\mathrm{U}$ & $01 / 06 / 03$ to $06 / 23 / 03$ & ${ }^{134} \mathrm{Cs}$ & $1.9 \mathrm{E}-05 \pm 8.0 \mathrm{E}-05$ & $\mathrm{U}$ \\
\hline & ${ }^{137} \mathrm{Cs}$ & $-1.2 \mathrm{E}-05 \pm 6.7 \mathrm{E}-05$ & $\mathrm{U}$ & & ${ }^{137} \mathrm{Cs}$ & 7.7E-05 $\pm 8.3 \mathrm{E}-05$ & U \\
\hline & ${ }^{152} \mathrm{Eu}$ & $-1.4 \mathrm{E}-04 \pm 1.7 \mathrm{E}-04$ & $\mathrm{U}$ & & ${ }^{152} \mathrm{Eu}$ & $-8.3 \mathrm{E}-06 \pm 8.3 \mathrm{E}-05$ & $\mathrm{U}$ \\
\hline & ${ }^{154} \mathrm{Eu}$ & $9.4 \mathrm{E}-05 \pm 2.0 \mathrm{E}-04$ & $\mathrm{U}$ & & ${ }^{154} \mathrm{Eu}$ & $1.5 \mathrm{E}-04 \pm 2.4 \mathrm{E}-04$ & $\mathrm{U}$ \\
\hline & ${ }^{155} \mathrm{Eu}$ & $1.6 \mathrm{E}-05 \pm 1.6 \mathrm{E}-04$ & U & & ${ }^{155} \mathrm{Eu}$ & $-2.2 \mathrm{E}-05 \pm 2.2 \mathrm{E}-04$ & $\mathrm{U}$ \\
\hline & ${ }^{238} \mathrm{Pu}$ & $-3.5 \mathrm{E}-06 \pm 8.8 \mathrm{E}-06$ & $\mathrm{U}$ & & ${ }^{238} \mathrm{Pu}$ & $1.7 \mathrm{E}-06 \pm 1.2 \mathrm{E}-05$ & U \\
\hline & ${ }^{239 / 240} \mathrm{Pu}$ & $4.4 \mathrm{E}-06 \pm 4.3 \mathrm{E}-06$ & & & ${ }^{239 / 240} \mathrm{Pu}$ & $1.7 \mathrm{E}-06 \pm 4.3 \mathrm{E}-06$ & $\mathrm{U}$ \\
\hline & ${ }^{103} \mathrm{Ru}$ & $-1.1 \mathrm{E}-07 \pm 1.1 \mathrm{E}-06$ & $\mathrm{U}$ & & ${ }^{103} \mathrm{Ru}$ & $5.1 \mathrm{E}-05 \pm 8.0 \mathrm{E}-05$ & $\mathrm{U}$ \\
\hline & ${ }^{106} \mathrm{Ru}$ & $1.7 \mathrm{E}-04 \pm 5.4 \mathrm{E}-04$ & $\mathrm{U}$ & & ${ }^{106} \mathrm{Ru}$ & $-1.4 \mathrm{E}-04 \pm 6.5 \mathrm{E}-04$ & $\mathrm{U}$ \\
\hline & ${ }^{125} \mathrm{Sb}$ & $-2.5 \mathrm{E}-05 \pm 1.6 \mathrm{E}-04$ & $\mathrm{U}$ & & ${ }^{125} \mathrm{Sb}$ & $-3.7 \mathrm{E}-05 \pm 1.8 \mathrm{E}-04$ & $\mathrm{U}$ \\
\hline & ${ }^{113} \mathrm{Sn}$ & $4.4 \mathrm{E}-06 \pm 4.4 \mathrm{E}-05$ & $\mathrm{U}$ & & ${ }^{113} \mathrm{Sn}$ & $-1.4 \mathrm{E}-05 \pm 9.2 \mathrm{E}-05$ & $\mathrm{U}$ \\
\hline & ${ }^{90} \mathrm{Sr}$ & $7.5 \mathrm{E}-06 \pm 7.5 \mathrm{E}-05$ & $\mathrm{U}$ & & ${ }^{90} \mathrm{Sr}$ & $-7.8 \mathrm{E}-05 \pm 8.8 \mathrm{E}-05$ & U \\
\hline & ${ }^{234} \mathrm{U}$ & $1.2 \mathrm{E}-05 \pm 7.4 \mathrm{E}-06$ & & & ${ }^{234} \mathrm{U}$ & $1.2 \mathrm{E}-05 \pm 7.5 \mathrm{E}-06$ & \\
\hline & ${ }^{235} \mathrm{U}$ & $7.5 \mathrm{E}-07 \pm 2.6 \mathrm{E}-06$ & $\mathrm{U}$ & & ${ }^{235} \mathrm{U}$ & $4.6 \mathrm{E}-06 \pm 4.1 \mathrm{E}-06$ & \\
\hline & ${ }^{238} \mathrm{U}$ & $1.4 \mathrm{E}-05 \pm 7.9 \mathrm{E}-06$ & & & ${ }^{238} \mathrm{U}$ & $6.3 \mathrm{E}-06 \pm 4.8 \mathrm{E}-06$ & \\
\hline & ${ }^{65} \mathrm{Zn}$ & $-5.9 \mathrm{E}-05 \pm 1.6 \mathrm{E}-04$ & $\mathrm{U}$ & & ${ }^{65} \mathrm{Zn}$ & $-1.6 \mathrm{E}-04 \pm 2.0 \mathrm{E}-04$ & $\mathrm{U}$ \\
\hline N965 (200-West) & ${ }^{144} \mathrm{Ce}$ & $9.9 \mathrm{E}-05 \pm 5.9 \mathrm{E}-04$ & $\mathrm{U}$ & N966 (200-West) & ${ }^{144} \mathrm{Ce}$ & $-4.8 \mathrm{E}-05 \pm 4.8 \mathrm{E}-04$ & $\mathrm{U}$ \\
\hline Composite Period & ${ }^{60} \mathrm{Co}$ & $-8.7 \mathrm{E}-06 \pm 6.8 \mathrm{E}-05$ & $\mathrm{U}$ & Composite Period & ${ }^{60} \mathrm{Co}$ & $-2.8 \mathrm{E}-05 \pm 9.3 \mathrm{E}-05$ & $\mathrm{U}$ \\
\hline \multirow[t]{16}{*}{$06 / 23 / 03$ to $12 / 22 / 03$} & ${ }^{134} \mathrm{Cs}$ & $5.4 \mathrm{E}-05 \pm 6.8 \mathrm{E}-05$ & $\mathrm{U}$ & $01 / 06 / 03$ to $06 / 23 / 03$ & ${ }^{134} \mathrm{Cs}$ & $4.7 \mathrm{E}-05 \pm 7.9 \mathrm{E}-05$ & $\mathrm{U}$ \\
\hline & ${ }^{137} \mathrm{Cs}$ & $7.2 \mathrm{E}-06 \pm 5.8 \mathrm{E}-05$ & $\mathrm{U}$ & & ${ }^{137} \mathrm{Cs}$ & $1.1 \mathrm{E}-07 \pm 1.1 \mathrm{E}-06$ & $\mathrm{U}$ \\
\hline & ${ }^{152} \mathrm{Eu}$ & $-1.0 \mathrm{E}-04 \pm 1.5 \mathrm{E}-04$ & $\mathrm{U}$ & & ${ }^{152} \mathrm{Eu}$ & $1.5 \mathrm{E}-04 \pm 2.0 \mathrm{E}-04$ & $\mathrm{U}$ \\
\hline & ${ }^{154} \mathrm{Eu}$ & $-6.8 \mathrm{E}-05 \pm 2.6 \mathrm{E}-04$ & $\mathrm{U}$ & & ${ }^{154} \mathrm{Eu}$ & $8.0 \mathrm{E}-05 \pm 2.6 \mathrm{E}-04$ & $\mathrm{U}$ \\
\hline & ${ }^{155} \mathrm{Eu}$ & $8.4 \mathrm{E}-05 \pm 1.5 \mathrm{E}-04$ & $\mathrm{U}$ & & ${ }^{155} \mathrm{Eu}$ & $4.5 \mathrm{E}-05 \pm 1.8 \mathrm{E}-04$ & $\mathrm{U}$ \\
\hline & ${ }^{238} \mathrm{Pu}$ & $3.5 \mathrm{E}-06 \pm 6.4 \mathrm{E}-06$ & $\mathrm{U}$ & & ${ }^{238} \mathrm{Pu}$ & $-5.2 \mathrm{E}-06 \pm 9.5 \mathrm{E}-06$ & $\mathrm{U}$ \\
\hline & ${ }^{239 / 240} \mathrm{Pu}$ & $2.6 \mathrm{E}-06 \pm 4.0 \mathrm{E}-06$ & $\mathrm{U}$ & & ${ }^{239 / 240} \mathrm{Pu}$ & $2.6 \mathrm{E}-06 \pm 4.0 \mathrm{E}-06$ & $\mathrm{U}$ \\
\hline & ${ }^{103} \mathrm{Ru}$ & $-1.9 \mathrm{E}-06 \pm 1.9 \mathrm{E}-05$ & $\mathrm{U}$ & & ${ }^{103} \mathrm{Ru}$ & $3.4 \mathrm{E}-05 \pm 7.7 \mathrm{E}-05$ & $\mathrm{U}$ \\
\hline & ${ }^{106} \mathrm{Ru}$ & $9.5 \mathrm{E}-05 \pm 4.9 \mathrm{E}-04$ & $\mathrm{U}$ & & ${ }^{106} \mathrm{Ru}$ & $3.7 \mathrm{E}-04 \pm 6.7 \mathrm{E}-04$ & $\mathrm{U}$ \\
\hline & ${ }^{125} \mathrm{Sb}$ & $-1.1 \mathrm{E}-05 \pm 1.1 \mathrm{E}-04$ & $\mathrm{U}$ & & ${ }^{125} \mathrm{Sb}$ & $-1.4 \mathrm{E}-04 \pm 1.9 \mathrm{E}-04$ & $\mathrm{U}$ \\
\hline & ${ }^{113} \mathrm{Sn}$ & $2.4 \mathrm{E}-04 \pm 2.0 \mathrm{E}-04$ & $\mathrm{U}$ & & ${ }^{113} \mathrm{Sn}$ & $-3.9 \mathrm{E}-05 \pm 8.1 \mathrm{E}-05$ & $\mathrm{U}$ \\
\hline & ${ }^{90} \mathrm{Sr}$ & $-2.9 \mathrm{E}-05 \pm 1.1 \mathrm{E}-04$ & $\mathrm{U}$ & & ${ }^{90} \mathrm{Sr}$ & $-2.2 \mathrm{E}-05 \pm 9.0 \mathrm{E}-05$ & $\mathrm{U}$ \\
\hline & ${ }^{234} \mathrm{U}$ & $9.4 \mathrm{E}-06 \pm 6.8 \mathrm{E}-06$ & & & ${ }^{234} \mathrm{U}$ & $9.7 \mathrm{E}-06 \pm 6.7 \mathrm{E}-06$ & \\
\hline & ${ }^{235} \mathrm{U}$ & $8.0 \mathrm{E}-07 \pm 8.3 \mathrm{E}-07$ & $\mathrm{U}$ & & ${ }^{235} \mathrm{U}$ & $-7.3 \mathrm{E}-07 \pm 1.5 \mathrm{E}-06$ & $\mathrm{U}$ \\
\hline & ${ }^{238} \mathrm{U}$ & $9.4 \mathrm{E}-06 \pm 6.5 \mathrm{E}-06$ & & & ${ }^{238} \mathrm{U}$ & $7.4 \mathrm{E}-06 \pm 5.9 \mathrm{E}-06$ & \\
\hline & ${ }^{65} \mathrm{Zn}$ & $1.4 \mathrm{E}-04 \pm 2.2 \mathrm{E}-04$ & $\mathrm{U}$ & & ${ }^{65} \mathrm{Zn}$ & $-1.5 \mathrm{E}-04 \pm 1.8 \mathrm{E}-04$ & $\mathrm{U}$ \\
\hline
\end{tabular}

$\overline{\mathrm{RQ}}=$ Result Qualifier. $\mathrm{U}=$ The analyte was analyzed for but not detected. 
Table 2-3. Near-Facility Air Sampling Results, 2003 (pCi/m³ \pm total analytical uncertainty). (cont)

\begin{tabular}{|c|c|c|c|c|c|c|c|}
\hline Location & Isotope & Result \pm Uncertainty & $\mathbf{R Q}^{*}$ & Location & Isotope & Result \pm Uncertainty & RQ* \\
\hline N966 (200-West) & ${ }^{144} \mathrm{Ce}$ & 7.9E-05 $\pm 6.6 \mathrm{E}-04$ & $\mathrm{U}$ & N974 (200-West) & ${ }^{144} \mathrm{Ce}$ & $-3.5 \mathrm{E}-04 \pm 7.6 \mathrm{E}-04$ & $\mathrm{U}$ \\
\hline Composite Period & ${ }^{60} \mathrm{Co}$ & $-1.2 \mathrm{E}-04 \pm 1.3 \mathrm{E}-04$ & $\mathrm{U}$ & Composite Period & ${ }^{60} \mathrm{Co}$ & 4.7E-06 $\pm 4.7 \mathrm{E}-05$ & $\mathrm{U}$ \\
\hline \multirow[t]{16}{*}{$06 / 23 / 03$ to $12 / 22 / 03$} & ${ }^{134} \mathrm{Cs}$ & $-4.4 \mathrm{E}-05 \pm 8.6 \mathrm{E}-05$ & $\mathrm{U}$ & $01 / 06 / 03$ to $06 / 23 / 03$ & ${ }^{134} \mathrm{Cs}$ & $5.6 \mathrm{E}-06 \pm 5.7 \mathrm{E}-05$ & $\mathrm{U}$ \\
\hline & ${ }^{137} \mathrm{Cs}$ & $-1.4 \mathrm{E}-05 \pm 7.5 \mathrm{E}-05$ & $\mathrm{U}$ & & ${ }^{137} \mathrm{Cs}$ & $8.1 \mathrm{E}-05 \pm 9.4 \mathrm{E}-05$ & $\mathrm{U}$ \\
\hline & ${ }^{152} \mathrm{Eu}$ & $7.4 \mathrm{E}-05 \pm 1.8 \mathrm{E}-04$ & U & & ${ }^{152} \mathrm{Eu}$ & $3.3 \mathrm{E}-05 \pm 1.9 \mathrm{E}-04$ & $\mathrm{U}$ \\
\hline & ${ }^{154} \mathrm{Eu}$ & $2.2 \mathrm{E}-04 \pm 2.4 \mathrm{E}-04$ & $\mathrm{U}$ & & ${ }^{154} \mathrm{Eu}$ & $1.2 \mathrm{E}-05 \pm 1.2 \mathrm{E}-04$ & $\mathrm{U}$ \\
\hline & ${ }^{155} \mathrm{Eu}$ & $-1.2 \mathrm{E}-04 \pm 1.7 \mathrm{E}-04$ & $\mathrm{U}$ & & ${ }^{155} \mathrm{Eu}$ & $4.0 \mathrm{E}-05 \pm 2.1 \mathrm{E}-04$ & $\mathrm{U}$ \\
\hline & ${ }^{238} \mathrm{Pu}$ & $7.8 \mathrm{E}-07 \pm 2.7 \mathrm{E}-06$ & $\mathrm{U}$ & & ${ }^{238} \mathrm{Pu}$ & $-4.7 \mathrm{E}-06 \pm 1.2 \mathrm{E}-05$ & $\mathrm{U}$ \\
\hline & ${ }^{239 / 240} \mathrm{Pu}$ & $4.0 \mathrm{E}-06 \pm 4.6 \mathrm{E}-06$ & $\mathrm{U}$ & & ${ }^{239 / 240} \mathrm{Pu}$ & $3.1 \mathrm{E}-06 \pm 4.4 \mathrm{E}-06$ & $\mathrm{U}$ \\
\hline & ${ }^{103} \mathrm{Ru}$ & $-1.8 \mathrm{E}-05 \pm 7.5 \mathrm{E}-05$ & $\mathrm{U}$ & & ${ }^{103} \mathrm{Ru}$ & $-2.0 \mathrm{E}-05 \pm 8.3 \mathrm{E}-05$ & $\mathrm{U}$ \\
\hline & ${ }^{106} \mathrm{Ru}$ & $-2.5 \mathrm{E}-04 \pm 6.0 \mathrm{E}-04$ & $\mathrm{U}$ & & ${ }^{106} \mathrm{Ru}$ & $-1.8 \mathrm{E}-04 \pm 7.9 \mathrm{E}-04$ & $\mathrm{U}$ \\
\hline & ${ }^{125} \mathrm{Sb}$ & $-5.8 \mathrm{E}-05 \pm 1.7 \mathrm{E}-04$ & $\mathrm{U}$ & & ${ }^{125} \mathrm{Sb}$ & $4.4 \mathrm{E}-05 \pm 2.0 \mathrm{E}-04$ & $\mathrm{U}$ \\
\hline & ${ }^{113} \mathrm{Sn}$ & $-2.1 \mathrm{E}-05 \pm 7.1 \mathrm{E}-05$ & $\mathrm{U}$ & & ${ }^{113} \mathrm{Sn}$ & $-5.8 \mathrm{E}-05 \pm 9.7 \mathrm{E}-05$ & $\mathrm{U}$ \\
\hline & ${ }^{90} \mathrm{Sr}$ & $-5.7 \mathrm{E}-05 \pm 9.2 \mathrm{E}-05$ & $\mathrm{U}$ & & ${ }^{90} \mathrm{Sr}$ & $-1.2 \mathrm{E}-04 \pm 1.2 \mathrm{E}-04$ & $\mathrm{U}$ \\
\hline & ${ }^{234} \mathrm{U}$ & $1.4 \mathrm{E}-05 \pm 9.4 \mathrm{E}-06$ & & & ${ }^{234} \mathrm{U}$ & $7.7 \mathrm{E}-06 \pm 5.8 \mathrm{E}-06$ & \\
\hline & ${ }^{235} \mathrm{U}$ & $8.5 \mathrm{E}-07 \pm 8.9 \mathrm{E}-07$ & $\mathrm{U}$ & & ${ }^{235} \mathrm{U}$ & $7.0 \mathrm{E}-07 \pm 7.3 \mathrm{E}-07$ & $\mathrm{U}$ \\
\hline & ${ }^{238} \mathrm{U}$ & $1.5 \mathrm{E}-05 \pm 9.0 \mathrm{E}-06$ & & & ${ }^{238} \mathrm{U}$ & $5.6 \mathrm{E}-06 \pm 4.9 \mathrm{E}-06$ & \\
\hline & ${ }^{65} \mathrm{Zn}$ & $5.0 \mathrm{E}-05 \pm 2.0 \mathrm{E}-04$ & $\mathrm{U}$ & & ${ }^{65} \mathrm{Zn}$ & $2.4 \mathrm{E}-04 \pm 2.3 \mathrm{E}-04$ & $\mathrm{U}$ \\
\hline N974 (200-West) & ${ }^{144} \mathrm{Ce}$ & $2.4 \mathrm{E}-04 \pm 6.2 \mathrm{E}-04$ & $\bar{U}$ & N975 (200-West) & ${ }^{144} \mathrm{Ce}$ & $3.6 \mathrm{E}-04 \pm 5.6 \mathrm{E}-04$ & $\mathrm{U}$ \\
\hline Composite Period & ${ }^{60} \mathrm{Co}$ & $-3.9 \mathrm{E}-05 \pm 8.1 \mathrm{E}-05$ & $\mathrm{U}$ & Composite Period & ${ }^{60} \mathrm{Co}$ & $-7.3 \mathrm{E}-06 \pm 7.3 \mathrm{E}-05$ & $\mathrm{U}$ \\
\hline \multirow[t]{16}{*}{$06 / 23 / 03$ to $12 / 22 / 03$} & ${ }^{134} \mathrm{Cs}$ & $-2.2 \mathrm{E}-05 \pm 7.0 \mathrm{E}-05$ & $\mathrm{U}$ & $01 / 06 / 03$ to $06 / 23 / 03$ & ${ }^{134} \mathrm{Cs}$ & $-8.4 \mathrm{E}-06 \pm 7.6 \mathrm{E}-05$ & $\mathrm{U}$ \\
\hline & ${ }^{137} \mathrm{Cs}$ & $-2.2 \mathrm{E}-05 \pm 6.1 \mathrm{E}-05$ & $\mathrm{U}$ & & ${ }^{137} \mathrm{Cs}$ & $5.3 \mathrm{E}-05 \pm 7.2 \mathrm{E}-05$ & $\mathrm{U}$ \\
\hline & ${ }^{152} \mathrm{Eu}$ & $-6.7 \mathrm{E}-05 \pm 1.6 \mathrm{E}-04$ & $\mathrm{U}$ & & ${ }^{152} \mathrm{Eu}$ & $1.0 \mathrm{E}-04 \pm 1.6 \mathrm{E}-04$ & $\mathrm{U}$ \\
\hline & ${ }^{154} \mathrm{Eu}$ & $2.5 \mathrm{E}-04 \pm 2.4 \mathrm{E}-04$ & $\mathrm{U}$ & & ${ }^{154} \mathrm{Eu}$ & $-8.2 \mathrm{E}-05 \pm 2.7 \mathrm{E}-04$ & $\mathrm{U}$ \\
\hline & ${ }^{155} \mathrm{Eu}$ & $-3.0 \mathrm{E}-05 \pm 1.5 \mathrm{E}-04$ & $\mathrm{U}$ & & ${ }^{155} \mathrm{Eu}$ & $-4.5 \mathrm{E}-06 \pm 4.5 \mathrm{E}-05$ & $\mathrm{U}$ \\
\hline & ${ }^{238} \mathrm{Pu}$ & $9.7 \mathrm{E}-06 \pm 1.4 \mathrm{E}-05$ & $\mathrm{U}$ & & ${ }^{238} \mathrm{Pu}$ & $7.7 \mathrm{E}-07 \pm 8.0 \mathrm{E}-07$ & $\mathrm{U}$ \\
\hline & ${ }^{239 / 240} \mathrm{Pu}$ & $3.7 \mathrm{E}-06 \pm 6.8 \mathrm{E}-06$ & $\mathrm{U}$ & & ${ }^{239 / 240} \mathrm{Pu}$ & $9.3 \mathrm{E}-06 \pm 6.7 \mathrm{E}-06$ & \\
\hline & ${ }^{103} \mathrm{Ru}$ & $-1.8 \mathrm{E}-05 \pm 5.9 \mathrm{E}-05$ & $\mathrm{U}$ & & ${ }^{103} \mathrm{Ru}$ & $-3.3 \mathrm{E}-05 \pm 7.0 \mathrm{E}-05$ & $\mathrm{U}$ \\
\hline & ${ }^{106} \mathrm{Ru}$ & $1.7 \mathrm{E}-04 \pm 4.7 \mathrm{E}-04$ & $\mathrm{U}$ & & ${ }^{106} \mathrm{Ru}$ & $3.2 \mathrm{E}-04 \pm 6.5 \mathrm{E}-04$ & $\mathrm{U}$ \\
\hline & ${ }^{125} \mathrm{Sb}$ & $-8.3 \mathrm{E}-05 \pm 1.5 \mathrm{E}-04$ & $\mathrm{U}$ & & ${ }^{125} \mathrm{Sb}$ & $3.9 \mathrm{E}-06 \pm 3.9 \mathrm{E}-05$ & $\mathrm{U}$ \\
\hline & ${ }^{113} \mathrm{Sn}$ & $-4.6 \mathrm{E}-05 \pm 6.6 \mathrm{E}-05$ & $\mathrm{U}$ & & ${ }^{113} \mathrm{Sn}$ & $-7.2 \mathrm{E}-05 \pm 8.1 \mathrm{E}-05$ & $\mathrm{U}$ \\
\hline & ${ }^{90} \mathrm{Sr}$ & $-1.7 \mathrm{E}-04 \pm 1.8 \mathrm{E}-04$ & $\mathrm{U}$ & & ${ }^{90} \mathrm{Sr}$ & $-3.9 \mathrm{E}-05 \pm 9.8 \mathrm{E}-05$ & $\mathrm{U}$ \\
\hline & ${ }^{234} \mathrm{U}$ & $1.2 \mathrm{E}-05 \pm 8.1 \mathrm{E}-06$ & & & ${ }^{234} \mathrm{U}$ & $1.4 \mathrm{E}-05 \pm 8.3 \mathrm{E}-06$ & \\
\hline & ${ }^{235} \mathrm{U}$ & $7.5 \mathrm{E}-07 \pm 1.5 \mathrm{E}-06$ & $\mathrm{U}$ & & ${ }^{235} \mathrm{U}$ & $3.1 \mathrm{E}-06 \pm 3.2 \mathrm{E}-06$ & \\
\hline & ${ }^{238} \mathrm{U}$ & $1.3 \mathrm{E}-05 \pm 8.1 \mathrm{E}-06$ & & & ${ }^{238} \mathrm{U}$ & $4.9 \mathrm{E}-06 \pm 4.6 \mathrm{E}-06$ & $\mathrm{U}$ \\
\hline & ${ }^{65} \mathrm{Zn}$ & $3.8 \mathrm{E}-05 \pm 1.6 \mathrm{E}-04$ & $\mathrm{U}$ & & ${ }^{65} \mathrm{Zn}$ & $-1.8 \mathrm{E}-04 \pm 1.9 \mathrm{E}-04$ & $\mathrm{U}$ \\
\hline
\end{tabular}

$\overline{\mathrm{RQ}}=$ Result Qualifier. $\mathrm{U}=$ The analyte was analyzed for but not detected. 
Table 2-3. Near-Facility Air Sampling Results, 2003 (pCi/m³ \pm total analytical uncertainty). (cont)

\begin{tabular}{|c|c|c|c|c|c|c|c|}
\hline Location & Isotope & Result \pm Uncertainty & $\mathbf{R Q}^{*}$ & Location & Isotope & Result \pm Uncertainty & $\mathbf{R Q}^{*}$ \\
\hline N975 (200-West) & ${ }^{144} \mathrm{Ce}$ & $3.8 \mathrm{E}-05 \pm 3.8 \mathrm{E}-04$ & $\mathrm{U}$ & N987 (200-West) & ${ }^{144} \mathrm{Ce}$ & $3.0 \mathrm{E}-04 \pm 8.0 \mathrm{E}-04$ & $\mathrm{U}$ \\
\hline Composite Period & ${ }^{60} \mathrm{Co}$ & $-4.3 \mathrm{E}-05 \pm 9.0 \mathrm{E}-05$ & $\mathrm{U}$ & Composite Period & ${ }^{60} \mathrm{Co}$ & $-3.9 \mathrm{E}-05 \pm 9.2 \mathrm{E}-05$ & $\mathrm{U}$ \\
\hline \multirow[t]{16}{*}{$06 / 23 / 03$ to $12 / 22 / 03$} & ${ }^{134} \mathrm{Cs}$ & $-4.5 \mathrm{E}-05 \pm 8.8 \mathrm{E}-05$ & $\mathrm{U}$ & $01 / 06 / 03$ to $06 / 23 / 03$ & ${ }^{134} \mathrm{Cs}$ & $-3.5 \mathrm{E}-05 \pm 8.0 \mathrm{E}-05$ & $\mathrm{U}$ \\
\hline & ${ }^{137} \mathrm{Cs}$ & $2.5 \mathrm{E}-05 \pm 7.5 \mathrm{E}-05$ & $\mathrm{U}$ & & ${ }^{137} \mathrm{Cs}$ & $5.8 \mathrm{E}-05 \pm 8.1 \mathrm{E}-05$ & $\mathrm{U}$ \\
\hline & ${ }^{152} \mathrm{Eu}$ & $-6.5 \mathrm{E}-05 \pm 2.1 \mathrm{E}-04$ & U & & ${ }^{152} \mathrm{Eu}$ & $-1.2 \mathrm{E}-04 \pm 2.4 \mathrm{E}-04$ & $\mathrm{U}$ \\
\hline & ${ }^{154} \mathrm{Eu}$ & $4.3 \mathrm{E}-05 \pm 2.6 \mathrm{E}-04$ & $\mathrm{U}$ & & ${ }^{154} \mathrm{Eu}$ & $1.6 \mathrm{E}-04 \pm 2.1 \mathrm{E}-04$ & $\mathrm{U}$ \\
\hline & ${ }^{155} \mathrm{Eu}$ & $6.2 \mathrm{E}-05 \pm 1.8 \mathrm{E}-04$ & $\mathrm{U}$ & & ${ }^{155} \mathrm{Eu}$ & $-1.7 \mathrm{E}-04 \pm 2.2 \mathrm{E}-04$ & $\mathrm{U}$ \\
\hline & ${ }^{238} \mathrm{Pu}$ & $8.8 \mathrm{E}-06 \pm 1.3 \mathrm{E}-05$ & $\mathrm{U}$ & & ${ }^{238} \mathrm{Pu}$ & $-5.7 \mathrm{E}-06 \pm 1.2 \mathrm{E}-05$ & $\mathrm{U}$ \\
\hline & ${ }^{239 / 240} \mathrm{Pu}$ & $3.3 \mathrm{E}-05 \pm 1.7 \mathrm{E}-05$ & & & ${ }^{239 / 240} \mathrm{Pu}$ & $3.2 \mathrm{E}-06 \pm 3.9 \mathrm{E}-06$ & $\mathrm{U}$ \\
\hline & ${ }^{103} \mathrm{Ru}$ & $1.4 \mathrm{E}-05 \pm 7.9 \mathrm{E}-05$ & $\mathrm{U}$ & & ${ }^{103} \mathrm{Ru}$ & $1.1 \mathrm{E}-05 \pm 8.1 \mathrm{E}-05$ & $\mathrm{U}$ \\
\hline & ${ }^{106} \mathrm{Ru}$ & $6.4 \mathrm{E}-04 \pm 6.8 \mathrm{E}-04$ & $\mathrm{U}$ & & ${ }^{106} \mathrm{Ru}$ & $2.9 \mathrm{E}-04 \pm 6.9 \mathrm{E}-04$ & $\mathrm{U}$ \\
\hline & ${ }^{125} \mathrm{Sb}$ & $-2.5 \mathrm{E}-04 \pm 2.6 \mathrm{E}-04$ & $\mathrm{U}$ & & ${ }^{125} \mathrm{Sb}$ & $-7.8 \mathrm{E}-05 \pm 1.8 \mathrm{E}-04$ & $\mathrm{U}$ \\
\hline & ${ }^{113} \mathrm{Sn}$ & $-3.9 \mathrm{E}-05 \pm 7.9 \mathrm{E}-05$ & $\mathrm{U}$ & & ${ }^{113} \mathrm{Sn}$ & $-4.6 \mathrm{E}-05 \pm 9.3 \mathrm{E}-05$ & $\mathrm{U}$ \\
\hline & ${ }^{90} \mathrm{Sr}$ & $-6.6 \mathrm{E}-05 \pm 1.0 \mathrm{E}-04$ & $\mathrm{U}$ & & ${ }^{90} \mathrm{Sr}$ & $-3.1 \mathrm{E}-05 \pm 9.7 \mathrm{E}-05$ & $\mathrm{U}$ \\
\hline & ${ }^{234} \mathrm{U}$ & $1.8 \mathrm{E}-05 \pm 1.0 \mathrm{E}-05$ & & & ${ }^{234} \mathrm{U}$ & $9.2 \mathrm{E}-06 \pm 6.5 \mathrm{E}-06$ & \\
\hline & ${ }^{235} \mathrm{U}$ & $5.2 \mathrm{E}-06 \pm 4.4 \mathrm{E}-06$ & & & ${ }^{235} \mathrm{U}$ & $-7.6 \mathrm{E}-07 \pm 3.4 \mathrm{E}-06$ & $\mathrm{U}$ \\
\hline & ${ }^{238} \mathrm{U}$ & $7.4 \mathrm{E}-06 \pm 6.1 \mathrm{E}-06$ & & & ${ }^{238} \mathrm{U}$ & $7.2 \mathrm{E}-06 \pm 5.7 \mathrm{E}-06$ & \\
\hline & ${ }^{65} \mathrm{Zn}$ & $1.0 \mathrm{E}-04 \pm 2.1 \mathrm{E}-04$ & $\mathrm{U}$ & & ${ }^{65} \mathrm{Zn}$ & $-7.0 \mathrm{E}-05 \pm 1.8 \mathrm{E}-04$ & $\mathrm{U}$ \\
\hline N987 (200-West) & ${ }^{144} \mathrm{Ce}$ & $6.2 \mathrm{E}-04 \pm 7.6 \mathrm{E}-04$ & $\bar{U}$ & N994 (200-West) & ${ }^{144} \mathrm{Ce}$ & $3.0 \mathrm{E}-04 \pm 7.7 \mathrm{E}-04$ & $\mathrm{U}$ \\
\hline Composite Period & ${ }^{60} \mathrm{Co}$ & $8.1 \mathrm{E}-07 \pm 8.1 \mathrm{E}-06$ & $\mathrm{U}$ & Composite Period & ${ }^{60} \mathrm{Co}$ & $-6.8 \mathrm{E}-05 \pm 9.5 \mathrm{E}-05$ & $\mathrm{U}$ \\
\hline \multirow[t]{16}{*}{$06 / 23 / 03$ to $12 / 22 / 03$} & ${ }^{134} \mathrm{Cs}$ & $-4.0 \mathrm{E}-05 \pm 9.1 \mathrm{E}-05$ & $\mathrm{U}$ & $01 / 06 / 03$ to $06 / 23 / 03$ & ${ }^{134} \mathrm{Cs}$ & $5.5 \mathrm{E}-05 \pm 5.8 \mathrm{E}-05$ & $\mathrm{U}$ \\
\hline & ${ }^{137} \mathrm{Cs}$ & $8.1 \mathrm{E}-05 \pm 8.3 \mathrm{E}-05$ & $\mathrm{U}$ & & ${ }^{137} \mathrm{Cs}$ & $-1.5 \mathrm{E}-05 \pm 7.6 \mathrm{E}-05$ & $\mathrm{U}$ \\
\hline & ${ }^{152} \mathrm{Eu}$ & $1.2 \mathrm{E}-04 \pm 2.0 \mathrm{E}-04$ & $\mathrm{U}$ & & ${ }^{152} \mathrm{Eu}$ & $2.4 \mathrm{E}-05 \pm 1.8 \mathrm{E}-04$ & $\mathrm{U}$ \\
\hline & ${ }^{154} \mathrm{Eu}$ & $2.3 \mathrm{E}-04 \pm 2.8 \mathrm{E}-04$ & $\mathrm{U}$ & & ${ }^{154} \mathrm{Eu}$ & $-2.4 \mathrm{E}-04 \pm 2.6 \mathrm{E}-04$ & $\mathrm{U}$ \\
\hline & ${ }^{155} \mathrm{Eu}$ & $5.7 \mathrm{E}-05 \pm 1.8 \mathrm{E}-04$ & $\mathrm{U}$ & & ${ }^{155} \mathrm{Eu}$ & $2.9 \mathrm{E}-05 \pm 1.9 \mathrm{E}-04$ & $\mathrm{U}$ \\
\hline & ${ }^{238} \mathrm{Pu}$ & $1.3 \mathrm{E}-06 \pm 1.3 \mathrm{E}-06$ & $\mathrm{U}$ & & ${ }^{238} \mathrm{Pu}$ & $-7.1 \mathrm{E}-06 \pm 1.4 \mathrm{E}-05$ & $\mathrm{U}$ \\
\hline & ${ }^{239 / 240} \mathrm{Pu}$ & $3.8 \mathrm{E}-06 \pm 8.4 \mathrm{E}-06$ & $\mathrm{U}$ & & ${ }^{239 / 240} \mathrm{Pu}$ & $9.1 \mathrm{E}-07 \pm 3.2 \mathrm{E}-06$ & $\mathrm{U}$ \\
\hline & ${ }^{103} \mathrm{Ru}$ & $1.5 \mathrm{E}-05 \pm 7.9 \mathrm{E}-05$ & $\mathrm{U}$ & & ${ }^{103} \mathrm{Ru}$ & $-6.1 \mathrm{E}-06 \pm 6.1 \mathrm{E}-05$ & $\mathrm{U}$ \\
\hline & ${ }^{106} \mathrm{Ru}$ & $-1.0 \mathrm{E}-04 \pm 6.6 \mathrm{E}-04$ & $\mathrm{U}$ & & ${ }^{106} \mathrm{Ru}$ & $-5.0 \mathrm{E}-04 \pm 7.4 \mathrm{E}-04$ & $\mathrm{U}$ \\
\hline & ${ }^{125} \mathrm{Sb}$ & $-8.9 \mathrm{E}-06 \pm 8.9 \mathrm{E}-05$ & $\mathrm{U}$ & & ${ }^{125} \mathrm{Sb}$ & $2.8 \mathrm{E}-04 \pm 2.1 \mathrm{E}-04$ & $\mathrm{U}$ \\
\hline & ${ }^{113} \mathrm{Sn}$ & 5.7E-06 \pm 5.7E-05 & $\mathrm{U}$ & & ${ }^{113} \mathrm{Sn}$ & $3.6 \mathrm{E}-05 \pm 9.2 \mathrm{E}-05$ & $\mathrm{U}$ \\
\hline & ${ }^{90} \mathrm{Sr}$ & $-1.1 \mathrm{E}-04 \pm 1.2 \mathrm{E}-04$ & $\mathrm{U}$ & & ${ }^{90} \mathrm{Sr}$ & $-3.8 \mathrm{E}-05 \pm 9.6 \mathrm{E}-05$ & $\mathrm{U}$ \\
\hline & ${ }^{234} \mathrm{U}$ & $1.1 \mathrm{E}-05 \pm 8.5 \mathrm{E}-06$ & & & ${ }^{234} \mathrm{U}$ & $5.2 \mathrm{E}-06 \pm 4.9 \mathrm{E}-06$ & $\mathrm{U}$ \\
\hline & ${ }^{235} \mathrm{U}$ & $5.2 \mathrm{E}-06 \pm 4.7 \mathrm{E}-06$ & & & ${ }^{235} \mathrm{U}$ & $4.9 \mathrm{E}-06 \pm 4.5 \mathrm{E}-06$ & \\
\hline & ${ }^{238} \mathrm{U}$ & $1.4 \mathrm{E}-05 \pm 8.5 \mathrm{E}-06$ & & & ${ }^{238} \mathrm{U}$ & $7.5 \mathrm{E}-06 \pm 5.9 \mathrm{E}-06$ & \\
\hline & ${ }^{65} \mathrm{Zn}$ & $1.2 \mathrm{E}-04 \pm 2.2 \mathrm{E}-04$ & $\mathrm{U}$ & & ${ }^{65} \mathrm{Zn}$ & $-2.2 \mathrm{E}-04 \pm 2.3 \mathrm{E}-04$ & $\mathrm{U}$ \\
\hline
\end{tabular}

$\overline{\mathrm{RQ}}=$ Result Qualifier. $\mathrm{U}=$ The analyte was analyzed for but not detected. 
Table 2-3. Near-Facility Air Sampling Results, 2003 (pCi/m³ \pm total analytical uncertainty). (cont)

\begin{tabular}{|c|c|c|c|c|c|c|c|}
\hline Location & Isotope & Result \pm Uncertainty & $\mathbf{R Q}^{*}$ & Location & Isotope & Result \pm Uncertainty & RQ* \\
\hline N994 (200-West) & ${ }^{144} \mathrm{Ce}$ & $-3.2 \mathrm{E}-04 \pm 6.0 \mathrm{E}-04$ & $\bar{U}$ & N485 (300-FF-1\&2) & ${ }^{144} \mathrm{Ce}$ & $-8.0 \mathrm{E}-05 \pm 8.0 \mathrm{E}-04$ & $\bar{U}$ \\
\hline Composite Period & ${ }^{60} \mathrm{Co}$ & $-5.5 \mathrm{E}-05 \pm 7.8 \mathrm{E}-05$ & $\mathrm{U}$ & Composite Period & ${ }^{60} \mathrm{Co}$ & $1.3 \mathrm{E}-05 \pm 8.3 \mathrm{E}-05$ & $\mathrm{U}$ \\
\hline \multirow[t]{16}{*}{$06 / 23 / 03$ to $12 / 22 / 03$} & ${ }^{134} \mathrm{Cs}$ & $-1.8 \mathrm{E}-05 \pm 7.4 \mathrm{E}-05$ & $\mathrm{U}$ & $01 / 07 / 03$ to $06 / 24 / 03$ & ${ }^{134} \mathrm{Cs}$ & $-5.3 \mathrm{E}-05 \pm 8.6 \mathrm{E}-05$ & $\mathrm{U}$ \\
\hline & ${ }^{137} \mathrm{Cs}$ & $-1.6 \mathrm{E}-05 \pm 6.0 \mathrm{E}-05$ & U & & ${ }^{137} \mathrm{Cs}$ & $8.4 \mathrm{E}-05 \pm 8.7 \mathrm{E}-05$ & $\mathrm{U}$ \\
\hline & ${ }^{152} \mathrm{Eu}$ & $9.2 \mathrm{E}-05 \pm 1.6 \mathrm{E}-04$ & U & & ${ }^{152} \mathrm{Eu}$ & $-1.6 \mathrm{E}-04 \pm 2.5 \mathrm{E}-04$ & $\mathrm{U}$ \\
\hline & ${ }^{154} \mathrm{Eu}$ & $-2.3 \mathrm{E}-04 \pm 2.7 \mathrm{E}-04$ & $\mathrm{U}$ & & ${ }^{154} \mathrm{Eu}$ & $3.3 \mathrm{E}-04 \pm 3.3 \mathrm{E}-04$ & $\mathrm{U}$ \\
\hline & ${ }^{155} \mathrm{Eu}$ & $-1.9 \mathrm{E}-05 \pm 1.5 \mathrm{E}-04$ & U & & ${ }^{155} \mathrm{Eu}$ & $-2.1 \mathrm{E}-05 \pm 2.1 \mathrm{E}-04$ & $\mathrm{U}$ \\
\hline & ${ }^{238} \mathrm{Pu}$ & $-1.2 \mathrm{E}-06 \pm 1.0 \mathrm{E}-05$ & $\mathrm{U}$ & & ${ }^{103} \mathrm{Ru}$ & $3.4 \mathrm{E}-05 \pm 8.9 \mathrm{E}-05$ & $\mathrm{U}$ \\
\hline & ${ }^{239 / 240} \mathrm{Pu}$ & $1.2 \mathrm{E}-06 \pm 1.3 \mathrm{E}-06$ & $\mathrm{U}$ & & ${ }^{106} \mathrm{Ru}$ & $2.2 \mathrm{E}-04 \pm 6.6 \mathrm{E}-04$ & $\mathrm{U}$ \\
\hline & ${ }^{103} \mathrm{Ru}$ & $5.3 \mathrm{E}-05 \pm 6.7 \mathrm{E}-05$ & $\mathrm{U}$ & & ${ }^{125} \mathrm{Sb}$ & $4.0 \mathrm{E}-05 \pm 1.8 \mathrm{E}-04$ & $\mathrm{U}$ \\
\hline & ${ }^{106} \mathrm{Ru}$ & $-1.8 \mathrm{E}-04 \pm 5.6 \mathrm{E}-04$ & U & & ${ }^{113} \mathrm{Sn}$ & $-2.5 \mathrm{E}-05 \pm 9.5 \mathrm{E}-05$ & $\mathrm{U}$ \\
\hline & ${ }^{125} \mathrm{Sb}$ & $-6.8 \mathrm{E}-05 \pm 1.6 \mathrm{E}-04$ & $\mathrm{U}$ & & ${ }^{234} \mathrm{U}$ & $3.6 \mathrm{E}-05 \pm 1.7 \mathrm{E}-05$ & \\
\hline & ${ }^{113} \mathrm{Sn}$ & $-1.9 \mathrm{E}-05 \pm 6.7 \mathrm{E}-05$ & $\mathrm{U}$ & & ${ }^{235} \mathrm{U}$ & $4.1 \mathrm{E}-06 \pm 4.2 \mathrm{E}-06$ & $\mathrm{U}$ \\
\hline & ${ }^{90} \mathrm{Sr}$ & $-1.3 \mathrm{E}-04 \pm 1.3 \mathrm{E}-04$ & $\mathrm{U}$ & & ${ }^{238} \mathrm{U}$ & $3.8 \mathrm{E}-05 \pm 1.8 \mathrm{E}-05$ & \\
\hline & ${ }^{234} \mathrm{U}$ & $1.4 \mathrm{E}-05 \pm 8.9 \mathrm{E}-06$ & & & ${ }^{65} \mathrm{Zn}$ & $-2.1 \mathrm{E}-04 \pm 2.2 \mathrm{E}-04$ & $\mathrm{U}$ \\
\hline & ${ }^{235} \mathrm{U}$ & $1.6 \mathrm{E}-06 \pm 3.2 \mathrm{E}-06$ & $\mathrm{U}$ & & & & \\
\hline & ${ }^{238} \mathrm{U}$ & $1.5 \mathrm{E}-05 \pm 8.8 \mathrm{E}-06$ & & & & & \\
\hline & ${ }^{65} \mathrm{Zn}$ & $-1.4 \mathrm{E}-04 \pm 1.8 \mathrm{E}-04$ & $\mathrm{U}$ & & & & \\
\hline N485 (300-FF-1\&2) & ${ }^{144} \mathrm{Ce}$ & $-9.2 \mathrm{E}-04 \pm 1.1 \mathrm{E}-03$ & $\mathrm{U}$ & N486 (300-FF-1\&2) & ${ }^{144} \mathrm{Ce}$ & $4.5 \mathrm{E}-04 \pm 8.3 \mathrm{E}-04$ & $\mathrm{U}$ \\
\hline Composite Period & ${ }^{60} \mathrm{Co}$ & $3.0 \mathrm{E}-04 \pm 1.3 \mathrm{E}-04$ & $\mathrm{U}$ & Composite Period & ${ }^{60} \mathrm{Co}$ & $-1.4 \mathrm{E}-05 \pm 7.5 \mathrm{E}-05$ & $\mathrm{U}$ \\
\hline \multirow[t]{13}{*}{$06 / 24 / 03$ to $09 / 30 / 03$} & ${ }^{134} \mathrm{Cs}$ & $4.7 \mathrm{E}-05 \pm 1.3 \mathrm{E}-04$ & $\mathrm{U}$ & $01 / 07 / 03$ to $06 / 24 / 03$ & ${ }^{134} \mathrm{Cs}$ & $-7.5 \mathrm{E}-05 \pm 9.0 \mathrm{E}-05$ & U \\
\hline & ${ }^{137} \mathrm{Cs}$ & $-9.9 \mathrm{E}-06 \pm 9.9 \mathrm{E}-05$ & $\mathrm{U}$ & & ${ }^{137} \mathrm{Cs}$ & $3.7 \mathrm{E}-05 \pm 7.1 \mathrm{E}-05$ & $\mathrm{U}$ \\
\hline & ${ }^{152} \mathrm{Eu}$ & $-7.6 \mathrm{E}-05 \pm 2.5 \mathrm{E}-04$ & $\mathrm{U}$ & & ${ }^{152} \mathrm{Eu}$ & $-6.8 \mathrm{E}-05 \pm 1.9 \mathrm{E}-04$ & $\mathrm{U}$ \\
\hline & ${ }^{154} \mathrm{Eu}$ & $-1.9 \mathrm{E}-04 \pm 3.9 \mathrm{E}-04$ & $\mathrm{U}$ & & ${ }^{154} \mathrm{Eu}$ & $-1.0 \mathrm{E}-04 \pm 2.2 \mathrm{E}-04$ & $\mathrm{U}$ \\
\hline & ${ }^{155} \mathrm{Eu}$ & $-4.5 E-05 \pm 2.5 E-04$ & $\mathrm{U}$ & & ${ }^{155} \mathrm{Eu}$ & $-2.7 \mathrm{E}-04 \pm 2.8 \mathrm{E}-04$ & $\mathrm{U}$ \\
\hline & ${ }^{103} \mathrm{Ru}$ & $-6.0 \mathrm{E}-05 \pm 1.2 \mathrm{E}-04$ & $\mathrm{U}$ & & ${ }^{103} \mathrm{Ru}$ & $-2.9 \mathrm{E}-05 \pm 8.6 \mathrm{E}-05$ & $\mathrm{U}$ \\
\hline & ${ }^{106} \mathrm{Ru}$ & $-3.2 \mathrm{E}-04 \pm 1.0 \mathrm{E}-03$ & $\mathrm{U}$ & & ${ }^{106} \mathrm{Ru}$ & $6.0 \mathrm{E}-04 \pm 6.7 \mathrm{E}-04$ & $\mathrm{U}$ \\
\hline & ${ }^{125} \mathrm{Sb}$ & $-2.1 \mathrm{E}-04 \pm 2.6 \mathrm{E}-04$ & $\mathrm{U}$ & & ${ }^{125} \mathrm{Sb}$ & $1.6 \mathrm{E}-04 \pm 1.8 \mathrm{E}-04$ & $\mathrm{U}$ \\
\hline & ${ }^{113} \mathrm{Sn}$ & $-8.1 \mathrm{E}-05 \pm 1.2 \mathrm{E}-04$ & $\mathrm{U}$ & & ${ }^{113} \mathrm{Sn}$ & $-3.2 \mathrm{E}-06 \pm 3.2 \mathrm{E}-05$ & $\mathrm{U}$ \\
\hline & ${ }^{234} \mathrm{U}$ & $3.6 \mathrm{E}-05 \pm 1.9 \mathrm{E}-05$ & & & ${ }^{234} \mathrm{U}$ & $2.4 \mathrm{E}-05 \pm 1.2 \mathrm{E}-05$ & \\
\hline & ${ }^{235} \mathrm{U}$ & $4.3 \mathrm{E}-06 \pm 5.2 \mathrm{E}-06$ & & & ${ }^{235} \mathrm{U}$ & $-7.6 \mathrm{E}-07 \pm 2.7 \mathrm{E}-06$ & $\mathrm{U}$ \\
\hline & ${ }^{238} \mathrm{U}$ & $2.0 \mathrm{E}-05 \pm 1.2 \mathrm{E}-05$ & & & ${ }^{238} \mathrm{U}$ & $1.3 \mathrm{E}-05 \pm 7.8 \mathrm{E}-06$ & \\
\hline & ${ }^{65} \mathrm{Zn}$ & $4.9 \mathrm{E}-04 \pm 3.9 \mathrm{E}-04$ & $\mathrm{U}$ & & ${ }^{65} \mathrm{Zn}$ & $1.1 \mathrm{E}-04 \pm 1.7 \mathrm{E}-04$ & $\mathrm{U}$ \\
\hline
\end{tabular}

$\overline{\mathrm{RQ}}=$ Result Qualifier. $\mathrm{U}=$ The analyte was analyzed for but not detected. 
Table 2-3. Near-Facility Air Sampling Results, 2003 (pCi/m³ \pm total analytical uncertainty). (cont)

\begin{tabular}{|c|c|c|c|}
\hline Location & Isotope & Result \pm Uncertainty & RQ* \\
\hline N486 (300-FF-1\&2) & ${ }^{144} \mathrm{Ce}$ & $1.3 \mathrm{E}-03 \pm 1.1 \mathrm{E}-03$ & $\bar{U}$ \\
\hline Composite Period & ${ }^{60} \mathrm{Co}$ & $-1.5 \mathrm{E}-05 \pm 1.4 \mathrm{E}-04$ & $\mathrm{U}$ \\
\hline \multirow[t]{13}{*}{$06 / 24 / 03$ to $09 / 30 / 03$} & ${ }^{134} \mathrm{Cs}$ & $-8.2 \mathrm{E}-06 \pm 8.2 \mathrm{E}-05$ & $\mathrm{U}$ \\
\hline & ${ }^{137} \mathrm{Cs}$ & $-5.0 \mathrm{E}-05 \pm 1.1 \mathrm{E}-04$ & U \\
\hline & ${ }^{152} \mathrm{Eu}$ & $-1.0 \mathrm{E}-04 \pm 2.7 \mathrm{E}-04$ & $\mathrm{U}$ \\
\hline & ${ }^{154} \mathrm{Eu}$ & $-6.1 \mathrm{E}-05 \pm 3.5 \mathrm{E}-04$ & $\mathrm{U}$ \\
\hline & ${ }^{155} \mathrm{Eu}$ & $-2.2 \mathrm{E}-06 \pm 2.2 \mathrm{E}-05$ & $\mathrm{U}$ \\
\hline & ${ }^{103} \mathrm{Ru}$ & $-5.5 \mathrm{E}-07 \pm 5.5 \mathrm{E}-06$ & $\mathrm{U}$ \\
\hline & ${ }^{106} \mathrm{Ru}$ & $1.2 \mathrm{E}-03 \pm 1.0 \mathrm{E}-03$ & $\mathrm{U}$ \\
\hline & ${ }^{125} \mathrm{Sb}$ & $2.0 \mathrm{E}-04 \pm 2.6 \mathrm{E}-04$ & $\mathrm{U}$ \\
\hline & ${ }^{113} \mathrm{Sn}$ & $-3.9 \mathrm{E}-05 \pm 1.4 \mathrm{E}-04$ & $\mathrm{U}$ \\
\hline & ${ }^{234} \mathrm{U}$ & $4.4 \mathrm{E}-05 \pm 2.2 \mathrm{E}-05$ & \\
\hline & ${ }^{235} \mathrm{U}$ & $2.9 \mathrm{E}-06 \pm 4.1 \mathrm{E}-06$ & $\mathrm{U}$ \\
\hline & ${ }^{238} \mathrm{U}$ & $3.4 \mathrm{E}-05 \pm 1.8 \mathrm{E}-05$ & \\
\hline & ${ }^{65} \mathrm{Zn}$ & $3.2 \mathrm{E}-04 \pm 3.6 \mathrm{E}-04$ & $\mathrm{U}$ \\
\hline N487 (300-FF-1\&2) & ${ }^{144} \mathrm{Ce}$ & $5.5 \mathrm{E}-04 \pm 1.2 \mathrm{E}-03$ & $\mathrm{U}$ \\
\hline Composite Period & ${ }^{60} \mathrm{Co}$ & $-4.0 \mathrm{E}-05 \pm 1.6 \mathrm{E}-04$ & $\mathrm{U}$ \\
\hline \multirow[t]{13}{*}{$06 / 24 / 03$ to $09 / 30 / 03$} & ${ }^{134} \mathrm{Cs}$ & $1.1 \mathrm{E}-04 \pm 1.5 \mathrm{E}-04$ & $\mathrm{U}$ \\
\hline & ${ }^{137} \mathrm{Cs}$ & $-1.4 \mathrm{E}-05 \pm 1.3 \mathrm{E}-04$ & $\mathrm{U}$ \\
\hline & ${ }^{152} \mathrm{Eu}$ & $-8.7 \mathrm{E}-05 \pm 2.6 \mathrm{E}-04$ & $\mathrm{U}$ \\
\hline & ${ }^{154} \mathrm{Eu}$ & $3.8 \mathrm{E}-05 \pm 3.8 \mathrm{E}-04$ & $\mathrm{U}$ \\
\hline & ${ }^{155} \mathrm{Eu}$ & $-1.1 \mathrm{E}-05 \pm 1.1 \mathrm{E}-04$ & $\mathrm{U}$ \\
\hline & ${ }^{103} \mathrm{Ru}$ & $-5.1 \mathrm{E}-06 \pm 5.1 \mathrm{E}-05$ & $\mathrm{U}$ \\
\hline & ${ }^{106} \mathrm{Ru}$ & $5.8 \mathrm{E}-04 \pm 1.0 \mathrm{E}-03$ & $\mathrm{U}$ \\
\hline & ${ }^{125} \mathrm{Sb}$ & $-2.0 \mathrm{E}-04 \pm 2.9 \mathrm{E}-04$ & $\mathrm{U}$ \\
\hline & ${ }^{113} \mathrm{Sn}$ & $-9.2 \mathrm{E}-05 \pm 1.3 \mathrm{E}-04$ & $\mathrm{U}$ \\
\hline & ${ }^{234} \mathrm{U}$ & $6.9 \mathrm{E}-05 \pm 2.8 \mathrm{E}-05$ & \\
\hline & ${ }^{235} \mathrm{U}$ & $7.1 \mathrm{E}-06 \pm 6.7 \mathrm{E}-06$ & \\
\hline & ${ }^{238} \mathrm{U}$ & $4.2 \mathrm{E}-05 \pm 1.9 \mathrm{E}-05$ & \\
\hline & ${ }^{65} \mathrm{Zn}$ & $-1.9 \mathrm{E}-04 \pm 3.4 \mathrm{E}-04$ & $\mathrm{U}$ \\
\hline
\end{tabular}

\begin{tabular}{|c|c|c|c|}
\hline Location & Isotope & Result \pm Uncertainty & RQ* \\
\hline N487 (300-FF-1\&2) & ${ }^{144} \mathrm{Ce}$ & $4.3 \mathrm{E}-04 \pm 8.1 \mathrm{E}-04$ & $\bar{U}$ \\
\hline Composite Period & ${ }^{60} \mathrm{Co}$ & 4.3E-05 $\pm 9.2 \mathrm{E}-05$ & $\mathrm{U}$ \\
\hline \multirow[t]{13}{*}{$01 / 07 / 03$ to $06 / 24 / 03$} & ${ }^{134} \mathrm{Cs}$ & $-2.6 \mathrm{E}-05 \pm 8.5 \mathrm{E}-05$ & $\mathrm{U}$ \\
\hline & ${ }^{137} \mathrm{Cs}$ & $2.2 \mathrm{E}-05 \pm 8.1 \mathrm{E}-05$ & $\mathrm{U}$ \\
\hline & ${ }^{152} \mathrm{Eu}$ & $1.3 \mathrm{E}-04 \pm 2.0 \mathrm{E}-04$ & $\mathrm{U}$ \\
\hline & ${ }^{154} \mathrm{Eu}$ & $1.7 \mathrm{E}-04 \pm 2.6 \mathrm{E}-04$ & $\mathrm{U}$ \\
\hline & ${ }^{155} \mathrm{Eu}$ & $-1.2 \mathrm{E}-04 \pm 1.9 \mathrm{E}-04$ & $\mathrm{U}$ \\
\hline & ${ }^{103} \mathrm{Ru}$ & $-1.1 \mathrm{E}-04 \pm 1.1 \mathrm{E}-04$ & $\mathrm{U}$ \\
\hline & ${ }^{106} \mathrm{Ru}$ & $-2.8 \mathrm{E}-04 \pm 6.8 \mathrm{E}-04$ & $\mathrm{U}$ \\
\hline & ${ }^{125} \mathrm{Sb}$ & $6.7 \mathrm{E}-05 \pm 1.8 \mathrm{E}-04$ & $\mathrm{U}$ \\
\hline & ${ }^{113} \mathrm{Sn}$ & $1.0 \mathrm{E}-04 \pm 1.0 \mathrm{E}-04$ & $\mathrm{U}$ \\
\hline & ${ }^{234} \mathrm{U}$ & $4.2 \mathrm{E}-05 \pm 1.9 \mathrm{E}-05$ & \\
\hline & ${ }^{235} \mathrm{U}$ & $5.0 \mathrm{E}-06 \pm 4.5 \mathrm{E}-06$ & \\
\hline & ${ }^{238} \mathrm{U}$ & $4.0 \mathrm{E}-05 \pm 1.8 \mathrm{E}-05$ & \\
\hline & ${ }^{65} \mathrm{Zn}$ & $-2.0 \mathrm{E}-05 \pm 2.0 \mathrm{E}-04$ & $\mathrm{U}$ \\
\hline N527 (300-FF-1\&2) & ${ }^{144} \mathrm{Ce}$ & $-4.0 \mathrm{E}-04 \pm 7.9 \mathrm{E}-04$ & $\mathrm{U}$ \\
\hline Composite Period & ${ }^{60} \mathrm{Co}$ & $-1.9 \mathrm{E}-05 \pm 9.5 \mathrm{E}-05$ & $\mathrm{U}$ \\
\hline \multirow[t]{13}{*}{$01 / 07 / 03$ to $06 / 24 / 03$} & ${ }^{134} \mathrm{Cs}$ & $-4.3 \mathrm{E}-05 \pm 8.7 \mathrm{E}-05$ & $\mathrm{U}$ \\
\hline & ${ }^{137} \mathrm{Cs}$ & $6.9 \mathrm{E}-05 \pm 7.9 \mathrm{E}-05$ & $\mathrm{U}$ \\
\hline & ${ }^{152} \mathrm{Eu}$ & $-3.6 \mathrm{E}-05 \pm 1.9 \mathrm{E}-04$ & $\mathrm{U}$ \\
\hline & ${ }^{154} \mathrm{Eu}$ & $1.5 \mathrm{E}-04 \pm 2.7 \mathrm{E}-04$ & $\mathrm{U}$ \\
\hline & ${ }^{155} \mathrm{Eu}$ & $1.0 \mathrm{E}-04 \pm 1.8 \mathrm{E}-04$ & $\mathrm{U}$ \\
\hline & ${ }^{103} \mathrm{Ru}$ & $-5.8 \mathrm{E}-05 \pm 9.5 \mathrm{E}-05$ & $\mathrm{U}$ \\
\hline & ${ }^{106} \mathrm{Ru}$ & $-5.8 \mathrm{E}-06 \pm 5.8 \mathrm{E}-05$ & $\mathrm{U}$ \\
\hline & ${ }^{125} \mathrm{Sb}$ & $-6.8 \mathrm{E}-05 \pm 1.8 \mathrm{E}-04$ & $\mathrm{U}$ \\
\hline & ${ }^{113} \mathrm{Sn}$ & $5.1 \mathrm{E}-05 \pm 8.6 \mathrm{E}-05$ & $\mathrm{U}$ \\
\hline & ${ }^{234} \mathrm{U}$ & $6.9 \mathrm{E}-05 \pm 3.0 \mathrm{E}-05$ & \\
\hline & ${ }^{235} \mathrm{U}$ & $5.1 \mathrm{E}-06 \pm 4.6 \mathrm{E}-06$ & \\
\hline & ${ }^{238} \mathrm{U}$ & $5.8 \mathrm{E}-05 \pm 2.5 \mathrm{E}-05$ & \\
\hline & ${ }^{65} \mathrm{Zn}$ & $-4.3 \mathrm{E}-06 \pm 4.3 \mathrm{E}-05$ & $\mathrm{U}$ \\
\hline
\end{tabular}

$\overline{\mathrm{RQ}}=$ Result Qualifier. $\mathrm{U}=$ The analyte was analyzed for but not detected. 
Table 2-3. Near-Facility Air Sampling Results, 2003 (pCi/m³ \pm total analytical uncertainty). (cont)

\begin{tabular}{|c|c|c|c|c|c|c|c|}
\hline Location & Isotope & Result \pm Uncertainty & $\mathbf{R Q}^{*}$ & Location & Isotope & Result \pm Uncertainty & $\mathbf{R Q}^{*}$ \\
\hline N130 (300 TEDF) & ${ }^{144} \mathrm{Ce}$ & $-1.6 \mathrm{E}-04 \pm 8.1 \mathrm{E}-04$ & $\mathrm{U}$ & N130 (300 TEDF) & ${ }^{144} \mathrm{Ce}$ & $1.4 \mathrm{E}-04 \pm 6.4 \mathrm{E}-04$ & $\bar{U}$ \\
\hline Composite Period & ${ }^{60} \mathrm{Co}$ & $-5.5 \mathrm{E}-05 \pm 7.3 \mathrm{E}-05$ & $\mathrm{U}$ & Composite Period & ${ }^{60} \mathrm{Co}$ & $5.8 \mathrm{E}-05 \pm 8.6 \mathrm{E}-05$ & $\mathrm{U}$ \\
\hline \multirow[t]{16}{*}{$01 / 07 / 03$ to $06 / 24 / 03$} & ${ }^{134} \mathrm{Cs}$ & $7.5 \mathrm{E}-05 \pm 8.5 \mathrm{E}-05$ & $\mathrm{U}$ & $06 / 24 / 03$ to $12 / 22 / 03$ & ${ }^{134} \mathrm{Cs}$ & $3.9 \mathrm{E}-06 \pm 3.9 \mathrm{E}-05$ & $\mathrm{U}$ \\
\hline & ${ }^{137} \mathrm{Cs}$ & $-5.5 \mathrm{E}-06 \pm 5.6 \mathrm{E}-05$ & $\mathrm{U}$ & & ${ }^{137} \mathrm{Cs}$ & $-1.8 \mathrm{E}-05 \pm 7.3 \mathrm{E}-05$ & $\mathrm{U}$ \\
\hline & ${ }^{152} \mathrm{Eu}$ & $-1.7 \mathrm{E}-04 \pm 2.1 \mathrm{E}-04$ & $\mathrm{U}$ & & ${ }^{152} \mathrm{Eu}$ & $4.4 \mathrm{E}-05 \pm 1.7 \mathrm{E}-04$ & $\mathrm{U}$ \\
\hline & ${ }^{154} \mathrm{Eu}$ & $2.4 \mathrm{E}-04 \pm 2.6 \mathrm{E}-04$ & $\mathrm{U}$ & & ${ }^{154} \mathrm{Eu}$ & $-8.6 \mathrm{E}-05 \pm 2.5 \mathrm{E}-04$ & $\mathrm{U}$ \\
\hline & ${ }^{155} \mathrm{Eu}$ & $-7.1 \mathrm{E}-05 \pm 2.1 \mathrm{E}-04$ & $\mathrm{U}$ & & ${ }^{155} \mathrm{Eu}$ & $-1.2 \mathrm{E}-04 \pm 1.9 \mathrm{E}-04$ & $\mathrm{U}$ \\
\hline & ${ }^{238} \mathrm{Pu}$ & $9.9 \mathrm{E}-06 \pm 2.4 \mathrm{E}-05$ & $\mathrm{U}$ & & ${ }^{238} \mathrm{Pu}$ & $1.6 \mathrm{E}-06 \pm 1.2 \mathrm{E}-05$ & $\mathrm{U}$ \\
\hline & ${ }^{239 / 240} \mathrm{Pu}$ & $4.1 \mathrm{E}-06 \pm 8.3 \mathrm{E}-06$ & $\mathrm{U}$ & & ${ }^{239 / 240} \mathrm{Pu}$ & $1.6 \mathrm{E}-06 \pm 4.5 \mathrm{E}-06$ & $\mathrm{U}$ \\
\hline & ${ }^{103} \mathrm{Ru}$ & $6.0 \mathrm{E}-05 \pm 7.4 \mathrm{E}-05$ & $\mathrm{U}$ & & ${ }^{103} \mathrm{Ru}$ & $-3.3 \mathrm{E}-05 \pm 6.5 \mathrm{E}-05$ & $\mathrm{U}$ \\
\hline & ${ }^{106} \mathrm{Ru}$ & $2.0 \mathrm{E}-04 \pm 6.7 \mathrm{E}-04$ & $\mathrm{U}$ & & ${ }^{106} \mathrm{Ru}$ & $4.6 \mathrm{E}-04 \pm 6.6 \mathrm{E}-04$ & $\mathrm{U}$ \\
\hline & ${ }^{125} \mathrm{Sb}$ & $-2.7 \mathrm{E}-05 \pm 1.9 \mathrm{E}-04$ & $\mathrm{U}$ & & ${ }^{125} \mathrm{Sb}$ & $-1.8 \mathrm{E}-06 \pm 1.8 \mathrm{E}-05$ & $\mathrm{U}$ \\
\hline & ${ }^{113} \mathrm{Sn}$ & $-2.8 \mathrm{E}-05 \pm 8.8 \mathrm{E}-05$ & $\mathrm{U}$ & & ${ }^{113} \mathrm{Sn}$ & $4.3 \mathrm{E}-05 \pm 7.5 \mathrm{E}-05$ & $\mathrm{U}$ \\
\hline & ${ }^{90} \mathrm{Sr}$ & $-2.6 \mathrm{E}-04 \pm 2.7 \mathrm{E}-04$ & $\mathrm{U}$ & & ${ }^{90} \mathrm{Sr}$ & $-7.0 \mathrm{E}-06 \pm 6.5 \mathrm{E}-05$ & $\mathrm{U}$ \\
\hline & ${ }^{234} \mathrm{U}$ & $1.5 \mathrm{E}-05 \pm 8.9 \mathrm{E}-06$ & & & ${ }^{234} \mathrm{U}$ & $2.5 \mathrm{E}-05 \pm 1.2 \mathrm{E}-05$ & \\
\hline & ${ }^{235} \mathrm{U}$ & $2.4 \mathrm{E}-06 \pm 3.0 \mathrm{E}-06$ & & & ${ }^{235} \mathrm{U}$ & $5.5 \mathrm{E}-06 \pm 4.4 \mathrm{E}-06$ & \\
\hline & ${ }^{238} \mathrm{U}$ & $6.6 \mathrm{E}-06 \pm 5.5 \mathrm{E}-06$ & & & ${ }^{238} \mathrm{U}$ & $1.3 \mathrm{E}-05 \pm 7.6 \mathrm{E}-06$ & \\
\hline & ${ }^{65} \mathrm{Zn}$ & $1.4 \mathrm{E}-05 \pm 1.4 \mathrm{E}-04$ & $\mathrm{U}$ & & ${ }^{65} \mathrm{Zn}$ & $7.6 \mathrm{E}-05 \pm 2.1 \mathrm{E}-04$ & $\mathrm{U}$ \\
\hline N981 (WYE & ${ }^{144} \mathrm{Ce}$ & $-2.3 \mathrm{E}-04 \pm 5.7 \mathrm{E}-04$ & $\mathrm{U}$ & N981 (WYE & ${ }^{144} \mathrm{Ce}$ & $-9.7 \mathrm{E}-05 \pm 5.4 \mathrm{E}-04$ & $\mathrm{U}$ \\
\hline Barricade) & ${ }^{60} \mathrm{Co}$ & $5.2 \mathrm{E}-06 \pm 5.2 \mathrm{E}-05$ & $\mathrm{U}$ & Barricade) & ${ }^{60} \mathrm{Co}$ & $-1.6 \mathrm{E}-05 \pm 7.5 \mathrm{E}-05$ & $\mathrm{U}$ \\
\hline Composite Period & ${ }^{134} \mathrm{Cs}$ & $-4.8 \mathrm{E}-05 \pm 6.7 \mathrm{E}-05$ & $\mathrm{U}$ & Composite Period & ${ }^{134} \mathrm{Cs}$ & $3.8 \mathrm{E}-05 \pm 7.3 \mathrm{E}-05$ & $\mathrm{U}$ \\
\hline \multirow[t]{15}{*}{$01 / 07 / 03$ to $06 / 24 / 03$} & ${ }^{137} \mathrm{Cs}$ & $-1.6 \mathrm{E}-05 \pm 6.7 \mathrm{E}-05$ & $\mathrm{U}$ & $06 / 24 / 03$ to $12 / 22 / 03$ & ${ }^{137} \mathrm{Cs}$ & $5.0 \mathrm{E}-05 \pm 7.3 \mathrm{E}-05$ & $\mathrm{U}$ \\
\hline & ${ }^{152} \mathrm{Eu}$ & $-1.9 \mathrm{E}-05 \pm 1.5 \mathrm{E}-04$ & $\mathrm{U}$ & & ${ }^{152} \mathrm{Eu}$ & $8.6 \mathrm{E}-06 \pm 8.6 \mathrm{E}-05$ & $\mathrm{U}$ \\
\hline & ${ }^{154} \mathrm{Eu}$ & $-1.2 \mathrm{E}-04 \pm 2.0 \mathrm{E}-04$ & $\mathrm{U}$ & & ${ }^{154} \mathrm{Eu}$ & $2.8 \mathrm{E}-04 \pm 2.3 \mathrm{E}-04$ & $\mathrm{U}$ \\
\hline & ${ }^{155} \mathrm{Eu}$ & $3.9 \mathrm{E}-05 \pm 1.5 \mathrm{E}-04$ & $\mathrm{U}$ & & ${ }^{155} \mathrm{Eu}$ & $-1.2 \mathrm{E}-04 \pm 1.4 \mathrm{E}-04$ & $\mathrm{U}$ \\
\hline & ${ }^{238} \mathrm{Pu}$ & $-5.6 \mathrm{E}-06 \pm 1.6 \mathrm{E}-05$ & $\mathrm{U}$ & & ${ }^{238} \mathrm{Pu}$ & $1.0 \mathrm{E}-05 \pm 1.6 \mathrm{E}-05$ & $\mathrm{U}$ \\
\hline & ${ }^{239 / 240} \mathrm{Pu}$ & $2.8 \mathrm{E}-06 \pm 3.4 \mathrm{E}-06$ & & & ${ }^{239 / 240} \mathrm{Pu}$ & $3.7 \mathrm{E}-05 \pm 1.9 \mathrm{E}-05$ & \\
\hline & ${ }^{103} \mathrm{Ru}$ & $-6.2 \mathrm{E}-05 \pm 7.1 \mathrm{E}-05$ & $\mathrm{U}$ & & ${ }^{103} \mathrm{Ru}$ & $-6.2 \mathrm{E}-06 \pm 6.2 \mathrm{E}-05$ & $\mathrm{U}$ \\
\hline & ${ }^{106} \mathrm{Ru}$ & $-1.5 \mathrm{E}-04 \pm 6.2 \mathrm{E}-04$ & $\mathrm{U}$ & & ${ }^{106} \mathrm{Ru}$ & $-3.3 \mathrm{E}-05 \pm 3.3 \mathrm{E}-04$ & $\mathrm{U}$ \\
\hline & ${ }^{125} \mathrm{Sb}$ & $7.3 \mathrm{E}-05 \pm 1.5 \mathrm{E}-04$ & $\mathrm{U}$ & & ${ }^{125} \mathrm{Sb}$ & $-7.5 \mathrm{E}-05 \pm 1.4 \mathrm{E}-04$ & $\mathrm{U}$ \\
\hline & ${ }^{113} \mathrm{Sn}$ & $1.5 \mathrm{E}-05 \pm 7.8 \mathrm{E}-05$ & $\mathrm{U}$ & & ${ }^{113} \mathrm{Sn}$ & $-3.9 \mathrm{E}-05 \pm 6.7 \mathrm{E}-05$ & $\mathrm{U}$ \\
\hline & ${ }^{90} \mathrm{Sr}$ & $1.3 \mathrm{E}-04 \pm 1.1 \mathrm{E}-04$ & & & ${ }^{90} \mathrm{Sr}$ & $2.9 \mathrm{E}-05 \pm 1.2 \mathrm{E}-04$ & $\mathrm{U}$ \\
\hline & ${ }^{234} \mathrm{U}$ & $5.8 \mathrm{E}-06 \pm 5.4 \mathrm{E}-06$ & $\mathrm{U}$ & & ${ }^{234} \mathrm{U}$ & $1.1 \mathrm{E}-05 \pm 7.1 \mathrm{E}-06$ & \\
\hline & ${ }^{235} \mathrm{U}$ & $5.5 \mathrm{E}-06 \pm 5.1 \mathrm{E}-06$ & $\mathrm{U}$ & & ${ }^{235} \mathrm{U}$ & $2.6 \mathrm{E}-06 \pm 3.9 \mathrm{E}-06$ & $\mathrm{U}$ \\
\hline & ${ }^{238} \mathrm{U}$ & $5.8 \mathrm{E}-06 \pm 4.6 \mathrm{E}-06$ & & & ${ }^{238} \mathrm{U}$ & $6.3 \mathrm{E}-06 \pm 5.5 \mathrm{E}-06$ & \\
\hline & ${ }^{65} \mathrm{Zn}$ & $-9.1 \mathrm{E}-05 \pm 1.6 \mathrm{E}-04$ & $\mathrm{U}$ & & ${ }^{65} \mathrm{Zn}$ & $2.2 \mathrm{E}-04 \pm 1.8 \mathrm{E}-04$ & $\mathrm{U}$ \\
\hline
\end{tabular}

$\overline{\mathrm{RQ}}=$ Result Qualifier. $\mathrm{U}=$ The analyte was analyzed for but not detected. 
Table 2-4. Pacific Northwest National Laboratory Air Sampling Data, 2003 $\left(\mathrm{pCi} / \mathrm{m}^{3} \pm\right.$ total analytical uncertainty).

\begin{tabular}{cccc}
\hline Sampler & Isotope & \multicolumn{2}{c}{ Result \pm Uncertainty } \\
\hline 200 W SE & ${ }^{60} \mathrm{Co}$ & $-3.1 \mathrm{E}-04 \pm 6.9 \mathrm{E}-04$ & $\mathrm{U}$ \\
Composite Period & ${ }^{134} \mathrm{Cs}$ & $-3.0 \mathrm{E}-04 \pm 6.8 \mathrm{E}-04$ & $\mathrm{U}$ \\
$12 / 31 / 02-04 / 08 / 03$ & ${ }^{137} \mathrm{Cs}$ & $2.2 \mathrm{E}-04 \pm 4.7 \mathrm{E}-04$ & $\mathrm{U}$ \\
& ${ }^{152} \mathrm{Eu}$ & $-4.2 \mathrm{E}-04 \pm 1.3 \mathrm{E}-03$ & $\mathrm{U}$ \\
& ${ }^{154} \mathrm{Eu}$ & $-4.8 \mathrm{E}-04 \pm 1.8 \mathrm{E}-03$ & $\mathrm{U}$ \\
& ${ }^{155} \mathrm{Eu}$ & $3.7 \mathrm{E}-04 \pm 9.3 \mathrm{E}-04$ & $\mathrm{U}$ \\
& ${ }^{238} \mathrm{Pu}$ & $-5.1 \mathrm{E}-07 \pm 1.5 \mathrm{E}-06$ & $\mathrm{U}$ \\
& ${ }^{239,240} \mathrm{Pu}$ & $2.0 \mathrm{E}-06 \pm 2.2 \mathrm{E}-06$ & $\mathrm{U}$ \\
& ${ }^{106} \mathrm{Ru}$ & $-1.8 \mathrm{E}-03 \pm 5.4 \mathrm{E}-03$ & $\mathrm{U}$ \\
& ${ }^{125} \mathrm{Sb}$ & $-8.3 \mathrm{E}-04 \pm 1.2 \mathrm{E}-03$ & $\mathrm{U}$ \\
& ${ }^{90} \mathrm{Sr}$ & $1.3 \mathrm{E}-04 \pm 9.2 \mathrm{E}-05$ & $\mathrm{U}$
\end{tabular}

\begin{tabular}{|c|c|c|c|}
\hline $200 \mathrm{~W}$ SE & ${ }^{60} \mathrm{Co}$ & $1.4 \mathrm{E}-04 \pm 6.4 \mathrm{E}-04$ & $\bar{U}$ \\
\hline Composite Period & ${ }^{134} \mathrm{Cs}$ & $2.3 \mathrm{E}-05 \pm 6.7 \mathrm{E}-04$ & $\mathrm{U}$ \\
\hline \multirow[t]{12}{*}{ 06/30/03 - 10/07/03 } & ${ }^{137} \mathrm{Cs}$ & $8.5 \mathrm{E}-06 \pm 6.1 \mathrm{E}-04$ & $\mathrm{U}$ \\
\hline & ${ }^{152} \mathrm{Eu}$ & $4.9 \mathrm{E}-04 \pm 1.6 \mathrm{E}-03$ & $\mathrm{U}$ \\
\hline & ${ }^{154} \mathrm{Eu}$ & $1.3 \mathrm{E}-03 \pm 1.9 \mathrm{E}-03$ & $\mathrm{U}$ \\
\hline & ${ }^{155} \mathrm{Eu}$ & $5.3 \mathrm{E}-04 \pm 1.6 \mathrm{E}-03$ & $\mathrm{U}$ \\
\hline & ${ }^{238} \mathrm{Pu}$ & $5.0 \mathrm{E}-07 \pm 1.6 \mathrm{E}-06$ & $\mathrm{U}$ \\
\hline & ${ }^{239,240} \mathrm{Pu}$ & $5.3 \mathrm{E}-06 \pm 3.4 \mathrm{E}-06$ & \\
\hline & ${ }^{106} \mathrm{Ru}$ & $4.0 \mathrm{E}-03 \pm 7.7 \mathrm{E}-03$ & $\mathrm{U}$ \\
\hline & ${ }^{125} \mathrm{Sb}$ & $-2.5 \mathrm{E}-04 \pm 1.3 \mathrm{E}-03$ & $\mathrm{U}$ \\
\hline & ${ }^{90} \mathrm{Sr}$ & $2.1 \mathrm{E}-05 \pm 4.3 \mathrm{E}-05$ & $\mathrm{U}$ \\
\hline & ${ }^{234} \mathrm{U}$ & $2.2 \mathrm{E}-05 \pm 9.3 \mathrm{E}-06$ & \\
\hline & ${ }^{235} \mathrm{U}$ & $-4.4 \mathrm{E}-07 \pm 2.5 \mathrm{E}-06$ & $\mathrm{U}$ \\
\hline & ${ }^{238} \mathrm{U}$ & $2.7 \mathrm{E}-05 \pm 1.0 \mathrm{E}-05$ & \\
\hline $300 \mathrm{NE}$ & ${ }^{60} \mathrm{Co}$ & $1.5 \mathrm{E}-04 \pm 6.3 \mathrm{E}-04$ & $\overline{\mathrm{U}}$ \\
\hline Composite Period & ${ }^{134} \mathrm{Cs}$ & $-4.8 \mathrm{E}-05 \pm 5.4 \mathrm{E}-04$ & $\mathrm{U}$ \\
\hline \multirow[t]{12}{*}{$01 / 08 / 03-04 / 02 / 03$} & ${ }^{137} \mathrm{Cs}$ & $9.7 \mathrm{E}-05 \pm 5.2 \mathrm{E}-04$ & $\mathrm{U}$ \\
\hline & ${ }^{152} \mathrm{Eu}$ & $9.6 \mathrm{E}-04 \pm 1.3 \mathrm{E}-03$ & $\mathrm{U}$ \\
\hline & ${ }^{154} \mathrm{Eu}$ & $8.7 \mathrm{E}-04 \pm 1.4 \mathrm{E}-03$ & $\mathrm{U}$ \\
\hline & ${ }^{155} \mathrm{Eu}$ & $-9.1 \mathrm{E}-04 \pm 8.3 \mathrm{E}-04$ & U \\
\hline & ${ }^{238} \mathrm{Pu}$ & $-3.3 \mathrm{E}-07 \pm 1.1 \mathrm{E}-06$ & U \\
\hline & ${ }^{239,240} \mathrm{Pu}$ & $6.2 \mathrm{E}-07 \pm 1.1 \mathrm{E}-06$ & $\mathrm{U}$ \\
\hline & ${ }^{106} \mathrm{Ru}$ & $7.1 \mathrm{E}-04 \pm 5.1 \mathrm{E}-03$ & $\mathrm{U}$ \\
\hline & ${ }^{125} \mathrm{Sb}$ & $1.1 \mathrm{E}-03 \pm 1.7 \mathrm{E}-03$ & $\mathrm{U}$ \\
\hline & ${ }^{90} \mathrm{Sr}$ & $2.5 \mathrm{E}-05 \pm 5.7 \mathrm{E}-05$ & $\mathrm{U}$ \\
\hline & ${ }^{234} \mathrm{U}$ & $4.1 \mathrm{E}-05 \pm 1.7 \mathrm{E}-05$ & \\
\hline & ${ }^{235} \mathrm{U}$ & $1.1 \mathrm{E}-06 \pm 5.5 \mathrm{E}-06$ & $\mathrm{U}$ \\
\hline & ${ }^{238} \mathrm{U}$ & $1.5 \mathrm{E}-05 \pm 1.2 \mathrm{E}-05$ & \\
\hline
\end{tabular}

$\overline{\mathrm{RQ}=\text { Result Qualifier. }} \mathrm{U}=$ The analyte was analyzed for but not detected.

\begin{tabular}{|c|c|c|c|}
\hline Sampler & Isotope & Result \pm Uncertainty & $\mathbf{R Q}^{*}$ \\
\hline $200 \mathrm{~W}$ SE & ${ }^{60} \mathrm{Co}$ & $-2.6 \mathrm{E}-04 \pm 5.5 \mathrm{E}-04$ & $\mathrm{U}$ \\
\hline Composite Period & ${ }^{134} \mathrm{Cs}$ & $2.5 \mathrm{E}-04 \pm 3.8 \mathrm{E}-04$ & $\mathrm{U}$ \\
\hline \multirow[t]{12}{*}{ 04/08/03 - 06/30/03 } & ${ }^{137} \mathrm{Cs}$ & $1.6 \mathrm{E}-04 \pm 4.5 \mathrm{E}-04$ & $\mathrm{U}$ \\
\hline & ${ }^{152} \mathrm{Eu}$ & $-1.4 \mathrm{E}-04 \pm 1.1 \mathrm{E}-03$ & $\mathrm{U}$ \\
\hline & ${ }^{154} \mathrm{Eu}$ & $-1.1 \mathrm{E}-03 \pm 1.5 \mathrm{E}-03$ & $\mathrm{U}$ \\
\hline & ${ }^{155} \mathrm{Eu}$ & $-3.6 \mathrm{E}-05 \pm 6.9 \mathrm{E}-04$ & $\mathrm{U}$ \\
\hline & ${ }^{238} \mathrm{Pu}$ & $-5.9 \mathrm{E}-07 \pm 2.5 \mathrm{E}-06$ & $\mathrm{U}$ \\
\hline & ${ }^{239,240} \mathrm{Pu}$ & $2.7 \mathrm{E}-06 \pm 3.4 \mathrm{E}-06$ & $\mathrm{U}$ \\
\hline & ${ }^{106} \mathrm{Ru}$ & $-7.9 \mathrm{E}-04 \pm 3.9 \mathrm{E}-03$ & $\mathrm{U}$ \\
\hline & ${ }^{125} \mathrm{Sb}$ & $4.1 \mathrm{E}-04 \pm 1.1 \mathrm{E}-03$ & $\mathrm{U}$ \\
\hline & ${ }^{90} \mathrm{Sr}$ & $-4.5 \mathrm{E}-05 \pm 1.1 \mathrm{E}-04$ & $\mathrm{U}$ \\
\hline & ${ }^{234} \mathrm{U}$ & $1.5 \mathrm{E}-05 \pm 8.3 \mathrm{E}-06$ & \\
\hline & ${ }^{235} \mathrm{U}$ & $-4.5 \mathrm{E}-07 \pm 2.1 \mathrm{E}-06$ & U \\
\hline & ${ }^{238} \mathrm{U}$ & $2.7 \mathrm{E}-05 \pm 1.1 \mathrm{E}-05$ & \\
\hline \multirow{14}{*}{$\begin{array}{c}\mathbf{2 0 0} \text { W SE } \\
\text { Composite Period } \\
10 / 07 / 03-12 / 30 / 03\end{array}$} & ${ }^{60} \mathrm{Co}$ & $-5.0 \mathrm{E}-04 \pm 8.3 \mathrm{E}-04$ & $\bar{U}$ \\
\hline & ${ }^{134} \mathrm{Cs}$ & $3.8 \mathrm{E}-05 \pm 7.7 \mathrm{E}-04$ & $\mathrm{U}$ \\
\hline & ${ }^{137} \mathrm{Cs}$ & $3.2 \mathrm{E}-06 \pm 5.7 \mathrm{E}-04$ & $\mathrm{U}$ \\
\hline & ${ }^{152} \mathrm{Eu}$ & $5.5 \mathrm{E}-04 \pm 1.5 \mathrm{E}-03$ & $\mathrm{U}$ \\
\hline & ${ }^{154} \mathrm{Eu}$ & $7.0 \mathrm{E}-04 \pm 1.5 \mathrm{E}-03$ & $\mathrm{U}$ \\
\hline & ${ }^{155} \mathrm{Eu}$ & $-6.8 \mathrm{E}-06 \pm 9.4 \mathrm{E}-04$ & $\mathrm{U}$ \\
\hline & ${ }^{238} \mathrm{Pu}$ & $9.0 \mathrm{E}-07 \pm 2.2 \mathrm{E}-06$ & $\mathrm{U}$ \\
\hline & ${ }^{239,240} \mathrm{Pu}$ & $2.0 \mathrm{E}-06 \pm 3.3 \mathrm{E}-06$ & $\mathrm{U}$ \\
\hline & ${ }^{106} \mathrm{Ru}$ & $-3.3 \mathrm{E}-04 \pm 6.3 \mathrm{E}-03$ & $\mathrm{U}$ \\
\hline & ${ }^{125} \mathrm{Sb}$ & $-5.4 \mathrm{E}-04 \pm 1.5 \mathrm{E}-03$ & $\mathrm{U}$ \\
\hline & ${ }^{90} \mathrm{Sr}$ & $8.3 \mathrm{E}-05 \pm 5.5 \mathrm{E}-05$ & \\
\hline & ${ }^{234} \mathrm{U}$ & $4.5 \mathrm{E}-05 \pm 1.6 \mathrm{E}-05$ & \\
\hline & ${ }^{235} \mathrm{U}$ & $-2.1 \mathrm{E}-07 \pm 4.3 \mathrm{E}-06$ & $\mathrm{U}$ \\
\hline & ${ }^{238} \mathrm{U}$ & $1.6 \mathrm{E}-04 \pm 3.7 \mathrm{E}-05$ & \\
\hline \multirow{14}{*}{$\begin{array}{c}\mathbf{3 0 0} \mathbf{N E} \\
\text { Composite Period } \\
04 / 02 / 03-07 / 10 / 03\end{array}$} & ${ }^{60} \mathrm{Co}$ & $4.7 \mathrm{E}-04 \pm 6.4 \mathrm{E}-04$ & $\bar{U}$ \\
\hline & ${ }^{134} \mathrm{Cs}$ & $1.5 \mathrm{E}-04 \pm 5.7 \mathrm{E}-04$ & $\mathrm{U}$ \\
\hline & ${ }^{137} \mathrm{Cs}$ & $2.1 \mathrm{E}-04 \pm 4.5 \mathrm{E}-04$ & $\mathrm{U}$ \\
\hline & ${ }^{152} \mathrm{Eu}$ & $-5.8 \mathrm{E}-04 \pm 1.3 \mathrm{E}-03$ & $\mathrm{U}$ \\
\hline & ${ }^{154} \mathrm{Eu}$ & $-1.1 \mathrm{E}-03 \pm 2.1 \mathrm{E}-03$ & $\mathrm{U}$ \\
\hline & ${ }^{155} \mathrm{Eu}$ & $9.0 \mathrm{E}-04 \pm 1.2 \mathrm{E}-03$ & $\mathrm{U}$ \\
\hline & ${ }^{238} \mathrm{Pu}$ & $-8.0 \mathrm{E}-07 \pm 1.1 \mathrm{E}-06$ & $\mathrm{U}$ \\
\hline & ${ }^{239,240} \mathrm{Pu}$ & $-6.2 \mathrm{E}-07 \pm 1.1 \mathrm{E}-06$ & U \\
\hline & ${ }^{106} \mathrm{Ru}$ & $4.0 \mathrm{E}-03 \pm 5.8 \mathrm{E}-03$ & $\mathrm{U}$ \\
\hline & ${ }^{125} \mathrm{Sb}$ & $1.8 \mathrm{E}-03 \pm 1.5 \mathrm{E}-03$ & $\mathrm{U}$ \\
\hline & ${ }^{90} \mathrm{Sr}$ & $-9.1 \mathrm{E}-06 \pm 5.2 \mathrm{E}-05$ & $\mathrm{U}$ \\
\hline & ${ }^{234} \mathrm{U}$ & $2.1 \mathrm{E}-05 \pm 1.5 \mathrm{E}-05$ & \\
\hline & ${ }^{235} \mathrm{U}$ & $4.0 \mathrm{E}-06 \pm 8.0 \mathrm{E}-06$ & $\mathrm{U}$ \\
\hline & ${ }^{238} U$ & $2.7 \mathrm{E}-05 \pm 1.8 \mathrm{E}-05$ & \\
\hline
\end{tabular}


Table 2-4. Pacific Northwest National Laboratory Air Sampling Data, 2003 ( $\mathrm{pCi} / \mathrm{m}^{3} \pm$ total analytical uncertainty). (cont)

\begin{tabular}{cccc}
\hline Sampler & Isotope & \multicolumn{2}{c}{ Result \pm Uncertainty } \\
\hline R00 NE & ${ }^{60} \mathrm{Co}$ & $7.3 \mathrm{E}-04 \pm 9.5 \mathrm{E}-04$ & $\mathrm{U}$ \\
Composite Period & ${ }^{134} \mathrm{Cs}$ & $2.5 \mathrm{E}-04 \pm 6.2 \mathrm{E}-04$ & $\mathrm{U}$ \\
$07 / 10 / 03-09 / 30 / 03$ & ${ }^{137} \mathrm{Cs}$ & $-1.5 \mathrm{E}-04 \pm 7.2 \mathrm{E}-04$ & $\mathrm{U}$ \\
& ${ }^{152} \mathrm{Eu}$ & $5.8 \mathrm{E}-04 \pm 1.6 \mathrm{E}-03$ & $\mathrm{U}$ \\
& ${ }^{154} \mathrm{Eu}$ & $1.1 \mathrm{E}-03 \pm 1.3 \mathrm{E}-03$ & $\mathrm{U}$ \\
& ${ }^{155} \mathrm{Eu}$ & $-6.4 \mathrm{E}-04 \pm 8.8 \mathrm{E}-04$ & $\mathrm{U}$ \\
& ${ }^{238} \mathrm{Pu}$ & $5.3 \mathrm{E}-07 \pm 1.0 \mathrm{E}-06$ & $\mathrm{U}$ \\
& ${ }^{239,240} \mathrm{Pu}$ & $1.7 \mathrm{E}-07 \pm 6.0 \mathrm{E}-07$ & $\mathrm{U}$ \\
& ${ }^{106} \mathrm{Ru}$ & $-1.3 \mathrm{E}-03 \pm 6.6 \mathrm{E}-03$ & $\mathrm{U}$ \\
& ${ }^{125} \mathrm{Sb}$ & $-1.4 \mathrm{E}-03 \pm 1.4 \mathrm{E}-03$ & $\mathrm{U}$ \\
& ${ }^{90} \mathrm{Sr}$ & $2.6 \mathrm{E}-05 \pm 2.9 \mathrm{E}-05$ & $\mathrm{U}$ \\
& ${ }^{234} \mathrm{U}$ & $6.0 \mathrm{E}-05 \pm 1.8 \mathrm{E}-05$ & \\
& ${ }^{235} \mathrm{U}$ & $1.3 \mathrm{E}-06 \pm 3.3 \mathrm{E}-06$ & $\mathrm{U}$ \\
& ${ }^{238} \mathrm{U}$ & $3.9 \mathrm{E}-05 \pm 1.4 \mathrm{E}-05$ & \\
\hline
\end{tabular}

\section{$300 \mathrm{NE}$}

Sample Period

$12 / 26 / 02-01 / 22 / 03 \quad{ }^{3} \mathrm{H} \quad 8.0 \mathrm{E}+00 \pm 1.4 \mathrm{E}+00$

$02 / 19 / 03-03 / 20 / 03 \quad{ }^{3} \mathrm{H} \quad 7.3 \mathrm{E}+00 \pm 1.5 \mathrm{E}+00$

$03 / 20 / 03-04 / 15 / 03 \quad{ }^{3} \mathrm{H} \quad 6.0 \mathrm{E}+00 \pm 1.2 \mathrm{E}+00$

$04 / 15 / 03-05 / 13 / 03 \quad{ }^{3} \mathrm{H} \quad 7.0 \mathrm{E}+00 \pm 1.3 \mathrm{E}+00$

$05 / 13 / 03-06 / 12 / 03 \quad{ }^{3} \mathrm{H} \quad 8.2 \mathrm{E}+00 \pm 1.6 \mathrm{E}+00$

$06 / 12 / 03-07 / 10 / 03 \quad{ }^{3} \mathrm{H} \quad 1.2 \mathrm{E}+01 \pm 1.2 \mathrm{E}+00$

Composite Period

01/08/03 - 04/02/03

\begin{tabular}{|c|c|c|}
\hline & & \\
\hline & ${ }^{152} \mathrm{Eu}$ & $-3.9 \mathrm{E}-04 \pm 1.2 \mathrm{E}-03$ \\
\hline & ${ }^{154} \mathrm{Eu}$ & $-1.1 \mathrm{E}-03 \pm 2.0 \mathrm{E}-03$ \\
\hline & ${ }^{155} \mathrm{Eu}$ & $6.0 \mathrm{E}-04 \pm 1.0 \mathrm{E}-03$ \\
\hline & ${ }^{106} \mathrm{Ru}$ & $-3.3 \mathrm{E}-03 \pm 5.1 \mathrm{E}-03$ \\
\hline & ${ }^{125} \mathrm{Sb}$ & $-5.8 \mathrm{E}-04 \pm 1.5 \mathrm{E}-03$ \\
\hline & ${ }^{234} \mathrm{U}$ & $1.5 \mathrm{E}-05 \pm 1.3 \mathrm{E}-05$ \\
\hline & ${ }^{235} \mathrm{U}$ & $4.7 \mathrm{E}-06 \pm 5.1 \mathrm{E}-06$ \\
\hline & ${ }^{238} \mathrm{U}$ & $1.9 \mathrm{E}-05 \pm 1.5 \mathrm{E}-05$ \\
\hline \multirow{11}{*}{$\begin{array}{c}\text { 300 TRENCH } \\
\text { Composite Period } \\
07 / 10 / 03-09 / 30 / 03\end{array}$} & ${ }^{60} \mathrm{Co}$ & $1.6 \mathrm{E}-04 \pm 8.6 \mathrm{E}-04$ \\
\hline & ${ }^{134} \mathrm{Cs}$ & $-4.5 \mathrm{E}-04 \pm 6.2 \mathrm{E}-04$ \\
\hline & ${ }^{137} \mathrm{Cs}$ & $-2.4 \mathrm{E}-04 \pm 5.9 \mathrm{E}-04$ \\
\hline & ${ }^{152} \mathrm{Eu}$ & $1.2 \mathrm{E}-03 \pm 1.8 \mathrm{E}-03$ \\
\hline & ${ }^{154} \mathrm{Eu}$ & $1.6 \mathrm{E}-03 \pm 2.9 \mathrm{E}-03$ \\
\hline & ${ }^{155} \mathrm{Eu}$ & $-3.5 \mathrm{E}-04 \pm 1.1 \mathrm{E}-03$ \\
\hline & ${ }^{106} \mathrm{Ru}$ & $5.5 \mathrm{E}-04 \pm 6.7 \mathrm{E}-03$ \\
\hline & ${ }^{125} \mathrm{Sb}$ & $-2.4 \mathrm{E}-04 \pm 1.7 \mathrm{E}-03$ \\
\hline & ${ }^{234} \mathrm{U}$ & $6.2 \mathrm{E}-05 \pm 2.0 \mathrm{E}-05$ \\
\hline & ${ }^{235} \mathrm{U}$ & $7.7 \mathrm{E}-07 \pm 3.2 \mathrm{E}-06$ \\
\hline & ${ }^{238} \mathrm{U}$ & $4.2 \mathrm{E}-05 \pm 1.6 \mathrm{E}-05$ \\
\hline
\end{tabular}

$\overline{\mathrm{RQ}=\text { Result Qualifier. }} \mathrm{U}=$ The analyte was analyzed for but not detected.

\begin{tabular}{|c|c|c|c|}
\hline Sampler & Isotope & Result \pm Uncertainty & $\mathbf{R Q}^{*}$ \\
\hline $300 \mathrm{NE}$ & ${ }^{60} \mathrm{Co}$ & $6.0 \mathrm{E}-04 \pm 7.5 \mathrm{E}-04$ & $\bar{U}$ \\
\hline Composite Period & ${ }^{134} \mathrm{Cs}$ & $7.8 \mathrm{E}-05 \pm 5.3 \mathrm{E}-04$ & $\mathrm{U}$ \\
\hline \multirow[t]{12}{*}{ 09/30/03 - 01/08/04 } & ${ }^{137} \mathrm{Cs}$ & $-1.9 \mathrm{E}-04 \pm 5.3 \mathrm{E}-04$ & $\mathrm{U}$ \\
\hline & ${ }^{152} \mathrm{Eu}$ & $-3.3 \mathrm{E}-04 \pm 1.4 \mathrm{E}-03$ & $\mathrm{U}$ \\
\hline & ${ }^{154} \mathrm{Eu}$ & $8.8 \mathrm{E}-05 \pm 1.7 \mathrm{E}-03$ & $\mathrm{U}$ \\
\hline & ${ }^{155} \mathrm{Eu}$ & $8.9 \mathrm{E}-04 \pm 9.8 \mathrm{E}-04$ & $\mathrm{U}$ \\
\hline & ${ }^{238} \mathrm{Pu}$ & $1.6 \mathrm{E}-07 \pm 9.5 \mathrm{E}-07$ & $\mathrm{U}$ \\
\hline & ${ }^{239,240} \mathrm{Pu}$ & $1.7 \mathrm{E}-06 \pm 1.8 \mathrm{E}-06$ & $\mathrm{U}$ \\
\hline & ${ }^{106} \mathrm{Ru}$ & $-2.6 \mathrm{E}-03 \pm 6.4 \mathrm{E}-03$ & $\mathrm{U}$ \\
\hline & ${ }^{125} \mathrm{Sb}$ & $-5.5 \mathrm{E}-04 \pm 1.2 \mathrm{E}-03$ & $\mathrm{U}$ \\
\hline & ${ }^{90} \mathrm{Sr}$ & $5.9 \mathrm{E}-05 \pm 3.4 \mathrm{E}-05$ & \\
\hline & ${ }^{234} \mathrm{U}$ & $1.2 \mathrm{E}-04 \pm 3.1 \mathrm{E}-05$ & \\
\hline & ${ }^{235} \mathrm{U}$ & $3.7 \mathrm{E}-06 \pm 7.0 \mathrm{E}-06$ & $\mathrm{U}$ \\
\hline & ${ }^{238} \mathrm{U}$ & $1.0 \mathrm{E}-04 \pm 2.8 \mathrm{E}-05$ & \\
\hline
\end{tabular}

$300 \mathrm{NE}$

Sample Period

07/10/03 - 08/08/03 $\quad{ }^{3} \mathrm{H} \quad 8.5 \mathrm{E}+00 \pm 1.7 \mathrm{E}+00$

$08 / 08 / 03-09 / 03 / 03 \quad{ }^{3} \mathrm{H} \quad 1.2 \mathrm{E}+01 \pm 2.1 \mathrm{E}+00$

09/03/03 - 09/30/03 $\quad{ }^{3} \mathrm{H} \quad 9.0 \mathrm{E}+00 \pm 1.1 \mathrm{E}+00$

09/30/03 - 10/29/03 $\quad{ }^{3} \mathrm{H} \quad 1.4 \mathrm{E}+01 \pm 2.3 \mathrm{E}+00$

$10 / 29 / 03-11 / 25 / 03 \quad{ }^{3} \mathrm{H} \quad 3.8 \mathrm{E}+00 \pm 7.4 \mathrm{E}-01$

$11 / 25 / 03-12 / 23 / 03 \quad{ }^{3} \mathrm{H} \quad 1.1 \mathrm{E}+01 \pm 1.8 \mathrm{E}+00$

300 TRENCH

Composite Period

04/02/03 - 07/10/03

${ }^{60} \mathrm{Co} \quad-2.8 \mathrm{E}-04 \pm 6.9 \mathrm{E}-04$

${ }^{134} \mathrm{Cs} \quad-1.9 \mathrm{E}-04 \pm 6.5 \mathrm{E}-04$

${ }^{137} \mathrm{Cs} \quad-1.6 \mathrm{E}-04 \pm 5.0 \mathrm{E}-04$

${ }^{152} \mathrm{Eu} \quad-3.3 \mathrm{E}-04 \pm 1.3 \mathrm{E}-03$

${ }^{154} \mathrm{Eu} \quad 1.4 \mathrm{E}-03 \pm 1.8 \mathrm{E}-03$

${ }^{155} \mathrm{Eu} \quad 2.8 \mathrm{E}-04 \pm 8.7 \mathrm{E}-04$

${ }^{106} \mathrm{Ru} \quad-8.7 \mathrm{E}-04 \pm 5.1 \mathrm{E}-03$

${ }^{125} \mathrm{Sb} \quad-2.6 \mathrm{E}-04 \pm 1.3 \mathrm{E}-03$

${ }^{234} \mathrm{U} \quad 2.1 \mathrm{E}-05 \pm 1.2 \mathrm{E}-05$

${ }^{235} \mathrm{U} \quad-4.3 \mathrm{E}-07 \pm 4.8 \mathrm{E}-06 \quad \mathrm{U}$

${ }^{238} \mathrm{U} \quad 4.3 \mathrm{E}-05 \pm 1.5 \mathrm{E}-05$

300 TRENCH

Composite Period

$\begin{array}{ll}{ }^{234} \mathrm{U} & 1.0 \mathrm{E}-04 \pm 3.0 \mathrm{E}-05 \\ { }^{235} \mathrm{U} & 8.9 \mathrm{E}-05 \pm 2.8 \mathrm{E}-05\end{array}$

09/30/03 - 01/08/04 
Table 2-4. Pacific Northwest National Laboratory Air Sampling Data, 2003 (pCi $/ \mathrm{m}^{3} \pm$ total analytical uncertainty). (cont)

\begin{tabular}{cccc}
\hline Sampler & Isotope & Result \pm Uncertainty & RQ* \\
\hline 300 TRENCH & & & \\
Sample Period & & & \\
12/26/02 - 01/22/03 & ${ }^{3} \mathrm{H}$ & $3.8 \mathrm{E}+00 \pm 9.1 \mathrm{E}-01$ & \\
$01 / 22 / 03$ - 02/19/03 & ${ }^{3} \mathrm{H}$ & $9.7 \mathrm{E}+00 \pm 1.8 \mathrm{E}+00$ & \\
$02 / 19 / 03$ - 03/20/03 & ${ }^{3} \mathrm{H}$ & $5.2 \mathrm{E}+00 \pm 1.1 \mathrm{E}+00$ & \\
$03 / 20 / 03$ - 04/15/03 & ${ }^{3} \mathrm{H}$ & $4.7 \mathrm{E}+00 \pm 1.0 \mathrm{E}+00$ & \\
$04 / 15 / 03$ - 05/13/03 & ${ }^{3} \mathrm{H}$ & $7.3 \mathrm{E}+00 \pm 1.4 \mathrm{E}+00$ & \\
$05 / 13 / 03$ - 06/12/03 & ${ }^{3} \mathrm{H}$ & $8.0 \mathrm{E}+00 \pm 1.5 \mathrm{E}+00$ & \\
\hline 300 WATER INTAKE & & & \\
Sample Period & & & \\
$12 / 26 / 02$ - 01/22/03 & ${ }^{3} \mathrm{H}$ & $1.2 \mathrm{E}+01 \pm 2.3 \mathrm{E}+00$ & \\
$01 / 22 / 03$ - 02/19/03 & ${ }^{3} \mathrm{H}$ & $6.9 \mathrm{E}+00 \pm 1.4 \mathrm{E}+00$ & \\
$02 / 19 / 03$ - 03/20/03 & ${ }^{3} \mathrm{H}$ & $2.8 \mathrm{E}+00 \pm 8.2 \mathrm{E}-01$ & \\
$03 / 20 / 03$ - 04/15/03 & ${ }^{3} \mathrm{H}$ & $9.4 \mathrm{E}+00 \pm 1.6 \mathrm{E}+00$ & \\
$04 / 15 / 03$ - 05/13/03 & ${ }^{3} \mathrm{H}$ & $1.1 \mathrm{E}+01 \pm 1.8 \mathrm{E}+00$ & \\
$05 / 13 / 03$ - 06/12/03 & ${ }^{3} \mathrm{H}$ & $9.0 \mathrm{E}+00 \pm 1.7 \mathrm{E}+00$ & \\
\hline Yakima Barricade & ${ }^{60} \mathrm{Co}$ & $2.3 \mathrm{E}-04 \pm 3.2 \mathrm{E}-04$ & $\mathrm{U}$ \\
Composite Period & ${ }^{134} \mathrm{Cs}$ & $-1.6 \mathrm{E}-04 \pm 3.2 \mathrm{E}-04$ & $\mathrm{U}$ \\
$01 / 08 / 03$ - 04/02/03 & ${ }^{137} \mathrm{Cs}$ & $1.8 \mathrm{E}-04 \pm 3.3 \mathrm{E}-04$ & $\mathrm{U}$ \\
& ${ }^{152} \mathrm{Eu}$ & $5.7 \mathrm{E}-04 \pm 6.4 \mathrm{E}-04$ & $\mathrm{U}$ \\
& ${ }^{154} \mathrm{Eu}$ & $5.1 \mathrm{E}-05 \pm 1.0 \mathrm{E}-03$ & $\mathrm{U}$ \\
& ${ }^{155} \mathrm{Sb}$ & $-5.3 \mathrm{E}-04 \pm 1.1 \mathrm{E}-03$ & $\mathrm{U}$
\end{tabular}

\begin{tabular}{cccc}
\hline Sampler & Isotope & Result \pm Uncertainty & RQ* \\
\hline 300 TRENCH & & & \\
Sample Period & & & \\
06/12/03 - 07/10/03 & ${ }^{3} \mathrm{H}$ & $5.5 \mathrm{E}+00 \pm 7.8 \mathrm{E}-01$ & \\
07/10/03 - 08/08/03 & ${ }^{3} \mathrm{H}$ & $8.8 \mathrm{E}+00 \pm 1.7 \mathrm{E}+00$ & \\
08/08/03 - 09/03/03 & ${ }^{3} \mathrm{H}$ & $1.0 \mathrm{E}+01 \pm 1.9 \mathrm{E}+00$ & \\
09/03/03 - 09/30/03 & ${ }^{3} \mathrm{H}$ & $6.8 \mathrm{E}+00 \pm 9.2 \mathrm{E}-01$ & \\
10/28/03 - 11/25/03 & ${ }^{3} \mathrm{H}$ & $2.5 \mathrm{E}+00 \pm 6.3 \mathrm{E}-01$ & \\
$11 / 25 / 03$ - 12/23/03 & ${ }^{3} \mathrm{H}$ & $3.8 \mathrm{E}+00 \pm 8.5 \mathrm{E}-01$ & \\
\hline 300 WATER INTAKE & & & \\
Sample Period & & & \\
$06 / 12 / 03$ - 07/10/03 & ${ }^{3} \mathrm{H}$ & $8.4 \mathrm{E}+00 \pm 9.5 \mathrm{E}-01$ & \\
08/08/03 - 09/03/03 & ${ }^{3} \mathrm{H}$ & $1.7 \mathrm{E}+01 \pm 3.2 \mathrm{E}+00$ & \\
09/03/03 - 09/30/03 & ${ }^{3} \mathrm{H}$ & $5.5 \mathrm{E}+00 \pm 8.5 \mathrm{E}-01$ & \\
$09 / 30 / 03$ - 10/29/03 & ${ }^{3} \mathrm{H}$ & $1.0 \mathrm{E}+01 \pm 1.9 \mathrm{E}+00$ & \\
$10 / 29 / 03$ - 11/25/03 & ${ }^{3} \mathrm{H}$ & $5.0 \mathrm{E}+00 \pm 9.4 \mathrm{E}-01$ & \\
$11 / 25 / 03$ - 12/23/03 & ${ }^{3} \mathrm{H}$ & $6.0 \mathrm{E}+00 \pm 1.1 \mathrm{E}+00$ & \\
\hline Yakima Barricade & ${ }^{60} \mathrm{Co}$ & $2.0 \mathrm{E}-04 \pm 3.0 \mathrm{E}-04$ & $\mathrm{U}$ \\
Composite Period & ${ }^{134} \mathrm{Cs}$ & $-9.5 \mathrm{E}-05 \pm 3.1 \mathrm{E}-04$ & $\mathrm{U}$ \\
$04 / 02 / 03$ - 07/11/03 & ${ }^{137} \mathrm{Cs}$ & $-2.1 \mathrm{E}-04 \pm 2.4 \mathrm{E}-04$ & $\mathrm{U}$ \\
& ${ }^{152} \mathrm{Eu}$ & $-1.7 \mathrm{E}-04 \pm 6.7 \mathrm{E}-04$ & $\mathrm{U}$ \\
& ${ }^{154} \mathrm{Eu}$ & $2.7 \mathrm{E}-04 \pm 7.8 \mathrm{E}-04$ & $\mathrm{U}$ \\
& ${ }^{155} \mathrm{Eu}$ & $-3.1 \mathrm{E}-04 \pm 5.4 \mathrm{E}-04$ & $\mathrm{U}$ \\
10/03/03 - 01/09/04 & ${ }^{125} \mathrm{Sb}$ & $2.0 \mathrm{E}-04 \pm 7.9 \mathrm{E}-04$ & $\mathrm{U}$
\end{tabular}

$\overline{\mathrm{RQ}}=$ Result Qualifier. $\mathrm{U}=$ The analyte was analyzed for but not detected. 


\subsection{SOIL AND VEGETATION MONITORING}

The radionuclide content of soil and vegetation was measured to evaluate long-term trends in environmental accumulation of radioactivity in the 100, 200/600, and 300/400 Areas. Soil and vegetation samples were collected on or near facilities that store, handle, or dispose of radioactive waste. The number of soil and vegetation samples collected in 2003 and their locations are shown in Table 3-1.

Table 3-1. Soil and Vegetation Samples Collected in 2003.

\begin{tabular}{lccccccccc} 
& Number of sample & \multicolumn{7}{c}{ Operational area } \\
\cline { 2 - 9 } Sample type & locations & $\mathbf{1 0 0 - B / C}$ & $\mathbf{1 0 0 - F}$ & $\mathbf{1 0 0 - K}$ & $\mathbf{1 0 0 - N}$ & $\mathbf{2 0 0 / 6 0 0}$ & $\mathbf{3 0 0 / 4 0 0}$ & ERDF $^{\text {a }}$ \\
\hline Soil & 82 & 5 & 2 & 2 & 1 & 57 & 14 & 1 \\
Vegetation & 65 & 0 & 0 & 0 & 4 & 48 & 13 & 0
\end{tabular}

a Odd-numbered soil and vegetation sampling locations in the 200/600 Areas are sampled in odd-numbered years.

${ }^{\mathrm{b}}$ Environmental Restoration Disposal Facility (ERDF).

Soil sampling locations are illustrated in Figures 3-1 through 3-9. Historical soil sampling results for the 100, 200/600, and 300/400 Areas are displayed in Table 3-2. The 2003 soil sampling results for all areas are provided in Table 3-3.

Vegetation sampling locations are illustrated in Figures 3-10 through 3-15. Historical vegetation sampling results for the 100-N, 200/600, and 300/400 Areas are displayed in Table 3-4. The 2003 vegetation sampling results for all areas are provided in Table 3-5.

Radionuclide analyses indicated that cobalt-60, strontium-90, cesium-137, plutonium-239/240, and uranium were consistently detectable in both soil and vegetation samples in 2003. Generally, the predominant radionuclides observed in soil samples were activation and fission products in the 100 Areas, fission products in the 200 Areas, and uranium in the 300 Area. For vegetation samples, the predominant radionuclides were generally activation and fission products in the 100 Areas, fission products in the 200 Areas, and uranium in the 300 Area.

Strontium-90 results for soil and vegetation samples for this report period showed a frequent occurrence of negative (i.e., less than zero) concentrations. This was primarily due to changes in laboratory background correction calculations that were implemented in 2003. Both historical and current values are within accepted statistical ranges as evidenced by laboratory QA and performance evaluation programs.

Additional discussion of the 2003 results can be found in Section 3.2 of PNNL-14687. 


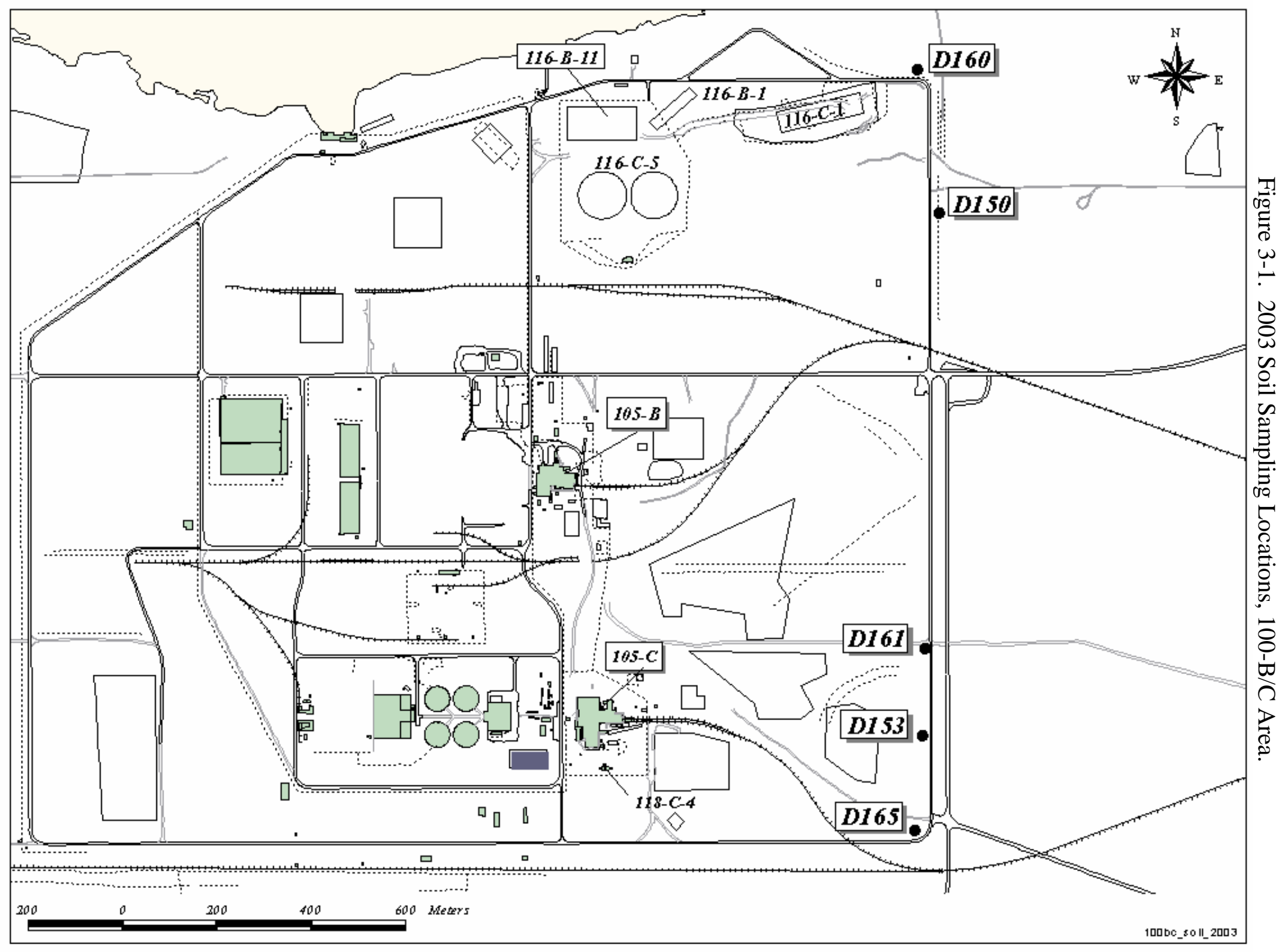




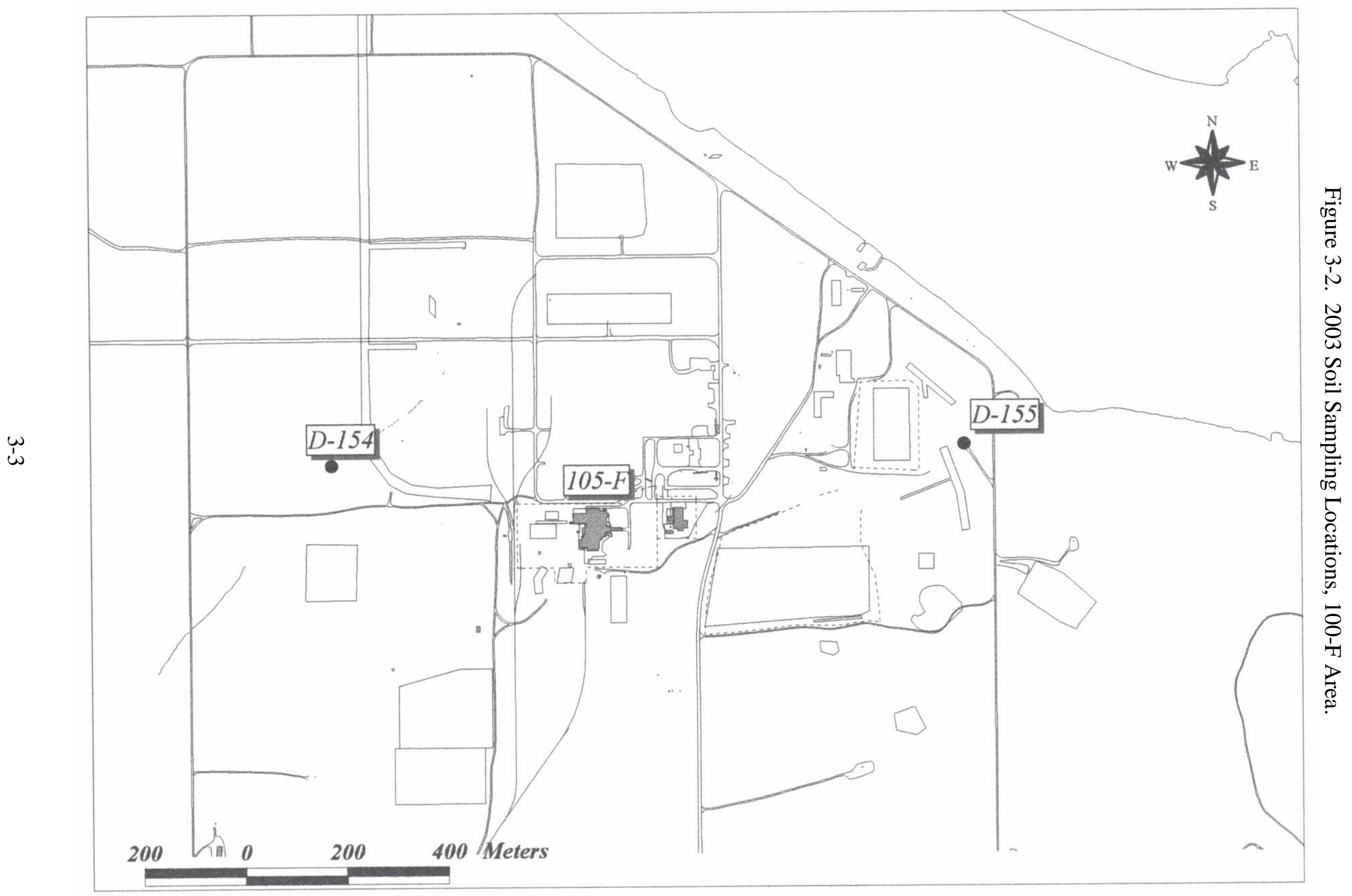




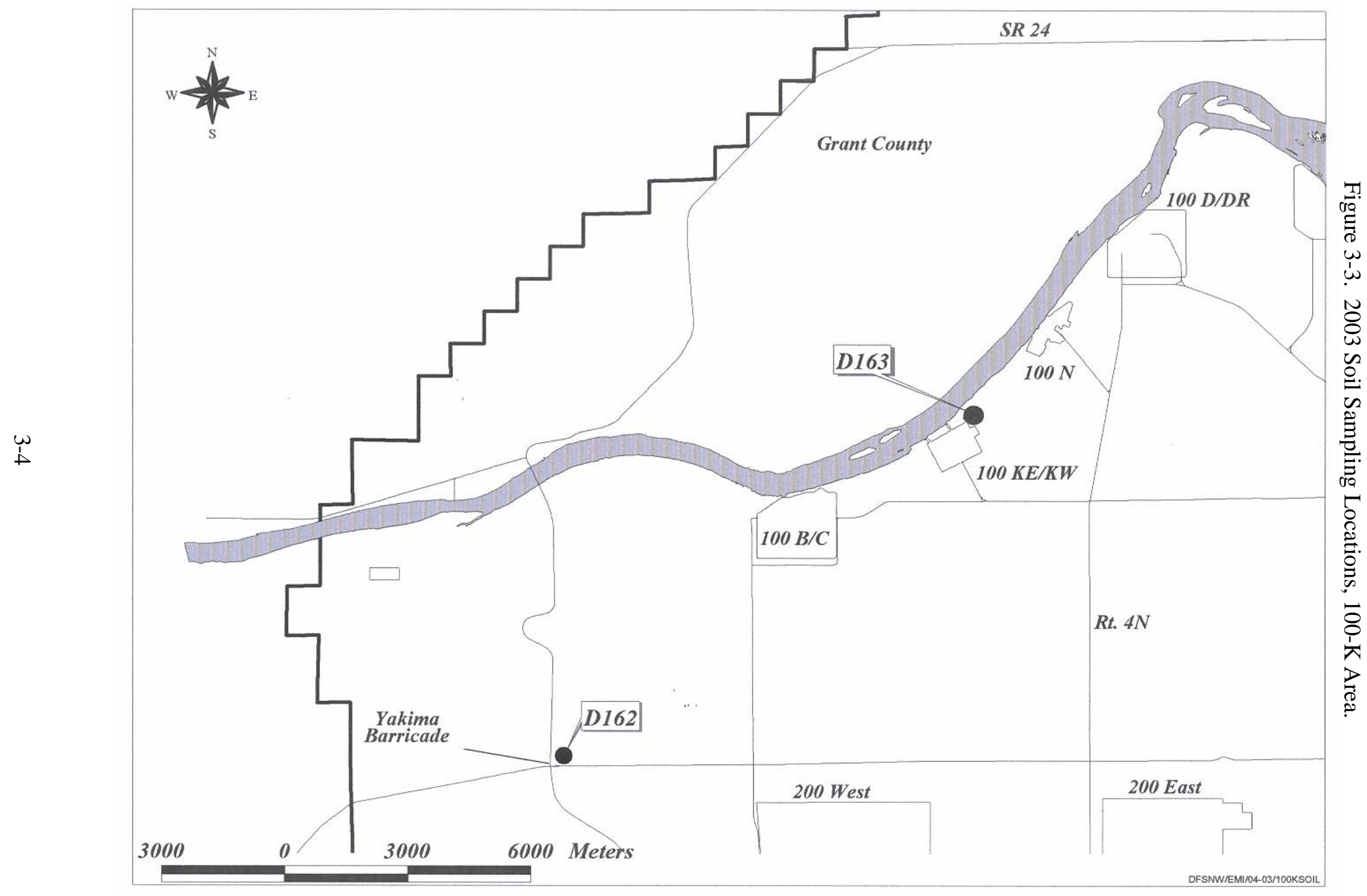




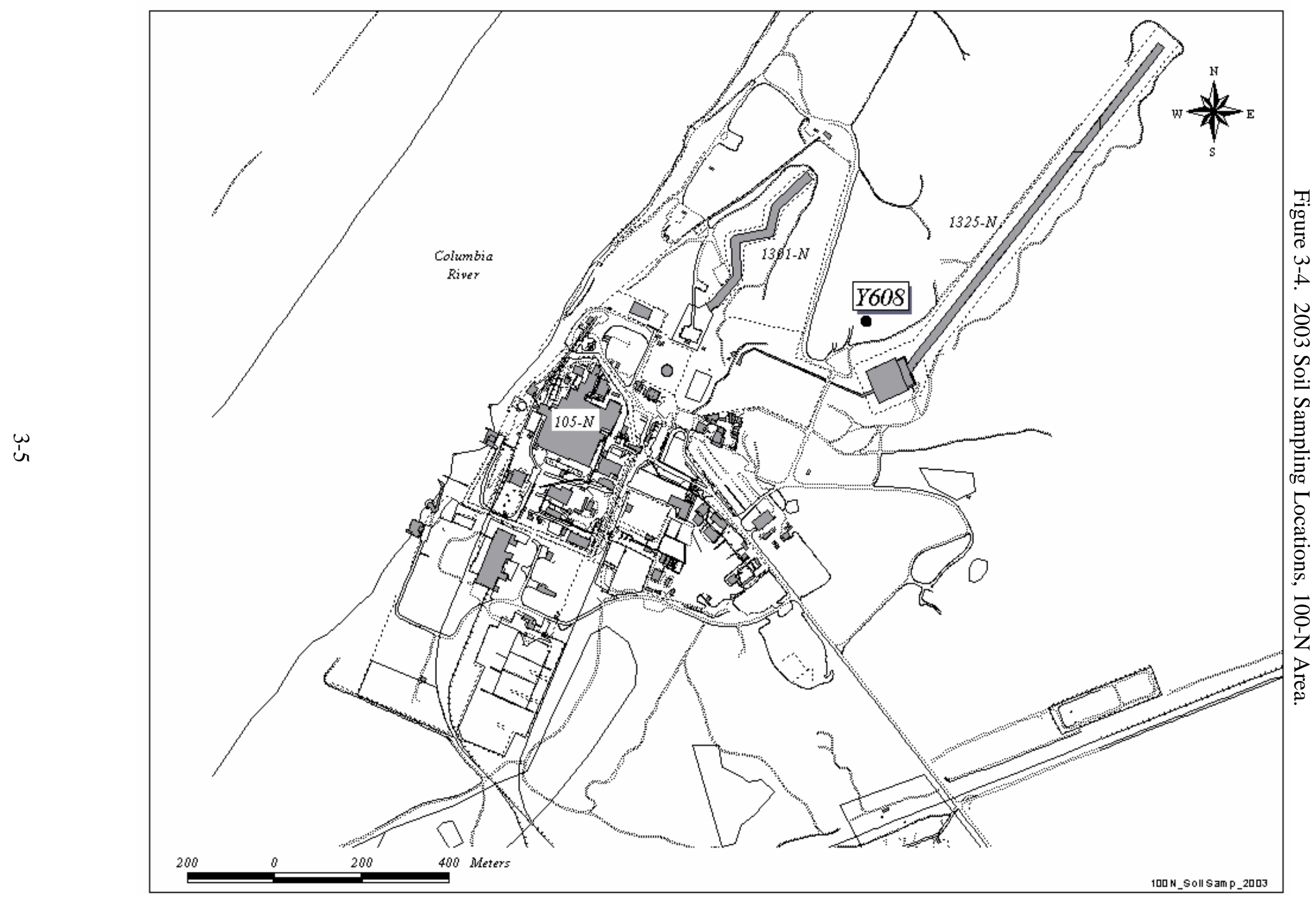




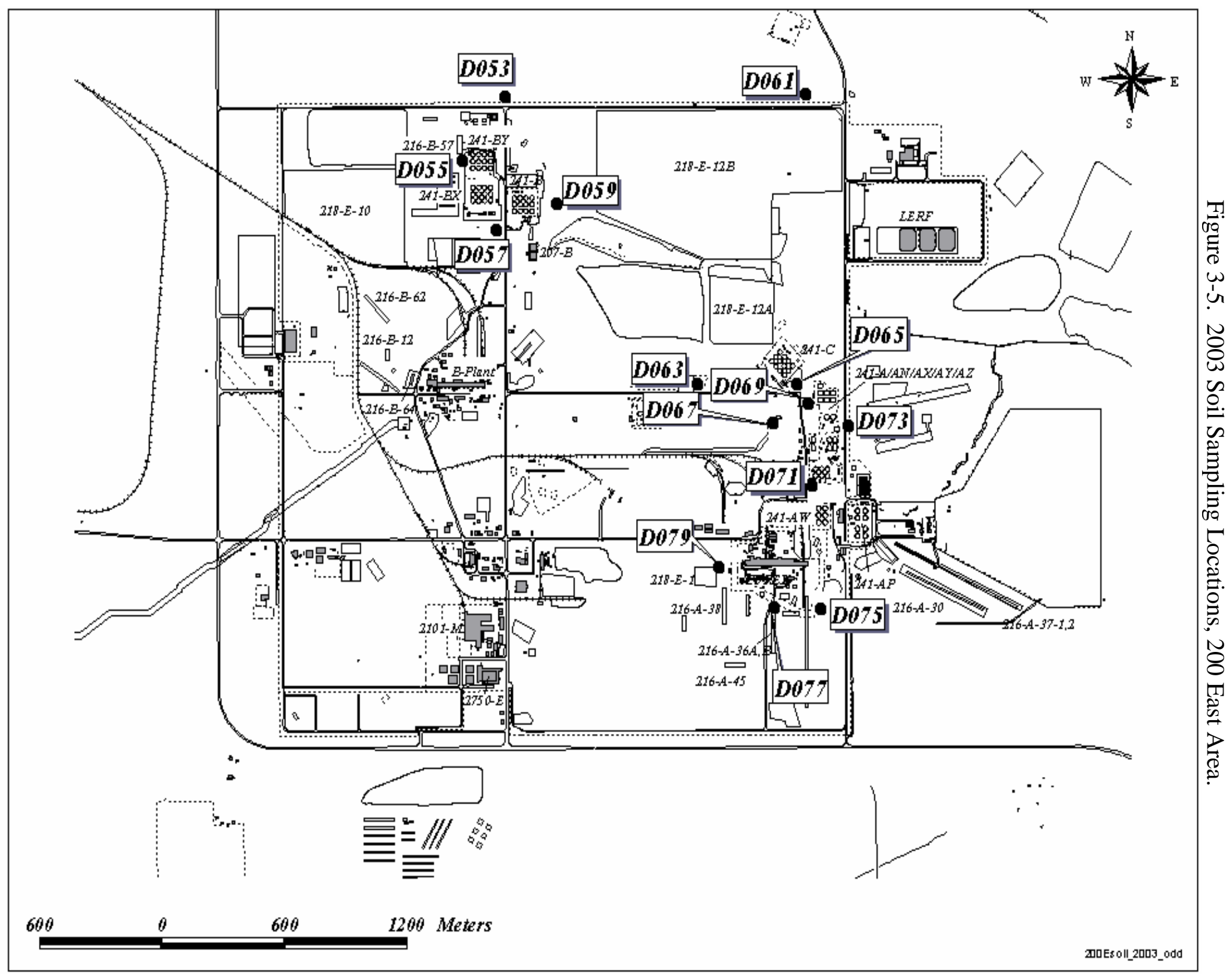




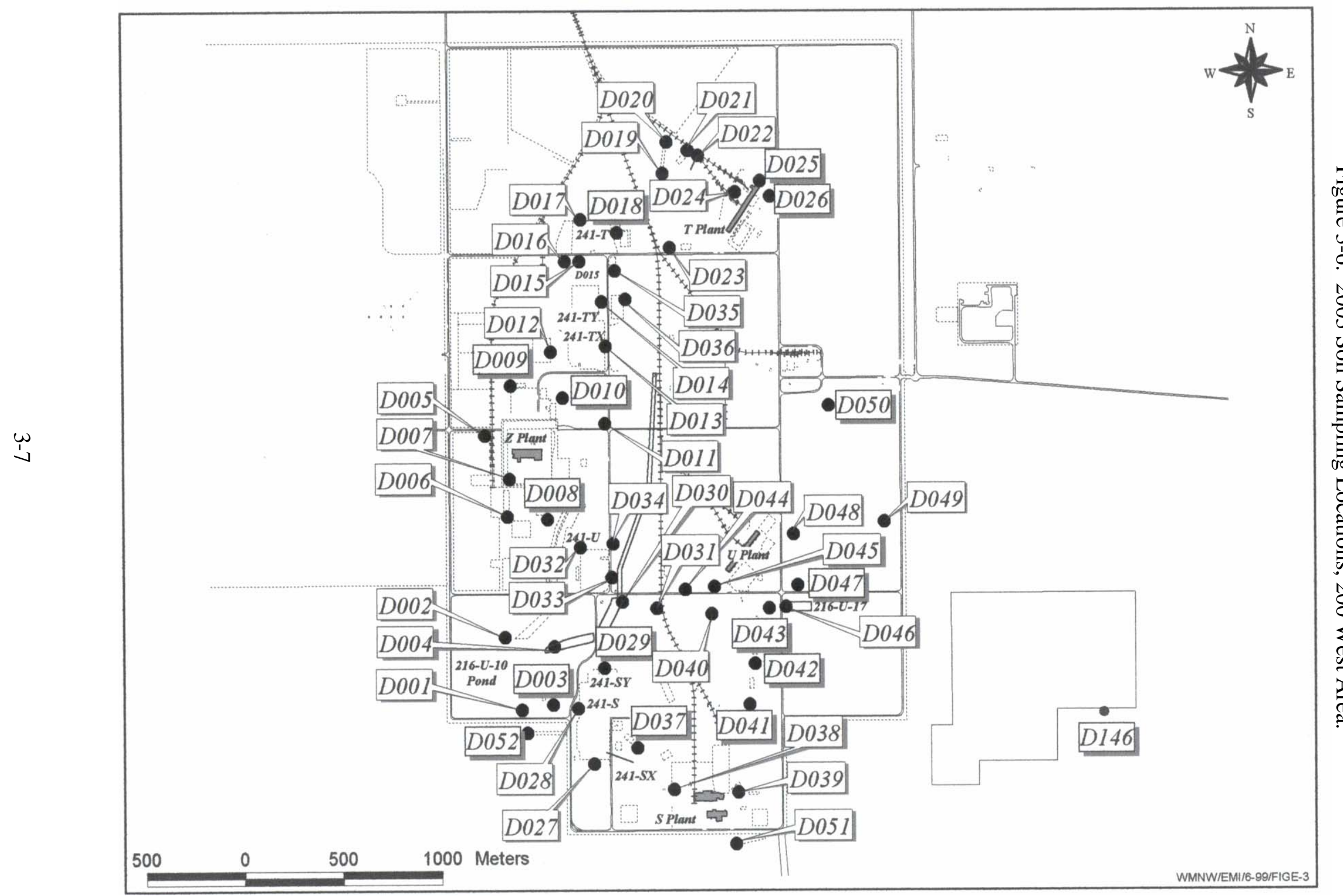

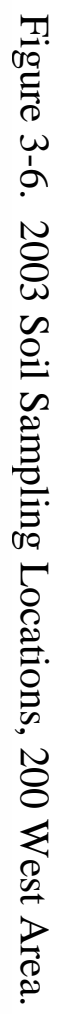




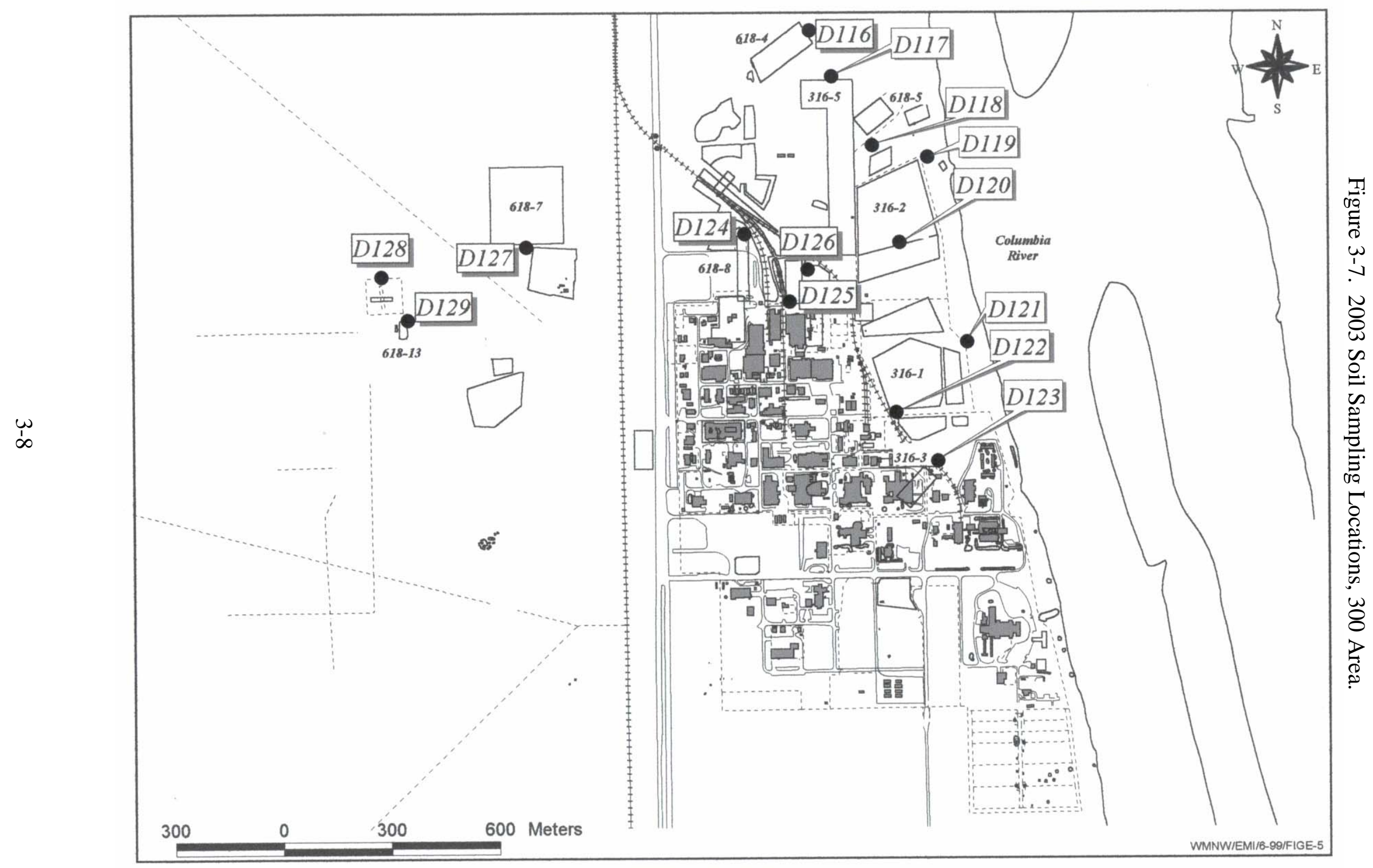




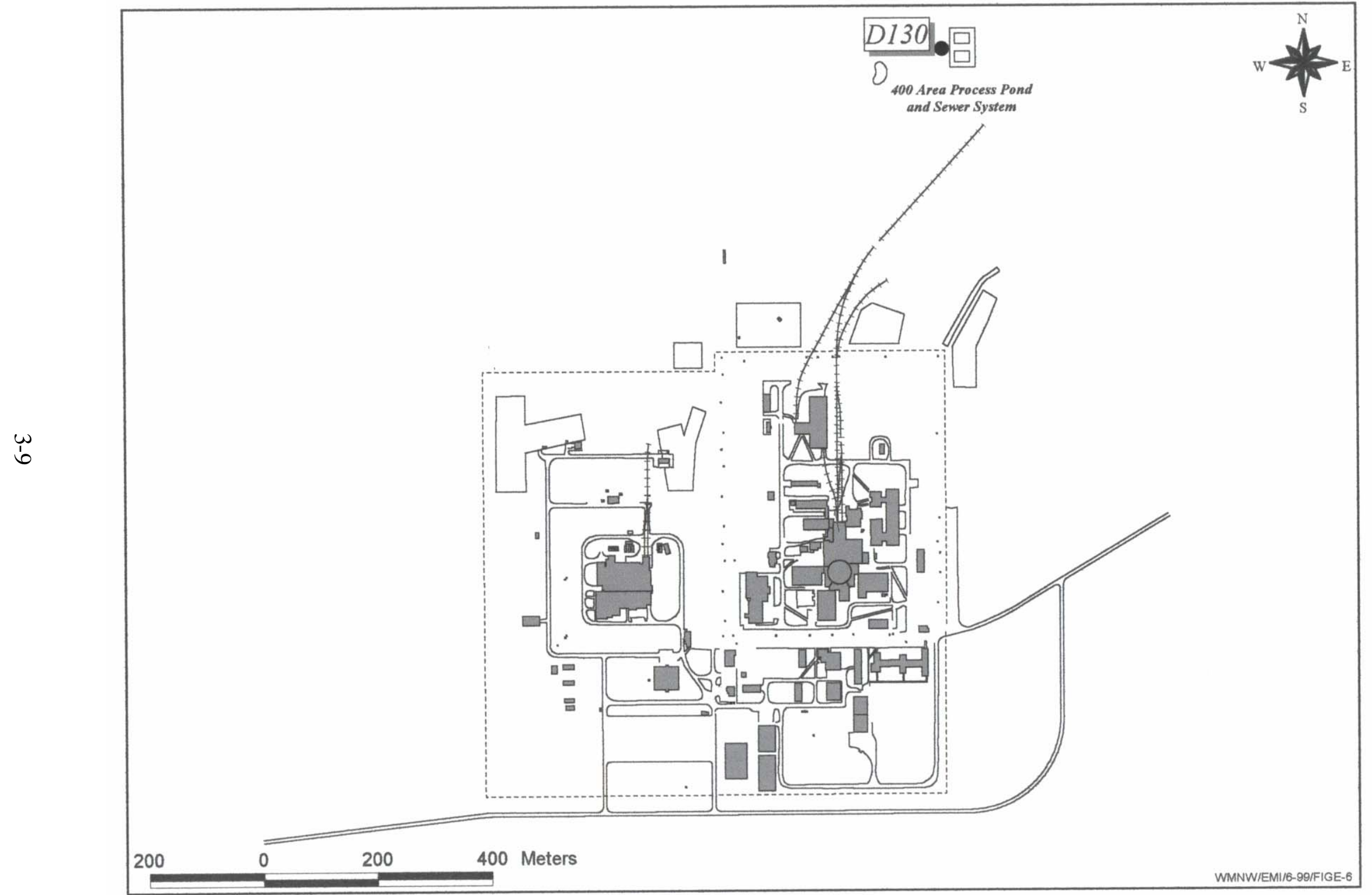

1
00
0
0
0
0
0
$N$
0
0
$\omega$
0
0
0
0
0
0
0
0
0
0
0
0
0
0
0
0
0
0
0
0
0
0 


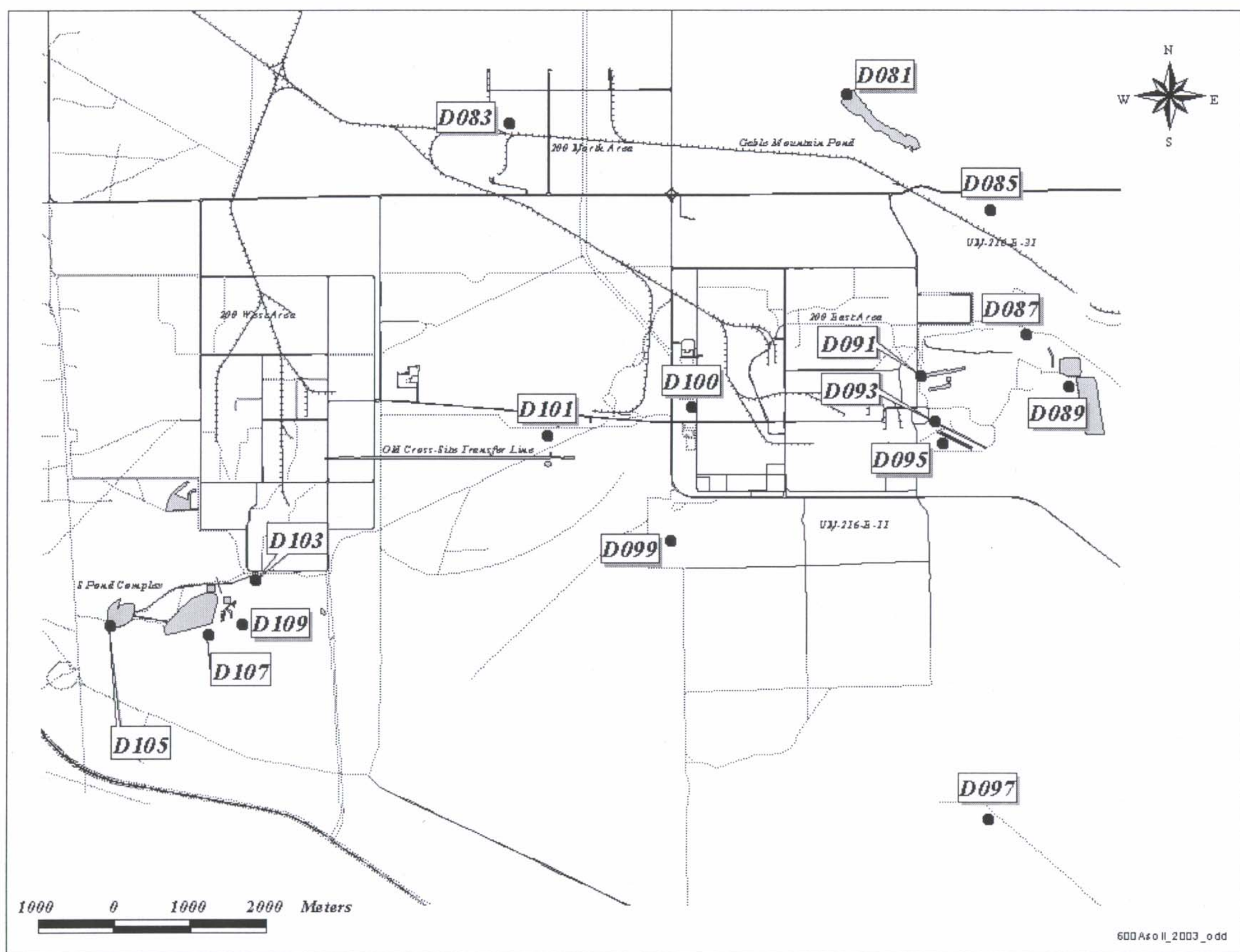

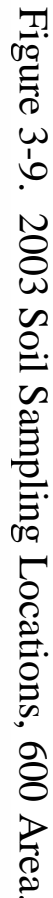




\section{Table 3-2. Average Radionuclide Concentrations ( $\mathrm{pCi} / \mathrm{g}^{\mathrm{a}}$ ) in Hanford Soils, 1995 through 2003.}

\begin{tabular}{|c|c|c|c|c|c|c|c|}
\hline \multicolumn{8}{|c|}{100 Areas } \\
\hline Year & ${ }^{60} \mathrm{Co}$ & & ${ }^{90} \mathrm{Sr}$ & ${ }^{137} \mathrm{Cs}$ & ${ }^{234} \mathrm{U}$ & ${ }^{238} \mathrm{U}$ & ${ }^{239,240} \mathrm{Pu}$ \\
\hline 1995 & $9.4 \mathrm{E}-01 \pm 9.9 \mathrm{E}+01$ & $1.3 \mathrm{E}-01$ & $\pm 6.9 \mathrm{E}-02$ & $5.1 \mathrm{E}-01 \pm 2.4 \mathrm{E}-01$ & $9.1 \mathrm{E}-02 \pm 1.0 \mathrm{E}-02$ & $9.7 \mathrm{E}-02 \pm 2.7 \mathrm{E}-02$ & $1.4 \mathrm{E}-02 \pm 9.3 \mathrm{E}-03$ \\
\hline 1996 & $1.5 \mathrm{E}+00 \pm 1.1 \mathrm{E}+00$ & $2.0 \mathrm{E}-01$ & $\pm 7.6 \mathrm{E}-02$ & $7.7 \mathrm{E}-01 \pm 4.1 \mathrm{E}-01$ & $5.7 \mathrm{E}-02 \pm 8.0 \mathrm{E}-03$ & $5.7 \mathrm{E}-01 \pm 1.2 \mathrm{E}-01$ & $4.3 \mathrm{E}-02 \pm 1.6 \mathrm{E}-02$ \\
\hline 1997 & $2.5 \mathrm{E}+00 \pm 3.0 \mathrm{E}-01$ & $3.9 \mathrm{E}-01$ & $\pm 6.5 \mathrm{E}-01$ & $8.9 \mathrm{E}-01 \pm 8.9 \mathrm{E}-01$ & $2.1 \mathrm{E}-01 \pm 3.8 \mathrm{E}-02$ & $2.1 \mathrm{E}-01 \pm 3.4 \mathrm{E}-02$ & $9.1 \mathrm{E}-01 \pm 1.6 \mathrm{E}+00$ \\
\hline 1998 & $4.9 \mathrm{E}+00 \pm 7.7 \mathrm{E}+00$ & $1.2 \mathrm{E}+00$ & $\pm 1.1 \mathrm{E}+00$ & $3.1 \mathrm{E}+00 \pm 4.1 \mathrm{E}+00$ & $2.1 \mathrm{E}-01 \pm 6.0 \mathrm{E}-02$ & $1.7 \mathrm{E}-01 \pm 3.0 \mathrm{E}-02$ & $1.5 \mathrm{E}-01 \pm 1.3 \mathrm{E}-01$ \\
\hline 1999 & $1.6 \mathrm{E}+00 \pm 2.1 \mathrm{E}+00$ & $2.0 \mathrm{E}+00$ & $\pm 2.0 \mathrm{E}+00$ & $8.4 \mathrm{E}-01 \pm 8.1 \mathrm{E}-01$ & $2.2 \mathrm{E}-01 \pm 3.0 \mathrm{E}-02$ & $2.0 \mathrm{E}-01 \pm 3.0 \mathrm{E}-02$ & $2.9 \mathrm{E}-02 \pm 2.3 \mathrm{E}-02$ \\
\hline 2000 & $3.1 \mathrm{E}+00 \pm 3.0 \mathrm{E}+00$ & 8.4E-01 & $\pm 4.5 \mathrm{E}-01$ & $2.5 \mathrm{E}+00 \pm 2.3 \mathrm{E}+00$ & $2.2 \mathrm{E}-01 \pm 8.7 \mathrm{E}-02$ & $2.2 \mathrm{E}-01 \pm 3.2 \mathrm{E}-02$ & $5.8 \mathrm{E}-02 \pm 3.3 \mathrm{E}-02$ \\
\hline 2001 & $4.0 \mathrm{E}-01 \pm 3.4 \mathrm{E}-01$ & $4.8 \mathrm{E}-01$ & $\pm 3.0 \mathrm{E}-01$ & $3.9 \mathrm{E}-01 \pm 1.6 \mathrm{E}-01$ & $2.4 \mathrm{E}-01 \pm 3.6 \mathrm{E}-02$ & $2.5 \mathrm{E}-01 \pm 2.7 \mathrm{E}-02$ & $3.1 \mathrm{E}-02 \pm 2.0 \mathrm{E}-02$ \\
\hline 2002 & $3.0 \mathrm{E}-01 \pm 1.1 \mathrm{E}+00$ & $1.5 \mathrm{E}-01$ & $\pm 4.7 \mathrm{E}-01$ & $2.6 \mathrm{E}-01 \pm 5.1 \mathrm{E}-01$ & $1.3 \mathrm{E}-01 \pm 4.7 \mathrm{E}-02$ & $1.1 \mathrm{E}-01 \pm 3.9 \mathrm{E}-02$ & $6.1 \mathrm{E}-03 \pm 6.1 \mathrm{E}-03$ \\
\hline 2003 & $1.8 \mathrm{E}-01 \pm 2.1 \mathrm{E}-02$ & $-8.2 \mathrm{E}-02$ & $\pm 2.4 \mathrm{E}-01$ & $2.1 \mathrm{E}-01 \pm 3.6 \mathrm{E}-02$ & $1.4 \mathrm{E}-01 \pm 4.8 \mathrm{E}-02$ & $1.5 \mathrm{E}-01 \pm 5.1 \mathrm{E}-02$ & $1.8 \mathrm{E}-03 \pm 6.3 \mathrm{E}-03$ \\
\hline
\end{tabular}

\begin{tabular}{ccccccc}
\multicolumn{7}{c}{$\mathbf{2 0 0 / 6 0 0}$ Areas } \\
Year & ${ }^{60} \mathbf{C o}$ & ${ }^{90} \mathbf{S r}$ & ${ }^{\mathbf{1 3 7}} \mathbf{C s}$ & ${ }^{234} \mathbf{U}$ & ${ }^{238} \mathbf{U}$ & ${ }^{239,240} \mathbf{P u}$ \\
\hline 1995 & $4.0 \mathrm{E}-03 \pm 4.0 \mathrm{E}-03$ & $4.9 \mathrm{E}-01 \pm 1.8 \mathrm{E}-01$ & $2.7 \mathrm{E}+00 \pm 1.1 \mathrm{E}+00$ & $1.2 \mathrm{E}-01 \pm 1.7 \mathrm{E}-02$ & $1.2 \mathrm{E}-01 \pm 1.6 \mathrm{E}-02$ & $7.0 \mathrm{E}-02 \pm 3.0 \mathrm{E}-02$ \\
1996 & $3.0 \mathrm{E}-03 \pm 3.0 \mathrm{E}-03$ & $3.5 \mathrm{E}-01 \pm 2.0 \mathrm{E}-01$ & $2.0 \mathrm{E}+00 \pm 7.0 \mathrm{E}-01$ & $1.0 \mathrm{E}-01 \pm 1.2 \mathrm{E}-02$ & $1.1 \mathrm{E}-01 \pm 1.2 \mathrm{E}-02$ & $1.6 \mathrm{E}-01 \pm 1.0 \mathrm{E}-01$ \\
1997 & $3.0 \mathrm{E}-02 \pm 2.0 \mathrm{E}-02$ & $6.7 \mathrm{E}-01 \pm 2.3 \mathrm{E}-01$ & $1.8 \mathrm{E}+00 \pm 4.0 \mathrm{E}-01$ & $2.0 \mathrm{E}-01 \pm 1.4 \mathrm{E}-02$ & $2.0 \mathrm{E}-01 \pm 1.4 \mathrm{E}-02$ & $1.0 \mathrm{E}-01 \pm 7.0 \mathrm{E}-02$ \\
1998 & $1.9 \mathrm{E}-02 \pm 6.0 \mathrm{E}-03$ & $5.0 \mathrm{E}-01 \pm 1.4 \mathrm{E}-01$ & $1.1 \mathrm{E}+00 \pm 4.0 \mathrm{E}-01$ & $1.9 \mathrm{E}-01 \pm 1.0 \mathrm{E}-02$ & $1.9 \mathrm{E}-01 \pm 1.0 \mathrm{E}-02$ & $1.3 \mathrm{E}-01 \pm 1.0 \mathrm{E}-02$ \\
1999 & Not Detected & $1.1 \mathrm{E}+00 \pm 5.0 \mathrm{E}-01$ & $1.4 \mathrm{E}+00 \pm 5.0 \mathrm{E}-01$ & $2.3 \mathrm{E}-01 \pm 2.0 \mathrm{E}-02$ & $2.2 \mathrm{E}-01 \pm 2.0 \mathrm{E}-02$ & $1.0 \mathrm{E}-01 \pm 5.0 \mathrm{E}-02$ \\
2000 & $6.0 \mathrm{E}-03 \pm 6.0 \mathrm{E}-03$ & $1.1 \mathrm{E}+00 \pm 2.0 \mathrm{E}-01$ & $1.4 \mathrm{E}+00 \pm 5.0 \mathrm{E}-01$ & $2.3 \mathrm{E}-01 \pm 3.0 \mathrm{E}-02$ & $2.3 \mathrm{E}-01 \pm 3.0 \mathrm{E}-02$ & $4.1 \mathrm{E}-01 \pm 4.2 \mathrm{E}-01$ \\
2001 & Not Detected & $5.5 \mathrm{E}-01 \pm 2.3 \mathrm{E}-01$ & $1.5 \mathrm{E}+00 \pm 5.4 \mathrm{E}-01$ & $2.2 \mathrm{E}-01 \pm 1.4 \mathrm{E}-02$ & $2.2 \mathrm{E}-01 \pm 1.4 \mathrm{E}-02$ & $1.3 \mathrm{E}-01 \pm 6.2 \mathrm{E}-02$ \\
2002 & Not Detected & $2.7 \mathrm{E}-01 \pm 6.6 \mathrm{E}-01$ & $1.4 \mathrm{E}+00 \pm 4.3 \mathrm{E}+00$ & $1.7 \mathrm{E}-01 \pm 1.0 \mathrm{E}-01$ & $1.7 \mathrm{E}-01 \pm 1.1 \mathrm{E}-01$ & $1.2 \mathrm{E}-01 \pm 7.2 \mathrm{E}-01$ \\
2003 & $2.4 \mathrm{E}-03 \pm 1.3 \mathrm{E}-02$ & $8.4 \mathrm{E}-02 \pm 6.3 \mathrm{E}-01$ & $1.8 \mathrm{E}+00 \pm 6.3 \mathrm{E}-01$ & $1.6 \mathrm{E}-01 \pm 9.6 \mathrm{E}-02$ & $1.7 \mathrm{E}-01 \pm 1.0 \mathrm{E}-01$ & $9.3 \mathrm{E}-02 \pm 5.0 \mathrm{E}-01$
\end{tabular}

\begin{tabular}{cccccccc}
\multicolumn{7}{c}{} & \multicolumn{2}{c}{$\mathbf{3 0 0 / 4 0 0}$ Areas } \\
Year & ${ }^{60} \mathbf{C o}$ & ${ }^{\mathbf{9 0}} \mathbf{S r}$ & ${ }^{137} \mathbf{C s}$ & ${ }^{234} \mathbf{U}$ & ${ }^{238} \mathbf{U}$ & ${ }^{239,240} \mathbf{P u}$ \\
\hline 1995 & $2.0 \mathrm{E}-03 \pm 1.0 \mathrm{E}-03$ & $5.0 \mathrm{E}-02 \pm 2.0 \mathrm{E}-02$ & $2.4 \mathrm{E}-01 \pm 1.1 \mathrm{E}-01$ & $2.1 \mathrm{E}+00 \pm 2.0 \mathrm{E}+00$ & $2.1 \mathrm{E}+00 \pm 2.1 \mathrm{E}+00$ & $2.6 \mathrm{E}-02 \pm 2.4 \mathrm{E}-02$ \\
1996 & $2.0 \mathrm{E}-03 \pm 6.0 \mathrm{E}-03$ & $4.0 \mathrm{E}-02 \pm 1.0 \mathrm{E}-02$ & $1.5 \mathrm{E}-01 \pm 7.0 \mathrm{E}-02$ & $1.3 \mathrm{E}+00 \pm 1.0 \mathrm{E}+00$ & $1.2 \mathrm{E}+00 \pm 1.0 \mathrm{E}+00$ & $2.5 \mathrm{E}-02 \pm 3.3 \mathrm{E}-02$ \\
1997 & Not Detected & $4.5 \mathrm{E}-01 \pm 1.9 \mathrm{E}-01$ & $7.0 \mathrm{E}-02 \pm 3.0 \mathrm{E}-02$ & $9.0 \mathrm{E}-01 \pm 1.0 \mathrm{E}-01$ & $9.0 \mathrm{E}-01 \pm 9.0 \mathrm{E}-01$ & $3.8 \mathrm{E}-02 \pm 4.9 \mathrm{E}-02$ \\
1998 & Not Detected & $2.4 \mathrm{E}-01 \pm 1.2 \mathrm{E}-01$ & $9.0 \mathrm{E}-02 \pm 8.0 \mathrm{E}-02$ & $8.5 \mathrm{E}-01 \pm 9.8 \mathrm{E}-01$ & $8.2 \mathrm{E}-01 \pm 9.8 \mathrm{E}-01$ & $4.5 \mathrm{E}-02 \pm 5.7 \mathrm{E}-02$ \\
1999 & Not Detected & $8.7 \mathrm{E}-01 \pm 1.9 \mathrm{E}-01$ & $9.0 \mathrm{E}-02 \pm 3.0 \mathrm{E}-02$ & $7.5 \mathrm{E}-01 \pm 5.4 \mathrm{E}-01$ & $7.1 \mathrm{E}-01 \pm 5.3 \mathrm{E}-01$ & $4.0 \mathrm{E}-02 \pm 2.0 \mathrm{E}-02$ \\
2000 & Not Detected & $5.9 \mathrm{E}-01 \pm 1.8 \mathrm{E}-01$ & $1.4 \mathrm{E}-01 \pm 6.0 \mathrm{E}-02$ & $5.4 \mathrm{E}+00 \pm 5.6 \mathrm{E}+00$ & $5.4 \mathrm{E}+00 \pm 5.7 \mathrm{E}+00$ & $1.7 \mathrm{E}-01 \pm 8.0 \mathrm{E}-02$ \\
2001 & Not Detected & Not Detected & $5.0 \mathrm{E}-02 \pm 2.1 \mathrm{E}-02$ & $9.4 \mathrm{E}-01 \pm 7.1 \mathrm{E}-01$ & $9.5 \mathrm{E}-01 \pm 7.3 \mathrm{E}-01$ & $4.1 \mathrm{E}-02 \pm 2.6 \mathrm{E}-02$ \\
2002 & Not Detected & $2.8 \mathrm{E}-02 \pm 2.9 \mathrm{E}-02$ & $7.4 \mathrm{E}-02 \pm 1.3 \mathrm{E}-01$ & $1.5 \mathrm{E}+00 \pm 6.4 \mathrm{E}+00$ & $1.5 \mathrm{E}+00 \pm 6.4 \mathrm{E}+00$ & $2.4 \mathrm{E}-02 \pm 9.9 \mathrm{E}-02$ \\
2003 & Not Detected & $5.6 \mathrm{E}-02 \pm 7.3 \mathrm{E}-02$ & $8.1 \mathrm{E}-02 \pm 1.4 \mathrm{E}-01$ & $1.3 \mathrm{E}+00 \pm 5.1 \mathrm{E}+00$ & $1.3 \mathrm{E}+00 \pm 5.2 \mathrm{E}+00$ & $7.5 \mathrm{E}-02 \pm 3.8 \mathrm{E}-01$
\end{tabular}

$\overline{{ }^{ \pm} \pm 2 \text { standard deviations }}$ 
Table 3-3. 2003 Soil Sampling Results (pCi/g \pm total analytical uncertainty).

\begin{tabular}{|c|c|c|c|c|c|c|c|}
\hline Location & Isotope & Result \pm Error & $\mathbf{R Q}^{*}$ & Location & Isotope & \pm Error & $\mathbf{R Q}^{*}$ \\
\hline D146 & ${ }^{144} \mathrm{Ce}$ & $-2.8 \mathrm{E}-02 \pm 9.8 \mathrm{E}-02$ & $\bar{U}$ & D150 & ${ }^{144} \mathrm{Ce}$ & $-7.9 \mathrm{E}-02 \pm 1.6 \mathrm{E}-01$ & $\overline{\mathrm{U}}$ \\
\hline \multirow[t]{17}{*}{ (ERDF) } & ${ }^{60} \mathrm{Co}$ & $1.1 \mathrm{E}-02 \pm 7.8 \mathrm{E}-03$ & & $(100-B / C$ & ${ }^{60} \mathrm{Co}$ & $1.6 \mathrm{E}-02 \pm 1.1 \mathrm{E}-02$ & $\mathrm{U}$ \\
\hline & ${ }^{134} \mathrm{Cs}$ & $2.6 \mathrm{E}-02 \pm 9.6 \mathrm{E}-03$ & $\mathrm{U}$ & Remedial & ${ }^{134} \mathrm{Cs}$ & $3.5 \mathrm{E}-02 \pm 1.1 \mathrm{E}-02$ & $\mathrm{U}$ \\
\hline & ${ }^{137} \mathrm{Cs}$ & $2.3 \mathrm{E}-02 \pm 8.4 \mathrm{E}-03$ & & Action) & ${ }^{137} \mathrm{Cs}$ & $3.8 \mathrm{E}-01 \pm 6.1 \mathrm{E}-02$ & \\
\hline & ${ }^{152} \mathrm{Eu}$ & $-6.3 \mathrm{E}-03 \pm 2.0 \mathrm{E}-02$ & $\mathrm{U}$ & & ${ }^{152} \mathrm{Eu}$ & $2.7 \mathrm{E}-01 \pm 3.0 \mathrm{E}-02$ & \\
\hline & ${ }^{154} \mathrm{Eu}$ & $-2.4 \mathrm{E}-03 \pm 1.9 \mathrm{E}-02$ & $\mathrm{U}$ & & ${ }^{154} \mathrm{Eu}$ & $1.6 \mathrm{E}-02 \pm 2.8 \mathrm{E}-02$ & $\mathrm{U}$ \\
\hline & ${ }^{155} \mathrm{Eu}$ & $1.9 \mathrm{E}-02 \pm 2.6 \mathrm{E}-02$ & $\mathrm{U}$ & & ${ }^{155} \mathrm{Eu}$ & $5.8 \mathrm{E}-02 \pm 4.8 \mathrm{E}-02$ & $\mathrm{U}$ \\
\hline & ${ }^{238} \mathrm{Pu}$ & $-4.8 \mathrm{E}-03 \pm 1.3 \mathrm{E}-02$ & $\mathrm{U}$ & & ${ }^{238} \mathrm{Pu}$ & $1.2 \mathrm{E}-02 \pm 1.9 \mathrm{E}-02$ & $\mathrm{U}$ \\
\hline & ${ }^{239,240} \mathrm{Pu}$ & $-3.2 \mathrm{E}-03 \pm 6.4 \mathrm{E}-03$ & $\mathrm{U}$ & & ${ }^{239,240} \mathrm{Pu}$ & $1.9 \mathrm{E}-03 \pm 8.6 \mathrm{E}-03$ & $\mathrm{U}$ \\
\hline & ${ }^{103} \mathrm{Ru}$ & $-1.2 \mathrm{E}-03 \pm 5.1 \mathrm{E}-03$ & $\mathrm{U}$ & & ${ }^{103} \mathrm{Ru}$ & $-1.6 \mathrm{E}-03 \pm 8.7 \mathrm{E}-03$ & $\mathrm{U}$ \\
\hline & ${ }^{106} \mathrm{Ru}$ & $1.0 \mathrm{E}-02 \pm 4.6 \mathrm{E}-02$ & $\mathrm{U}$ & & ${ }^{106} \mathrm{Ru}$ & $9.9 \mathrm{E}-03 \pm 7.2 \mathrm{E}-02$ & $\mathrm{U}$ \\
\hline & ${ }^{125} \mathrm{Sb}$ & $-4.3 \mathrm{E}-03 \pm 1.5 \mathrm{E}-02$ & $\mathrm{U}$ & & ${ }^{125} \mathrm{Sb}$ & $8.3 \mathrm{E}-03 \pm 2.2 \mathrm{E}-02$ & $\mathrm{U}$ \\
\hline & ${ }^{113} \mathrm{Sn}$ & $-2.7 \mathrm{E}-03 \pm 6.8 \mathrm{E}-03$ & $\mathrm{U}$ & & ${ }^{113} \mathrm{Sn}$ & $1.8 \mathrm{E}-03 \pm 1.1 \mathrm{E}-02$ & $\mathrm{U}$ \\
\hline & ${ }^{90} \mathrm{Sr}$ & $3.9 \mathrm{E}-02 \pm 2.2 \mathrm{E}-01$ & $\mathrm{U}$ & & ${ }^{90} \mathrm{Sr}$ & $-2.5 \mathrm{E}-01 \pm 2.5 \mathrm{E}-01$ & $\mathrm{U}$ \\
\hline & ${ }^{234} \mathrm{U}$ & $1.8 \mathrm{E}-01 \pm 5.9 \mathrm{E}-02$ & & & & $1.3 \mathrm{E}-01 \pm 4.7 \mathrm{E}-02$ & \\
\hline & ${ }^{235} \mathrm{U}$ & $1.1 \mathrm{E}-02 \pm 1.0 \mathrm{E}-02$ & & & ${ }^{235} \mathrm{U}$ & $2.1 \mathrm{E}-02 \pm 1.6 \mathrm{E}-02$ & \\
\hline & ${ }^{238} \mathrm{U}$ & $2.0 \mathrm{E}-01 \pm 6.4 \mathrm{E}-02$ & & & ${ }^{238} \mathrm{U}$ & $1.6 \mathrm{E}-01 \pm 5.4 \mathrm{E}-02$ & \\
\hline & ${ }^{65} \mathrm{Zn}$ & $-9.4 \mathrm{E}-03 \pm 1.5 \mathrm{E}-02$ & $\mathrm{U}$ & & ${ }^{65} \mathrm{Zn}$ & $1.5 \mathrm{E}-02 \pm 3.8 \mathrm{E}-02$ & $\mathrm{U}$ \\
\hline D153 & ${ }^{144} \mathrm{Ce}$ & $-2.1 \mathrm{E}-02 \pm 1.0 \mathrm{E}-01$ & $\overline{\mathrm{U}}$ & D160 & ${ }^{144} \mathrm{Ce}$ & $-6.9 \mathrm{E}-03 \pm 6.9 \mathrm{E}-02$ & $\overline{\mathrm{U}}$ \\
\hline$(100-B / C$ & ${ }^{60} \mathrm{Co}$ & $-4.9 \mathrm{E}-03 \pm 5.2 \mathrm{E}-03$ & $\mathrm{U}$ & $(100-B / C$ & ${ }^{60} \mathrm{Co}$ & $2.2 \mathrm{E}-03 \pm 8.1 \mathrm{E}-03$ & $\mathrm{U}$ \\
\hline Remedial & ${ }^{134} \mathrm{Cs}$ & $3.0 \mathrm{E}-02 \pm 9.7 \mathrm{E}-03$ & $\mathrm{U}$ & Remedial & ${ }^{134} \mathrm{Cs}$ & $2.1 \mathrm{E}-02 \pm 1.2 \mathrm{E}-02$ & $\mathrm{U}$ \\
\hline \multirow[t]{15}{*}{ Action) } & ${ }^{137} \mathrm{Cs}$ & $2.6 \mathrm{E}-01 \pm 4.4 \mathrm{E}-02$ & & Action) & ${ }^{137} \mathrm{Cs}$ & $1.3 \mathrm{E}-01 \pm 2.7 \mathrm{E}-02$ & \\
\hline & ${ }^{152} \mathrm{Eu}$ & $-5.5 \mathrm{E}-03 \pm 1.9 \mathrm{E}-02$ & $\mathrm{U}$ & & ${ }^{152} \mathrm{Eu}$ & $6.4 \mathrm{E}-02 \pm 2.5 \mathrm{E}-02$ & \\
\hline & ${ }^{154} \mathrm{Eu}$ & $2.3 \mathrm{E}-03 \pm 1.7 \mathrm{E}-02$ & $\mathrm{U}$ & & ${ }^{154} \mathrm{Eu}$ & $-1.1 \mathrm{E}-02 \pm 2.6 \mathrm{E}-02$ & $\mathrm{U}$ \\
\hline & ${ }^{155} \mathrm{Eu}$ & $4.7 \mathrm{E}-02 \pm 2.6 \mathrm{E}-02$ & $\mathrm{U}$ & & ${ }^{155} \mathrm{Eu}$ & $-6.1 \mathrm{E}-03 \pm 4.4 \mathrm{E}-02$ & $\mathrm{U}$ \\
\hline & ${ }^{238} \mathrm{Pu}$ & $2.4 \mathrm{E}-02 \pm 4.1 \mathrm{E}-02$ & $\mathrm{U}$ & & ${ }^{238} \mathrm{Pu}$ & $-2.4 \mathrm{E}-02 \pm 3.4 \mathrm{E}-02$ & $\mathrm{U}$ \\
\hline & ${ }^{239,240} \mathrm{Pu}$ & $1.8 \mathrm{E}-01 \pm 6.1 \mathrm{E}-02$ & & & ${ }^{239,240} \mathrm{Pu}$ & $7.9 \mathrm{E}-03 \pm 9.5 \mathrm{E}-03$ & $\mathrm{U}$ \\
\hline & ${ }^{103} \mathrm{Ru}$ & $-7.2 \mathrm{E}-04 \pm 5.3 \mathrm{E}-03$ & $\mathrm{U}$ & & ${ }^{103} \mathrm{Ru}$ & $2.9 \mathrm{E}-03 \pm 9.0 \mathrm{E}-03$ & $\mathrm{U}$ \\
\hline & ${ }^{106} \mathrm{Ru}$ & $2.4 \mathrm{E}-02 \pm 4.4 \mathrm{E}-02$ & $\mathrm{U}$ & & ${ }^{106} \mathrm{Ru}$ & $-3.2 \mathrm{E}-02 \pm 7.6 \mathrm{E}-02$ & $\mathrm{U}$ \\
\hline & ${ }^{125} \mathrm{Sb}$ & 4.7E-03 $\pm 1.5 \mathrm{E}-02$ & $\mathrm{U}$ & & ${ }^{125} \mathrm{Sb}$ & $6.8 \mathrm{E}-03 \pm 2.4 \mathrm{E}-02$ & $\mathrm{U}$ \\
\hline & ${ }^{113} \mathrm{Sn}$ & $-1.9 \mathrm{E}-03 \pm 7.0 \mathrm{E}-03$ & $\mathrm{U}$ & & ${ }^{113} \mathrm{Sn}$ & $1.5 \mathrm{E}-02 \pm 1.3 \mathrm{E}-02$ & $\mathrm{U}$ \\
\hline & ${ }^{90} \mathrm{Sr}$ & $-1.0 \mathrm{E}-01 \pm 2.4 \mathrm{E}-01$ & $\mathrm{U}$ & & ${ }^{90} \mathrm{Sr}$ & $3.4 \mathrm{E}-01 \pm 3.2 \mathrm{E}-01$ & \\
\hline & ${ }^{234} \mathrm{U}$ & $1.6 \mathrm{E}-01 \pm 5.4 \mathrm{E}-02$ & & & ${ }^{234} \mathrm{U}$ & $1.7 \mathrm{E}-01 \pm 5.6 \mathrm{E}-02$ & \\
\hline & ${ }^{235} \mathrm{U}$ & $8.5 \mathrm{E}-03 \pm 1.0 \mathrm{E}-02$ & $\mathrm{U}$ & & ${ }^{235} \mathrm{U}$ & $4.1 \mathrm{E}-03 \pm 8.2 \mathrm{E}-03$ & $\mathrm{U}$ \\
\hline & ${ }^{238} \mathrm{U}$ & $1.6 \mathrm{E}-01 \pm 5.4 \mathrm{E}-02$ & & & ${ }^{238} \mathrm{U}$ & $1.6 \mathrm{E}-01 \pm 5.4 \mathrm{E}-02$ & \\
\hline & ${ }^{65} \mathrm{Zn}$ & $-3.8 \mathrm{E}-03 \pm 1.4 \mathrm{E}-02$ & $\mathrm{U}$ & & ${ }^{65} \mathrm{Zn}$ & $2.0 \mathrm{E}-02 \pm 2.4 \mathrm{E}-02$ & $\mathrm{U}$ \\
\hline
\end{tabular}

$\overline{\mathrm{RQ}}=$ Result Qualifier. U $=$ The analyte was analyzed for but not detected. 
Table 3-3. 2003 Soil Sampling Results (pCi/g \pm total analytical uncertainty). (cont)

\begin{tabular}{|c|c|c|c|c|c|c|c|}
\hline Location & Isotope & Result \pm Error & $\mathbf{R Q}^{*}$ & Location & Isotope & \pm Error & $\mathbf{R Q}^{*}$ \\
\hline D161 & ${ }^{144} \mathrm{Ce}$ & $1.4 \mathrm{E}-01 \pm 1.6 \mathrm{E}-01$ & $\bar{U}$ & D165 & ${ }^{144} \mathrm{Ce}$ & $-5.1 \mathrm{E}-02 \pm 1.1 \mathrm{E}-01$ & $\mathrm{U}$ \\
\hline$(100-B / C$ & ${ }^{60} \mathrm{Co}$ & $-1.3 \mathrm{E}-02 \pm 1.3 \mathrm{E}-02$ & $\mathrm{U}$ & $(100-B / C$ & ${ }^{60} \mathrm{Co}$ & $4.3 \mathrm{E}-03 \pm 5.6 \mathrm{E}-03$ & $\mathrm{U}$ \\
\hline Remedial & ${ }^{134} \mathrm{Cs}$ & $3.1 \mathrm{E}-02 \pm 1.2 \mathrm{E}-02$ & $\mathrm{U}$ & Remedial & ${ }^{134} \mathrm{Cs}$ & $4.3 \mathrm{E}-02 \pm 1.3 \mathrm{E}-02$ & $\mathrm{U}$ \\
\hline \multirow[t]{15}{*}{ Action) } & ${ }^{137} \mathrm{Cs}$ & $1.7 \mathrm{E}-01 \pm 3.2 \mathrm{E}-02$ & & Action) & ${ }^{137} \mathrm{Cs}$ & $1.8 \mathrm{E}-01 \pm 3.0 \mathrm{E}-02$ & \\
\hline & ${ }^{152} \mathrm{Eu}$ & 2.9E-01 $\pm 2.9 \mathrm{E}-02$ & & & ${ }^{152} \mathrm{Eu}$ & $7.1 \mathrm{E}-04 \pm 7.1 \mathrm{E}-03$ & $\mathrm{U}$ \\
\hline & ${ }^{154} \mathrm{Eu}$ & $1.6 \mathrm{E}-02 \pm 3.3 \mathrm{E}-02$ & $\mathrm{U}$ & & ${ }^{154} \mathrm{Eu}$ & $7.8 \mathrm{E}-03 \pm 1.8 \mathrm{E}-02$ & $\mathrm{U}$ \\
\hline & ${ }^{155} \mathrm{Eu}$ & $4.9 \mathrm{E}-02 \pm 4.3 \mathrm{E}-02$ & $\mathrm{U}$ & & ${ }^{155} \mathrm{Eu}$ & $5.6 \mathrm{E}-02 \pm 3.9 \mathrm{E}-02$ & \\
\hline & ${ }^{238} \mathrm{Pu}$ & $1.5 \mathrm{E}-02 \pm 3.6 \mathrm{E}-02$ & $\mathrm{U}$ & & ${ }^{238} \mathrm{Pu}$ & $-5.6 \mathrm{E}-03 \pm 2.5 \mathrm{E}-02$ & $\mathrm{U}$ \\
\hline & ${ }^{239,240} \mathrm{Pu}$ & $1.3 \mathrm{E}-02 \pm 1.6 \mathrm{E}-02$ & $\mathrm{U}$ & & ${ }^{239,240} \mathrm{Pu}$ & $1.8 \mathrm{E}-03 \pm 9.5 \mathrm{E}-03$ & $\mathrm{U}$ \\
\hline & ${ }^{103} \mathrm{Ru}$ & $-6.1 \mathrm{E}-03 \pm 8.5 \mathrm{E}-03$ & $\mathrm{U}$ & & ${ }^{103} \mathrm{Ru}$ & $-7.2 \mathrm{E}-04 \pm 5.4 \mathrm{E}-03$ & $\mathrm{U}$ \\
\hline & ${ }^{106} \mathrm{Ru}$ & $-1.3 \mathrm{E}-02 \pm 7.8 \mathrm{E}-02$ & $\mathrm{U}$ & & ${ }^{106} \mathrm{Ru}$ & $1.9 \mathrm{E}-02 \pm 4.5 \mathrm{E}-02$ & $\mathrm{U}$ \\
\hline & ${ }^{125} \mathrm{Sb}$ & $-3.9 \mathrm{E}-03 \pm 2.3 \mathrm{E}-02$ & $\mathrm{U}$ & & ${ }^{125} \mathrm{Sb}$ & $3.2 \mathrm{E}-03 \pm 1.6 \mathrm{E}-02$ & $\mathrm{U}$ \\
\hline & ${ }^{113} \mathrm{Sn}$ & $-8.8 \mathrm{E}-03 \pm 1.1 \mathrm{E}-02$ & $\mathrm{U}$ & & ${ }^{113} \mathrm{Sn}$ & $-4.7 \mathrm{E}-03 \pm 7.4 \mathrm{E}-03$ & $\mathrm{U}$ \\
\hline & ${ }^{90} \mathrm{Sr}$ & $-7.3 \mathrm{E}-02 \pm 2.6 \mathrm{E}-01$ & $\mathrm{U}$ & & ${ }^{90} \mathrm{Sr}$ & $3.4 \mathrm{E}-01 \pm 2.7 \mathrm{E}-01$ & \\
\hline & ${ }^{234} \mathrm{U}$ & $1.5 \mathrm{E}-01 \pm 5.3 \mathrm{E}-02$ & & & & $1.3 \mathrm{E}-01 \pm 4.7 \mathrm{E}-02$ & \\
\hline & ${ }^{235} \mathrm{U}$ & $6.4 \mathrm{E}-03 \pm 7.7 \mathrm{E}-03$ & & & ${ }^{235} \mathrm{U}$ & $6.7 \mathrm{E}-03 \pm 8.0 \mathrm{E}-03$ & \\
\hline & ${ }^{238} \mathrm{U}$ & $1.4 \mathrm{E}-01 \pm 4.9 \mathrm{E}-02$ & & & ${ }^{238} \mathrm{U}$ & $9.6 \mathrm{E}-02 \pm 3.7 \mathrm{E}-02$ & \\
\hline & ${ }^{65} \mathrm{Zn}$ & $1.8 \mathrm{E}-03 \pm 1.8 \mathrm{E}-02$ & $\mathrm{U}$ & & ${ }^{65} \mathrm{Zn}$ & $1.1 \mathrm{E}-03 \pm 1.1 \mathrm{E}-02$ & $\mathrm{U}$ \\
\hline D154 & ${ }^{144} \mathrm{Ce}$ & $-1.4 \mathrm{E}-03 \pm 1.4 \mathrm{E}-02$ & $\bar{U}$ & D155 & ${ }^{144} \mathrm{Ce}$ & $5.0 \mathrm{E}-02 \pm 1.3 \mathrm{E}-01$ & $\mathrm{U}$ \\
\hline$(100-F$ & ${ }^{60} \mathrm{Co}$ & $-7.1 \mathrm{E}-03 \pm 8.2 \mathrm{E}-03$ & $\mathrm{U}$ & $(100-\mathrm{F}$ & ${ }^{60} \mathrm{Co}$ & $6.8 \mathrm{E}-03 \pm 6.5 \mathrm{E}-03$ & $\mathrm{U}$ \\
\hline Remedial & ${ }^{134} \mathrm{Cs}$ & $3.5 \mathrm{E}-02 \pm 1.3 \mathrm{E}-02$ & $\mathrm{U}$ & Remedial & ${ }^{134} \mathrm{Cs}$ & $3.6 \mathrm{E}-02 \pm 1.2 \mathrm{E}-02$ & $\mathrm{U}$ \\
\hline \multirow[t]{15}{*}{ Action) } & ${ }^{137} \mathrm{Cs}$ & $8.9 \mathrm{E}-02 \pm 2.2 \mathrm{E}-02$ & & Action) & ${ }^{137} \mathrm{Cs}$ & $2.5 \mathrm{E}-01 \pm 3.6 \mathrm{E}-02$ & \\
\hline & ${ }^{152} \mathrm{Eu}$ & $-1.8 \mathrm{E}-02 \pm 4.0 \mathrm{E}-02$ & $\mathrm{U}$ & & ${ }^{152} \mathrm{Eu}$ & $3.2 \mathrm{E}-01 \pm 3.0 \mathrm{E}-02$ & \\
\hline & ${ }^{154} \mathrm{Eu}$ & $-9.3 \mathrm{E}-03 \pm 2.6 \mathrm{E}-02$ & $\mathrm{U}$ & & ${ }^{154} \mathrm{Eu}$ & $4.8 \mathrm{E}-02 \pm 3.0 \mathrm{E}-02$ & $\mathrm{U}$ \\
\hline & ${ }^{155} \mathrm{Eu}$ & $4.4 \mathrm{E}-02 \pm 3.0 \mathrm{E}-02$ & $\mathrm{U}$ & & ${ }^{155} \mathrm{Eu}$ & $2.2 \mathrm{E}-03 \pm 2.2 \mathrm{E}-02$ & $\mathrm{U}$ \\
\hline & ${ }^{238} \mathrm{Pu}$ & $9.2 \mathrm{E}-03 \pm 3.2 \mathrm{E}-02$ & $\mathrm{U}$ & & ${ }^{238} \mathrm{Pu}$ & $1.9 \mathrm{E}-03 \pm 1.9 \mathrm{E}-02$ & $\mathrm{U}$ \\
\hline & ${ }^{239,240} \mathrm{Pu}$ & $2.3 \mathrm{E}-03 \pm 2.3 \mathrm{E}-02$ & $\mathrm{U}$ & & ${ }^{239,240} \mathrm{Pu}$ & $5.7 \mathrm{E}-03 \pm 1.0 \mathrm{E}-02$ & $\mathrm{U}$ \\
\hline & ${ }^{103} \mathrm{Ru}$ & $1.2 \mathrm{E}-03 \pm 7.1 \mathrm{E}-03$ & $\mathrm{U}$ & & ${ }^{103} \mathrm{Ru}$ & $-2.1 \mathrm{E}-03 \pm 6.4 \mathrm{E}-03$ & $\mathrm{U}$ \\
\hline & ${ }^{106} \mathrm{Ru}$ & $3.4 \mathrm{E}-02 \pm 6.8 \mathrm{E}-02$ & $\mathrm{U}$ & & ${ }^{106} \mathrm{Ru}$ & $-1.2 \mathrm{E}-02 \pm 5.9 \mathrm{E}-02$ & $\mathrm{U}$ \\
\hline & ${ }^{125} \mathrm{Sb}$ & $1.1 \mathrm{E}-02 \pm 2.1 \mathrm{E}-02$ & $\mathrm{U}$ & & ${ }^{125} \mathrm{Sb}$ & $6.0 \mathrm{E}-03 \pm 2.0 \mathrm{E}-02$ & $\mathrm{U}$ \\
\hline & ${ }^{113} \mathrm{Sn}$ & $1.0 \mathrm{E}-03 \pm 9.6 \mathrm{E}-03$ & $\mathrm{U}$ & & ${ }^{113} \mathrm{Sn}$ & $-6.2 \mathrm{E}-03 \pm 9.3 \mathrm{E}-03$ & $\mathrm{U}$ \\
\hline & ${ }^{90} \mathrm{Sr}$ & $5.7 \mathrm{E}-02 \pm 2.2 \mathrm{E}-01$ & $\mathrm{U}$ & & ${ }^{90} \mathrm{Sr}$ & $-1.4 \mathrm{E}-01 \pm 2.3 \mathrm{E}-01$ & $\mathrm{U}$ \\
\hline & ${ }^{234} \mathrm{U}$ & $1.2 \mathrm{E}-01 \pm 3.8 \mathrm{E}-02$ & & & ${ }^{234} \mathrm{U}$ & $6.7 \mathrm{E}-02 \pm 2.8 \mathrm{E}-02$ & \\
\hline & ${ }^{235} \mathrm{U}$ & $6.5 \mathrm{E}-03 \pm 7.8 \mathrm{E}-03$ & & & ${ }^{235} \mathrm{U}$ & 7.9E-03 $\pm 7.9 \mathrm{E}-03$ & \\
\hline & ${ }^{238} \mathrm{U}$ & $1.1 \mathrm{E}-01 \pm 3.6 \mathrm{E}-02$ & & & ${ }^{238} \mathrm{U}$ & $9.2 \mathrm{E}-02 \pm 3.1 \mathrm{E}-02$ & \\
\hline & ${ }^{65} \mathrm{Zn}$ & $4.4 \mathrm{E}-02 \pm 2.3 \mathrm{E}-02$ & $\mathrm{U}$ & & ${ }^{65} \mathrm{Zn}$ & $-7.3 \mathrm{E}-03 \pm 2.9 \mathrm{E}-02$ & $\mathrm{U}$ \\
\hline
\end{tabular}

$\overline{\mathrm{RQ}}=$ Result Qualifier. U $=$ The analyte was analyzed for but not detected. 
Table 3-3. 2003 Soil Sampling Results (pCi/g \pm total analytical uncertainty). (cont)

\begin{tabular}{|c|c|c|c|c|c|c|c|}
\hline Location & Isotope & Result \pm Error & $\overline{\mathbf{R Q}^{*}}$ & Location & Isotope & \pm Error & $\overline{\mathbf{R Q}^{*}}$ \\
\hline D162 & ${ }^{144} \mathrm{Ce}$ & $-1.3 \mathrm{E}-01 \pm 2.0 \mathrm{E}-01$ & $\overline{\mathrm{U}}$ & D163 & ${ }^{144} \mathrm{Ce}$ & $-1.8 \mathrm{E}-02 \pm 1.5 \mathrm{E}-01$ & $\bar{U}$ \\
\hline (100-KR-1 & ${ }^{60} \mathrm{Co}$ & $4.2 \mathrm{E}-03 \pm 8.4 \mathrm{E}-03$ & $\mathrm{U}$ & (100-KR-1 & ${ }^{60} \mathrm{Co}$ & $5.1 \mathrm{E}-03 \pm 7.8 \mathrm{E}-03$ & U \\
\hline Remedial & ${ }^{134} \mathrm{Cs}$ & $3.2 \mathrm{E}-02 \pm 1.6 \mathrm{E}-02$ & $\mathrm{U}$ & Remedial & ${ }^{134} \mathrm{Cs}$ & $4.0 \mathrm{E}-02 \pm 1.5 \mathrm{E}-02$ & $\mathrm{U}$ \\
\hline \multirow[t]{15}{*}{ Action) } & ${ }^{137} \mathrm{Cs}$ & $1.3 \mathrm{E}-01 \pm 2.8 \mathrm{E}-02$ & & Action) & ${ }^{137} \mathrm{Cs}$ & $2.1 \mathrm{E}-01 \pm 3.0 \mathrm{E}-02$ & \\
\hline & ${ }^{152} \mathrm{Eu}$ & $1.3 \mathrm{E}-02 \pm 5.5 \mathrm{E}-02$ & $\mathrm{U}$ & & ${ }^{152} \mathrm{Eu}$ & $1.2 \mathrm{E}-01 \pm 2.6 \mathrm{E}-02$ & \\
\hline & ${ }^{154} \mathrm{Eu}$ & $-5.8 \mathrm{E}-03 \pm 2.8 \mathrm{E}-02$ & $\mathrm{U}$ & & ${ }^{154} \mathrm{Eu}$ & 4.3E-02 $\pm 3.5 \mathrm{E}-02$ & $\mathrm{U}$ \\
\hline & ${ }^{155} \mathrm{Eu}$ & $1.7 \mathrm{E}-02 \pm 4.7 \mathrm{E}-02$ & $\mathrm{U}$ & & ${ }^{155} \mathrm{Eu}$ & $8.2 \mathrm{E}-02 \pm 5.5 \mathrm{E}-02$ & $\mathrm{U}$ \\
\hline & ${ }^{238} \mathrm{Pu}$ & $-3.4 \mathrm{E}-02 \pm 3.4 \mathrm{E}-02$ & $\mathrm{U}$ & & ${ }^{238} \mathrm{Pu}$ & $1.5 \mathrm{E}-02 \pm 2.1 \mathrm{E}-02$ & U \\
\hline & ${ }^{239,240} \mathrm{Pu}$ & $2.1 \mathrm{E}-03 \pm 1.1 \mathrm{E}-02$ & $\mathrm{U}$ & & ${ }^{239,240} \mathrm{Pu}$ & $1.1 \mathrm{E}-02 \pm 1.3 \mathrm{E}-02$ & $\mathrm{U}$ \\
\hline & ${ }^{103} \mathrm{Ru}$ & $-2.9 \mathrm{E}-03 \pm 9.7 \mathrm{E}-03$ & $\mathrm{U}$ & & ${ }^{103} \mathrm{Ru}$ & $8.8 \mathrm{E}-04 \pm 8.0 \mathrm{E}-03$ & U \\
\hline & ${ }^{106} \mathrm{Ru}$ & $6.0 \mathrm{E}-02 \pm 8.0 \mathrm{E}-02$ & $\mathrm{U}$ & & ${ }^{106} \mathrm{Ru}$ & $-1.4 \mathrm{E}-02 \pm 7.2 \mathrm{E}-02$ & $\mathrm{U}$ \\
\hline & ${ }^{125} \mathrm{Sb}$ & $-1.3 \mathrm{E}-02 \pm 2.6 \mathrm{E}-02$ & $\mathrm{U}$ & & ${ }^{125} \mathrm{Sb}$ & $-3.9 \mathrm{E}-03 \pm 2.1 \mathrm{E}-02$ & $\mathrm{U}$ \\
\hline & ${ }^{113} \mathrm{Sn}$ & $-5.6 \mathrm{E}-03 \pm 1.2 \mathrm{E}-02$ & $\mathrm{U}$ & & ${ }^{113} \mathrm{Sn}$ & $-1.7 \mathrm{E}-02 \pm 1.7 \mathrm{E}-02$ & $\mathrm{U}$ \\
\hline & ${ }^{90} \mathrm{Sr}$ & $2.3 \mathrm{E}-01 \pm 2.6 \mathrm{E}-01$ & & & ${ }^{90} \mathrm{Sr}$ & $-3.1 \mathrm{E}-01 \pm 3.1 \mathrm{E}-01$ & $\mathrm{U}$ \\
\hline & ${ }^{234} \mathrm{U}$ & $1.3 \mathrm{E}-01 \pm 4.7 \mathrm{E}-02$ & & & ${ }^{234} \mathrm{U}$ & $2.1 \mathrm{E}-01 \pm 6.9 \mathrm{E}-02$ & \\
\hline & ${ }^{235} \mathrm{U}$ & $1.2 \mathrm{E}-02 \pm 1.2 \mathrm{E}-02$ & $\mathrm{U}$ & & ${ }^{235} \mathrm{U}$ & $2.0 \mathrm{E}-02 \pm 1.6 \mathrm{E}-02$ & \\
\hline & ${ }^{238} \mathrm{U}$ & $1.4 \mathrm{E}-01 \pm 4.9 \mathrm{E}-02$ & & & ${ }^{238} \mathrm{U}$ & $2.0 \mathrm{E}-01 \pm 6.6 \mathrm{E}-02$ & \\
\hline & ${ }^{65} \mathrm{Zn}$ & $3.8 \mathrm{E}-03 \pm 2.5 \mathrm{E}-02$ & $\mathrm{U}$ & & ${ }^{65} \mathrm{Zn}$ & $-1.4 \mathrm{E}-02 \pm 2.2 \mathrm{E}-02$ & $\mathrm{U}$ \\
\hline Y608 & ${ }^{144} \mathrm{Ce}$ & $-2.6 \mathrm{E}-02 \pm 1.5 \mathrm{E}-01$ & $\overline{\mathrm{U}}$ & D001 & ${ }^{144} \mathrm{Ce}$ & $-1.1 \mathrm{E}-01 \pm 1.3 \mathrm{E}-01$ & $\bar{U}$ \\
\hline \multirow[t]{17}{*}{$(100-N)$} & ${ }^{60} \mathrm{Co}$ & $1.8 \mathrm{E}-01 \pm 2.2 \mathrm{E}-02$ & & (200 West) & ${ }^{60} \mathrm{Co}$ & $1.6 \mathrm{E}-03 \pm 6.5 \mathrm{E}-03$ & $\mathrm{U}$ \\
\hline & ${ }^{134} \mathrm{Cs}$ & $4.0 \mathrm{E}-02 \pm 1.4 \mathrm{E}-02$ & $\mathrm{U}$ & & ${ }^{134} \mathrm{Cs}$ & $2.4 \mathrm{E}-02 \pm 1.1 \mathrm{E}-02$ & \\
\hline & ${ }^{137} \mathrm{Cs}$ & $2.1 \mathrm{E}-01 \pm 3.7 \mathrm{E}-02$ & & & ${ }^{137} \mathrm{Cs}$ & $5.4 \mathrm{E}-01 \pm 8.8 \mathrm{E}-02$ & \\
\hline & ${ }^{152} \mathrm{Eu}$ & $-1.1 \mathrm{E}-02 \pm 3.2 \mathrm{E}-02$ & $\mathrm{U}$ & & ${ }^{152} \mathrm{Eu}$ & $-1.2 \mathrm{E}-02 \pm 2.3 \mathrm{E}-02$ & $\mathrm{U}$ \\
\hline & ${ }^{154} \mathrm{Eu}$ & $-1.6 \mathrm{E}-02 \pm 2.3 \mathrm{E}-02$ & $\mathrm{U}$ & & ${ }^{154} \mathrm{Eu}$ & $1.2 \mathrm{E}-02 \pm 2.3 \mathrm{E}-02$ & $\mathrm{U}$ \\
\hline & ${ }^{155} \mathrm{Eu}$ & $3.3 \mathrm{E}-02 \pm 3.6 \mathrm{E}-02$ & $\mathrm{U}$ & & ${ }^{155} \mathrm{Eu}$ & $3.2 \mathrm{E}-02 \pm 3.1 \mathrm{E}-02$ & $\mathrm{U}$ \\
\hline & ${ }^{238} \mathrm{Pu}$ & $-1.8 \mathrm{E}-03 \pm 1.7 \mathrm{E}-02$ & $\mathrm{U}$ & & ${ }^{238} \mathrm{Pu}$ & $3.3 \mathrm{E}-03 \pm 1.2 \mathrm{E}-02$ & $\mathrm{U}$ \\
\hline & ${ }^{239,240} \mathrm{Pu}$ & $1.8 \mathrm{E}-03 \pm 6.3 \mathrm{E}-03$ & $\mathrm{U}$ & & ${ }^{239,240} \mathrm{Pu}$ & $6.6 \mathrm{E}-02 \pm 2.2 \mathrm{E}-02$ & \\
\hline & ${ }^{103} \mathrm{Ru}$ & $-2.4 \mathrm{E}-03 \pm 7.5 \mathrm{E}-03$ & $\mathrm{U}$ & & ${ }^{103} \mathrm{Ru}$ & $-4.0 \mathrm{E}-04 \pm 4.1 \mathrm{E}-03$ & $\mathrm{U}$ \\
\hline & ${ }^{106} \mathrm{Ru}$ & $-4.1 \mathrm{E}-02 \pm 6.6 \mathrm{E}-02$ & $\mathrm{U}$ & & ${ }^{106} \mathrm{Ru}$ & $1.5 \mathrm{E}-02 \pm 5.6 \mathrm{E}-02$ & $\mathrm{U}$ \\
\hline & ${ }^{125} \mathrm{Sb}$ & $2.4 \mathrm{E}-04 \pm 2.4 \mathrm{E}-03$ & $\mathrm{U}$ & & ${ }^{125} \mathrm{Sb}$ & $1.1 \mathrm{E}-02 \pm 2.0 \mathrm{E}-02$ & U \\
\hline & ${ }^{113} \mathrm{Sn}$ & $-3.0 \mathrm{E}-04 \pm 3.0 \mathrm{E}-03$ & $\mathrm{U}$ & & ${ }^{113} \mathrm{Sn}$ & $-1.8 \mathrm{E}-03 \pm 8.9 \mathrm{E}-03$ & U \\
\hline & ${ }^{90} \mathrm{Sr}$ & $-8.2 \mathrm{E}-02 \pm 2.4 \mathrm{E}-01$ & $\mathrm{U}$ & & ${ }^{90} \mathrm{Sr}$ & $6.6 \mathrm{E}-02 \pm 2.0 \mathrm{E}-01$ & $\mathrm{U}$ \\
\hline & ${ }^{234} \mathrm{U}$ & $1.4 \mathrm{E}-01 \pm 4.9 \mathrm{E}-02$ & & & ${ }^{234} \mathrm{U}$ & $1.2 \mathrm{E}-01 \pm 3.7 \mathrm{E}-02$ & \\
\hline & ${ }^{235} \mathrm{U}$ & $6.5 \mathrm{E}-03 \pm 1.2 \mathrm{E}-02$ & $\mathrm{U}$ & & ${ }^{235} \mathrm{U}$ & $1.2 \mathrm{E}-02 \pm 1.2 \mathrm{E}-02$ & U \\
\hline & ${ }^{238} \mathrm{U}$ & $1.5 \mathrm{E}-01 \pm 5.1 \mathrm{E}-02$ & & & ${ }^{238} \mathrm{U}$ & $1.4 \mathrm{E}-01 \pm 4.1 \mathrm{E}-02$ & \\
\hline & ${ }^{65} \mathrm{Zn}$ & $-2.9 \mathrm{E}-03 \pm 2.0 \mathrm{E}-02$ & $\mathrm{U}$ & & ${ }^{65} \mathrm{Zn}$ & $-6.5 \mathrm{E}-03 \pm 1.7 \mathrm{E}-02$ & U \\
\hline
\end{tabular}

$\overline{\mathrm{RQ}}=$ Result Qualifier. U $=$ The analyte was analyzed for but not detected. 
Table 3-3. 2003 Soil Sampling Results (pCi/g \pm total analytical uncertainty). (cont)

\begin{tabular}{|c|c|c|c|c|c|c|c|c|}
\hline Location & Isotope & Result \pm Error & $\overline{\mathbf{R Q}^{*}}$ & Location & Isotope & Result & \pm Error & $\overline{\mathbf{R Q}^{*}}$ \\
\hline D003 & ${ }^{144} \mathrm{Ce}$ & $-1.7 \mathrm{E}-02 \pm 1.3 \mathrm{E}-01$ & $\overline{\mathrm{U}}$ & D005 & ${ }^{144} \mathrm{Ce}$ & $-1.2 \mathrm{E}-01$ & $\pm 1.3 \mathrm{E}-01$ & $\bar{U}$ \\
\hline \multirow[t]{17}{*}{ (200 West) } & ${ }^{60} \mathrm{Co}$ & $-1.5 \mathrm{E}-03 \pm 6.3 \mathrm{E}-03$ & $\mathrm{U}$ & (200 West) & ${ }^{60} \mathrm{Co}$ & $1.1 \mathrm{E}-03$ & $\pm 8.3 \mathrm{E}-03$ & U \\
\hline & ${ }^{134} \mathrm{Cs}$ & $3.5 \mathrm{E}-02 \pm 1.0 \mathrm{E}-02$ & & & ${ }^{134} \mathrm{Cs}$ & $4.0 \mathrm{E}-02$ & $\pm 1.6 \mathrm{E}-02$ & \\
\hline & ${ }^{137} \mathrm{Cs}$ & $8.6 \mathrm{E}-01 \pm 1.4 \mathrm{E}-01$ & & & ${ }^{137} \mathrm{Cs}$ & $1.1 \mathrm{E}-01$ & $\pm 2.5 \mathrm{E}-02$ & \\
\hline & ${ }^{152} \mathrm{Eu}$ & $-1.3 \mathrm{E}-02 \pm 2.6 \mathrm{E}-02$ & $\mathrm{U}$ & & ${ }^{152} \mathrm{Eu}$ & $1.9 \mathrm{E}-02$ & $\pm 3.9 \mathrm{E}-02$ & $\mathrm{U}$ \\
\hline & ${ }^{154} \mathrm{Eu}$ & $-8.5 \mathrm{E}-03 \pm 2.0 \mathrm{E}-02$ & $\mathrm{U}$ & & ${ }^{154} \mathrm{Eu}$ & $1.1 \mathrm{E}-02$ & $\pm 3.1 \mathrm{E}-02$ & $\mathrm{U}$ \\
\hline & ${ }^{155} \mathrm{Eu}$ & $3.9 \mathrm{E}-02 \pm 4.1 \mathrm{E}-02$ & $\mathrm{U}$ & & ${ }^{155} \mathrm{Eu}$ & $3.9 \mathrm{E}-02$ & $\pm 4.4 \mathrm{E}-02$ & $\mathrm{U}$ \\
\hline & ${ }^{238} \mathrm{Pu}$ & $-1.7 \mathrm{E}-03 \pm 1.0 \mathrm{E}-02$ & $\mathrm{U}$ & & ${ }^{238} \mathrm{Pu}$ & $3.4 \mathrm{E}-03$ & $\pm 1.1 \mathrm{E}-02$ & $\mathrm{U}$ \\
\hline & ${ }^{239,240} \mathrm{Pu}$ & $3.8 \mathrm{E}-02 \pm 1.7 \mathrm{E}-02$ & & & ${ }^{239,240} \mathrm{Pu}$ & $1.2 \mathrm{E}-02$ & $\pm 9.2 \mathrm{E}-03$ & \\
\hline & ${ }^{103} \mathrm{Ru}$ & $-1.8 \mathrm{E}-03 \pm 6.5 \mathrm{E}-03$ & $\mathrm{U}$ & & ${ }^{103} \mathrm{Ru}$ & $-1.2 \mathrm{E}-03$ & $\pm 7.0 \mathrm{E}-03$ & U \\
\hline & ${ }^{106} \mathrm{Ru}$ & $6.8 \mathrm{E}-03 \pm 5.5 \mathrm{E}-02$ & $\mathrm{U}$ & & ${ }^{106} \mathrm{Ru}$ & $-3.4 \mathrm{E}-02$ & $\pm 7.1 \mathrm{E}-02$ & $\mathrm{U}$ \\
\hline & ${ }^{125} \mathrm{Sb}$ & $2.0 \mathrm{E}-02 \pm 2.1 \mathrm{E}-02$ & $\mathrm{U}$ & & ${ }^{125} \mathrm{Sb}$ & $1.4 \mathrm{E}-02$ & $\pm 2.1 \mathrm{E}-02$ & $\mathrm{U}$ \\
\hline & ${ }^{113} \mathrm{Sn}$ & $-1.3 \mathrm{E}-03 \pm 9.1 \mathrm{E}-03$ & $\mathrm{U}$ & & ${ }^{113} \mathrm{Sn}$ & $-8.0 \mathrm{E}-03$ & $\pm 1.0 \mathrm{E}-02$ & $\mathrm{U}$ \\
\hline & ${ }^{90} \mathrm{Sr}$ & $2.1 \mathrm{E}-01 \pm 2.3 \mathrm{E}-01$ & $\mathrm{U}$ & & ${ }^{90} \mathrm{Sr}$ & $-1.9 \mathrm{E}-01$ & $\pm 1.9 \mathrm{E}-01$ & $\mathrm{U}$ \\
\hline & ${ }^{234} \mathrm{U}$ & $1.8 \mathrm{E}-01 \pm 5.0 \mathrm{E}-02$ & & & ${ }^{234} \mathrm{U}$ & $1.8 \mathrm{E}-01$ & $\pm 5.0 \mathrm{E}-02$ & \\
\hline & ${ }^{235} \mathrm{U}$ & $8.1 \mathrm{E}-03 \pm 8.1 \mathrm{E}-03$ & & & ${ }^{235} \mathrm{U}$ & 8.5E-03 & $\pm 8.5 \mathrm{E}-03$ & \\
\hline & ${ }^{238} \mathrm{U}$ & $2.0 \mathrm{E}-01 \pm 5.4 \mathrm{E}-02$ & & & ${ }^{238} U$ & $1.9 \mathrm{E}-01$ & $\pm 5.3 \mathrm{E}-02$ & \\
\hline & ${ }^{65} \mathrm{Zn}$ & $-3.6 \mathrm{E}-03 \pm 1.7 \mathrm{E}-02$ & $\mathrm{U}$ & & ${ }^{65} \mathrm{Zn}$ & 4.7E-02 & $\pm 2.4 \mathrm{E}-02$ & \\
\hline D007 & ${ }^{144} \mathrm{Ce}$ & $5.8 \mathrm{E}-02 \pm 1.2 \mathrm{E}-01$ & $\overline{\mathrm{U}}$ & D009 & ${ }^{144} \mathrm{Ce}$ & $-1.1 \mathrm{E}-01$ & $\pm 1.9 \mathrm{E}-01$ & $\bar{U}$ \\
\hline \multirow[t]{17}{*}{ (200 West) } & ${ }^{60} \mathrm{Co}$ & $-9.1 \mathrm{E}-04 \pm 8.0 \mathrm{E}-03$ & $\mathrm{U}$ & (200 West) & ${ }^{60} \mathrm{Co}$ & $-1.3 \mathrm{E}-04$ & $\pm 1.3 \mathrm{E}-03$ & $\mathrm{U}$ \\
\hline & ${ }^{134} \mathrm{Cs}$ & $4.5 \mathrm{E}-02 \pm 1.6 \mathrm{E}-02$ & & & ${ }^{134} \mathrm{Cs}$ & 4.3E-02 & $\pm 1.5 \mathrm{E}-02$ & \\
\hline & ${ }^{137} \mathrm{Cs}$ & $5.1 \mathrm{E}-01 \pm 8.6 \mathrm{E}-02$ & & & ${ }^{137} \mathrm{Cs}$ & $5.9 \mathrm{E}-01$ & $\pm 1.0 \mathrm{E}-01$ & \\
\hline & ${ }^{152} \mathrm{Eu}$ & $-1.1 \mathrm{E}-02 \pm 2.4 \mathrm{E}-02$ & $\mathrm{U}$ & & ${ }^{152} \mathrm{Eu}$ & $1.2 \mathrm{E}-02$ & $\pm 5.3 \mathrm{E}-02$ & $\mathrm{U}$ \\
\hline & ${ }^{154} \mathrm{Eu}$ & $1.4 \mathrm{E}-02 \pm 3.0 \mathrm{E}-02$ & $\mathrm{U}$ & & ${ }^{154} \mathrm{Eu}$ & $-5.4 \mathrm{E}-02$ & $\pm 5.4 \mathrm{E}-02$ & U \\
\hline & ${ }^{155} \mathrm{Eu}$ & $8.6 \mathrm{E}-02 \pm 4.0 \mathrm{E}-02$ & $\mathrm{U}$ & & ${ }^{155} \mathrm{Eu}$ & $1.4 \mathrm{E}-03$ & $\pm 1.4 \mathrm{E}-02$ & $\mathrm{U}$ \\
\hline & ${ }^{238} \mathrm{Pu}$ & $6.2 \mathrm{E}-03 \pm 9.3 \mathrm{E}-03$ & $\mathrm{U}$ & & ${ }^{238} \mathrm{Pu}$ & $-1.0 \mathrm{E}-02$ & $\pm 2.9 \mathrm{E}-02$ & $\mathrm{U}$ \\
\hline & ${ }^{239,240} \mathrm{Pu}$ & $8.6 \mathrm{E}-02 \pm 2.9 \mathrm{E}-02$ & & & ${ }^{239,240} \mathrm{Pu}$ & $5.8 \mathrm{E}-02$ & $\pm 2.8 \mathrm{E}-02$ & \\
\hline & ${ }^{103} \mathrm{Ru}$ & $2.0 \mathrm{E}-03 \pm 7.3 \mathrm{E}-03$ & $\mathrm{U}$ & & ${ }^{103} \mathrm{Ru}$ & $-1.1 \mathrm{E}-03$ & $\pm 8.8 \mathrm{E}-03$ & $\mathrm{U}$ \\
\hline & ${ }^{106} \mathrm{Ru}$ & $3.1 \mathrm{E}-02 \pm 6.8 \mathrm{E}-02$ & $\mathrm{U}$ & & ${ }^{106} \mathrm{Ru}$ & $-2.0 \mathrm{E}-02$ & $\pm 7.4 \mathrm{E}-02$ & $\mathrm{U}$ \\
\hline & ${ }^{125} \mathrm{Sb}$ & $-2.0 \mathrm{E}-02 \pm 2.4 \mathrm{E}-02$ & $\mathrm{U}$ & & ${ }^{125} \mathrm{Sb}$ & $-5.6 \mathrm{E}-03$ & $\pm 2.6 \mathrm{E}-02$ & U \\
\hline & ${ }^{113} \mathrm{Sn}$ & $3.7 \mathrm{E}-03 \pm 9.8 \mathrm{E}-03$ & $\mathrm{U}$ & & ${ }^{113} \mathrm{Sn}$ & $9.9 \mathrm{E}-03$ & $\pm 1.2 \mathrm{E}-02$ & $\mathrm{U}$ \\
\hline & ${ }^{90} \mathrm{Sr}$ & $-2.7 \mathrm{E}-01 \pm 2.7 \mathrm{E}-01$ & $\mathrm{U}$ & & ${ }^{90} \mathrm{Sr}$ & $5.8 \mathrm{E}-01$ & $\pm 2.6 \mathrm{E}-01$ & \\
\hline & ${ }^{234} \mathrm{U}$ & $1.4 \mathrm{E}-01 \pm 4.3 \mathrm{E}-02$ & & & ${ }^{234} \mathrm{U}$ & $1.2 \mathrm{E}-01$ & $\pm 3.7 \mathrm{E}-02$ & \\
\hline & ${ }^{235} \mathrm{U}$ & $2.5 \mathrm{E}-02 \pm 1.7 \mathrm{E}-02$ & & & ${ }^{235} \mathrm{U}$ & 1.3E-02 & $\pm 1.1 \mathrm{E}-02$ & \\
\hline & ${ }^{238} \mathrm{U}$ & $1.4 \mathrm{E}-01 \pm 4.2 \mathrm{E}-02$ & & & ${ }^{238} \mathrm{U}$ & $1.2 \mathrm{E}-01$ & \pm 3.7E-02 & \\
\hline & ${ }^{65} \mathrm{Zn}$ & $2.3 \mathrm{E}-02 \pm 2.2 \mathrm{E}-02$ & U & & ${ }^{65} \mathrm{Zn}$ & $-5.1 \mathrm{E}-03$ & $\pm 2.3 \mathrm{E}-02$ & U \\
\hline
\end{tabular}

$\overline{\mathrm{RQ}}=$ Result Qualifier. U $=$ The analyte was analyzed for but not detected. 
Table 3-3. 2003 Soil Sampling Results (pCi/g \pm total analytical uncertainty). (cont)

\begin{tabular}{|c|c|c|c|c|c|c|c|}
\hline Location & Isotope & Result \pm Error & $\mathbf{R Q}^{*}$ & Location & Isotope & \pm Error & $\mathbf{R Q}^{*}$ \\
\hline D011 & ${ }^{144} \mathrm{Ce}$ & $4.7 \mathrm{E}-02 \pm 1.8 \mathrm{E}-01$ & $\bar{U}$ & D013 & ${ }^{144} \mathrm{Ce}$ & $2.3 \mathrm{E}-02 \pm 1.9 \mathrm{E}-01$ & $\overline{\mathrm{U}}$ \\
\hline \multirow[t]{17}{*}{ (200 West) } & ${ }^{60} \mathrm{Co}$ & $-6.5 \mathrm{E}-03 \pm 8.5 \mathrm{E}-03$ & $\mathrm{U}$ & (200 West) & ${ }^{60} \mathrm{Co}$ & $-6.7 \mathrm{E}-04 \pm 6.7 \mathrm{E}-03$ & $\mathrm{U}$ \\
\hline & ${ }^{134} \mathrm{Cs}$ & $3.3 \mathrm{E}-02 \pm 1.5 \mathrm{E}-02$ & & & ${ }^{134} \mathrm{Cs}$ & $2.4 \mathrm{E}-02 \pm 1.2 \mathrm{E}-02$ & \\
\hline & ${ }^{137} \mathrm{Cs}$ & $2.6 \mathrm{E}+00 \pm 4.7 \mathrm{E}-01$ & & & ${ }^{137} \mathrm{Cs}$ & $9.0 \mathrm{E}+00 \pm 1.4 \mathrm{E}+00$ & \\
\hline & ${ }^{152} \mathrm{Eu}$ & $-2.2 \mathrm{E}-02 \pm 4.5 \mathrm{E}-02$ & $\mathrm{U}$ & & ${ }^{152} \mathrm{Eu}$ & $-1.2 \mathrm{E}-02 \pm 4.7 \mathrm{E}-02$ & $\mathrm{U}$ \\
\hline & ${ }^{154} \mathrm{Eu}$ & $-1.3 \mathrm{E}-02 \pm 2.7 \mathrm{E}-02$ & $\mathrm{U}$ & & ${ }^{154} \mathrm{Eu}$ & $6.9 \mathrm{E}-03 \pm 2.7 \mathrm{E}-02$ & $\mathrm{U}$ \\
\hline & ${ }^{155} \mathrm{Eu}$ & $1.8 \mathrm{E}-03 \pm 1.8 \mathrm{E}-02$ & $\mathrm{U}$ & & ${ }^{155} \mathrm{Eu}$ & $3.5 \mathrm{E}-02 \pm 4.5 \mathrm{E}-02$ & $\mathrm{U}$ \\
\hline & ${ }^{238} \mathrm{Pu}$ & $-9.4 \mathrm{E}-03 \pm 1.5 \mathrm{E}-02$ & $\mathrm{U}$ & & ${ }^{238} \mathrm{Pu}$ & $5.5 \mathrm{E}-03 \pm 1.3 \mathrm{E}-02$ & $\mathrm{U}$ \\
\hline & ${ }^{239,240} \mathrm{Pu}$ & $3.3 \mathrm{E}-01 \pm 7.9 \mathrm{E}-02$ & & & ${ }^{239,240} \mathrm{Pu}$ & $1.1 \mathrm{E}-02 \pm 9.2 \mathrm{E}-03$ & \\
\hline & ${ }^{103} \mathrm{Ru}$ & $5.7 \mathrm{E}-03 \pm 9.8 \mathrm{E}-03$ & $\mathrm{U}$ & & ${ }^{103} \mathrm{Ru}$ & $1.9 \mathrm{E}-03 \pm 1.2 \mathrm{E}-02$ & $\mathrm{U}$ \\
\hline & ${ }^{106} \mathrm{Ru}$ & $-7.5 \mathrm{E}-02 \pm 8.5 \mathrm{E}-02$ & $\mathrm{U}$ & & ${ }^{106} \mathrm{Ru}$ & $-2.8 \mathrm{E}-02 \pm 9.0 \mathrm{E}-02$ & $\mathrm{U}$ \\
\hline & ${ }^{125} \mathrm{Sb}$ & $-2.4 \mathrm{E}-04 \pm 2.4 \mathrm{E}-03$ & $\mathrm{U}$ & & ${ }^{125} \mathrm{Sb}$ & $2.9 \mathrm{E}-02 \pm 4.0 \mathrm{E}-02$ & $\mathrm{U}$ \\
\hline & ${ }^{113} \mathrm{Sn}$ & $5.0 \mathrm{E}-03 \pm 1.3 \mathrm{E}-02$ & $\mathrm{U}$ & & ${ }^{113} \mathrm{Sn}$ & $1.8 \mathrm{E}-04 \pm 1.8 \mathrm{E}-03$ & $\mathrm{U}$ \\
\hline & ${ }^{90} \mathrm{Sr}$ & $-7.2 \mathrm{E}-02 \pm 1.7 \mathrm{E}-01$ & $\mathrm{U}$ & & ${ }^{90} \mathrm{Sr}$ & $-1.3 \mathrm{E}-01 \pm 1.8 \mathrm{E}-01$ & $\mathrm{U}$ \\
\hline & ${ }^{234} \mathrm{U}$ & $1.4 \mathrm{E}-01 \pm 4.3 \mathrm{E}-02$ & & & & $1.3 \mathrm{E}-01 \pm 4.0 \mathrm{E}-02$ & \\
\hline & ${ }^{235} \mathrm{U}$ & $2.1 \mathrm{E}-02 \pm 1.4 \mathrm{E}-02$ & & & ${ }^{235} \mathrm{U}$ & $9.9 \mathrm{E}-03 \pm 1.2 \mathrm{E}-02$ & $\mathrm{U}$ \\
\hline & ${ }^{238} \mathrm{U}$ & $1.4 \mathrm{E}-01 \pm 4.3 \mathrm{E}-02$ & & & ${ }^{238} \mathrm{U}$ & $1.4 \mathrm{E}-01 \pm 4.2 \mathrm{E}-02$ & \\
\hline & ${ }^{65} \mathrm{Zn}$ & $-1.5 \mathrm{E}-02 \pm 2.2 \mathrm{E}-02$ & $\mathrm{U}$ & & ${ }^{65} \mathrm{Zn}$ & $-1.2 \mathrm{E}-02 \pm 1.9 \mathrm{E}-02$ & $\mathrm{U}$ \\
\hline D015 & ${ }^{144} \mathrm{Ce}$ & $-2.0 \mathrm{E}-02 \pm 1.3 \mathrm{E}-01$ & $\overline{\mathrm{U}}$ & D017 & ${ }^{144} \mathrm{Ce}$ & $-8.2 \mathrm{E}-02 \pm 1.6 \mathrm{E}-01$ & $\overline{\mathrm{U}}$ \\
\hline \multirow[t]{17}{*}{ (200 West) } & ${ }^{60} \mathrm{Co}$ & $1.0 \mathrm{E}-03 \pm 6.2 \mathrm{E}-03$ & $\mathrm{U}$ & (200 West) & ${ }^{60} \mathrm{Co}$ & $-9.8 \mathrm{E}-04 \pm 7.7 \mathrm{E}-03$ & $\mathrm{U}$ \\
\hline & ${ }^{134} \mathrm{Cs}$ & $3.4 \mathrm{E}-02 \pm 1.0 \mathrm{E}-02$ & & & ${ }^{134} \mathrm{Cs}$ & $3.7 \mathrm{E}-02 \pm 1.2 \mathrm{E}-02$ & \\
\hline & ${ }^{137} \mathrm{Cs}$ & $1.8 \mathrm{E}+00 \pm 2.9 \mathrm{E}-01$ & & & ${ }^{137} \mathrm{Cs}$ & $2.1 \mathrm{E}+00 \pm 3.4 \mathrm{E}-01$ & \\
\hline & ${ }^{152} \mathrm{Eu}$ & $2.4 \mathrm{E}-02 \pm 3.1 \mathrm{E}-02$ & $\mathrm{U}$ & & ${ }^{152} \mathrm{Eu}$ & $-1.5 \mathrm{E}-02 \pm 4.7 \mathrm{E}-02$ & $\mathrm{U}$ \\
\hline & ${ }^{154} \mathrm{Eu}$ & $6.9 \mathrm{E}-03 \pm 2.2 \mathrm{E}-02$ & $\mathrm{U}$ & & ${ }^{154} \mathrm{Eu}$ & $-1.2 \mathrm{E}-02 \pm 3.0 \mathrm{E}-02$ & $\mathrm{U}$ \\
\hline & ${ }^{155} \mathrm{Eu}$ & $6.9 \mathrm{E}-03 \pm 3.2 \mathrm{E}-02$ & $\mathrm{U}$ & & ${ }^{155} \mathrm{Eu}$ & $6.3 \mathrm{E}-02 \pm 3.9 \mathrm{E}-02$ & $\mathrm{U}$ \\
\hline & ${ }^{238} \mathrm{Pu}$ & $4.2 \mathrm{E}-03 \pm 3.4 \mathrm{E}-02$ & $\mathrm{U}$ & & ${ }^{238} \mathrm{Pu}$ & $1.7 \mathrm{E}-02 \pm 1.3 \mathrm{E}-02$ & \\
\hline & ${ }^{239,240} \mathrm{Pu}$ & $1.3 \mathrm{E}-02 \pm 1.4 \mathrm{E}-02$ & $\mathrm{U}$ & & ${ }^{239,240} \mathrm{Pu}$ & $6.9 \mathrm{E}-02 \pm 2.6 \mathrm{E}-02$ & \\
\hline & ${ }^{103} \mathrm{Ru}$ & $-2.0 \mathrm{E}-04 \pm 1.9 \mathrm{E}-03$ & $\mathrm{U}$ & & ${ }^{103} \mathrm{Ru}$ & $3.4 \mathrm{E}-03 \pm 9.0 \mathrm{E}-03$ & $\mathrm{U}$ \\
\hline & ${ }^{106} \mathrm{Ru}$ & $-2.2 \mathrm{E}-02 \pm 6.3 \mathrm{E}-02$ & $\mathrm{U}$ & & ${ }^{106} \mathrm{Ru}$ & $4.0 \mathrm{E}-02 \pm 7.8 \mathrm{E}-02$ & $\mathrm{U}$ \\
\hline & ${ }^{125} \mathrm{Sb}$ & $1.9 \mathrm{E}-03 \pm 1.9 \mathrm{E}-02$ & $\mathrm{U}$ & & ${ }^{125} \mathrm{Sb}$ & $1.0 \mathrm{E}-02 \pm 2.7 \mathrm{E}-02$ & $\mathrm{U}$ \\
\hline & ${ }^{113} \mathrm{Sn}$ & $2.7 \mathrm{E}-03 \pm 1.0 \mathrm{E}-02$ & $\mathrm{U}$ & & ${ }^{113} \mathrm{Sn}$ & $8.2 \mathrm{E}-03 \pm 1.2 \mathrm{E}-02$ & $\mathrm{U}$ \\
\hline & ${ }^{90} \mathrm{Sr}$ & $2.0 \mathrm{E}-01 \pm 2.0 \mathrm{E}-01$ & $\mathrm{U}$ & & ${ }^{90} \mathrm{Sr}$ & $4.7 \mathrm{E}-02 \pm 2.1 \mathrm{E}-01$ & $\mathrm{U}$ \\
\hline & ${ }^{234} \mathrm{U}$ & $1.2 \mathrm{E}-01 \pm 4.0 \mathrm{E}-02$ & & & ${ }^{234} \mathrm{U}$ & $1.7 \mathrm{E}-01 \pm 4.9 \mathrm{E}-02$ & \\
\hline & ${ }^{235} \mathrm{U}$ & $9.5 \mathrm{E}-03 \pm 9.5 \mathrm{E}-03$ & & & ${ }^{235} \mathrm{U}$ & $1.9 \mathrm{E}-02 \pm 1.3 \mathrm{E}-02$ & \\
\hline & ${ }^{238} \mathrm{U}$ & 8.7E-02 $\pm 3.2 \mathrm{E}-02$ & & & ${ }^{238} \mathrm{U}$ & $1.5 \mathrm{E}-01 \pm 4.4 \mathrm{E}-02$ & \\
\hline & ${ }^{65} \mathrm{Zn}$ & $-1.7 \mathrm{E}-03 \pm 1.7 \mathrm{E}-02$ & $\mathrm{U}$ & & ${ }^{65} \mathrm{Zn}$ & $6.1 \mathrm{E}-02 \pm 2.4 \mathrm{E}-02$ & \\
\hline
\end{tabular}

$\overline{\mathrm{RQ}}=$ Result Qualifier. U $=$ The analyte was analyzed for but not detected. 
Table 3-3. 2003 Soil Sampling Results (pCi/g \pm total analytical uncertainty). (cont)

\begin{tabular}{|c|c|c|c|c|c|c|c|c|}
\hline Location & Isotope & Result \pm Error & $\mathbf{R Q}^{*}$ & Location & Isotope & Result & \pm Error & $\mathbf{R Q}^{*}$ \\
\hline D019 & ${ }^{144} \mathrm{Ce}$ & $6.9 \mathrm{E}-02 \pm 1.2 \mathrm{E}-01$ & $\bar{U}$ & D021 & ${ }^{144} \mathrm{Ce}$ & $-7.3 \mathrm{E}-02$ & $\pm 2.1 \mathrm{E}-01$ & $\mathrm{U}$ \\
\hline \multirow[t]{17}{*}{ (200 West) } & ${ }^{60} \mathrm{Co}$ & $-2.7 \mathrm{E}-04 \pm 2.7 \mathrm{E}-03$ & U & (200 West) & ${ }^{60} \mathrm{Co}$ & $-9.4 \mathrm{E}-04$ & $\pm 8.8 \mathrm{E}-03$ & U \\
\hline & ${ }^{134} \mathrm{Cs}$ & $3.7 \mathrm{E}-02 \pm 1.1 \mathrm{E}-02$ & & & ${ }^{134} \mathrm{Cs}$ & 4.7E-02 & $\pm 1.7 \mathrm{E}-02$ & \\
\hline & ${ }^{137} \mathrm{Cs}$ & $1.8 \mathrm{E}+00 \pm 3.0 \mathrm{E}-01$ & & & ${ }^{137} \mathrm{Cs}$ & $2.0 \mathrm{E}-01$ & $\pm 4.0 \mathrm{E}-02$ & \\
\hline & ${ }^{152} \mathrm{Eu}$ & $2.6 \mathrm{E}-02 \pm 2.8 \mathrm{E}-02$ & $\mathrm{U}$ & & ${ }^{152} \mathrm{Eu}$ & $-1.1 \mathrm{E}-02$ & $\pm 5.6 \mathrm{E}-02$ & $\mathrm{U}$ \\
\hline & ${ }^{154} \mathrm{Eu}$ & $-2.6 \mathrm{E}-02 \pm 2.9 \mathrm{E}-02$ & $\mathrm{U}$ & & ${ }^{154} \mathrm{Eu}$ & $-7.8 \mathrm{E}-03$ & $\pm 3.3 \mathrm{E}-02$ & $\mathrm{U}$ \\
\hline & ${ }^{155} \mathrm{Eu}$ & $2.1 \mathrm{E}-02 \pm 3.2 \mathrm{E}-02$ & $\mathrm{U}$ & & ${ }^{155} \mathrm{Eu}$ & 5.6E-02 & $\pm 6.0 \mathrm{E}-02$ & $\mathrm{U}$ \\
\hline & ${ }^{238} \mathrm{Pu}$ & $1.8 \mathrm{E}-03 \pm 1.3 \mathrm{E}-02$ & U & & ${ }^{238} \mathrm{Pu}$ & $2.0 \mathrm{E}-02$ & $\pm 3.2 \mathrm{E}-02$ & $\mathrm{U}$ \\
\hline & ${ }^{239,240} \mathrm{Pu}$ & $5.7 \mathrm{E}-02 \pm 2.4 \mathrm{E}-02$ & & & ${ }^{239,240} \mathrm{Pu}$ & $1.6 \mathrm{E}-02$ & $\pm 1.3 \mathrm{E}-02$ & \\
\hline & ${ }^{103} \mathrm{Ru}$ & $-7.2 \mathrm{E}-03 \pm 8.3 \mathrm{E}-03$ & U & & ${ }^{103} \mathrm{Ru}$ & $-3.0 \mathrm{E}-03$ & $\pm 9.5 \mathrm{E}-03$ & $\mathrm{U}$ \\
\hline & ${ }^{106} \mathrm{Ru}$ & $-1.4 \mathrm{E}-02 \pm 7.3 \mathrm{E}-02$ & $\mathrm{U}$ & & ${ }^{106} \mathrm{Ru}$ & $-4.6 \mathrm{E}-02$ & $\pm 8.2 \mathrm{E}-02$ & $\mathrm{U}$ \\
\hline & ${ }^{125} \mathrm{Sb}$ & $-1.5 \mathrm{E}-02 \pm 2.7 \mathrm{E}-02$ & $\mathrm{U}$ & & ${ }^{125} \mathrm{Sb}$ & $1.6 \mathrm{E}-02$ & $\pm 2.8 \mathrm{E}-02$ & $\mathrm{U}$ \\
\hline & ${ }^{113} \mathrm{Sn}$ & $-7.4 \mathrm{E}-03 \pm 1.1 \mathrm{E}-02$ & $\mathrm{U}$ & & ${ }^{113} \mathrm{Sn}$ & $1.2 \mathrm{E}-02$ & $\pm 1.3 \mathrm{E}-02$ & $\mathrm{U}$ \\
\hline & ${ }^{90} \mathrm{Sr}$ & $2.1 \mathrm{E}-01 \pm 2.5 \mathrm{E}-01$ & $\mathrm{U}$ & & ${ }^{90} \mathrm{Sr}$ & 6.7E-02 & $\pm 2.2 \mathrm{E}-01$ & $\mathrm{U}$ \\
\hline & ${ }^{234} \mathrm{U}$ & $1.4 \mathrm{E}-01 \pm 4.1 \mathrm{E}-02$ & & & & $1.3 \mathrm{E}-01$ & $\pm 4.0 \mathrm{E}-02$ & \\
\hline & ${ }^{235} \mathrm{U}$ & $7.4 \mathrm{E}-03 \pm 7.4 \mathrm{E}-03$ & & & ${ }^{235} \mathrm{U}$ & $9.7 \mathrm{E}-03$ & $\pm 8.8 \mathrm{E}-03$ & \\
\hline & ${ }^{238} \mathrm{U}$ & $1.3 \mathrm{E}-01 \pm 3.8 \mathrm{E}-02$ & & & ${ }^{238} \mathrm{U}$ & $1.1 \mathrm{E}-01$ & $\pm 3.4 \mathrm{E}-02$ & \\
\hline & ${ }^{65} \mathrm{Zn}$ & $8.8 \mathrm{E}-03 \pm 2.2 \mathrm{E}-02$ & U & & ${ }^{65} \mathrm{Zn}$ & $1.2 \mathrm{E}-02$ & $\pm 2.5 \mathrm{E}-02$ & $\mathrm{U}$ \\
\hline D023 & ${ }^{144} \mathrm{Ce}$ & $-1.9 \mathrm{E}-01 \pm 2.2 \mathrm{E}-01$ & $\bar{U}$ & D025 & ${ }^{144} \mathrm{Ce}$ & $-1.2 \mathrm{E}-01$ & $\pm 1.6 \mathrm{E}-01$ & $\bar{U}$ \\
\hline \multirow[t]{17}{*}{ (200 West) } & ${ }^{60} \mathrm{Co}$ & $6.1 \mathrm{E}-03 \pm 1.1 \mathrm{E}-02$ & $\mathrm{U}$ & (200 West) & ${ }^{60} \mathrm{Co}$ & $1.8 \mathrm{E}-01$ & $\pm 2.0 \mathrm{E}-02$ & \\
\hline & ${ }^{134} \mathrm{Cs}$ & $3.7 \mathrm{E}-02 \pm 1.5 \mathrm{E}-02$ & & & ${ }^{134} \mathrm{Cs}$ & 3.2E-02 & $\pm 1.1 \mathrm{E}-02$ & \\
\hline & ${ }^{137} \mathrm{Cs}$ & $2.9 \mathrm{E}+00 \pm 5.3 \mathrm{E}-01$ & & & ${ }^{137} \mathrm{Cs}$ & $6.1 \mathrm{E}+00$ & $\pm 9.8 \mathrm{E}-01$ & \\
\hline & ${ }^{152} \mathrm{Eu}$ & $-5.3 \mathrm{E}-02 \pm 5.3 \mathrm{E}-02$ & U & & ${ }^{152} \mathrm{Eu}$ & $2.0 \mathrm{E}-02$ & $\pm 3.4 \mathrm{E}-02$ & $\mathrm{U}$ \\
\hline & ${ }^{154} \mathrm{Eu}$ & $-6.6 \mathrm{E}-03 \pm 3.6 \mathrm{E}-02$ & U & & ${ }^{154} \mathrm{Eu}$ & $-2.7 \mathrm{E}-02$ & $\pm 2.7 \mathrm{E}-02$ & $\mathrm{U}$ \\
\hline & ${ }^{155} \mathrm{Eu}$ & $5.6 \mathrm{E}-02 \pm 5.4 \mathrm{E}-02$ & $\mathrm{U}$ & & ${ }^{155} \mathrm{Eu}$ & 4.7E-02 & $\pm 4.2 \mathrm{E}-02$ & $\mathrm{U}$ \\
\hline & ${ }^{238} \mathrm{Pu}$ & $2.2 \mathrm{E}-03 \pm 2.2 \mathrm{E}-02$ & $\mathrm{U}$ & & ${ }^{238} \mathrm{Pu}$ & $-1.2 \mathrm{E}-02$ & \pm 3.7E-02 & $\mathrm{U}$ \\
\hline & ${ }^{239,240} \mathrm{Pu}$ & $4.0 \mathrm{E}-02 \pm 2.2 \mathrm{E}-02$ & & & ${ }^{239,240} \mathrm{Pu}$ & $3.9 \mathrm{E}-01$ & $\pm 9.4 \mathrm{E}-02$ & \\
\hline & ${ }^{103} \mathrm{Ru}$ & $-1.1 \mathrm{E}-02 \pm 1.3 \mathrm{E}-02$ & $\mathrm{U}$ & & ${ }^{103} \mathrm{Ru}$ & $1.6 \mathrm{E}-03$ & $\pm 1.0 \mathrm{E}-02$ & $\mathrm{U}$ \\
\hline & ${ }^{106} \mathrm{Ru}$ & $5.1 \mathrm{E}-02 \pm 1.1 \mathrm{E}-01$ & U & & ${ }^{106} \mathrm{Ru}$ & 4.2E-04 & $\pm 4.2 \mathrm{E}-03$ & $\mathrm{U}$ \\
\hline & ${ }^{125} \mathrm{Sb}$ & $1.4 \mathrm{E}-02 \pm 3.7 \mathrm{E}-02$ & U & & ${ }^{125} \mathrm{Sb}$ & 4.4E-02 & $\pm 3.4 \mathrm{E}-02$ & $\mathrm{U}$ \\
\hline & ${ }^{113} \mathrm{Sn}$ & $1.0 \mathrm{E}-02 \pm 1.7 \mathrm{E}-02$ & U & & ${ }^{113} \mathrm{Sn}$ & $-4.1 E-03$ & $\pm 1.4 \mathrm{E}-02$ & U \\
\hline & ${ }^{90} \mathrm{Sr}$ & $6.9 \mathrm{E}-02 \pm 2.6 \mathrm{E}-01$ & U & & ${ }^{90} \mathrm{Sr}$ & $3.1 \mathrm{E}-01$ & $\pm 2.5 \mathrm{E}-01$ & $\mathrm{U}$ \\
\hline & ${ }^{234} \mathrm{U}$ & $1.1 \mathrm{E}-01 \pm 3.6 \mathrm{E}-02$ & & & ${ }^{234} \mathrm{U}$ & $1.6 \mathrm{E}-01$ & $\pm 4.6 \mathrm{E}-02$ & \\
\hline & ${ }^{235} \mathrm{U}$ & $1.0 \mathrm{E}-02 \pm 1.1 \mathrm{E}-02$ & $\mathrm{U}$ & & ${ }^{235} \mathrm{U}$ & 8.6E-03 & $\pm 8.6 \mathrm{E}-03$ & \\
\hline & ${ }^{238} \mathrm{U}$ & $1.2 \mathrm{E}-01 \pm 3.7 \mathrm{E}-02$ & & & ${ }^{238} \mathrm{U}$ & $1.6 \mathrm{E}-01$ & $\pm 4.6 \mathrm{E}-02$ & \\
\hline & ${ }^{65} \mathrm{Zn}$ & $2.2 \mathrm{E}-02 \pm 2.8 \mathrm{E}-02$ & $\mathrm{U}$ & & ${ }^{65} \mathrm{Zn}$ & $-7.9 \mathrm{E}-03$ & $\pm 1.9 \mathrm{E}-02$ & $\mathrm{U}$ \\
\hline
\end{tabular}

$\overline{\mathrm{RQ}}=$ Result Qualifier. U $=$ The analyte was analyzed for but not detected. 
Table 3-3. 2003 Soil Sampling Results (pCi/g \pm total analytical uncertainty). (cont)

\begin{tabular}{|c|c|c|c|c|c|c|c|c|}
\hline Location & Isotope & Result \pm Error & $\overline{\mathbf{R Q}^{*}}$ & Location & Isotope & Result & \pm Error & $\overline{\mathbf{R Q}^{*}}$ \\
\hline D027 & ${ }^{144} \mathrm{Ce}$ & $-1.5 \mathrm{E}-01 \pm 1.5 \mathrm{E}-01$ & $\overline{\mathrm{U}}$ & D029 & ${ }^{144} \mathrm{Ce}$ & $6.6 \mathrm{E}-03$ & $\pm 6.6 \mathrm{E}-02$ & $\bar{U}$ \\
\hline \multirow[t]{17}{*}{ (200 West) } & ${ }^{60} \mathrm{Co}$ & $-4.5 \mathrm{E}-03 \pm 5.8 \mathrm{E}-03$ & $\mathrm{U}$ & (200 West) & ${ }^{60} \mathrm{Co}$ & $-4.3 E-03$ & $\pm 5.8 \mathrm{E}-03$ & U \\
\hline & ${ }^{134} \mathrm{Cs}$ & $3.5 \mathrm{E}-02 \pm 1.2 \mathrm{E}-02$ & & & ${ }^{134} \mathrm{Cs}$ & 3.3E-02 & $\pm 1.1 \mathrm{E}-02$ & \\
\hline & ${ }^{137} \mathrm{Cs}$ & $9.5 \mathrm{E}-01 \pm 1.5 \mathrm{E}-01$ & & & ${ }^{137} \mathrm{Cs}$ & $1.3 \mathrm{E}+00$ & $\pm 2.1 \mathrm{E}-01$ & \\
\hline & ${ }^{152} \mathrm{Eu}$ & $-2.0 \mathrm{E}-02 \pm 2.3 \mathrm{E}-02$ & $\mathrm{U}$ & & ${ }^{152} \mathrm{Eu}$ & $-1.1 \mathrm{E}-03$ & $\pm 1.1 \mathrm{E}-02$ & $\mathrm{U}$ \\
\hline & ${ }^{154} \mathrm{Eu}$ & $-1.1 \mathrm{E}-04 \pm 1.1 \mathrm{E}-03$ & $\mathrm{U}$ & & ${ }^{154} \mathrm{Eu}$ & $-2.7 \mathrm{E}-02$ & $\pm 2.7 \mathrm{E}-02$ & $\mathrm{U}$ \\
\hline & ${ }^{155} \mathrm{Eu}$ & $3.1 \mathrm{E}-02 \pm 3.4 \mathrm{E}-02$ & $\mathrm{U}$ & & ${ }^{155} \mathrm{Eu}$ & $1.6 \mathrm{E}-02$ & $\pm 3.1 \mathrm{E}-02$ & $\mathrm{U}$ \\
\hline & ${ }^{238} \mathrm{Pu}$ & $8.1 \mathrm{E}-03 \pm 3.2 \mathrm{E}-02$ & $\mathrm{U}$ & & ${ }^{238} \mathrm{Pu}$ & $1.8 \mathrm{E}-03$ & $\pm 1.8 \mathrm{E}-02$ & U \\
\hline & ${ }^{239,240} \mathrm{Pu}$ & $6.0 \mathrm{E}-03 \pm 9.0 \mathrm{E}-03$ & $\mathrm{U}$ & & ${ }^{239,240} \mathrm{Pu}$ & $4.1 \mathrm{E}-02$ & $\pm 2.1 \mathrm{E}-02$ & \\
\hline & ${ }^{103} \mathrm{Ru}$ & $-2.7 \mathrm{E}-04 \pm 2.7 \mathrm{E}-03$ & $\mathrm{U}$ & & ${ }^{103} \mathrm{Ru}$ & $1.7 \mathrm{E}-03$ & $\pm 6.6 \mathrm{E}-03$ & $\mathrm{U}$ \\
\hline & ${ }^{106} \mathrm{Ru}$ & $-2.0 \mathrm{E}-02 \pm 5.3 \mathrm{E}-02$ & $\mathrm{U}$ & & ${ }^{106} \mathrm{Ru}$ & $-8.5 E-03$ & $\pm 5.7 \mathrm{E}-02$ & $\mathrm{U}$ \\
\hline & ${ }^{125} \mathrm{Sb}$ & $1.1 \mathrm{E}-02 \pm 1.9 \mathrm{E}-02$ & $\mathrm{U}$ & & ${ }^{125} \mathrm{Sb}$ & $3.8 \mathrm{E}-03$ & $\pm 2.1 \mathrm{E}-02$ & $\mathrm{U}$ \\
\hline & ${ }^{113} \mathrm{Sn}$ & $1.3 \mathrm{E}-03 \pm 8.9 \mathrm{E}-03$ & $\mathrm{U}$ & & ${ }^{113} \mathrm{Sn}$ & $-3.7 E-03$ & $\pm 9.7 \mathrm{E}-03$ & $\mathrm{U}$ \\
\hline & ${ }^{90} \mathrm{Sr}$ & $-2.4 \mathrm{E}-01 \pm 2.4 \mathrm{E}-01$ & $\mathrm{U}$ & & ${ }^{90} \mathrm{Sr}$ & $-2.0 \mathrm{E}-01$ & $\pm 2.4 \mathrm{E}-01$ & $\mathrm{U}$ \\
\hline & ${ }^{234} \mathrm{U}$ & $1.9 \mathrm{E}-01 \pm 5.3 \mathrm{E}-02$ & & & & $1.4 \mathrm{E}-01$ & $\pm 4.5 \mathrm{E}-02$ & \\
\hline & ${ }^{235} \mathrm{U}$ & $2.5 \mathrm{E}-02 \pm 1.5 \mathrm{E}-02$ & & & ${ }^{235} \mathrm{U}$ & $1.4 \mathrm{E}-02$ & $\pm 1.5 \mathrm{E}-02$ & $\mathrm{U}$ \\
\hline & ${ }^{238} U$ & $1.8 \mathrm{E}-01 \pm 5.0 \mathrm{E}-02$ & & & ${ }^{238} U$ & $1.2 \mathrm{E}-01$ & $\pm 4.0 \mathrm{E}-02$ & \\
\hline & ${ }^{65} \mathrm{Zn}$ & $8.0 \mathrm{E}-03 \pm 2.2 \mathrm{E}-02$ & $\mathrm{U}$ & & ${ }^{65} \mathrm{Zn}$ & $-2.4 \mathrm{E}-03$ & $\pm 1.7 \mathrm{E}-02$ & $\mathrm{U}$ \\
\hline D031 & ${ }^{144} \mathrm{Ce}$ & $-1.7 \mathrm{E}-02 \pm 1.5 \mathrm{E}-01$ & $\overline{\mathrm{U}}$ & D033 & ${ }^{144} \mathrm{Ce}$ & $-4.2 \mathrm{E}-02$ & $\pm 1.6 \mathrm{E}-01$ & $\bar{U}$ \\
\hline \multirow[t]{17}{*}{ (200 West) } & ${ }^{60} \mathrm{Co}$ & $1.9 \mathrm{E}-03 \pm 7.2 \mathrm{E}-03$ & $\mathrm{U}$ & (200 West) & ${ }^{60} \mathrm{Co}$ & $-5.7 E-03$ & $\pm 7.4 \mathrm{E}-03$ & $\mathrm{U}$ \\
\hline & ${ }^{134} \mathrm{Cs}$ & $2.2 \mathrm{E}-02 \pm 1.1 \mathrm{E}-02$ & & & ${ }^{134} \mathrm{Cs}$ & 4.8E-02 & $\pm 1.4 \mathrm{E}-02$ & \\
\hline & ${ }^{137} \mathrm{Cs}$ & $3.1 \mathrm{E}+00 \pm 5.1 \mathrm{E}-01$ & & & ${ }^{137} \mathrm{Cs}$ & $7.6 \mathrm{E}+00$ & $\pm 1.2 \mathrm{E}+00$ & \\
\hline & ${ }^{152} \mathrm{Eu}$ & $1.3 \mathrm{E}-03 \pm 1.3 \mathrm{E}-02$ & $\mathrm{U}$ & & ${ }^{152} \mathrm{Eu}$ & $3.4 \mathrm{E}-02$ & $\pm 3.8 \mathrm{E}-02$ & $\mathrm{U}$ \\
\hline & ${ }^{154} \mathrm{Eu}$ & $-4.0 \mathrm{E}-02 \pm 4.0 \mathrm{E}-02$ & $\mathrm{U}$ & & ${ }^{154} \mathrm{Eu}$ & $-5.0 \mathrm{E}-03$ & $\pm 2.4 \mathrm{E}-02$ & U \\
\hline & ${ }^{155} \mathrm{Eu}$ & $2.2 \mathrm{E}-02 \pm 3.4 \mathrm{E}-02$ & $\mathrm{U}$ & & ${ }^{155} \mathrm{Eu}$ & $5.0 \mathrm{E}-02$ & $\pm 5.0 \mathrm{E}-02$ & $\mathrm{U}$ \\
\hline & ${ }^{238} \mathrm{Pu}$ & $6.0 \mathrm{E}-03 \pm 9.0 \mathrm{E}-03$ & $\mathrm{U}$ & & ${ }^{238} \mathrm{Pu}$ & 7.2E-03 & $\pm 1.4 \mathrm{E}-02$ & $\mathrm{U}$ \\
\hline & ${ }^{239,240} \mathrm{Pu}$ & $1.2 \mathrm{E}-01 \pm 3.8 \mathrm{E}-02$ & & & ${ }^{239,240} \mathrm{Pu}$ & 4.8E-02 & $\pm 2.2 \mathrm{E}-02$ & \\
\hline & ${ }^{103} \mathrm{Ru}$ & $-4.8 \mathrm{E}-04 \pm 4.8 \mathrm{E}-03$ & $\mathrm{U}$ & & ${ }^{103} \mathrm{Ru}$ & $-1.3 \mathrm{E}-02$ & $\pm 1.3 \mathrm{E}-02$ & $\mathrm{U}$ \\
\hline & ${ }^{106} \mathrm{Ru}$ & $-3.4 \mathrm{E}-02 \pm 8.1 \mathrm{E}-02$ & $\mathrm{U}$ & & ${ }^{106} \mathrm{Ru}$ & 8.4E-02 & $\pm 8.9 \mathrm{E}-02$ & $\mathrm{U}$ \\
\hline & ${ }^{125} \mathrm{Sb}$ & $-1.5 \mathrm{E}-02 \pm 2.9 \mathrm{E}-02$ & $\mathrm{U}$ & & ${ }^{125} \mathrm{Sb}$ & $-1.6 \mathrm{E}-02$ & $\pm 3.4 \mathrm{E}-02$ & U \\
\hline & ${ }^{113} \mathrm{Sn}$ & $3.3 \mathrm{E}-03 \pm 1.2 \mathrm{E}-02$ & $\mathrm{U}$ & & ${ }^{113} \mathrm{Sn}$ & $8.0 \mathrm{E}-03$ & $\pm 1.5 \mathrm{E}-02$ & $\mathrm{U}$ \\
\hline & ${ }^{90} \mathrm{Sr}$ & $4.2 \mathrm{E}-01 \pm 2.7 \mathrm{E}-01$ & & & ${ }^{90} \mathrm{Sr}$ & $9.5 \mathrm{E}-01$ & $\pm 2.9 \mathrm{E}-01$ & \\
\hline & ${ }^{234} \mathrm{U}$ & $1.3 \mathrm{E}-01 \pm 4.2 \mathrm{E}-02$ & & & ${ }^{234} \mathrm{U}$ & $1.6 \mathrm{E}-01$ & $\pm 4.6 \mathrm{E}-02$ & \\
\hline & ${ }^{235} \mathrm{U}$ & $6.5 \mathrm{E}-03 \pm 1.2 \mathrm{E}-02$ & $\mathrm{U}$ & & ${ }^{235} \mathrm{U}$ & $1.2 \mathrm{E}-02$ & $\pm 1.4 \mathrm{E}-02$ & $\mathrm{U}$ \\
\hline & ${ }^{238} \mathrm{U}$ & $1.7 \mathrm{E}-01 \pm 4.9 \mathrm{E}-02$ & & & ${ }^{238} \mathrm{U}$ & $1.5 \mathrm{E}-01$ & $\pm 4.5 \mathrm{E}-02$ & \\
\hline & ${ }^{65} \mathrm{Zn}$ & $5.4 \mathrm{E}-02 \pm 2.2 \mathrm{E}-02$ & & & ${ }^{65} \mathrm{Zn}$ & $2.8 \mathrm{E}-02$ & $\pm 1.9 \mathrm{E}-02$ & $\mathrm{U}$ \\
\hline
\end{tabular}

$\overline{\mathrm{RQ}}=$ Result Qualifier. U $=$ The analyte was analyzed for but not detected. 
Table 3-3. 2003 Soil Sampling Results (pCi/g \pm total analytical uncertainty). (cont)

\begin{tabular}{|c|c|c|c|c|c|c|c|c|}
\hline Location & Isotope & Result \pm Error & $\overline{\mathbf{R Q}^{*}}$ & Location & Isotope & Result & \pm Error & $\mathbf{R Q}^{*}$ \\
\hline D035 & ${ }^{144} \mathrm{Ce}$ & $1.1 \mathrm{E}-01 \pm 1.9 \mathrm{E}-01$ & $\bar{U}$ & D037 & ${ }^{144} \mathrm{Ce}$ & $5.9 \mathrm{E}-02$ & $\pm 1.9 \mathrm{E}-01$ & $\mathrm{U}$ \\
\hline \multirow[t]{17}{*}{ (200 West) } & ${ }^{60} \mathrm{Co}$ & $-1.1 \mathrm{E}-03 \pm 6.0 \mathrm{E}-03$ & $\mathrm{U}$ & (200 West) & ${ }^{60} \mathrm{Co}$ & $-1.2 \mathrm{E}-03$ & $\pm 7.6 \mathrm{E}-03$ & $\mathrm{U}$ \\
\hline & ${ }^{134} \mathrm{Cs}$ & $2.7 \mathrm{E}-02 \pm 1.1 \mathrm{E}-02$ & & & ${ }^{134} \mathrm{Cs}$ & 2.8E-02 & $\pm 1.1 \mathrm{E}-02$ & \\
\hline & ${ }^{137} \mathrm{Cs}$ & $4.0 \mathrm{E}+00 \pm 6.7 \mathrm{E}-01$ & & & ${ }^{137} \mathrm{Cs}$ & $6.8 \mathrm{E}+00$ & $\pm 1.2 \mathrm{E}+00$ & \\
\hline & ${ }^{152} \mathrm{Eu}$ & $-9.2 \mathrm{E}-03 \pm 4.8 \mathrm{E}-02$ & $\mathrm{U}$ & & ${ }^{152} \mathrm{Eu}$ & $-7.2 \mathrm{E}-03$ & \pm 3.9E-02 & $\mathrm{U}$ \\
\hline & ${ }^{154} \mathrm{Eu}$ & $-4.1 \mathrm{E}-03 \pm 2.3 \mathrm{E}-02$ & $\mathrm{U}$ & & ${ }^{154} \mathrm{Eu}$ & $-3.6 \mathrm{E}-02$ & $\pm 3.6 \mathrm{E}-02$ & $\mathrm{U}$ \\
\hline & ${ }^{155} \mathrm{Eu}$ & $2.0 \mathrm{E}-02 \pm 4.5 \mathrm{E}-02$ & $\mathrm{U}$ & & ${ }^{155} \mathrm{Eu}$ & $-3.2 \mathrm{E}-02$ & $\pm 4.4 \mathrm{E}-02$ & $\mathrm{U}$ \\
\hline & ${ }^{238} \mathrm{Pu}$ & 8.3E-03 $\pm 3.1 \mathrm{E}-02$ & $\mathrm{U}$ & & ${ }^{238} \mathrm{Pu}$ & 1.7E-03 & $\pm 1.7 \mathrm{E}-02$ & $\mathrm{U}$ \\
\hline & ${ }^{239,240} \mathrm{Pu}$ & $3.3 \mathrm{E}-02 \pm 1.9 \mathrm{E}-02$ & & & ${ }^{239,240} \mathrm{Pu}$ & 4.4E-02 & $\pm 2.0 \mathrm{E}-02$ & \\
\hline & ${ }^{103} \mathrm{Ru}$ & $-3.0 \mathrm{E}-04 \pm 3.0 \mathrm{E}-03$ & $\mathrm{U}$ & & ${ }^{103} \mathrm{Ru}$ & 8.5E-03 & $\pm 1.2 \mathrm{E}-02$ & $\mathrm{U}$ \\
\hline & ${ }^{106} \mathrm{Ru}$ & $3.6 \mathrm{E}-02 \pm 8.7 \mathrm{E}-02$ & $\mathrm{U}$ & & ${ }^{106} \mathrm{Ru}$ & 6.6E-02 & $\pm 8.9 \mathrm{E}-02$ & $\mathrm{U}$ \\
\hline & ${ }^{125} \mathrm{Sb}$ & $-7.3 \mathrm{E}-03 \pm 3.0 \mathrm{E}-02$ & $\mathrm{U}$ & & ${ }^{125} \mathrm{Sb}$ & $-2.8 \mathrm{E}-02$ & $\pm 3.6 \mathrm{E}-02$ & U \\
\hline & ${ }^{113} \mathrm{Sn}$ & $-3.2 \mathrm{E}-03 \pm 1.3 \mathrm{E}-02$ & $\mathrm{U}$ & & ${ }^{113} \mathrm{Sn}$ & 9.3E-03 & $\pm 1.5 \mathrm{E}-02$ & $\mathrm{U}$ \\
\hline & ${ }^{90} \mathrm{Sr}$ & $-6.8 \mathrm{E}-02 \pm 1.9 \mathrm{E}-01$ & $\mathrm{U}$ & & ${ }^{90} \mathrm{Sr}$ & 8.2E-01 & $\pm 2.5 \mathrm{E}-01$ & \\
\hline & ${ }^{234} \mathrm{U}$ & $1.3 \mathrm{E}-01 \pm 4.0 \mathrm{E}-02$ & & & ${ }^{234} \mathrm{U}$ & $1.1 \mathrm{E}-01$ & $\pm 3.5 \mathrm{E}-02$ & \\
\hline & ${ }^{235} \mathrm{U}$ & $6.4 \mathrm{E}-03 \pm 9.6 \mathrm{E}-03$ & $\mathrm{U}$ & & ${ }^{235} \mathrm{U}$ & 1.9E-03 & $\pm 1.9 \mathrm{E}-02$ & $\mathrm{U}$ \\
\hline & ${ }^{238} \mathrm{U}$ & $1.5 \mathrm{E}-01 \pm 4.4 \mathrm{E}-02$ & & & ${ }^{238} \mathrm{U}$ & $1.0 \mathrm{E}-01$ & \pm 3.3E-02 & \\
\hline & ${ }^{65} \mathrm{Zn}$ & $9.3 \mathrm{E}-04 \pm 9.3 \mathrm{E}-03$ & $\mathrm{U}$ & & ${ }^{65} \mathrm{Zn}$ & 1.5E-02 & $\pm 1.9 \mathrm{E}-02$ & $\mathrm{U}$ \\
\hline D039 & ${ }^{144} \mathrm{Ce}$ & $2.5 \mathrm{E}-02 \pm 1.2 \mathrm{E}-01$ & $\overline{\mathrm{U}}$ & D041 & ${ }^{144} \mathrm{Ce}$ & $6.6 \mathrm{E}-04$ & $\pm 6.6 \mathrm{E}-03$ & $\bar{U}$ \\
\hline \multirow[t]{17}{*}{ (200 West) } & ${ }^{60} \mathrm{Co}$ & $-7.9 \mathrm{E}-04 \pm 5.6 \mathrm{E}-03$ & $\mathrm{U}$ & (200 West) & ${ }^{60} \mathrm{Co}$ & $-8.0 \mathrm{E}-03$ & $\pm 8.3 \mathrm{E}-03$ & $\mathrm{U}$ \\
\hline & ${ }^{134} \mathrm{Cs}$ & $2.4 \mathrm{E}-02 \pm 8.6 \mathrm{E}-03$ & & & ${ }^{134} \mathrm{Cs}$ & 4.0E-02 & $\pm 1.6 \mathrm{E}-02$ & \\
\hline & ${ }^{137} \mathrm{Cs}$ & $3.6 \mathrm{E}+00 \pm 5.7 \mathrm{E}-01$ & & & ${ }^{137} \mathrm{Cs}$ & 4.9E-01 & $\pm 8.2 \mathrm{E}-02$ & \\
\hline & ${ }^{152} \mathrm{Eu}$ & $-6.3 \mathrm{E}-03 \pm 3.0 \mathrm{E}-02$ & $\mathrm{U}$ & & ${ }^{152} \mathrm{Eu}$ & 7.5E-03 & $\pm 3.1 \mathrm{E}-02$ & $\mathrm{U}$ \\
\hline & ${ }^{154} \mathrm{Eu}$ & $5.3 \mathrm{E}-03 \pm 2.1 \mathrm{E}-02$ & $\mathrm{U}$ & & ${ }^{154} \mathrm{Eu}$ & $-1.3 \mathrm{E}-03$ & $\pm 1.3 \mathrm{E}-02$ & $\mathrm{U}$ \\
\hline & ${ }^{155} \mathrm{Eu}$ & $2.6 \mathrm{E}-02 \pm 3.2 \mathrm{E}-02$ & $\mathrm{U}$ & & ${ }^{155} \mathrm{Eu}$ & 3.6E-02 & $\pm 4.3 \mathrm{E}-02$ & $\mathrm{U}$ \\
\hline & ${ }^{238} \mathrm{Pu}$ & $1.0 \mathrm{E}-02 \pm 3.3 \mathrm{E}-02$ & $\mathrm{U}$ & & ${ }^{238} \mathrm{Pu}$ & $-1.4 \mathrm{E}-02$ & $\pm 2.5 \mathrm{E}-02$ & $\mathrm{U}$ \\
\hline & ${ }^{239,240} \mathrm{Pu}$ & $6.7 \mathrm{E}-01 \pm 1.5 \mathrm{E}-01$ & & & ${ }^{239,240} \mathrm{Pu}$ & 3.9E-02 & $\pm 2.0 \mathrm{E}-02$ & \\
\hline & ${ }^{103} \mathrm{Ru}$ & $-2.0 \mathrm{E}-03 \pm 7.9 \mathrm{E}-03$ & $\mathrm{U}$ & & ${ }^{103} \mathrm{Ru}$ & $-1.0 \mathrm{E}-03$ & $\pm 9.1 \mathrm{E}-03$ & $\mathrm{U}$ \\
\hline & ${ }^{106} \mathrm{Ru}$ & $-2.3 \mathrm{E}-02 \pm 6.1 \mathrm{E}-02$ & $\mathrm{U}$ & & ${ }^{106} \mathrm{Ru}$ & $-4.2 E-02$ & $\pm 7.8 \mathrm{E}-02$ & $\mathrm{U}$ \\
\hline & ${ }^{125} \mathrm{Sb}$ & $-1.3 \mathrm{E}-02 \pm 2.5 \mathrm{E}-02$ & $\mathrm{U}$ & & ${ }^{125} \mathrm{Sb}$ & $1.6 \mathrm{E}-02$ & $\pm 2.5 \mathrm{E}-02$ & $\mathrm{U}$ \\
\hline & ${ }^{113} \mathrm{Sn}$ & $-2.2 \mathrm{E}-03 \pm 1.1 \mathrm{E}-02$ & $\mathrm{U}$ & & ${ }^{113} \mathrm{Sn}$ & $-4.0 \mathrm{E}-03$ & $\pm 1.2 \mathrm{E}-02$ & $\mathrm{U}$ \\
\hline & ${ }^{90} \mathrm{Sr}$ & $5.3 \mathrm{E}-01 \pm 2.2 \mathrm{E}-01$ & & & ${ }^{90} \mathrm{Sr}$ & $-3.2 \mathrm{E}-01$ & $\pm 3.2 \mathrm{E}-01$ & $\mathrm{U}$ \\
\hline & ${ }^{234} \mathrm{U}$ & $1.2 \mathrm{E}-01 \pm 3.8 \mathrm{E}-02$ & & & ${ }^{234} \mathrm{U}$ & $1.4 \mathrm{E}-01$ & $\pm 4.6 \mathrm{E}-02$ & \\
\hline & ${ }^{235} \mathrm{U}$ & $1.3 \mathrm{E}-02 \pm 1.2 \mathrm{E}-02$ & $\mathrm{U}$ & & ${ }^{235} \mathrm{U}$ & 2.2E-02 & $\pm 1.6 \mathrm{E}-02$ & \\
\hline & ${ }^{238} U$ & $1.5 \mathrm{E}-01 \pm 4.5 \mathrm{E}-02$ & & & ${ }^{238} U$ & $1.8 \mathrm{E}-01$ & $\pm 5.4 \mathrm{E}-02$ & \\
\hline & ${ }^{65} \mathrm{Zn}$ & $-1.7 \mathrm{E}-02 \pm 1.7 \mathrm{E}-02$ & $\mathrm{U}$ & & ${ }^{65} \mathrm{Zn}$ & $4.0 \mathrm{E}-02$ & $\pm 2.5 \mathrm{E}-02$ & \\
\hline
\end{tabular}

$\overline{\mathrm{RQ}}=$ Result Qualifier. U $=$ The analyte was analyzed for but not detected. 
Table 3-3. 2003 Soil Sampling Results (pCi/g \pm total analytical uncertainty). (cont)

\begin{tabular}{|c|c|c|c|c|c|c|c|c|}
\hline Location & Isotope & Result \pm Error & $\overline{\mathbf{R Q}^{*}}$ & Location & Isotope & Result & \pm Error & $\mathbf{R Q}^{*}$ \\
\hline D043 & ${ }^{144} \mathrm{Ce}$ & $-3.9 \mathrm{E}-02 \pm 1.2 \mathrm{E}-01$ & $\bar{U}$ & D045 & ${ }^{144} \mathrm{Ce}$ & $-2.2 \mathrm{E}-01$ & $\pm 2.2 \mathrm{E}-01$ & $\mathrm{U}$ \\
\hline \multirow[t]{17}{*}{ (200 West) } & ${ }^{60} \mathrm{Co}$ & $-1.4 \mathrm{E}-03 \pm 7.9 \mathrm{E}-03$ & $\mathrm{U}$ & (200 West) & ${ }^{60} \mathrm{Co}$ & $-2.9 E-03$ & $\pm 6.7 \mathrm{E}-03$ & $\mathrm{U}$ \\
\hline & ${ }^{134} \mathrm{Cs}$ & $3.8 \mathrm{E}-02 \pm 1.4 \mathrm{E}-02$ & & & ${ }^{134} \mathrm{Cs}$ & 2.9E-02 & $\pm 1.1 \mathrm{E}-02$ & \\
\hline & ${ }^{137} \mathrm{Cs}$ & $1.2 \mathrm{E}+00 \pm 2.0 \mathrm{E}-01$ & & & ${ }^{137} \mathrm{Cs}$ & $6.2 \mathrm{E}+00$ & $\pm 1.0 \mathrm{E}+00$ & \\
\hline & ${ }^{152} \mathrm{Eu}$ & $-1.4 \mathrm{E}-02 \pm 2.6 \mathrm{E}-02$ & $\mathrm{U}$ & & ${ }^{152} \mathrm{Eu}$ & $-4.8 \mathrm{E}-02$ & $\pm 6.0 \mathrm{E}-02$ & $\mathrm{U}$ \\
\hline & ${ }^{154} \mathrm{Eu}$ & $-1.6 \mathrm{E}-02 \pm 2.6 \mathrm{E}-02$ & $\mathrm{U}$ & & ${ }^{154} \mathrm{Eu}$ & $-1.6 \mathrm{E}-02$ & $\pm 2.2 \mathrm{E}-02$ & $\mathrm{U}$ \\
\hline & ${ }^{155} \mathrm{Eu}$ & $3.8 \mathrm{E}-02 \pm 4.0 \mathrm{E}-02$ & $\mathrm{U}$ & & ${ }^{155} \mathrm{Eu}$ & $-2.1 \mathrm{E}-02$ & $\pm 5.1 \mathrm{E}-02$ & U \\
\hline & ${ }^{238} \mathrm{Pu}$ & $5.6 \mathrm{E}-03 \pm 2.9 \mathrm{E}-02$ & $\mathrm{U}$ & & ${ }^{238} \mathrm{Pu}$ & 7.8E-03 & $\pm 2.7 \mathrm{E}-02$ & $\mathrm{U}$ \\
\hline & ${ }^{239,240} \mathrm{Pu}$ & $1.0 \mathrm{E}-01 \pm 3.3 \mathrm{E}-02$ & & & ${ }^{239,240} \mathrm{Pu}$ & $1.2 \mathrm{E}-01$ & \pm 3.8E-02 & \\
\hline & ${ }^{103} \mathrm{Ru}$ & $-4.1 \mathrm{E}-03 \pm 8.5 \mathrm{E}-03$ & $\mathrm{U}$ & & ${ }^{103} \mathrm{Ru}$ & $6.0 \mathrm{E}-03$ & $\pm 1.4 \mathrm{E}-02$ & $\mathrm{U}$ \\
\hline & ${ }^{106} \mathrm{Ru}$ & $-4.8 \mathrm{E}-02 \pm 6.9 \mathrm{E}-02$ & $\mathrm{U}$ & & ${ }^{106} \mathrm{Ru}$ & $-2.8 \mathrm{E}-02$ & $\pm 8.7 \mathrm{E}-02$ & $\mathrm{U}$ \\
\hline & ${ }^{125} \mathrm{Sb}$ & $-1.2 \mathrm{E}-02 \pm 2.2 \mathrm{E}-02$ & $\mathrm{U}$ & & ${ }^{125} \mathrm{Sb}$ & $1.8 \mathrm{E}-03$ & $\pm 1.8 \mathrm{E}-02$ & U \\
\hline & ${ }^{113} \mathrm{Sn}$ & $-3.0 \mathrm{E}-03 \pm 1.1 \mathrm{E}-02$ & $\mathrm{U}$ & & ${ }^{113} \mathrm{Sn}$ & $-8.3 \mathrm{E}-03$ & $\pm 1.7 \mathrm{E}-02$ & $\mathrm{U}$ \\
\hline & ${ }^{90} \mathrm{Sr}$ & $-1.3 \mathrm{E}-01 \pm 1.6 \mathrm{E}-01$ & $\mathrm{U}$ & & ${ }^{90} \mathrm{Sr}$ & $1.5 \mathrm{E}+00$ & $\pm 3.0 \mathrm{E}-01$ & \\
\hline & ${ }^{234} \mathrm{U}$ & $3.5 \mathrm{E}-01 \pm 1.0 \mathrm{E}-01$ & & & ${ }^{234} \mathrm{U}$ & 1.8E-01 & $\pm 5.9 \mathrm{E}-02$ & \\
\hline & ${ }^{235} \mathrm{U}$ & $3.5 \mathrm{E}-02 \pm 2.1 \mathrm{E}-02$ & & & ${ }^{235} \mathrm{U}$ & $1.4 \mathrm{E}-02$ & $\pm 1.1 \mathrm{E}-02$ & \\
\hline & ${ }^{238} \mathrm{U}$ & $4.1 \mathrm{E}-01 \pm 1.2 \mathrm{E}-01$ & & & ${ }^{238} \mathrm{U}$ & 1.7E-01 & $\pm 5.6 \mathrm{E}-02$ & \\
\hline & ${ }^{65} \mathrm{Zn}$ & $1.7 \mathrm{E}-02 \pm 2.1 \mathrm{E}-02$ & $\mathrm{U}$ & & ${ }^{65} \mathrm{Zn}$ & $5.2 \mathrm{E}-03$ & $\pm 1.9 \mathrm{E}-02$ & $\mathrm{U}$ \\
\hline D047 & ${ }^{144} \mathrm{Ce}$ & $6.8 \mathrm{E}-03 \pm 6.8 \mathrm{E}-02$ & $\overline{\mathrm{U}}$ & D049 & ${ }^{144} \mathrm{Ce}$ & $2.1 \mathrm{E}-02$ & $\pm 1.5 \mathrm{E}-01$ & $\bar{U}$ \\
\hline \multirow[t]{17}{*}{ (200 West) } & ${ }^{60} \mathrm{Co}$ & $3.3 \mathrm{E}-04 \pm 3.3 \mathrm{E}-03$ & $\mathrm{U}$ & (200 West) & ${ }^{60} \mathrm{Co}$ & $-5.2 E-03$ & $\pm 9.4 \mathrm{E}-03$ & $\mathrm{U}$ \\
\hline & ${ }^{134} \mathrm{Cs}$ & $4.6 \mathrm{E}-02 \pm 1.3 \mathrm{E}-02$ & & & ${ }^{134} \mathrm{Cs}$ & 4.2E-02 & $\pm 1.8 \mathrm{E}-02$ & \\
\hline & ${ }^{137} \mathrm{Cs}$ & $1.6 \mathrm{E}+00 \pm 2.9 \mathrm{E}-01$ & & & ${ }^{137} \mathrm{Cs}$ & 4.9E-01 & $\pm 8.5 \mathrm{E}-02$ & \\
\hline & ${ }^{152} \mathrm{Eu}$ & $-4.6 \mathrm{E}-02 \pm 4.6 \mathrm{E}-02$ & $\mathrm{U}$ & & ${ }^{152} \mathrm{Eu}$ & $3.9 \mathrm{E}-02$ & $\pm 4.0 \mathrm{E}-02$ & $\mathrm{U}$ \\
\hline & ${ }^{154} \mathrm{Eu}$ & $-1.5 \mathrm{E}-02 \pm 2.7 \mathrm{E}-02$ & $\mathrm{U}$ & & ${ }^{154} \mathrm{Eu}$ & $-2.0 \mathrm{E}-02$ & $\pm 3.3 \mathrm{E}-02$ & $\mathrm{U}$ \\
\hline & ${ }^{155} \mathrm{Eu}$ & $4.5 \mathrm{E}-02 \pm 5.0 \mathrm{E}-02$ & $\mathrm{U}$ & & ${ }^{155} \mathrm{Eu}$ & 5.9E-02 & $\pm 4.7 \mathrm{E}-02$ & $\mathrm{U}$ \\
\hline & ${ }^{238} \mathrm{Pu}$ & $1.9 \mathrm{E}-03 \pm 1.9 \mathrm{E}-03$ & $\mathrm{U}$ & & ${ }^{238} \mathrm{Pu}$ & $-2.5 E-02$ & $\pm 3.0 \mathrm{E}-02$ & $\mathrm{U}$ \\
\hline & ${ }^{239,240} \mathrm{Pu}$ & $1.9 \mathrm{E}-02 \pm 1.6 \mathrm{E}-02$ & $\mathrm{U}$ & & ${ }^{239,240} \mathrm{Pu}$ & $4.0 \mathrm{E}-02$ & $\pm 1.9 \mathrm{E}-02$ & \\
\hline & ${ }^{103} \mathrm{Ru}$ & $-3.6 \mathrm{E}-03 \pm 1.0 \mathrm{E}-02$ & $\mathrm{U}$ & & ${ }^{103} \mathrm{Ru}$ & $-1.6 \mathrm{E}-03$ & $\pm 1.0 \mathrm{E}-02$ & $\mathrm{U}$ \\
\hline & ${ }^{106} \mathrm{Ru}$ & $-1.8 \mathrm{E}-03 \pm 1.8 \mathrm{E}-02$ & $\mathrm{U}$ & & ${ }^{106} \mathrm{Ru}$ & $-5.4 \mathrm{E}-03$ & $\pm 5.4 \mathrm{E}-02$ & $\mathrm{U}$ \\
\hline & ${ }^{125} \mathrm{Sb}$ & $4.0 \mathrm{E}-03 \pm 2.6 \mathrm{E}-02$ & $\mathrm{U}$ & & ${ }^{125} \mathrm{Sb}$ & $6.8 \mathrm{E}-03$ & $\pm 2.7 \mathrm{E}-02$ & $\mathrm{U}$ \\
\hline & ${ }^{113} \mathrm{Sn}$ & $-3.4 \mathrm{E}-03 \pm 1.2 \mathrm{E}-02$ & $\mathrm{U}$ & & ${ }^{113} \mathrm{Sn}$ & $1.4 \mathrm{E}-02$ & $\pm 1.3 \mathrm{E}-02$ & $\mathrm{U}$ \\
\hline & ${ }^{90} \mathrm{Sr}$ & $-1.2 \mathrm{E}-02 \pm 1.2 \mathrm{E}-01$ & $\mathrm{U}$ & & ${ }^{90} \mathrm{Sr}$ & $-5.9 E-02$ & $\pm 2.2 \mathrm{E}-01$ & $\mathrm{U}$ \\
\hline & ${ }^{234} \mathrm{U}$ & $2.4 \mathrm{E}-01 \pm 7.4 \mathrm{E}-02$ & & & ${ }^{234} \mathrm{U}$ & $1.5 \mathrm{E}-01$ & $\pm 4.9 \mathrm{E}-02$ & \\
\hline & ${ }^{235} \mathrm{U}$ & $2.2 \mathrm{E}-02 \pm 1.5 \mathrm{E}-02$ & & & ${ }^{235} \mathrm{U}$ & 1.5E-02 & $\pm 1.1 \mathrm{E}-02$ & \\
\hline & ${ }^{238} U$ & $2.4 \mathrm{E}-01 \pm 7.4 \mathrm{E}-02$ & & & ${ }^{238} U$ & $1.9 \mathrm{E}-01$ & $\pm 6.1 \mathrm{E}-02$ & \\
\hline & ${ }^{65} \mathrm{Zn}$ & $-5.6 \mathrm{E}-03 \pm 2.2 \mathrm{E}-02$ & $\mathrm{U}$ & & ${ }^{65} \mathrm{Zn}$ & $6.0 \mathrm{E}-02$ & $\pm 2.9 \mathrm{E}-02$ & \\
\hline
\end{tabular}

$\overline{\mathrm{RQ}}=$ Result Qualifier. U $=$ The analyte was analyzed for but not detected. 
Table 3-3. 2003 Soil Sampling Results (pCi/g \pm total analytical uncertainty). (cont)

\begin{tabular}{|c|c|c|c|c|c|c|c|c|}
\hline Location & Isotope & Result \pm Error & $\overline{\mathbf{R Q}^{*}}$ & Location & Isotope & Result & \pm Error & $\mathbf{R Q}^{*}$ \\
\hline D051 & ${ }^{144} \mathrm{Ce}$ & $-1.0 \mathrm{E}-01 \pm 1.1 \mathrm{E}-01$ & $\bar{U}$ & D053 & ${ }^{144} \mathrm{Ce}$ & $6.1 \mathrm{E}-02$ & $\pm 1.3 \mathrm{E}-01$ & $\mathrm{U}$ \\
\hline \multirow[t]{17}{*}{ (200 West) } & ${ }^{60} \mathrm{Co}$ & $2.6 \mathrm{E}-03 \pm 7.5 \mathrm{E}-03$ & $\mathrm{U}$ & (200 East) & ${ }^{60} \mathrm{Co}$ & $-3.9 E-03$ & $\pm 6.6 \mathrm{E}-03$ & $\mathrm{U}$ \\
\hline & ${ }^{134} \mathrm{Cs}$ & $3.4 \mathrm{E}-02 \pm 1.5 \mathrm{E}-02$ & & & ${ }^{134} \mathrm{Cs}$ & 4.4E-02 & $\pm 1.4 \mathrm{E}-02$ & \\
\hline & ${ }^{137} \mathrm{Cs}$ & $2.8 \mathrm{E}-01 \pm 5.0 \mathrm{E}-02$ & & & ${ }^{137} \mathrm{Cs}$ & 7.1E-01 & $\pm 1.1 \mathrm{E}-01$ & \\
\hline & ${ }^{152} \mathrm{Eu}$ & $-1.6 \mathrm{E}-02 \pm 2.2 \mathrm{E}-02$ & $\mathrm{U}$ & & ${ }^{152} \mathrm{Eu}$ & $-4.2 \mathrm{E}-03$ & $\pm 2.5 \mathrm{E}-02$ & $\mathrm{U}$ \\
\hline & ${ }^{154} \mathrm{Eu}$ & $-2.7 \mathrm{E}-03 \pm 2.4 \mathrm{E}-02$ & $\mathrm{U}$ & & ${ }^{154} \mathrm{Eu}$ & $1.4 \mathrm{E}-03$ & $\pm 1.4 \mathrm{E}-02$ & $\mathrm{U}$ \\
\hline & ${ }^{155} \mathrm{Eu}$ & $4.8 \mathrm{E}-02 \pm 4.0 \mathrm{E}-02$ & $\mathrm{U}$ & & ${ }^{155} \mathrm{Eu}$ & 5.8E-02 & $\pm 4.2 \mathrm{E}-02$ & U \\
\hline & ${ }^{238} \mathrm{Pu}$ & $-1.3 \mathrm{E}-02 \pm 2.7 \mathrm{E}-02$ & $\mathrm{U}$ & & ${ }^{238} \mathrm{Pu}$ & $-1.2 \mathrm{E}-02$ & $\pm 2.9 \mathrm{E}-02$ & U \\
\hline & ${ }^{239,240} \mathrm{Pu}$ & $2.8 \mathrm{E}-02 \pm 1.5 \mathrm{E}-02$ & & & ${ }^{239,240} \mathrm{Pu}$ & $1.9 \mathrm{E}-03$ & $\pm 1.9 \mathrm{E}-02$ & $\mathrm{U}$ \\
\hline & ${ }^{103} \mathrm{Ru}$ & $-4.5 \mathrm{E}-03 \pm 7.5 \mathrm{E}-03$ & $\mathrm{U}$ & & ${ }^{103} \mathrm{Ru}$ & 3.0E-03 & $\pm 7.4 \mathrm{E}-03$ & $\mathrm{U}$ \\
\hline & ${ }^{106} \mathrm{Ru}$ & $1.2 \mathrm{E}-02 \pm 6.3 \mathrm{E}-02$ & $\mathrm{U}$ & & ${ }^{106} \mathrm{Ru}$ & $-2.0 \mathrm{E}-03$ & $\pm 2.0 \mathrm{E}-02$ & $\mathrm{U}$ \\
\hline & ${ }^{125} \mathrm{Sb}$ & $-1.9 \mathrm{E}-03 \pm 1.9 \mathrm{E}-02$ & $\mathrm{U}$ & & ${ }^{125} \mathrm{Sb}$ & 3.0E-04 & $\pm 3.0 \mathrm{E}-03$ & U \\
\hline & ${ }^{113} \mathrm{Sn}$ & $3.7 \mathrm{E}-03 \pm 1.0 \mathrm{E}-02$ & $\mathrm{U}$ & & ${ }^{113} \mathrm{Sn}$ & $-5.8 \mathrm{E}-03$ & $\pm 9.7 \mathrm{E}-03$ & $\mathrm{U}$ \\
\hline & ${ }^{90} \mathrm{Sr}$ & $-1.1 \mathrm{E}-01 \pm 1.8 \mathrm{E}-01$ & $\mathrm{U}$ & & ${ }^{90} \mathrm{Sr}$ & $-1.1 \mathrm{E}-01$ & $\pm 2.1 \mathrm{E}-01$ & $\mathrm{U}$ \\
\hline & ${ }^{234} \mathrm{U}$ & $1.8 \mathrm{E}-01 \pm 5.9 \mathrm{E}-02$ & & & ${ }^{234} \mathrm{U}$ & 2.0E-01 & $\pm 6.6 \mathrm{E}-02$ & \\
\hline & ${ }^{235} \mathrm{U}$ & $8.7 \mathrm{E}-03 \pm 1.4 \mathrm{E}-02$ & $\mathrm{U}$ & & ${ }^{235} \mathrm{U}$ & $1.4 \mathrm{E}-02$ & $\pm 1.4 \mathrm{E}-02$ & $\mathrm{U}$ \\
\hline & ${ }^{238} \mathrm{U}$ & $2.2 \mathrm{E}-01 \pm 7.0 \mathrm{E}-02$ & & & ${ }^{238} \mathrm{U}$ & $1.4 \mathrm{E}-01$ & $\pm 4.9 \mathrm{E}-02$ & \\
\hline & ${ }^{65} \mathrm{Zn}$ & $2.5 \mathrm{E}-02 \pm 2.1 \mathrm{E}-02$ & $\mathrm{U}$ & & ${ }^{65} \mathrm{Zn}$ & $-7.3 \mathrm{E}-04$ & $\pm 7.3 \mathrm{E}-03$ & $\mathrm{U}$ \\
\hline D055 & ${ }^{144} \mathrm{Ce}$ & $1.4 \mathrm{E}-02 \pm 1.4 \mathrm{E}-01$ & $\overline{\mathrm{U}}$ & D057 & ${ }^{144} \mathrm{Ce}$ & $-2.5 \mathrm{E}-01$ & $\pm 2.5 \mathrm{E}-01$ & $\bar{U}$ \\
\hline \multirow[t]{17}{*}{ (200 East) } & ${ }^{60} \mathrm{Co}$ & $7.8 \mathrm{E}-04 \pm 6.7 \mathrm{E}-03$ & $\mathrm{U}$ & (200 East) & ${ }^{60} \mathrm{Co}$ & 1.3E-03 & $\pm 6.5 \mathrm{E}-03$ & $\mathrm{U}$ \\
\hline & ${ }^{134} \mathrm{Cs}$ & $3.1 \mathrm{E}-02 \pm 1.0 \mathrm{E}-02$ & & & ${ }^{134} \mathrm{Cs}$ & 4.2E-02 & $\pm 1.5 \mathrm{E}-02$ & \\
\hline & ${ }^{137} \mathrm{Cs}$ & $2.2 \mathrm{E}+00 \pm 4.1 \mathrm{E}-01$ & & & ${ }^{137} \mathrm{Cs}$ & $1.4 \mathrm{E}+01$ & $\pm 2.3 \mathrm{E}+00$ & \\
\hline & ${ }^{152} \mathrm{Eu}$ & $-1.8 \mathrm{E}-03 \pm 1.8 \mathrm{E}-02$ & $\mathrm{U}$ & & ${ }^{152} \mathrm{Eu}$ & $-8.0 \mathrm{E}-03$ & $\pm 7.5 \mathrm{E}-02$ & $\mathrm{U}$ \\
\hline & ${ }^{154} \mathrm{Eu}$ & $5.4 \mathrm{E}-03 \pm 2.5 \mathrm{E}-02$ & $\mathrm{U}$ & & ${ }^{154} \mathrm{Eu}$ & $-2.0 \mathrm{E}-02$ & $\pm 2.2 \mathrm{E}-02$ & $\mathrm{U}$ \\
\hline & ${ }^{155} \mathrm{Eu}$ & $1.9 \mathrm{E}-02 \pm 3.3 \mathrm{E}-02$ & $\mathrm{U}$ & & ${ }^{155} \mathrm{Eu}$ & $-1.1 \mathrm{E}-02$ & $\pm 6.1 \mathrm{E}-02$ & $\mathrm{U}$ \\
\hline & ${ }^{238} \mathrm{Pu}$ & $2.1 \mathrm{E}-02 \pm 2.9 \mathrm{E}-02$ & $\mathrm{U}$ & & ${ }^{238} \mathrm{Pu}$ & 2.7E-02 & $\pm 3.8 \mathrm{E}-02$ & $\mathrm{U}$ \\
\hline & ${ }^{239,240} \mathrm{Pu}$ & $7.5 \mathrm{E}-03 \pm 1.0 \mathrm{E}-02$ & $\mathrm{U}$ & & ${ }^{239,240} \mathrm{Pu}$ & $5.4 \mathrm{E}-02$ & $\pm 2.6 \mathrm{E}-02$ & \\
\hline & ${ }^{103} \mathrm{Ru}$ & $-4.4 \mathrm{E}-03 \pm 8.9 \mathrm{E}-03$ & $\mathrm{U}$ & & ${ }^{103} \mathrm{Ru}$ & $-9.9 E-03$ & $\pm 1.8 \mathrm{E}-02$ & $\mathrm{U}$ \\
\hline & ${ }^{106} \mathrm{Ru}$ & $-1.5 \mathrm{E}-02 \pm 6.6 \mathrm{E}-02$ & $\mathrm{U}$ & & ${ }^{106} \mathrm{Ru}$ & $1.2 \mathrm{E}-01$ & $\pm 1.1 \mathrm{E}-01$ & $\mathrm{U}$ \\
\hline & ${ }^{125} \mathrm{Sb}$ & $3.7 \mathrm{E}-03 \pm 2.4 \mathrm{E}-02$ & $\mathrm{U}$ & & ${ }^{125} \mathrm{Sb}$ & 3.0E-02 & $\pm 5.0 \mathrm{E}-02$ & $\mathrm{U}$ \\
\hline & ${ }^{113} \mathrm{Sn}$ & $-7.4 \mathrm{E}-03 \pm 1.1 \mathrm{E}-02$ & $\mathrm{U}$ & & ${ }^{113} \mathrm{Sn}$ & $-2.3 \mathrm{E}-02$ & $\pm 2.3 \mathrm{E}-02$ & $\mathrm{U}$ \\
\hline & ${ }^{90} \mathrm{Sr}$ & $1.1 \mathrm{E}-01 \pm 1.9 \mathrm{E}-01$ & $\mathrm{U}$ & & ${ }^{90} \mathrm{Sr}$ & 1.6E-01 & $\pm 1.6 \mathrm{E}-01$ & $\mathrm{U}$ \\
\hline & ${ }^{234} \mathrm{U}$ & $1.9 \mathrm{E}-01 \pm 6.3 \mathrm{E}-02$ & & & ${ }^{234} \mathrm{U}$ & $1.6 \mathrm{E}-01$ & $\pm 5.6 \mathrm{E}-02$ & \\
\hline & ${ }^{235} \mathrm{U}$ & $4.5 \mathrm{E}-03 \pm 6.3 \mathrm{E}-03$ & $\mathrm{U}$ & & ${ }^{235} \mathrm{U}$ & $9.1 \mathrm{E}-03$ & $\pm 9.1 \mathrm{E}-03$ & \\
\hline & ${ }^{238} U$ & $1.9 \mathrm{E}-01 \pm 6.3 \mathrm{E}-02$ & & & ${ }^{238} U$ & 1.7E-01 & $\pm 5.8 \mathrm{E}-02$ & \\
\hline & ${ }^{65} \mathrm{Zn}$ & $2.6 \mathrm{E}-03 \pm 1.8 \mathrm{E}-02$ & $\mathrm{U}$ & & ${ }^{65} \mathrm{Zn}$ & 3.0E-02 & $\pm 1.8 \mathrm{E}-02$ & \\
\hline
\end{tabular}

$\overline{\mathrm{RQ}}=$ Result Qualifier. U $=$ The analyte was analyzed for but not detected. 
Table 3-3. 2003 Soil Sampling Results (pCi/g \pm total analytical uncertainty). (cont)

\begin{tabular}{|c|c|c|c|c|c|c|c|c|}
\hline Location & Isotope & Result \pm Error & $\overline{\mathbf{R Q}^{*}}$ & Location & Isotope & Result & \pm Error & $\overline{\mathbf{R Q}^{*}}$ \\
\hline D059 & ${ }^{144} \mathrm{Ce}$ & $-5.6 \mathrm{E}-02 \pm 1.6 \mathrm{E}-01$ & $\overline{\mathrm{U}}$ & D061 & ${ }^{144} \mathrm{Ce}$ & $-1.2 \mathrm{E}-01$ & $\pm 1.5 \mathrm{E}-01$ & $\bar{U}$ \\
\hline \multirow[t]{17}{*}{ (200 East) } & ${ }^{60} \mathrm{Co}$ & $-1.6 \mathrm{E}-03 \pm 6.7 \mathrm{E}-03$ & $\mathrm{U}$ & (200 East) & ${ }^{60} \mathrm{Co}$ & $-2.5 E-05$ & $\pm 2.5 \mathrm{E}-04$ & U \\
\hline & ${ }^{134} \mathrm{Cs}$ & $5.0 \mathrm{E}-02 \pm 1.5 \mathrm{E}-02$ & & & ${ }^{134} \mathrm{Cs}$ & 3.9E-02 & $\pm 1.3 \mathrm{E}-02$ & \\
\hline & ${ }^{137} \mathrm{Cs}$ & $4.2 \mathrm{E}+00 \pm 6.7 \mathrm{E}-01$ & & & ${ }^{137} \mathrm{Cs}$ & $1.4 \mathrm{E}+00$ & $\pm 2.3 \mathrm{E}-01$ & \\
\hline & ${ }^{152} \mathrm{Eu}$ & $-1.5 \mathrm{E}-03 \pm 1.5 \mathrm{E}-02$ & $\mathrm{U}$ & & ${ }^{152} \mathrm{Eu}$ & $-2.1 \mathrm{E}-02$ & $\pm 2.7 \mathrm{E}-02$ & $\mathrm{U}$ \\
\hline & ${ }^{154} \mathrm{Eu}$ & $-1.4 \mathrm{E}-02 \pm 2.5 \mathrm{E}-02$ & $\mathrm{U}$ & & ${ }^{154} \mathrm{Eu}$ & $-1.3 \mathrm{E}-02$ & $\pm 2.2 \mathrm{E}-02$ & $\mathrm{U}$ \\
\hline & ${ }^{155} \mathrm{Eu}$ & $1.5 \mathrm{E}-03 \pm 1.5 \mathrm{E}-02$ & $\mathrm{U}$ & & ${ }^{155} \mathrm{Eu}$ & 8.3E-02 & $\pm 5.0 \mathrm{E}-02$ & $\mathrm{U}$ \\
\hline & ${ }^{238} \mathrm{Pu}$ & $3.7 \mathrm{E}-03 \pm 9.3 \mathrm{E}-03$ & $\mathrm{U}$ & & ${ }^{238} \mathrm{Pu}$ & $2.0 \mathrm{E}-03$ & $\pm 2.0 \mathrm{E}-02$ & $\mathrm{U}$ \\
\hline & ${ }^{239,240} \mathrm{Pu}$ & $1.1 \mathrm{E}-02 \pm 9.2 \mathrm{E}-03$ & & & ${ }^{239,240} \mathrm{Pu}$ & $4.0 \mathrm{E}-03$ & $\pm 5.6 \mathrm{E}-03$ & $\mathrm{U}$ \\
\hline & ${ }^{103} \mathrm{Ru}$ & $-5.5 \mathrm{E}-04 \pm 5.5 \mathrm{E}-03$ & $\mathrm{U}$ & & ${ }^{103} \mathrm{Ru}$ & 2.8E-04 & $\pm 2.8 \mathrm{E}-03$ & U \\
\hline & ${ }^{106} \mathrm{Ru}$ & $3.5 \mathrm{E}-02 \pm 7.3 \mathrm{E}-02$ & $\mathrm{U}$ & & ${ }^{106} \mathrm{Ru}$ & $-1.2 \mathrm{E}-02$ & $\pm 6.4 \mathrm{E}-02$ & $\mathrm{U}$ \\
\hline & ${ }^{125} \mathrm{Sb}$ & $4.1 \mathrm{E}-03 \pm 3.0 \mathrm{E}-02$ & $\mathrm{U}$ & & ${ }^{125} \mathrm{Sb}$ & $-3.0 \mathrm{E}-03$ & $\pm 2.3 \mathrm{E}-02$ & $\mathrm{U}$ \\
\hline & ${ }^{113} \mathrm{Sn}$ & $-8.4 \mathrm{E}-03 \pm 1.3 \mathrm{E}-02$ & $\mathrm{U}$ & & ${ }^{113} \mathrm{Sn}$ & $-4.2 \mathrm{E}-03$ & $\pm 1.1 \mathrm{E}-02$ & $\mathrm{U}$ \\
\hline & ${ }^{90} \mathrm{Sr}$ & $-3.5 \mathrm{E}-03 \pm 3.5 \mathrm{E}-02$ & $\mathrm{U}$ & & ${ }^{90} \mathrm{Sr}$ & $-1.5 \mathrm{E}-01$ & $\pm 1.8 \mathrm{E}-01$ & $\mathrm{U}$ \\
\hline & ${ }^{234} \mathrm{U}$ & $1.4 \mathrm{E}-01 \pm 4.9 \mathrm{E}-02$ & & & & $1.3 \mathrm{E}-01$ & $\pm 4.7 \mathrm{E}-02$ & \\
\hline & ${ }^{235} \mathrm{U}$ & $1.7 \mathrm{E}-02 \pm 1.3 \mathrm{E}-02$ & & & ${ }^{235} \mathrm{U}$ & $2.1 \mathrm{E}-03$ & $\pm 4.2 \mathrm{E}-03$ & $\mathrm{U}$ \\
\hline & ${ }^{238} \mathrm{U}$ & $1.6 \mathrm{E}-01 \pm 5.4 \mathrm{E}-02$ & & & ${ }^{238} \mathrm{U}$ & $1.7 \mathrm{E}-01$ & $\pm 5.6 \mathrm{E}-02$ & \\
\hline & ${ }^{65} \mathrm{Zn}$ & $-1.2 \mathrm{E}-02 \pm 1.9 \mathrm{E}-02$ & $\mathrm{U}$ & & ${ }^{65} \mathrm{Zn}$ & $-6.9 \mathrm{E}-03$ & $\pm 1.9 \mathrm{E}-02$ & $\mathrm{U}$ \\
\hline D063 & ${ }^{144} \mathrm{Ce}$ & $1.4 \mathrm{E}-02 \pm 1.4 \mathrm{E}-01$ & $\overline{\mathrm{U}}$ & D065 & ${ }^{144} \mathrm{Ce}$ & $-7.7 \mathrm{E}-04$ & $\pm 7.7 \mathrm{E}-03$ & $\bar{U}$ \\
\hline \multirow[t]{17}{*}{ (200 East) } & ${ }^{60} \mathrm{Co}$ & $-3.8 \mathrm{E}-04 \pm 3.8 \mathrm{E}-03$ & $\mathrm{U}$ & (200 East) & ${ }^{60} \mathrm{Co}$ & $-8.3 E-04$ & $\pm 6.9 \mathrm{E}-03$ & $\mathrm{U}$ \\
\hline & ${ }^{134} \mathrm{Cs}$ & $4.7 \mathrm{E}-02 \pm 1.4 \mathrm{E}-02$ & & & ${ }^{134} \mathrm{Cs}$ & 4.8E-02 & $\pm 1.5 \mathrm{E}-02$ & \\
\hline & ${ }^{137} \mathrm{Cs}$ & $5.2 \mathrm{E}-01 \pm 8.8 \mathrm{E}-02$ & & & ${ }^{137} \mathrm{Cs}$ & $1.7 \mathrm{E}+00$ & $\pm 3.0 \mathrm{E}-01$ & \\
\hline & ${ }^{152} \mathrm{Eu}$ & $-6.4 \mathrm{E}-03 \pm 3.8 \mathrm{E}-02$ & $\mathrm{U}$ & & ${ }^{152} \mathrm{Eu}$ & $1.5 \mathrm{E}-02$ & $\pm 4.0 \mathrm{E}-02$ & $\mathrm{U}$ \\
\hline & ${ }^{154} \mathrm{Eu}$ & $-3.3 \mathrm{E}-02 \pm 3.3 \mathrm{E}-02$ & $\mathrm{U}$ & & ${ }^{154} \mathrm{Eu}$ & $-3.1 \mathrm{E}-02$ & $\pm 3.1 \mathrm{E}-02$ & U \\
\hline & ${ }^{155} \mathrm{Eu}$ & $4.1 \mathrm{E}-02 \pm 4.3 \mathrm{E}-02$ & $\mathrm{U}$ & & ${ }^{155} \mathrm{Eu}$ & $5.0 \mathrm{E}-02$ & $\pm 3.6 \mathrm{E}-02$ & $\mathrm{U}$ \\
\hline & ${ }^{238} \mathrm{Pu}$ & $1.9 \mathrm{E}-03 \pm 1.9 \mathrm{E}-02$ & $\mathrm{U}$ & & ${ }^{238} \mathrm{Pu}$ & $-1.4 \mathrm{E}-02$ & $\pm 3.1 \mathrm{E}-02$ & $\mathrm{U}$ \\
\hline & ${ }^{239,240} \mathrm{Pu}$ & $3.5 \mathrm{E}-02 \pm 2.2 \mathrm{E}-02$ & & & ${ }^{239,240} \mathrm{Pu}$ & $2.0 \mathrm{E}-03$ & $\pm 2.0 \mathrm{E}-02$ & $\mathrm{U}$ \\
\hline & ${ }^{103} \mathrm{Ru}$ & $-6.3 \mathrm{E}-03 \pm 9.4 \mathrm{E}-03$ & $\mathrm{U}$ & & ${ }^{103} \mathrm{Ru}$ & $2.5 \mathrm{E}-03$ & $\pm 9.0 \mathrm{E}-03$ & $\mathrm{U}$ \\
\hline & ${ }^{106} \mathrm{Ru}$ & $-1.4 \mathrm{E}-02 \pm 7.9 \mathrm{E}-02$ & $\mathrm{U}$ & & ${ }^{106} \mathrm{Ru}$ & $1.7 \mathrm{E}-02$ & $\pm 6.6 \mathrm{E}-02$ & $\mathrm{U}$ \\
\hline & ${ }^{125} \mathrm{Sb}$ & $2.0 \mathrm{E}-02 \pm 2.4 \mathrm{E}-02$ & $\mathrm{U}$ & & ${ }^{125} \mathrm{Sb}$ & 8.9E-03 & $\pm 2.4 \mathrm{E}-02$ & U \\
\hline & ${ }^{113} \mathrm{Sn}$ & $-5.3 \mathrm{E}-03 \pm 1.2 \mathrm{E}-02$ & $\mathrm{U}$ & & ${ }^{113} \mathrm{Sn}$ & 8.6E-03 & $\pm 1.1 \mathrm{E}-02$ & U \\
\hline & ${ }^{90} \mathrm{Sr}$ & $3.0 \mathrm{E}-01 \pm 2.1 \mathrm{E}-01$ & & & ${ }^{90} \mathrm{Sr}$ & 2.3E-01 & $\pm 2.4 \mathrm{E}-01$ & $\mathrm{U}$ \\
\hline & ${ }^{234} \mathrm{U}$ & $2.6 \mathrm{E}-01 \pm 8.1 \mathrm{E}-02$ & & & ${ }^{234} \mathrm{U}$ & $1.5 \mathrm{E}-01$ & $\pm 5.4 \mathrm{E}-02$ & \\
\hline & ${ }^{235} \mathrm{U}$ & $1.6 \mathrm{E}-02 \pm 1.2 \mathrm{E}-02$ & & & ${ }^{235} \mathrm{U}$ & 4.8E-03 & $\pm 9.6 \mathrm{E}-03$ & U \\
\hline & ${ }^{238} \mathrm{U}$ & $2.2 \mathrm{E}-01 \pm 6.8 \mathrm{E}-02$ & & & ${ }^{238} \mathrm{U}$ & 1.7E-01 & $\pm 5.8 \mathrm{E}-02$ & \\
\hline & ${ }^{65} \mathrm{Zn}$ & $7.7 \mathrm{E}-02 \pm 2.8 \mathrm{E}-02$ & & & ${ }^{65} \mathrm{Zn}$ & $1.1 \mathrm{E}-02$ & $\pm 1.9 \mathrm{E}-02$ & U \\
\hline
\end{tabular}

$\overline{\mathrm{RQ}}=$ Result Qualifier. U $=$ The analyte was analyzed for but not detected. 
Table 3-3. 2003 Soil Sampling Results (pCi/g \pm total analytical uncertainty). (cont)

\begin{tabular}{|c|c|c|c|c|c|c|c|c|}
\hline Location & Isotope & Result \pm Error & $\overline{\mathbf{R Q}^{*}}$ & Location & Isotope & Result & \pm Error & $\overline{\mathbf{R Q}^{*}}$ \\
\hline D067 & ${ }^{144} \mathrm{Ce}$ & $9.4 \mathrm{E}-02 \pm 1.7 \mathrm{E}-01$ & $\overline{\mathrm{U}}$ & D069 & ${ }^{144} \mathrm{Ce}$ & $6.4 \mathrm{E}-02$ & $\pm 1.2 \mathrm{E}-01$ & $\bar{U}$ \\
\hline \multirow[t]{17}{*}{ (200 East) } & ${ }^{60} \mathrm{Co}$ & $1.9 \mathrm{E}-03 \pm 7.0 \mathrm{E}-03$ & $\mathrm{U}$ & (200 East) & ${ }^{60} \mathrm{Co}$ & $-3.1 E-03$ & $\pm 6.3 \mathrm{E}-03$ & U \\
\hline & ${ }^{134} \mathrm{Cs}$ & $4.7 \mathrm{E}-02 \pm 1.5 \mathrm{E}-02$ & & & ${ }^{134} \mathrm{Cs}$ & 2.7E-02 & $\pm 1.1 \mathrm{E}-02$ & \\
\hline & ${ }^{137} \mathrm{Cs}$ & $1.2 \mathrm{E}-02 \pm 8.4 \mathrm{E}-03$ & $\mathrm{U}$ & & ${ }^{137} \mathrm{Cs}$ & 5.3E-01 & $\pm 8.6 \mathrm{E}-02$ & \\
\hline & ${ }^{152} \mathrm{Eu}$ & $7.2 \mathrm{E}-03 \pm 4.6 \mathrm{E}-02$ & $\mathrm{U}$ & & ${ }^{152} \mathrm{Eu}$ & $-1.7 \mathrm{E}-02$ & $\pm 2.2 \mathrm{E}-02$ & $\mathrm{U}$ \\
\hline & ${ }^{154} \mathrm{Eu}$ & $-2.9 \mathrm{E}-02 \pm 2.9 \mathrm{E}-02$ & $\mathrm{U}$ & & ${ }^{154} \mathrm{Eu}$ & $-1.0 \mathrm{E}-02$ & $\pm 2.0 \mathrm{E}-02$ & $\mathrm{U}$ \\
\hline & ${ }^{155} \mathrm{Eu}$ & $2.3 \mathrm{E}-02 \pm 4.8 \mathrm{E}-02$ & $\mathrm{U}$ & & ${ }^{155} \mathrm{Eu}$ & $5.1 \mathrm{E}-02$ & $\pm 3.4 \mathrm{E}-02$ & $\mathrm{U}$ \\
\hline & ${ }^{238} \mathrm{Pu}$ & $1.4 \mathrm{E}-02 \pm 2.5 \mathrm{E}-02$ & $\mathrm{U}$ & & ${ }^{238} \mathrm{Pu}$ & 3.3E-02 & $\pm 3.3 \mathrm{E}-02$ & U \\
\hline & ${ }^{239,240} \mathrm{Pu}$ & $6.4 \mathrm{E}-03 \pm 9.0 \mathrm{E}-03$ & $\mathrm{U}$ & & ${ }^{239,240} \mathrm{Pu}$ & $1.7 \mathrm{E}-03$ & $\pm 1.7 \mathrm{E}-03$ & $\mathrm{U}$ \\
\hline & ${ }^{103} \mathrm{Ru}$ & $1.4 \mathrm{E}-03 \pm 8.6 \mathrm{E}-03$ & $\mathrm{U}$ & & ${ }^{103} \mathrm{Ru}$ & 2.7E-03 & $\pm 7.8 \mathrm{E}-03$ & U \\
\hline & ${ }^{106} \mathrm{Ru}$ & $-2.8 \mathrm{E}-02 \pm 6.8 \mathrm{E}-02$ & $\mathrm{U}$ & & ${ }^{106} \mathrm{Ru}$ & $-5.6 \mathrm{E}-02$ & $\pm 5.6 \mathrm{E}-02$ & $\mathrm{U}$ \\
\hline & ${ }^{125} \mathrm{Sb}$ & $1.3 \mathrm{E}-02 \pm 2.2 \mathrm{E}-02$ & $\mathrm{U}$ & & ${ }^{125} \mathrm{Sb}$ & $1.1 \mathrm{E}-02$ & $\pm 1.9 \mathrm{E}-02$ & $\mathrm{U}$ \\
\hline & ${ }^{113} \mathrm{Sn}$ & $2.1 \mathrm{E}-03 \pm 1.1 \mathrm{E}-02$ & $\mathrm{U}$ & & ${ }^{113} \mathrm{Sn}$ & $-7.9 \mathrm{E}-03$ & $\pm 9.1 \mathrm{E}-03$ & $\mathrm{U}$ \\
\hline & ${ }^{90} \mathrm{Sr}$ & $-2.0 \mathrm{E}-01 \pm 2.0 \mathrm{E}-01$ & $\mathrm{U}$ & & ${ }^{90} \mathrm{Sr}$ & $-2.4 \mathrm{E}-02$ & $\pm 1.6 \mathrm{E}-01$ & $\mathrm{U}$ \\
\hline & ${ }^{234} \mathrm{U}$ & $1.9 \mathrm{E}-01 \pm 6.1 \mathrm{E}-02$ & & & ${ }^{234} \mathrm{~J}$ & 3.1E-01 & $\pm 9.3 \mathrm{E}-02$ & \\
\hline & ${ }^{235} \mathrm{U}$ & $9.6 \mathrm{E}-03 \pm 1.3 \mathrm{E}-02$ & $\mathrm{U}$ & & ${ }^{235} \mathrm{U}$ & 3.7E-02 & $\pm 2.0 \mathrm{E}-02$ & \\
\hline & ${ }^{238} \mathrm{U}$ & $1.7 \mathrm{E}-01 \pm 5.6 \mathrm{E}-02$ & & & ${ }^{238} \mathrm{U}$ & $3.0 \mathrm{E}-01$ & $\pm 9.0 \mathrm{E}-02$ & \\
\hline & ${ }^{65} \mathrm{Zn}$ & $1.9 \mathrm{E}-02 \pm 2.0 \mathrm{E}-02$ & $\mathrm{U}$ & & ${ }^{65} \mathrm{Zn}$ & $-5.3 \mathrm{E}-03$ & $\pm 1.6 \mathrm{E}-02$ & $\mathrm{U}$ \\
\hline D071 & ${ }^{144} \mathrm{Ce}$ & $5.7 \mathrm{E}-02 \pm 1.4 \mathrm{E}-01$ & $\overline{\mathrm{U}}$ & D073 & ${ }^{144} \mathrm{Ce}$ & $-8.7 \mathrm{E}-02$ & $\pm 1.2 \mathrm{E}-01$ & $\bar{U}$ \\
\hline \multirow[t]{17}{*}{ (200 East) } & ${ }^{60} \mathrm{Co}$ & $-4.1 \mathrm{E}-03 \pm 8.8 \mathrm{E}-03$ & $\mathrm{U}$ & (200 East) & ${ }^{60} \mathrm{Co}$ & $-3.6 \mathrm{E}-03$ & $\pm 6.8 \mathrm{E}-03$ & $\mathrm{U}$ \\
\hline & ${ }^{134} \mathrm{Cs}$ & $3.9 \mathrm{E}-02 \pm 1.5 \mathrm{E}-02$ & & & ${ }^{134} \mathrm{Cs}$ & 3.1E-02 & $\pm 1.0 \mathrm{E}-02$ & \\
\hline & ${ }^{137} \mathrm{Cs}$ & $1.9 \mathrm{E}-01 \pm 3.8 \mathrm{E}-02$ & & & ${ }^{137} \mathrm{Cs}$ & $1.9 \mathrm{E}+00$ & $\pm 3.2 \mathrm{E}-01$ & \\
\hline & ${ }^{152} \mathrm{Eu}$ & $-1.5 \mathrm{E}-02 \pm 2.9 \mathrm{E}-02$ & $\mathrm{U}$ & & ${ }^{152} \mathrm{Eu}$ & $-3.8 \mathrm{E}-03$ & $\pm 2.5 \mathrm{E}-02$ & $\mathrm{U}$ \\
\hline & ${ }^{154} \mathrm{Eu}$ & $-3.2 \mathrm{E}-02 \pm 3.2 \mathrm{E}-02$ & $\mathrm{U}$ & & ${ }^{154} \mathrm{Eu}$ & $-3.0 \mathrm{E}-02$ & $\pm 3.0 \mathrm{E}-02$ & U \\
\hline & ${ }^{155} \mathrm{Eu}$ & $4.0 \mathrm{E}-02 \pm 4.4 \mathrm{E}-02$ & $\mathrm{U}$ & & ${ }^{155} \mathrm{Eu}$ & 3.5E-02 & $\pm 2.9 \mathrm{E}-02$ & $\mathrm{U}$ \\
\hline & ${ }^{238} \mathrm{Pu}$ & $-2.9 \mathrm{E}-02 \pm 3.5 \mathrm{E}-02$ & $\mathrm{U}$ & & ${ }^{238} \mathrm{Pu}$ & $-4.3 E-03$ & $\pm 2.3 \mathrm{E}-02$ & $\mathrm{U}$ \\
\hline & ${ }^{239,240} \mathrm{Pu}$ & $6.2 \mathrm{E}-02 \pm 2.9 \mathrm{E}-02$ & & & ${ }^{239,240} \mathrm{Pu}$ & $1.1 \mathrm{E}-02$ & $\pm 1.0 \mathrm{E}-02$ & \\
\hline & ${ }^{103} \mathrm{Ru}$ & $-1.1 \mathrm{E}-02 \pm 1.1 \mathrm{E}-02$ & $\mathrm{U}$ & & ${ }^{103} \mathrm{Ru}$ & $-4.2 \mathrm{E}-03$ & $\pm 8.7 \mathrm{E}-03$ & $\mathrm{U}$ \\
\hline & ${ }^{106} \mathrm{Ru}$ & $6.7 \mathrm{E}-02 \pm 7.6 \mathrm{E}-02$ & $\mathrm{U}$ & & ${ }^{106} \mathrm{Ru}$ & 1.6E-02 & $\pm 6.4 \mathrm{E}-02$ & $\mathrm{U}$ \\
\hline & ${ }^{125} \mathrm{Sb}$ & $1.8 \mathrm{E}-02 \pm 2.1 \mathrm{E}-02$ & $\mathrm{U}$ & & ${ }^{125} \mathrm{Sb}$ & $-7.7 \mathrm{E}-03$ & $\pm 2.1 \mathrm{E}-02$ & U \\
\hline & ${ }^{113} \mathrm{Sn}$ & $2.3 \mathrm{E}-03 \pm 1.1 \mathrm{E}-02$ & $\mathrm{U}$ & & ${ }^{113} \mathrm{Sn}$ & $-7.6 \mathrm{E}-04$ & $\pm 7.6 \mathrm{E}-03$ & U \\
\hline & ${ }^{90} \mathrm{Sr}$ & $7.3 \mathrm{E}-02 \pm 1.6 \mathrm{E}-01$ & $\mathrm{U}$ & & ${ }^{90} \mathrm{Sr}$ & $-8.5 \mathrm{E}-02$ & $\pm 1.7 \mathrm{E}-01$ & $\mathrm{U}$ \\
\hline & ${ }^{234} \mathrm{U}$ & $1.6 \mathrm{E}-01 \pm 5.3 \mathrm{E}-02$ & & & ${ }^{234} U$ & 2.0E-01 & $\pm 6.6 \mathrm{E}-02$ & \\
\hline & ${ }^{235} \mathrm{U}$ & $1.2 \mathrm{E}-02 \pm 1.0 \mathrm{E}-02$ & & & ${ }^{235} \mathrm{U}$ & $1.1 \mathrm{E}-02$ & $\pm 1.0 \mathrm{E}-02$ & \\
\hline & ${ }^{238} \mathrm{U}$ & $1.4 \mathrm{E}-01 \pm 4.9 \mathrm{E}-02$ & & & ${ }^{238} \mathrm{U}$ & 2.1E-01 & $\pm 6.7 \mathrm{E}-02$ & \\
\hline & ${ }^{65} \mathrm{Zn}$ & $1.5 \mathrm{E}-02 \pm 2.4 \mathrm{E}-02$ & $\mathrm{U}$ & & ${ }^{65} \mathrm{Zn}$ & 1.7E-02 & $\pm 1.9 \mathrm{E}-02$ & $\mathrm{U}$ \\
\hline
\end{tabular}

$\overline{\mathrm{RQ}}=$ Result Qualifier. U $=$ The analyte was analyzed for but not detected. 
Table 3-3. 2003 Soil Sampling Results (pCi/g \pm total analytical uncertainty). (cont)

\begin{tabular}{|c|c|c|c|c|c|c|c|c|}
\hline Location & Isotope & Result \pm Error & $\mathbf{R Q}^{*}$ & Location & Isotope & Result & \pm Error & $\mathbf{R Q}^{*}$ \\
\hline D075 & ${ }^{144} \mathrm{Ce}$ & $1.2 \mathrm{E}-01 \pm 1.3 \mathrm{E}-01$ & $\bar{U}$ & D077 & ${ }^{144} \mathrm{Ce}$ & $9.1 \mathrm{E}-03$ & $\pm 9.1 \mathrm{E}-02$ & $\overline{\mathrm{U}}$ \\
\hline \multirow[t]{17}{*}{ (200 East) } & ${ }^{60} \mathrm{Co}$ & $-1.1 \mathrm{E}-03 \pm 7.5 \mathrm{E}-03$ & $\mathrm{U}$ & (200 East) & ${ }^{60} \mathrm{Co}$ & 2.7E-03 & $\pm 6.3 \mathrm{E}-03$ & $\mathrm{U}$ \\
\hline & ${ }^{134} \mathrm{Cs}$ & $3.2 \mathrm{E}-02 \pm 1.4 \mathrm{E}-02$ & & & ${ }^{134} \mathrm{Cs}$ & 3.2E-02 & $\pm 1.4 \mathrm{E}-02$ & \\
\hline & ${ }^{137} \mathrm{Cs}$ & $2.9 \mathrm{E}-01 \pm 5.5 \mathrm{E}-02$ & & & ${ }^{137} \mathrm{Cs}$ & 3.6E-01 & $\pm 6.5 \mathrm{E}-02$ & \\
\hline & ${ }^{152} \mathrm{Eu}$ & $-2.5 \mathrm{E}-02 \pm 3.0 \mathrm{E}-02$ & $\mathrm{U}$ & & ${ }^{152} \mathrm{Eu}$ & $-1.4 \mathrm{E}-02$ & $\pm 4.3 \mathrm{E}-02$ & $\mathrm{U}$ \\
\hline & ${ }^{154} \mathrm{Eu}$ & $-3.7 \mathrm{E}-02 \pm 3.7 \mathrm{E}-02$ & $\mathrm{U}$ & & ${ }^{154} \mathrm{Eu}$ & $-1.4 \mathrm{E}-02$ & $\pm 2.1 \mathrm{E}-02$ & $\mathrm{U}$ \\
\hline & ${ }^{155} \mathrm{Eu}$ & $3.6 \mathrm{E}-02 \pm 3.4 \mathrm{E}-02$ & $\mathrm{U}$ & & ${ }^{155} \mathrm{Eu}$ & $-6.4 \mathrm{E}-03$ & $\pm 4.3 \mathrm{E}-02$ & $\mathrm{U}$ \\
\hline & ${ }^{238} \mathrm{Pu}$ & $3.5 \mathrm{E}-03 \pm 1.7 \mathrm{E}-02$ & $\mathrm{U}$ & & ${ }^{238} \mathrm{Pu}$ & $2.4 \mathrm{E}-02$ & $\pm 2.6 \mathrm{E}-02$ & $\mathrm{U}$ \\
\hline & ${ }^{239,240} \mathrm{Pu}$ & $3.0 \mathrm{E}-02 \pm 1.7 \mathrm{E}-02$ & & & ${ }^{239,240} \mathrm{Pu}$ & 2.9E-02 & $\pm 1.8 \mathrm{E}-02$ & \\
\hline & ${ }^{103} \mathrm{Ru}$ & $-5.4 \mathrm{E}-03 \pm 9.0 \mathrm{E}-03$ & $\mathrm{U}$ & & ${ }^{103} \mathrm{Ru}$ & $-6.5 E-03$ & $\pm 8.2 \mathrm{E}-03$ & $\mathrm{U}$ \\
\hline & ${ }^{106} \mathrm{Ru}$ & $-3.9 \mathrm{E}-02 \pm 6.7 \mathrm{E}-02$ & $\mathrm{U}$ & & ${ }^{106} \mathrm{Ru}$ & 4.7E-02 & $\pm 6.1 \mathrm{E}-02$ & $\mathrm{U}$ \\
\hline & ${ }^{125} \mathrm{Sb}$ & $-6.4 \mathrm{E}-03 \pm 2.1 \mathrm{E}-02$ & $\mathrm{U}$ & & ${ }^{125} \mathrm{Sb}$ & $-5.2 \mathrm{E}-03$ & $\pm 2.1 \mathrm{E}-02$ & $\mathrm{U}$ \\
\hline & ${ }^{113} \mathrm{Sn}$ & $2.6 \mathrm{E}-03 \pm 1.0 \mathrm{E}-02$ & $\mathrm{U}$ & & ${ }^{113} \mathrm{Sn}$ & 5.3E-03 & $\pm 1.0 \mathrm{E}-02$ & $\mathrm{U}$ \\
\hline & ${ }^{90} \mathrm{Sr}$ & $4.2 \mathrm{E}-01 \pm 2.1 \mathrm{E}-01$ & & & ${ }^{90} \mathrm{Sr}$ & $-1.6 \mathrm{E}-01$ & $\pm 2.1 \mathrm{E}-01$ & $\mathrm{U}$ \\
\hline & ${ }^{234} \mathrm{U}$ & $2.0 \mathrm{E}-01 \pm 6.4 \mathrm{E}-02$ & & & ${ }^{234} \mathrm{U}$ & $1.2 \mathrm{E}-01$ & $\pm 4.3 \mathrm{E}-02$ & \\
\hline & ${ }^{235} \mathrm{U}$ & $9.6 \mathrm{E}-03 \pm 8.9 \mathrm{E}-03$ & & & ${ }^{235} \mathrm{U}$ & $6.0 \mathrm{E}-03$ & $\pm 9.0 \mathrm{E}-03$ & $\mathrm{U}$ \\
\hline & ${ }^{238} \mathrm{U}$ & $2.3 \mathrm{E}-01 \pm 7.1 \mathrm{E}-02$ & & & ${ }^{238} \mathrm{U}$ & $1.2 \mathrm{E}-01$ & $\pm 4.3 \mathrm{E}-02$ & \\
\hline & ${ }^{65} \mathrm{Zn}$ & $1.2 \mathrm{E}-02 \pm 2.1 \mathrm{E}-02$ & $\mathrm{U}$ & & ${ }^{65} \mathrm{Zn}$ & $-5.1 \mathrm{E}-03$ & $\pm 1.8 \mathrm{E}-02$ & $\mathrm{U}$ \\
\hline D079 & ${ }^{144} \mathrm{Ce}$ & $9.4 \mathrm{E}-02 \pm 1.4 \mathrm{E}-01$ & $\bar{U}$ & D081 & ${ }^{144} \mathrm{Ce}$ & $-7.4 \mathrm{E}-02$ & $\pm 1.2 \mathrm{E}-01$ & $\bar{U}$ \\
\hline \multirow[t]{17}{*}{ (200 East) } & ${ }^{60} \mathrm{Co}$ & $1.5 \mathrm{E}-03 \pm 7.0 \mathrm{E}-03$ & $\mathrm{U}$ & (600 Area) & ${ }^{60} \mathrm{Co}$ & $-6.2 \mathrm{E}-03$ & $\pm 6.2 \mathrm{E}-03$ & $\mathrm{U}$ \\
\hline & ${ }^{134} \mathrm{Cs}$ & $3.3 \mathrm{E}-02 \pm 9.6 \mathrm{E}-03$ & & & ${ }^{134} \mathrm{Cs}$ & 3.8E-02 & $\pm 1.3 \mathrm{E}-02$ & \\
\hline & ${ }^{137} \mathrm{Cs}$ & $6.1 \mathrm{E}-01 \pm 1.1 \mathrm{E}-01$ & & & ${ }^{137} \mathrm{Cs}$ & 6.6E-02 & $\pm 1.5 \mathrm{E}-02$ & \\
\hline & ${ }^{152} \mathrm{Eu}$ & $-1.4 \mathrm{E}-02 \pm 3.0 \mathrm{E}-02$ & $\mathrm{U}$ & & ${ }^{152} \mathrm{Eu}$ & $-2.4 \mathrm{E}-02$ & $\pm 2.4 \mathrm{E}-02$ & $\mathrm{U}$ \\
\hline & ${ }^{154} \mathrm{Eu}$ & $-2.1 \mathrm{E}-02 \pm 2.1 \mathrm{E}-02$ & $\mathrm{U}$ & & ${ }^{154} \mathrm{Eu}$ & $-9.6 \mathrm{E}-03$ & $\pm 2.0 \mathrm{E}-02$ & $\mathrm{U}$ \\
\hline & ${ }^{155} \mathrm{Eu}$ & $4.8 \mathrm{E}-02 \pm 4.0 \mathrm{E}-02$ & $\mathrm{U}$ & & ${ }^{155} \mathrm{Eu}$ & $3.4 \mathrm{E}-02$ & $\pm 2.8 \mathrm{E}-02$ & $\mathrm{U}$ \\
\hline & ${ }^{238} \mathrm{Pu}$ & $-6.0 \mathrm{E}-03 \pm 1.9 \mathrm{E}-02$ & $\mathrm{U}$ & & ${ }^{238} \mathrm{Pu}$ & 2.0E-03 & $\pm 1.6 \mathrm{E}-02$ & $\mathrm{U}$ \\
\hline & ${ }^{239,240} \mathrm{Pu}$ & $3.2 \mathrm{E}-02 \pm 1.9 \mathrm{E}-02$ & & & ${ }^{239,240} \mathrm{Pu}$ & $4.0 \mathrm{E}-03$ & $\pm 5.6 \mathrm{E}-03$ & $\mathrm{U}$ \\
\hline & ${ }^{103} \mathrm{Ru}$ & $-3.0 \mathrm{E}-03 \pm 7.6 \mathrm{E}-03$ & $\mathrm{U}$ & & ${ }^{103} \mathrm{Ru}$ & $3.6 \mathrm{E}-03$ & $\pm 6.7 \mathrm{E}-03$ & $\mathrm{U}$ \\
\hline & ${ }^{106} \mathrm{Ru}$ & $1.4 \mathrm{E}-02 \pm 6.0 \mathrm{E}-02$ & $\mathrm{U}$ & & ${ }^{106} \mathrm{Ru}$ & 2.3E-02 & $\pm 5.5 \mathrm{E}-02$ & $\mathrm{U}$ \\
\hline & ${ }^{125} \mathrm{Sb}$ & $1.2 \mathrm{E}-02 \pm 2.0 \mathrm{E}-02$ & $\mathrm{U}$ & & ${ }^{125} \mathrm{Sb}$ & $-4.7 E-03$ & $\pm 1.8 \mathrm{E}-02$ & $\mathrm{U}$ \\
\hline & ${ }^{113} \mathrm{Sn}$ & $1.9 \mathrm{E}-04 \pm 1.9 \mathrm{E}-03$ & $\mathrm{U}$ & & ${ }^{113} \mathrm{Sn}$ & $-4.1 \mathrm{E}-03$ & $\pm 8.4 \mathrm{E}-03$ & $\mathrm{U}$ \\
\hline & ${ }^{90} \mathrm{Sr}$ & $1.2 \mathrm{E}-02 \pm 1.2 \mathrm{E}-01$ & $\mathrm{U}$ & & ${ }^{90} \mathrm{Sr}$ & $-2.1 \mathrm{E}-02$ & $\pm 2.1 \mathrm{E}-01$ & $\mathrm{U}$ \\
\hline & ${ }^{234} \mathrm{U}$ & $1.8 \mathrm{E}-01 \pm 6.1 \mathrm{E}-02$ & & & ${ }^{234} \mathrm{U}$ & $1.2 \mathrm{E}-01$ & $\pm 4.3 \mathrm{E}-02$ & \\
\hline & ${ }^{235} \mathrm{U}$ & $2.2 \mathrm{E}-02 \pm 1.7 \mathrm{E}-02$ & & & ${ }^{235} \mathrm{U}$ & $1.1 \mathrm{E}-02$ & $\pm 1.2 \mathrm{E}-02$ & $\mathrm{U}$ \\
\hline & ${ }^{238} \mathrm{U}$ & $2.0 \mathrm{E}-01 \pm 6.6 \mathrm{E}-02$ & & & ${ }^{238} \mathrm{U}$ & $1.2 \mathrm{E}-01$ & $\pm 4.3 \mathrm{E}-02$ & \\
\hline & ${ }^{65} \mathrm{Zn}$ & $-4.0 \mathrm{E}-03 \pm 1.8 \mathrm{E}-02$ & $\mathrm{U}$ & & ${ }^{65} \mathrm{Zn}$ & $-1.5 \mathrm{E}-03$ & $\pm 1.5 \mathrm{E}-02$ & $\mathrm{U}$ \\
\hline
\end{tabular}

$\overline{\mathrm{RQ}}=$ Result Qualifier. U $=$ The analyte was analyzed for but not detected. 
Table 3-3. 2003 Soil Sampling Results (pCi/g \pm total analytical uncertainty). (cont)

\begin{tabular}{|c|c|c|c|c|c|c|c|c|}
\hline Location & Isotope & Result \pm Error & $\overline{\mathbf{R Q}^{*}}$ & Location & Isotope & Result & \pm Error & $\overline{\mathbf{R Q}^{*}}$ \\
\hline D083 & ${ }^{144} \mathrm{Ce}$ & $-1.1 \mathrm{E}-01 \pm 1.6 \mathrm{E}-01$ & $\overline{\mathrm{U}}$ & D085 & ${ }^{144} \mathrm{Ce}$ & $-5.0 \mathrm{E}-02$ & $\pm 1.6 \mathrm{E}-01$ & $\bar{U}$ \\
\hline \multirow[t]{17}{*}{ (600 Area) } & ${ }^{60} \mathrm{Co}$ & $6.6 \mathrm{E}-03 \pm 9.0 \mathrm{E}-03$ & $\mathrm{U}$ & (600 Area) & ${ }^{60} \mathrm{Co}$ & 1.6E-03 & $\pm 8.8 \mathrm{E}-03$ & U \\
\hline & ${ }^{134} \mathrm{Cs}$ & $4.7 \mathrm{E}-02 \pm 1.6 \mathrm{E}-02$ & & & ${ }^{134} \mathrm{Cs}$ & 4.5E-02 & $\pm 1.9 \mathrm{E}-02$ & \\
\hline & ${ }^{137} \mathrm{Cs}$ & $1.4 \mathrm{E}+00 \pm 2.3 \mathrm{E}-01$ & & & ${ }^{137} \mathrm{Cs}$ & 4.5E-01 & $\pm 7.8 \mathrm{E}-02$ & \\
\hline & ${ }^{152} \mathrm{Eu}$ & $-2.6 \mathrm{E}-02 \pm 3.6 \mathrm{E}-02$ & $\mathrm{U}$ & & ${ }^{152} \mathrm{Eu}$ & 3.6E-02 & $\pm 3.7 \mathrm{E}-02$ & $\mathrm{U}$ \\
\hline & ${ }^{154} \mathrm{Eu}$ & $-2.8 \mathrm{E}-02 \pm 3.1 \mathrm{E}-02$ & $\mathrm{U}$ & & ${ }^{154} \mathrm{Eu}$ & $-2.3 E-02$ & $\pm 3.0 \mathrm{E}-02$ & $\mathrm{U}$ \\
\hline & ${ }^{155} \mathrm{Eu}$ & $1.5 \mathrm{E}-02 \pm 3.8 \mathrm{E}-02$ & $\mathrm{U}$ & & ${ }^{155} \mathrm{Eu}$ & 1.9E-02 & $\pm 3.6 \mathrm{E}-02$ & $\mathrm{U}$ \\
\hline & ${ }^{238} \mathrm{Pu}$ & $1.9 \mathrm{E}-02 \pm 2.7 \mathrm{E}-02$ & $\mathrm{U}$ & & ${ }^{238} \mathrm{Pu}$ & 7.5E-03 & $\pm 1.9 \mathrm{E}-02$ & $\mathrm{U}$ \\
\hline & ${ }^{239,240} \mathrm{Pu}$ & $7.6 \mathrm{E}-02 \pm 3.6 \mathrm{E}-02$ & & & ${ }^{239,240} \mathrm{Pu}$ & $2.8 \mathrm{E}-02$ & $\pm 1.8 \mathrm{E}-02$ & \\
\hline & ${ }^{103} \mathrm{Ru}$ & $4.0 \mathrm{E}-04 \pm 4.1 \mathrm{E}-03$ & $\mathrm{U}$ & & ${ }^{103} \mathrm{Ru}$ & 2.6E-03 & $\pm 1.0 \mathrm{E}-02$ & U \\
\hline & ${ }^{106} \mathrm{Ru}$ & $-2.1 \mathrm{E}-02 \pm 8.5 \mathrm{E}-02$ & $\mathrm{U}$ & & ${ }^{106} \mathrm{Ru}$ & $-3.0 \mathrm{E}-03$ & $\pm 3.0 \mathrm{E}-02$ & $\mathrm{U}$ \\
\hline & ${ }^{125} \mathrm{Sb}$ & $-7.9 \mathrm{E}-03 \pm 2.8 \mathrm{E}-02$ & $\mathrm{U}$ & & ${ }^{125} \mathrm{Sb}$ & $-1.4 \mathrm{E}-02$ & $\pm 2.5 \mathrm{E}-02$ & $\mathrm{U}$ \\
\hline & ${ }^{113} \mathrm{Sn}$ & $3.2 \mathrm{E}-03 \pm 1.3 \mathrm{E}-02$ & $\mathrm{U}$ & & ${ }^{113} \mathrm{Sn}$ & $2.2 \mathrm{E}-03$ & $\pm 1.2 \mathrm{E}-02$ & $\mathrm{U}$ \\
\hline & ${ }^{90} \mathrm{Sr}$ & $-1.4 \mathrm{E}-01 \pm 1.9 \mathrm{E}-01$ & $\mathrm{U}$ & & ${ }^{90} \mathrm{Sr}$ & $-1.2 \mathrm{E}-01$ & $\pm 2.0 \mathrm{E}-01$ & $\mathrm{U}$ \\
\hline & ${ }^{234} \mathrm{U}$ & $1.5 \mathrm{E}-01 \pm 5.3 \mathrm{E}-02$ & & & & 1.3E-01 & $\pm 4.5 \mathrm{E}-02$ & \\
\hline & ${ }^{235} \mathrm{U}$ & $2.7 \mathrm{E}-02 \pm 2.0 \mathrm{E}-02$ & & & ${ }^{235} \mathrm{U}$ & 2.1E-02 & $\pm 1.6 \mathrm{E}-02$ & \\
\hline & ${ }^{238} \mathrm{U}$ & $1.2 \mathrm{E}-01 \pm 4.6 \mathrm{E}-02$ & & & ${ }^{238} \mathrm{U}$ & 1.7E-01 & $\pm 5.6 \mathrm{E}-02$ & \\
\hline & ${ }^{65} \mathrm{Zn}$ & $5.4 \mathrm{E}-02 \pm 2.7 \mathrm{E}-02$ & & & ${ }^{65} \mathrm{Zn}$ & 4.7E-02 & $\pm 2.7 \mathrm{E}-02$ & \\
\hline D087 & ${ }^{144} \mathrm{Ce}$ & $6.7 \mathrm{E}-02 \pm 1.9 \mathrm{E}-01$ & $\overline{\mathrm{U}}$ & D089 & ${ }^{144} \mathrm{Ce}$ & $-7.6 \mathrm{E}-02$ & $\pm 1.4 \mathrm{E}-01$ & $\bar{U}$ \\
\hline \multirow[t]{17}{*}{ (600 Area) } & ${ }^{60} \mathrm{Co}$ & $9.0 \mathrm{E}-03 \pm 8.8 \mathrm{E}-03$ & $\mathrm{U}$ & (600 Area) & ${ }^{60} \mathrm{Co}$ & $2.2 \mathrm{E}-03$ & $\pm 6.4 \mathrm{E}-03$ & $\mathrm{U}$ \\
\hline & ${ }^{134} \mathrm{Cs}$ & $3.1 \mathrm{E}-02 \pm 1.2 \mathrm{E}-02$ & & & ${ }^{134} \mathrm{Cs}$ & 4.1E-02 & $\pm 1.2 \mathrm{E}-02$ & \\
\hline & ${ }^{137} \mathrm{Cs}$ & $2.1 \mathrm{E}-02 \pm 1.1 \mathrm{E}-02$ & & & ${ }^{137} \mathrm{Cs}$ & 4.0E-01 & $\pm 6.5 \mathrm{E}-02$ & \\
\hline & ${ }^{152} \mathrm{Eu}$ & $-5.1 \mathrm{E}-03 \pm 5.0 \mathrm{E}-02$ & $\mathrm{U}$ & & ${ }^{152} \mathrm{Eu}$ & $-3.9 \mathrm{E}-02$ & $\pm 3.9 \mathrm{E}-02$ & $\mathrm{U}$ \\
\hline & ${ }^{154} \mathrm{Eu}$ & $-5.8 \mathrm{E}-03 \pm 2.9 \mathrm{E}-02$ & $\mathrm{U}$ & & ${ }^{154} \mathrm{Eu}$ & $-6.4 \mathrm{E}-03$ & $\pm 4.0 \mathrm{E}-02$ & U \\
\hline & ${ }^{155} \mathrm{Eu}$ & $2.7 \mathrm{E}-03 \pm 2.7 \mathrm{E}-02$ & $\mathrm{U}$ & & ${ }^{155} \mathrm{Eu}$ & 3.2E-02 & $\pm 3.6 \mathrm{E}-02$ & $\mathrm{U}$ \\
\hline & ${ }^{238} \mathrm{Pu}$ & $1.8 \mathrm{E}-03 \pm 1.8 \mathrm{E}-03$ & $\mathrm{U}$ & & ${ }^{238} \mathrm{Pu}$ & $-1.5 \mathrm{E}-02$ & $\pm 2.7 \mathrm{E}-02$ & $\mathrm{U}$ \\
\hline & ${ }^{239,240} \mathrm{Pu}$ & $1.8 \mathrm{E}-03 \pm 3.6 \mathrm{E}-03$ & $\mathrm{U}$ & & ${ }^{239,240} \mathrm{Pu}$ & $5.8 \mathrm{E}-03$ & $\pm 8.7 \mathrm{E}-03$ & U \\
\hline & ${ }^{103} \mathrm{Ru}$ & $-9.4 \mathrm{E}-03 \pm 9.9 \mathrm{E}-03$ & $\mathrm{U}$ & & ${ }^{103} \mathrm{Ru}$ & $-2.4 \mathrm{E}-03$ & $\pm 7.2 \mathrm{E}-03$ & $\mathrm{U}$ \\
\hline & ${ }^{106} \mathrm{Ru}$ & $2.0 \mathrm{E}-03 \pm 2.0 \mathrm{E}-02$ & $\mathrm{U}$ & & ${ }^{106} \mathrm{Ru}$ & $-3.3 E-02$ & $\pm 5.7 \mathrm{E}-02$ & $\mathrm{U}$ \\
\hline & ${ }^{125} \mathrm{Sb}$ & $9.4 \mathrm{E}-03 \pm 2.4 \mathrm{E}-02$ & $\mathrm{U}$ & & ${ }^{125} \mathrm{Sb}$ & 4.4E-03 & $\pm 1.9 \mathrm{E}-02$ & U \\
\hline & ${ }^{113} \mathrm{Sn}$ & $-4.8 \mathrm{E}-03 \pm 1.2 \mathrm{E}-02$ & $\mathrm{U}$ & & ${ }^{113} \mathrm{Sn}$ & $-2.7 \mathrm{E}-03$ & $\pm 9.2 \mathrm{E}-03$ & U \\
\hline & ${ }^{90} \mathrm{Sr}$ & $-8.9 \mathrm{E}-02 \pm 1.6 \mathrm{E}-01$ & $\mathrm{U}$ & & ${ }^{90} \mathrm{Sr}$ & $-1.7 \mathrm{E}-01$ & $\pm 1.7 \mathrm{E}-01$ & $\mathrm{U}$ \\
\hline & ${ }^{234} \mathrm{U}$ & $2.3 \mathrm{E}-01 \pm 7.4 \mathrm{E}-02$ & & & ${ }^{234} \mathrm{U}$ & $1.4 \mathrm{E}-01$ & $\pm 5.0 \mathrm{E}-02$ & \\
\hline & ${ }^{235} \mathrm{U}$ & $3.1 \mathrm{E}-02 \pm 1.8 \mathrm{E}-02$ & & & ${ }^{235} \mathrm{U}$ & 6.8E-03 & $\pm 8.2 \mathrm{E}-03$ & \\
\hline & ${ }^{238} \mathrm{U}$ & $1.6 \mathrm{E}-01 \pm 5.4 \mathrm{E}-02$ & & & ${ }^{238} \mathrm{U}$ & 1.4E-01 & $\pm 4.9 \mathrm{E}-02$ & \\
\hline & ${ }^{65} \mathrm{Zn}$ & $9.1 \mathrm{E}-03 \pm 2.2 \mathrm{E}-02$ & $\mathrm{U}$ & & ${ }^{65} \mathrm{Zn}$ & $1.2 \mathrm{E}-02$ & $\pm 1.6 \mathrm{E}-02$ & $\mathrm{U}$ \\
\hline
\end{tabular}

$\overline{\mathrm{RQ}}=$ Result Qualifier. U $=$ The analyte was analyzed for but not detected. 
Table 3-3. 2003 Soil Sampling Results (pCi/g \pm total analytical uncertainty). (cont)

\begin{tabular}{|c|c|c|c|c|c|c|c|c|}
\hline Location & Isotope & Result \pm Error & $\overline{\mathbf{R Q}^{*}}$ & Location & Isotope & Result & \pm Error & $\overline{\mathbf{R Q}^{*}}$ \\
\hline D091 & ${ }^{144} \mathrm{Ce}$ & $1.2 \mathrm{E}-01 \pm 1.5 \mathrm{E}-01$ & $\overline{\mathrm{U}}$ & D093 & ${ }^{144} \mathrm{Ce}$ & $-5.8 \mathrm{E}-02$ & $\pm 1.1 \mathrm{E}-01$ & $\bar{U}$ \\
\hline \multirow[t]{17}{*}{ (600 Area) } & ${ }^{60} \mathrm{Co}$ & $-1.1 \mathrm{E}-03 \pm 7.1 \mathrm{E}-03$ & $\mathrm{U}$ & (600 Area) & ${ }^{60} \mathrm{Co}$ & 2.0E-03 & $\pm 5.5 \mathrm{E}-03$ & U \\
\hline & ${ }^{134} \mathrm{Cs}$ & $4.2 \mathrm{E}-02 \pm 1.2 \mathrm{E}-02$ & & & ${ }^{134} \mathrm{Cs}$ & 2.9E-02 & $\pm 1.2 \mathrm{E}-02$ & \\
\hline & ${ }^{137} \mathrm{Cs}$ & $2.0 \mathrm{E}+00 \pm 3.5 \mathrm{E}-01$ & & & ${ }^{137} \mathrm{Cs}$ & $1.1 \mathrm{E}+00$ & $\pm 1.5 \mathrm{E}-01$ & \\
\hline & ${ }^{152} \mathrm{Eu}$ & $-2.6 \mathrm{E}-02 \pm 3.3 \mathrm{E}-02$ & $\mathrm{U}$ & & ${ }^{152} \mathrm{Eu}$ & $-7.9 \mathrm{E}-03$ & $\pm 2.5 \mathrm{E}-02$ & $\mathrm{U}$ \\
\hline & ${ }^{154} \mathrm{Eu}$ & $-5.4 \mathrm{E}-03 \pm 2.3 \mathrm{E}-02$ & $\mathrm{U}$ & & ${ }^{154} \mathrm{Eu}$ & 4.9E-03 & $\pm 3.2 \mathrm{E}-02$ & $\mathrm{U}$ \\
\hline & ${ }^{155} \mathrm{Eu}$ & $2.1 \mathrm{E}-02 \pm 3.5 \mathrm{E}-02$ & $\mathrm{U}$ & & ${ }^{155} \mathrm{Eu}$ & 2.6E-02 & $\pm 3.0 \mathrm{E}-02$ & $\mathrm{U}$ \\
\hline & ${ }^{238} \mathrm{Pu}$ & $1.4 \mathrm{E}-02 \pm 2.0 \mathrm{E}-02$ & $\mathrm{U}$ & & ${ }^{238} \mathrm{Pu}$ & $-5.3 E-03$ & $\pm 1.5 \mathrm{E}-02$ & U \\
\hline & ${ }^{239,240} \mathrm{Pu}$ & $3.2 \mathrm{E}-03 \pm 4.5 \mathrm{E}-03$ & $\mathrm{U}$ & & ${ }^{239,240} \mathrm{Pu}$ & 4.2E-02 & $\pm 2.0 \mathrm{E}-02$ & \\
\hline & ${ }^{103} \mathrm{Ru}$ & $8.5 \mathrm{E}-03 \pm 9.6 \mathrm{E}-03$ & $\mathrm{U}$ & & ${ }^{103} \mathrm{Ru}$ & $-7.6 \mathrm{E}-03$ & $\pm 7.6 \mathrm{E}-03$ & $\mathrm{U}$ \\
\hline & ${ }^{106} \mathrm{Ru}$ & $2.3 \mathrm{E}-02 \pm 7.0 \mathrm{E}-02$ & $\mathrm{U}$ & & ${ }^{106} \mathrm{Ru}$ & $1.6 \mathrm{E}-02$ & $\pm 5.3 \mathrm{E}-02$ & $\mathrm{U}$ \\
\hline & ${ }^{125} \mathrm{Sb}$ & $-8.8 \mathrm{E}-03 \pm 2.4 \mathrm{E}-02$ & $\mathrm{U}$ & & ${ }^{125} \mathrm{Sb}$ & $-5.7 \mathrm{E}-03$ & $\pm 1.9 \mathrm{E}-02$ & $\mathrm{U}$ \\
\hline & ${ }^{113} \mathrm{Sn}$ & $-9.1 \mathrm{E}-04 \pm 9.1 \mathrm{E}-03$ & $\mathrm{U}$ & & ${ }^{113} \mathrm{Sn}$ & $-5.1 \mathrm{E}-03$ & $\pm 9.0 \mathrm{E}-03$ & $\mathrm{U}$ \\
\hline & ${ }^{90} \mathrm{Sr}$ & $-9.1 \mathrm{E}-03 \pm 9.1 \mathrm{E}-02$ & $\mathrm{U}$ & & ${ }^{90} \mathrm{Sr}$ & 2.5E-01 & $\pm 2.2 \mathrm{E}-01$ & \\
\hline & ${ }^{234} \mathrm{U}$ & $1.7 \mathrm{E}-01 \pm 5.4 \mathrm{E}-02$ & & & ${ }^{234} \mathrm{U}$ & $1.8 \mathrm{E}-01$ & $\pm 6.1 \mathrm{E}-02$ & \\
\hline & ${ }^{235} \mathrm{U}$ & $3.7 \mathrm{E}-03 \pm 7.4 \mathrm{E}-03$ & $\mathrm{U}$ & & ${ }^{235} \mathrm{U}$ & $9.6 \mathrm{E}-03$ & $\pm 1.2 \mathrm{E}-02$ & $\mathrm{U}$ \\
\hline & ${ }^{238} \mathrm{U}$ & $2.0 \mathrm{E}-01 \pm 6.4 \mathrm{E}-02$ & & & ${ }^{238} \mathrm{U}$ & $1.9 \mathrm{E}-01$ & $\pm 6.5 \mathrm{E}-02$ & \\
\hline & ${ }^{65} \mathrm{Zn}$ & $3.0 \mathrm{E}-03 \pm 1.9 \mathrm{E}-02$ & $\mathrm{U}$ & & ${ }^{65} \mathrm{Zn}$ & $-6.7 \mathrm{E}-03$ & $\pm 1.5 \mathrm{E}-02$ & $\mathrm{U}$ \\
\hline D095 & ${ }^{144} \mathrm{Ce}$ & $1.6 \mathrm{E}-02 \pm 1.2 \mathrm{E}-01$ & $\overline{\mathrm{U}}$ & D097 & ${ }^{144} \mathrm{Ce}$ & $7.5 \mathrm{E}-03$ & $\pm 7.5 \mathrm{E}-02$ & $\bar{U}$ \\
\hline \multirow[t]{17}{*}{ (600 Area) } & ${ }^{60} \mathrm{Co}$ & $-1.4 \mathrm{E}-04 \pm 1.4 \mathrm{E}-03$ & $\mathrm{U}$ & (600 Area) & ${ }^{60} \mathrm{Co}$ & $-3.0 \mathrm{E}-03$ & $\pm 7.4 \mathrm{E}-03$ & $\mathrm{U}$ \\
\hline & ${ }^{134} \mathrm{Cs}$ & $3.1 \mathrm{E}-02 \pm 9.0 \mathrm{E}-03$ & & & ${ }^{134} \mathrm{Cs}$ & 4.6E-02 & $\pm 1.4 \mathrm{E}-02$ & \\
\hline & ${ }^{137} \mathrm{Cs}$ & $5.7 \mathrm{E}-01 \pm 9.1 \mathrm{E}-02$ & & & ${ }^{137} \mathrm{Cs}$ & 8.6E-02 & $\pm 1.9 \mathrm{E}-02$ & \\
\hline & ${ }^{152} \mathrm{Eu}$ & $-1.4 \mathrm{E}-02 \pm 2.1 \mathrm{E}-02$ & $\mathrm{U}$ & & ${ }^{152} \mathrm{Eu}$ & $-2.3 \mathrm{E}-02$ & $\pm 2.9 \mathrm{E}-02$ & $\mathrm{U}$ \\
\hline & ${ }^{154} \mathrm{Eu}$ & $1.5 \mathrm{E}-02 \pm 1.6 \mathrm{E}-02$ & $\mathrm{U}$ & & ${ }^{154} \mathrm{Eu}$ & $-1.9 \mathrm{E}-02$ & $\pm 2.8 \mathrm{E}-02$ & U \\
\hline & ${ }^{155} \mathrm{Eu}$ & $5.4 \mathrm{E}-02 \pm 3.6 \mathrm{E}-02$ & $\mathrm{U}$ & & ${ }^{155} \mathrm{Eu}$ & 3.6E-02 & $\pm 3.8 \mathrm{E}-02$ & $\mathrm{U}$ \\
\hline & ${ }^{238} \mathrm{Pu}$ & $-2.0 \mathrm{E}-03 \pm 2.0 \mathrm{E}-02$ & $\mathrm{U}$ & & ${ }^{238} \mathrm{Pu}$ & 1.7E-03 & $\pm 1.7 \mathrm{E}-02$ & $\mathrm{U}$ \\
\hline & ${ }^{239,240} \mathrm{Pu}$ & $1.2 \mathrm{E}-02 \pm 1.2 \mathrm{E}-02$ & $\mathrm{U}$ & & ${ }^{239,240} \mathrm{Pu}$ & 8.5E-03 & $\pm 7.9 \mathrm{E}-03$ & \\
\hline & ${ }^{103} \mathrm{Ru}$ & $-3.9 \mathrm{E}-03 \pm 6.7 \mathrm{E}-03$ & $\mathrm{U}$ & & ${ }^{103} \mathrm{Ru}$ & $-1.7 \mathrm{E}-03$ & $\pm 8.3 \mathrm{E}-03$ & $\mathrm{U}$ \\
\hline & ${ }^{106} \mathrm{Ru}$ & $2.0 \mathrm{E}-02 \pm 4.8 \mathrm{E}-02$ & $\mathrm{U}$ & & ${ }^{106} \mathrm{Ru}$ & 3.8E-02 & $\pm 6.5 \mathrm{E}-02$ & $\mathrm{U}$ \\
\hline & ${ }^{125} \mathrm{Sb}$ & $-2.7 \mathrm{E}-03 \pm 1.7 \mathrm{E}-02$ & $\mathrm{U}$ & & ${ }^{125} \mathrm{Sb}$ & 6.5E-03 & $\pm 1.9 \mathrm{E}-02$ & U \\
\hline & ${ }^{113} \mathrm{Sn}$ & $-8.2 \mathrm{E}-04 \pm 8.2 \mathrm{E}-03$ & $\mathrm{U}$ & & ${ }^{113} \mathrm{Sn}$ & $-7.1 \mathrm{E}-03$ & $\pm 9.7 \mathrm{E}-03$ & $\mathrm{U}$ \\
\hline & ${ }^{90} \mathrm{Sr}$ & $1.1 \mathrm{E}-01 \pm 1.6 \mathrm{E}-01$ & $\mathrm{U}$ & & ${ }^{90} \mathrm{Sr}$ & $-9.3 E-02$ & $\pm 2.0 \mathrm{E}-01$ & $\mathrm{U}$ \\
\hline & ${ }^{234} \mathrm{U}$ & $2.0 \mathrm{E}-01 \pm 6.6 \mathrm{E}-02$ & & & ${ }^{234} \mathrm{U}$ & $1.8 \mathrm{E}-01$ & $\pm 6.1 \mathrm{E}-02$ & \\
\hline & ${ }^{235} \mathrm{U}$ & $1.8 \mathrm{E}-02 \pm 1.4 \mathrm{E}-02$ & & & ${ }^{235} \mathrm{U}$ & $9.2 \mathrm{E}-03$ & $\pm 9.2 \mathrm{E}-03$ & \\
\hline & ${ }^{238} \mathrm{U}$ & $1.7 \mathrm{E}-01 \pm 5.9 \mathrm{E}-02$ & & & ${ }^{238} \mathrm{U}$ & 1.4E-01 & $\pm 5.0 \mathrm{E}-02$ & \\
\hline & ${ }^{65} \mathrm{Zn}$ & $-7.2 \mathrm{E}-04 \pm 7.2 \mathrm{E}-03$ & $\mathrm{U}$ & & ${ }^{65} \mathrm{Zn}$ & 2.0E-02 & $\pm 2.0 \mathrm{E}-02$ & $\mathrm{U}$ \\
\hline
\end{tabular}

$\overline{\mathrm{RQ}}=$ Result Qualifier. U $=$ The analyte was analyzed for but not detected. 
Table 3-3. 2003 Soil Sampling Results (pCi/g \pm total analytical uncertainty). (cont)

\begin{tabular}{|c|c|c|c|c|c|c|c|c|}
\hline Location & Isotope & Result \pm Error & $\mathbf{R Q}^{*}$ & Location & Isotope & Result & \pm Error & $\mathbf{R Q}^{*}$ \\
\hline D099 & ${ }^{144} \mathrm{Ce}$ & $-8.7 \mathrm{E}-02 \pm 1.4 \mathrm{E}-01$ & $\overline{\mathrm{U}}$ & D101 & ${ }^{144} \mathrm{Ce}$ & $1.4 \mathrm{E}-02$ & $\pm 1.2 \mathrm{E}-01$ & $\mathrm{U}$ \\
\hline \multirow[t]{17}{*}{ (600 Area) } & ${ }^{60} \mathrm{Co}$ & $1.2 \mathrm{E}-03 \pm 8.0 \mathrm{E}-03$ & $\mathrm{U}$ & (600 Area) & ${ }^{60} \mathrm{Co}$ & $-1.4 \mathrm{E}-03$ & $\pm 7.0 \mathrm{E}-03$ & $\mathrm{U}$ \\
\hline & ${ }^{134} \mathrm{Cs}$ & $1.3 \mathrm{E}-02 \pm 1.1 \mathrm{E}-02$ & $\mathrm{U}$ & & ${ }^{134} \mathrm{Cs}$ & 3.4E-02 & $\pm 1.1 \mathrm{E}-02$ & \\
\hline & ${ }^{137} \mathrm{Cs}$ & $3.4 \mathrm{E}-01 \pm 5.9 \mathrm{E}-02$ & & & ${ }^{137} \mathrm{Cs}$ & 2.5E-01 & $\pm 4.4 \mathrm{E}-02$ & \\
\hline & ${ }^{152} \mathrm{Eu}$ & $-3.8 \mathrm{E}-02 \pm 3.8 \mathrm{E}-02$ & $\mathrm{U}$ & & ${ }^{152} \mathrm{Eu}$ & $-1.7 \mathrm{E}-02$ & $\pm 2.0 \mathrm{E}-02$ & $\mathrm{U}$ \\
\hline & ${ }^{154} \mathrm{Eu}$ & $-2.4 \mathrm{E}-02 \pm 2.7 \mathrm{E}-02$ & $\mathrm{U}$ & & ${ }^{154} \mathrm{Eu}$ & $3.6 \mathrm{E}-02$ & $\pm 3.0 \mathrm{E}-02$ & U \\
\hline & ${ }^{155} \mathrm{Eu}$ & $6.1 \mathrm{E}-02 \pm 3.8 \mathrm{E}-02$ & $\mathrm{U}$ & & ${ }^{155} \mathrm{Eu}$ & 6.1E-02 & $\pm 4.1 \mathrm{E}-02$ & U \\
\hline & ${ }^{238} \mathrm{Pu}$ & $1.8 \mathrm{E}-03 \pm 1.5 \mathrm{E}-02$ & $\mathrm{U}$ & & ${ }^{238} \mathrm{Pu}$ & 7.8E-03 & $\pm 8.6 \mathrm{E}-03$ & $\mathrm{U}$ \\
\hline & ${ }^{239,240} \mathrm{Pu}$ & $1.1 \mathrm{E}-02 \pm 1.2 \mathrm{E}-02$ & U & & ${ }^{239,240} \mathrm{Pu}$ & $2.0 \mathrm{E}-02$ & $\pm 1.2 \mathrm{E}-02$ & \\
\hline & ${ }^{103} \mathrm{Ru}$ & $4.2 \mathrm{E}-03 \pm 8.9 \mathrm{E}-03$ & $\mathrm{U}$ & & ${ }^{103} \mathrm{Ru}$ & $5.2 \mathrm{E}-03$ & $\pm 7.8 \mathrm{E}-03$ & $\mathrm{U}$ \\
\hline & ${ }^{106} \mathrm{Ru}$ & $-1.9 \mathrm{E}-02 \pm 6.9 \mathrm{E}-02$ & $\mathrm{U}$ & & ${ }^{106} \mathrm{Ru}$ & $-2.1 \mathrm{E}-03$ & $\pm 2.1 \mathrm{E}-02$ & $\mathrm{U}$ \\
\hline & ${ }^{125} \mathrm{Sb}$ & $8.6 \mathrm{E}-03 \pm 2.2 \mathrm{E}-02$ & U & & ${ }^{125} \mathrm{Sb}$ & $-3.4 \mathrm{E}-03$ & $\pm 1.8 \mathrm{E}-02$ & $\mathrm{U}$ \\
\hline & ${ }^{113} \mathrm{Sn}$ & $-2.7 \mathrm{E}-03 \pm 1.1 \mathrm{E}-02$ & $\mathrm{U}$ & & ${ }^{113} \mathrm{Sn}$ & $6.9 \mathrm{E}-03$ & $\pm 9.2 \mathrm{E}-03$ & $\mathrm{U}$ \\
\hline & ${ }^{90} \mathrm{Sr}$ & $2.8 \mathrm{E}-01 \pm 2.2 \mathrm{E}-01$ & & & ${ }^{90} \mathrm{Sr}$ & 2.7E-01 & $\pm 2.2 \mathrm{E}-01$ & $\mathrm{U}$ \\
\hline & ${ }^{234} \mathrm{U}$ & $2.4 \mathrm{E}-01 \pm 7.7 \mathrm{E}-02$ & & & ${ }^{234} \mathrm{U}$ & $1.1 \mathrm{E}-01$ & $\pm 4.0 \mathrm{E}-02$ & \\
\hline & ${ }^{235} \mathrm{U}$ & $5.6 \mathrm{E}-02 \pm 2.7 \mathrm{E}-02$ & & & ${ }^{235} \mathrm{U}$ & 2.1E-02 & $\pm 1.4 \mathrm{E}-02$ & \\
\hline & ${ }^{238} \mathrm{U}$ & 1.9E-01 $\pm 6.5 \mathrm{E}-02$ & & & ${ }^{238} \mathrm{U}$ & $1.5 \mathrm{E}-01$ & $\pm 5.1 \mathrm{E}-02$ & \\
\hline & ${ }^{65} \mathrm{Zn}$ & $5.2 \mathrm{E}-02 \pm 2.5 \mathrm{E}-02$ & & & ${ }^{65} \mathrm{Zn}$ & 4.2E-02 & $\pm 1.9 \mathrm{E}-02$ & \\
\hline D103 & ${ }^{144} \mathrm{Ce}$ & $-3.6 \mathrm{E}-02 \pm 1.6 \mathrm{E}-01$ & $\overline{\mathrm{U}}$ & D105 & ${ }^{144} \mathrm{Ce}$ & $-9.4 \mathrm{E}-02$ & $\pm 1.3 \mathrm{E}-01$ & $\overline{\mathrm{U}}$ \\
\hline \multirow[t]{17}{*}{ (600 Area) } & ${ }^{60} \mathrm{Co}$ & $-6.8 \mathrm{E}-03 \pm 6.8 \mathrm{E}-03$ & $\mathrm{U}$ & (600 Area) & ${ }^{60} \mathrm{Co}$ & 3.1E-04 & $\pm 3.1 \mathrm{E}-03$ & U \\
\hline & ${ }^{134} \mathrm{Cs}$ & $3.0 \mathrm{E}-02 \pm 1.1 \mathrm{E}-02$ & & & ${ }^{134} \mathrm{Cs}$ & 4.2E-02 & $\pm 1.5 \mathrm{E}-02$ & \\
\hline & ${ }^{137} \mathrm{Cs}$ & $9.3 \mathrm{E}-01 \pm 1.6 \mathrm{E}-01$ & & & ${ }^{137} \mathrm{Cs}$ & $2.0 \mathrm{E}-01$ & $\pm 3.8 \mathrm{E}-02$ & \\
\hline & ${ }^{152} \mathrm{Eu}$ & $1.4 \mathrm{E}-02 \pm 4.8 \mathrm{E}-02$ & U & & ${ }^{152} \mathrm{Eu}$ & $-2.3 \mathrm{E}-02$ & $\pm 4.3 \mathrm{E}-02$ & U \\
\hline & ${ }^{154} \mathrm{Eu}$ & $4.2 \mathrm{E}-03 \pm 2.6 \mathrm{E}-02$ & $\mathrm{U}$ & & ${ }^{154} \mathrm{Eu}$ & $-8.4 \mathrm{E}-03$ & $\pm 3.4 \mathrm{E}-02$ & U \\
\hline & ${ }^{155} \mathrm{Eu}$ & $3.8 \mathrm{E}-02 \pm 4.2 \mathrm{E}-02$ & $\mathrm{U}$ & & ${ }^{155} \mathrm{Eu}$ & 1.3E-02 & $\pm 3.4 \mathrm{E}-02$ & $\mathrm{U}$ \\
\hline & ${ }^{238} \mathrm{Pu}$ & $3.1 \mathrm{E}-03 \pm 6.2 \mathrm{E}-03$ & $\mathrm{U}$ & & ${ }^{238} \mathrm{Pu}$ & $1.4 \mathrm{E}-02$ & $\pm 3.8 \mathrm{E}-02$ & $\mathrm{U}$ \\
\hline & ${ }^{239,240} \mathrm{Pu}$ & $9.9 \mathrm{E}-02 \pm 3.4 \mathrm{E}-02$ & & & ${ }^{239,240} \mathrm{Pu}$ & 2.2E-01 & $\pm 7.0 \mathrm{E}-02$ & \\
\hline & ${ }^{103} \mathrm{Ru}$ & $-1.6 \mathrm{E}-03 \pm 9.9 \mathrm{E}-03$ & $\mathrm{U}$ & & ${ }^{103} \mathrm{Ru}$ & $-2.5 \mathrm{E}-03$ & $\pm 9.7 \mathrm{E}-03$ & $\mathrm{U}$ \\
\hline & ${ }^{106} \mathrm{Ru}$ & $2.5 \mathrm{E}-02 \pm 6.9 \mathrm{E}-02$ & $\mathrm{U}$ & & ${ }^{106} \mathrm{Ru}$ & $5.4 \mathrm{E}-02$ & $\pm 7.6 \mathrm{E}-02$ & $\mathrm{U}$ \\
\hline & ${ }^{125} \mathrm{Sb}$ & $1.5 \mathrm{E}-02 \pm 2.4 \mathrm{E}-02$ & $\mathrm{U}$ & & ${ }^{125} \mathrm{Sb}$ & 3.1E-03 & $\pm 2.3 \mathrm{E}-02$ & U \\
\hline & ${ }^{113} \mathrm{Sn}$ & $-7.3 \mathrm{E}-03 \pm 1.2 \mathrm{E}-02$ & $\mathrm{U}$ & & ${ }^{113} \mathrm{Sn}$ & 4.1E-03 & $\pm 1.3 \mathrm{E}-02$ & $\mathrm{U}$ \\
\hline & ${ }^{90} \mathrm{Sr}$ & $-2.0 \mathrm{E}-02 \pm 1.8 \mathrm{E}-01$ & $\mathrm{U}$ & & ${ }^{90} \mathrm{Sr}$ & $-2.6 \mathrm{E}-01$ & $\pm 2.6 \mathrm{E}-01$ & $\mathrm{U}$ \\
\hline & ${ }^{234} \mathrm{U}$ & $1.5 \mathrm{E}-01 \pm 5.1 \mathrm{E}-02$ & & & ${ }^{234} \mathrm{U}$ & 9.3E-02 & $\pm 3.6 \mathrm{E}-02$ & \\
\hline & ${ }^{235} \mathrm{U}$ & 7.3E-03 \pm 8.8E-03 & $\mathrm{U}$ & & ${ }^{235} \mathrm{U}$ & $1.1 \mathrm{E}-02$ & $\pm 1.0 \mathrm{E}-02$ & \\
\hline & ${ }^{238} \mathrm{U}$ & $1.4 \mathrm{E}-01 \pm 4.6 \mathrm{E}-02$ & & & ${ }^{238} \mathrm{U}$ & $1.5 \mathrm{E}-01$ & $\pm 5.1 \mathrm{E}-02$ & \\
\hline & ${ }^{65} \mathrm{Zn}$ & $2.8 \mathrm{E}-02 \pm 1.9 \mathrm{E}-02$ & U & & ${ }^{65} \mathrm{Zn}$ & 8.6E-02 & $\pm 4.0 \mathrm{E}-02$ & \\
\hline
\end{tabular}

$\overline{\mathrm{RQ}}=$ Result Qualifier. U $=$ The analyte was analyzed for but not detected. 
Table 3-3. 2003 Soil Sampling Results (pCi/g \pm total analytical uncertainty). (cont)

\begin{tabular}{|c|c|c|c|c|c|c|c|c|}
\hline Location & Isotope & Result \pm Error & $\overline{\mathbf{R Q}^{*}}$ & Location & Isotope & Result & \pm Error & $\overline{\mathbf{R Q}^{*}}$ \\
\hline D107 & ${ }^{144} \mathrm{Ce}$ & $3.1 \mathrm{E}-02 \pm 1.0 \mathrm{E}-01$ & $\overline{\mathrm{U}}$ & D109 & ${ }^{144} \mathrm{Ce}$ & $2.5 \mathrm{E}-02$ & $\pm 1.7 \mathrm{E}-01$ & $\bar{U}$ \\
\hline \multirow[t]{17}{*}{ (600 Area) } & ${ }^{60} \mathrm{Co}$ & $2.0 \mathrm{E}-03 \pm 6.9 \mathrm{E}-03$ & $\mathrm{U}$ & (600 Area) & ${ }^{60} \mathrm{Co}$ & $9.6 \mathrm{E}-04$ & $\pm 6.7 \mathrm{E}-03$ & U \\
\hline & ${ }^{134} \mathrm{Cs}$ & $2.6 \mathrm{E}-02 \pm 8.8 \mathrm{E}-03$ & & & ${ }^{134} \mathrm{Cs}$ & 4.1E-02 & $\pm 1.5 \mathrm{E}-02$ & \\
\hline & ${ }^{137} \mathrm{Cs}$ & $8.6 \mathrm{E}-02 \pm 1.8 \mathrm{E}-02$ & & & ${ }^{137} \mathrm{Cs}$ & 6.7E-01 & $\pm 1.2 \mathrm{E}-01$ & \\
\hline & ${ }^{152} \mathrm{Eu}$ & $-2.9 \mathrm{E}-02 \pm 2.9 \mathrm{E}-02$ & $\mathrm{U}$ & & ${ }^{152} \mathrm{Eu}$ & $-9.7 E-05$ & $\pm 9.7 \mathrm{E}-04$ & $\mathrm{U}$ \\
\hline & ${ }^{154} \mathrm{Eu}$ & $-7.1 \mathrm{E}-04 \pm 7.1 \mathrm{E}-03$ & $\mathrm{U}$ & & ${ }^{154} \mathrm{Eu}$ & $-1.0 \mathrm{E}-02$ & $\pm 2.2 \mathrm{E}-02$ & $\mathrm{U}$ \\
\hline & ${ }^{155} \mathrm{Eu}$ & $6.4 \mathrm{E}-02 \pm 3.9 \mathrm{E}-02$ & $\mathrm{U}$ & & ${ }^{155} \mathrm{Eu}$ & $1.8 \mathrm{E}-02$ & $\pm 4.1 \mathrm{E}-02$ & $\mathrm{U}$ \\
\hline & ${ }^{238} \mathrm{Pu}$ & $1.8 \mathrm{E}-03 \pm 1.8 \mathrm{E}-02$ & $\mathrm{U}$ & & ${ }^{238} \mathrm{Pu}$ & 3.3E-02 & $\pm 3.3 \mathrm{E}-02$ & $\mathrm{U}$ \\
\hline & ${ }^{239,240} \mathrm{Pu}$ & $1.2 \mathrm{E}-01 \pm 4.3 \mathrm{E}-02$ & & & ${ }^{239,240} \mathrm{Pu}$ & $1.8 \mathrm{E}+00$ & $\pm 4.7 \mathrm{E}-01$ & \\
\hline & ${ }^{103} \mathrm{Ru}$ & $-2.2 \mathrm{E}-03 \pm 7.4 \mathrm{E}-03$ & $\mathrm{U}$ & & ${ }^{103} \mathrm{Ru}$ & $-5.7 E-03$ & $\pm 1.0 \mathrm{E}-02$ & U \\
\hline & ${ }^{106} \mathrm{Ru}$ & $1.2 \mathrm{E}-02 \pm 6.0 \mathrm{E}-02$ & $\mathrm{U}$ & & ${ }^{106} \mathrm{Ru}$ & $3.0 \mathrm{E}-02$ & $\pm 6.8 \mathrm{E}-02$ & $\mathrm{U}$ \\
\hline & ${ }^{125} \mathrm{Sb}$ & $6.3 \mathrm{E}-03 \pm 1.7 \mathrm{E}-02$ & $\mathrm{U}$ & & ${ }^{125} \mathrm{Sb}$ & $1.1 \mathrm{E}-03$ & $\pm 1.1 \mathrm{E}-02$ & U \\
\hline & ${ }^{113} \mathrm{Sn}$ & $-5.2 \mathrm{E}-03 \pm 8.8 \mathrm{E}-03$ & $\mathrm{U}$ & & ${ }^{113} \mathrm{Sn}$ & $-6.2 \mathrm{E}-03$ & $\pm 1.2 \mathrm{E}-02$ & $\mathrm{U}$ \\
\hline & ${ }^{90} \mathrm{Sr}$ & $2.8 \mathrm{E}-03 \pm 2.8 \mathrm{E}-02$ & $\mathrm{U}$ & & ${ }^{90} \mathrm{Sr}$ & $1.4 \mathrm{E}-01$ & $\pm 2.0 \mathrm{E}-01$ & $\mathrm{U}$ \\
\hline & ${ }^{234} \mathrm{U}$ & $1.3 \mathrm{E}-01 \pm 4.4 \mathrm{E}-02$ & & & ${ }^{234} \mathrm{U}$ & $1.4 \mathrm{E}-01$ & $\pm 4.8 \mathrm{E}-02$ & \\
\hline & ${ }^{235} \mathrm{U}$ & $1.8 \mathrm{E}-02 \pm 1.2 \mathrm{E}-02$ & & & ${ }^{235} \mathrm{U}$ & $1.1 \mathrm{E}-02$ & $\pm 9.5 \mathrm{E}-03$ & \\
\hline & ${ }^{238} \mathrm{U}$ & $1.6 \mathrm{E}-01 \pm 5.1 \mathrm{E}-02$ & & & ${ }^{238} \mathrm{U}$ & $1.3 \mathrm{E}-01$ & $\pm 4.5 \mathrm{E}-02$ & \\
\hline & ${ }^{65} \mathrm{Zn}$ & $2.1 \mathrm{E}-02 \pm 1.8 \mathrm{E}-02$ & $\mathrm{U}$ & & ${ }^{65} \mathrm{Zn}$ & $3.2 \mathrm{E}-02$ & $\pm 2.0 \mathrm{E}-02$ & \\
\hline \multirow{18}{*}{$\begin{array}{c}\text { D111 } \\
\text { (Duplicate } \\
\text { of D051, } \\
\text { 200 West) }\end{array}$} & ${ }^{144} \mathrm{Ce}$ & $3.2 \mathrm{E}-03 \pm 3.2 \mathrm{E}-02$ & $\overline{\mathrm{U}}$ & D113 & ${ }^{144} \mathrm{Ce}$ & $1.4 \mathrm{E}-02$ & $\pm 1.3 \mathrm{E}-01$ & $\bar{U}$ \\
\hline & ${ }^{60} \mathrm{Co}$ & $-1.2 \mathrm{E}-03 \pm 7.7 \mathrm{E}-03$ & $\mathrm{U}$ & (Duplicate & ${ }^{60} \mathrm{Co}$ & $-1.0 \mathrm{E}-03$ & $\pm 5.7 \mathrm{E}-03$ & $\mathrm{U}$ \\
\hline & ${ }^{134} \mathrm{Cs}$ & $5.3 \mathrm{E}-02 \pm 1.7 \mathrm{E}-02$ & & of D083, & ${ }^{134} \mathrm{Cs}$ & $3.2 \mathrm{E}-02$ & $\pm 1.1 \mathrm{E}-02$ & \\
\hline & ${ }^{137} \mathrm{Cs}$ & $2.0 \mathrm{E}-01 \pm 4.0 \mathrm{E}-02$ & & 600 Area) & ${ }^{137} \mathrm{Cs}$ & $3.1 \mathrm{E}-01$ & $\pm 5.1 \mathrm{E}-02$ & \\
\hline & ${ }^{152} \mathrm{Eu}$ & $9.1 \mathrm{E}-03 \pm 3.0 \mathrm{E}-02$ & $\mathrm{U}$ & & ${ }^{152} \mathrm{Eu}$ & $-7.2 \mathrm{E}-03$ & $\pm 2.1 \mathrm{E}-02$ & $\mathrm{U}$ \\
\hline & ${ }^{154} \mathrm{Eu}$ & $-3.3 \mathrm{E}-03 \pm 2.9 \mathrm{E}-02$ & $\mathrm{U}$ & & ${ }^{154} \mathrm{Eu}$ & $-4.7 \mathrm{E}-03$ & $\pm 2.1 \mathrm{E}-02$ & U \\
\hline & ${ }^{155} \mathrm{Eu}$ & $3.3 \mathrm{E}-02 \pm 3.9 \mathrm{E}-02$ & $\mathrm{U}$ & & ${ }^{155} \mathrm{Eu}$ & $2.2 \mathrm{E}-02$ & $\pm 2.7 \mathrm{E}-02$ & $\mathrm{U}$ \\
\hline & ${ }^{238} \mathrm{Pu}$ & $-8.6 \mathrm{E}-03 \pm 2.7 \mathrm{E}-02$ & $\mathrm{U}$ & & ${ }^{238} \mathrm{Pu}$ & $-1.8 \mathrm{E}-03$ & $\pm 1.2 \mathrm{E}-02$ & $\mathrm{U}$ \\
\hline & ${ }^{239,240} \mathrm{Pu}$ & $1.9 \mathrm{E}-02 \pm 1.5 \mathrm{E}-02$ & & & ${ }^{239,240} \mathrm{Pu}$ & 2.3E-02 & $\pm 1.6 \mathrm{E}-02$ & \\
\hline & ${ }^{103} \mathrm{Ru}$ & $-3.7 \mathrm{E}-03 \pm 9.1 \mathrm{E}-03$ & $\mathrm{U}$ & & ${ }^{103} \mathrm{Ru}$ & $-1.3 \mathrm{E}-03$ & $\pm 6.8 \mathrm{E}-03$ & $\mathrm{U}$ \\
\hline & ${ }^{106} \mathrm{Ru}$ & $-1.2 \mathrm{E}-02 \pm 6.8 \mathrm{E}-02$ & $\mathrm{U}$ & & ${ }^{106} \mathrm{Ru}$ & $-3.4 \mathrm{E}-02$ & $\pm 4.9 \mathrm{E}-02$ & $\mathrm{U}$ \\
\hline & ${ }^{125} \mathrm{Sb}$ & $-1.0 \mathrm{E}-02 \pm 2.1 \mathrm{E}-02$ & $\mathrm{U}$ & & ${ }^{125} \mathrm{Sb}$ & $1.0 \mathrm{E}-02$ & $\pm 1.7 \mathrm{E}-02$ & U \\
\hline & ${ }^{113} \mathrm{Sn}$ & $-3.1 \mathrm{E}-03 \pm 1.0 \mathrm{E}-02$ & $\mathrm{U}$ & & ${ }^{113} \mathrm{Sn}$ & $-8.8 \mathrm{E}-03$ & $\pm 8.8 \mathrm{E}-03$ & $\mathrm{U}$ \\
\hline & ${ }^{90} \mathrm{Sr}$ & $5.2 \mathrm{E}-02 \pm 1.9 \mathrm{E}-01$ & $\mathrm{U}$ & & ${ }^{90} \mathrm{Sr}$ & $-1.4 \mathrm{E}-01$ & $\pm 1.6 \mathrm{E}-01$ & $\mathrm{U}$ \\
\hline & ${ }^{234} \mathrm{U}$ & $1.6 \mathrm{E}-01 \pm 5.3 \mathrm{E}-02$ & & & ${ }^{234} \mathrm{U}$ & $1.3 \mathrm{E}-01$ & $\pm 4.5 \mathrm{E}-02$ & \\
\hline & ${ }^{235} \mathrm{U}$ & $1.1 \mathrm{E}-02 \pm 9.5 \mathrm{E}-03$ & & & ${ }^{235} \mathrm{U}$ & 1.7E-02 & $\pm 1.3 \mathrm{E}-02$ & \\
\hline & ${ }^{238} \mathrm{U}$ & $1.4 \mathrm{E}-01 \pm 4.8 \mathrm{E}-02$ & & & ${ }^{238} \mathrm{U}$ & $1.2 \mathrm{E}-01$ & $\pm 4.2 \mathrm{E}-02$ & \\
\hline & ${ }^{65} \mathrm{Zn}$ & $-1.9 \mathrm{E}-02 \pm 2.2 \mathrm{E}-02$ & $\mathrm{U}$ & & ${ }^{65} \mathrm{Zn}$ & $-8.8 \mathrm{E}-03$ & $\pm 1.5 \mathrm{E}-02$ & $\mathrm{U}$ \\
\hline
\end{tabular}

$\overline{\mathrm{RQ}}=$ Result Qualifier. U $=$ The analyte was analyzed for but not detected. 
Table 3-3. 2003 Soil Sampling Results (pCi/g \pm total analytical uncertainty). (cont)

\begin{tabular}{|c|c|c|c|c|c|c|c|c|}
\hline Location & Isotope & Result \pm Error & $\mathbf{R Q}^{*}$ & Location & Isotope & Result & \pm Error & $\mathbf{R Q}^{*}$ \\
\hline D116 & ${ }^{144} \mathrm{Ce}$ & $-6.1 \mathrm{E}-03 \pm 6.1 \mathrm{E}-02$ & $\bar{U}$ & D117 & ${ }^{144} \mathrm{Ce}$ & $-1.1 \mathrm{E}-01$ & $\pm 1.1 \mathrm{E}-01$ & $\mathrm{U}$ \\
\hline \multirow[t]{17}{*}{ (300 Area) } & ${ }^{60} \mathrm{Co}$ & $-2.8 \mathrm{E}-03 \pm 4.6 \mathrm{E}-03$ & $\mathrm{U}$ & (300 Area) & ${ }^{60} \mathrm{Co}$ & $-1.2 \mathrm{E}-03$ & $\pm 5.0 \mathrm{E}-03$ & U \\
\hline & ${ }^{134} \mathrm{Cs}$ & $2.8 \mathrm{E}-02 \pm 7.5 \mathrm{E}-03$ & & & ${ }^{134} \mathrm{Cs}$ & 2.5E-02 & $\pm 7.4 \mathrm{E}-03$ & \\
\hline & ${ }^{137} \mathrm{Cs}$ & $2.4 \mathrm{E}-02 \pm 1.1 \mathrm{E}-02$ & & & ${ }^{137} \mathrm{Cs}$ & $2.4 \mathrm{E}-02$ & $\pm 8.3 \mathrm{E}-03$ & \\
\hline & ${ }^{152} \mathrm{Eu}$ & $-9.8 \mathrm{E}-03 \pm 1.8 \mathrm{E}-02$ & $\mathrm{U}$ & & ${ }^{152} \mathrm{Eu}$ & $3.4 \mathrm{E}-03$ & $\pm 1.7 \mathrm{E}-02$ & $\mathrm{U}$ \\
\hline & ${ }^{154} \mathrm{Eu}$ & $-2.0 \mathrm{E}-03 \pm 1.6 \mathrm{E}-02$ & $\mathrm{U}$ & & ${ }^{154} \mathrm{Eu}$ & $-2.1 \mathrm{E}-03$ & $\pm 1.7 \mathrm{E}-02$ & $\mathrm{U}$ \\
\hline & ${ }^{155} \mathrm{Eu}$ & $2.3 \mathrm{E}-02 \pm 2.7 \mathrm{E}-02$ & $\mathrm{U}$ & & ${ }^{155} \mathrm{Eu}$ & $2.4 \mathrm{E}-02$ & $\pm 2.5 \mathrm{E}-02$ & $\mathrm{U}$ \\
\hline & ${ }^{238} \mathrm{Pu}$ & $-2.3 \mathrm{E}-02 \pm 3.0 \mathrm{E}-02$ & $\mathrm{U}$ & & ${ }^{238} \mathrm{Pu}$ & 1.3E-02 & $\pm 1.1 \mathrm{E}-02$ & $\mathrm{U}$ \\
\hline & ${ }^{239,240} \mathrm{Pu}$ & $1.8 \mathrm{E}-03 \pm 8.1 \mathrm{E}-03$ & $\mathrm{U}$ & & ${ }^{239,240} \mathrm{Pu}$ & $9.6 \mathrm{E}-03$ & $\pm 1.1 \mathrm{E}-02$ & $\mathrm{U}$ \\
\hline & ${ }^{103} \mathrm{Ru}$ & $-7.9 \mathrm{E}-04 \pm 4.9 \mathrm{E}-03$ & U & & ${ }^{103} \mathrm{Ru}$ & 4.3E-04 & $\pm 4.3 \mathrm{E}-03$ & U \\
\hline & ${ }^{106} \mathrm{Ru}$ & $1.5 \mathrm{E}-02 \pm 4.1 \mathrm{E}-02$ & $\mathrm{U}$ & & ${ }^{106} \mathrm{Ru}$ & $2.8 \mathrm{E}-02$ & $\pm 4.6 \mathrm{E}-02$ & $\mathrm{U}$ \\
\hline & ${ }^{125} \mathrm{Sb}$ & $3.7 \mathrm{E}-03 \pm 1.4 \mathrm{E}-02$ & $\mathrm{U}$ & & ${ }^{125} \mathrm{Sb}$ & 4.6E-03 & $\pm 1.4 \mathrm{E}-02$ & $\mathrm{U}$ \\
\hline & ${ }^{113} \mathrm{Sn}$ & $-4.7 \mathrm{E}-03 \pm 6.8 \mathrm{E}-03$ & $\mathrm{U}$ & & ${ }^{113} \mathrm{Sn}$ & $-9.9 \mathrm{E}-04$ & $\pm 6.9 \mathrm{E}-03$ & $\mathrm{U}$ \\
\hline & ${ }^{90} \mathrm{Sr}$ & $1.6 \mathrm{E}-02 \pm 8.8 \mathrm{E}-02$ & $\mathrm{U}$ & & ${ }^{90} \mathrm{Sr}$ & $-6.8 \mathrm{E}-02$ & $\pm 9.9 \mathrm{E}-02$ & $\mathrm{U}$ \\
\hline & ${ }^{234} \mathrm{U}$ & $2.2 \mathrm{E}-01 \pm 5.9 \mathrm{E}-02$ & & & & $1.5 \mathrm{E}-01$ & $\pm 4.4 \mathrm{E}-02$ & \\
\hline & ${ }^{235} \mathrm{U}$ & $1.7 \mathrm{E}-02 \pm 1.2 \mathrm{E}-02$ & & & ${ }^{235} \mathrm{U}$ & 2.2E-02 & $\pm 1.5 \mathrm{E}-02$ & \\
\hline & ${ }^{238} \mathrm{U}$ & $1.8 \mathrm{E}-01 \pm 5.0 \mathrm{E}-02$ & & & ${ }^{238} \mathrm{U}$ & $1.4 \mathrm{E}-01$ & $\pm 4.2 \mathrm{E}-02$ & \\
\hline & ${ }^{65} \mathrm{Zn}$ & $-8.3 \mathrm{E}-03 \pm 1.3 \mathrm{E}-02$ & $\mathrm{U}$ & & ${ }^{65} \mathrm{Zn}$ & 6.1E-03 & $\pm 1.4 \mathrm{E}-02$ & $\mathrm{U}$ \\
\hline D119 & ${ }^{144} \mathrm{Ce}$ & $-1.1 \mathrm{E}-01 \pm 1.2 \mathrm{E}-01$ & $\bar{U}$ & D121 & ${ }^{144} \mathrm{Ce}$ & $6.6 \mathrm{E}-02$ & $\pm 1.1 \mathrm{E}-01$ & $\bar{U}$ \\
\hline \multirow[t]{17}{*}{ (300 Area) } & ${ }^{60} \mathrm{Co}$ & $2.2 \mathrm{E}-03 \pm 6.3 \mathrm{E}-03$ & $\mathrm{U}$ & (300 Area) & ${ }^{60} \mathrm{Co}$ & $-3.3 E-03$ & $\pm 6.2 \mathrm{E}-03$ & $\mathrm{U}$ \\
\hline & ${ }^{134} \mathrm{Cs}$ & $2.4 \mathrm{E}-02 \pm 1.0 \mathrm{E}-02$ & & & ${ }^{134} \mathrm{Cs}$ & 2.5E-02 & $\pm 1.2 \mathrm{E}-02$ & \\
\hline & ${ }^{137} \mathrm{Cs}$ & $1.6 \mathrm{E}-01 \pm 2.6 \mathrm{E}-02$ & & & ${ }^{137} \mathrm{Cs}$ & $-2.2 \mathrm{E}-03$ & $\pm 6.4 \mathrm{E}-03$ & $\mathrm{U}$ \\
\hline & ${ }^{152} \mathrm{Eu}$ & $-2.5 \mathrm{E}-02 \pm 2.6 \mathrm{E}-02$ & $\mathrm{U}$ & & ${ }^{152} \mathrm{Eu}$ & $-3.8 E-03$ & $\pm 2.3 \mathrm{E}-02$ & $\mathrm{U}$ \\
\hline & ${ }^{154} \mathrm{Eu}$ & $-2.3 \mathrm{E}-03 \pm 2.1 \mathrm{E}-02$ & $\mathrm{U}$ & & ${ }^{154} \mathrm{Eu}$ & $-1.4 \mathrm{E}-02$ & $\pm 2.1 \mathrm{E}-02$ & U \\
\hline & ${ }^{155} \mathrm{Eu}$ & $4.1 \mathrm{E}-02 \pm 2.9 \mathrm{E}-02$ & $\mathrm{U}$ & & ${ }^{155} \mathrm{Eu}$ & 2.2E-02 & $\pm 2.7 \mathrm{E}-02$ & $\mathrm{U}$ \\
\hline & ${ }^{238} \mathrm{Pu}$ & $2.3 \mathrm{E}-02 \pm 1.6 \mathrm{E}-02$ & & & ${ }^{238} \mathrm{Pu}$ & $-3.7 \mathrm{E}-03$ & $\pm 3.1 \mathrm{E}-02$ & $\mathrm{U}$ \\
\hline & ${ }^{239,240} \mathrm{Pu}$ & $7.3 \mathrm{E}-01 \pm 1.5 \mathrm{E}-01$ & & & ${ }^{239,240} \mathrm{Pu}$ & 3.7E-03 & $\pm 9.3 \mathrm{E}-03$ & $\mathrm{U}$ \\
\hline & ${ }^{103} \mathrm{Ru}$ & $5.6 \mathrm{E}-03 \pm 6.5 \mathrm{E}-03$ & $\mathrm{U}$ & & ${ }^{103} \mathrm{Ru}$ & $-5.2 \mathrm{E}-05$ & $\pm 5.2 \mathrm{E}-04$ & $\mathrm{U}$ \\
\hline & ${ }^{106} \mathrm{Ru}$ & $1.0 \mathrm{E}-03 \pm 1.0 \mathrm{E}-02$ & $\mathrm{U}$ & & ${ }^{106} \mathrm{Ru}$ & $-6.4 \mathrm{E}-03$ & $\pm 5.4 \mathrm{E}-02$ & $\mathrm{U}$ \\
\hline & ${ }^{125} \mathrm{Sb}$ & $-4.4 \mathrm{E}-04 \pm 4.4 \mathrm{E}-03$ & $\mathrm{U}$ & & ${ }^{125} \mathrm{Sb}$ & $-6.1 \mathrm{E}-03$ & $\pm 1.6 \mathrm{E}-02$ & U \\
\hline & ${ }^{113} \mathrm{Sn}$ & $4.4 \mathrm{E}-03 \pm 8.2 \mathrm{E}-03$ & U & & ${ }^{113} \mathrm{Sn}$ & $-3.4 \mathrm{E}-03$ & $\pm 7.8 \mathrm{E}-03$ & $\mathrm{U}$ \\
\hline & ${ }^{90} \mathrm{Sr}$ & $6.7 \mathrm{E}-03 \pm 6.7 \mathrm{E}-02$ & U & & ${ }^{90} \mathrm{Sr}$ & 7.1E-02 & $\pm 1.3 \mathrm{E}-01$ & U \\
\hline & ${ }^{234} \mathrm{U}$ & $8.5 \mathrm{E}+00 \pm 1.6 \mathrm{E}+00$ & & & ${ }^{234} \mathrm{U}$ & 4.0E-01 & $\pm 9.2 \mathrm{E}-02$ & \\
\hline & ${ }^{235} \mathrm{U}$ & $4.1 \mathrm{E}-01 \pm 9.8 \mathrm{E}-02$ & & & ${ }^{235} \mathrm{U}$ & 2.0E-02 & $\pm 1.4 \mathrm{E}-02$ & \\
\hline & ${ }^{238} \mathrm{U}$ & $8.6 \mathrm{E}+00 \pm 1.6 \mathrm{E}+00$ & & & ${ }^{238} \mathrm{U}$ & 3.9E-01 & $\pm 9.0 \mathrm{E}-02$ & \\
\hline & ${ }^{65} \mathrm{Zn}$ & $5.3 \mathrm{E}-02 \pm 1.9 \mathrm{E}-02$ & & & ${ }^{65} \mathrm{Zn}$ & 1.7E-03 & $\pm 1.6 \mathrm{E}-02$ & $\mathrm{U}$ \\
\hline
\end{tabular}

$\overline{\mathrm{RQ}}=$ Result Qualifier. U $=$ The analyte was analyzed for but not detected. 
Table 3-3. 2003 Soil Sampling Results (pCi/g \pm total analytical uncertainty). (cont)

\begin{tabular}{|c|c|c|c|c|c|c|c|c|}
\hline Location & $\begin{array}{l}\text { Isotope } \\
\end{array}$ & Result \pm Error & $\overline{\mathbf{R Q}^{*}}$ & Location & Isotope & Result & \pm Error & $\mathbf{R Q}^{*}$ \\
\hline D123 & ${ }^{144} \mathrm{Ce}$ & $-8.1 \mathrm{E}-02 \pm 9.5 \mathrm{E}-02$ & $\bar{U}$ & D124 & ${ }^{144} \mathrm{Ce}$ & $6.0 \mathrm{E}-02$ & $\pm 1.0 \mathrm{E}-01$ & $\mathrm{U}$ \\
\hline \multirow[t]{17}{*}{ (300 Area) } & ${ }^{60} \mathrm{Co}$ & $5.6 \mathrm{E}-03 \pm 5.2 \mathrm{E}-03$ & $\mathrm{U}$ & (300 Area) & ${ }^{60} \mathrm{Co}$ & $-2.2 \mathrm{E}-03$ & $\pm 5.0 \mathrm{E}-03$ & $\mathrm{U}$ \\
\hline & ${ }^{134} \mathrm{Cs}$ & $2.6 \mathrm{E}-02 \pm 1.1 \mathrm{E}-02$ & & & ${ }^{134} \mathrm{Cs}$ & 2.9E-02 & $\pm 8.6 \mathrm{E}-03$ & \\
\hline & ${ }^{137} \mathrm{Cs}$ & $2.3 \mathrm{E}-02 \pm 9.8 \mathrm{E}-03$ & & & ${ }^{137} \mathrm{Cs}$ & $5.1 \mathrm{E}-02$ & $\pm 1.0 \mathrm{E}-02$ & \\
\hline & ${ }^{152} \mathrm{Eu}$ & $1.3 \mathrm{E}-03 \pm 1.3 \mathrm{E}-02$ & $\mathrm{U}$ & & ${ }^{152} \mathrm{Eu}$ & 5.0E-03 & $\pm 1.8 \mathrm{E}-02$ & $\mathrm{U}$ \\
\hline & ${ }^{154} \mathrm{Eu}$ & $-9.0 \mathrm{E}-03 \pm 1.8 \mathrm{E}-02$ & $\mathrm{U}$ & & ${ }^{154} \mathrm{Eu}$ & $-8.7 \mathrm{E}-03$ & $\pm 1.7 \mathrm{E}-02$ & $\mathrm{U}$ \\
\hline & ${ }^{155} \mathrm{Eu}$ & $1.5 \mathrm{E}-02 \pm 2.5 \mathrm{E}-02$ & $\mathrm{U}$ & & ${ }^{155} \mathrm{Eu}$ & 2.9E-02 & $\pm 2.9 \mathrm{E}-02$ & $\mathrm{U}$ \\
\hline & ${ }^{238} \mathrm{Pu}$ & $5.4 \mathrm{E}-03 \pm 3.0 \mathrm{E}-02$ & $\mathrm{U}$ & & ${ }^{238} \mathrm{Pu}$ & 1.7E-03 & $\pm 1.7 \mathrm{E}-02$ & $\mathrm{U}$ \\
\hline & ${ }^{239,240} \mathrm{Pu}$ & $3.6 \mathrm{E}-03 \pm 7.2 \mathrm{E}-03$ & $\mathrm{U}$ & & ${ }^{239,240} \mathrm{Pu}$ & $-1.7 \mathrm{E}-03$ & $\pm 9.0 \mathrm{E}-03$ & $\mathrm{U}$ \\
\hline & ${ }^{103} \mathrm{Ru}$ & $-2.8 \mathrm{E}-03 \pm 5.4 \mathrm{E}-03$ & $\mathrm{U}$ & & ${ }^{103} \mathrm{Ru}$ & $-3.5 E-04$ & $\pm 3.5 \mathrm{E}-03$ & $\mathrm{U}$ \\
\hline & ${ }^{106} \mathrm{Ru}$ & $3.6 \mathrm{E}-03 \pm 3.6 \mathrm{E}-02$ & $\mathrm{U}$ & & ${ }^{106} \mathrm{Ru}$ & $-2.3 \mathrm{E}-03$ & $\pm 2.3 \mathrm{E}-02$ & $\mathrm{U}$ \\
\hline & ${ }^{125} \mathrm{Sb}$ & $-5.8 \mathrm{E}-03 \pm 1.5 \mathrm{E}-02$ & $\mathrm{U}$ & & ${ }^{125} \mathrm{Sb}$ & 1.5E-02 & $\pm 1.5 \mathrm{E}-02$ & U \\
\hline & ${ }^{113} \mathrm{Sn}$ & $-1.1 \mathrm{E}-03 \pm 7.9 \mathrm{E}-03$ & $\mathrm{U}$ & & ${ }^{113} \mathrm{Sn}$ & $-4.5 E-03$ & $\pm 7.2 \mathrm{E}-03$ & $\mathrm{U}$ \\
\hline & ${ }^{90} \mathrm{Sr}$ & $-7.4 \mathrm{E}-02 \pm 8.9 \mathrm{E}-02$ & $\mathrm{U}$ & & ${ }^{90} \mathrm{Sr}$ & $-1.2 \mathrm{E}-02$ & $\pm 1.1 \mathrm{E}-01$ & $\mathrm{U}$ \\
\hline & ${ }^{234} \mathrm{U}$ & $1.6 \mathrm{E}-01 \pm 4.6 \mathrm{E}-02$ & & & ${ }^{234} \mathrm{U}$ & 2.2E-01 & $\pm 5.7 \mathrm{E}-02$ & \\
\hline & ${ }^{235} \mathrm{U}$ & $2.1 \mathrm{E}-03 \pm 4.2 \mathrm{E}-03$ & $\mathrm{U}$ & & ${ }^{235} \mathrm{U}$ & 7.2E-03 & $\pm 1.2 \mathrm{E}-02$ & $\mathrm{U}$ \\
\hline & ${ }^{238} \mathrm{U}$ & $2.0 \mathrm{E}-01 \pm 5.4 \mathrm{E}-02$ & & & ${ }^{238} \mathrm{U}$ & $1.8 \mathrm{E}-01$ & $\pm 4.9 \mathrm{E}-02$ & \\
\hline & ${ }^{65} \mathrm{Zn}$ & $1.4 \mathrm{E}-02 \pm 1.3 \mathrm{E}-02$ & $\mathrm{U}$ & & ${ }^{65} \mathrm{Zn}$ & $-6.2 \mathrm{E}-03$ & $\pm 1.4 \mathrm{E}-02$ & $\mathrm{U}$ \\
\hline D125 & ${ }^{144} \mathrm{Ce}$ & $1.3 \mathrm{E}-01 \pm 1.0 \mathrm{E}-01$ & $\overline{\mathrm{U}}$ & D126 & ${ }^{144} \mathrm{Ce}$ & $-7.5 \mathrm{E}-02$ & $\pm 1.0 \mathrm{E}-01$ & $\bar{U}$ \\
\hline \multirow[t]{17}{*}{ (300 Area) } & ${ }^{60} \mathrm{Co}$ & $1.1 \mathrm{E}-03 \pm 5.7 \mathrm{E}-03$ & $\mathrm{U}$ & (300 Area) & ${ }^{60} \mathrm{Co}$ & $-2.1 E-03$ & $\pm 6.4 \mathrm{E}-03$ & $\mathrm{U}$ \\
\hline & ${ }^{134} \mathrm{Cs}$ & $2.9 \mathrm{E}-02 \pm 1.0 \mathrm{E}-02$ & & & ${ }^{134} \mathrm{Cs}$ & 2.5E-02 & $\pm 1.2 \mathrm{E}-02$ & \\
\hline & ${ }^{137} \mathrm{Cs}$ & $2.0 \mathrm{E}-01 \pm 2.8 \mathrm{E}-02$ & & & ${ }^{137} \mathrm{Cs}$ & 1.3E-02 & $\pm 8.1 \mathrm{E}-03$ & \\
\hline & ${ }^{152} \mathrm{Eu}$ & $-1.4 \mathrm{E}-02 \pm 1.8 \mathrm{E}-02$ & $\mathrm{U}$ & & ${ }^{152} \mathrm{Eu}$ & $-1.4 \mathrm{E}-02$ & $\pm 1.9 \mathrm{E}-02$ & $\mathrm{U}$ \\
\hline & ${ }^{154} \mathrm{Eu}$ & $9.8 \mathrm{E}-03 \pm 1.9 \mathrm{E}-02$ & $\mathrm{U}$ & & ${ }^{154} \mathrm{Eu}$ & 4.8E-03 & $\pm 2.2 \mathrm{E}-02$ & $\mathrm{U}$ \\
\hline & ${ }^{155} \mathrm{Eu}$ & $3.5 \mathrm{E}-03 \pm 2.4 \mathrm{E}-02$ & $\mathrm{U}$ & & ${ }^{155} \mathrm{Eu}$ & 2.7E-02 & $\pm 2.4 \mathrm{E}-02$ & $\mathrm{U}$ \\
\hline & ${ }^{238} \mathrm{Pu}$ & $-2.1 \mathrm{E}-02 \pm 2.5 \mathrm{E}-02$ & $\mathrm{U}$ & & ${ }^{238} \mathrm{Pu}$ & $-2.9 \mathrm{E}-02$ & $\pm 2.9 \mathrm{E}-02$ & U \\
\hline & ${ }^{239,240} \mathrm{Pu}$ & $4.6 \mathrm{E}-03 \pm 5.5 \mathrm{E}-03$ & & & ${ }^{239,240} \mathrm{Pu}$ & $1.9 \mathrm{E}-03$ & $\pm 8.6 \mathrm{E}-03$ & $\mathrm{U}$ \\
\hline & ${ }^{103} \mathrm{Ru}$ & $-2.1 \mathrm{E}-03 \pm 5.5 \mathrm{E}-03$ & $\mathrm{U}$ & & ${ }^{103} \mathrm{Ru}$ & $-1.7 \mathrm{E}-03$ & $\pm 6.1 \mathrm{E}-03$ & $\mathrm{U}$ \\
\hline & ${ }^{106} \mathrm{Ru}$ & $1.9 \mathrm{E}-03 \pm 1.9 \mathrm{E}-02$ & $\mathrm{U}$ & & ${ }^{106} \mathrm{Ru}$ & $-3.6 \mathrm{E}-02$ & $\pm 6.2 \mathrm{E}-02$ & $\mathrm{U}$ \\
\hline & ${ }^{125} \mathrm{Sb}$ & $-7.7 \mathrm{E}-03 \pm 1.5 \mathrm{E}-02$ & $\mathrm{U}$ & & ${ }^{125} \mathrm{Sb}$ & 1.7E-02 & $\pm 2.0 \mathrm{E}-02$ & $\mathrm{U}$ \\
\hline & ${ }^{113} \mathrm{Sn}$ & $-6.2 \mathrm{E}-03 \pm 7.3 \mathrm{E}-03$ & $\mathrm{U}$ & & ${ }^{113} \mathrm{Sn}$ & $-2.3 \mathrm{E}-03$ & $\pm 7.9 \mathrm{E}-03$ & $\mathrm{U}$ \\
\hline & ${ }^{90} \mathrm{Sr}$ & $1.3 \mathrm{E}-01 \pm 1.2 \mathrm{E}-01$ & $\mathrm{U}$ & & ${ }^{90} \mathrm{Sr}$ & $1.2 \mathrm{E}-02$ & $\pm 1.1 \mathrm{E}-01$ & $\mathrm{U}$ \\
\hline & ${ }^{234} \mathrm{U}$ & $7.6 \mathrm{E}-01 \pm 1.6 \mathrm{E}-01$ & & & ${ }^{234} \mathrm{U}$ & $1.8 \mathrm{E}-01$ & $\pm 5.2 \mathrm{E}-02$ & \\
\hline & ${ }^{235} \mathrm{U}$ & $5.8 \mathrm{E}-02 \pm 2.3 \mathrm{E}-02$ & & & ${ }^{235} \mathrm{U}$ & 1.3E-02 & $\pm 1.2 \mathrm{E}-02$ & $\mathrm{U}$ \\
\hline & ${ }^{238} U$ & $6.5 \mathrm{E}-01 \pm 1.4 \mathrm{E}-01$ & & & ${ }^{238} \mathrm{U}$ & 1.5E-01 & $\pm 4.5 \mathrm{E}-02$ & \\
\hline & ${ }^{65} \mathrm{Zn}$ & $6.6 \mathrm{E}-04 \pm 6.6 \mathrm{E}-03$ & $\mathrm{U}$ & & ${ }^{65} \mathrm{Zn}$ & 2.9E-02 & $\pm 1.9 \mathrm{E}-02$ & \\
\hline
\end{tabular}

$\overline{\mathrm{RQ}}=$ Result Qualifier. U $=$ The analyte was analyzed for but not detected. 
Table 3-3. 2003 Soil Sampling Results (pCi/g \pm total analytical uncertainty). (cont)

\begin{tabular}{|c|c|c|c|c|c|c|c|c|}
\hline Location & Isotope & Result \pm Error & $\overline{\mathbf{R Q}^{*}}$ & Location & Isotope & Result & \pm Error & $\overline{\mathbf{R Q}^{*}}$ \\
\hline D127 & ${ }^{144} \mathrm{Ce}$ & $-2.1 \mathrm{E}-02 \pm 1.0 \mathrm{E}-01$ & $\overline{\mathrm{U}}$ & D128 & ${ }^{144} \mathrm{Ce}$ & $-8.3 \mathrm{E}-03$ & $\pm 8.3 \mathrm{E}-02$ & $\bar{U}$ \\
\hline \multirow[t]{17}{*}{ (300 Area) } & ${ }^{60} \mathrm{Co}$ & $2.9 \mathrm{E}-03 \pm 6.4 \mathrm{E}-03$ & $\mathrm{U}$ & (300 Area) & ${ }^{60} \mathrm{Co}$ & $1.2 \mathrm{E}-03$ & $\pm 5.2 \mathrm{E}-03$ & U \\
\hline & ${ }^{134} \mathrm{Cs}$ & $1.7 \mathrm{E}-02 \pm 8.8 \mathrm{E}-03$ & & & ${ }^{134} \mathrm{Cs}$ & 2.1E-02 & $\pm 7.3 \mathrm{E}-03$ & \\
\hline & ${ }^{137} \mathrm{Cs}$ & $2.1 \mathrm{E}-01 \pm 3.4 \mathrm{E}-02$ & & & ${ }^{137} \mathrm{Cs}$ & $1.2 \mathrm{E}-01$ & $\pm 2.2 \mathrm{E}-02$ & \\
\hline & ${ }^{152} \mathrm{Eu}$ & $-1.4 \mathrm{E}-02 \pm 2.7 \mathrm{E}-02$ & $\mathrm{U}$ & & ${ }^{152} \mathrm{Eu}$ & $-1.2 \mathrm{E}-02$ & $\pm 1.9 \mathrm{E}-02$ & $\mathrm{U}$ \\
\hline & ${ }^{154} \mathrm{Eu}$ & $-4.6 \mathrm{E}-03 \pm 2.0 \mathrm{E}-02$ & $\mathrm{U}$ & & ${ }^{154} \mathrm{Eu}$ & $-9.0 \mathrm{E}-03$ & $\pm 1.7 \mathrm{E}-02$ & $\mathrm{U}$ \\
\hline & ${ }^{155} \mathrm{Eu}$ & $6.2 \mathrm{E}-02 \pm 2.8 \mathrm{E}-02$ & & & ${ }^{155} \mathrm{Eu}$ & 4.8E-02 & $\pm 3.4 \mathrm{E}-02$ & $\mathrm{U}$ \\
\hline & ${ }^{238} \mathrm{Pu}$ & $1.7 \mathrm{E}-03 \pm 6.0 \mathrm{E}-03$ & $\mathrm{U}$ & & ${ }^{238} \mathrm{Pu}$ & 4.0E-03 & $\pm 1.3 \mathrm{E}-02$ & $\mathrm{U}$ \\
\hline & ${ }^{239,240} \mathrm{Pu}$ & $1.4 \mathrm{E}-02 \pm 1.1 \mathrm{E}-02$ & & & ${ }^{239,240} \mathrm{Pu}$ & $6.0 \mathrm{E}-03$ & $\pm 7.2 \mathrm{E}-03$ & \\
\hline & ${ }^{103} \mathrm{Ru}$ & $1.4 \mathrm{E}-03 \pm 6.6 \mathrm{E}-03$ & $\mathrm{U}$ & & ${ }^{103} \mathrm{Ru}$ & 4.4E-04 & $\pm 4.4 \mathrm{E}-03$ & $\mathrm{U}$ \\
\hline & ${ }^{106} \mathrm{Ru}$ & $-9.0 \mathrm{E}-03 \pm 5.5 \mathrm{E}-02$ & $\mathrm{U}$ & & ${ }^{106} \mathrm{Ru}$ & 2.9E-03 & $\pm 2.9 \mathrm{E}-02$ & $\mathrm{U}$ \\
\hline & ${ }^{125} \mathrm{Sb}$ & $7.2 \mathrm{E}-03 \pm 1.7 \mathrm{E}-02$ & $\mathrm{U}$ & & ${ }^{125} \mathrm{Sb}$ & $1.9 \mathrm{E}-02$ & $\pm 1.7 \mathrm{E}-02$ & $\mathrm{U}$ \\
\hline & ${ }^{113} \mathrm{Sn}$ & $2.5 \mathrm{E}-04 \pm 2.5 \mathrm{E}-03$ & $\mathrm{U}$ & & ${ }^{113} \mathrm{Sn}$ & $-3.0 \mathrm{E}-03$ & $\pm 8.1 \mathrm{E}-03$ & $\mathrm{U}$ \\
\hline & ${ }^{90} \mathrm{Sr}$ & $3.0 \mathrm{E}-01 \pm 1.2 \mathrm{E}-01$ & & & ${ }^{90} \mathrm{Sr}$ & $6.2 \mathrm{E}-02$ & $\pm 9.3 \mathrm{E}-02$ & $\mathrm{U}$ \\
\hline & ${ }^{234} \mathrm{U}$ & $2.2 \mathrm{E}-01 \pm 5.7 \mathrm{E}-02$ & & & ${ }^{234} \mathrm{U}$ & $1.6 \mathrm{E}-01$ & $\pm 4.6 \mathrm{E}-02$ & \\
\hline & ${ }^{235} \mathrm{U}$ & $1.0 \mathrm{E}-02 \pm 1.1 \mathrm{E}-02$ & $\mathrm{U}$ & & ${ }^{235} \mathrm{U}$ & 1.7E-02 & $\pm 1.2 \mathrm{E}-02$ & \\
\hline & ${ }^{238} \mathrm{U}$ & $1.7 \mathrm{E}-01 \pm 4.8 \mathrm{E}-02$ & & & ${ }^{238} \mathrm{U}$ & $2.0 \mathrm{E}-01$ & $\pm 5.4 \mathrm{E}-02$ & \\
\hline & ${ }^{65} \mathrm{Zn}$ & $4.9 \mathrm{E}-02 \pm 1.9 \mathrm{E}-02$ & & & ${ }^{65} \mathrm{Zn}$ & 3.5E-02 & $\pm 1.5 \mathrm{E}-02$ & \\
\hline D129 & ${ }^{144} \mathrm{Ce}$ & $-3.6 \mathrm{E}-02 \pm 1.0 \mathrm{E}-01$ & $\overline{\mathrm{U}}$ & D130 & ${ }^{144} \mathrm{Ce}$ & $8.2 \mathrm{E}-05$ & $\pm 8.1 \mathrm{E}-04$ & $\bar{U}$ \\
\hline \multirow[t]{17}{*}{ (300 Area) } & ${ }^{60} \mathrm{Co}$ & $-5.1 \mathrm{E}-04 \pm 4.8 \mathrm{E}-03$ & $\mathrm{U}$ & (400 Area) & ${ }^{60} \mathrm{Co}$ & $-2.8 \mathrm{E}-03$ & $\pm 5.5 \mathrm{E}-03$ & $\mathrm{U}$ \\
\hline & ${ }^{134} \mathrm{Cs}$ & $2.0 \mathrm{E}-02 \pm 7.2 \mathrm{E}-03$ & & & ${ }^{134} \mathrm{Cs}$ & 2.2E-02 & $\pm 8.7 \mathrm{E}-03$ & \\
\hline & ${ }^{137} \mathrm{Cs}$ & $1.3 \mathrm{E}-01 \pm 2.1 \mathrm{E}-02$ & & & ${ }^{137} \mathrm{Cs}$ & 1.2E-02 & $\pm 7.4 \mathrm{E}-03$ & \\
\hline & ${ }^{152} \mathrm{Eu}$ & $1.5 \mathrm{E}-02 \pm 2.1 \mathrm{E}-02$ & $\mathrm{U}$ & & ${ }^{152} \mathrm{Eu}$ & $-1.0 \mathrm{E}-02$ & $\pm 1.9 \mathrm{E}-02$ & $\mathrm{U}$ \\
\hline & ${ }^{154} \mathrm{Eu}$ & $-6.6 \mathrm{E}-03 \pm 1.6 \mathrm{E}-02$ & $\mathrm{U}$ & & ${ }^{154} \mathrm{Eu}$ & 8.0E-03 & $\pm 2.0 \mathrm{E}-02$ & U \\
\hline & ${ }^{155} \mathrm{Eu}$ & $2.7 \mathrm{E}-02 \pm 2.9 \mathrm{E}-02$ & $\mathrm{U}$ & & ${ }^{155} \mathrm{Eu}$ & $1.8 \mathrm{E}-02$ & $\pm 2.7 \mathrm{E}-02$ & $\mathrm{U}$ \\
\hline & ${ }^{238} \mathrm{Pu}$ & $-1.4 \mathrm{E}-02 \pm 2.8 \mathrm{E}-02$ & $\mathrm{U}$ & & ${ }^{238} \mathrm{Pu}$ & 8.6E-03 & $\pm 2.9 \mathrm{E}-02$ & $\mathrm{U}$ \\
\hline & ${ }^{239,240} \mathrm{Pu}$ & $1.1 \mathrm{E}-02 \pm 1.2 \mathrm{E}-02$ & $\mathrm{U}$ & & ${ }^{239,240} \mathrm{Pu}$ & 3.4E-03 & $\pm 6.8 \mathrm{E}-03$ & U \\
\hline & ${ }^{103} \mathrm{Ru}$ & $-5.1 \mathrm{E}-04 \pm 5.1 \mathrm{E}-03$ & $\mathrm{U}$ & & ${ }^{103} \mathrm{Ru}$ & $-2.0 \mathrm{E}-03$ & $\pm 6.4 \mathrm{E}-03$ & $\mathrm{U}$ \\
\hline & ${ }^{106} \mathrm{Ru}$ & $1.6 \mathrm{E}-02 \pm 4.3 \mathrm{E}-02$ & $\mathrm{U}$ & & ${ }^{106} \mathrm{Ru}$ & $-3.2 E-02$ & $\pm 4.9 \mathrm{E}-02$ & $\mathrm{U}$ \\
\hline & ${ }^{125} \mathrm{Sb}$ & $9.4 \mathrm{E}-03 \pm 1.5 \mathrm{E}-02$ & $\mathrm{U}$ & & ${ }^{125} \mathrm{Sb}$ & 1.6E-03 & $\pm 1.6 \mathrm{E}-02$ & U \\
\hline & ${ }^{113} \mathrm{Sn}$ & $-2.5 \mathrm{E}-03 \pm 7.3 \mathrm{E}-03$ & $\mathrm{U}$ & & ${ }^{113} \mathrm{Sn}$ & $-1.7 \mathrm{E}-03$ & $\pm 7.9 \mathrm{E}-03$ & U \\
\hline & ${ }^{90} \mathrm{Sr}$ & $1.7 \mathrm{E}-02 \pm 8.8 \mathrm{E}-02$ & $\mathrm{U}$ & & ${ }^{90} \mathrm{Sr}$ & $1.8 \mathrm{E}-01$ & $\pm 1.3 \mathrm{E}-01$ & $\mathrm{U}$ \\
\hline & ${ }^{234} \mathrm{U}$ & $3.2 \mathrm{E}-01 \pm 7.7 \mathrm{E}-02$ & & & ${ }^{234} \mathrm{U}$ & $9.0 \mathrm{E}-02$ & $\pm 3.2 \mathrm{E}-02$ & \\
\hline & ${ }^{235} \mathrm{U}$ & $2.7 \mathrm{E}-02 \pm 1.5 \mathrm{E}-02$ & & & ${ }^{235} \mathrm{U}$ & 4.0E-03 & $\pm 5.6 \mathrm{E}-03$ & U \\
\hline & ${ }^{238} \mathrm{U}$ & $4.6 \mathrm{E}-01 \pm 1.0 \mathrm{E}-01$ & & & ${ }^{238} \mathrm{U}$ & $1.2 \mathrm{E}-01$ & $\pm 3.8 \mathrm{E}-02$ & \\
\hline & ${ }^{65} \mathrm{Zn}$ & $4.4 \mathrm{E}-03 \pm 1.4 \mathrm{E}-02$ & $\mathrm{U}$ & & ${ }^{65} \mathrm{Zn}$ & $-7.2 \mathrm{E}-03$ & $\pm 1.5 \mathrm{E}-02$ & U \\
\hline
\end{tabular}

$\overline{\mathrm{RQ}}=$ Result Qualifier. U $=$ The analyte was analyzed for but not detected. 
Table 3-3. 2003 Soil Sampling Results (pCi/g \pm total analytical uncertainty). (cont)

\begin{tabular}{|c|c|c|c|c|c|c|c|c|}
\hline Location & Isotope & Result \pm Error & $\overline{\mathrm{RQ}^{*}}$ & Location & Isotope & Result & \pm Error & $\mathbf{R Q}^{*}$ \\
\hline D131 & ${ }^{144} \mathrm{Ce}$ & $-6.8 \mathrm{E}-03 \pm 6.8 \mathrm{E}-02$ & $\bar{U}$ & D140 & ${ }^{144} \mathrm{Ce}$ & $2.4 \mathrm{E}-02$ & $\pm 1.2 \mathrm{E}-01$ & $\mathrm{U}$ \\
\hline (Duplicate & ${ }^{60} \mathrm{Co}$ & $-2.0 \mathrm{E}-03 \pm 4.8 \mathrm{E}-03$ & $\mathrm{U}$ & (Duplicate & ${ }^{60} \mathrm{Co}$ & 4.7E-03 & $\pm 6.2 \mathrm{E}-03$ & $\mathrm{U}$ \\
\hline of D119, & ${ }^{134} \mathrm{Cs}$ & $2.8 \mathrm{E}-02 \pm 9.2 \mathrm{E}-03$ & & of D123, & ${ }^{134} \mathrm{Cs}$ & 2.8E-02 & $\pm 8.6 \mathrm{E}-03$ & \\
\hline 300 Area) & ${ }^{137} \mathrm{Cs}$ & $1.3 \mathrm{E}-01 \pm 1.9 \mathrm{E}-02$ & & 300 Area) & ${ }^{137} \mathrm{Cs}$ & $3.8 \mathrm{E}-02$ & $\pm 1.1 \mathrm{E}-02$ & \\
\hline & ${ }^{152} \mathrm{Eu}$ & $7.1 \mathrm{E}-04 \pm 7.1 \mathrm{E}-03$ & $\mathrm{U}$ & & ${ }^{152} \mathrm{Eu}$ & $-1.4 \mathrm{E}-04$ & $\pm 1.4 \mathrm{E}-03$ & $\mathrm{U}$ \\
\hline & ${ }^{154} \mathrm{Eu}$ & $-1.2 \mathrm{E}-02 \pm 1.9 \mathrm{E}-02$ & $\mathrm{U}$ & & ${ }^{154} \mathrm{Eu}$ & $-3.7 E-03$ & $\pm 1.9 \mathrm{E}-02$ & $\mathrm{U}$ \\
\hline & ${ }^{155} \mathrm{Eu}$ & $6.7 \mathrm{E}-02 \pm 3.6 \mathrm{E}-02$ & $\mathrm{U}$ & & ${ }^{155} \mathrm{Eu}$ & 3.7E-02 & $\pm 3.3 \mathrm{E}-02$ & $\mathrm{U}$ \\
\hline & ${ }^{238} \mathrm{Pu}$ & $5.2 \mathrm{E}-03 \pm 2.8 \mathrm{E}-02$ & $\mathrm{U}$ & & ${ }^{238} \mathrm{Pu}$ & $-4.9 E-03$ & $\pm 1.5 \mathrm{E}-02$ & $\mathrm{U}$ \\
\hline & ${ }^{239,240} \mathrm{Pu}$ & $2.5 \mathrm{E}-01 \pm 6.3 \mathrm{E}-02$ & & & ${ }^{239,240} \mathrm{Pu}$ & $6.6 \mathrm{E}-03$ & $\pm 7.9 \mathrm{E}-03$ & $\mathrm{U}$ \\
\hline & ${ }^{103} \mathrm{Ru}$ & $3.2 \mathrm{E}-03 \pm 6.1 \mathrm{E}-03$ & $\mathrm{U}$ & & ${ }^{103} \mathrm{Ru}$ & $1.1 \mathrm{E}-03$ & $\pm 6.7 \mathrm{E}-03$ & $\mathrm{U}$ \\
\hline & ${ }^{106} \mathrm{Ru}$ & $5.9 \mathrm{E}-03 \pm 4.6 \mathrm{E}-02$ & $\mathrm{U}$ & & ${ }^{106} \mathrm{Ru}$ & $-6.0 \mathrm{E}-02$ & $\pm 6.0 \mathrm{E}-02$ & $\mathrm{U}$ \\
\hline & ${ }^{125} \mathrm{Sb}$ & $1.4 \mathrm{E}-02 \pm 1.5 \mathrm{E}-02$ & $\mathrm{U}$ & & ${ }^{125} \mathrm{Sb}$ & $1.5 \mathrm{E}-02$ & $\pm 1.6 \mathrm{E}-02$ & $\mathrm{U}$ \\
\hline & ${ }^{113} \mathrm{Sn}$ & $-5.2 \mathrm{E}-03 \pm 7.7 \mathrm{E}-03$ & $\mathrm{U}$ & & ${ }^{113} \mathrm{Sn}$ & $-3.6 \mathrm{E}-03$ & $\pm 8.0 \mathrm{E}-03$ & $\mathrm{U}$ \\
\hline & ${ }^{90} \mathrm{Sr}$ & $6.7 \mathrm{E}-02 \pm 1.1 \mathrm{E}-01$ & $\mathrm{U}$ & & ${ }^{90} \mathrm{Sr}$ & 7.2E-02 & $\pm 9.4 \mathrm{E}-02$ & $\mathrm{U}$ \\
\hline & ${ }^{234} \mathrm{U}$ & $6.4 \mathrm{E}+00 \pm 1.2 \mathrm{E}+00$ & & & ${ }^{234} \mathrm{U}$ & $2.0 \mathrm{E}-01$ & $\pm 5.2 \mathrm{E}-02$ & \\
\hline & ${ }^{235} \mathrm{U}$ & $3.1 \mathrm{E}-01 \pm 7.8 \mathrm{E}-02$ & & & ${ }^{235} \mathrm{U}$ & 7.8E-03 & $\pm 7.8 \mathrm{E}-03$ & \\
\hline & ${ }^{238} \mathrm{U}$ & $6.5 \mathrm{E}+00 \pm 1.2 \mathrm{E}+00$ & & & ${ }^{238} \mathrm{U}$ & 2.2E-01 & $\pm 5.7 \mathrm{E}-02$ & \\
\hline & ${ }^{65} \mathrm{Zn}$ & $-5.5 \mathrm{E}-03 \pm 1.5 \mathrm{E}-02$ & $\mathrm{U}$ & & ${ }^{65} \mathrm{Zn}$ & 3.3E-02 & $\pm 2.3 \mathrm{E}-02$ & \\
\hline
\end{tabular}

$\overline{\mathrm{RQ}}=$ Result Qualifier. $\mathrm{U}=$ The analyte was analyzed for but not detected. 


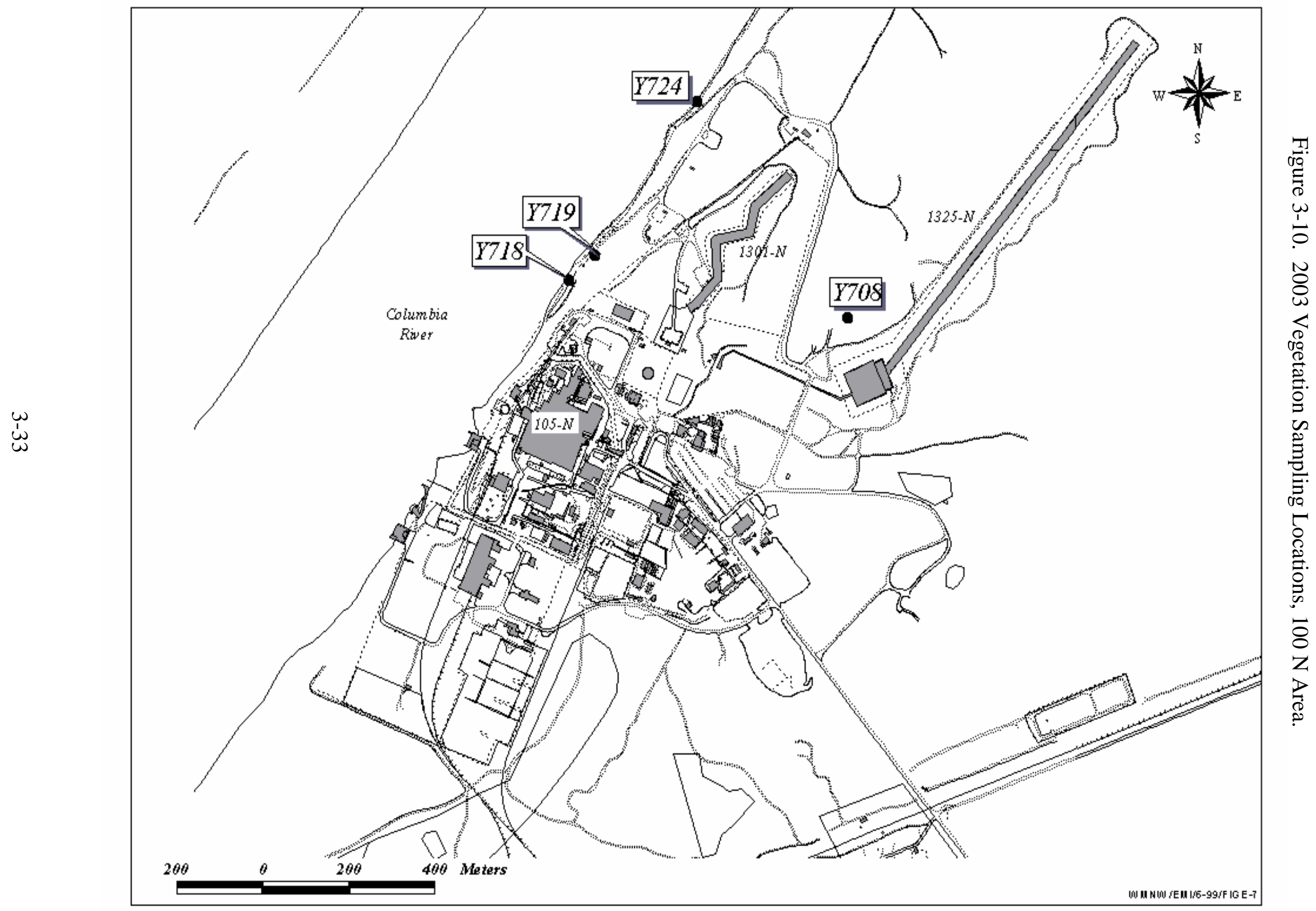




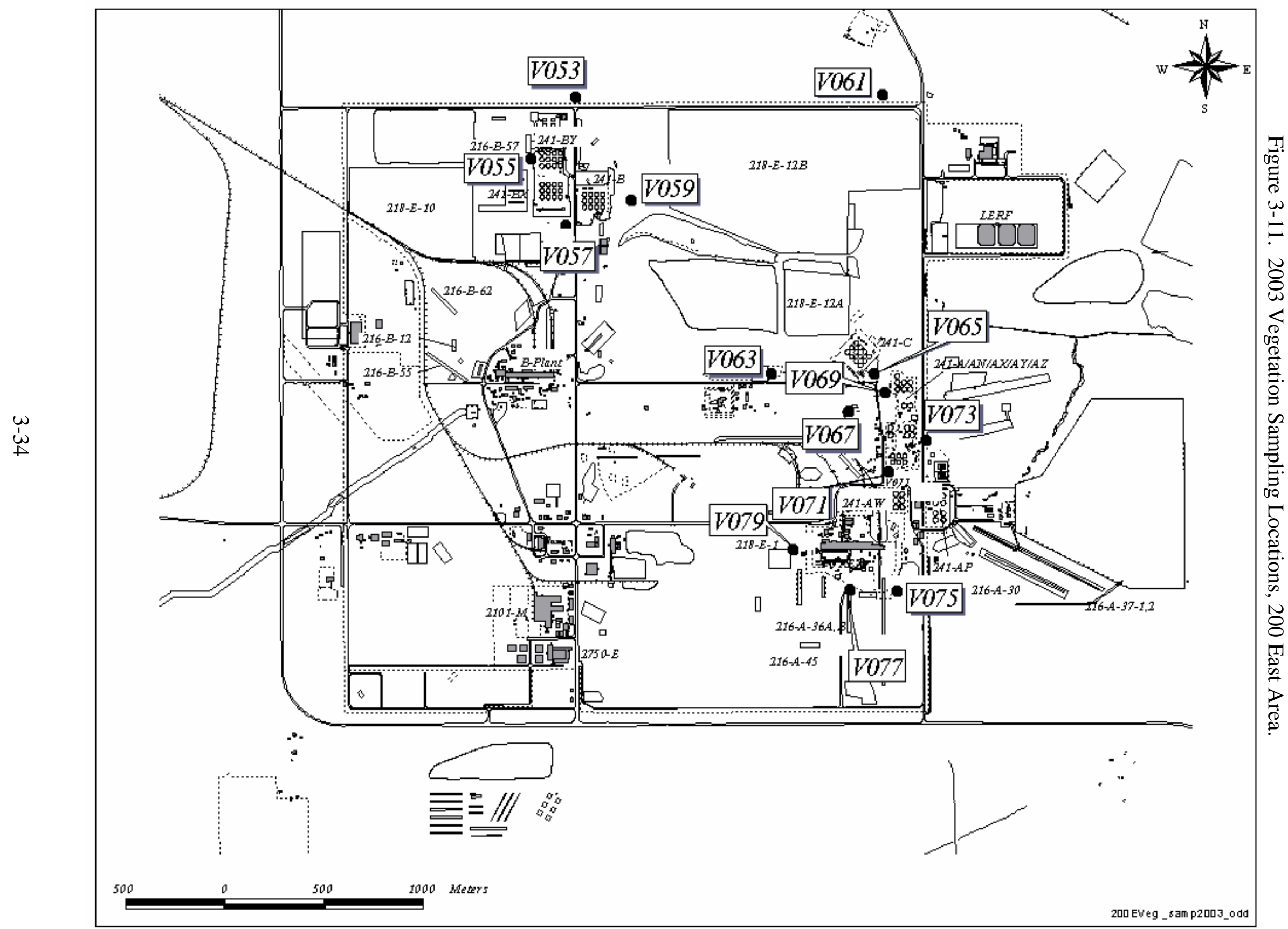




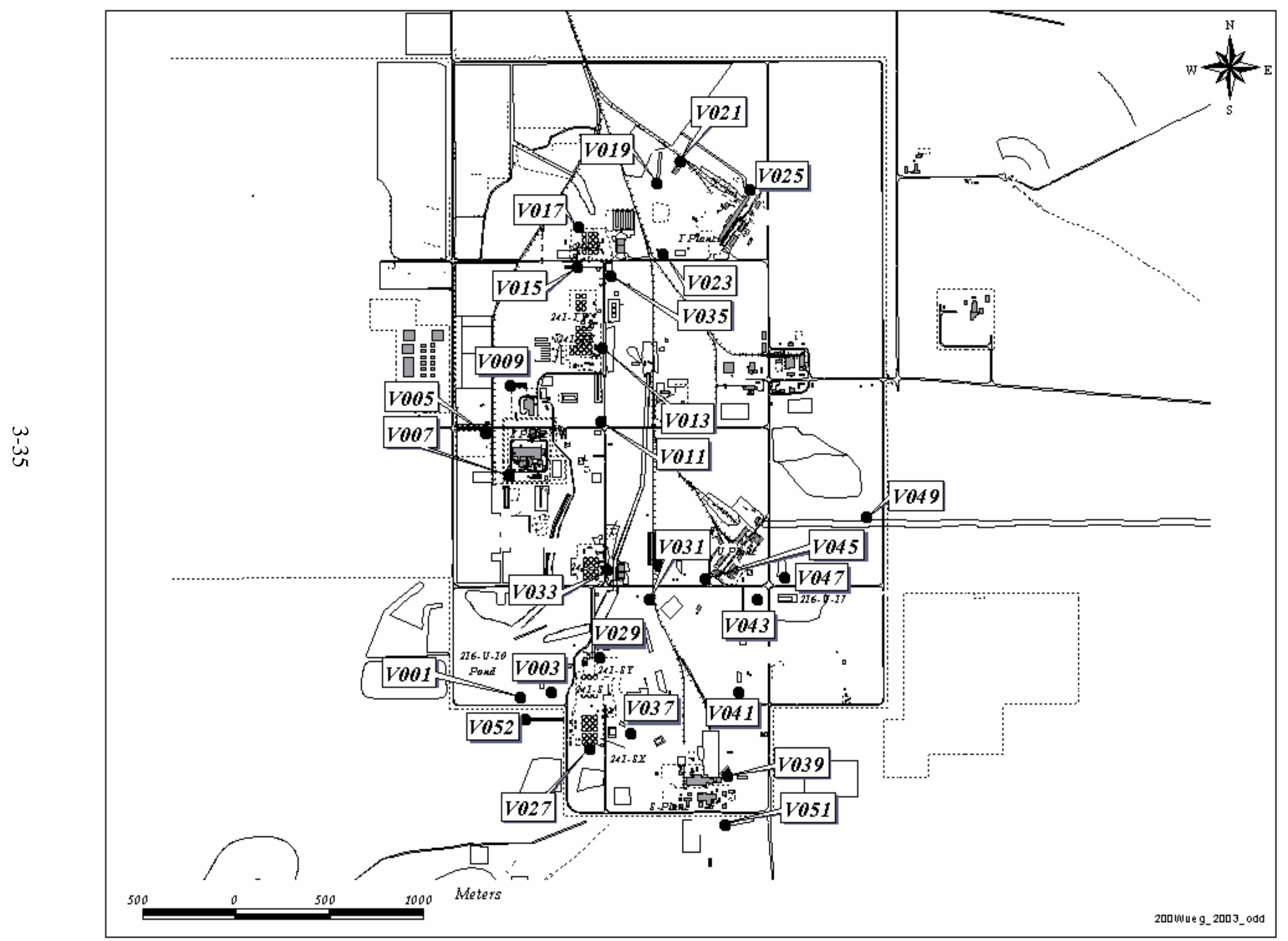

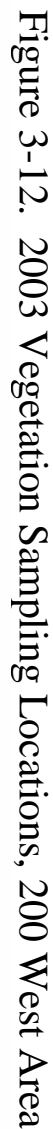




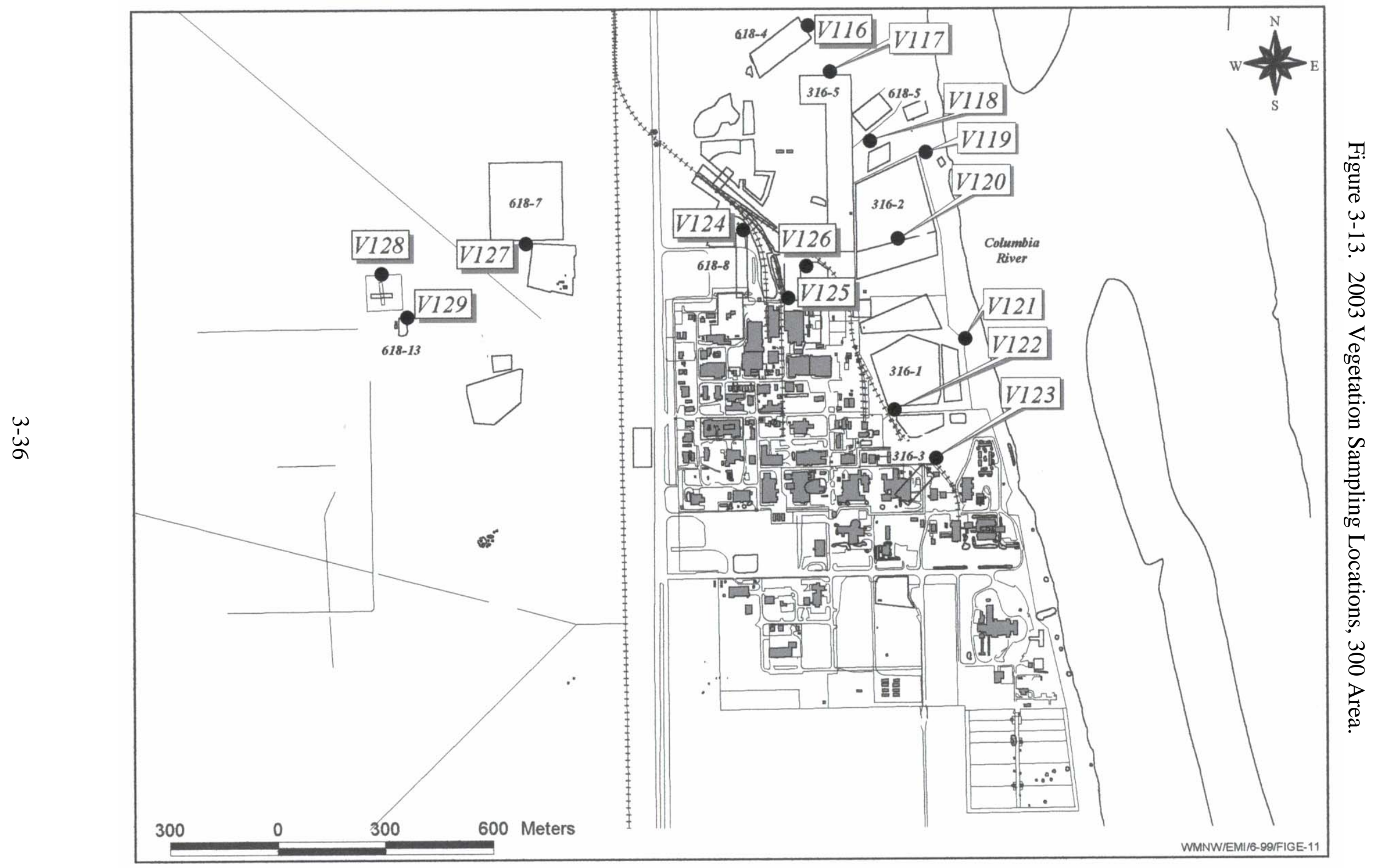




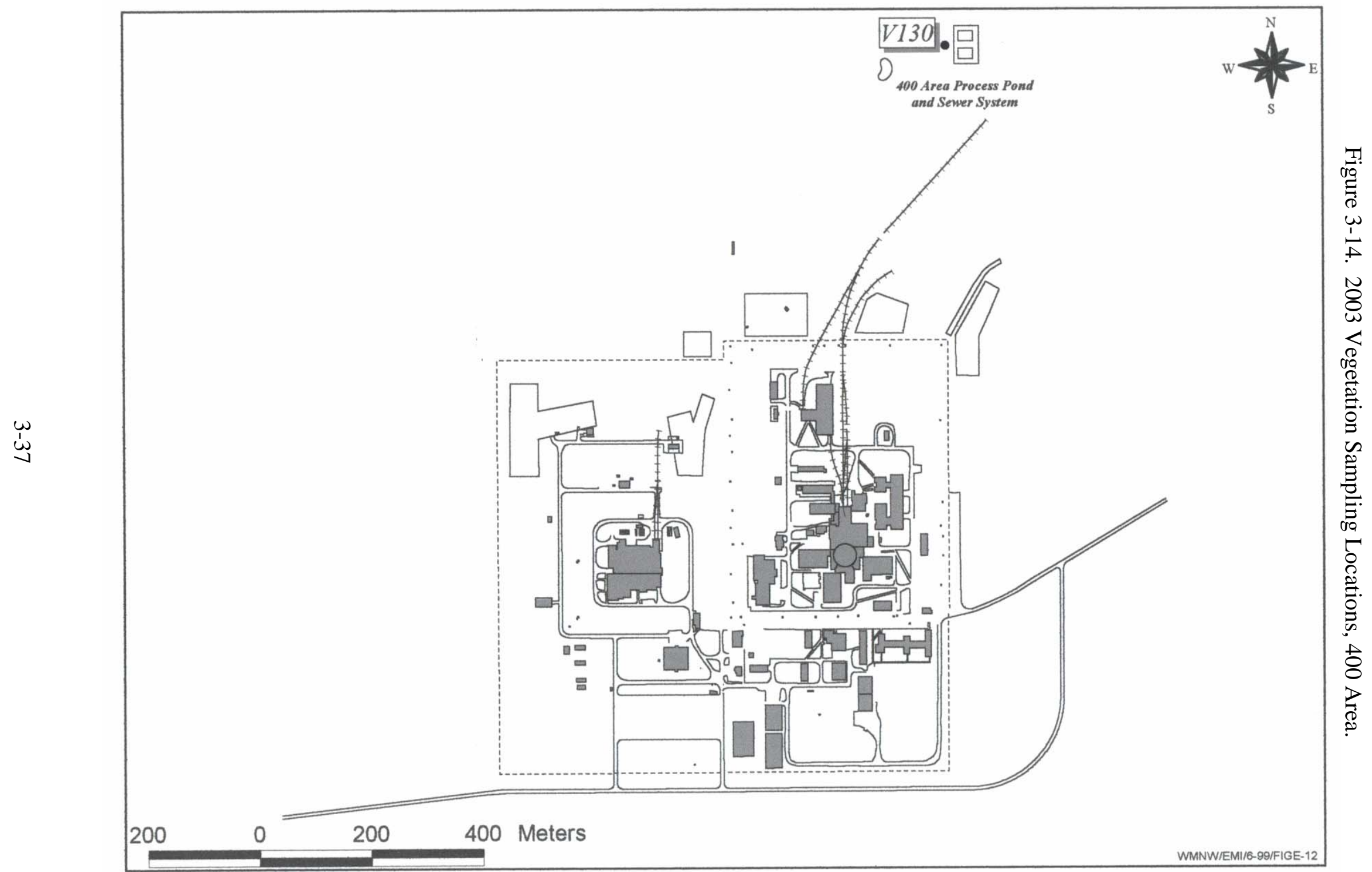




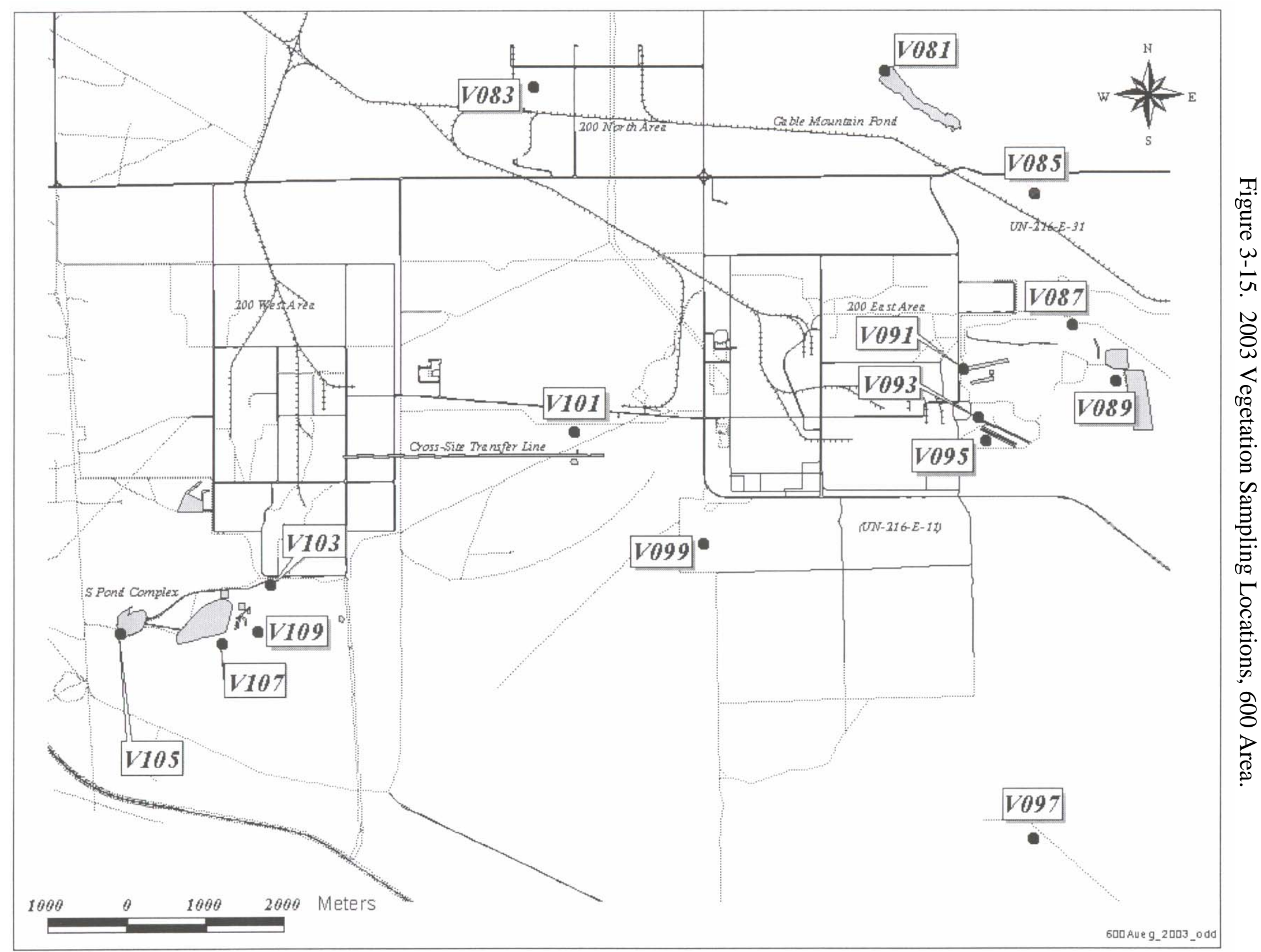




\section{Table 3-4. Average Radionuclide Concentrations ( $\mathrm{pCi} / \mathrm{g}^{\mathrm{a}}$ ) in Hanford Vegetation, 1995 through 2003.}

\begin{tabular}{|c|c|c|c|c|c|c|}
\hline \multirow[b]{2}{*}{ Year } & \multicolumn{6}{|c|}{ 100-N Area } \\
\hline & ${ }^{60} \mathrm{Co}$ & ${ }^{90} \mathrm{Sr}$ & ${ }^{137} \mathrm{Cs}$ & ${ }^{234} \mathbf{U}$ & ${ }^{238} \mathrm{U}$ & ${ }^{239,240} \mathbf{P u}$ \\
\hline 1995 & $3.0 \mathrm{E}-02 \pm 5.1 \mathrm{E}-02$ & $5.4 \mathrm{E}+00 \pm 4.8 \mathrm{E}+00$ & $8.1 \mathrm{E}-02 \pm 4.4 \mathrm{E}-02$ & $1.1 \mathrm{E}-02 \pm 6.6 \mathrm{E}-03$ & $9.2 \mathrm{E}-03 \pm 4.8 \mathrm{E}-03$ & $3.3 \mathrm{E}-03 \pm 1.6 \mathrm{E}-03$ \\
\hline 1996 & $2.4 \mathrm{E}+00 \pm 4.5 \mathrm{E}+00$ & $2.3 \mathrm{E}+02 \pm 4.4 \mathrm{E}+02$ & $2.3 \mathrm{E}+02 \pm 2.0 \mathrm{E}+02$ & $2.6 \mathrm{E}-02 \pm 3.2 \mathrm{E}-02$ & $2.2 \mathrm{E}-02 \pm 1.7 \mathrm{E}-01$ & $-5.1 \mathrm{E}-03 \pm 0.0 \mathrm{E}+00$ \\
\hline 1997 & $4.2 \mathrm{E}-01 \pm 5.0 \mathrm{E}-02$ & $3.6 \mathrm{E}+00 \pm 5.3 \mathrm{E}+00$ & $1.6 \mathrm{E}-01 \pm 7.7 \mathrm{E}-02$ & $1.3 \mathrm{E}-02 \pm 2.9 \mathrm{E}-03$ & $9.7 \mathrm{E}-03 \pm 4.7 \mathrm{E}-03$ & Not Detected \\
\hline 1998 & $6.2 \mathrm{E}-01 \pm 6.5 \mathrm{E}-01$ & $1.2 \mathrm{E}+01 \pm 6.0 \mathrm{E}+00$ & $3.8 \mathrm{E}+01 \pm 6.5 \mathrm{E}+01$ & $1.4 \mathrm{E}-02 \pm 6.0 \mathrm{E}-03$ & $8.7 \mathrm{E}-03 \pm 4.4 \mathrm{E}-03$ & $4.2 \mathrm{E}-03 \pm 2.3 \mathrm{E}-03$ \\
\hline 1999 & $6.1 \mathrm{E}-01 \pm 5.9 \mathrm{E}-01$ & $9.1 \mathrm{E}+01 \pm 1.0 \mathrm{E}+02$ & $2.5 \mathrm{E}+02 \pm 2.5 \mathrm{E}+02$ & $2.8 \mathrm{E}-02 \pm 1.0 \mathrm{E}-03$ & $2.1 \mathrm{E}-02 \pm 7.0 \mathrm{E}-03$ & $2.2 \mathrm{E}-02 \pm 1.0 \mathrm{E}-02$ \\
\hline 2000 & $4.8 \mathrm{E}-02 \pm 3.2 \mathrm{E}-02$ & $5.7 \mathrm{E}+00 \pm 8.7 \mathrm{E}+00$ & $2.0 \mathrm{E}-01 \pm 1.2 \mathrm{E}-01$ & $3.3 \mathrm{E}-02 \pm 2.7 \mathrm{E}-02$ & $2.4 \mathrm{E}-02 \pm 1.8 \mathrm{E}-02$ & $9.1 \mathrm{E}-03 \pm 8.3 \mathrm{E}-03$ \\
\hline 2001 & $8.9 \mathrm{E}-01 \pm 1.3 \mathrm{E}+00$ & $3.5 \mathrm{E}+00 \pm 3.4 \mathrm{E}+00$ & $3.8 \mathrm{E}-01 \pm 2.2 \mathrm{E}-01$ & $9.8 \mathrm{E}-03 \pm 2.4 \mathrm{E}-03$ & $9.2 \mathrm{E}-03 \pm 2.9 \mathrm{E}-03$ & $2.4 \mathrm{E}-02 \pm 2.5 \mathrm{E}-02$ \\
\hline 2002 & $3.7 \mathrm{E}-03 \pm 3.7 \mathrm{E}-02$ & $5.4 \mathrm{E}+00 \pm 1.8 \mathrm{E}+01$ & $2.4 \mathrm{E}-03 \pm 8.4 \mathrm{E}-03$ & $9.8 \mathrm{E}-03 \pm 4.5 \mathrm{E}-03$ & $5.1 \mathrm{E}-03 \pm 2.9 \mathrm{E}-03$ & $1.9 \mathrm{E}-03 \pm 5.3 \mathrm{E}-03$ \\
\hline 2003 & $6.6 \mathrm{E}-02 \pm 6.8 \mathrm{E}-02$ & $1.4 \mathrm{E}+01 \pm 4.5 \mathrm{E}+01$ & $1.5 \mathrm{E}-01 \pm 1.5 \mathrm{E}-01$ & $6.8 \mathrm{E}-03 \pm 2.1 \mathrm{E}-03$ & $4.6 \mathrm{E}-03 \pm 2.9 \mathrm{E}-03$ & $-2.8 \mathrm{E}-04 \pm 7.0 \mathrm{E}-03$ \\
\hline
\end{tabular}

\begin{tabular}{|c|c|c|c|c|c|c|}
\hline \multicolumn{7}{|c|}{$\underline{200 / 600 \text { Areas }}$} \\
\hline Year & ${ }^{60} \mathrm{Co}$ & ${ }^{90} \mathrm{Sr}$ & ${ }^{137} \mathrm{Cs}$ & ${ }^{234} \mathbf{U}$ & ${ }^{238} \mathrm{U}$ & ${ }^{239,240} \mathbf{P u}$ \\
\hline 1995 & $1.4 \mathrm{E}-02 \pm 2.1 \mathrm{E}-02$ & $1.4 \mathrm{E}-02 \pm 2.1 \mathrm{E}-02$ & $1.6 \mathrm{E}-01 \pm 1.4 \mathrm{E}-01$ & $1.1 \mathrm{E}-02 \pm 6.3 \mathrm{E}-03$ & $7.9 \mathrm{E}-03 \pm 4.4 \mathrm{E}-03$ & $4.9 \mathrm{E}-03 \pm 2.9 \mathrm{E}-03$ \\
\hline 1996 & $2.6 \mathrm{E}-02 \pm 2.4 \mathrm{E}-02$ & $3.7 \mathrm{E}-01 \pm 1.8 \mathrm{E}-01$ & $6.9 \mathrm{E}-02 \pm 3.0 \mathrm{E}-02$ & $5.0 \mathrm{E}-03 \pm 1.0 \mathrm{E}-03$ & $5.0 \mathrm{E}-03 \pm 1.0 \mathrm{E}-03$ & $4.1 \mathrm{E}-03 \pm 3.1 \mathrm{E}-03$ \\
\hline 1997 & Not Detected & $2.9 \mathrm{E}+00 \pm 2.5 \mathrm{E}+00$ & $1.3 \mathrm{E}-01 \pm 6.0 \mathrm{E}-02$ & $1.5 \mathrm{E}-02 \pm 2.4 \mathrm{E}-03$ & $1.1 \mathrm{E}-02 \pm 2.1 \mathrm{E}-03$ & $6.6 \mathrm{E}-03 \pm 1.0 \mathrm{E}-04$ \\
\hline 1998 & Not Detected & $3.3 \mathrm{E}-01 \pm 1.3 \mathrm{E}-01$ & $2.1 \mathrm{E}-01 \pm 9.0 \mathrm{E}-02$ & $1.6 \mathrm{E}-02 \pm 3.0 \mathrm{E}-03$ & $9.7 \mathrm{E}-03 \pm 1.3 \mathrm{E}-03$ & $1.8 \mathrm{E}-02 \pm 8.0 \mathrm{E}-03$ \\
\hline 1999 & Not Detected & 7.9E-01 \pm 3.8E-01 & $1.3 \mathrm{E}-01 \pm 4.0 \mathrm{E}-02$ & $3.3 \mathrm{E}-02 \pm 6.0 \mathrm{E}-03$ & $2.3 \mathrm{E}-02 \pm 4.0 \mathrm{E}-03$ & $1.4 \mathrm{E}-02 \pm 4.0 \mathrm{E}-03$ \\
\hline 2000 & Not Detected & $1.3 \mathrm{E}+00 \pm 8.0 \mathrm{E}-01$ & $1.6 \mathrm{E}-01 \pm 6.0 \mathrm{E}-02$ & $2.0 \mathrm{E}-02 \pm 3.0 \mathrm{E}-02$ & $1.4 \mathrm{E}-02 \pm 2.0 \mathrm{E}-03$ & $3.3 \mathrm{E}-02 \pm 2.8 \mathrm{E}-02$ \\
\hline 2001 & Not Detected & $1.0 \mathrm{E}+00 \pm 6.2 \mathrm{E}-01$ & $1.7 \mathrm{E}-01 \pm 6.5 \mathrm{E}-02$ & $1.9 \mathrm{E}-02 \pm 2.8 \mathrm{E}-03$ & $1.8 \mathrm{E}-02 \pm 2.6 \mathrm{E}-03$ & $2.1 \mathrm{E}-02 \pm 7.1 \mathrm{E}-03$ \\
\hline 2002 & $3.2 \mathrm{E}-04 \pm 1.8 \mathrm{E}-03$ & $3.2 \mathrm{E}-01 \pm 1.1 \mathrm{E}+00$ & $8.9 \mathrm{E}-02 \pm 4.2 \mathrm{E}-01$ & $1.6 \mathrm{E}-02 \pm 1.6 \mathrm{E}-02$ & $1.4 \mathrm{E}-02 \pm 1.5 \mathrm{E}-02$ & $8.8 \mathrm{E}-03 \pm 2.4 \mathrm{E}-02$ \\
\hline 2003 & $1.6 \mathrm{E}-02 \pm 2.1 \mathrm{E}-01$ & $1.5 \mathrm{E}+00 \pm 1.0 \mathrm{E}+01$ & $2.7 \mathrm{E}-01 \pm 2.0 \mathrm{E}+00$ & $1.0 \mathrm{E}-02 \pm 9.7 \mathrm{E}-03$ & $8.4 \mathrm{E}-03 \pm 9.0 \mathrm{E}-03$ & $2.7 \mathrm{E}-03 \pm 7.9 \mathrm{E}-03$ \\
\hline
\end{tabular}

\begin{tabular}{|c|c|c|c|c|c|c|}
\hline \multicolumn{7}{|c|}{$\underline{300 / 400 \text { Areas }}$} \\
\hline Year & ${ }^{60} \mathrm{Co}$ & ${ }^{90} \mathrm{Sr}$ & ${ }^{137}$ Cs & ${ }^{234} \mathbf{U}$ & ${ }^{238} \mathrm{U}$ & ${ }^{239,240} \mathrm{Pu}$ \\
\hline 1995 & $4.0 \mathrm{E}-02 \pm 3.0 \mathrm{E}-02$ & $5.1 \mathrm{E}-02 \pm 2.4 \mathrm{E}-02$ & Not Detected & $5.6 \mathrm{E}-02 \pm 4.1 \mathrm{E}-02$ & $5.6 \mathrm{E}-02 \pm 4.1 \mathrm{E}-02$ & $3.5 \mathrm{E}-04 \pm 1.9 \mathrm{E}-04$ \\
\hline 1996 & $7.1 \mathrm{E}-03 \pm 2.0 \mathrm{E}-02$ & $6.3 \mathrm{E}-02 \pm 2.5 \mathrm{E}-02$ & $1.6 \mathrm{E}-02 \pm 1.6 \mathrm{E}-02$ & $4.9 \mathrm{E}-02 \pm 3.9 \mathrm{E}-02$ & $4.7 \mathrm{E}-02 \pm 3.8 \mathrm{E}-02$ & $3.8 \mathrm{E}-04 \pm 1.9 \mathrm{E}-04$ \\
\hline 1997 & Not Detected & $6.6 \mathrm{E}-01 \pm 3.9 \mathrm{E}-01$ & Not Detected & $6.9 \mathrm{E}-02 \pm 4.8 \mathrm{E}-02$ & $6.2 \mathrm{E}-02 \pm 4.5 \mathrm{E}-02$ & $4.4 \mathrm{E}-04 \pm 2.9 \mathrm{E}-04$ \\
\hline 1998 & Not Detected & $1.0 \mathrm{E}-01 \pm 6.0 \mathrm{E}-02$ & Not Detected & $4.6 \mathrm{E}-02 \pm 3.3 \mathrm{E}-02$ & $4.4 \mathrm{E}-02 \pm 3.6 \mathrm{E}-02$ & $8.4 \mathrm{E}-03 \pm 4.5 \mathrm{E}-03$ \\
\hline 1999 & Not Detected & $4.5 \mathrm{E}-01 \pm 7.0 \mathrm{E}-02$ & Not Detected & $9.4 \mathrm{E}-02 \pm 5.3 \mathrm{E}-02$ & $8.9 \mathrm{E}-01 \pm 5.9 \mathrm{E}-02$ & $7.1 \mathrm{E}-03 \pm 3.2 \mathrm{E}-03$ \\
\hline 2000 & Not Detected & $2.1 \mathrm{E}-01 \pm 3.0 \mathrm{E}-02$ & Not Detected & $1.8 \mathrm{E}-02 \pm 1.9 \mathrm{E}-02$ & $1.7 \mathrm{E}-02 \pm 1.9 \mathrm{E}-02$ & $9.1 \mathrm{E}-03 \pm 2.4 \mathrm{E}-03$ \\
\hline 2001 & Not Detected & $2.6 \mathrm{E}-01 \pm 1.1 \mathrm{E}-01$ & Not Detected & $9.8 \mathrm{E}-02 \pm 8.0 \mathrm{E}-02$ & $1.1 \mathrm{E}-01 \pm 8.8 \mathrm{E}-02$ & $5.8 \mathrm{E}-03 \pm 1.5 \mathrm{E}-03$ \\
\hline 2002 & Not Detected & $2.1 \mathrm{E}-01 \pm 4.7 \mathrm{E}-01$ & $1.1 \mathrm{E}-02 \pm 7.9 \mathrm{E}-02$ & $3.2 \mathrm{E}-02 \pm 5.5 \mathrm{E}-02$ & $2.9 \mathrm{E}-02 \pm 5.8 \mathrm{E}-02$ & $-3.6 \mathrm{E}-04 \pm 7.2 \mathrm{E}-04$ \\
\hline 2003 & $5.0 \mathrm{E}-03 \pm 3.8 \mathrm{E}-02$ & $-8.2 \mathrm{E}-02 \pm 2.0 \mathrm{E}-01$ & $-9.4 \mathrm{E}-03 \pm 4.4 \mathrm{E}-02$ & $4.3 \mathrm{E}-02 \pm 1.1 \mathrm{E}-01$ & $3.6 \mathrm{E}-02 \pm 1.9 \mathrm{E}-01$ & $1.7 \mathrm{E}-03 \pm 1.7 \mathrm{E}-02$ \\
\hline
\end{tabular}

$\overline{\mathrm{a} \pm 2 \text { standard deviations }}$ 
Table 3-5. 2003 Vegetation Sampling Results (pCi/g \pm total analytical uncertainty).

\begin{tabular}{|c|c|c|c|c|c|c|c|}
\hline Location & Isotope & Result \pm Error & $\mathbf{R Q}^{*}$ & Location & Isotope & Result \pm Error & $\mathbf{R Q}^{*}$ \\
\hline Y708 & ${ }^{144} \mathrm{Ce}$ & $8.1 \mathrm{E}-02 \pm 5.6 \mathrm{E}-01$ & $\mathrm{U}$ & Y718 & ${ }^{144} \mathrm{Ce}$ & $-1.5 \mathrm{E}-01 \pm 4.8 \mathrm{E}-01$ & $\bar{U}$ \\
\hline \multirow[t]{17}{*}{$(100-N)$} & ${ }^{60} \mathrm{Co}$ & $1.7 \mathrm{E}-01 \pm 6.4 \mathrm{E}-02$ & & (N Springs & ${ }^{60} \mathrm{Co}$ & 2.3E-02 \pm 4.3E-02 & $\mathrm{U}$ \\
\hline & ${ }^{134} \mathrm{Cs}$ & $4.0 \mathrm{E}-02 \pm 4.6 \mathrm{E}-02$ & $\mathrm{U}$ & Shoreline) & ${ }^{134} \mathrm{Cs}$ & $1.2 \mathrm{E}-02 \pm 4.4 \mathrm{E}-02$ & $\mathrm{U}$ \\
\hline & ${ }^{137} \mathrm{Cs}$ & $5.1 \mathrm{E}-01 \pm 1.3 \mathrm{E}-01$ & & & ${ }^{137} \mathrm{Cs}$ & $-2.0 \mathrm{E}-02 \pm 4.2 \mathrm{E}-02$ & $\mathrm{U}$ \\
\hline & ${ }^{152} \mathrm{Eu}$ & $-1.2 \mathrm{E}-01 \pm 1.3 \mathrm{E}-01$ & $\mathrm{U}$ & & ${ }^{152} \mathrm{Eu}$ & $-8.6 \mathrm{E}-02 \pm 1.4 \mathrm{E}-01$ & $\mathrm{U}$ \\
\hline & ${ }^{154} \mathrm{Eu}$ & $-8.3 \mathrm{E}-02 \pm 1.4 \mathrm{E}-01$ & $\mathrm{U}$ & & ${ }^{154} \mathrm{Eu}$ & $-9.3 \mathrm{E}-02 \pm 1.4 \mathrm{E}-01$ & $\mathrm{U}$ \\
\hline & ${ }^{155} \mathrm{Eu}$ & $-7.6 \mathrm{E}-02 \pm 1.4 \mathrm{E}-01$ & $\mathrm{U}$ & & ${ }^{155} \mathrm{Eu}$ & $-3.5 \mathrm{E}-02 \pm 1.5 \mathrm{E}-01$ & $\mathrm{U}$ \\
\hline & ${ }^{238} \mathrm{Pu}$ & $3.0 \mathrm{E}-03 \pm 1.5 \mathrm{E}-02$ & $\mathrm{U}$ & & ${ }^{238} \mathrm{Pu}$ & $-1.9 \mathrm{E}-03 \pm 7.6 \mathrm{E}-03$ & $\mathrm{U}$ \\
\hline & ${ }^{239,240} \mathrm{Pu}$ & $4.0 \mathrm{E}-03 \pm 6.4 \mathrm{E}-03$ & $\mathrm{U}$ & & ${ }^{239,240} \mathrm{Pu}$ & $9.5 \mathrm{E}-04 \pm 3.3 \mathrm{E}-03$ & $\mathrm{U}$ \\
\hline & ${ }^{103} \mathrm{Ru}$ & $-2.4 \mathrm{E}-02 \pm 3.9 \mathrm{E}-02$ & $\mathrm{U}$ & & ${ }^{103} \mathrm{Ru}$ & 4.2E-03 $\pm 3.7 \mathrm{E}-02$ & $\mathrm{U}$ \\
\hline & ${ }^{106} \mathrm{Ru}$ & $-3.1 \mathrm{E}-01 \pm 3.9 \mathrm{E}-01$ & $\mathrm{U}$ & & ${ }^{106} \mathrm{Ru}$ & $1.7 \mathrm{E}-01 \pm 4.6 \mathrm{E}-01$ & $\mathrm{U}$ \\
\hline & ${ }^{125} \mathrm{Sb}$ & $-1.6 \mathrm{E}-02 \pm 1.1 \mathrm{E}-01$ & $\mathrm{U}$ & & ${ }^{125} \mathrm{Sb}$ & $3.6 \mathrm{E}-03 \pm 3.6 \mathrm{E}-02$ & $\mathrm{U}$ \\
\hline & ${ }^{113} \mathrm{Sn}$ & $-6.8 \mathrm{E}-03 \pm 4.9 \mathrm{E}-02$ & $\mathrm{U}$ & & ${ }^{113} \mathrm{Sn}$ & $7.0 \mathrm{E}-03 \pm 4.7 \mathrm{E}-02$ & $\mathrm{U}$ \\
\hline & ${ }^{90} \mathrm{Sr}$ & $5.1 \mathrm{E}-03 \pm 5.1 \mathrm{E}-02$ & $\mathrm{U}$ & & ${ }^{90} \mathrm{Sr}$ & $1.7 \mathrm{E}+00 \pm 3.4 \mathrm{E}-01$ & \\
\hline & ${ }^{234} \mathrm{U}$ & $5.7 \mathrm{E}-03 \pm 5.1 \mathrm{E}-03$ & $\mathrm{U}$ & & & $7.2 \mathrm{E}-03 \pm 5.1 \mathrm{E}-03$ & \\
\hline & ${ }^{235} \mathrm{U}$ & $8.9 \mathrm{E}-04 \pm 3.1 \mathrm{E}-03$ & $\mathrm{U}$ & & ${ }^{235} \mathrm{U}$ & $2.6 \mathrm{E}-03 \pm 4.7 \mathrm{E}-03$ & $\mathrm{U}$ \\
\hline & ${ }^{238} \mathrm{U}$ & $4.1 \mathrm{E}-03 \pm 4.5 \mathrm{E}-03$ & U & & ${ }^{238} \mathrm{U}$ & $2.4 \mathrm{E}-03 \pm 4.3 \mathrm{E}-03$ & $\mathrm{U}$ \\
\hline & ${ }^{65} \mathrm{Zn}$ & $-1.9 \mathrm{E}-01 \pm 1.9 \mathrm{E}-01$ & $\mathrm{U}$ & & ${ }^{65} \mathrm{Zn}$ & $1.4 \mathrm{E}-01 \pm 1.2 \mathrm{E}-01$ & $\mathrm{U}$ \\
\hline Y719 & ${ }^{144} \mathrm{Ce}$ & 4.9E-01 \pm 5.3E-01 & $\bar{U}$ & Y724 & ${ }^{144} \mathrm{Ce}$ & 1.9E-01 $\pm 6.3 \mathrm{E}-01$ & $\mathrm{U}$ \\
\hline (N Springs & ${ }^{60} \mathrm{Co}$ & $2.5 \mathrm{E}-02 \pm 4.6 \mathrm{E}-02$ & $\mathrm{U}$ & (N Springs & ${ }^{60} \mathrm{Co}$ & 4.4E-02 $\pm 4.6 \mathrm{E}-02$ & $\mathrm{U}$ \\
\hline \multirow[t]{16}{*}{ Shoreline) } & ${ }^{134} \mathrm{Cs}$ & $8.0 \mathrm{E}-03 \pm 5.1 \mathrm{E}-02$ & $\mathrm{U}$ & Shoreline) & ${ }^{134} \mathrm{Cs}$ & $1.8 \mathrm{E}-03 \pm 1.8 \mathrm{E}-02$ & $\mathrm{U}$ \\
\hline & ${ }^{137} \mathrm{Cs}$ & $4.2 \mathrm{E}-02 \pm 4.3 \mathrm{E}-02$ & $\mathrm{U}$ & & ${ }^{137} \mathrm{Cs}$ & $4.9 \mathrm{E}-02 \pm 4.8 \mathrm{E}-02$ & $\mathrm{U}$ \\
\hline & ${ }^{152} \mathrm{Eu}$ & $-7.6 \mathrm{E}-03 \pm 7.6 \mathrm{E}-02$ & $\mathrm{U}$ & & ${ }^{152} \mathrm{Eu}$ & $3.6 \mathrm{E}-02 \pm 2.5 \mathrm{E}-01$ & $\mathrm{U}$ \\
\hline & ${ }^{154} \mathrm{Eu}$ & $-9.6 \mathrm{E}-02 \pm 1.4 \mathrm{E}-01$ & $\mathrm{U}$ & & ${ }^{154} \mathrm{Eu}$ & $7.5 \mathrm{E}-03 \pm 7.5 \mathrm{E}-02$ & $\mathrm{U}$ \\
\hline & ${ }^{155} \mathrm{Eu}$ & $7.5 \mathrm{E}-02 \pm 1.5 \mathrm{E}-01$ & $\mathrm{U}$ & & ${ }^{155} \mathrm{Eu}$ & $-8.3 \mathrm{E}-02 \pm 1.7 \mathrm{E}-01$ & $\mathrm{U}$ \\
\hline & ${ }^{238} \mathrm{Pu}$ & $9.4 \mathrm{E}-04 \pm 9.4 \mathrm{E}-03$ & $\mathrm{U}$ & & ${ }^{238} \mathrm{Pu}$ & $9.0 \mathrm{E}-03 \pm 1.6 \mathrm{E}-02$ & $\mathrm{U}$ \\
\hline & ${ }^{239,240} \mathrm{Pu}$ & $-1.9 \mathrm{E}-03 \pm 3.8 \mathrm{E}-03$ & $\mathrm{U}$ & & ${ }^{239,240} \mathrm{Pu}$ & $-4.0 \mathrm{E}-03 \pm 4.0 \mathrm{E}-03$ & $\mathrm{U}$ \\
\hline & ${ }^{103} \mathrm{Ru}$ & $-1.7 \mathrm{E}-02 \pm 3.8 \mathrm{E}-02$ & $\mathrm{U}$ & & ${ }^{103} \mathrm{Ru}$ & $2.3 \mathrm{E}-02 \pm 4.3 \mathrm{E}-02$ & $\mathrm{U}$ \\
\hline & ${ }^{106} \mathrm{Ru}$ & $2.2 \mathrm{E}-01 \pm 3.9 \mathrm{E}-01$ & $\mathrm{U}$ & & ${ }^{106} \mathrm{Ru}$ & $-1.8 \mathrm{E}-01 \pm 4.2 \mathrm{E}-01$ & $\mathrm{U}$ \\
\hline & ${ }^{125} \mathrm{Sb}$ & $-7.8 \mathrm{E}-02 \pm 1.0 \mathrm{E}-01$ & $\mathrm{U}$ & & ${ }^{125} \mathrm{Sb}$ & $2.9 \mathrm{E}-02 \pm 1.2 \mathrm{E}-01$ & $\mathrm{U}$ \\
\hline & ${ }^{113} \mathrm{Sn}$ & $1.9 \mathrm{E}-02 \pm 5.1 \mathrm{E}-02$ & $\mathrm{U}$ & & ${ }^{113} \mathrm{Sn}$ & $3.4 \mathrm{E}-02 \pm 5.5 \mathrm{E}-02$ & $\mathrm{U}$ \\
\hline & ${ }^{90} \mathrm{Sr}$ & $5.3 \mathrm{E}+01 \pm 7.9 \mathrm{E}+00$ & & & ${ }^{90} \mathrm{Sr}$ & $2.4 \mathrm{E}-01 \pm 1.2 \mathrm{E}-01$ & \\
\hline & ${ }^{234} \mathrm{U}$ & $5.9 \mathrm{E}-03 \pm 5.3 \mathrm{E}-03$ & $\mathrm{U}$ & & ${ }^{234} \mathrm{U}$ & $8.3 \mathrm{E}-03 \pm 5.6 \mathrm{E}-03$ & \\
\hline & ${ }^{235} \mathrm{U}$ & $2.8 \mathrm{E}-03 \pm 4.2 \mathrm{E}-03$ & $\mathrm{U}$ & & ${ }^{235} \mathrm{U}$ & $2.7 \mathrm{E}-03 \pm 3.2 \mathrm{E}-03$ & \\
\hline & ${ }^{238} \mathrm{U}$ & $5.9 \mathrm{E}-03 \pm 4.7 \mathrm{E}-03$ & & & ${ }^{238} \mathrm{U}$ & $5.8 \mathrm{E}-03 \pm 4.6 \mathrm{E}-03$ & \\
\hline & ${ }^{65} \mathrm{Zn}$ & $8.5 \mathrm{E}-02 \pm 1.1 \mathrm{E}-01$ & $\mathrm{U}$ & & ${ }^{65} \mathrm{Zn}$ & $1.3 \mathrm{E}-01 \pm 1.1 \mathrm{E}-01$ & $\mathrm{U}$ \\
\hline
\end{tabular}

$\overline{\mathrm{RQ}}=$ Result Qualifier. U $=$ The analyte was analyzed for but not detected. 
Table 3-5. 2003 Vegetation Sampling Results (pCi/g \pm total analytical uncertainty). (cont)

\begin{tabular}{|c|c|c|c|c|c|c|c|}
\hline Location & Isotope & Result \pm Error & $\mathbf{R Q}^{*}$ & Location & Isotope & Result \pm Error & $\mathbf{R Q}^{*}$ \\
\hline V001 & ${ }^{144} \mathrm{Ce}$ & $-5.6 \mathrm{E}-01 \pm 8.5 \mathrm{E}-01$ & $\mathrm{U}$ & V003 & ${ }^{144} \mathrm{Ce}$ & $-1.0 \mathrm{E}-01 \pm 7.3 \mathrm{E}-01$ & $\overline{\mathrm{U}}$ \\
\hline \multirow[t]{17}{*}{ (200 West) } & ${ }^{60} \mathrm{Co}$ & $-2.3 \mathrm{E}-03 \pm 2.3 \mathrm{E}-02$ & $\mathrm{U}$ & (200 West) & ${ }^{60} \mathrm{Co}$ & $-1.4 \mathrm{E}-02 \pm 6.2 \mathrm{E}-02$ & $\mathrm{U}$ \\
\hline & ${ }^{134} \mathrm{Cs}$ & $-5.8 \mathrm{E}-03 \pm 5.8 \mathrm{E}-02$ & $\mathrm{U}$ & & ${ }^{134} \mathrm{Cs}$ & $3.4 \mathrm{E}-02 \pm 5.8 \mathrm{E}-02$ & $\mathrm{U}$ \\
\hline & ${ }^{137} \mathrm{Cs}$ & $1.3 \mathrm{E}-01 \pm 7.0 \mathrm{E}-02$ & & & ${ }^{137} \mathrm{Cs}$ & $1.3 \mathrm{E}+00 \pm 2.6 \mathrm{E}-01$ & \\
\hline & ${ }^{152} \mathrm{Eu}$ & $-1.0 \mathrm{E}-01 \pm 2.2 \mathrm{E}-01$ & $\mathrm{U}$ & & ${ }^{152} \mathrm{Eu}$ & $-5.4 \mathrm{E}-02 \pm 1.6 \mathrm{E}-01$ & $\mathrm{U}$ \\
\hline & ${ }^{154} \mathrm{Eu}$ & $-2.8 \mathrm{E}-01 \pm 2.8 \mathrm{E}-01$ & $\mathrm{U}$ & & ${ }^{154} \mathrm{Eu}$ & $1.6 \mathrm{E}-01 \pm 2.1 \mathrm{E}-01$ & $\mathrm{U}$ \\
\hline & ${ }^{155} \mathrm{Eu}$ & $1.3 \mathrm{E}-02 \pm 1.3 \mathrm{E}-01$ & $\mathrm{U}$ & & ${ }^{155} \mathrm{Eu}$ & $1.1 \mathrm{E}-01 \pm 2.0 \mathrm{E}-01$ & $\mathrm{U}$ \\
\hline & ${ }^{238} \mathrm{Pu}$ & $1.9 \mathrm{E}-03 \pm 1.4 \mathrm{E}-02$ & $\mathrm{U}$ & & ${ }^{238} \mathrm{Pu}$ & $-9.7 \mathrm{E}-03 \pm 1.4 \mathrm{E}-02$ & $\mathrm{U}$ \\
\hline & ${ }^{239,240} \mathrm{Pu}$ & $-9.4 \mathrm{E}-04 \pm 4.2 \mathrm{E}-03$ & $\mathrm{U}$ & & ${ }^{239,240} \mathrm{Pu}$ & $2.9 \mathrm{E}-03 \pm 6.4 \mathrm{E}-03$ & $\mathrm{U}$ \\
\hline & ${ }^{103} \mathrm{Ru}$ & $-1.3 \mathrm{E}-02 \pm 6.1 \mathrm{E}-02$ & $\mathrm{U}$ & & ${ }^{103} \mathrm{Ru}$ & $1.5 \mathrm{E}-02 \pm 5.1 \mathrm{E}-02$ & $\mathrm{U}$ \\
\hline & ${ }^{106} \mathrm{Ru}$ & $1.6 \mathrm{E}-01 \pm 5.5 \mathrm{E}-01$ & $\mathrm{U}$ & & ${ }^{106} \mathrm{Ru}$ & $-1.7 \mathrm{E}-01 \pm 5.0 \mathrm{E}-01$ & $\mathrm{U}$ \\
\hline & ${ }^{125} \mathrm{Sb}$ & $7.3 \mathrm{E}-02 \pm 1.6 \mathrm{E}-01$ & $\mathrm{U}$ & & ${ }^{125} \mathrm{Sb}$ & $7.8 \mathrm{E}-02 \pm 1.4 \mathrm{E}-01$ & $\mathrm{U}$ \\
\hline & ${ }^{113} \mathrm{Sn}$ & $1.0 \mathrm{E}-02 \pm 7.2 \mathrm{E}-02$ & $\mathrm{U}$ & & ${ }^{113} \mathrm{Sn}$ & $-2.7 \mathrm{E}-03 \pm 2.7 \mathrm{E}-02$ & $\mathrm{U}$ \\
\hline & ${ }^{90} \mathrm{Sr}$ & $2.2 \mathrm{E}+00 \pm 4.6 \mathrm{E}-01$ & & & ${ }^{90} \mathrm{Sr}$ & $2.5 \mathrm{E}+01 \pm 3.8 \mathrm{E}+00$ & \\
\hline & ${ }^{234} \mathrm{U}$ & $2.0 \mathrm{E}-02 \pm 1.1 \mathrm{E}-02$ & & & ${ }^{234} \mathrm{U}$ & $1.7 \mathrm{E}-02 \pm 1.0 \mathrm{E}-02$ & \\
\hline & ${ }^{235} \mathrm{U}$ & $1.1 \mathrm{E}-03 \pm 2.2 \mathrm{E}-03$ & $\mathrm{U}$ & & ${ }^{235} \mathrm{U}$ & $4.4 \mathrm{E}-03 \pm 6.6 \mathrm{E}-03$ & $\mathrm{U}$ \\
\hline & ${ }^{238} \mathrm{U}$ & $1.0 \mathrm{E}-02 \pm 6.6 \mathrm{E}-03$ & & & ${ }^{238} \mathrm{U}$ & $1.5 \mathrm{E}-02 \pm 9.4 \mathrm{E}-03$ & \\
\hline & ${ }^{65} \mathrm{Zn}$ & $-2.2 \mathrm{E}-01 \pm 2.2 \mathrm{E}-01$ & $\mathrm{U}$ & & ${ }^{65} \mathrm{Zn}$ & $1.6 \mathrm{E}-01 \pm 1.6 \mathrm{E}-01$ & $\mathrm{U}$ \\
\hline V007 & ${ }^{144} \mathrm{Ce}$ & $-2.0 \mathrm{E}-01 \pm 3.8 \mathrm{E}-01$ & $\overline{\mathrm{U}}$ & V009 & ${ }^{144} \mathrm{Ce}$ & $-1.9 \mathrm{E}-01 \pm 4.9 \mathrm{E}-01$ & $\bar{U}$ \\
\hline \multirow[t]{17}{*}{ (200 West) } & ${ }^{60} \mathrm{Co}$ & $-6.6 \mathrm{E}-03 \pm 2.9 \mathrm{E}-02$ & $\mathrm{U}$ & (200 West) & ${ }^{60} \mathrm{Co}$ & $1.6 \mathrm{E}-02 \pm 4.4 \mathrm{E}-02$ & $\mathrm{U}$ \\
\hline & ${ }^{134} \mathrm{Cs}$ & $1.5 \mathrm{E}-02 \pm 2.9 \mathrm{E}-02$ & $\mathrm{U}$ & & ${ }^{134} \mathrm{Cs}$ & $3.2 \mathrm{E}-03 \pm 3.2 \mathrm{E}-02$ & $\mathrm{U}$ \\
\hline & ${ }^{137} \mathrm{Cs}$ & $2.4 \mathrm{E}-02 \pm 2.8 \mathrm{E}-02$ & $\mathrm{U}$ & & ${ }^{137} \mathrm{Cs}$ & $5.3 \mathrm{E}-03 \pm 4.5 \mathrm{E}-02$ & $\mathrm{U}$ \\
\hline & ${ }^{152} \mathrm{Eu}$ & $8.7 \mathrm{E}-02 \pm 8.5 \mathrm{E}-02$ & $\mathrm{U}$ & & ${ }^{152} \mathrm{Eu}$ & $-2.5 \mathrm{E}-02 \pm 1.1 \mathrm{E}-01$ & $\mathrm{U}$ \\
\hline & ${ }^{154} \mathrm{Eu}$ & $-7.3 \mathrm{E}-02 \pm 8.9 \mathrm{E}-02$ & $\mathrm{U}$ & & ${ }^{154} \mathrm{Eu}$ & $-7.7 \mathrm{E}-02 \pm 1.4 \mathrm{E}-01$ & $\mathrm{U}$ \\
\hline & ${ }^{155} \mathrm{Eu}$ & $-2.5 \mathrm{E}-02 \pm 9.6 \mathrm{E}-02$ & $\mathrm{U}$ & & ${ }^{155} \mathrm{Eu}$ & $-5.1 \mathrm{E}-02 \pm 1.3 \mathrm{E}-01$ & $\mathrm{U}$ \\
\hline & ${ }^{238} \mathrm{Pu}$ & $1.3 \mathrm{E}-02 \pm 1.2 \mathrm{E}-02$ & $\mathrm{U}$ & & ${ }^{238} \mathrm{Pu}$ & $3.7 \mathrm{E}-03 \pm 1.6 \mathrm{E}-02$ & $\mathrm{U}$ \\
\hline & ${ }^{239,240} \mathrm{Pu}$ & $3.5 \mathrm{E}-03 \pm 4.9 \mathrm{E}-03$ & $\mathrm{U}$ & & ${ }^{239,240} \mathrm{Pu}$ & $6.5 \mathrm{E}-03 \pm 6.3 \mathrm{E}-03$ & $\mathrm{U}$ \\
\hline & ${ }^{103} \mathrm{Ru}$ & $-4.3 \mathrm{E}-03 \pm 2.6 \mathrm{E}-02$ & $\mathrm{U}$ & & ${ }^{103} \mathrm{Ru}$ & $1.3 \mathrm{E}-02 \pm 4.0 \mathrm{E}-02$ & $\mathrm{U}$ \\
\hline & ${ }^{106} \mathrm{Ru}$ & $5.8 \mathrm{E}-02 \pm 2.4 \mathrm{E}-01$ & $\mathrm{U}$ & & ${ }^{106} \mathrm{Ru}$ & $6.3 \mathrm{E}-02 \pm 3.9 \mathrm{E}-01$ & $\mathrm{U}$ \\
\hline & ${ }^{125} \mathrm{Sb}$ & 4.3E-02 $\pm 8.2 \mathrm{E}-02$ & $\mathrm{U}$ & & ${ }^{125} \mathrm{Sb}$ & $3.4 \mathrm{E}-02 \pm 1.1 \mathrm{E}-01$ & $\mathrm{U}$ \\
\hline & ${ }^{113} \mathrm{Sn}$ & $-5.2 \mathrm{E}-03 \pm 3.5 \mathrm{E}-02$ & $\mathrm{U}$ & & ${ }^{113} \mathrm{Sn}$ & $-2.9 \mathrm{E}-03 \pm 2.9 \mathrm{E}-02$ & $\mathrm{U}$ \\
\hline & ${ }^{90} \mathrm{Sr}$ & $-8.6 \mathrm{E}-02 \pm 8.6 \mathrm{E}-02$ & $\mathrm{U}$ & & ${ }^{90} \mathrm{Sr}$ & $-7.2 \mathrm{E}-02 \pm 7.9 \mathrm{E}-02$ & $\mathrm{U}$ \\
\hline & ${ }^{234} \mathrm{U}$ & $6.4 \mathrm{E}-03 \pm 8.3 \mathrm{E}-03$ & $\mathrm{U}$ & & ${ }^{234} \mathrm{U}$ & $1.6 \mathrm{E}-02 \pm 8.8 \mathrm{E}-03$ & \\
\hline & ${ }^{235} \mathrm{U}$ & $3.0 \mathrm{E}-03 \pm 3.6 \mathrm{E}-03$ & & & ${ }^{235} \mathrm{U}$ & $4.7 \mathrm{E}-03 \pm 4.3 \mathrm{E}-03$ & \\
\hline & ${ }^{238} \mathrm{U}$ & $1.0 \mathrm{E}-02 \pm 6.8 \mathrm{E}-03$ & & & ${ }^{238} \mathrm{U}$ & $9.5 \mathrm{E}-03 \pm 6.0 \mathrm{E}-03$ & \\
\hline & ${ }^{65} \mathrm{Zn}$ & $-2.9 \mathrm{E}-01 \pm 2.9 \mathrm{E}-01$ & $\mathrm{U}$ & & ${ }^{65} \mathrm{Zn}$ & $-2.0 \mathrm{E}-02 \pm 1.0 \mathrm{E}-01$ & $\mathrm{U}$ \\
\hline
\end{tabular}

$\overline{\mathrm{RQ}}=$ Result Qualifier. U $=$ The analyte was analyzed for but not detected. 
Table 3-5. 2003 Vegetation Sampling Results (pCi/g \pm total analytical uncertainty). (cont)

\begin{tabular}{|c|c|c|c|c|c|c|c|}
\hline Location & Isotope & Result \pm Error & $\mathbf{R Q}^{*}$ & Location & Isotope & Result \pm Error & $\mathbf{R Q}^{*}$ \\
\hline V011 & ${ }^{144} \mathrm{Ce}$ & $2.2 \mathrm{E}-01 \pm 3.3 \mathrm{E}-01$ & $\bar{U}$ & V015 & ${ }^{144} \mathrm{Ce}$ & $-7.1 \mathrm{E}-02 \pm 5.6 \mathrm{E}-01$ & $\overline{\mathrm{U}}$ \\
\hline \multirow[t]{17}{*}{ (200 West) } & ${ }^{60} \mathrm{Co}$ & $-5.5 \mathrm{E}-03 \pm 3.4 \mathrm{E}-02$ & $\mathrm{U}$ & (200 West) & ${ }^{60} \mathrm{Co}$ & $-1.5 \mathrm{E}-04 \pm 1.5 \mathrm{E}-03$ & $\mathrm{U}$ \\
\hline & ${ }^{134} \mathrm{Cs}$ & $3.1 \mathrm{E}-02 \pm 3.3 \mathrm{E}-02$ & $\mathrm{U}$ & & ${ }^{134} \mathrm{Cs}$ & $-3.9 \mathrm{E}-02 \pm 4.3 \mathrm{E}-02$ & $\mathrm{U}$ \\
\hline & ${ }^{137} \mathrm{Cs}$ & $2.1 \mathrm{E}-02 \pm 3.0 \mathrm{E}-02$ & $\mathrm{U}$ & & ${ }^{137} \mathrm{Cs}$ & $7.1 \mathrm{E}-02 \pm 6.4 \mathrm{E}-02$ & \\
\hline & ${ }^{152} \mathrm{Eu}$ & $-3.2 \mathrm{E}-02 \pm 8.5 \mathrm{E}-02$ & $\mathrm{U}$ & & ${ }^{152} \mathrm{Eu}$ & $-1.8 \mathrm{E}-01 \pm 1.8 \mathrm{E}-01$ & $\mathrm{U}$ \\
\hline & ${ }^{154} \mathrm{Eu}$ & $1.6 \mathrm{E}-02 \pm 1.0 \mathrm{E}-01$ & $\mathrm{U}$ & & ${ }^{154} \mathrm{Eu}$ & $7.8 \mathrm{E}-02 \pm 1.4 \mathrm{E}-01$ & $\mathrm{U}$ \\
\hline & ${ }^{155} \mathrm{Eu}$ & $-8.2 \mathrm{E}-02 \pm 9.0 \mathrm{E}-02$ & $\mathrm{U}$ & & ${ }^{155} \mathrm{Eu}$ & $-6.8 \mathrm{E}-02 \pm 1.6 \mathrm{E}-01$ & $\mathrm{U}$ \\
\hline & ${ }^{238} \mathrm{Pu}$ & $1.2 \mathrm{E}-02 \pm 1.7 \mathrm{E}-02$ & $\mathrm{U}$ & & ${ }^{238} \mathrm{Pu}$ & $1.2 \mathrm{E}-02 \pm 1.4 \mathrm{E}-02$ & $\mathrm{U}$ \\
\hline & ${ }^{239,240} \mathrm{Pu}$ & $4.2 \mathrm{E}-03 \pm 7.1 \mathrm{E}-03$ & $\mathrm{U}$ & & ${ }^{239,240} \mathrm{Pu}$ & $5.8 \mathrm{E}-03 \pm 6.4 \mathrm{E}-03$ & $\mathrm{U}$ \\
\hline & ${ }^{103} \mathrm{Ru}$ & $7.4 \mathrm{E}-03 \pm 2.8 \mathrm{E}-02$ & $\mathrm{U}$ & & ${ }^{103} \mathrm{Ru}$ & $-4.1 \mathrm{E}-03 \pm 3.8 \mathrm{E}-02$ & $\mathrm{U}$ \\
\hline & ${ }^{106} \mathrm{Ru}$ & $-1.5 \mathrm{E}-01 \pm 2.9 \mathrm{E}-01$ & $\mathrm{U}$ & & ${ }^{106} \mathrm{Ru}$ & $-1.9 \mathrm{E}-01 \pm 3.5 \mathrm{E}-01$ & $\mathrm{U}$ \\
\hline & ${ }^{125} \mathrm{Sb}$ & $6.6 \mathrm{E}-02 \pm 7.1 \mathrm{E}-02$ & $\mathrm{U}$ & & ${ }^{125} \mathrm{Sb}$ & $1.4 \mathrm{E}-02 \pm 1.0 \mathrm{E}-01$ & $\mathrm{U}$ \\
\hline & ${ }^{113} \mathrm{Sn}$ & $-1.5 \mathrm{E}-02 \pm 3.6 \mathrm{E}-02$ & $\mathrm{U}$ & & ${ }^{113} \mathrm{Sn}$ & $-7.9 \mathrm{E}-03 \pm 4.7 \mathrm{E}-02$ & $\mathrm{U}$ \\
\hline & ${ }^{90} \mathrm{Sr}$ & $-2.3 \mathrm{E}-02 \pm 9.7 \mathrm{E}-02$ & $\mathrm{U}$ & & ${ }^{90} \mathrm{Sr}$ & $4.9 \mathrm{E}-01 \pm 1.5 \mathrm{E}-01$ & \\
\hline & ${ }^{234} \mathrm{U}$ & $1.1 \mathrm{E}-02 \pm 8.0 \mathrm{E}-03$ & & & ${ }^{234} \mathrm{U}$ & $7.6 \mathrm{E}-03 \pm 6.2 \mathrm{E}-03$ & \\
\hline & ${ }^{235} \mathrm{U}$ & $5.6 \mathrm{E}-03 \pm 5.4 \mathrm{E}-03$ & $\mathrm{U}$ & & ${ }^{235} \mathrm{U}$ & $1.0 \mathrm{E}-03 \pm 2.0 \mathrm{E}-03$ & $\mathrm{U}$ \\
\hline & ${ }^{238} \mathrm{U}$ & $1.1 \mathrm{E}-02 \pm 6.8 \mathrm{E}-03$ & & & ${ }^{238} \mathrm{U}$ & $7.6 \mathrm{E}-03 \pm 6.8 \mathrm{E}-03$ & $\mathrm{U}$ \\
\hline & ${ }^{65} \mathrm{Zn}$ & $-2.9 \mathrm{E}-02 \pm 8.2 \mathrm{E}-02$ & $\mathrm{U}$ & & ${ }^{65} \mathrm{Zn}$ & $-8.1 \mathrm{E}-02 \pm 9.9 \mathrm{E}-02$ & $\mathrm{U}$ \\
\hline V017 & ${ }^{144} \mathrm{Ce}$ & $5.7 \mathrm{E}-02 \pm 3.5 \mathrm{E}-01$ & $\bar{U}$ & V019 & ${ }^{144} \mathrm{Ce}$ & $1.5 \mathrm{E}-01 \pm 4.2 \mathrm{E}-01$ & $\bar{U}$ \\
\hline \multirow[t]{17}{*}{ (200 West) } & ${ }^{60} \mathrm{Co}$ & $-6.1 \mathrm{E}-03 \pm 2.6 \mathrm{E}-02$ & $\mathrm{U}$ & (200 West) & ${ }^{60} \mathrm{Co}$ & 2.7E-02 \pm 3.9E-02 & $\mathrm{U}$ \\
\hline & ${ }^{134} \mathrm{Cs}$ & $-5.6 \mathrm{E}-03 \pm 2.4 \mathrm{E}-02$ & $\mathrm{U}$ & & ${ }^{134} \mathrm{Cs}$ & $8.5 \mathrm{E}-03 \pm 3.9 \mathrm{E}-02$ & $\mathrm{U}$ \\
\hline & ${ }^{137} \mathrm{Cs}$ & $8.0 \mathrm{E}-02 \pm 4.4 \mathrm{E}-02$ & & & ${ }^{137} \mathrm{Cs}$ & $3.5 \mathrm{E}-02 \pm 3.7 \mathrm{E}-02$ & $\mathrm{U}$ \\
\hline & ${ }^{152} \mathrm{Eu}$ & $-1.3 \mathrm{E}-02 \pm 7.9 \mathrm{E}-02$ & $\mathrm{U}$ & & ${ }^{152} \mathrm{Eu}$ & $-3.3 \mathrm{E}-02 \pm 9.9 \mathrm{E}-02$ & $\mathrm{U}$ \\
\hline & ${ }^{154} \mathrm{Eu}$ & $6.2 \mathrm{E}-03 \pm 6.2 \mathrm{E}-02$ & $\mathrm{U}$ & & ${ }^{154} \mathrm{Eu}$ & $4.9 \mathrm{E}-02 \pm 1.2 \mathrm{E}-01$ & $\mathrm{U}$ \\
\hline & ${ }^{155} \mathrm{Eu}$ & $-2.1 \mathrm{E}-02 \pm 9.0 \mathrm{E}-02$ & $\mathrm{U}$ & & ${ }^{155} \mathrm{Eu}$ & $8.3 \mathrm{E}-02 \pm 1.1 \mathrm{E}-01$ & $\mathrm{U}$ \\
\hline & ${ }^{238} \mathrm{Pu}$ & $-1.8 \mathrm{E}-03 \pm 1.4 \mathrm{E}-02$ & $\mathrm{U}$ & & ${ }^{238} \mathrm{Pu}$ & $-9.0 \mathrm{E}-04 \pm 9.0 \mathrm{E}-03$ & $\mathrm{U}$ \\
\hline & ${ }^{239,240} \mathrm{Pu}$ & $2.6 \mathrm{E}-03 \pm 3.9 \mathrm{E}-03$ & $\mathrm{U}$ & & ${ }^{239,240} \mathrm{Pu}$ & $9.0 \mathrm{E}-04 \pm 4.0 \mathrm{E}-03$ & $\mathrm{U}$ \\
\hline & ${ }^{103} \mathrm{Ru}$ & $-1.6 \mathrm{E}-03 \pm 1.6 \mathrm{E}-02$ & $\mathrm{U}$ & & ${ }^{103} \mathrm{Ru}$ & $-1.9 \mathrm{E}-02 \pm 3.3 \mathrm{E}-02$ & $\mathrm{U}$ \\
\hline & ${ }^{106} \mathrm{Ru}$ & $8.6 \mathrm{E}-02 \pm 2.3 \mathrm{E}-01$ & $\mathrm{U}$ & & ${ }^{106} \mathrm{Ru}$ & $-2.1 \mathrm{E}-01 \pm 3.6 \mathrm{E}-01$ & $\mathrm{U}$ \\
\hline & ${ }^{125} \mathrm{Sb}$ & $-3.7 \mathrm{E}-03 \pm 3.7 \mathrm{E}-02$ & $\mathrm{U}$ & & ${ }^{125} \mathrm{Sb}$ & $-4.8 \mathrm{E}-02 \pm 9.4 \mathrm{E}-02$ & $\mathrm{U}$ \\
\hline & ${ }^{113} \mathrm{Sn}$ & $-1.5 \mathrm{E}-02 \pm 3.2 \mathrm{E}-02$ & $\mathrm{U}$ & & ${ }^{113} \mathrm{Sn}$ & $-2.9 \mathrm{E}-02 \pm 4.1 \mathrm{E}-02$ & $\mathrm{U}$ \\
\hline & ${ }^{90} \mathrm{Sr}$ & $-1.2 \mathrm{E}-01 \pm 1.2 \mathrm{E}-01$ & $\mathrm{U}$ & & ${ }^{90} \mathrm{Sr}$ & $-1.0 \mathrm{E}-01 \pm 1.0 \mathrm{E}-01$ & $\mathrm{U}$ \\
\hline & ${ }^{234} \mathrm{U}$ & $3.6 \mathrm{E}-03 \pm 5.8 \mathrm{E}-03$ & $\mathrm{U}$ & & ${ }^{234} \mathrm{U}$ & $4.7 \mathrm{E}-03 \pm 4.3 \mathrm{E}-03$ & \\
\hline & ${ }^{235} \mathrm{U}$ & $2.0 \mathrm{E}-03 \pm 2.8 \mathrm{E}-03$ & $\mathrm{U}$ & & ${ }^{235} \mathrm{U}$ & $3.1 \mathrm{E}-03 \pm 3.7 \mathrm{E}-03$ & \\
\hline & ${ }^{238} \mathrm{U}$ & $8.9 \mathrm{E}-03 \pm 6.4 \mathrm{E}-03$ & & & ${ }^{238} \mathrm{U}$ & $2.8 \mathrm{E}-03 \pm 4.2 \mathrm{E}-03$ & $\mathrm{U}$ \\
\hline & ${ }^{65} \mathrm{Zn}$ & $-1.3 \mathrm{E}-01 \pm 1.3 \mathrm{E}-01$ & $\mathrm{U}$ & & ${ }^{65} \mathrm{Zn}$ & $-2.4 \mathrm{E}-02 \pm 8.9 \mathrm{E}-02$ & $\mathrm{U}$ \\
\hline
\end{tabular}

$\overline{\mathrm{RQ}}=$ Result Qualifier. U $=$ The analyte was analyzed for but not detected. 
Table 3-5. 2003 Vegetation Sampling Results (pCi/g \pm total analytical uncertainty). (cont)

\begin{tabular}{|c|c|c|c|c|c|c|c|}
\hline Location & Isotope & Result \pm Error & $\mathbf{R Q}^{*}$ & Location & Isotope & Result \pm Error & $\mathbf{R Q}^{*}$ \\
\hline \multirow[t]{18}{*}{ V021 } & ${ }^{144} \mathrm{Ce}$ & $3.4 \mathrm{E}-01 \pm 5.1 \mathrm{E}-01$ & $\bar{U}$ & \multirow[t]{18}{*}{ V023 } & ${ }^{144} \mathrm{Ce}$ & $7.1 \mathrm{E}-02 \pm 4.9 \mathrm{E}-01$ & $\overline{\mathrm{U}}$ \\
\hline & ${ }^{60} \mathrm{Co}$ & $4.6 \mathrm{E}-02 \pm 5.8 \mathrm{E}-02$ & $\mathrm{U}$ & & ${ }^{60} \mathrm{Co}$ & $1.2 \mathrm{E}-02 \pm 3.6 \mathrm{E}-02$ & $\mathrm{U}$ \\
\hline & ${ }^{134} \mathrm{Cs}$ & $-2.3 \mathrm{E}-02 \pm 5.0 \mathrm{E}-02$ & $\mathrm{U}$ & & ${ }^{134} \mathrm{Cs}$ & $-4.3 \mathrm{E}-02 \pm 4.3 \mathrm{E}-02$ & $\mathrm{U}$ \\
\hline & ${ }^{137} \mathrm{Cs}$ & $1.2 \mathrm{E}-02 \pm 4.8 \mathrm{E}-02$ & $\mathrm{U}$ & & ${ }^{137} \mathrm{Cs}$ & $5.7 \mathrm{E}-02 \pm 5.8 \mathrm{E}-02$ & $\mathrm{U}$ \\
\hline & ${ }^{152} \mathrm{Eu}$ & $-8.9 \mathrm{E}-03 \pm 8.9 \mathrm{E}-02$ & $\mathrm{U}$ & & ${ }^{152} \mathrm{Eu}$ & $-1.2 \mathrm{E}-01 \pm 1.2 \mathrm{E}-01$ & $\mathrm{U}$ \\
\hline & ${ }^{154} \mathrm{Eu}$ & $-3.1 \mathrm{E}-02 \pm 1.9 \mathrm{E}-01$ & $\mathrm{U}$ & & ${ }^{154} \mathrm{Eu}$ & $-4.1 \mathrm{E}-02 \pm 1.2 \mathrm{E}-01$ & $\mathrm{U}$ \\
\hline & ${ }^{155} \mathrm{Eu}$ & $1.9 \mathrm{E}-02 \pm 1.4 \mathrm{E}-01$ & $\mathrm{U}$ & & ${ }^{155} \mathrm{Eu}$ & $-5.9 \mathrm{E}-02 \pm 1.4 \mathrm{E}-01$ & $\mathrm{U}$ \\
\hline & ${ }^{238} \mathrm{Pu}$ & $1.9 \mathrm{E}-03 \pm 4.7 \mathrm{E}-03$ & $\mathrm{U}$ & & ${ }^{238} \mathrm{Pu}$ & $-2.7 \mathrm{E}-03 \pm 7.8 \mathrm{E}-03$ & $\mathrm{U}$ \\
\hline & ${ }^{239,240} \mathrm{Pu}$ & $2.8 \mathrm{E}-03 \pm 3.4 \mathrm{E}-03$ & & & ${ }^{239,240} \mathrm{Pu}$ & $9.0 \mathrm{E}-04 \pm 1.8 \mathrm{E}-03$ & $\mathrm{U}$ \\
\hline & ${ }^{103} \mathrm{Ru}$ & $-2.3 \mathrm{E}-02 \pm 4.2 \mathrm{E}-02$ & $\mathrm{U}$ & & ${ }^{103} \mathrm{Ru}$ & $1.2 \mathrm{E}-02 \pm 3.5 \mathrm{E}-02$ & $\mathrm{U}$ \\
\hline & ${ }^{106} \mathrm{Ru}$ & $-2.5 \mathrm{E}-01 \pm 4.2 \mathrm{E}-01$ & $\mathrm{U}$ & & ${ }^{106} \mathrm{Ru}$ & $2.8 \mathrm{E}-01 \pm 3.2 \mathrm{E}-01$ & $\mathrm{U}$ \\
\hline & ${ }^{125} \mathrm{Sb}$ & $-1.7 \mathrm{E}-02 \pm 1.1 \mathrm{E}-01$ & $\mathrm{U}$ & & ${ }^{125} \mathrm{Sb}$ & $-1.5 \mathrm{E}-02 \pm 9.4 \mathrm{E}-02$ & $\mathrm{U}$ \\
\hline & ${ }^{113} \mathrm{Sn}$ & $-1.8 \mathrm{E}-02 \pm 5.1 \mathrm{E}-02$ & $\mathrm{U}$ & & ${ }^{113} \mathrm{Sn}$ & $-7.5 \mathrm{E}-03 \pm 4.3 \mathrm{E}-02$ & $\mathrm{U}$ \\
\hline & ${ }^{90} \mathrm{Sr}$ & $8.3 \mathrm{E}-01 \pm 1.7 \mathrm{E}-01$ & & & ${ }^{90} \mathrm{Sr}$ & $9.3 \mathrm{E}-02 \pm 8.4 \mathrm{E}-02$ & $\mathrm{U}$ \\
\hline & ${ }^{234} \mathrm{U}$ & $1.4 \mathrm{E}-02 \pm 8.4 \mathrm{E}-03$ & & & ${ }^{234} \mathrm{U}$ & $8.5 \mathrm{E}-03 \pm 7.0 \mathrm{E}-03$ & $\mathrm{U}$ \\
\hline & ${ }^{235} \mathrm{U}$ & $9.8 \mathrm{E}-04 \pm 9.8 \mathrm{E}-03$ & $\mathrm{U}$ & & ${ }^{235} \mathrm{U}$ & $-9.2 \mathrm{E}-04 \pm 1.8 \mathrm{E}-03$ & $\mathrm{U}$ \\
\hline & ${ }^{238} \mathrm{U}$ & $5.9 \mathrm{E}-03 \pm 6.5 \mathrm{E}-03$ & $\mathrm{U}$ & & ${ }^{238} \mathrm{U}$ & $8.5 \mathrm{E}-03 \pm 5.6 \mathrm{E}-03$ & \\
\hline & ${ }^{65} \mathrm{Zn}$ & $1.2 \mathrm{E}-01 \pm 1.3 \mathrm{E}-01$ & $\mathrm{U}$ & & ${ }^{65} \mathrm{Zn}$ & $-4.7 \mathrm{E}-02 \pm 9.0 \mathrm{E}-02$ & $\mathrm{U}$ \\
\hline V025 & ${ }^{144} \mathrm{Ce}$ & $-9.6 \mathrm{E}-02 \pm 4.3 \mathrm{E}-01$ & $\bar{U}$ & V027 & ${ }^{144} \mathrm{Ce}$ & $2.0 \mathrm{E}-02 \pm 2.0 \mathrm{E}-01$ & $\bar{U}$ \\
\hline \multirow[t]{17}{*}{ (200 West) } & ${ }^{60} \mathrm{Co}$ & $-3.5 \mathrm{E}-03 \pm 3.5 \mathrm{E}-02$ & $\mathrm{U}$ & (200 West) & ${ }^{60} \mathrm{Co}$ & $2.7 \mathrm{E}-03 \pm 2.7 \mathrm{E}-02$ & $\mathrm{U}$ \\
\hline & ${ }^{134} \mathrm{Cs}$ & $-1.7 \mathrm{E}-02 \pm 3.2 \mathrm{E}-02$ & $\mathrm{U}$ & & ${ }^{134} \mathrm{Cs}$ & $-1.1 \mathrm{E}-02 \pm 2.7 \mathrm{E}-02$ & $\mathrm{U}$ \\
\hline & ${ }^{137} \mathrm{Cs}$ & $1.1 \mathrm{E}-01 \pm 5.2 \mathrm{E}-02$ & & & ${ }^{137} \mathrm{Cs}$ & $2.3 \mathrm{E}-02 \pm 2.9 \mathrm{E}-02$ & $\mathrm{U}$ \\
\hline & ${ }^{152} \mathrm{Eu}$ & $2.2 \mathrm{E}-02 \pm 9.3 \mathrm{E}-02$ & $\mathrm{U}$ & & ${ }^{152} \mathrm{Eu}$ & $-4.9 \mathrm{E}-02 \pm 8.3 \mathrm{E}-02$ & $\mathrm{U}$ \\
\hline & ${ }^{154} \mathrm{Eu}$ & $-1.3 \mathrm{E}-01 \pm 1.3 \mathrm{E}-01$ & $\mathrm{U}$ & & ${ }^{154} \mathrm{Eu}$ & $9.3 \mathrm{E}-04 \pm 9.3 \mathrm{E}-03$ & $\mathrm{U}$ \\
\hline & ${ }^{155} \mathrm{Eu}$ & $-2.9 \mathrm{E}-02 \pm 1.1 \mathrm{E}-01$ & $\mathrm{U}$ & & ${ }^{155} \mathrm{Eu}$ & $5.3 \mathrm{E}-02 \pm 9.0 \mathrm{E}-02$ & $\mathrm{U}$ \\
\hline & ${ }^{238} \mathrm{Pu}$ & $-9.9 \mathrm{E}-04 \pm 9.9 \mathrm{E}-03$ & $\mathrm{U}$ & & ${ }^{238} \mathrm{Pu}$ & $7.7 \mathrm{E}-03 \pm 1.4 \mathrm{E}-02$ & $\mathrm{U}$ \\
\hline & ${ }^{239,240} \mathrm{Pu}$ & $9.9 \mathrm{E}-04 \pm 6.5 \mathrm{E}-03$ & $\mathrm{U}$ & & ${ }^{239,240} \mathrm{Pu}$ & $1.7 \mathrm{E}-03 \pm 4.2 \mathrm{E}-03$ & $\mathrm{U}$ \\
\hline & ${ }^{103} \mathrm{Ru}$ & $-1.7 \mathrm{E}-02 \pm 3.5 \mathrm{E}-02$ & $\mathrm{U}$ & & ${ }^{103} \mathrm{Ru}$ & $4.2 \mathrm{E}-03 \pm 2.5 \mathrm{E}-02$ & $\mathrm{U}$ \\
\hline & ${ }^{106} \mathrm{Ru}$ & $4.7 \mathrm{E}-02 \pm 3.0 \mathrm{E}-01$ & $\mathrm{U}$ & & ${ }^{106} \mathrm{Ru}$ & $7.0 \mathrm{E}-02 \pm 2.4 \mathrm{E}-01$ & $\mathrm{U}$ \\
\hline & ${ }^{125} \mathrm{Sb}$ & $-2.0 \mathrm{E}-02 \pm 8.3 \mathrm{E}-02$ & $\mathrm{U}$ & & ${ }^{125} \mathrm{Sb}$ & $-2.8 \mathrm{E}-03 \pm 2.8 \mathrm{E}-02$ & $\mathrm{U}$ \\
\hline & ${ }^{113} \mathrm{Sn}$ & $-3.1 \mathrm{E}-02 \pm 3.9 \mathrm{E}-02$ & $\mathrm{U}$ & & ${ }^{113} \mathrm{Sn}$ & $1.6 \mathrm{E}-02 \pm 3.4 \mathrm{E}-02$ & $\mathrm{U}$ \\
\hline & ${ }^{90} \mathrm{Sr}$ & $-6.4 \mathrm{E}-02 \pm 7.7 \mathrm{E}-02$ & $\mathrm{U}$ & & ${ }^{90} \mathrm{Sr}$ & $-1.4 \mathrm{E}-02 \pm 8.9 \mathrm{E}-02$ & $\mathrm{U}$ \\
\hline & ${ }^{234} \mathrm{U}$ & $1.2 \mathrm{E}-02 \pm 7.0 \mathrm{E}-03$ & & & ${ }^{234} \mathrm{U}$ & $9.0 \mathrm{E}-03 \pm 7.4 \mathrm{E}-03$ & $\mathrm{U}$ \\
\hline & ${ }^{235} \mathrm{U}$ & $3.6 \mathrm{E}-03 \pm 3.6 \mathrm{E}-03$ & & & ${ }^{235} \mathrm{U}$ & 8.9E-04 \pm 8.9E-03 & $\mathrm{U}$ \\
\hline & ${ }^{238} \mathrm{U}$ & $2.4 \mathrm{E}-03 \pm 4.3 \mathrm{E}-03$ & $\mathrm{U}$ & & ${ }^{238} \mathrm{U}$ & $1.5 \mathrm{E}-02 \pm 8.2 \mathrm{E}-03$ & \\
\hline & ${ }^{65} \mathrm{Zn}$ & $-2.2 \mathrm{E}-02 \pm 8.9 \mathrm{E}-02$ & $\mathrm{U}$ & & ${ }^{65} \mathrm{Zn}$ & $-2.0 \mathrm{E}-01 \pm 2.0 \mathrm{E}-01$ & $\mathrm{U}$ \\
\hline
\end{tabular}

$\overline{\mathrm{RQ}}=$ Result Qualifier. U $=$ The analyte was analyzed for but not detected. 
Table 3-5. 2003 Vegetation Sampling Results (pCi/g \pm total analytical uncertainty). (cont)

\begin{tabular}{|c|c|c|c|c|c|c|c|}
\hline Location & Isotope & Result \pm Error & $\mathbf{R Q}^{*}$ & Location & Isotope & Result \pm Error & $\mathbf{R Q}^{*}$ \\
\hline V029 & ${ }^{144} \mathrm{Ce}$ & $-6.6 \mathrm{E}-03 \pm 6.6 \mathrm{E}-02$ & $\bar{U}$ & V031 & ${ }^{144} \mathrm{Ce}$ & $-1.5 \mathrm{E}-01 \pm 3.9 \mathrm{E}-01$ & $\overline{\mathrm{U}}$ \\
\hline \multirow[t]{17}{*}{ (200 West) } & ${ }^{60} \mathrm{Co}$ & $-2.3 \mathrm{E}-02 \pm 2.8 \mathrm{E}-02$ & $\mathrm{U}$ & (200 West) & ${ }^{60} \mathrm{Co}$ & $-4.3 \mathrm{E}-03 \pm 2.9 \mathrm{E}-02$ & $\mathrm{U}$ \\
\hline & ${ }^{134} \mathrm{Cs}$ & $2.2 \mathrm{E}-02 \pm 2.8 \mathrm{E}-02$ & $\mathrm{U}$ & & ${ }^{134} \mathrm{Cs}$ & $7.0 \mathrm{E}-03 \pm 3.3 \mathrm{E}-02$ & $\mathrm{U}$ \\
\hline & ${ }^{137} \mathrm{Cs}$ & $5.0 \mathrm{E}-02 \pm 3.6 \mathrm{E}-02$ & $\mathrm{U}$ & & ${ }^{137} \mathrm{Cs}$ & $8.8 \mathrm{E}-02 \pm 4.4 \mathrm{E}-02$ & \\
\hline & ${ }^{152} \mathrm{Eu}$ & $-7.6 \mathrm{E}-02 \pm 8.4 \mathrm{E}-02$ & $\mathrm{U}$ & & ${ }^{152} \mathrm{Eu}$ & $-3.7 \mathrm{E}-02 \pm 8.9 \mathrm{E}-02$ & $\mathrm{U}$ \\
\hline & ${ }^{154} \mathrm{Eu}$ & $-2.7 \mathrm{E}-02 \pm 8.5 \mathrm{E}-02$ & $\mathrm{U}$ & & ${ }^{154} \mathrm{Eu}$ & $2.7 \mathrm{E}-02 \pm 9.8 \mathrm{E}-02$ & $\mathrm{U}$ \\
\hline & ${ }^{155} \mathrm{Eu}$ & $3.8 \mathrm{E}-02 \pm 9.5 \mathrm{E}-02$ & $\mathrm{U}$ & & ${ }^{155} \mathrm{Eu}$ & $2.0 \mathrm{E}-02 \pm 1.1 \mathrm{E}-01$ & $\mathrm{U}$ \\
\hline & ${ }^{238} \mathrm{Pu}$ & $-1.4 \mathrm{E}-02 \pm 1.7 \mathrm{E}-02$ & $\mathrm{U}$ & & ${ }^{238} \mathrm{Pu}$ & $-1.1 \mathrm{E}-03 \pm 1.1 \mathrm{E}-02$ & $\mathrm{U}$ \\
\hline & ${ }^{239,240} \mathrm{Pu}$ & $6.0 \mathrm{E}-03 \pm 5.8 \mathrm{E}-03$ & $\mathrm{U}$ & & ${ }^{239,240} \mathrm{Pu}$ & $1.1 \mathrm{E}-03 \pm 1.1 \mathrm{E}-03$ & $\mathrm{U}$ \\
\hline & ${ }^{103} \mathrm{Ru}$ & $-3.1 \mathrm{E}-02 \pm 3.1 \mathrm{E}-02$ & $\mathrm{U}$ & & ${ }^{103} \mathrm{Ru}$ & $-1.9 \mathrm{E}-02 \pm 3.0 \mathrm{E}-02$ & $\mathrm{U}$ \\
\hline & ${ }^{106} \mathrm{Ru}$ & $2.2 \mathrm{E}-01 \pm 2.5 \mathrm{E}-01$ & $\mathrm{U}$ & & ${ }^{106} \mathrm{Ru}$ & $5.2 \mathrm{E}-03 \pm 5.2 \mathrm{E}-02$ & $\mathrm{U}$ \\
\hline & ${ }^{125} \mathrm{Sb}$ & $-5.9 \mathrm{E}-02 \pm 7.6 \mathrm{E}-02$ & $\mathrm{U}$ & & ${ }^{125} \mathrm{Sb}$ & $-4.9 \mathrm{E}-02 \pm 8.0 \mathrm{E}-02$ & $\mathrm{U}$ \\
\hline & ${ }^{113} \mathrm{Sn}$ & $-1.1 \mathrm{E}-02 \pm 3.6 \mathrm{E}-02$ & $\mathrm{U}$ & & ${ }^{113} \mathrm{Sn}$ & $3.5 \mathrm{E}-03 \pm 3.5 \mathrm{E}-02$ & $\mathrm{U}$ \\
\hline & ${ }^{90} \mathrm{Sr}$ & $-1.8 \mathrm{E}-01 \pm 1.8 \mathrm{E}-01$ & $\mathrm{U}$ & & ${ }^{90} \mathrm{Sr}$ & $2.1 \mathrm{E}-01 \pm 1.0 \mathrm{E}-01$ & \\
\hline & ${ }^{234} \mathrm{U}$ & $6.4 \mathrm{E}-03 \pm 5.0 \mathrm{E}-03$ & & & ${ }^{234} \mathrm{U}$ & $9.3 \mathrm{E}-03 \pm 6.1 \mathrm{E}-03$ & \\
\hline & ${ }^{235} \mathrm{U}$ & $3.0 \mathrm{E}-03 \pm 4.5 \mathrm{E}-03$ & $\mathrm{U}$ & & ${ }^{235} \mathrm{U}$ & $4.1 \mathrm{E}-03 \pm 4.9 \mathrm{E}-03$ & $\mathrm{U}$ \\
\hline & ${ }^{238} \mathrm{U}$ & 7.3E-03 \pm 5.3E-03 & & & ${ }^{238} \mathrm{U}$ & $1.0 \mathrm{E}-02 \pm 6.3 \mathrm{E}-03$ & \\
\hline & ${ }^{65} \mathrm{Zn}$ & $4.1 \mathrm{E}-02 \pm 6.7 \mathrm{E}-02$ & $\mathrm{U}$ & & ${ }^{65} \mathrm{Zn}$ & $-1.1 \mathrm{E}-02 \pm 7.7 \mathrm{E}-02$ & $\mathrm{U}$ \\
\hline V033 & ${ }^{144} \mathrm{Ce}$ & $1.3 \mathrm{E}+00 \pm 1.4 \mathrm{E}+00$ & $\overline{\mathrm{U}}$ & V035 & ${ }^{144} \mathrm{Ce}$ & $3.0 \mathrm{E}-02 \pm 3.0 \mathrm{E}-01$ & $\bar{U}$ \\
\hline \multirow[t]{17}{*}{ (200 West) } & ${ }^{60} \mathrm{Co}$ & $-2.0 \mathrm{E}-02 \pm 1.0 \mathrm{E}-01$ & $\mathrm{U}$ & (200 West) & ${ }^{60} \mathrm{Co}$ & $2.4 \mathrm{E}-02 \pm 3.0 \mathrm{E}-02$ & $\mathrm{U}$ \\
\hline & ${ }^{134} \mathrm{Cs}$ & $1.1 \mathrm{E}-02 \pm 1.0 \mathrm{E}-01$ & $\mathrm{U}$ & & ${ }^{134} \mathrm{Cs}$ & $-3.4 \mathrm{E}-03 \pm 3.4 \mathrm{E}-02$ & $\mathrm{U}$ \\
\hline & ${ }^{137} \mathrm{Cs}$ & $3.3 \mathrm{E}+00 \pm 6.1 \mathrm{E}-01$ & & & ${ }^{137} \mathrm{Cs}$ & $1.0 \mathrm{E}-01 \pm 5.1 \mathrm{E}-02$ & \\
\hline & ${ }^{152} \mathrm{Eu}$ & $-1.4 \mathrm{E}-02 \pm 1.4 \mathrm{E}-01$ & $\mathrm{U}$ & & ${ }^{152} \mathrm{Eu}$ & $3.2 \mathrm{E}-03 \pm 3.2 \mathrm{E}-02$ & $\mathrm{U}$ \\
\hline & ${ }^{154} \mathrm{Eu}$ & $-8.5 \mathrm{E}-02 \pm 3.1 \mathrm{E}-01$ & $\mathrm{U}$ & & ${ }^{154} \mathrm{Eu}$ & $4.9 \mathrm{E}-02 \pm 9.6 \mathrm{E}-02$ & $\mathrm{U}$ \\
\hline & ${ }^{155} \mathrm{Eu}$ & $-1.3 \mathrm{E}-04 \pm 1.3 \mathrm{E}-03$ & $\mathrm{U}$ & & ${ }^{155} \mathrm{Eu}$ & $4.7 \mathrm{E}-02 \pm 1.0 \mathrm{E}-01$ & $\mathrm{U}$ \\
\hline & ${ }^{238} \mathrm{Pu}$ & $9.4 \mathrm{E}-04 \pm 9.4 \mathrm{E}-03$ & $\mathrm{U}$ & & ${ }^{238} \mathrm{Pu}$ & $1.0 \mathrm{E}-03 \pm 1.0 \mathrm{E}-03$ & $\mathrm{U}$ \\
\hline & ${ }^{239,240} \mathrm{Pu}$ & $3.8 \mathrm{E}-03 \pm 5.3 \mathrm{E}-03$ & $\mathrm{U}$ & & ${ }^{239,240} \mathrm{Pu}$ & $2.0 \mathrm{E}-03 \pm 2.8 \mathrm{E}-03$ & $\mathrm{U}$ \\
\hline & ${ }^{103} \mathrm{Ru}$ & $4.8 \mathrm{E}-02 \pm 1.0 \mathrm{E}-01$ & $\mathrm{U}$ & & ${ }^{103} \mathrm{Ru}$ & $8.4 \mathrm{E}-03 \pm 3.1 \mathrm{E}-02$ & $\mathrm{U}$ \\
\hline & ${ }^{106} \mathrm{Ru}$ & $4.0 \mathrm{E}-01 \pm 8.5 \mathrm{E}-01$ & $\mathrm{U}$ & & ${ }^{106} \mathrm{Ru}$ & $-7.9 \mathrm{E}-02 \pm 2.7 \mathrm{E}-01$ & $\mathrm{U}$ \\
\hline & ${ }^{125} \mathrm{Sb}$ & $-9.7 \mathrm{E}-02 \pm 2.6 \mathrm{E}-01$ & $\mathrm{U}$ & & ${ }^{125} \mathrm{Sb}$ & $5.5 \mathrm{E}-02 \pm 7.8 \mathrm{E}-02$ & $\mathrm{U}$ \\
\hline & ${ }^{113} \mathrm{Sn}$ & $6.1 \mathrm{E}-03 \pm 6.1 \mathrm{E}-02$ & $\mathrm{U}$ & & ${ }^{113} \mathrm{Sn}$ & $-4.2 \mathrm{E}-03 \pm 3.8 \mathrm{E}-02$ & $\mathrm{U}$ \\
\hline & ${ }^{90} \mathrm{Sr}$ & $2.4 \mathrm{E}+01 \pm 3.0 \mathrm{E}+00$ & & & ${ }^{90} \mathrm{Sr}$ & $5.5 \mathrm{E}-02 \pm 1.3 \mathrm{E}-01$ & $\mathrm{U}$ \\
\hline & ${ }^{234} \mathrm{U}$ & $2.0 \mathrm{E}-02 \pm 1.1 \mathrm{E}-02$ & & & ${ }^{234} \mathrm{U}$ & $7.8 \mathrm{E}-03 \pm 6.5 \mathrm{E}-03$ & \\
\hline & ${ }^{235} \mathrm{U}$ & $1.1 \mathrm{E}-03 \pm 2.2 \mathrm{E}-03$ & $\mathrm{U}$ & & ${ }^{235} \mathrm{U}$ & $2.1 \mathrm{E}-03 \pm 2.9 \mathrm{E}-03$ & $\mathrm{U}$ \\
\hline & ${ }^{238} \mathrm{U}$ & $1.3 \mathrm{E}-02 \pm 7.9 \mathrm{E}-03$ & & & ${ }^{238} \mathrm{U}$ & $1.2 \mathrm{E}-02 \pm 8.0 \mathrm{E}-03$ & \\
\hline & ${ }^{65} \mathrm{Zn}$ & $-1.3 \mathrm{E}-01 \pm 2.3 \mathrm{E}-01$ & $\mathrm{U}$ & & ${ }^{65} \mathrm{Zn}$ & $-1.2 \mathrm{E}-01 \pm 1.2 \mathrm{E}-01$ & $\mathrm{U}$ \\
\hline
\end{tabular}

$\overline{\mathrm{RQ}}=$ Result Qualifier. U $=$ The analyte was analyzed for but not detected. 
Table 3-5. 2003 Vegetation Sampling Results (pCi/g \pm total analytical uncertainty). (cont)

\begin{tabular}{|c|c|c|c|c|c|c|c|}
\hline Location & Isotope & Result \pm Error & $\mathbf{R Q}^{*}$ & Location & Isotope & Result \pm Error & $\mathbf{R Q}^{*}$ \\
\hline V039 & ${ }^{144} \mathrm{Ce}$ & $1.4 \mathrm{E}-02 \pm 1.4 \mathrm{E}-01$ & $\bar{U}$ & V041 & ${ }^{144} \mathrm{Ce}$ & $6.8 \mathrm{E}-02 \pm 4.3 \mathrm{E}-01$ & $\overline{\mathrm{U}}$ \\
\hline \multirow[t]{17}{*}{ (200 West) } & ${ }^{60} \mathrm{Co}$ & $1.9 \mathrm{E}-02 \pm 2.5 \mathrm{E}-02$ & $\mathrm{U}$ & (200 West) & ${ }^{60} \mathrm{Co}$ & $-2.2 \mathrm{E}-02 \pm 3.4 \mathrm{E}-02$ & $\mathrm{U}$ \\
\hline & ${ }^{134} \mathrm{Cs}$ & $-6.6 \mathrm{E}-03 \pm 2.6 \mathrm{E}-02$ & $\mathrm{U}$ & & ${ }^{134} \mathrm{Cs}$ & $1.4 \mathrm{E}-02 \pm 3.4 \mathrm{E}-02$ & $\mathrm{U}$ \\
\hline & ${ }^{137} \mathrm{Cs}$ & $1.5 \mathrm{E}-02 \pm 2.6 \mathrm{E}-02$ & $\mathrm{U}$ & & ${ }^{137} \mathrm{Cs}$ & $6.5 \mathrm{E}-02 \pm 3.6 \mathrm{E}-02$ & \\
\hline & ${ }^{152} \mathrm{Eu}$ & $-1.4 \mathrm{E}-03 \pm 1.4 \mathrm{E}-02$ & $\mathrm{U}$ & & ${ }^{152} \mathrm{Eu}$ & $-4.9 \mathrm{E}-02 \pm 9.9 \mathrm{E}-02$ & $\mathrm{U}$ \\
\hline & ${ }^{154} \mathrm{Eu}$ & $1.3 \mathrm{E}-02 \pm 8.0 \mathrm{E}-02$ & $\mathrm{U}$ & & ${ }^{154} \mathrm{Eu}$ & $2.7 \mathrm{E}-02 \pm 1.0 \mathrm{E}-01$ & $\mathrm{U}$ \\
\hline & ${ }^{155} \mathrm{Eu}$ & $3.1 \mathrm{E}-02 \pm 8.5 \mathrm{E}-02$ & $\mathrm{U}$ & & ${ }^{155} \mathrm{Eu}$ & $-1.9 \mathrm{E}-03 \pm 1.9 \mathrm{E}-02$ & $\mathrm{U}$ \\
\hline & ${ }^{238} \mathrm{Pu}$ & $1.0 \mathrm{E}-03 \pm 1.0 \mathrm{E}-03$ & $\mathrm{U}$ & & ${ }^{238} \mathrm{Pu}$ & $2.8 \mathrm{E}-03 \pm 1.4 \mathrm{E}-02$ & $\mathrm{U}$ \\
\hline & ${ }^{239,240} \mathrm{Pu}$ & $6.2 \mathrm{E}-03 \pm 5.3 \mathrm{E}-03$ & & & ${ }^{239,240} \mathrm{Pu}$ & $-3.6 \mathrm{E}-03 \pm 4.3 \mathrm{E}-03$ & $\mathrm{U}$ \\
\hline & ${ }^{103} \mathrm{Ru}$ & $1.3 \mathrm{E}-02 \pm 2.6 \mathrm{E}-02$ & $\mathrm{U}$ & & ${ }^{103} \mathrm{Ru}$ & $2.3 \mathrm{E}-02 \pm 3.4 \mathrm{E}-02$ & $\mathrm{U}$ \\
\hline & ${ }^{106} \mathrm{Ru}$ & $1.5 \mathrm{E}-01 \pm 2.3 \mathrm{E}-01$ & $\mathrm{U}$ & & ${ }^{106} \mathrm{Ru}$ & $1.0 \mathrm{E}-01 \pm 3.0 \mathrm{E}-01$ & $\mathrm{U}$ \\
\hline & ${ }^{125} \mathrm{Sb}$ & $-6.2 \mathrm{E}-03 \pm 6.2 \mathrm{E}-02$ & $\mathrm{U}$ & & ${ }^{125} \mathrm{Sb}$ & $-7.3 \mathrm{E}-02 \pm 8.4 \mathrm{E}-02$ & $\mathrm{U}$ \\
\hline & ${ }^{113} \mathrm{Sn}$ & $-5.7 \mathrm{E}-04 \pm 5.7 \mathrm{E}-03$ & $\mathrm{U}$ & & ${ }^{113} \mathrm{Sn}$ & $9.5 \mathrm{E}-03 \pm 4.0 \mathrm{E}-02$ & $\mathrm{U}$ \\
\hline & ${ }^{90} \mathrm{Sr}$ & $9.3 \mathrm{E}-02 \pm 1.1 \mathrm{E}-01$ & $\mathrm{U}$ & & ${ }^{90} \mathrm{Sr}$ & $5.8 \mathrm{E}-02 \pm 1.3 \mathrm{E}-01$ & $\mathrm{U}$ \\
\hline & ${ }^{234} \mathrm{U}$ & $9.3 \mathrm{E}-04 \pm 1.9 \mathrm{E}-03$ & $\mathrm{U}$ & & ${ }^{234} \mathrm{U}$ & $9.7 \mathrm{E}-03 \pm 6.3 \mathrm{E}-03$ & \\
\hline & ${ }^{235} \mathrm{U}$ & $2.0 \mathrm{E}-03 \pm 2.8 \mathrm{E}-03$ & $\mathrm{U}$ & & ${ }^{235} \mathrm{U}$ & $3.9 \mathrm{E}-03 \pm 3.9 \mathrm{E}-03$ & \\
\hline & ${ }^{238} \mathrm{U}$ & 4.6E-03 \pm 4.3E-03 & & & ${ }^{238} \mathrm{U}$ & $8.0 \mathrm{E}-03 \pm 6.2 \mathrm{E}-03$ & \\
\hline & ${ }^{65} \mathrm{Zn}$ & $-1.6 \mathrm{E}-01 \pm 1.6 \mathrm{E}-01$ & $\mathrm{U}$ & & ${ }^{65} \mathrm{Zn}$ & $2.0 \mathrm{E}-02 \pm 8.5 \mathrm{E}-02$ & $\mathrm{U}$ \\
\hline V043 & ${ }^{144} \mathrm{Ce}$ & $6.7 \mathrm{E}-02 \pm 5.1 \mathrm{E}-01$ & $\bar{U}$ & V045 & ${ }^{144} \mathrm{Ce}$ & $-2.8 \mathrm{E}+00 \pm 2.8 \mathrm{E}+01$ & $\bar{U}$ \\
\hline \multirow[t]{17}{*}{ (200 West) } & ${ }^{60} \mathrm{Co}$ & $-1.7 \mathrm{E}-02 \pm 4.3 \mathrm{E}-02$ & $\mathrm{U}$ & (200 West) & ${ }^{60} \mathrm{Co}$ & $7.2 \mathrm{E}-01 \pm 3.1 \mathrm{E}+00$ & $\mathrm{U}$ \\
\hline & ${ }^{134} \mathrm{Cs}$ & $-2.0 \mathrm{E}-02 \pm 4.2 \mathrm{E}-02$ & $\mathrm{U}$ & & ${ }^{134} \mathrm{Cs}$ & $1.1 \mathrm{E}+00 \pm 3.2 \mathrm{E}+00$ & $\mathrm{U}$ \\
\hline & ${ }^{137} \mathrm{Cs}$ & $4.2 \mathrm{E}-02 \pm 4.4 \mathrm{E}-02$ & $\mathrm{U}$ & & ${ }^{137} \mathrm{Cs}$ & $6.0 \mathrm{E}+00 \pm 4.3 \mathrm{E}+00$ & \\
\hline & ${ }^{152} \mathrm{Eu}$ & $7.1 \mathrm{E}-02 \pm 1.1 \mathrm{E}-01$ & $\mathrm{U}$ & & ${ }^{152} \mathrm{Eu}$ & $-6.3 \mathrm{E}+00 \pm 7.7 \mathrm{E}+00$ & $\mathrm{U}$ \\
\hline & ${ }^{154} \mathrm{Eu}$ & $-2.3 \mathrm{E}-02 \pm 1.2 \mathrm{E}-01$ & $\mathrm{U}$ & & ${ }^{154} \mathrm{Eu}$ & $3.8 \mathrm{E}-01 \pm 3.8 \mathrm{E}+00$ & $\mathrm{U}$ \\
\hline & ${ }^{155} \mathrm{Eu}$ & $-7.6 \mathrm{E}-02 \pm 1.4 \mathrm{E}-01$ & $\mathrm{U}$ & & ${ }^{155} \mathrm{Eu}$ & $-3.4 \mathrm{E}-01 \pm 3.4 \mathrm{E}+00$ & $\mathrm{U}$ \\
\hline & ${ }^{238} \mathrm{Pu}$ & $3.5 \mathrm{E}-03 \pm 1.3 \mathrm{E}-02$ & $\mathrm{U}$ & & ${ }^{238} \mathrm{Pu}$ & $-6.9 \mathrm{E}-03 \pm 1.5 \mathrm{E}-02$ & $\mathrm{U}$ \\
\hline & ${ }^{239,240} \mathrm{Pu}$ & $1.8 \mathrm{E}-03 \pm 4.5 \mathrm{E}-03$ & $\mathrm{U}$ & & ${ }^{239,240} \mathrm{Pu}$ & $6.0 \mathrm{E}-03 \pm 5.3 \mathrm{E}-03$ & $\mathrm{U}$ \\
\hline & ${ }^{103} \mathrm{Ru}$ & $-1.2 \mathrm{E}-02 \pm 4.0 \mathrm{E}-02$ & $\mathrm{U}$ & & ${ }^{103} \mathrm{Ru}$ & $1.3 \mathrm{E}-01 \pm 1.3 \mathrm{E}+00$ & $\mathrm{U}$ \\
\hline & ${ }^{106} \mathrm{Ru}$ & $-2.2 \mathrm{E}-03 \pm 2.2 \mathrm{E}-02$ & $\mathrm{U}$ & & ${ }^{106} \mathrm{Ru}$ & $3.0 \mathrm{E}+00 \pm 3.0 \mathrm{E}+01$ & $\mathrm{U}$ \\
\hline & ${ }^{125} \mathrm{Sb}$ & $-2.5 \mathrm{E}-02 \pm 1.0 \mathrm{E}-01$ & $\mathrm{U}$ & & ${ }^{125} \mathrm{Sb}$ & $-3.8 \mathrm{E}+00 \pm 7.5 \mathrm{E}+00$ & $\mathrm{U}$ \\
\hline & ${ }^{113} \mathrm{Sn}$ & $-3.0 \mathrm{E}-02 \pm 4.8 \mathrm{E}-02$ & $\mathrm{U}$ & & ${ }^{113} \mathrm{Sn}$ & $-8.8 \mathrm{E}-01 \pm 3.7 \mathrm{E}+00$ & $\mathrm{U}$ \\
\hline & ${ }^{90} \mathrm{Sr}$ & $-2.1 \mathrm{E}-01 \pm 2.1 \mathrm{E}-01$ & $\mathrm{U}$ & & ${ }^{90} \mathrm{Sr}$ & $-4.5 \mathrm{E}-02 \pm 1.2 \mathrm{E}-01$ & $\mathrm{U}$ \\
\hline & ${ }^{234} \mathrm{U}$ & $1.6 \mathrm{E}-02 \pm 9.0 \mathrm{E}-03$ & & & ${ }^{234} \mathrm{U}$ & $2.0 \mathrm{E}-02 \pm 9.4 \mathrm{E}-03$ & \\
\hline & ${ }^{235} \mathrm{U}$ & $4.7 \mathrm{E}-03 \pm 4.4 \mathrm{E}-03$ & & & ${ }^{235} \mathrm{U}$ & $2.7 \mathrm{E}-03 \pm 3.2 \mathrm{E}-03$ & \\
\hline & ${ }^{238} \mathrm{U}$ & $1.4 \mathrm{E}-02 \pm 7.8 \mathrm{E}-03$ & & & ${ }^{238} \mathrm{U}$ & $9.8 \mathrm{E}-03 \pm 6.6 \mathrm{E}-03$ & \\
\hline & ${ }^{65} \mathrm{Zn}$ & $9.6 \mathrm{E}-02 \pm 9.8 \mathrm{E}-02$ & $\mathrm{U}$ & & ${ }^{65} \mathrm{Zn}$ & $-1.2 \mathrm{E}+01 \pm 1.2 \mathrm{E}+01$ & $\mathrm{U}$ \\
\hline
\end{tabular}

$\overline{\mathrm{RQ}}=$ Result Qualifier. U $=$ The analyte was analyzed for but not detected. 
Table 3-5. 2003 Vegetation Sampling Results (pCi/g \pm total analytical uncertainty). (cont)

\begin{tabular}{|c|c|c|c|c|c|c|c|}
\hline Location & Isotope & Result \pm Error & $\mathbf{R Q}^{*}$ & Location & Isotope & Result \pm Error & $\mathbf{R Q}^{*}$ \\
\hline V047 & ${ }^{144} \mathrm{Ce}$ & $-1.3 \mathrm{E}-01 \pm 6.8 \mathrm{E}-01$ & $\bar{U}$ & V049 & ${ }^{144} \mathrm{Ce}$ & $-4.6 \mathrm{E}-01 \pm 6.9 \mathrm{E}-01$ & $\overline{\mathrm{U}}$ \\
\hline \multirow[t]{17}{*}{ (200 West) } & ${ }^{60} \mathrm{Co}$ & $5.2 \mathrm{E}-02 \pm 5.0 \mathrm{E}-02$ & $\mathrm{U}$ & (200 West) & ${ }^{60} \mathrm{Co}$ & $-1.8 \mathrm{E}-03 \pm 1.8 \mathrm{E}-02$ & $\mathrm{U}$ \\
\hline & ${ }^{134} \mathrm{Cs}$ & $2.1 \mathrm{E}-03 \pm 2.1 \mathrm{E}-02$ & $\mathrm{U}$ & & ${ }^{134} \mathrm{Cs}$ & $5.9 \mathrm{E}-03 \pm 5.2 \mathrm{E}-02$ & $\mathrm{U}$ \\
\hline & ${ }^{137} \mathrm{Cs}$ & $1.3 \mathrm{E}-01 \pm 5.6 \mathrm{E}-02$ & & & ${ }^{137} \mathrm{Cs}$ & $-3.0 \mathrm{E}-02 \pm 5.3 \mathrm{E}-02$ & $\mathrm{U}$ \\
\hline & ${ }^{152} \mathrm{Eu}$ & $-8.9 \mathrm{E}-02 \pm 1.5 \mathrm{E}-01$ & $\mathrm{U}$ & & ${ }^{152} \mathrm{Eu}$ & $-5.5 \mathrm{E}-03 \pm 5.5 \mathrm{E}-02$ & $\mathrm{U}$ \\
\hline & ${ }^{154} \mathrm{Eu}$ & $8.0 \mathrm{E}-02 \pm 1.4 \mathrm{E}-01$ & $\mathrm{U}$ & & ${ }^{154} \mathrm{Eu}$ & $3.7 \mathrm{E}-02 \pm 1.4 \mathrm{E}-01$ & $\mathrm{U}$ \\
\hline & ${ }^{155} \mathrm{Eu}$ & $-2.5 \mathrm{E}-02 \pm 1.9 \mathrm{E}-01$ & $\mathrm{U}$ & & ${ }^{155} \mathrm{Eu}$ & $-4.1 \mathrm{E}-02 \pm 1.7 \mathrm{E}-01$ & $\mathrm{U}$ \\
\hline & ${ }^{238} \mathrm{Pu}$ & $-8.6 \mathrm{E}-04 \pm 8.6 \mathrm{E}-03$ & $\mathrm{U}$ & & ${ }^{238} \mathrm{Pu}$ & $-2.0 \mathrm{E}-03 \pm 1.7 \mathrm{E}-02$ & $\mathrm{U}$ \\
\hline & ${ }^{239,240} \mathrm{Pu}$ & $8.6 \mathrm{E}-04 \pm 4.6 \mathrm{E}-03$ & $\mathrm{U}$ & & ${ }^{239,240} \mathrm{Pu}$ & $5.0 \mathrm{E}-03 \pm 6.0 \mathrm{E}-03$ & $\mathrm{U}$ \\
\hline & ${ }^{103} \mathrm{Ru}$ & $-4.7 \mathrm{E}-03 \pm 4.7 \mathrm{E}-02$ & $\mathrm{U}$ & & ${ }^{103} \mathrm{Ru}$ & $-5.6 \mathrm{E}-02 \pm 5.6 \mathrm{E}-02$ & $\mathrm{U}$ \\
\hline & ${ }^{106} \mathrm{Ru}$ & $-2.9 \mathrm{E}-01 \pm 4.5 \mathrm{E}-01$ & $\mathrm{U}$ & & ${ }^{106} \mathrm{Ru}$ & $6.9 \mathrm{E}-02 \pm 4.7 \mathrm{E}-01$ & $\mathrm{U}$ \\
\hline & ${ }^{125} \mathrm{Sb}$ & 4.3E-02 $\pm 1.3 \mathrm{E}-01$ & $\mathrm{U}$ & & ${ }^{125} \mathrm{Sb}$ & $-4.7 \mathrm{E}-02 \pm 1.4 \mathrm{E}-01$ & $\mathrm{U}$ \\
\hline & ${ }^{113} \mathrm{Sn}$ & $-1.2 \mathrm{E}-02 \pm 6.3 \mathrm{E}-02$ & $\mathrm{U}$ & & ${ }^{113} \mathrm{Sn}$ & $1.3 \mathrm{E}-02 \pm 6.8 \mathrm{E}-02$ & $\mathrm{U}$ \\
\hline & ${ }^{90} \mathrm{Sr}$ & $2.4 \mathrm{E}-01 \pm 1.5 \mathrm{E}-01$ & & & ${ }^{90} \mathrm{Sr}$ & $-1.3 \mathrm{E}-01 \pm 1.3 \mathrm{E}-01$ & $\mathrm{U}$ \\
\hline & ${ }^{234} \mathrm{U}$ & $2.2 \mathrm{E}-02 \pm 1.0 \mathrm{E}-02$ & & & ${ }^{234} \mathrm{U}$ & $1.4 \mathrm{E}-02 \pm 9.2 \mathrm{E}-03$ & \\
\hline & ${ }^{235} \mathrm{U}$ & $4.6 \mathrm{E}-03 \pm 4.3 \mathrm{E}-03$ & & & ${ }^{235} \mathrm{U}$ & $7.2 \mathrm{E}-03 \pm 5.8 \mathrm{E}-03$ & \\
\hline & ${ }^{238} \mathrm{U}$ & $1.8 \mathrm{E}-02 \pm 9.4 \mathrm{E}-03$ & & & ${ }^{238} \mathrm{U}$ & $1.0 \mathrm{E}-02 \pm 7.0 \mathrm{E}-03$ & \\
\hline & ${ }^{65} \mathrm{Zn}$ & $-1.5 \mathrm{E}-01 \pm 1.5 \mathrm{E}-01$ & $\mathrm{U}$ & & ${ }^{65} \mathrm{Zn}$ & $-3.9 \mathrm{E}-01 \pm 3.9 \mathrm{E}-01$ & $\mathrm{U}$ \\
\hline V051 & ${ }^{144} \mathrm{Ce}$ & $-2.9 \mathrm{E}-01 \pm 3.8 \mathrm{E}-01$ & $\overline{\mathrm{U}}$ & V053 & ${ }^{144} \mathrm{Ce}$ & $1.4 \mathrm{E}-02 \pm 1.4 \mathrm{E}-01$ & $\bar{U}$ \\
\hline \multirow[t]{17}{*}{ (200 West) } & ${ }^{60} \mathrm{Co}$ & $-1.5 \mathrm{E}-02 \pm 2.8 \mathrm{E}-02$ & $\mathrm{U}$ & (200 East) & ${ }^{60} \mathrm{Co}$ & $2.3 \mathrm{E}-02 \pm 3.4 \mathrm{E}-02$ & $\mathrm{U}$ \\
\hline & ${ }^{134} \mathrm{Cs}$ & $-5.2 \mathrm{E}-03 \pm 2.9 \mathrm{E}-02$ & $\mathrm{U}$ & & ${ }^{134} \mathrm{Cs}$ & $8.8 \mathrm{E}-03 \pm 3.5 \mathrm{E}-02$ & $\mathrm{U}$ \\
\hline & ${ }^{137} \mathrm{Cs}$ & $3.2 \mathrm{E}-02 \pm 3.0 \mathrm{E}-02$ & $\mathrm{U}$ & & ${ }^{137} \mathrm{Cs}$ & $-1.1 \mathrm{E}-02 \pm 3.5 \mathrm{E}-02$ & $\mathrm{U}$ \\
\hline & ${ }^{152} \mathrm{Eu}$ & $-3.9 \mathrm{E}-02 \pm 8.2 \mathrm{E}-02$ & $\mathrm{U}$ & & ${ }^{152} \mathrm{Eu}$ & $-4.1 \mathrm{E}-02 \pm 8.5 \mathrm{E}-02$ & $\mathrm{U}$ \\
\hline & ${ }^{154} \mathrm{Eu}$ & $-4.6 \mathrm{E}-02 \pm 8.7 \mathrm{E}-02$ & $\mathrm{U}$ & & ${ }^{154} \mathrm{Eu}$ & $-1.4 \mathrm{E}-01 \pm 1.4 \mathrm{E}-01$ & $\mathrm{U}$ \\
\hline & ${ }^{155} \mathrm{Eu}$ & $5.6 \mathrm{E}-02 \pm 9.4 \mathrm{E}-02$ & $\mathrm{U}$ & & ${ }^{155} \mathrm{Eu}$ & $2.1 \mathrm{E}-02 \pm 1.1 \mathrm{E}-01$ & $\mathrm{U}$ \\
\hline & ${ }^{238} \mathrm{Pu}$ & $-3.5 \mathrm{E}-03 \pm 4.9 \mathrm{E}-03$ & $\mathrm{U}$ & & ${ }^{238} \mathrm{Pu}$ & $-9.4 \mathrm{E}-04 \pm 8.2 \mathrm{E}-03$ & $\mathrm{U}$ \\
\hline & ${ }^{239,240} \mathrm{Pu}$ & $8.8 \mathrm{E}-04 \pm 4.0 \mathrm{E}-03$ & $\mathrm{U}$ & & ${ }^{239,240} \mathrm{Pu}$ & $9.4 \mathrm{E}-04 \pm 9.4 \mathrm{E}-03$ & $\mathrm{U}$ \\
\hline & ${ }^{103} \mathrm{Ru}$ & $-1.3 \mathrm{E}-02 \pm 2.9 \mathrm{E}-02$ & $\mathrm{U}$ & & ${ }^{103} \mathrm{Ru}$ & $2.8 \mathrm{E}-02 \pm 3.4 \mathrm{E}-02$ & $\mathrm{U}$ \\
\hline & ${ }^{106} \mathrm{Ru}$ & $-2.0 \mathrm{E}-02 \pm 2.0 \mathrm{E}-01$ & $\mathrm{U}$ & & ${ }^{106} \mathrm{Ru}$ & $9.0 \mathrm{E}-02 \pm 2.9 \mathrm{E}-01$ & $\mathrm{U}$ \\
\hline & ${ }^{125} \mathrm{Sb}$ & $5.6 \mathrm{E}-02 \pm 7.7 \mathrm{E}-02$ & $\mathrm{U}$ & & ${ }^{125} \mathrm{Sb}$ & $-9.8 \mathrm{E}-03 \pm 8.1 \mathrm{E}-02$ & $\mathrm{U}$ \\
\hline & ${ }^{113} \mathrm{Sn}$ & $2.3 \mathrm{E}-02 \pm 3.7 \mathrm{E}-02$ & $\mathrm{U}$ & & ${ }^{113} \mathrm{Sn}$ & $-1.0 \mathrm{E}-02 \pm 3.8 \mathrm{E}-02$ & $\mathrm{U}$ \\
\hline & ${ }^{90} \mathrm{Sr}$ & $9.9 \mathrm{E}-03 \pm 9.9 \mathrm{E}-02$ & $\mathrm{U}$ & & ${ }^{90} \mathrm{Sr}$ & $-7.6 \mathrm{E}-02 \pm 1.1 \mathrm{E}-01$ & $\mathrm{U}$ \\
\hline & ${ }^{234} \mathrm{U}$ & $1.5 \mathrm{E}-02 \pm 8.4 \mathrm{E}-03$ & & & ${ }^{234} \mathrm{U}$ & $8.2 \mathrm{E}-03 \pm 6.1 \mathrm{E}-03$ & \\
\hline & ${ }^{235} \mathrm{U}$ & $7.3 \mathrm{E}-03 \pm 5.5 \mathrm{E}-03$ & & & ${ }^{235} \mathrm{U}$ & $2.7 \mathrm{E}-03 \pm 4.1 \mathrm{E}-03$ & $\mathrm{U}$ \\
\hline & ${ }^{238} \mathrm{U}$ & $1.8 \mathrm{E}-02 \pm 9.0 \mathrm{E}-03$ & & & ${ }^{238} \mathrm{U}$ & $7.4 \mathrm{E}-03 \pm 5.8 \mathrm{E}-03$ & \\
\hline & ${ }^{65} \mathrm{Zn}$ & $-1.4 \mathrm{E}-01 \pm 1.4 \mathrm{E}-01$ & $\mathrm{U}$ & & ${ }^{65} \mathrm{Zn}$ & $-4.4 \mathrm{E}-02 \pm 7.9 \mathrm{E}-02$ & $\mathrm{U}$ \\
\hline
\end{tabular}

$\overline{\mathrm{RQ}}=$ Result Qualifier. U $=$ The analyte was analyzed for but not detected. 
Table 3-5. 2003 Vegetation Sampling Results (pCi/g \pm total analytical uncertainty). (cont)

\begin{tabular}{|c|c|c|c|c|c|c|c|}
\hline Location & Isotope & Result \pm Error & $\mathbf{R Q}^{*}$ & Location & Isotope & Result \pm Error & $\mathbf{R Q}^{*}$ \\
\hline V055 & ${ }^{144} \mathrm{Ce}$ & $1.0 \mathrm{E}-01 \pm 3.6 \mathrm{E}-01$ & $\bar{U}$ & V057 & ${ }^{144} \mathrm{Ce}$ & $9.3 \mathrm{E}-02 \pm 3.2 \mathrm{E}-01$ & $\overline{\mathrm{U}}$ \\
\hline \multirow[t]{17}{*}{ (200 East) } & ${ }^{60} \mathrm{Co}$ & $-1.1 \mathrm{E}-02 \pm 2.8 \mathrm{E}-02$ & $\mathrm{U}$ & (200 East) & ${ }^{60} \mathrm{Co}$ & $-4.1 \mathrm{E}-02 \pm 4.1 \mathrm{E}-02$ & $\mathrm{U}$ \\
\hline & ${ }^{134} \mathrm{Cs}$ & $-1.7 \mathrm{E}-02 \pm 2.8 \mathrm{E}-02$ & $\mathrm{U}$ & & ${ }^{134} \mathrm{Cs}$ & $-3.6 \mathrm{E}-03 \pm 3.6 \mathrm{E}-02$ & $\mathrm{U}$ \\
\hline & ${ }^{137} \mathrm{Cs}$ & $8.5 \mathrm{E}-02 \pm 6.0 \mathrm{E}-02$ & & & ${ }^{137} \mathrm{Cs}$ & $3.4 \mathrm{E}-02 \pm 3.7 \mathrm{E}-02$ & $\mathrm{U}$ \\
\hline & ${ }^{152} \mathrm{Eu}$ & $-1.6 \mathrm{E}-02 \pm 8.3 \mathrm{E}-02$ & $\mathrm{U}$ & & ${ }^{152} \mathrm{Eu}$ & $8.2 \mathrm{E}-03 \pm 8.0 \mathrm{E}-02$ & $\mathrm{U}$ \\
\hline & ${ }^{154} \mathrm{Eu}$ & $-6.8 \mathrm{E}-02 \pm 8.4 \mathrm{E}-02$ & $\mathrm{U}$ & & ${ }^{154} \mathrm{Eu}$ & $-3.8 \mathrm{E}-02 \pm 1.0 \mathrm{E}-01$ & $\mathrm{U}$ \\
\hline & ${ }^{155} \mathrm{Eu}$ & $-1.8 \mathrm{E}-02 \pm 8.9 \mathrm{E}-02$ & $\mathrm{U}$ & & ${ }^{155} \mathrm{Eu}$ & $3.6 \mathrm{E}-02 \pm 9.0 \mathrm{E}-02$ & $\mathrm{U}$ \\
\hline & ${ }^{238} \mathrm{Pu}$ & $4.8 \mathrm{E}-03 \pm 1.5 \mathrm{E}-02$ & $\mathrm{U}$ & & ${ }^{238} \mathrm{Pu}$ & $1.4 \mathrm{E}-02 \pm 1.4 \mathrm{E}-02$ & $\mathrm{U}$ \\
\hline & ${ }^{239,240} \mathrm{Pu}$ & $9.6 \mathrm{E}-04 \pm 5.8 \mathrm{E}-03$ & $\mathrm{U}$ & & ${ }^{239,240} \mathrm{Pu}$ & $9.2 \mathrm{E}-04 \pm 9.2 \mathrm{E}-03$ & $\mathrm{U}$ \\
\hline & ${ }^{103} \mathrm{Ru}$ & $-9.1 \mathrm{E}-03 \pm 2.6 \mathrm{E}-02$ & $\mathrm{U}$ & & ${ }^{103} \mathrm{Ru}$ & $4.9 \mathrm{E}-04 \pm 4.9 \mathrm{E}-03$ & $\mathrm{U}$ \\
\hline & ${ }^{106} \mathrm{Ru}$ & $2.5 \mathrm{E}-02 \pm 2.4 \mathrm{E}-01$ & $\mathrm{U}$ & & ${ }^{106} \mathrm{Ru}$ & $-2.0 \mathrm{E}-02 \pm 2.0 \mathrm{E}-01$ & $\mathrm{U}$ \\
\hline & ${ }^{125} \mathrm{Sb}$ & $2.2 \mathrm{E}-02 \pm 7.2 \mathrm{E}-02$ & $\mathrm{U}$ & & ${ }^{125} \mathrm{Sb}$ & $-1.1 \mathrm{E}-02 \pm 7.3 \mathrm{E}-02$ & $\mathrm{U}$ \\
\hline & ${ }^{113} \mathrm{Sn}$ & $-1.2 \mathrm{E}-03 \pm 1.2 \mathrm{E}-02$ & $\mathrm{U}$ & & ${ }^{113} \mathrm{Sn}$ & $-1.6 \mathrm{E}-02 \pm 3.5 \mathrm{E}-02$ & $\mathrm{U}$ \\
\hline & ${ }^{90} \mathrm{Sr}$ & $1.8 \mathrm{E}-01 \pm 1.3 \mathrm{E}-01$ & & & ${ }^{90} \mathrm{Sr}$ & $1.7 \mathrm{E}-01 \pm 1.4 \mathrm{E}-01$ & \\
\hline & ${ }^{234} \mathrm{U}$ & $1.0 \mathrm{E}-02 \pm 7.5 \mathrm{E}-03$ & & & ${ }^{234} \mathrm{U}$ & $1.2 \mathrm{E}-02 \pm 7.4 \mathrm{E}-03$ & \\
\hline & ${ }^{235} \mathrm{U}$ & $5.1 \mathrm{E}-03 \pm 5.6 \mathrm{E}-03$ & $\mathrm{U}$ & & ${ }^{235} \mathrm{U}$ & $9.1 \mathrm{E}-04 \pm 3.2 \mathrm{E}-03$ & $\mathrm{U}$ \\
\hline & ${ }^{238} \mathrm{U}$ & $1.0 \mathrm{E}-02 \pm 6.6 \mathrm{E}-03$ & & & ${ }^{238} \mathrm{U}$ & $6.7 \mathrm{E}-03 \pm 5.0 \mathrm{E}-03$ & \\
\hline & ${ }^{65} \mathrm{Zn}$ & $-1.1 \mathrm{E}-01 \pm 1.1 \mathrm{E}-01$ & $\mathrm{U}$ & & ${ }^{65} \mathrm{Zn}$ & $3.8 \mathrm{E}-02 \pm 8.2 \mathrm{E}-02$ & $\mathrm{U}$ \\
\hline V059 & ${ }^{144} \mathrm{Ce}$ & $-1.3 \mathrm{E}-01 \pm 4.4 \mathrm{E}-01$ & $\overline{\mathrm{U}}$ & V061 & ${ }^{144} \mathrm{Ce}$ & $-6.4 \mathrm{E}-02 \pm 4.1 \mathrm{E}-01$ & $\bar{U}$ \\
\hline \multirow[t]{17}{*}{ (200 East) } & ${ }^{60} \mathrm{Co}$ & $9.7 \mathrm{E}-03 \pm 3.2 \mathrm{E}-02$ & $\mathrm{U}$ & (200 East) & ${ }^{60} \mathrm{Co}$ & $1.0 \mathrm{E}-02 \pm 3.5 \mathrm{E}-02$ & $\mathrm{U}$ \\
\hline & ${ }^{134} \mathrm{Cs}$ & $1.2 \mathrm{E}-02 \pm 3.5 \mathrm{E}-02$ & $\mathrm{U}$ & & ${ }^{134} \mathrm{Cs}$ & $-6.9 \mathrm{E}-03 \pm 3.2 \mathrm{E}-02$ & $\mathrm{U}$ \\
\hline & ${ }^{137} \mathrm{Cs}$ & $1.2 \mathrm{E}-01 \pm 6.0 \mathrm{E}-02$ & & & ${ }^{137} \mathrm{Cs}$ & $2.4 \mathrm{E}-02 \pm 3.1 \mathrm{E}-02$ & $\mathrm{U}$ \\
\hline & ${ }^{152} \mathrm{Eu}$ & $-1.6 \mathrm{E}-02 \pm 9.9 \mathrm{E}-02$ & $\mathrm{U}$ & & ${ }^{152} \mathrm{Eu}$ & $-7.9 \mathrm{E}-02 \pm 1.0 \mathrm{E}-01$ & $\mathrm{U}$ \\
\hline & ${ }^{154} \mathrm{Eu}$ & $-8.6 \mathrm{E}-04 \pm 8.6 \mathrm{E}-03$ & $\mathrm{U}$ & & ${ }^{154} \mathrm{Eu}$ & $6.4 \mathrm{E}-03 \pm 6.4 \mathrm{E}-02$ & $\mathrm{U}$ \\
\hline & ${ }^{155} \mathrm{Eu}$ & $-4.5 \mathrm{E}-02 \pm 1.1 \mathrm{E}-01$ & $\mathrm{U}$ & & ${ }^{155} \mathrm{Eu}$ & $-2.5 \mathrm{E}-02 \pm 1.1 \mathrm{E}-01$ & $\mathrm{U}$ \\
\hline & ${ }^{238} \mathrm{Pu}$ & $-8.6 \mathrm{E}-03 \pm 1.7 \mathrm{E}-02$ & $\mathrm{U}$ & & ${ }^{238} \mathrm{Pu}$ & $-2.7 \mathrm{E}-02 \pm 2.7 \mathrm{E}-02$ & $\mathrm{U}$ \\
\hline & ${ }^{239,240} \mathrm{Pu}$ & $2.2 \mathrm{E}-03 \pm 3.1 \mathrm{E}-03$ & $\mathrm{U}$ & & ${ }^{239,240} \mathrm{Pu}$ & $4.7 \mathrm{E}-03 \pm 5.2 \mathrm{E}-03$ & $\mathrm{U}$ \\
\hline & ${ }^{103} \mathrm{Ru}$ & $2.3 \mathrm{E}-03 \pm 2.3 \mathrm{E}-02$ & $\mathrm{U}$ & & ${ }^{103} \mathrm{Ru}$ & $-1.9 \mathrm{E}-02 \pm 2.9 \mathrm{E}-02$ & $\mathrm{U}$ \\
\hline & ${ }^{106} \mathrm{Ru}$ & $-3.4 \mathrm{E}-01 \pm 3.4 \mathrm{E}-01$ & $\mathrm{U}$ & & ${ }^{106} \mathrm{Ru}$ & $-1.0 \mathrm{E}-01 \pm 2.7 \mathrm{E}-01$ & $\mathrm{U}$ \\
\hline & ${ }^{125} \mathrm{Sb}$ & 4.3E-02 $\pm 8.8 \mathrm{E}-02$ & $\mathrm{U}$ & & ${ }^{125} \mathrm{Sb}$ & 3.0E-02 $\pm 8.3 \mathrm{E}-02$ & $\mathrm{U}$ \\
\hline & ${ }^{113} \mathrm{Sn}$ & $-4.4 \mathrm{E}-02 \pm 4.4 \mathrm{E}-02$ & $\mathrm{U}$ & & ${ }^{113} \mathrm{Sn}$ & $1.2 \mathrm{E}-02 \pm 3.7 \mathrm{E}-02$ & $\mathrm{U}$ \\
\hline & ${ }^{90} \mathrm{Sr}$ & $7.1 \mathrm{E}-02 \pm 1.2 \mathrm{E}-01$ & $\mathrm{U}$ & & ${ }^{90} \mathrm{Sr}$ & $-1.2 \mathrm{E}-01 \pm 1.2 \mathrm{E}-01$ & $\mathrm{U}$ \\
\hline & ${ }^{234} \mathrm{U}$ & $6.5 \mathrm{E}-03 \pm 6.4 \mathrm{E}-03$ & $\mathrm{U}$ & & ${ }^{234} \mathrm{U}$ & $6.3 \mathrm{E}-03 \pm 5.0 \mathrm{E}-03$ & \\
\hline & ${ }^{235} \mathrm{U}$ & $2.0 \mathrm{E}-03 \pm 5.6 \mathrm{E}-03$ & $\mathrm{U}$ & & ${ }^{235} \mathrm{U}$ & $2.0 \mathrm{E}-03 \pm 4.0 \mathrm{E}-03$ & $\mathrm{U}$ \\
\hline & ${ }^{238} \mathrm{U}$ & 9.3E-04 \pm 9.3Е-04 & $\mathrm{U}$ & & ${ }^{238} \mathrm{U}$ & $1.2 \mathrm{E}-02 \pm 7.3 \mathrm{E}-03$ & \\
\hline & ${ }^{65} \mathrm{Zn}$ & $-8.1 \mathrm{E}-02 \pm 8.1 \mathrm{E}-02$ & $\mathrm{U}$ & & ${ }^{65} \mathrm{Zn}$ & $-9.6 \mathrm{E}-02 \pm 9.6 \mathrm{E}-02$ & $\mathrm{U}$ \\
\hline
\end{tabular}

$\overline{\mathrm{RQ}}=$ Result Qualifier. U $=$ The analyte was analyzed for but not detected. 
Table 3-5. 2003 Vegetation Sampling Results (pCi/g \pm total analytical uncertainty). (cont)

\begin{tabular}{|c|c|c|c|c|c|c|c|}
\hline Location & Isotope & Result \pm Error & $\mathbf{R Q}^{*}$ & Location & Isotope & Result \pm Error & $\mathbf{R Q}^{*}$ \\
\hline V063 & ${ }^{144} \mathrm{Ce}$ & $2.9 \mathrm{E}-01 \pm 7.9 \mathrm{E}-01$ & $\bar{U}$ & V079 & ${ }^{144} \mathrm{Ce}$ & $-3.8 \mathrm{E}-02 \pm 3.3 \mathrm{E}-01$ & $\overline{\mathrm{U}}$ \\
\hline \multirow[t]{17}{*}{ (200 East) } & ${ }^{60} \mathrm{Co}$ & $3.4 \mathrm{E}-02 \pm 5.8 \mathrm{E}-02$ & $\mathrm{U}$ & (200 East) & ${ }^{60} \mathrm{Co}$ & $2.7 \mathrm{E}-04 \pm 2.7 \mathrm{E}-03$ & $\mathrm{U}$ \\
\hline & ${ }^{134} \mathrm{Cs}$ & $2.4 \mathrm{E}-02 \pm 5.8 \mathrm{E}-02$ & $\mathrm{U}$ & & ${ }^{134} \mathrm{Cs}$ & $8.3 \mathrm{E}-03 \pm 2.6 \mathrm{E}-02$ & $\mathrm{U}$ \\
\hline & ${ }^{137} \mathrm{Cs}$ & $-2.4 \mathrm{E}-02 \pm 5.9 \mathrm{E}-02$ & $\mathrm{U}$ & & ${ }^{137} \mathrm{Cs}$ & $1.1 \mathrm{E}-02 \pm 2.6 \mathrm{E}-02$ & $\mathrm{U}$ \\
\hline & ${ }^{152} \mathrm{Eu}$ & $-1.4 \mathrm{E}-01 \pm 1.7 \mathrm{E}-01$ & $\mathrm{U}$ & & ${ }^{152} \mathrm{Eu}$ & $8.7 \mathrm{E}-03 \pm 7.5 \mathrm{E}-02$ & $\mathrm{U}$ \\
\hline & ${ }^{154} \mathrm{Eu}$ & $-1.5 \mathrm{E}-01 \pm 2.0 \mathrm{E}-01$ & $\mathrm{U}$ & & ${ }^{154} \mathrm{Eu}$ & $-1.0 \mathrm{E}-02 \pm 8.4 \mathrm{E}-02$ & $\mathrm{U}$ \\
\hline & ${ }^{155} \mathrm{Eu}$ & $-4.4 \mathrm{E}-02 \pm 2.0 \mathrm{E}-01$ & $\mathrm{U}$ & & ${ }^{155} \mathrm{Eu}$ & $3.8 \mathrm{E}-03 \pm 3.8 \mathrm{E}-02$ & $\mathrm{U}$ \\
\hline & ${ }^{238} \mathrm{Pu}$ & $-6.4 \mathrm{E}-03 \pm 1.8 \mathrm{E}-02$ & $\mathrm{U}$ & & ${ }^{238} \mathrm{Pu}$ & $2.3 \mathrm{E}-03 \pm 8.7 \mathrm{E}-03$ & $\mathrm{U}$ \\
\hline & ${ }^{239,240} \mathrm{Pu}$ & $4.2 \mathrm{E}-03 \pm 5.9 \mathrm{E}-03$ & $\mathrm{U}$ & & ${ }^{239,240} \mathrm{Pu}$ & $2.3 \mathrm{E}-03 \pm 5.8 \mathrm{E}-03$ & $\mathrm{U}$ \\
\hline & ${ }^{103} \mathrm{Ru}$ & $-1.9 \mathrm{E}-03 \pm 1.9 \mathrm{E}-02$ & $\mathrm{U}$ & & ${ }^{103} \mathrm{Ru}$ & $-2.6 \mathrm{E}-02 \pm 2.6 \mathrm{E}-02$ & $\mathrm{U}$ \\
\hline & ${ }^{106} \mathrm{Ru}$ & $-7.0 \mathrm{E}-02 \pm 5.2 \mathrm{E}-01$ & $\mathrm{U}$ & & ${ }^{106} \mathrm{Ru}$ & $-1.5 \mathrm{E}-01 \pm 2.3 \mathrm{E}-01$ & $\mathrm{U}$ \\
\hline & ${ }^{125} \mathrm{Sb}$ & $-3.9 \mathrm{E}-02 \pm 1.5 \mathrm{E}-01$ & $\mathrm{U}$ & & ${ }^{125} \mathrm{Sb}$ & $-7.7 \mathrm{E}-03 \pm 6.5 \mathrm{E}-02$ & $\mathrm{U}$ \\
\hline & ${ }^{113} \mathrm{Sn}$ & 7.4E-02 $\pm 7.3 \mathrm{E}-02$ & $\mathrm{U}$ & & ${ }^{113} \mathrm{Sn}$ & $8.9 \mathrm{E}-04 \pm 8.9 \mathrm{E}-03$ & $\mathrm{U}$ \\
\hline & ${ }^{90} \mathrm{Sr}$ & $1.2 \mathrm{E}+01 \pm 1.8 \mathrm{E}+00$ & & & ${ }^{90} \mathrm{Sr}$ & $-5.9 \mathrm{E}-02 \pm 1.2 \mathrm{E}-01$ & $\mathrm{U}$ \\
\hline & ${ }^{234} \mathrm{U}$ & $1.8 \mathrm{E}-02 \pm 1.0 \mathrm{E}-02$ & & & ${ }^{234} \mathrm{U}$ & $1.2 \mathrm{E}-02 \pm 7.4 \mathrm{E}-03$ & \\
\hline & ${ }^{235} \mathrm{U}$ & $2.4 \mathrm{E}-03 \pm 3.4 \mathrm{E}-03$ & $\mathrm{U}$ & & ${ }^{235} \mathrm{U}$ & $2.9 \mathrm{E}-03 \pm 3.5 \mathrm{E}-03$ & \\
\hline & ${ }^{238} \mathrm{U}$ & $2.2 \mathrm{E}-03 \pm 3.1 \mathrm{E}-03$ & $\mathrm{U}$ & & ${ }^{238} \mathrm{U}$ & $1.3 \mathrm{E}-02 \pm 7.5 \mathrm{E}-03$ & \\
\hline & ${ }^{65} \mathrm{Zn}$ & $-4.3 \mathrm{E}-01 \pm 4.3 \mathrm{E}-01$ & $\mathrm{U}$ & & ${ }^{65} \mathrm{Zn}$ & $-9.9 \mathrm{E}-02 \pm 9.9 \mathrm{E}-02$ & $\mathrm{U}$ \\
\hline V081 & ${ }^{144} \mathrm{Ce}$ & $1.4 \mathrm{E}-01 \pm 3.3 \mathrm{E}-01$ & $\bar{U}$ & V083 & ${ }^{144} \mathrm{Ce}$ & $-1.6 \mathrm{E}-01 \pm 3.7 \mathrm{E}-01$ & $\bar{U}$ \\
\hline \multirow[t]{17}{*}{ (600 Area) } & ${ }^{60} \mathrm{Co}$ & $-1.7 \mathrm{E}-02 \pm 2.4 \mathrm{E}-02$ & $\mathrm{U}$ & (600 Area) & ${ }^{60} \mathrm{Co}$ & $1.0 \mathrm{E}-02 \pm 3.6 \mathrm{E}-02$ & $\mathrm{U}$ \\
\hline & ${ }^{134} \mathrm{Cs}$ & $1.1 \mathrm{E}-02 \pm 2.4 \mathrm{E}-02$ & $\mathrm{U}$ & & ${ }^{134} \mathrm{Cs}$ & $-2.2 \mathrm{E}-02 \pm 3.6 \mathrm{E}-02$ & $\mathrm{U}$ \\
\hline & ${ }^{137} \mathrm{Cs}$ & $9.5 \mathrm{E}-03 \pm 2.3 \mathrm{E}-02$ & $\mathrm{U}$ & & ${ }^{137} \mathrm{Cs}$ & $-4.1 \mathrm{E}-03 \pm 3.3 \mathrm{E}-02$ & $\mathrm{U}$ \\
\hline & ${ }^{152} \mathrm{Eu}$ & $-3.3 \mathrm{E}-02 \pm 7.0 \mathrm{E}-02$ & $\mathrm{U}$ & & ${ }^{152} \mathrm{Eu}$ & $-7.6 \mathrm{E}-02 \pm 9.1 \mathrm{E}-02$ & $\mathrm{U}$ \\
\hline & ${ }^{154} \mathrm{Eu}$ & $-2.1 \mathrm{E}-02 \pm 8.4 \mathrm{E}-02$ & $\mathrm{U}$ & & ${ }^{154} \mathrm{Eu}$ & $-7.4 \mathrm{E}-02 \pm 1.1 \mathrm{E}-01$ & $\mathrm{U}$ \\
\hline & ${ }^{155} \mathrm{Eu}$ & $-1.8 \mathrm{E}-02 \pm 8.0 \mathrm{E}-02$ & $\mathrm{U}$ & & ${ }^{155} \mathrm{Eu}$ & $1.6 \mathrm{E}-01 \pm 9.9 \mathrm{E}-02$ & $\mathrm{U}$ \\
\hline & ${ }^{238} \mathrm{Pu}$ & $-1.0 \mathrm{E}-03 \pm 8.7 \mathrm{E}-03$ & $\mathrm{U}$ & & ${ }^{238} \mathrm{Pu}$ & $1.3 \mathrm{E}-02 \pm 1.8 \mathrm{E}-02$ & $\mathrm{U}$ \\
\hline & ${ }^{239,240} \mathrm{Pu}$ & $1.1 \mathrm{E}-03 \pm 1.1 \mathrm{E}-02$ & $\mathrm{U}$ & & ${ }^{239,240} \mathrm{Pu}$ & $1.1 \mathrm{E}-03 \pm 5.8 \mathrm{E}-03$ & $\mathrm{U}$ \\
\hline & ${ }^{103} \mathrm{Ru}$ & $-4.7 \mathrm{E}-03 \pm 2.3 \mathrm{E}-02$ & $\mathrm{U}$ & & ${ }^{103} \mathrm{Ru}$ & $2.5 \mathrm{E}-02 \pm 3.1 \mathrm{E}-02$ & $\mathrm{U}$ \\
\hline & ${ }^{106} \mathrm{Ru}$ & $1.2 \mathrm{E}-01 \pm 2.1 \mathrm{E}-01$ & $\mathrm{U}$ & & ${ }^{106} \mathrm{Ru}$ & $-4.1 \mathrm{E}-02 \pm 2.9 \mathrm{E}-01$ & $\mathrm{U}$ \\
\hline & ${ }^{125} \mathrm{Sb}$ & $-1.3 \mathrm{E}-03 \pm 1.3 \mathrm{E}-02$ & $\mathrm{U}$ & & ${ }^{125} \mathrm{Sb}$ & $-1.4 \mathrm{E}-02 \pm 8.0 \mathrm{E}-02$ & $\mathrm{U}$ \\
\hline & ${ }^{113} \mathrm{Sn}$ & $-1.8 \mathrm{E}-02 \pm 3.0 \mathrm{E}-02$ & $\mathrm{U}$ & & ${ }^{113} \mathrm{Sn}$ & $-3.1 \mathrm{E}-02 \pm 4.0 \mathrm{E}-02$ & $\mathrm{U}$ \\
\hline & ${ }^{90} \mathrm{Sr}$ & $-8.9 \mathrm{E}-02 \pm 1.2 \mathrm{E}-01$ & $\mathrm{U}$ & & ${ }^{90} \mathrm{Sr}$ & $-5.9 \mathrm{E}-02 \pm 1.4 \mathrm{E}-01$ & $\mathrm{U}$ \\
\hline & ${ }^{234} \mathrm{U}$ & $9.6 \mathrm{E}-03 \pm 7.6 \mathrm{E}-03$ & & & ${ }^{234} \mathrm{U}$ & $8.6 \mathrm{E}-03 \pm 7.1 \mathrm{E}-03$ & $\mathrm{U}$ \\
\hline & ${ }^{235} \mathrm{U}$ & $1.9 \mathrm{E}-03 \pm 4.7 \mathrm{E}-03$ & $\mathrm{U}$ & & ${ }^{235} \mathrm{U}$ & $2.4 \mathrm{E}-03 \pm 3.4 \mathrm{E}-03$ & $\mathrm{U}$ \\
\hline & ${ }^{238} \mathrm{U}$ & $7.0 \mathrm{E}-03 \pm 5.2 \mathrm{E}-03$ & & & ${ }^{238} \mathrm{U}$ & $3.8 \mathrm{E}-03 \pm 4.6 \mathrm{E}-03$ & $\mathrm{U}$ \\
\hline & ${ }^{65} \mathrm{Zn}$ & $-1.5 \mathrm{E}-01 \pm 1.5 \mathrm{E}-01$ & $\mathrm{U}$ & & ${ }^{65} \mathrm{Zn}$ & $-1.8 \mathrm{E}-01 \pm 1.8 \mathrm{E}-01$ & $\mathrm{U}$ \\
\hline
\end{tabular}

$\overline{\mathrm{RQ}}=$ Result Qualifier. U $=$ The analyte was analyzed for but not detected. 
Table 3-5. 2003 Vegetation Sampling Results (pCi/g \pm total analytical uncertainty). (cont)

\begin{tabular}{|c|c|c|c|c|c|c|c|}
\hline Location & Isotope & Result \pm Error & $\mathbf{R Q}^{*}$ & Location & Isotope & Result \pm Error & $\mathbf{R Q}^{*}$ \\
\hline V085 & ${ }^{144} \mathrm{Ce}$ & $-4.6 \mathrm{E}-03 \pm 4.6 \mathrm{E}-02$ & $\mathrm{U}$ & V087 & ${ }^{144} \mathrm{Ce}$ & $-4.2 \mathrm{E}-01 \pm 7.8 \mathrm{E}-01$ & $\overline{\mathrm{U}}$ \\
\hline \multirow[t]{16}{*}{ (600 Area) } & ${ }^{60} \mathrm{Co}$ & $-8.6 \mathrm{E}-03 \pm 3.7 \mathrm{E}-02$ & $\mathrm{U}$ & (600 Area) & ${ }^{60} \mathrm{Co}$ & $-2.7 \mathrm{E}-03 \pm 2.7 \mathrm{E}-02$ & $\mathrm{U}$ \\
\hline & ${ }^{134} \mathrm{Cs}$ & $-1.3 \mathrm{E}-02 \pm 4.1 \mathrm{E}-02$ & $\mathrm{U}$ & & ${ }^{134} \mathrm{Cs}$ & $-4.0 \mathrm{E}-02 \pm 7.2 \mathrm{E}-02$ & $\mathrm{U}$ \\
\hline & ${ }^{137} \mathrm{Cs}$ & $9.4 \mathrm{E}-03 \pm 3.8 \mathrm{E}-02$ & $\mathrm{U}$ & & ${ }^{137} \mathrm{Cs}$ & $1.8 \mathrm{E}-01 \pm 1.1 \mathrm{E}-01$ & \\
\hline & ${ }^{152} \mathrm{Eu}$ & $3.9 \mathrm{E}-02 \pm 1.6 \mathrm{E}-01$ & $\mathrm{U}$ & & ${ }^{152} \mathrm{Eu}$ & $-4.1 \mathrm{E}-02 \pm 1.7 \mathrm{E}-01$ & $\mathrm{U}$ \\
\hline & ${ }^{154} \mathrm{Eu}$ & $-3.9 \mathrm{E}-02 \pm 1.1 \mathrm{E}-01$ & $\mathrm{U}$ & & ${ }^{154} \mathrm{Eu}$ & $-2.2 \mathrm{E}-02 \pm 1.8 \mathrm{E}-01$ & $\mathrm{U}$ \\
\hline & ${ }^{155} \mathrm{Eu}$ & $-2.7 \mathrm{E}-02 \pm 1.5 \mathrm{E}-01$ & $\mathrm{U}$ & & ${ }^{155} \mathrm{Eu}$ & $-6.6 \mathrm{E}-02 \pm 2.1 \mathrm{E}-01$ & $\mathrm{U}$ \\
\hline & ${ }^{238} \mathrm{Pu}$ & $1.8 \mathrm{E}-02 \pm 1.4 \mathrm{E}-02$ & $\mathrm{U}$ & & ${ }^{238} \mathrm{Pu}$ & $-9.1 \mathrm{E}-03 \pm 1.6 \mathrm{E}-02$ & $\mathrm{U}$ \\
\hline & ${ }^{239,240} \mathrm{Pu}$ & $9.5 \mathrm{E}-04 \pm 1.9 \mathrm{E}-03$ & $\mathrm{U}$ & & ${ }^{239,240} \mathrm{Pu}$ & $1.0 \mathrm{E}-03 \pm 2.0 \mathrm{E}-03$ & $\mathrm{U}$ \\
\hline & ${ }^{103} \mathrm{Ru}$ & $5.1 \mathrm{E}-03 \pm 3.8 \mathrm{E}-02$ & $\mathrm{U}$ & & ${ }^{103} \mathrm{Ru}$ & $1.4 \mathrm{E}-02 \pm 5.9 \mathrm{E}-02$ & $\mathrm{U}$ \\
\hline & ${ }^{106} \mathrm{Ru}$ & $-3.0 \mathrm{E}-01 \pm 3.5 \mathrm{E}-01$ & $\mathrm{U}$ & & ${ }^{106} \mathrm{Ru}$ & $1.6 \mathrm{E}-01 \pm 5.6 \mathrm{E}-01$ & $\mathrm{U}$ \\
\hline & ${ }^{125} \mathrm{Sb}$ & $-3.6 \mathrm{E}-02 \pm 1.0 \mathrm{E}-01$ & $\mathrm{U}$ & & ${ }^{125} \mathrm{Sb}$ & $1.5 \mathrm{E}-02 \pm 1.5 \mathrm{E}-01$ & $\mathrm{U}$ \\
\hline & ${ }^{113} \mathrm{Sn}$ & $-8.9 \mathrm{E}-03 \pm 4.6 \mathrm{E}-02$ & $\mathrm{U}$ & & ${ }^{113} \mathrm{Sn}$ & $2.0 \mathrm{E}-02 \pm 7.6 \mathrm{E}-02$ & $\mathrm{U}$ \\
\hline & ${ }^{90} \mathrm{Sr}$ & $-9.2 \mathrm{E}-02 \pm 1.4 \mathrm{E}-01$ & $\mathrm{U}$ & & ${ }^{90} \mathrm{Sr}$ & $1.1 \mathrm{E}-01 \pm 1.6 \mathrm{E}-01$ & $\mathrm{U}$ \\
\hline & ${ }^{234} \mathrm{U}$ & $8.4 \mathrm{E}-03 \pm 5.4 \mathrm{E}-03$ & & & & $5.1 \mathrm{E}-03 \pm 5.6 \mathrm{E}-03$ & $\mathrm{U}$ \\
\hline & ${ }^{235} \mathrm{U}$ & $2.8 \mathrm{E}-03 \pm 3.4 \mathrm{E}-03$ & & & ${ }^{235} \mathrm{U}$ & $4.6 \mathrm{E}-03 \pm 5.1 \mathrm{E}-03$ & $\mathrm{U}$ \\
\hline & ${ }^{238} \mathrm{U}$ & $6.1 \mathrm{E}-03 \pm 4.5 \mathrm{E}-03$ & & & ${ }^{238} \mathrm{U}$ & $6.8 \mathrm{E}-03 \pm 5.1 \mathrm{E}-03$ & \\
\hline V089 & ${ }^{144} \mathrm{Ce}$ & $2.5 \mathrm{E}-01 \pm 4.4 \mathrm{E}-01$ & $\overline{\mathrm{U}}$ & V091 & ${ }^{144} \mathrm{Ce}$ & $5.7 \mathrm{E}-02 \pm 3.6 \mathrm{E}-01$ & $\overline{\mathrm{U}}$ \\
\hline \multirow[t]{17}{*}{ (600 Area) } & ${ }^{60} \mathrm{Co}$ & $-1.4 \mathrm{E}-03 \pm 1.4 \mathrm{E}-02$ & $\mathrm{U}$ & (600 Area) & ${ }^{60} \mathrm{Co}$ & $3.2 \mathrm{E}-03 \pm 3.1 \mathrm{E}-02$ & $\mathrm{U}$ \\
\hline & ${ }^{134} \mathrm{Cs}$ & $-1.6 \mathrm{E}-02 \pm 3.5 \mathrm{E}-02$ & $\mathrm{U}$ & & ${ }^{134} \mathrm{Cs}$ & $1.2 \mathrm{E}-02 \pm 3.0 \mathrm{E}-02$ & $\mathrm{U}$ \\
\hline & ${ }^{137} \mathrm{Cs}$ & $-8.2 \mathrm{E}-03 \pm 3.2 \mathrm{E}-02$ & $\mathrm{U}$ & & ${ }^{137} \mathrm{Cs}$ & $4.4 \mathrm{E}-01 \pm 1.0 \mathrm{E}-01$ & \\
\hline & ${ }^{152} \mathrm{Eu}$ & $-3.3 \mathrm{E}-02 \pm 1.0 \mathrm{E}-01$ & $\mathrm{U}$ & & ${ }^{152} \mathrm{Eu}$ & $-3.6 \mathrm{E}-03 \pm 3.6 \mathrm{E}-02$ & $\mathrm{U}$ \\
\hline & ${ }^{154} \mathrm{Eu}$ & $3.1 \mathrm{E}-02 \pm 1.1 \mathrm{E}-01$ & $\mathrm{U}$ & & ${ }^{154} \mathrm{Eu}$ & $7.6 \mathrm{E}-03 \pm 7.6 \mathrm{E}-02$ & $\mathrm{U}$ \\
\hline & ${ }^{155} \mathrm{Eu}$ & $-5.4 \mathrm{E}-02 \pm 1.1 \mathrm{E}-01$ & $\mathrm{U}$ & & ${ }^{155} \mathrm{Eu}$ & $-7.4 \mathrm{E}-02 \pm 9.8 \mathrm{E}-02$ & $\mathrm{U}$ \\
\hline & ${ }^{238} \mathrm{Pu}$ & $-2.1 \mathrm{E}-02 \pm 2.3 \mathrm{E}-02$ & $\mathrm{U}$ & & ${ }^{238} \mathrm{Pu}$ & $8.3 \mathrm{E}-04 \pm 8.3 \mathrm{E}-03$ & $\mathrm{U}$ \\
\hline & ${ }^{239,240} \mathrm{Pu}$ & $3.9 \mathrm{E}-03 \pm 6.2 \mathrm{E}-03$ & $\mathrm{U}$ & & ${ }^{239,240} \mathrm{Pu}$ & $-1.7 \mathrm{E}-03 \pm 2.4 \mathrm{E}-03$ & $\mathrm{U}$ \\
\hline & ${ }^{103} \mathrm{Ru}$ & $1.9 \mathrm{E}-02 \pm 3.2 \mathrm{E}-02$ & $\mathrm{U}$ & & ${ }^{103} \mathrm{Ru}$ & $-1.9 \mathrm{E}-03 \pm 1.9 \mathrm{E}-02$ & $\mathrm{U}$ \\
\hline & ${ }^{106} \mathrm{Ru}$ & $-2.7 \mathrm{E}-03 \pm 2.7 \mathrm{E}-02$ & $\mathrm{U}$ & & ${ }^{106} \mathrm{Ru}$ & $-5.1 \mathrm{E}-02 \pm 2.7 \mathrm{E}-01$ & $\mathrm{U}$ \\
\hline & ${ }^{125} \mathrm{Sb}$ & $-1.1 \mathrm{E}-01 \pm 1.1 \mathrm{E}-01$ & $\mathrm{U}$ & & ${ }^{125} \mathrm{Sb}$ & $-2.7 \mathrm{E}-02 \pm 7.3 \mathrm{E}-02$ & $\mathrm{U}$ \\
\hline & ${ }^{113} \mathrm{Sn}$ & $-2.2 \mathrm{E}-02 \pm 3.9 \mathrm{E}-02$ & $\mathrm{U}$ & & ${ }^{113} \mathrm{Sn}$ & $-3.9 \mathrm{E}-03 \pm 3.3 \mathrm{E}-02$ & $\mathrm{U}$ \\
\hline & ${ }^{90} \mathrm{Sr}$ & $-9.2 \mathrm{E}-02 \pm 1.2 \mathrm{E}-01$ & $\mathrm{U}$ & & ${ }^{90} \mathrm{Sr}$ & $9.3 \mathrm{E}-01 \pm 1.9 \mathrm{E}-01$ & \\
\hline & ${ }^{234} \mathrm{U}$ & $5.8 \mathrm{E}-03 \pm 5.2 \mathrm{E}-03$ & $\mathrm{U}$ & & ${ }^{234} \mathrm{U}$ & $1.6 \mathrm{E}-02 \pm 8.3 \mathrm{E}-03$ & \\
\hline & ${ }^{235} \mathrm{U}$ & $3.6 \mathrm{E}-03 \pm 3.6 \mathrm{E}-03$ & & & ${ }^{235} \mathrm{U}$ & $1.2 \mathrm{E}-02 \pm 7.3 \mathrm{E}-03$ & \\
\hline & ${ }^{238} \mathrm{U}$ & $5.8 \mathrm{E}-03 \pm 4.6 \mathrm{E}-03$ & & & ${ }^{238} \mathrm{U}$ & $2.2 \mathrm{E}-02 \pm 1.0 \mathrm{E}-02$ & \\
\hline & ${ }^{65} \mathrm{Zn}$ & $1.4 \mathrm{E}-03 \pm 1.4 \mathrm{E}-02$ & $\mathrm{U}$ & & ${ }^{65} \mathrm{Zn}$ & $-1.3 \mathrm{E}-02 \pm 7.5 \mathrm{E}-02$ & $\mathrm{U}$ \\
\hline
\end{tabular}

$\overline{\mathrm{RQ}}=$ Result Qualifier. U $=$ The analyte was analyzed for but not detected. 
Table 3-5. 2003 Vegetation Sampling Results (pCi/g \pm total analytical uncertainty). (cont)

\begin{tabular}{|c|c|c|c|c|c|c|c|}
\hline Location & Isotope & Result \pm Error & $\mathbf{R Q}^{*}$ & Location & Isotope & Result \pm Error & $\mathbf{R Q}^{*}$ \\
\hline V093 & ${ }^{144} \mathrm{Ce}$ & $7.0 \mathrm{E}-01 \pm 7.4 \mathrm{E}-01$ & $\bar{U}$ & V095 & ${ }^{144} \mathrm{Ce}$ & $-8.4 \mathrm{E}-02 \pm 3.7 \mathrm{E}-01$ & $\overline{\mathrm{U}}$ \\
\hline \multirow[t]{17}{*}{ (600 Area) } & ${ }^{60} \mathrm{Co}$ & $3.3 \mathrm{E}-02 \pm 4.9 \mathrm{E}-02$ & $\mathrm{U}$ & (600 Area) & ${ }^{60} \mathrm{Co}$ & $-2.4 \mathrm{E}-02 \pm 3.0 \mathrm{E}-02$ & $\mathrm{U}$ \\
\hline & ${ }^{134} \mathrm{Cs}$ & $-4.6 \mathrm{E}-02 \pm 5.5 \mathrm{E}-02$ & $\mathrm{U}$ & & ${ }^{134} \mathrm{Cs}$ & $1.7 \mathrm{E}-02 \pm 3.0 \mathrm{E}-02$ & $\mathrm{U}$ \\
\hline & ${ }^{137} \mathrm{Cs}$ & $2.2 \mathrm{E}-02 \pm 5.1 \mathrm{E}-02$ & $\mathrm{U}$ & & ${ }^{137} \mathrm{Cs}$ & $6.0 \mathrm{E}-02 \pm 5.8 \mathrm{E}-02$ & \\
\hline & ${ }^{152} \mathrm{Eu}$ & $-1.8 \mathrm{E}-01 \pm 1.8 \mathrm{E}-01$ & $\mathrm{U}$ & & ${ }^{152} \mathrm{Eu}$ & $-6.3 \mathrm{E}-03 \pm 6.3 \mathrm{E}-02$ & $\mathrm{U}$ \\
\hline & ${ }^{154} \mathrm{Eu}$ & $8.2 \mathrm{E}-02 \pm 1.4 \mathrm{E}-01$ & $\mathrm{U}$ & & ${ }^{154} \mathrm{Eu}$ & $1.4 \mathrm{E}-02 \pm 1.2 \mathrm{E}-01$ & $\mathrm{U}$ \\
\hline & ${ }^{155} \mathrm{Eu}$ & $1.9 \mathrm{E}-01 \pm 2.0 \mathrm{E}-01$ & $\mathrm{U}$ & & ${ }^{155} \mathrm{Eu}$ & $4.4 \mathrm{E}-02 \pm 9.7 \mathrm{E}-02$ & $\mathrm{U}$ \\
\hline & ${ }^{238} \mathrm{Pu}$ & $-1.8 \mathrm{E}-03 \pm 1.8 \mathrm{E}-02$ & $\mathrm{U}$ & & ${ }^{238} \mathrm{Pu}$ & $-6.0 \mathrm{E}-03 \pm 1.4 \mathrm{E}-02$ & $\mathrm{U}$ \\
\hline & ${ }^{239,240} \mathrm{Pu}$ & $-1.8 \mathrm{E}-03 \pm 3.6 \mathrm{E}-03$ & $\mathrm{U}$ & & ${ }^{239,240} \mathrm{Pu}$ & $2.4 \mathrm{E}-03 \pm 3.4 \mathrm{E}-03$ & $\mathrm{U}$ \\
\hline & ${ }^{103} \mathrm{Ru}$ & $1.1 \mathrm{E}-02 \pm 5.7 \mathrm{E}-02$ & $\mathrm{U}$ & & ${ }^{103} \mathrm{Ru}$ & $2.1 \mathrm{E}-03 \pm 2.1 \mathrm{E}-02$ & $\mathrm{U}$ \\
\hline & ${ }^{106} \mathrm{Ru}$ & $-5.5 \mathrm{E}-01 \pm 5.5 \mathrm{E}-01$ & $\mathrm{U}$ & & ${ }^{106} \mathrm{Ru}$ & $-8.2 \mathrm{E}-02 \pm 2.4 \mathrm{E}-01$ & $\mathrm{U}$ \\
\hline & ${ }^{125} \mathrm{Sb}$ & $-6.7 \mathrm{E}-02 \pm 1.4 \mathrm{E}-01$ & $\mathrm{U}$ & & ${ }^{125} \mathrm{Sb}$ & $2.4 \mathrm{E}-02 \pm 7.3 \mathrm{E}-02$ & $\mathrm{U}$ \\
\hline & ${ }^{113} \mathrm{Sn}$ & $-4.5 \mathrm{E}-02 \pm 6.3 \mathrm{E}-02$ & $\mathrm{U}$ & & ${ }^{113} \mathrm{Sn}$ & $-1.4 \mathrm{E}-02 \pm 3.4 \mathrm{E}-02$ & $\mathrm{U}$ \\
\hline & ${ }^{90} \mathrm{Sr}$ & $-1.2 \mathrm{E}-01 \pm 1.2 \mathrm{E}-01$ & $\mathrm{U}$ & & ${ }^{90} \mathrm{Sr}$ & $7.1 \mathrm{E}-01 \pm 1.8 \mathrm{E}-01$ & \\
\hline & ${ }^{234} \mathrm{U}$ & $4.7 \mathrm{E}-03 \pm 4.0 \mathrm{E}-03$ & & & ${ }^{234} \mathrm{U}$ & $9.8 \mathrm{E}-03 \pm 6.6 \mathrm{E}-03$ & \\
\hline & ${ }^{235} \mathrm{U}$ & $4.3 \mathrm{E}-03 \pm 5.2 \mathrm{E}-03$ & $\mathrm{U}$ & & ${ }^{235} \mathrm{U}$ & $1.8 \mathrm{E}-03 \pm 3.6 \mathrm{E}-03$ & $\mathrm{U}$ \\
\hline & ${ }^{238} \mathrm{U}$ & $1.6 \mathrm{E}-03 \pm 4.0 \mathrm{E}-03$ & $\mathrm{U}$ & & ${ }^{238} \mathrm{U}$ & $5.7 \mathrm{E}-03 \pm 4.6 \mathrm{E}-03$ & \\
\hline & ${ }^{65} \mathrm{Zn}$ & $-7.9 \mathrm{E}-02 \pm 1.1 \mathrm{E}-01$ & $\mathrm{U}$ & & ${ }^{65} \mathrm{Zn}$ & $-2.0 \mathrm{E}-01 \pm 2.0 \mathrm{E}-01$ & $\mathrm{U}$ \\
\hline V097 & ${ }^{144} \mathrm{Ce}$ & $8.2 \mathrm{E}-02 \pm 2.9 \mathrm{E}-01$ & $\bar{U}$ & V099 & ${ }^{144} \mathrm{Ce}$ & $1.8 \mathrm{E}-02 \pm 1.8 \mathrm{E}-01$ & $\bar{U}$ \\
\hline \multirow[t]{17}{*}{ (600 Area) } & ${ }^{60} \mathrm{Co}$ & $3.2 \mathrm{E}-03 \pm 1.9 \mathrm{E}-02$ & $\mathrm{U}$ & (600 Area) & ${ }^{60} \mathrm{Co}$ & $2.4 \mathrm{E}-02 \pm 2.6 \mathrm{E}-02$ & $\mathrm{U}$ \\
\hline & ${ }^{134} \mathrm{Cs}$ & $1.3 \mathrm{E}-02 \pm 2.6 \mathrm{E}-02$ & $\mathrm{U}$ & & ${ }^{134} \mathrm{Cs}$ & $-2.2 \mathrm{E}-02 \pm 2.6 \mathrm{E}-02$ & $\mathrm{U}$ \\
\hline & ${ }^{137} \mathrm{Cs}$ & $-5.1 \mathrm{E}-03 \pm 2.1 \mathrm{E}-02$ & $\mathrm{U}$ & & ${ }^{137} \mathrm{Cs}$ & $-8.0 \mathrm{E}-03 \pm 2.5 \mathrm{E}-02$ & $\mathrm{U}$ \\
\hline & ${ }^{152} \mathrm{Eu}$ & $-4.9 \mathrm{E}-03 \pm 4.9 \mathrm{E}-02$ & $\mathrm{U}$ & & ${ }^{152} \mathrm{Eu}$ & $8.9 \mathrm{E}-03 \pm 6.4 \mathrm{E}-02$ & $\mathrm{U}$ \\
\hline & ${ }^{154} \mathrm{Eu}$ & $2.4 \mathrm{E}-02 \pm 6.5 \mathrm{E}-02$ & $\mathrm{U}$ & & ${ }^{154} \mathrm{Eu}$ & $-1.7 \mathrm{E}-02 \pm 7.9 \mathrm{E}-02$ & $\mathrm{U}$ \\
\hline & ${ }^{155} \mathrm{Eu}$ & $5.6 \mathrm{E}-02 \pm 7.2 \mathrm{E}-02$ & $\mathrm{U}$ & & ${ }^{155} \mathrm{Eu}$ & $7.1 \mathrm{E}-02 \pm 8.2 \mathrm{E}-02$ & $\mathrm{U}$ \\
\hline & ${ }^{238} \mathrm{Pu}$ & $1.6 \mathrm{E}-02 \pm 1.6 \mathrm{E}-02$ & $\mathrm{U}$ & & ${ }^{238} \mathrm{Pu}$ & $1.6 \mathrm{E}-02 \pm 1.4 \mathrm{E}-02$ & $\mathrm{U}$ \\
\hline & ${ }^{239,240} \mathrm{Pu}$ & $-8.8 \mathrm{E}-04 \pm 3.1 \mathrm{E}-03$ & $\mathrm{U}$ & & ${ }^{239,240} \mathrm{Pu}$ & $1.0 \mathrm{E}-03 \pm 1.0 \mathrm{E}-02$ & $\mathrm{U}$ \\
\hline & ${ }^{103} \mathrm{Ru}$ & $6.6 \mathrm{E}-03 \pm 2.0 \mathrm{E}-02$ & $\mathrm{U}$ & & ${ }^{103} \mathrm{Ru}$ & $-1.3 \mathrm{E}-02 \pm 2.3 \mathrm{E}-02$ & $\mathrm{U}$ \\
\hline & ${ }^{106} \mathrm{Ru}$ & $5.1 \mathrm{E}-02 \pm 1.8 \mathrm{E}-01$ & $\mathrm{U}$ & & ${ }^{106} \mathrm{Ru}$ & $2.6 \mathrm{E}-01 \pm 2.2 \mathrm{E}-01$ & $\mathrm{U}$ \\
\hline & ${ }^{125} \mathrm{Sb}$ & $-7.7 \mathrm{E}-02 \pm 7.7 \mathrm{E}-02$ & $\mathrm{U}$ & & ${ }^{125} \mathrm{Sb}$ & $-2.8 \mathrm{E}-02 \pm 5.9 \mathrm{E}-02$ & $\mathrm{U}$ \\
\hline & ${ }^{113} \mathrm{Sn}$ & $-3.0 \mathrm{E}-02 \pm 3.0 \mathrm{E}-02$ & $\mathrm{U}$ & & ${ }^{113} \mathrm{Sn}$ & $-1.1 \mathrm{E}-04 \pm 1.1 \mathrm{E}-03$ & $\mathrm{U}$ \\
\hline & ${ }^{90} \mathrm{Sr}$ & $6.5 \mathrm{E}-02 \pm 1.2 \mathrm{E}-01$ & $\mathrm{U}$ & & ${ }^{90} \mathrm{Sr}$ & $4.8 \mathrm{E}+00 \pm 7.2 \mathrm{E}-01$ & \\
\hline & ${ }^{234} \mathrm{U}$ & $9.8 \mathrm{E}-03 \pm 6.2 \mathrm{E}-03$ & & & ${ }^{234} \mathrm{U}$ & $4.9 \mathrm{E}-03 \pm 4.8 \mathrm{E}-03$ & $\mathrm{U}$ \\
\hline & ${ }^{235} \mathrm{U}$ & $2.7 \mathrm{E}-03 \pm 3.2 \mathrm{E}-03$ & & & ${ }^{235} \mathrm{U}$ & $1.8 \mathrm{E}-03 \pm 3.6 \mathrm{E}-03$ & $\mathrm{U}$ \\
\hline & ${ }^{238} \mathrm{U}$ & $5.7 \mathrm{E}-03 \pm 4.6 \mathrm{E}-03$ & & & ${ }^{238} \mathrm{U}$ & $1.6 \mathrm{E}-03 \pm 3.2 \mathrm{E}-03$ & $\mathrm{U}$ \\
\hline & ${ }^{65} \mathrm{Zn}$ & $-5.1 \mathrm{E}-02 \pm 5.3 \mathrm{E}-02$ & $\mathrm{U}$ & & ${ }^{65} \mathrm{Zn}$ & $1.4 \mathrm{E}-03 \pm 1.4 \mathrm{E}-02$ & $\mathrm{U}$ \\
\hline
\end{tabular}

$\overline{\mathrm{RQ}}=$ Result Qualifier. U $=$ The analyte was analyzed for but not detected. 
Table 3-5. 2003 Vegetation Sampling Results (pCi/g \pm total analytical uncertainty). (cont)

\begin{tabular}{|c|c|c|c|c|c|c|c|}
\hline Location & Isotope & Result \pm Error & $\mathbf{R Q}^{*}$ & Location & Isotope & Result \pm Error & $\mathbf{R Q}^{*}$ \\
\hline V101 & ${ }^{144} \mathrm{Ce}$ & $-1.2 \mathrm{E}-02 \pm 1.2 \mathrm{E}-01$ & $\mathrm{U}$ & V103 & ${ }^{144} \mathrm{Ce}$ & $2.5 \mathrm{E}-01 \pm 3.8 \mathrm{E}-01$ & $\mathrm{U}$ \\
\hline \multirow[t]{17}{*}{ (600 Area) } & ${ }^{60} \mathrm{Co}$ & $-8.0 \mathrm{E}-03 \pm 2.5 \mathrm{E}-02$ & $\mathrm{U}$ & (600 Area) & ${ }^{60} \mathrm{Co}$ & $-2.9 \mathrm{E}-03 \pm 2.6 \mathrm{E}-02$ & $\mathrm{U}$ \\
\hline & ${ }^{134} \mathrm{Cs}$ & $-2.2 \mathrm{E}-02 \pm 2.6 \mathrm{E}-02$ & $\mathrm{U}$ & & ${ }^{134} \mathrm{Cs}$ & $-8.1 \mathrm{E}-03 \pm 2.7 \mathrm{E}-02$ & $\mathrm{U}$ \\
\hline & ${ }^{137} \mathrm{Cs}$ & $1.4 \mathrm{E}-02 \pm 3.0 \mathrm{E}-02$ & $\mathrm{U}$ & & ${ }^{137} \mathrm{Cs}$ & $1.2 \mathrm{E}-02 \pm 2.8 \mathrm{E}-02$ & $\mathrm{U}$ \\
\hline & ${ }^{152} \mathrm{Eu}$ & $-5.4 \mathrm{E}-02 \pm 7.6 \mathrm{E}-02$ & $\mathrm{U}$ & & ${ }^{152} \mathrm{Eu}$ & $-2.0 \mathrm{E}-02 \pm 8.1 \mathrm{E}-02$ & $\mathrm{U}$ \\
\hline & ${ }^{154} \mathrm{Eu}$ & $-5.2 \mathrm{E}-02 \pm 9.4 \mathrm{E}-02$ & $\mathrm{U}$ & & ${ }^{154} \mathrm{Eu}$ & $3.0 \mathrm{E}-02 \pm 8.5 \mathrm{E}-02$ & $\mathrm{U}$ \\
\hline & ${ }^{155} \mathrm{Eu}$ & $-1.1 \mathrm{E}-02 \pm 8.6 \mathrm{E}-02$ & $\mathrm{U}$ & & ${ }^{155} \mathrm{Eu}$ & $3.2 \mathrm{E}-02 \pm 9.2 \mathrm{E}-02$ & $\mathrm{U}$ \\
\hline & ${ }^{238} \mathrm{Pu}$ & 9.3E-04 \pm 9.3Е-03 & $\mathrm{U}$ & & ${ }^{238} \mathrm{Pu}$ & $4.5 \mathrm{E}-03 \pm 1.9 \mathrm{E}-02$ & $\mathrm{U}$ \\
\hline & ${ }^{239,240} \mathrm{Pu}$ & $-1.9 \mathrm{E}-03 \pm 3.8 \mathrm{E}-03$ & $\mathrm{U}$ & & ${ }^{239,240} \mathrm{Pu}$ & $6.8 \mathrm{E}-03 \pm 5.8 \mathrm{E}-03$ & \\
\hline & ${ }^{103} \mathrm{Ru}$ & $-3.7 \mathrm{E}-03 \pm 2.5 \mathrm{E}-02$ & $\mathrm{U}$ & & ${ }^{103} \mathrm{Ru}$ & $-5.8 \mathrm{E}-04 \pm 5.8 \mathrm{E}-03$ & $\mathrm{U}$ \\
\hline & ${ }^{106} \mathrm{Ru}$ & $-1.2 \mathrm{E}-01 \pm 2.1 \mathrm{E}-01$ & $\mathrm{U}$ & & ${ }^{106} \mathrm{Ru}$ & $-1.8 \mathrm{E}-01 \pm 2.2 \mathrm{E}-01$ & $\mathrm{U}$ \\
\hline & ${ }^{125} \mathrm{Sb}$ & $2.7 \mathrm{E}-02 \pm 7.1 \mathrm{E}-02$ & $\mathrm{U}$ & & ${ }^{125} \mathrm{Sb}$ & $6.8 \mathrm{E}-02 \pm 7.6 \mathrm{E}-02$ & $\mathrm{U}$ \\
\hline & ${ }^{113} \mathrm{Sn}$ & $-3.3 \mathrm{E}-02 \pm 3.4 \mathrm{E}-02$ & $\mathrm{U}$ & & ${ }^{113} \mathrm{Sn}$ & $-4.4 \mathrm{E}-03 \pm 3.5 \mathrm{E}-02$ & U \\
\hline & ${ }^{90} \mathrm{Sr}$ & $-1.8 \mathrm{E}-02 \pm 1.1 \mathrm{E}-01$ & $\mathrm{U}$ & & ${ }^{90} \mathrm{Sr}$ & $1.3 \mathrm{E}-02 \pm 1.2 \mathrm{E}-01$ & $\mathrm{U}$ \\
\hline & ${ }^{234} \mathrm{U}$ & $8.4 \mathrm{E}-03 \pm 6.0 \mathrm{E}-03$ & & & ${ }^{234} \mathrm{U}$ & $1.0 \mathrm{E}-02 \pm 6.3 \mathrm{E}-03$ & \\
\hline & ${ }^{235} \mathrm{U}$ & $-1.0 \mathrm{E}-03 \pm 3.5 \mathrm{E}-03$ & $\mathrm{U}$ & & ${ }^{235} \mathrm{U}$ & 8.3E-04 \pm 8.3E-03 & $\mathrm{U}$ \\
\hline & ${ }^{238} \mathrm{U}$ & $4.7 \mathrm{E}-03 \pm 4.4 \mathrm{E}-03$ & & & ${ }^{238} \mathrm{U}$ & $6.7 \mathrm{E}-03 \pm 5.0 \mathrm{E}-03$ & \\
\hline & ${ }^{65} \mathrm{Zn}$ & $-6.3 \mathrm{E}-03 \pm 6.3 \mathrm{E}-02$ & $\mathrm{U}$ & & ${ }^{65} \mathrm{Zn}$ & $5.2 \mathrm{E}-02 \pm 7.1 \mathrm{E}-02$ & $\mathrm{U}$ \\
\hline V105 & ${ }^{144} \mathrm{Ce}$ & $4.3 \mathrm{E}-02 \pm 4.3 \mathrm{E}-01$ & $\bar{U}$ & V107 & ${ }^{144} \mathrm{Ce}$ & $-4.8 \mathrm{E}-02 \pm 3.4 \mathrm{E}-01$ & $\bar{U}$ \\
\hline \multirow[t]{17}{*}{ (600 Area) } & ${ }^{60} \mathrm{Co}$ & $-5.7 \mathrm{E}-03 \pm 3.8 \mathrm{E}-02$ & $\mathrm{U}$ & (600 Area) & ${ }^{60} \mathrm{Co}$ & $-1.0 \mathrm{E}-02 \pm 3.4 \mathrm{E}-02$ & $\mathrm{U}$ \\
\hline & ${ }^{134} \mathrm{Cs}$ & $6.6 \mathrm{E}-05 \pm 6.6 \mathrm{E}-04$ & $\mathrm{U}$ & & ${ }^{134} \mathrm{Cs}$ & $2.3 \mathrm{E}-02 \pm 3.2 \mathrm{E}-02$ & $\mathrm{U}$ \\
\hline & ${ }^{137} \mathrm{Cs}$ & $1.1 \mathrm{E}-02 \pm 3.8 \mathrm{E}-02$ & $\mathrm{U}$ & & ${ }^{137} \mathrm{Cs}$ & $4.6 \mathrm{E}-03 \pm 3.1 \mathrm{E}-02$ & $\mathrm{U}$ \\
\hline & ${ }^{152} \mathrm{Eu}$ & 8.7E-03 $\pm 8.6 \mathrm{E}-02$ & $\mathrm{U}$ & & ${ }^{152} \mathrm{Eu}$ & $7.6 \mathrm{E}-04 \pm 7.6 \mathrm{E}-03$ & $\mathrm{U}$ \\
\hline & ${ }^{154} \mathrm{Eu}$ & $-7.5 \mathrm{E}-02 \pm 1.3 \mathrm{E}-01$ & $\mathrm{U}$ & & ${ }^{154} \mathrm{Eu}$ & $-1.5 \mathrm{E}-01 \pm 1.5 \mathrm{E}-01$ & $\mathrm{U}$ \\
\hline & ${ }^{155} \mathrm{Eu}$ & $1.7 \mathrm{E}-01 \pm 1.1 \mathrm{E}-01$ & $\mathrm{U}$ & & ${ }^{155} \mathrm{Eu}$ & $2.3 \mathrm{E}-02 \pm 1.0 \mathrm{E}-01$ & $\mathrm{U}$ \\
\hline & ${ }^{238} \mathrm{Pu}$ & $-6.2 \mathrm{E}-03 \pm 8.7 \mathrm{E}-03$ & $\mathrm{U}$ & & ${ }^{238} \mathrm{Pu}$ & $-2.5 \mathrm{E}-03 \pm 6.2 \mathrm{E}-03$ & $\mathrm{U}$ \\
\hline & ${ }^{239,240} \mathrm{Pu}$ & $1.0 \mathrm{E}-02 \pm 7.4 \mathrm{E}-03$ & & & ${ }^{239,240} \mathrm{Pu}$ & $1.2 \mathrm{E}-03 \pm 1.2 \mathrm{E}-03$ & $\mathrm{U}$ \\
\hline & ${ }^{103} \mathrm{Ru}$ & $-1.1 \mathrm{E}-02 \pm 4.2 \mathrm{E}-02$ & $\mathrm{U}$ & & ${ }^{103} \mathrm{Ru}$ & $1.1 \mathrm{E}-02 \pm 3.2 \mathrm{E}-02$ & $\mathrm{U}$ \\
\hline & ${ }^{106} \mathrm{Ru}$ & 2.3E-02 $\pm 2.3 \mathrm{E}-01$ & $\mathrm{U}$ & & ${ }^{106} \mathrm{Ru}$ & $-1.1 \mathrm{E}-01 \pm 2.9 \mathrm{E}-01$ & $\mathrm{U}$ \\
\hline & ${ }^{125} \mathrm{Sb}$ & $-4.4 \mathrm{E}-02 \pm 9.3 \mathrm{E}-02$ & $\mathrm{U}$ & & ${ }^{125} \mathrm{Sb}$ & $-3.6 \mathrm{E}-03 \pm 3.6 \mathrm{E}-02$ & $\mathrm{U}$ \\
\hline & ${ }^{113} \mathrm{Sn}$ & $-4.3 \mathrm{E}-04 \pm 4.2 \mathrm{E}-03$ & $\mathrm{U}$ & & ${ }^{113} \mathrm{Sn}$ & $-2.6 \mathrm{E}-02 \pm 3.7 \mathrm{E}-02$ & $\mathrm{U}$ \\
\hline & ${ }^{90} \mathrm{Sr}$ & $6.0 \mathrm{E}-02 \pm 1.4 \mathrm{E}-01$ & $\mathrm{U}$ & & ${ }^{90} \mathrm{Sr}$ & $-1.6 \mathrm{E}-01 \pm 1.6 \mathrm{E}-01$ & $\mathrm{U}$ \\
\hline & ${ }^{234} \mathrm{U}$ & $9.5 \mathrm{E}-03 \pm 6.2 \mathrm{E}-03$ & & & ${ }^{234} \mathrm{U}$ & $2.6 \mathrm{E}-03 \pm 3.1 \mathrm{E}-03$ & \\
\hline & ${ }^{235} \mathrm{U}$ & $1.9 \mathrm{E}-03 \pm 2.7 \mathrm{E}-03$ & $\mathrm{U}$ & & ${ }^{235} \mathrm{U}$ & $8.8 \mathrm{E}-04 \pm 8.8 \mathrm{E}-04$ & $\mathrm{U}$ \\
\hline & ${ }^{238} \mathrm{U}$ & $6.9 \mathrm{E}-03 \pm 5.2 \mathrm{E}-03$ & & & ${ }^{238} \mathrm{U}$ & $6.2 \mathrm{E}-03 \pm 5.0 \mathrm{E}-03$ & \\
\hline & ${ }^{65} \mathrm{Zn}$ & $1.0 \mathrm{E}-01 \pm 1.0 \mathrm{E}-01$ & $\mathrm{U}$ & & ${ }^{65} \mathrm{Zn}$ & $2.7 \mathrm{E}-02 \pm 8.7 \mathrm{E}-02$ & $\mathrm{U}$ \\
\hline
\end{tabular}

$\overline{\mathrm{RQ}}=$ Result Qualifier. U $=$ The analyte was analyzed for but not detected. 
Table 3-5. 2003 Vegetation Sampling Results (pCi/g \pm total analytical uncertainty). (cont)

\begin{tabular}{|c|c|c|c|c|c|c|c|}
\hline Location & Isotope & Result \pm Error & $\mathbf{R Q}^{*}$ & Location & Isotope & Result \pm Error & $\mathbf{R Q}^{*}$ \\
\hline V109 & ${ }^{144} \mathrm{Ce}$ & $-1.3 \mathrm{E}-01 \pm 5.5 \mathrm{E}-01$ & $\bar{U}$ & V111 & ${ }^{144} \mathrm{Ce}$ & $-2.2 \mathrm{E}-01 \pm 5.2 \mathrm{E}-01$ & $\overline{\mathrm{U}}$ \\
\hline \multirow[t]{17}{*}{ (600 Area) } & ${ }^{60} \mathrm{Co}$ & $-1.7 \mathrm{E}-02 \pm 3.5 \mathrm{E}-02$ & $\mathrm{U}$ & (Duplicate & ${ }^{60} \mathrm{Co}$ & $-1.2 \mathrm{E}-02 \pm 4.1 \mathrm{E}-02$ & $\mathrm{U}$ \\
\hline & ${ }^{134} \mathrm{Cs}$ & $-7.0 \mathrm{E}-03 \pm 3.9 \mathrm{E}-02$ & $\mathrm{U}$ & of V007, & ${ }^{134} \mathrm{Cs}$ & $1.3 \mathrm{E}-02 \pm 4.2 \mathrm{E}-02$ & $\mathrm{U}$ \\
\hline & ${ }^{137} \mathrm{Cs}$ & $3.2 \mathrm{E}-02 \pm 4.3 \mathrm{E}-02$ & $\mathrm{U}$ & 200 West) & ${ }^{137} \mathrm{Cs}$ & $6.3 \mathrm{E}-03 \pm 4.2 \mathrm{E}-02$ & $\mathrm{U}$ \\
\hline & ${ }^{152} \mathrm{Eu}$ & $-8.6 \mathrm{E}-02 \pm 1.3 \mathrm{E}-01$ & $\mathrm{U}$ & & ${ }^{152} \mathrm{Eu}$ & $1.0 \mathrm{E}-01 \pm 1.2 \mathrm{E}-01$ & $\mathrm{U}$ \\
\hline & ${ }^{154} \mathrm{Eu}$ & $6.1 \mathrm{E}-02 \pm 1.1 \mathrm{E}-01$ & $\mathrm{U}$ & & ${ }^{154} \mathrm{Eu}$ & $-1.6 \mathrm{E}-01 \pm 1.6 \mathrm{E}-01$ & $\mathrm{U}$ \\
\hline & ${ }^{155} \mathrm{Eu}$ & $7.6 \mathrm{E}-02 \pm 1.5 \mathrm{E}-01$ & $\mathrm{U}$ & & ${ }^{155} \mathrm{Eu}$ & $9.7 \mathrm{E}-02 \pm 1.4 \mathrm{E}-01$ & $\mathrm{U}$ \\
\hline & ${ }^{238} \mathrm{Pu}$ & $9.2 \mathrm{E}-04 \pm 9.2 \mathrm{E}-04$ & $\mathrm{U}$ & & ${ }^{238} \mathrm{Pu}$ & $-2.1 \mathrm{E}-03 \pm 4.2 \mathrm{E}-03$ & $\mathrm{U}$ \\
\hline & ${ }^{239,240} \mathrm{Pu}$ & $2.3 \mathrm{E}-02 \pm 1.1 \mathrm{E}-02$ & & & ${ }^{239,240} \mathrm{Pu}$ & $1.0 \mathrm{E}-03 \pm 4.5 \mathrm{E}-03$ & $\mathrm{U}$ \\
\hline & ${ }^{103} \mathrm{Ru}$ & $-2.1 \mathrm{E}-02 \pm 4.0 \mathrm{E}-02$ & $\mathrm{U}$ & & ${ }^{103} \mathrm{Ru}$ & $7.6 \mathrm{E}-03 \pm 3.9 \mathrm{E}-02$ & $\mathrm{U}$ \\
\hline & ${ }^{106} \mathrm{Ru}$ & $7.1 \mathrm{E}-02 \pm 3.5 \mathrm{E}-01$ & $\mathrm{U}$ & & ${ }^{106} \mathrm{Ru}$ & $1.1 \mathrm{E}-01 \pm 3.5 \mathrm{E}-01$ & $\mathrm{U}$ \\
\hline & ${ }^{125} \mathrm{Sb}$ & $6.8 \mathrm{E}-03 \pm 6.8 \mathrm{E}-02$ & $\mathrm{U}$ & & ${ }^{125} \mathrm{Sb}$ & $5.0 \mathrm{E}-02 \pm 1.0 \mathrm{E}-01$ & $\mathrm{U}$ \\
\hline & ${ }^{113} \mathrm{Sn}$ & $-2.2 \mathrm{E}-02 \pm 4.8 \mathrm{E}-02$ & $\mathrm{U}$ & & ${ }^{113} \mathrm{Sn}$ & $-1.6 \mathrm{E}-02 \pm 4.9 \mathrm{E}-02$ & $\mathrm{U}$ \\
\hline & ${ }^{90} \mathrm{Sr}$ & $-1.0 \mathrm{E}-02 \pm 1.0 \mathrm{E}-01$ & $\mathrm{U}$ & & ${ }^{90} \mathrm{Sr}$ & $-1.0 \mathrm{E}-01 \pm 1.0 \mathrm{E}-01$ & $\mathrm{U}$ \\
\hline & ${ }^{234} \mathrm{U}$ & $1.1 \mathrm{E}-02 \pm 6.7 \mathrm{E}-03$ & & & & $9.9 \mathrm{E}-03 \pm 6.9 \mathrm{E}-03$ & \\
\hline & ${ }^{235} \mathrm{U}$ & $9.2 \mathrm{E}-04 \pm 3.2 \mathrm{E}-03$ & $\mathrm{U}$ & & ${ }^{235} \mathrm{U}$ & $2.0 \mathrm{E}-03 \pm 4.0 \mathrm{E}-03$ & $\mathrm{U}$ \\
\hline & ${ }^{238} \mathrm{U}$ & $6.8 \mathrm{E}-03 \pm 6.1 \mathrm{E}-03$ & $\mathrm{U}$ & & ${ }^{238} \mathrm{U}$ & $5.4 \mathrm{E}-03 \pm 4.6 \mathrm{E}-03$ & \\
\hline & ${ }^{65} \mathrm{Zn}$ & $-8.7 \mathrm{E}-02 \pm 8.9 \mathrm{E}-02$ & $\mathrm{U}$ & & ${ }^{65} \mathrm{Zn}$ & $-9.9 \mathrm{E}-03 \pm 9.7 \mathrm{E}-02$ & $\mathrm{U}$ \\
\hline \multirow{18}{*}{$\begin{array}{c}\text { V113 } \\
\text { (Duplicate } \\
\text { of V083, } \\
600 \text { Area) }\end{array}$} & ${ }^{144} \mathrm{Ce}$ & $-3.1 \mathrm{E}-02 \pm 3.1 \mathrm{E}-01$ & $\bar{U}$ & V116 & ${ }^{144} \mathrm{Ce}$ & $-6.9 \mathrm{E}-02 \pm 4.5 \mathrm{E}-01$ & $\bar{U}$ \\
\hline & ${ }^{60} \mathrm{Co}$ & $-8.5 \mathrm{E}-03 \pm 3.2 \mathrm{E}-02$ & $\mathrm{U}$ & (300 Area) & ${ }^{60} \mathrm{Co}$ & $1.5 \mathrm{E}-02 \pm 3.0 \mathrm{E}-02$ & $\mathrm{U}$ \\
\hline & ${ }^{134} \mathrm{Cs}$ & $-2.4 \mathrm{E}-02 \pm 3.5 \mathrm{E}-02$ & $\mathrm{U}$ & & ${ }^{134} \mathrm{Cs}$ & $2.7 \mathrm{E}-04 \pm 2.7 \mathrm{E}-03$ & $\mathrm{U}$ \\
\hline & ${ }^{137} \mathrm{Cs}$ & $-4.1 \mathrm{E}-04 \pm 4.1 \mathrm{E}-03$ & $\mathrm{U}$ & & ${ }^{137} \mathrm{Cs}$ & $4.1 \mathrm{E}-03 \pm 3.1 \mathrm{E}-02$ & $\mathrm{U}$ \\
\hline & ${ }^{152} \mathrm{Eu}$ & $-8.2 \mathrm{E}-02 \pm 1.0 \mathrm{E}-01$ & $\mathrm{U}$ & & ${ }^{152} \mathrm{Eu}$ & $-7.2 \mathrm{E}-02 \pm 1.2 \mathrm{E}-01$ & $\mathrm{U}$ \\
\hline & ${ }^{154} \mathrm{Eu}$ & $4.1 \mathrm{E}-03 \pm 4.1 \mathrm{E}-02$ & $\mathrm{U}$ & & ${ }^{154} \mathrm{Eu}$ & $5.6 \mathrm{E}-02 \pm 9.3 \mathrm{E}-02$ & $\mathrm{U}$ \\
\hline & ${ }^{155} \mathrm{Eu}$ & $-2.8 \mathrm{E}-02 \pm 1.1 \mathrm{E}-01$ & $\mathrm{U}$ & & ${ }^{155} \mathrm{Eu}$ & $4.9 \mathrm{E}-02 \pm 1.1 \mathrm{E}-01$ & $\mathrm{U}$ \\
\hline & ${ }^{238} \mathrm{Pu}$ & $-1.0 \mathrm{E}-03 \pm 4.5 \mathrm{E}-03$ & $\mathrm{U}$ & & ${ }^{238} \mathrm{Pu}$ & $-2.6 \mathrm{E}-03 \pm 3.9 \mathrm{E}-03$ & $\mathrm{U}$ \\
\hline & ${ }^{239,240} \mathrm{Pu}$ & $1.0 \mathrm{E}-03 \pm 1.0 \mathrm{E}-03$ & $\mathrm{U}$ & & ${ }^{239,240} \mathrm{Pu}$ & $8.6 \mathrm{E}-04 \pm 8.6 \mathrm{E}-03$ & $\mathrm{U}$ \\
\hline & ${ }^{103} \mathrm{Ru}$ & $-6.8 \mathrm{E}-03 \pm 3.2 \mathrm{E}-02$ & $\mathrm{U}$ & & ${ }^{103} \mathrm{Ru}$ & $-3.4 \mathrm{E}-04 \pm 3.4 \mathrm{E}-03$ & $\mathrm{U}$ \\
\hline & ${ }^{106} \mathrm{Ru}$ & $-6.6 \mathrm{E}-02 \pm 2.7 \mathrm{E}-01$ & $\mathrm{U}$ & & ${ }^{106} \mathrm{Ru}$ & $1.8 \mathrm{E}-01 \pm 3.3 \mathrm{E}-01$ & $\mathrm{U}$ \\
\hline & ${ }^{125} \mathrm{Sb}$ & $2.1 \mathrm{E}-02 \pm 8.4 \mathrm{E}-02$ & $\mathrm{U}$ & & ${ }^{125} \mathrm{Sb}$ & $7.2 \mathrm{E}-03 \pm 7.2 \mathrm{E}-02$ & $\mathrm{U}$ \\
\hline & ${ }^{113} \mathrm{Sn}$ & $-6.7 \mathrm{E}-04 \pm 6.7 \mathrm{E}-03$ & $\mathrm{U}$ & & ${ }^{113} \mathrm{Sn}$ & $1.3 \mathrm{E}-02 \pm 4.4 \mathrm{E}-02$ & $\mathrm{U}$ \\
\hline & ${ }^{90} \mathrm{Sr}$ & $2.0 \mathrm{E}+00 \pm 3.0 \mathrm{E}-01$ & & & ${ }^{90} \mathrm{Sr}$ & $-6.1 \mathrm{E}-02 \pm 1.2 \mathrm{E}-01$ & $\mathrm{U}$ \\
\hline & ${ }^{234} \mathrm{U}$ & $1.2 \mathrm{E}-02 \pm 7.1 \mathrm{E}-03$ & & & ${ }^{234} \mathrm{U}$ & $6.0 \mathrm{E}-02 \pm 2.0 \mathrm{E}-02$ & \\
\hline & ${ }^{235} \mathrm{U}$ & $1.9 \mathrm{E}-03 \pm 2.7 \mathrm{E}-03$ & $\mathrm{U}$ & & ${ }^{235} \mathrm{U}$ & $5.0 \mathrm{E}-03 \pm 4.3 \mathrm{E}-03$ & \\
\hline & ${ }^{238} \mathrm{U}$ & $7.1 \mathrm{E}-03 \pm 5.9 \mathrm{E}-03$ & & & ${ }^{238} \mathrm{U}$ & $5.3 \mathrm{E}-02 \pm 1.9 \mathrm{E}-02$ & \\
\hline & ${ }^{65} \mathrm{Zn}$ & $-3.4 \mathrm{E}-01 \pm 3.4 \mathrm{E}-01$ & $\mathrm{U}$ & & ${ }^{65} \mathrm{Zn}$ & $-1.2 \mathrm{E}-01 \pm 1.2 \mathrm{E}-01$ & $\mathrm{U}$ \\
\hline
\end{tabular}

$\overline{\mathrm{RQ}}=$ Result Qualifier. U $=$ The analyte was analyzed for but not detected. 
Table 3-5. 2003 Vegetation Sampling Results (pCi/g \pm total analytical uncertainty). (cont)

\begin{tabular}{|c|c|c|c|c|c|c|c|}
\hline Location & Isotope & Result \pm Error & $\mathbf{R Q}^{*}$ & Location & Isotope & Result \pm Error & $\mathbf{R Q}^{*}$ \\
\hline V117 & ${ }^{144} \mathrm{Ce}$ & $-2.7 \mathrm{E}-01 \pm 5.3 \mathrm{E}-01$ & $\bar{U}$ & V119 & ${ }^{144} \mathrm{Ce}$ & $5.1 \mathrm{E}-02 \pm 3.5 \mathrm{E}-01$ & $\overline{\mathrm{U}}$ \\
\hline \multirow[t]{17}{*}{ (300 Area) } & ${ }^{60} \mathrm{Co}$ & $2.4 \mathrm{E}-02 \pm 3.6 \mathrm{E}-02$ & $\mathrm{U}$ & (300 Area) & ${ }^{60} \mathrm{Co}$ & $2.0 \mathrm{E}-02 \pm 3.4 \mathrm{E}-02$ & $\mathrm{U}$ \\
\hline & ${ }^{134} \mathrm{Cs}$ & $-2.8 \mathrm{E}-02 \pm 3.3 \mathrm{E}-02$ & $\mathrm{U}$ & & ${ }^{134} \mathrm{Cs}$ & $-4.4 \mathrm{E}-03 \pm 4.1 \mathrm{E}-02$ & $\mathrm{U}$ \\
\hline & ${ }^{137} \mathrm{Cs}$ & $-1.5 \mathrm{E}-02 \pm 3.4 \mathrm{E}-02$ & $\mathrm{U}$ & & ${ }^{137} \mathrm{Cs}$ & $-8.4 \mathrm{E}-03 \pm 3.2 \mathrm{E}-02$ & $\mathrm{U}$ \\
\hline & ${ }^{152} \mathrm{Eu}$ & $4.9 \mathrm{E}-02 \pm 9.1 \mathrm{E}-02$ & $\mathrm{U}$ & & ${ }^{152} \mathrm{Eu}$ & $-2.9 \mathrm{E}-02 \pm 8.6 \mathrm{E}-02$ & $\mathrm{U}$ \\
\hline & ${ }^{154} \mathrm{Eu}$ & $-6.1 \mathrm{E}-02 \pm 1.0 \mathrm{E}-01$ & $\mathrm{U}$ & & ${ }^{154} \mathrm{Eu}$ & $-4.3 \mathrm{E}-02 \pm 1.0 \mathrm{E}-01$ & $\mathrm{U}$ \\
\hline & ${ }^{155} \mathrm{Eu}$ & $1.6 \mathrm{E}-02 \pm 1.1 \mathrm{E}-01$ & $\mathrm{U}$ & & ${ }^{155} \mathrm{Eu}$ & $-5.5 \mathrm{E}-02 \pm 9.1 \mathrm{E}-02$ & $\mathrm{U}$ \\
\hline & ${ }^{238} \mathrm{Pu}$ & $-4.2 \mathrm{E}-03 \pm 1.4 \mathrm{E}-02$ & $\mathrm{U}$ & & ${ }^{238} \mathrm{Pu}$ & $-1.8 \mathrm{E}-03 \pm 1.3 \mathrm{E}-02$ & $\mathrm{U}$ \\
\hline & ${ }^{239,240} \mathrm{Pu}$ & $3.3 \mathrm{E}-03 \pm 4.6 \mathrm{E}-03$ & $\mathrm{U}$ & & ${ }^{239,240} \mathrm{Pu}$ & $2.6 \mathrm{E}-03 \pm 3.9 \mathrm{E}-03$ & $\mathrm{U}$ \\
\hline & ${ }^{103} \mathrm{Ru}$ & $9.5 \mathrm{E}-03 \pm 3.4 \mathrm{E}-02$ & $\mathrm{U}$ & & ${ }^{103} \mathrm{Ru}$ & $2.3 \mathrm{E}-02 \pm 3.2 \mathrm{E}-02$ & $\mathrm{U}$ \\
\hline & ${ }^{106} \mathrm{Ru}$ & $-5.2 \mathrm{E}-02 \pm 3.1 \mathrm{E}-01$ & $\mathrm{U}$ & & ${ }^{106} \mathrm{Ru}$ & $1.1 \mathrm{E}-01 \pm 2.9 \mathrm{E}-01$ & $\mathrm{U}$ \\
\hline & ${ }^{125} \mathrm{Sb}$ & $-4.0 \mathrm{E}-03 \pm 4.0 \mathrm{E}-02$ & $\mathrm{U}$ & & ${ }^{125} \mathrm{Sb}$ & $-5.2 \mathrm{E}-02 \pm 8.7 \mathrm{E}-02$ & $\mathrm{U}$ \\
\hline & ${ }^{113} \mathrm{Sn}$ & $5.5 \mathrm{E}-02 \pm 4.1 \mathrm{E}-02$ & $\mathrm{U}$ & & ${ }^{113} \mathrm{Sn}$ & $-1.3 \mathrm{E}-02 \pm 3.9 \mathrm{E}-02$ & $\mathrm{U}$ \\
\hline & ${ }^{90} \mathrm{Sr}$ & $-1.5 \mathrm{E}-01 \pm 1.5 \mathrm{E}-01$ & $\mathrm{U}$ & & ${ }^{90} \mathrm{Sr}$ & $-2.4 \mathrm{E}-01 \pm 2.4 \mathrm{E}-01$ & $\mathrm{U}$ \\
\hline & ${ }^{234} \mathrm{U}$ & $2.0 \mathrm{E}-02 \pm 9.6 \mathrm{E}-03$ & & & & $2.2 \mathrm{E}-01 \pm 4.8 \mathrm{E}-02$ & \\
\hline & ${ }^{235} \mathrm{U}$ & $1.8 \mathrm{E}-03 \pm 4.5 \mathrm{E}-03$ & $\mathrm{U}$ & & ${ }^{235} \mathrm{U}$ & $1.8 \mathrm{E}-02 \pm 9.0 \mathrm{E}-03$ & \\
\hline & ${ }^{238} \mathrm{U}$ & $1.7 \mathrm{E}-02 \pm 8.5 \mathrm{E}-03$ & & & ${ }^{238} \mathrm{U}$ & $1.9 \mathrm{E}-01 \pm 4.4 \mathrm{E}-02$ & \\
\hline & ${ }^{65} \mathrm{Zn}$ & $-4.0 \mathrm{E}-03 \pm 4.0 \mathrm{E}-02$ & $\mathrm{U}$ & & ${ }^{65} \mathrm{Zn}$ & $7.6 \mathrm{E}-03 \pm 7.6 \mathrm{E}-02$ & $\mathrm{U}$ \\
\hline V121 & ${ }^{144} \mathrm{Ce}$ & $-2.0 \mathrm{E}-01 \pm 3.1 \mathrm{E}-01$ & $\overline{\mathrm{U}}$ & V123 & ${ }^{144} \mathrm{Ce}$ & $-2.8 \mathrm{E}-01 \pm 5.0 \mathrm{E}-01$ & $\bar{U}$ \\
\hline \multirow[t]{17}{*}{ (300 Area) } & ${ }^{60} \mathrm{Co}$ & $3.1 \mathrm{E}-03 \pm 3.1 \mathrm{E}-02$ & $\mathrm{U}$ & (300 Area) & ${ }^{60} \mathrm{Co}$ & $2.7 \mathrm{E}-02 \pm 3.8 \mathrm{E}-02$ & $\mathrm{U}$ \\
\hline & ${ }^{134} \mathrm{Cs}$ & $4.2 \mathrm{E}-03 \pm 3.8 \mathrm{E}-02$ & $\mathrm{U}$ & & ${ }^{134} \mathrm{Cs}$ & $-1.8 \mathrm{E}-02 \pm 3.8 \mathrm{E}-02$ & $\mathrm{U}$ \\
\hline & ${ }^{137} \mathrm{Cs}$ & $-9.8 \mathrm{E}-03 \pm 2.9 \mathrm{E}-02$ & $\mathrm{U}$ & & ${ }^{137} \mathrm{Cs}$ & $2.3 \mathrm{E}-02 \pm 3.6 \mathrm{E}-02$ & $\mathrm{U}$ \\
\hline & ${ }^{152} \mathrm{Eu}$ & $-2.9 \mathrm{E}-02 \pm 8.0 \mathrm{E}-02$ & $\mathrm{U}$ & & ${ }^{152} \mathrm{Eu}$ & $-1.1 \mathrm{E}-01 \pm 1.1 \mathrm{E}-01$ & $\mathrm{U}$ \\
\hline & ${ }^{154} \mathrm{Eu}$ & $5.2 \mathrm{E}-02 \pm 9.6 \mathrm{E}-02$ & $\mathrm{U}$ & & ${ }^{154} \mathrm{Eu}$ & $-1.3 \mathrm{E}-02 \pm 1.2 \mathrm{E}-01$ & $\mathrm{U}$ \\
\hline & ${ }^{155} \mathrm{Eu}$ & $6.7 \mathrm{E}-03 \pm 6.7 \mathrm{E}-02$ & $\mathrm{U}$ & & ${ }^{155} \mathrm{Eu}$ & $-9.7 \mathrm{E}-02 \pm 1.4 \mathrm{E}-01$ & $\mathrm{U}$ \\
\hline & ${ }^{238} \mathrm{Pu}$ & $9.7 \mathrm{E}-03 \pm 1.6 \mathrm{E}-02$ & $\mathrm{U}$ & & ${ }^{238} \mathrm{Pu}$ & $-6.1 \mathrm{E}-03 \pm 1.8 \mathrm{E}-02$ & $\mathrm{U}$ \\
\hline & ${ }^{239,240} \mathrm{Pu}$ & $2.0 \mathrm{E}-03 \pm 4.0 \mathrm{E}-03$ & $\mathrm{U}$ & & ${ }^{239,240} \mathrm{Pu}$ & 8.7E-04 \pm 8.7E-03 & $\mathrm{U}$ \\
\hline & ${ }^{103} \mathrm{Ru}$ & $2.5 \mathrm{E}-03 \pm 2.5 \mathrm{E}-02$ & $\mathrm{U}$ & & ${ }^{103} \mathrm{Ru}$ & $3.1 \mathrm{E}-03 \pm 3.1 \mathrm{E}-02$ & $\mathrm{U}$ \\
\hline & ${ }^{106} \mathrm{Ru}$ & $-4.9 \mathrm{E}-02 \pm 2.7 \mathrm{E}-01$ & $\mathrm{U}$ & & ${ }^{106} \mathrm{Ru}$ & $-2.6 \mathrm{E}-01 \pm 3.3 \mathrm{E}-01$ & $\mathrm{U}$ \\
\hline & ${ }^{125} \mathrm{Sb}$ & $1.1 \mathrm{E}-02 \pm 7.3 \mathrm{E}-02$ & $\mathrm{U}$ & & ${ }^{125} \mathrm{Sb}$ & $-9.7 \mathrm{E}-03 \pm 9.7 \mathrm{E}-02$ & $\mathrm{U}$ \\
\hline & ${ }^{113} \mathrm{Sn}$ & $2.1 \mathrm{E}-02 \pm 3.7 \mathrm{E}-02$ & $\mathrm{U}$ & & ${ }^{113} \mathrm{Sn}$ & $-4.5 \mathrm{E}-03 \pm 4.5 \mathrm{E}-02$ & $\mathrm{U}$ \\
\hline & ${ }^{90} \mathrm{Sr}$ & $-6.8 \mathrm{E}-02 \pm 1.1 \mathrm{E}-01$ & $\mathrm{U}$ & & ${ }^{90} \mathrm{Sr}$ & $-9.5 \mathrm{E}-02 \pm 1.2 \mathrm{E}-01$ & $\mathrm{U}$ \\
\hline & ${ }^{234} \mathrm{U}$ & $4.0 \mathrm{E}-02 \pm 1.4 \mathrm{E}-02$ & & & ${ }^{234} \mathrm{U}$ & $1.1 \mathrm{E}-02 \pm 7.7 \mathrm{E}-03$ & \\
\hline & ${ }^{235} \mathrm{U}$ & $3.8 \mathrm{E}-03 \pm 3.8 \mathrm{E}-03$ & & & ${ }^{235} \mathrm{U}$ & $4.5 \mathrm{E}-03 \pm 4.9 \mathrm{E}-03$ & $\mathrm{U}$ \\
\hline & ${ }^{238} \mathrm{U}$ & $3.5 \mathrm{E}-02 \pm 1.3 \mathrm{E}-02$ & & & ${ }^{238} \mathrm{U}$ & $7.4 \mathrm{E}-03 \pm 5.6 \mathrm{E}-03$ & \\
\hline & ${ }^{65} \mathrm{Zn}$ & $-2.6 \mathrm{E}-02 \pm 7.7 \mathrm{E}-02$ & $\mathrm{U}$ & & ${ }^{65} \mathrm{Zn}$ & $-1.5 \mathrm{E}-01 \pm 1.5 \mathrm{E}-01$ & $\mathrm{U}$ \\
\hline
\end{tabular}

$\overline{\mathrm{RQ}}=$ Result Qualifier. U $=$ The analyte was analyzed for but not detected. 
Table 3-5. 2003 Vegetation Sampling Results (pCi/g \pm total analytical uncertainty). (cont)

\begin{tabular}{|c|c|c|c|c|c|c|c|}
\hline Location & Isotope & Result \pm Error & $\mathbf{R Q}^{*}$ & Location & Isotope & Result \pm Error & $\mathbf{R Q}^{*}$ \\
\hline V124 & ${ }^{144} \mathrm{Ce}$ & $-1.7 \mathrm{E}-01 \pm 3.1 \mathrm{E}-01$ & $\mathrm{U}$ & V125 & ${ }^{144} \mathrm{Ce}$ & $2.1 \mathrm{E}-01 \pm 4.6 \mathrm{E}-01$ & $\mathrm{U}$ \\
\hline \multirow[t]{17}{*}{ (300 Area) } & ${ }^{60} \mathrm{Co}$ & $-2.7 \mathrm{E}-03 \pm 2.2 \mathrm{E}-02$ & $\mathrm{U}$ & (300 Area) & ${ }^{60} \mathrm{Co}$ & $4.2 \mathrm{E}-03 \pm 3.2 \mathrm{E}-02$ & $\mathrm{U}$ \\
\hline & ${ }^{134} \mathrm{Cs}$ & $1.4 \mathrm{E}-04 \pm 1.4 \mathrm{E}-03$ & $\mathrm{U}$ & & ${ }^{134} \mathrm{Cs}$ & $-1.1 \mathrm{E}-03 \pm 1.1 \mathrm{E}-02$ & $\mathrm{U}$ \\
\hline & ${ }^{137} \mathrm{Cs}$ & $-9.1 \mathrm{E}-03 \pm 2.3 \mathrm{E}-02$ & $\mathrm{U}$ & & ${ }^{137} \mathrm{Cs}$ & $-3.6 \mathrm{E}-03 \pm 3.1 \mathrm{E}-02$ & $\mathrm{U}$ \\
\hline & ${ }^{152} \mathrm{Eu}$ & $1.9 \mathrm{E}-02 \pm 6.9 \mathrm{E}-02$ & $\mathrm{U}$ & & ${ }^{152} \mathrm{Eu}$ & $-5.5 \mathrm{E}-02 \pm 8.2 \mathrm{E}-02$ & $\mathrm{U}$ \\
\hline & ${ }^{154} \mathrm{Eu}$ & $-1.7 \mathrm{E}-02 \pm 6.7 \mathrm{E}-02$ & $\mathrm{U}$ & & ${ }^{154} \mathrm{Eu}$ & $-9.3 \mathrm{E}-02 \pm 9.4 \mathrm{E}-02$ & $\mathrm{U}$ \\
\hline & ${ }^{155} \mathrm{Eu}$ & $-7.1 \mathrm{E}-02 \pm 8.0 \mathrm{E}-02$ & $\mathrm{U}$ & & ${ }^{155} \mathrm{Eu}$ & $-8.0 \mathrm{E}-03 \pm 8.0 \mathrm{E}-02$ & $\mathrm{U}$ \\
\hline & ${ }^{238} \mathrm{Pu}$ & $6.6 \mathrm{E}-03 \pm 1.2 \mathrm{E}-02$ & $\mathrm{U}$ & & ${ }^{238} \mathrm{Pu}$ & $-2.5 \mathrm{E}-03 \pm 1.0 \mathrm{E}-02$ & $\mathrm{U}$ \\
\hline & ${ }^{239,240} \mathrm{Pu}$ & $3.3 \mathrm{E}-03 \pm 3.3 \mathrm{E}-03$ & & & ${ }^{239,240} \mathrm{Pu}$ & $4.1 \mathrm{E}-03 \pm 3.7 \mathrm{E}-03$ & \\
\hline & ${ }^{103} \mathrm{Ru}$ & $1.0 \mathrm{E}-02 \pm 2.3 \mathrm{E}-02$ & $\mathrm{U}$ & & ${ }^{103} \mathrm{Ru}$ & $-2.2 \mathrm{E}-02 \pm 3.1 \mathrm{E}-02$ & $\mathrm{U}$ \\
\hline & ${ }^{106} \mathrm{Ru}$ & $-1.4 \mathrm{E}-01 \pm 2.0 \mathrm{E}-01$ & $\mathrm{U}$ & & ${ }^{106} \mathrm{Ru}$ & $8.8 \mathrm{E}-02 \pm 2.8 \mathrm{E}-01$ & $\mathrm{U}$ \\
\hline & ${ }^{125} \mathrm{Sb}$ & $5.6 \mathrm{E}-02 \pm 6.4 \mathrm{E}-02$ & $\mathrm{U}$ & & ${ }^{125} \mathrm{Sb}$ & $3.4 \mathrm{E}-02 \pm 8.0 \mathrm{E}-02$ & $\mathrm{U}$ \\
\hline & ${ }^{113} \mathrm{Sn}$ & $2.6 \mathrm{E}-02 \pm 3.4 \mathrm{E}-02$ & $\mathrm{U}$ & & ${ }^{113} \mathrm{Sn}$ & $-1.0 \mathrm{E}-02 \pm 3.7 \mathrm{E}-02$ & U \\
\hline & ${ }^{90} \mathrm{Sr}$ & $-7.1 \mathrm{E}-02 \pm 9.4 \mathrm{E}-02$ & $\mathrm{U}$ & & ${ }^{90} \mathrm{Sr}$ & $-1.4 \mathrm{E}-01 \pm 1.4 \mathrm{E}-01$ & U \\
\hline & ${ }^{234} \mathrm{U}$ & $2.5 \mathrm{E}-02 \pm 1.1 \mathrm{E}-02$ & & & ${ }^{234} \mathrm{U}$ & $3.4 \mathrm{E}-02 \pm 1.3 \mathrm{E}-02$ & \\
\hline & ${ }^{235} \mathrm{U}$ & $8.2 \mathrm{E}-03 \pm 5.7 \mathrm{E}-03$ & & & ${ }^{235} \mathrm{U}$ & $1.2 \mathrm{E}-02 \pm 7.4 \mathrm{E}-03$ & \\
\hline & ${ }^{238} \mathrm{U}$ & $2.3 \mathrm{E}-02 \pm 9.9 \mathrm{E}-03$ & & & ${ }^{238} \mathrm{U}$ & $2.2 \mathrm{E}-02 \pm 1.1 \mathrm{E}-02$ & \\
\hline & ${ }^{65} \mathrm{Zn}$ & $-1.4 \mathrm{E}-01 \pm 1.4 \mathrm{E}-01$ & $\mathrm{U}$ & & ${ }^{65} \mathrm{Zn}$ & $-3.3 \mathrm{E}-03 \pm 3.3 \mathrm{E}-02$ & $\mathrm{U}$ \\
\hline V126 & ${ }^{144} \mathrm{Ce}$ & $-3.4 \mathrm{E}-02 \pm 3.2 \mathrm{E}-01$ & $\bar{U}$ & V127 & ${ }^{144} \mathrm{Ce}$ & $2.4 \mathrm{E}-01 \pm 4.9 \mathrm{E}-01$ & $\bar{U}$ \\
\hline \multirow[t]{17}{*}{ (300 Area) } & ${ }^{60} \mathrm{Co}$ & $-9.4 \mathrm{E}-03 \pm 2.5 \mathrm{E}-02$ & $\mathrm{U}$ & (300 Area) & ${ }^{60} \mathrm{Co}$ & $-1.5 \mathrm{E}-02 \pm 2.8 \mathrm{E}-02$ & $\mathrm{U}$ \\
\hline & ${ }^{134} \mathrm{Cs}$ & $-3.8 \mathrm{E}-03 \pm 2.3 \mathrm{E}-02$ & $\mathrm{U}$ & & ${ }^{134} \mathrm{Cs}$ & $2.7 \mathrm{E}-03 \pm 2.7 \mathrm{E}-02$ & $\mathrm{U}$ \\
\hline & ${ }^{137} \mathrm{Cs}$ & $-2.6 \mathrm{E}-02 \pm 2.6 \mathrm{E}-02$ & $\mathrm{U}$ & & ${ }^{137} \mathrm{Cs}$ & $-6.5 \mathrm{E}-02 \pm 6.5 \mathrm{E}-02$ & $\mathrm{U}$ \\
\hline & ${ }^{152} \mathrm{Eu}$ & $3.3 \mathrm{E}-02 \pm 7.3 \mathrm{E}-02$ & $\mathrm{U}$ & & ${ }^{152} \mathrm{Eu}$ & $-6.7 \mathrm{E}-02 \pm 1.1 \mathrm{E}-01$ & $\mathrm{U}$ \\
\hline & ${ }^{154} \mathrm{Eu}$ & $-6.7 \mathrm{E}-02 \pm 6.7 \mathrm{E}-02$ & $\mathrm{U}$ & & ${ }^{154} \mathrm{Eu}$ & $5.1 \mathrm{E}-02 \pm 9.8 \mathrm{E}-02$ & $\mathrm{U}$ \\
\hline & ${ }^{155} \mathrm{Eu}$ & $-3.9 \mathrm{E}-02 \pm 8.2 \mathrm{E}-02$ & $\mathrm{U}$ & & ${ }^{155} \mathrm{Eu}$ & $-1.2 \mathrm{E}-01 \pm 1.2 \mathrm{E}-01$ & $\mathrm{U}$ \\
\hline & ${ }^{238} \mathrm{Pu}$ & $4.8 \mathrm{E}-03 \pm 1.0 \mathrm{E}-02$ & $\mathrm{U}$ & & ${ }^{238} \mathrm{Pu}$ & $-8.7 \mathrm{E}-04 \pm 8.7 \mathrm{E}-03$ & $\mathrm{U}$ \\
\hline & ${ }^{239,240} \mathrm{Pu}$ & $-1.9 \mathrm{E}-03 \pm 3.8 \mathrm{E}-03$ & $\mathrm{U}$ & & ${ }^{239,240} \mathrm{Pu}$ & 8.7E-04 \pm 8.7E-03 & $\mathrm{U}$ \\
\hline & ${ }^{103} \mathrm{Ru}$ & $5.8 \mathrm{E}-04 \pm 5.8 \mathrm{E}-03$ & $\mathrm{U}$ & & ${ }^{103} \mathrm{Ru}$ & $-1.6 \mathrm{E}-02 \pm 3.6 \mathrm{E}-02$ & $\mathrm{U}$ \\
\hline & ${ }^{106} \mathrm{Ru}$ & $-4.1 \mathrm{E}-02 \pm 2.0 \mathrm{E}-01$ & $\mathrm{U}$ & & ${ }^{106} \mathrm{Ru}$ & $-9.3 \mathrm{E}-02 \pm 3.1 \mathrm{E}-01$ & $\mathrm{U}$ \\
\hline & ${ }^{125} \mathrm{Sb}$ & 7.6E-02 $\pm 6.9 \mathrm{E}-02$ & $\mathrm{U}$ & & ${ }^{125} \mathrm{Sb}$ & 5.0E-02 $\pm 9.7 \mathrm{E}-02$ & $\mathrm{U}$ \\
\hline & ${ }^{113} \mathrm{Sn}$ & $-1.3 \mathrm{E}-02 \pm 3.3 \mathrm{E}-02$ & $\mathrm{U}$ & & ${ }^{113} \mathrm{Sn}$ & $-1.3 \mathrm{E}-02 \pm 5.0 \mathrm{E}-02$ & $\mathrm{U}$ \\
\hline & ${ }^{90} \mathrm{Sr}$ & $-1.9 \mathrm{E}-02 \pm 9.9 \mathrm{E}-02$ & $\mathrm{U}$ & & ${ }^{90} \mathrm{Sr}$ & $-4.9 \mathrm{E}-02 \pm 1.3 \mathrm{E}-01$ & $\mathrm{U}$ \\
\hline & ${ }^{234} \mathrm{U}$ & $2.6 \mathrm{E}-02 \pm 1.1 \mathrm{E}-02$ & & & ${ }^{234} \mathrm{U}$ & $1.3 \mathrm{E}-02 \pm 7.1 \mathrm{E}-03$ & \\
\hline & ${ }^{235} \mathrm{U}$ & $4.6 \mathrm{E}-03 \pm 4.2 \mathrm{E}-03$ & & & ${ }^{235} \mathrm{U}$ & $1.7 \mathrm{E}-03 \pm 2.4 \mathrm{E}-03$ & $\mathrm{U}$ \\
\hline & ${ }^{238} \mathrm{U}$ & $1.8 \mathrm{E}-02 \pm 8.5 \mathrm{E}-03$ & & & ${ }^{238} \mathrm{U}$ & $1.0 \mathrm{E}-02 \pm 6.5 \mathrm{E}-03$ & \\
\hline & ${ }^{65} \mathrm{Zn}$ & $-1.2 \mathrm{E}-02 \pm 5.8 \mathrm{E}-02$ & $\mathrm{U}$ & & ${ }^{65} \mathrm{Zn}$ & $2.2 \mathrm{E}-02 \pm 8.2 \mathrm{E}-02$ & $\mathrm{U}$ \\
\hline
\end{tabular}

$\overline{\mathrm{RQ}}=$ Result Qualifier. U $=$ The analyte was analyzed for but not detected. 
Table 3-5. 2003 Vegetation Sampling Results (pCi/g \pm total analytical uncertainty). (cont)

\begin{tabular}{|c|c|c|c|c|c|c|c|}
\hline Location & Isotope & Result \pm Error & $\mathbf{R Q}^{*}$ & Location & Isotope & Result \pm Error & $\mathbf{R Q}^{*}$ \\
\hline V128 & ${ }^{144} \mathrm{Ce}$ & $9.5 \mathrm{E}-02 \pm 3.6 \mathrm{E}-01$ & $\bar{U}$ & V129 & ${ }^{144} \mathrm{Ce}$ & $-2.9 \mathrm{E}-01 \pm 4.4 \mathrm{E}-01$ & $\overline{\mathrm{U}}$ \\
\hline \multirow[t]{17}{*}{ (300 Area) } & ${ }^{60} \mathrm{Co}$ & $-3.1 \mathrm{E}-02 \pm 3.6 \mathrm{E}-02$ & $\mathrm{U}$ & (300 Area) & ${ }^{60} \mathrm{Co}$ & $8.1 \mathrm{E}-03 \pm 3.0 \mathrm{E}-02$ & $\mathrm{U}$ \\
\hline & ${ }^{134} \mathrm{Cs}$ & $3.2 \mathrm{E}-02 \pm 3.6 \mathrm{E}-02$ & $\mathrm{U}$ & & ${ }^{134} \mathrm{Cs}$ & $-1.5 \mathrm{E}-02 \pm 3.2 \mathrm{E}-02$ & $\mathrm{U}$ \\
\hline & ${ }^{137} \mathrm{Cs}$ & $-1.6 \mathrm{E}-02 \pm 3.3 \mathrm{E}-02$ & $\mathrm{U}$ & & ${ }^{137} \mathrm{Cs}$ & $1.8 \mathrm{E}-02 \pm 3.1 \mathrm{E}-02$ & $\mathrm{U}$ \\
\hline & ${ }^{152} \mathrm{Eu}$ & $-6.9 \mathrm{E}-02 \pm 8.9 \mathrm{E}-02$ & $\mathrm{U}$ & & ${ }^{152} \mathrm{Eu}$ & $1.4 \mathrm{E}-02 \pm 1.1 \mathrm{E}-01$ & $\mathrm{U}$ \\
\hline & ${ }^{154} \mathrm{Eu}$ & $4.4 \mathrm{E}-02 \pm 1.1 \mathrm{E}-01$ & U & & ${ }^{154} \mathrm{Eu}$ & $-3.4 \mathrm{E}-03 \pm 3.4 \mathrm{E}-02$ & $\mathrm{U}$ \\
\hline & ${ }^{155} \mathrm{Eu}$ & $1.4 \mathrm{E}-02 \pm 9.2 \mathrm{E}-02$ & $\mathrm{U}$ & & ${ }^{155} \mathrm{Eu}$ & $-6.7 \mathrm{E}-02 \pm 1.2 \mathrm{E}-01$ & $\mathrm{U}$ \\
\hline & ${ }^{238} \mathrm{Pu}$ & $6.2 \mathrm{E}-03 \pm 8.1 \mathrm{E}-03$ & $\mathrm{U}$ & & ${ }^{238} \mathrm{Pu}$ & $-3.2 \mathrm{E}-03 \pm 3.8 \mathrm{E}-03$ & $\mathrm{U}$ \\
\hline & ${ }^{239,240} \mathrm{Pu}$ & $2.6 \mathrm{E}-03 \pm 4.7 \mathrm{E}-03$ & $\mathrm{U}$ & & ${ }^{239,240} \mathrm{Pu}$ & $8.0 \mathrm{E}-04 \pm 1.6 \mathrm{E}-03$ & $\mathrm{U}$ \\
\hline & ${ }^{103} \mathrm{Ru}$ & $-4.4 \mathrm{E}-02 \pm 4.4 \mathrm{E}-02$ & $\mathrm{U}$ & & ${ }^{103} \mathrm{Ru}$ & $-1.0 \mathrm{E}-02 \pm 3.6 \mathrm{E}-02$ & $\mathrm{U}$ \\
\hline & ${ }^{106} \mathrm{Ru}$ & $1.1 \mathrm{E}-01 \pm 3.1 \mathrm{E}-01$ & $\mathrm{U}$ & & ${ }^{106} \mathrm{Ru}$ & $-1.0 \mathrm{E}-01 \pm 2.7 \mathrm{E}-01$ & $\mathrm{U}$ \\
\hline & ${ }^{125} \mathrm{Sb}$ & $-4.5 \mathrm{E}-03 \pm 4.5 \mathrm{E}-02$ & $\mathrm{U}$ & & ${ }^{125} \mathrm{Sb}$ & $2.8 \mathrm{E}-02 \pm 8.3 \mathrm{E}-02$ & $\mathrm{U}$ \\
\hline & ${ }^{113} \mathrm{Sn}$ & $-3.6 \mathrm{E}-03 \pm 3.6 \mathrm{E}-02$ & $\mathrm{U}$ & & ${ }^{113} \mathrm{Sn}$ & $-3.6 \mathrm{E}-02 \pm 4.2 \mathrm{E}-02$ & $\mathrm{U}$ \\
\hline & ${ }^{90} \mathrm{Sr}$ & $1.7 \mathrm{E}-01 \pm 1.5 \mathrm{E}-01$ & $\mathrm{U}$ & & ${ }^{90} \mathrm{Sr}$ & $-1.2 \mathrm{E}-01 \pm 1.2 \mathrm{E}-01$ & $\mathrm{U}$ \\
\hline & ${ }^{234} \mathrm{U}$ & $8.7 \mathrm{E}-03 \pm 5.7 \mathrm{E}-03$ & & & & $1.2 \mathrm{E}-02 \pm 7.3 \mathrm{E}-03$ & \\
\hline & ${ }^{235} \mathrm{U}$ & $4.8 \mathrm{E}-03 \pm 4.4 \mathrm{E}-03$ & & & ${ }^{235} \mathrm{U}$ & $3.5 \mathrm{E}-03 \pm 4.2 \mathrm{E}-03$ & $\mathrm{U}$ \\
\hline & ${ }^{238} \mathrm{U}$ & $5.2 \mathrm{E}-03 \pm 6.2 \mathrm{E}-03$ & $\mathrm{U}$ & & ${ }^{238} \mathrm{U}$ & $7.2 \mathrm{E}-03 \pm 5.0 \mathrm{E}-03$ & \\
\hline & ${ }^{65} \mathrm{Zn}$ & $-7.5 \mathrm{E}-03 \pm 7.5 \mathrm{E}-02$ & $\mathrm{U}$ & & ${ }^{65} \mathrm{Zn}$ & $-1.2 \mathrm{E}-02 \pm 8.8 \mathrm{E}-02$ & $\mathrm{U}$ \\
\hline \multirow{18}{*}{$\begin{array}{c}\text { V131 } \\
\text { (Duplicate } \\
\text { of V116, } \\
\text { 300 Area) }\end{array}$} & ${ }^{144} \mathrm{Ce}$ & $2.8 \mathrm{E}-01 \pm 4.6 \mathrm{E}-01$ & $\bar{U}$ & V132 & ${ }^{144} \mathrm{Ce}$ & $4.4 \mathrm{E}-01 \pm 7.0 \mathrm{E}-01$ & $\bar{U}$ \\
\hline & ${ }^{60} \mathrm{Co}$ & $1.4 \mathrm{E}-02 \pm 3.0 \mathrm{E}-02$ & $\mathrm{U}$ & (Duplicate & ${ }^{60} \mathrm{Co}$ & $8.2 \mathrm{E}-03 \pm 5.8 \mathrm{E}-02$ & $\mathrm{U}$ \\
\hline & ${ }^{134} \mathrm{Cs}$ & $5.9 \mathrm{E}-03 \pm 3.2 \mathrm{E}-02$ & $\mathrm{U}$ & of V123, & ${ }^{134} \mathrm{Cs}$ & $-1.6 \mathrm{E}-03 \pm 1.6 \mathrm{E}-02$ & $\mathrm{U}$ \\
\hline & ${ }^{137} \mathrm{Cs}$ & $-1.8 \mathrm{E}-02 \pm 3.2 \mathrm{E}-02$ & $\mathrm{U}$ & 300 Area) & ${ }^{137} \mathrm{Cs}$ & $4.0 \mathrm{E}-03 \pm 4.0 \mathrm{E}-02$ & $\mathrm{U}$ \\
\hline & ${ }^{152} \mathrm{Eu}$ & $1.0 \mathrm{E}-03 \pm 1.0 \mathrm{E}-02$ & $\mathrm{U}$ & & ${ }^{152} \mathrm{Eu}$ & $-7.5 \mathrm{E}-02 \pm 1.8 \mathrm{E}-01$ & $\mathrm{U}$ \\
\hline & ${ }^{154} \mathrm{Eu}$ & $1.7 \mathrm{E}-02 \pm 8.0 \mathrm{E}-02$ & $\mathrm{U}$ & & ${ }^{154} \mathrm{Eu}$ & $-7.1 \mathrm{E}-02 \pm 2.0 \mathrm{E}-01$ & $\mathrm{U}$ \\
\hline & ${ }^{155} \mathrm{Eu}$ & $-3.1 \mathrm{E}-02 \pm 1.1 \mathrm{E}-01$ & $\mathrm{U}$ & & ${ }^{155} \mathrm{Eu}$ & $-1.7 \mathrm{E}-01 \pm 1.7 \mathrm{E}-01$ & $\mathrm{U}$ \\
\hline & ${ }^{238} \mathrm{Pu}$ & $-2.5 \mathrm{E}-03 \pm 5.0 \mathrm{E}-03$ & $\mathrm{U}$ & & ${ }^{238} \mathrm{Pu}$ & $6.7 \mathrm{E}-03 \pm 5.8 \mathrm{E}-03$ & $\mathrm{U}$ \\
\hline & ${ }^{239,240} \mathrm{Pu}$ & 8.3E-04 $\pm 8.3 \mathrm{E}-03$ & $\mathrm{U}$ & & ${ }^{239,240} \mathrm{Pu}$ & $1.5 \mathrm{E}-03 \pm 3.0 \mathrm{E}-03$ & $\mathrm{U}$ \\
\hline & ${ }^{103} \mathrm{Ru}$ & $1.9 \mathrm{E}-02 \pm 5.8 \mathrm{E}-02$ & $\mathrm{U}$ & & ${ }^{103} \mathrm{Ru}$ & $-1.7 \mathrm{E}-02 \pm 9.8 \mathrm{E}-02$ & $\mathrm{U}$ \\
\hline & ${ }^{106} \mathrm{Ru}$ & $2.8 \mathrm{E}-01 \pm 2.9 \mathrm{E}-01$ & $\mathrm{U}$ & & ${ }^{106} \mathrm{Ru}$ & $-1.1 \mathrm{E}-01 \pm 5.4 \mathrm{E}-01$ & $\mathrm{U}$ \\
\hline & ${ }^{125} \mathrm{Sb}$ & $5.6 \mathrm{E}-02 \pm 9.2 \mathrm{E}-02$ & $\mathrm{U}$ & & ${ }^{125} \mathrm{Sb}$ & $8.4 \mathrm{E}-02 \pm 1.5 \mathrm{E}-01$ & $\mathrm{U}$ \\
\hline & ${ }^{113} \mathrm{Sn}$ & $2.0 \mathrm{E}-02 \pm 5.0 \mathrm{E}-02$ & $\mathrm{U}$ & & ${ }^{113} \mathrm{Sn}$ & $-3.5 \mathrm{E}-02 \pm 7.9 \mathrm{E}-02$ & $\mathrm{U}$ \\
\hline & ${ }^{90} \mathrm{Sr}$ & $-2.1 \mathrm{E}-01 \pm 2.1 \mathrm{E}-01$ & $\mathrm{U}$ & & ${ }^{90} \mathrm{Sr}$ & $-1.4 \mathrm{E}-02 \pm 1.2 \mathrm{E}-01$ & $\mathrm{U}$ \\
\hline & ${ }^{234} \mathrm{U}$ & $5.9 \mathrm{E}-02 \pm 1.8 \mathrm{E}-02$ & & & ${ }^{234} \mathrm{U}$ & $2.4 \mathrm{E}-02 \pm 1.3 \mathrm{E}-02$ & \\
\hline & ${ }^{235} \mathrm{U}$ & $9.2 \mathrm{E}-03 \pm 6.6 \mathrm{E}-03$ & & & ${ }^{235} \mathrm{U}$ & $8.4 \mathrm{E}-03 \pm 7.6 \mathrm{E}-03$ & \\
\hline & ${ }^{238} \mathrm{U}$ & $7.3 \mathrm{E}-02 \pm 2.0 \mathrm{E}-02$ & & & ${ }^{238} \mathrm{U}$ & $1.2 \mathrm{E}-02 \pm 1.1 \mathrm{E}-02$ & $\mathrm{U}$ \\
\hline & ${ }^{65} \mathrm{Zn}$ & $4.0 \mathrm{E}-02 \pm 1.2 \mathrm{E}-01$ & $\mathrm{U}$ & & ${ }^{65} \mathrm{Zn}$ & $1.3 \mathrm{E}-01 \pm 1.6 \mathrm{E}-01$ & $\mathrm{U}$ \\
\hline
\end{tabular}

$\overline{\mathrm{RQ}}=$ Result Qualifier. U $=$ The analyte was analyzed for but not detected. 
This page intentionally left blank. 


\subsection{EXTERNAL RADIATION}

External radiation fields were monitored near facilities and waste handling, storage, and disposal sites to measure and assess the impacts of operations. TLDs were used at numerous fixed locations to gather dose rate information over extended periods of time, typically three months.

In 2003, there were 134 TLD locations collecting external radiation information. At three of the operational areas, the dosimeter results showed a decrease of $6 \%$ or more in external radiation from 2002 levels. In the 100-K Area, there was temporary, significant increase in the annual average dose rate, attributable to the transfer and storage of radioactive materials associated with fuel storage basins clean-up activities (see details below). At the remaining operational areas, changes in the external radiation levels from 2002 to 2003 were 5\% or less. The number of TLD locations and a comparison summary between the 2002 and 2003 TLD results for each of the operational areas is provided in Table 4-1. Individual TLD results for 2003 are provided in Table 4-2. Maps illustrating TLD locations in 2003 are provided in Figures 4-1 through 4-10.

Table 4-1. Thermoluminescent Dosimeter Results (mrem/yr

\pm 2 standard deviations) for 2002 and 2003.

\begin{tabular}{|c|c|c|c|c|c|c|c|}
\hline \multirow[b]{2}{*}{ Area } & \multirow{2}{*}{$\begin{array}{c}\text { Number of } \\
\text { locations, } 2003\end{array}$} & \multicolumn{3}{|c|}{2002} & \multicolumn{2}{|c|}{2003} & \multirow[b]{2}{*}{${ }^{\%}$ Change $^{\mathrm{a}}$} \\
\hline & & Maximum & Mean & \pm 2SD & Maximum & Mean \pm 2SD & \\
\hline $100-\mathrm{B} / \mathrm{C}$ & 4 & 93 & 87 & \pm 9.0 & 88 & $85 \pm 5.0$ & -2 \\
\hline $100-\mathrm{F}$ & 5 & 93 & 86 & \pm 9.0 & 80 & $76 \pm 4.0$ & -12 \\
\hline $100-K$ & 11 & 439 & 129 & \pm 210 & 523 & $162 \pm 304$ & 26 \\
\hline $\mathrm{CVDF}^{\mathrm{b}}$ & 4 & 83 & 79 & \pm 5.0 & 82 & $80 \pm 6.0$ & 1 \\
\hline 100-KR-1 & 5 & 106 & 96 & \pm 20 & 103 & $95 \pm 15$ & -1 \\
\hline $100-\mathrm{N}$ & 14 & 1042 & 274 & \pm 543 & 993 & $261 \pm 485$ & -5 \\
\hline 200 East Area & 42 & 289 & 113 & \pm 97 & 482 & $118 \pm 138$ & 4 \\
\hline 200 West Area & 24 & 215 & 108 & \pm 64 & 189 & $106 \pm 52$ & -2 \\
\hline 200 North (212-R) & 1 & 3400 & 3200 & \pm 400 & 3400 & $3000 \pm 570$ & -6 \\
\hline 300 Area & 8 & 129 & 99 & \pm 39 & 112 & $92 \pm 24$ & -7 \\
\hline 300 TEDF $^{\mathrm{c}}$ & 6 & 88 & 85 & \pm 4.0 & 90 & $85 \pm 5.0$ & 0 \\
\hline 400 Area & 7 & 86 & 82 & \pm 5.0 & 85 & $81 \pm 5.0$ & -1 \\
\hline $\mathrm{ERDF}^{\mathrm{d}}$ & 3 & 95 & 90 & \pm 10 & 99 & $94 \pm 11$ & 4 \\
\hline
\end{tabular}

\footnotetext{
${ }^{\mathrm{a}}$ Numbers indicate a decrease (-) or increase from the 2002 mean.

${ }^{\mathrm{b}} \mathrm{CVDF}=$ Cold Vacuum Drying Facility (100-K Area).

${ }^{\mathrm{c}}$ TEDF $=300$ Area Treated Effluent Disposal Facility.

${ }^{\mathrm{d}} \mathrm{ERDF}=$ Environmental Restoration Disposal Facility.
}

Noteworthy observations in dose rate monitoring during 2003 included:

- In the $100-\mathrm{K}$ Area, compared to 2002, there was an overall $26 \%$ increase in the 2003 annual average dose rate. This increase was due to temporary, elevated dose rates at two monitoring locations situated near radioactive materials transfer and 
storage areas -- one near the 105-K East load-out station and the other near the 105-K West fuel storage basin. Dose rates at both locations decreased by year's end to typical Site background levels. The 2003 quarterly results from these locations as compared to the 100-K Area dose rate average are shown in graph form in Figure 4-11.

- $\quad$ In the 100-N Area, three of the five monitoring locations near the retired $116-\mathrm{N}-1$ Liquid Waste Disposal Facility (LWDF) Trench showed a slight increase of approximately $17 \%$ in annual average dose rate levels compared to those measured at these locations in 2002. This increase may be ascribed to the selective removal of low-level, radioactively contaminated material from selected portions of the Trench soil column. Removal of this layer of natural shielding from atop the residual, slightly higher-level radioactively contaminated subsurface materials led to the moderate increases observed in dose rates in the immediate vicinity of the excavation work. Remedial action activities will resume in mid-2004 to remove additional contamination with a scheduled completion date of fiscal year 2005. Figure 4-12 provides four year trend plots of quarterly dose rates from these three monitoring locations.

- In the 100-N Area, the 2003 annual average dose rate levels at the three monitoring locations at the 116-N-3 Facility showed a decrease of approximately $12 \%$ from 2002 levels. This reduction in dose rates was directly attributable to the removal of source material from the waste disposal facilities by the ERC. Overall, the average dose rate measured in the 100-N Area in 2003 was 5\% lower than that measured in 2002. Figure 4-13 provides four year trend plots of quarterly dose rates from these three monitoring locations. 


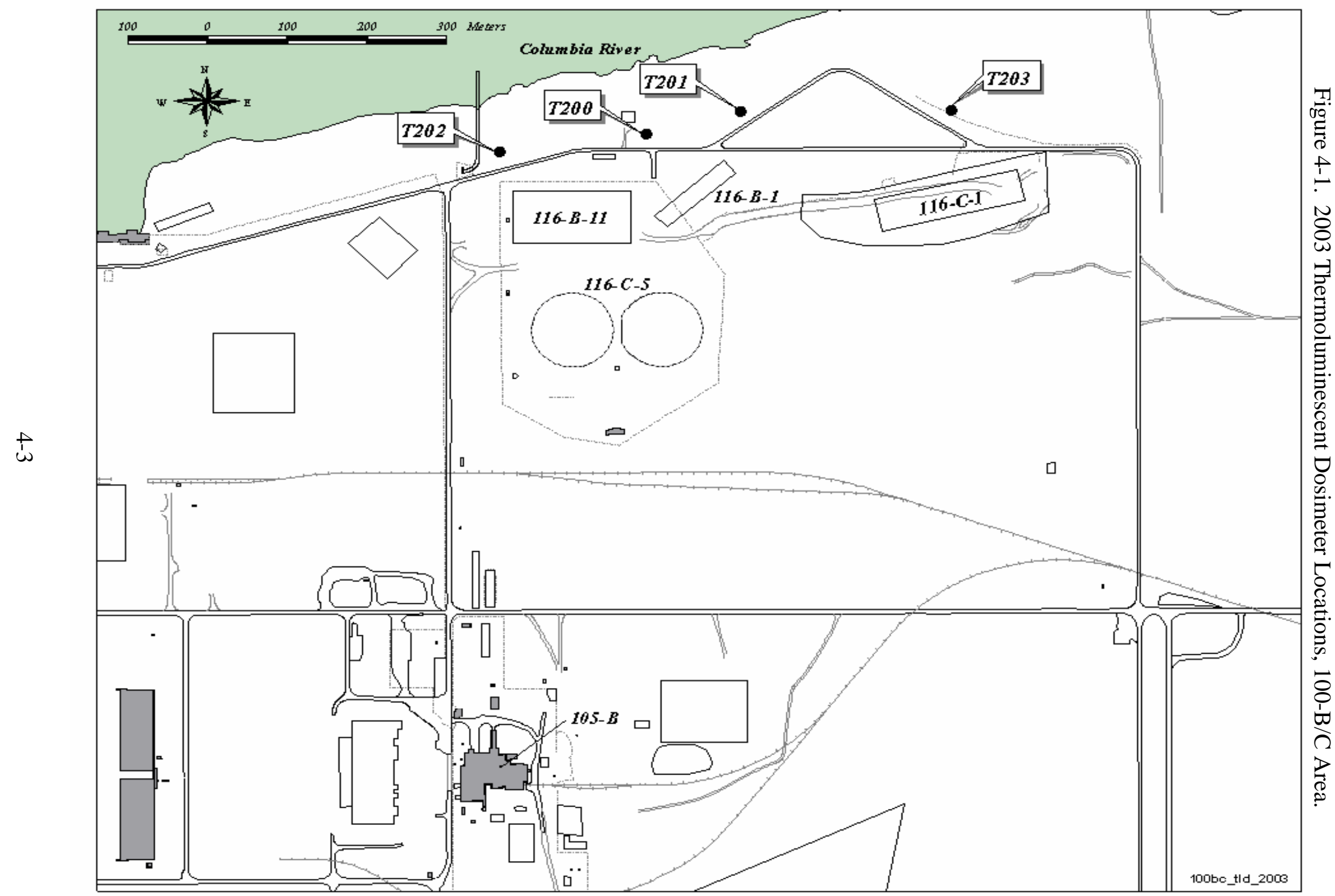




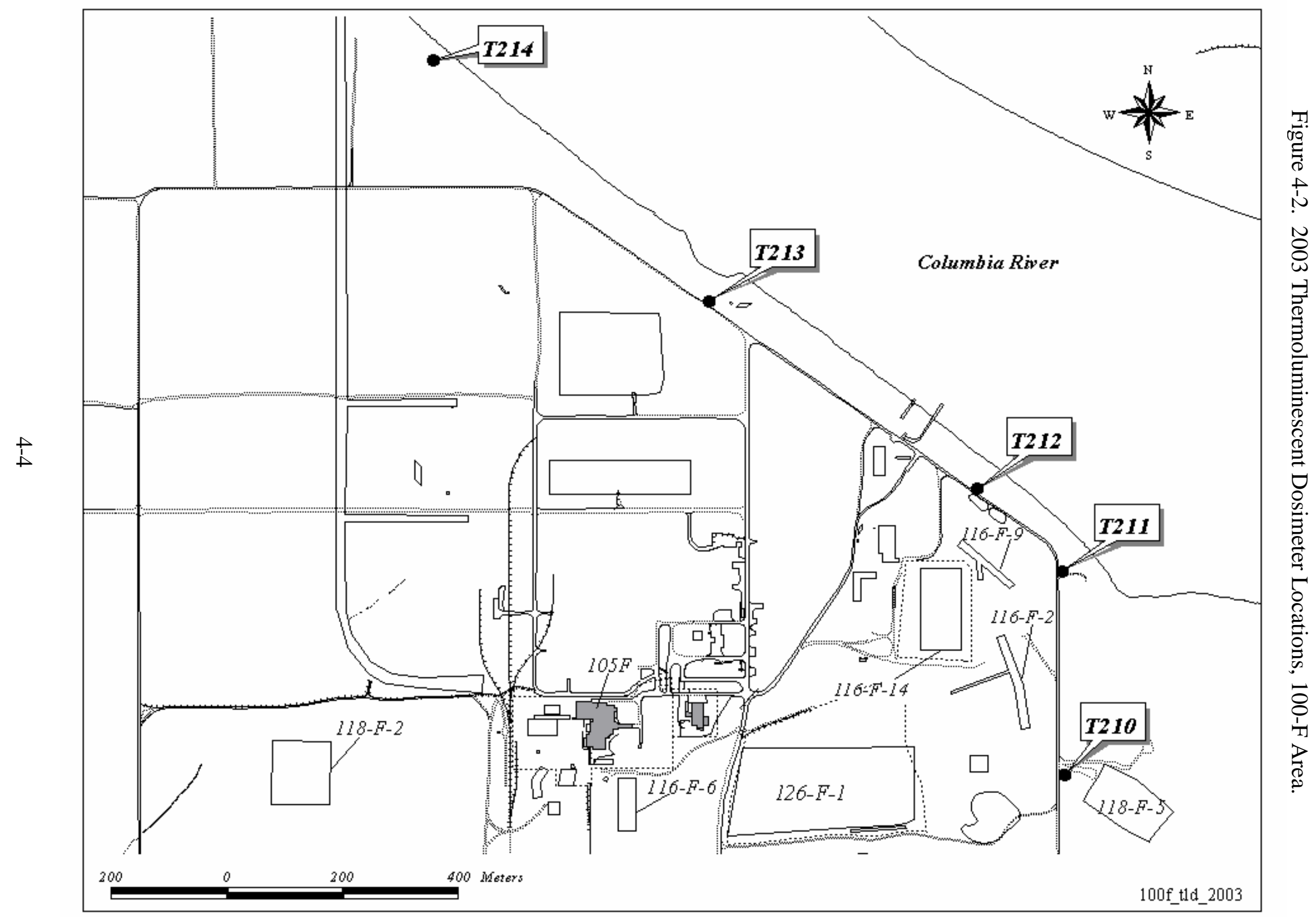




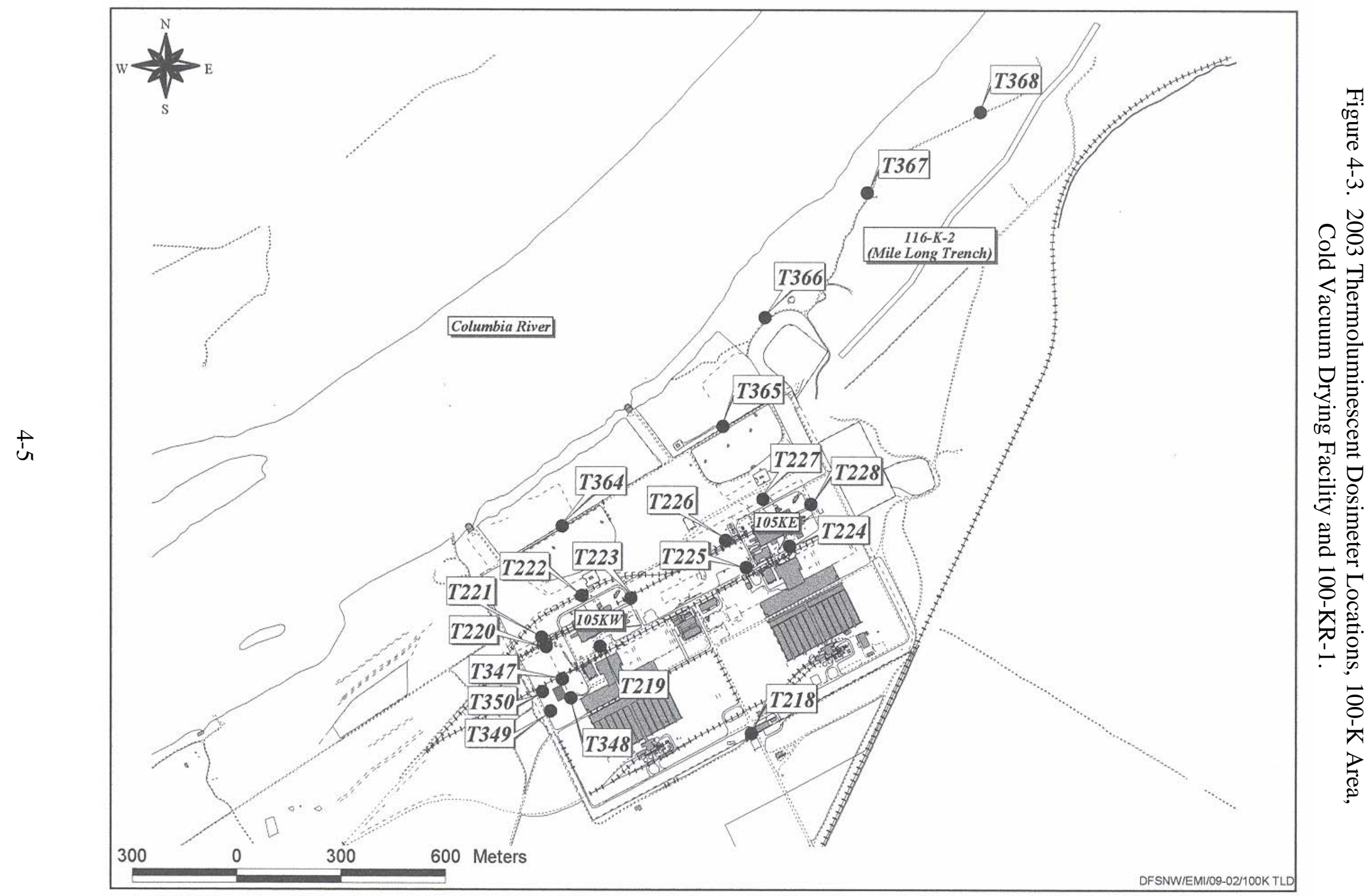




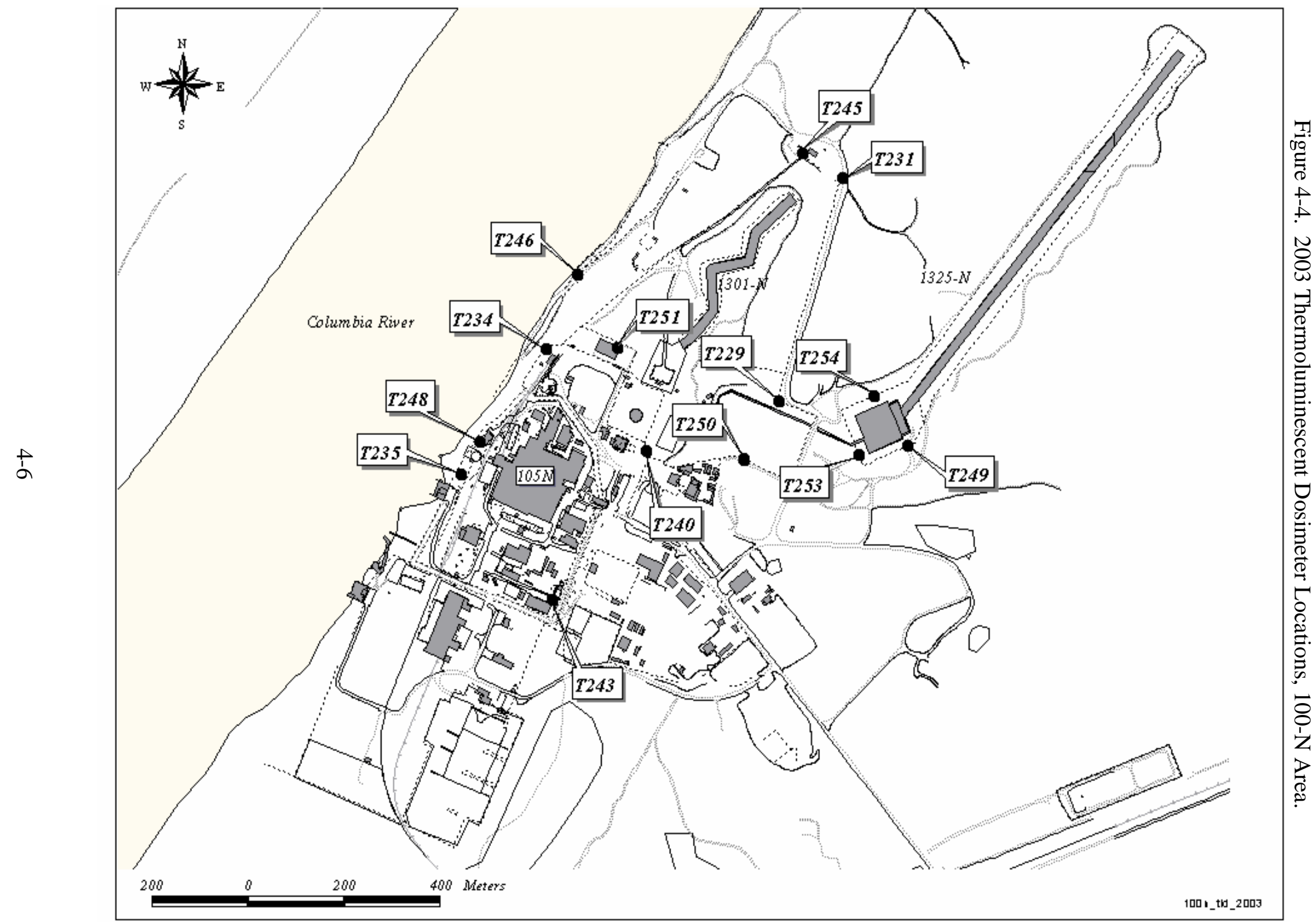




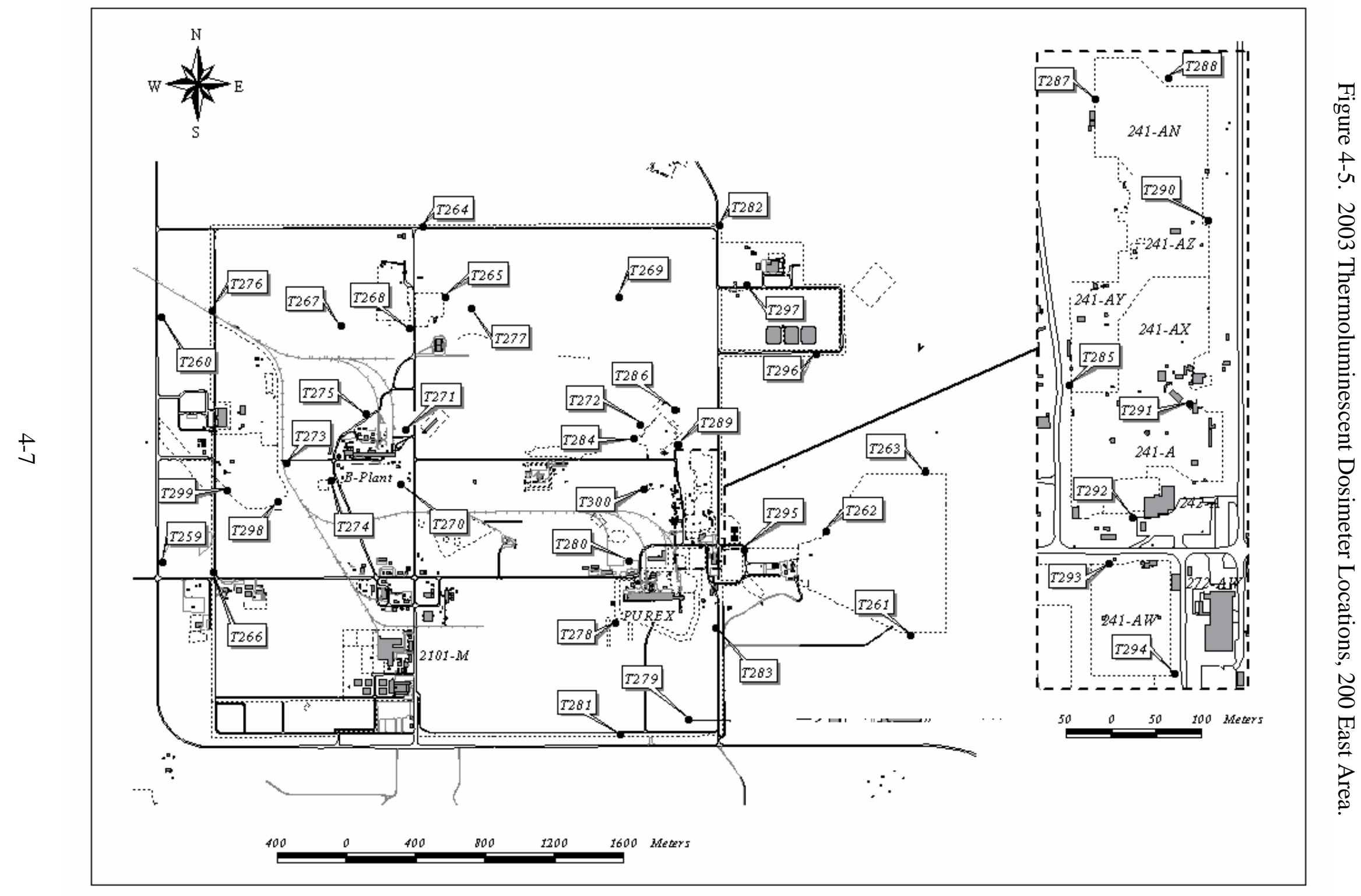




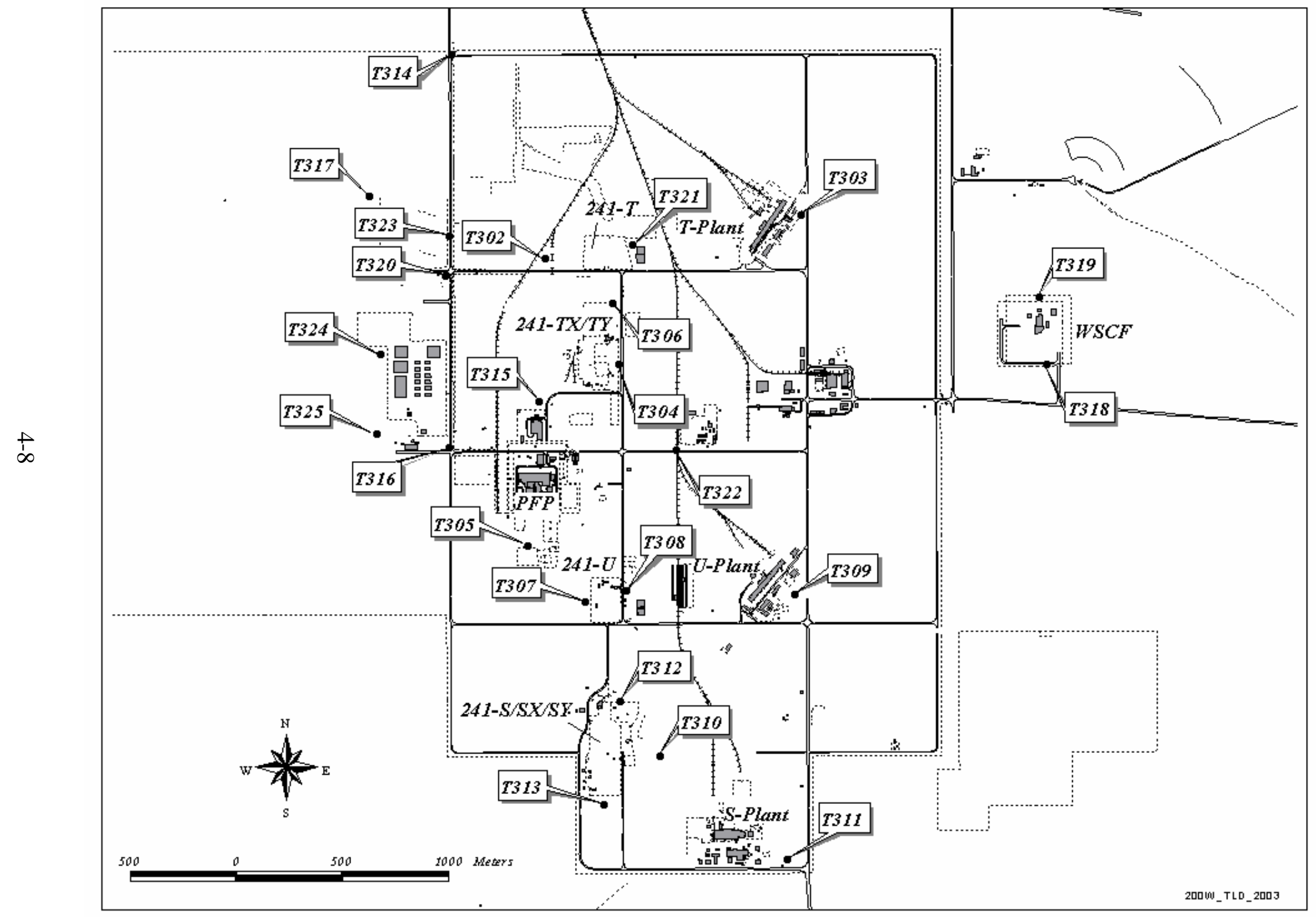

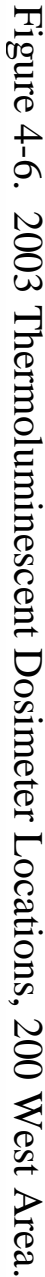




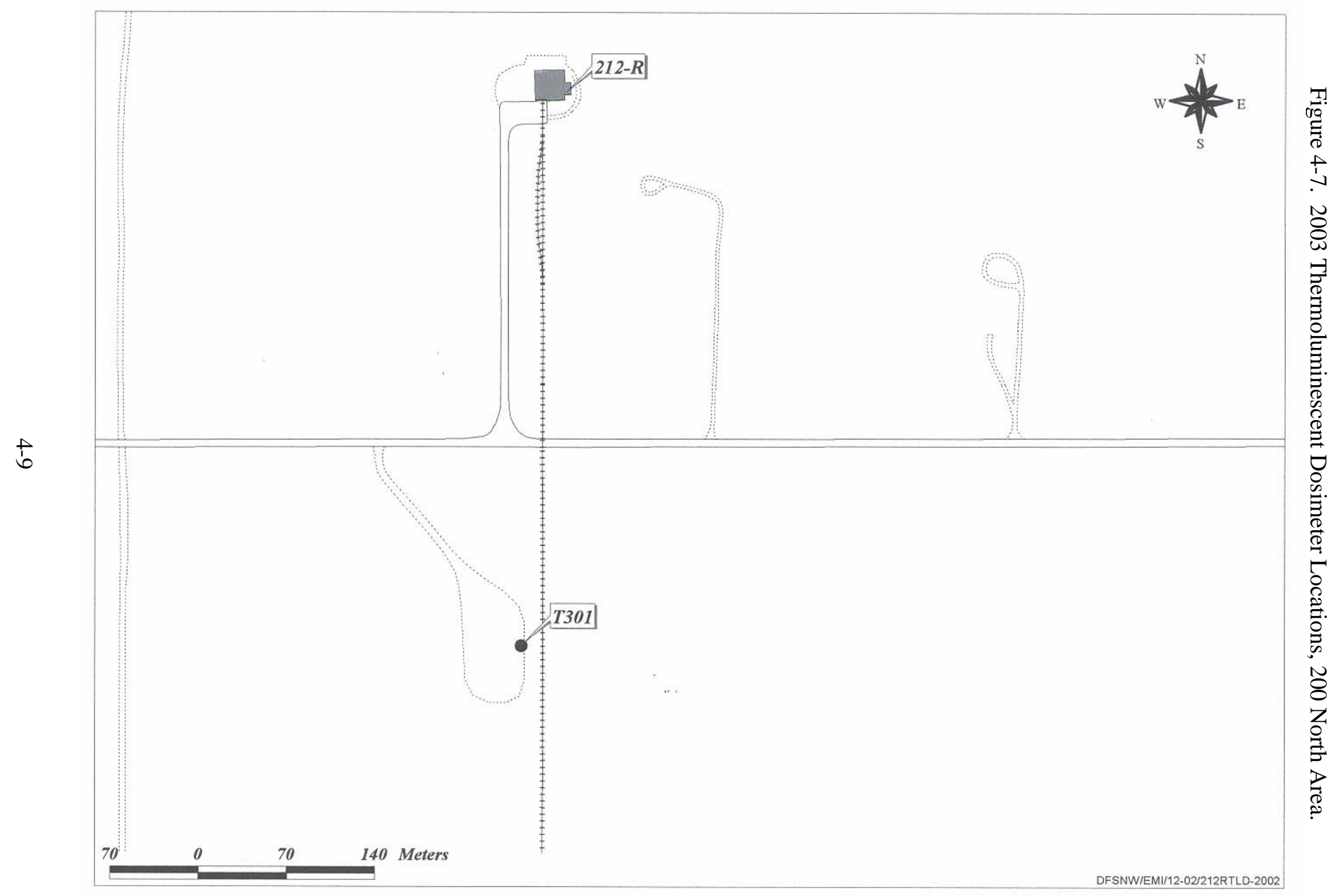




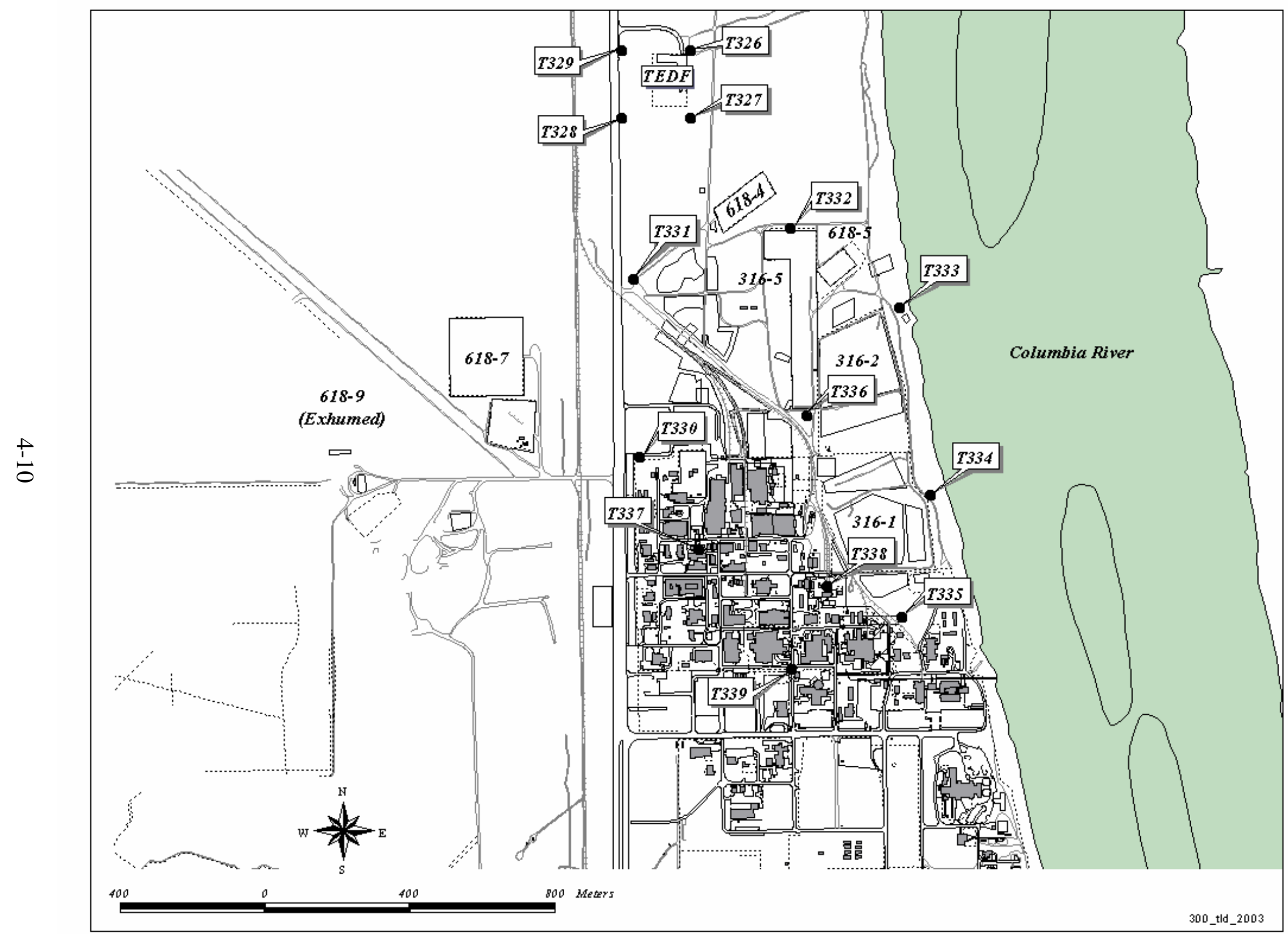

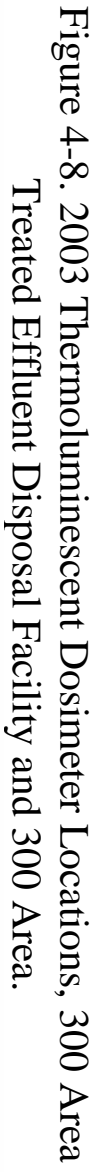




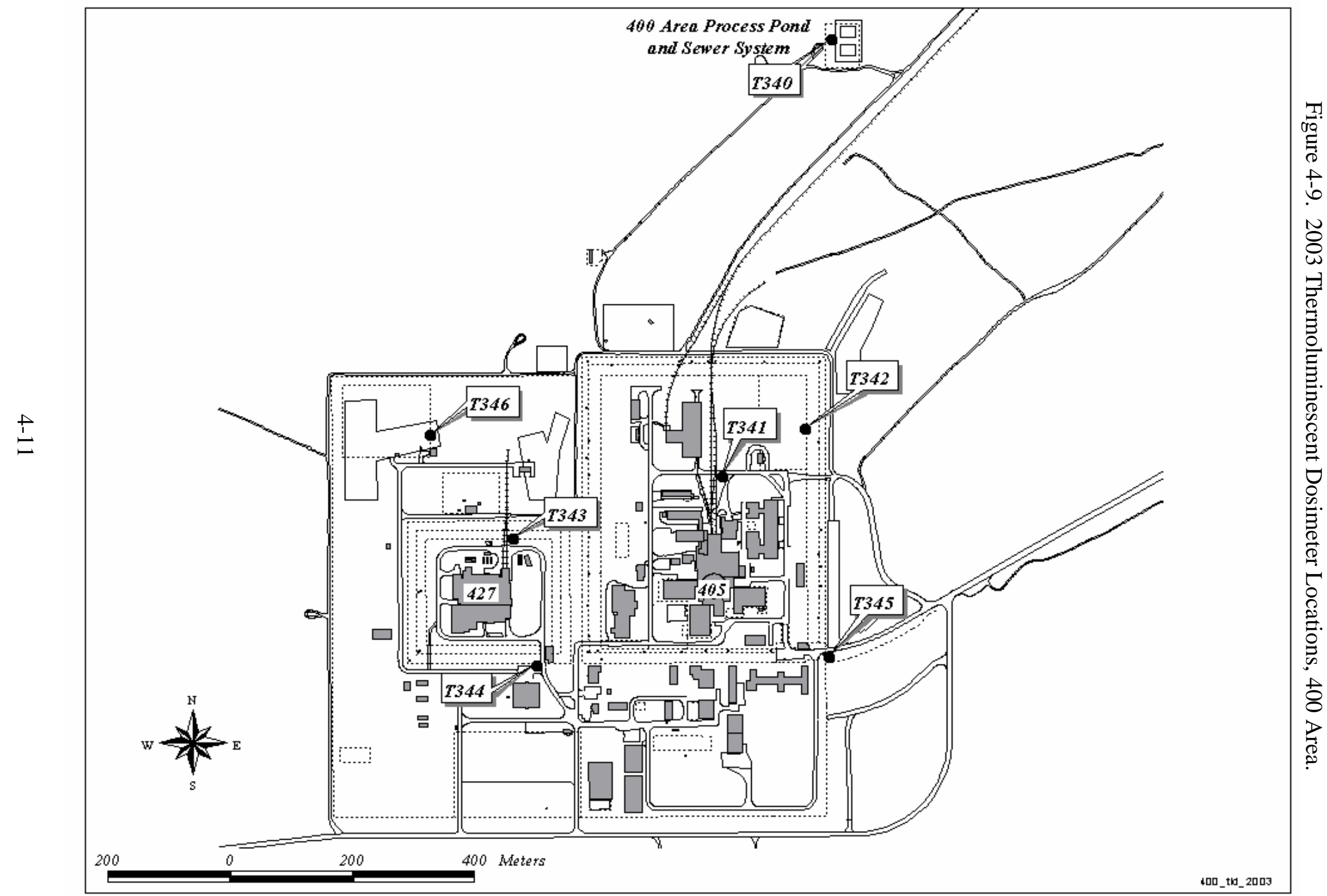




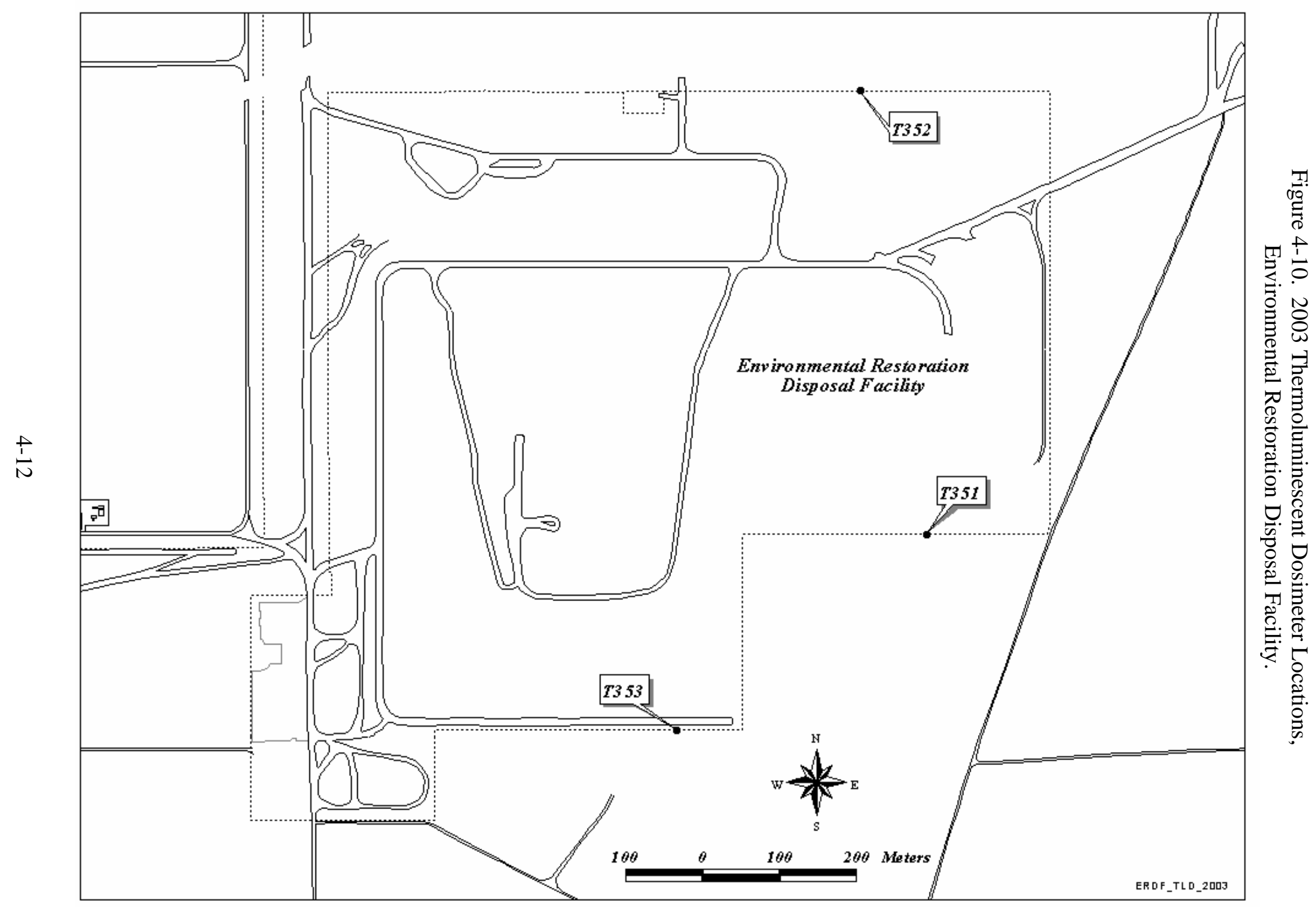


Figure 4-11. Thermoluminescent Dosimeter Results, 100-K Area

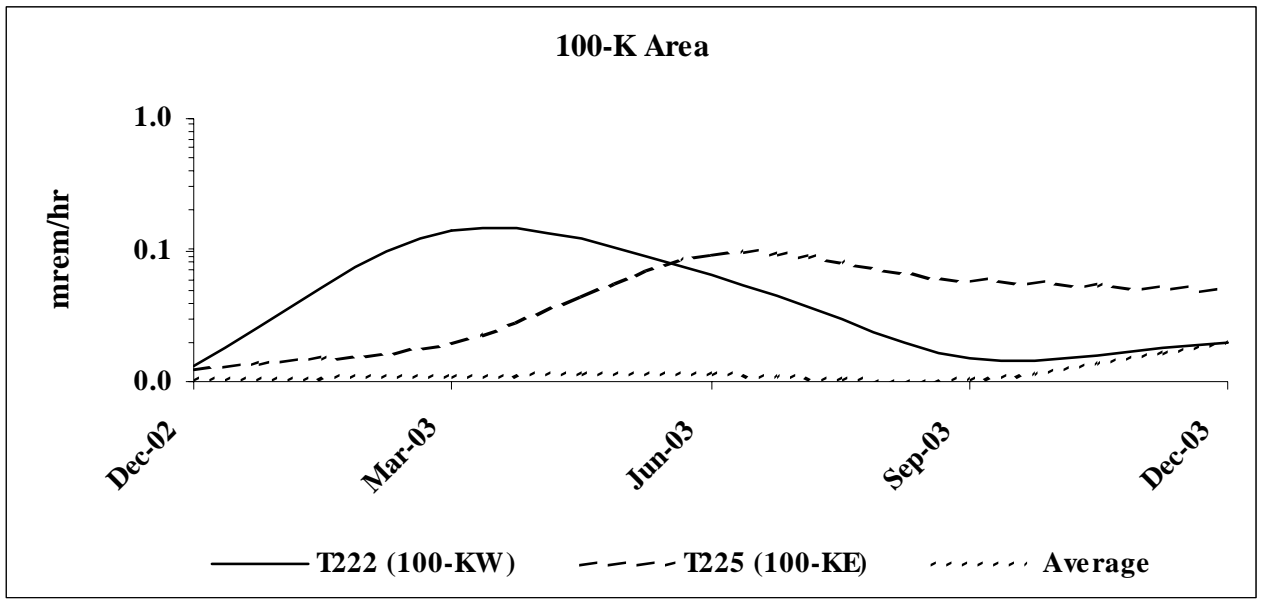

note: $100-\mathrm{K}$ area, "average" values do not include T222 and T225 results.

Figure 4-12. Thermoluminescent Dosimeter Results, 100-N Area

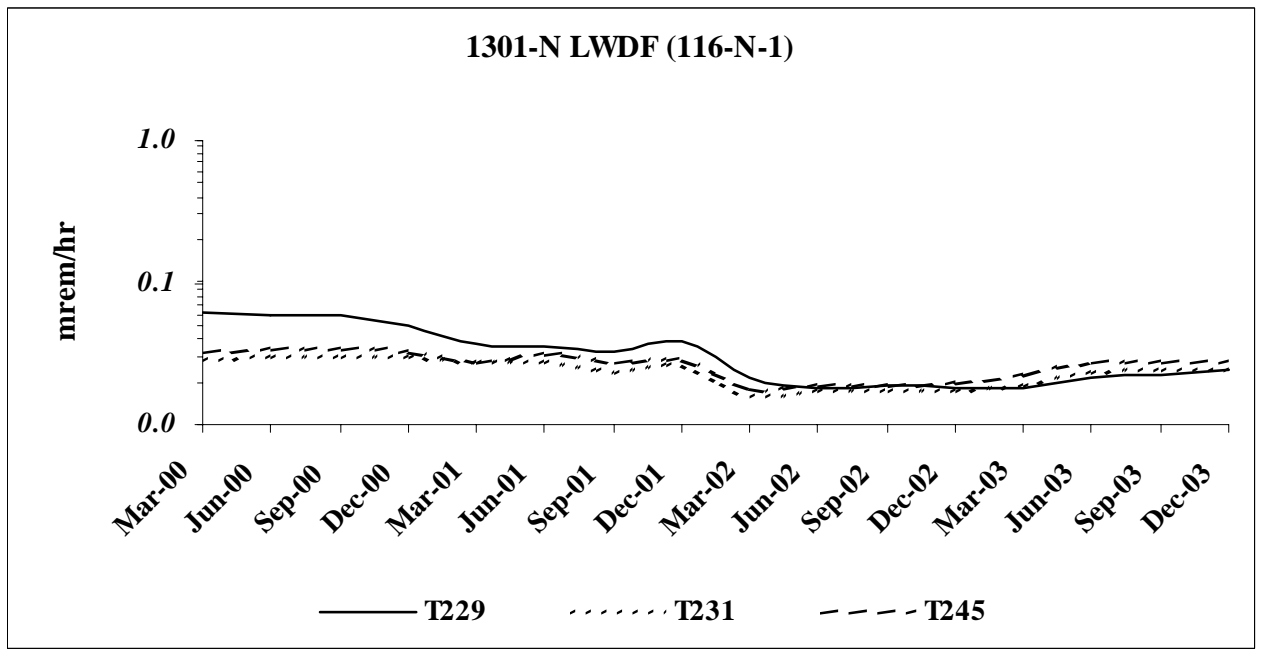

Figure 4-13. Thermoluminescent Dosimeter Results, 100-N Area

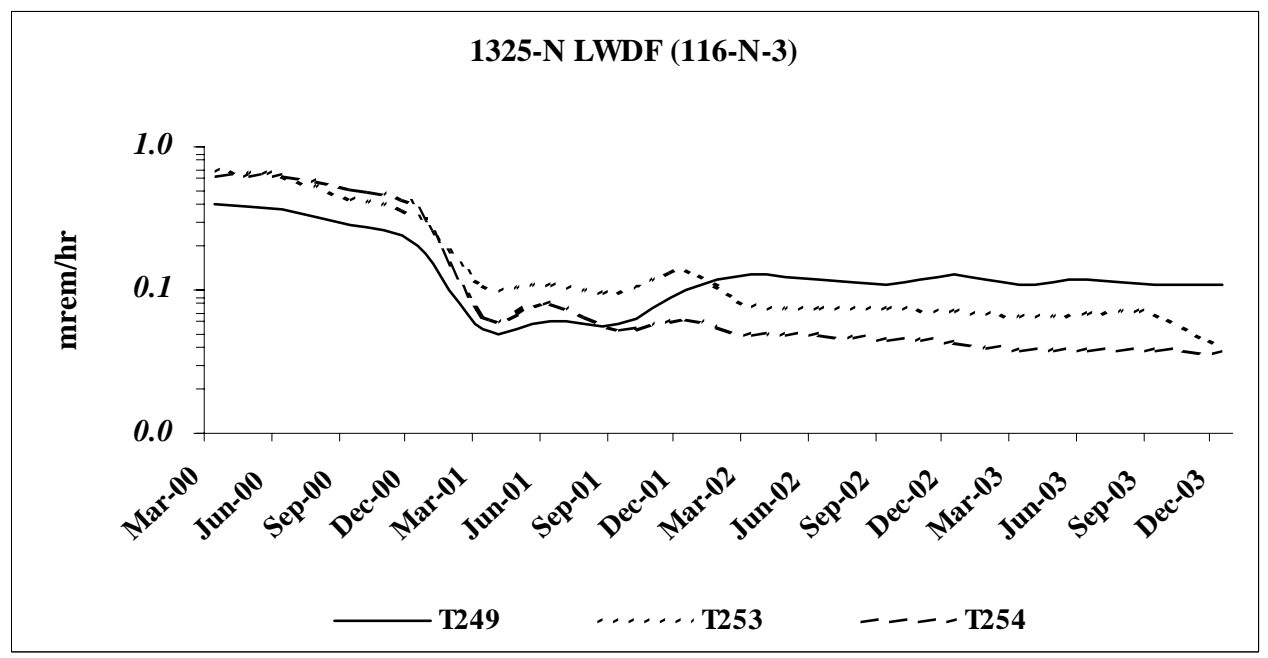


Table 4-2. 2003 Thermoluminescent Dosimeter Results.

\begin{tabular}{|c|c|c|c|c|c|c|c|}
\hline Location & Sample Period & EDP Code & mrem/hr & mrem/day & mrem/qtr & mrem/year & Days in Field \\
\hline \multirow[t]{16}{*}{ 100-B/C RA } & 1st Quarter '03 & T200 & 0.01 & 0.23 & 22.0 & 86 & 124 \\
\hline & & T201 & 0.01 & 0.23 & 21.2 & 82 & 124 \\
\hline & & T202 & 0.01 & 0.23 & 21.4 & 83 & 124 \\
\hline & & T203 & 0.01 & 0.21 & 19.8 & 77 & 124 \\
\hline & 2nd Quarter '03 & T200 & 0.01 & 0.24 & 21.6 & 88 & 119 \\
\hline & & T201 & \multicolumn{5}{|c|}{ TLD not found } \\
\hline & & T202 & 0.01 & 0.23 & 21.0 & 85 & 119 \\
\hline & & T203 & 0.01 & 0.23 & 20.4 & 83 & 119 \\
\hline & 3rd Quarter '03 & T200 & 0.01 & 0.25 & 24.4 & 92 & 127 \\
\hline & & T201 & 0.01 & 0.24 & 23.2 & 88 & 126 \\
\hline & & T202 & 0.01 & 0.24 & 22.8 & 86 & 127 \\
\hline & & T203 & 0.01 & 0.22 & 21.6 & 81 & 127 \\
\hline & 4th Quarter '03 & T200 & \multicolumn{5}{|c|}{ TLD not found } \\
\hline & & T201 & 0.01 & 0.23 & 20.9 & 85 & 120 \\
\hline & & T202 & 0.01 & 0.24 & 21.3 & 86 & 120 \\
\hline & & T203 & 0.01 & 0.24 & 21.2 & 86 & 120 \\
\hline \multicolumn{8}{|c|}{ 100-B/C RA, Annual Average \pm 2 Standard Deviation (2SD) } \\
\hline EDP Code & \multicolumn{2}{|c|}{$\mathrm{mrem} / \mathrm{hr} \pm \mathbf{2 S D}$} & \multicolumn{2}{|c|}{ mrem/day $\pm 2 S D$} & \multicolumn{2}{|c|}{ mrem/qtr \pm 2 SD } & $\mathrm{mrem} / \mathrm{yr} \pm 2 \mathrm{SD}$ \\
\hline T200 & \multicolumn{2}{|c|}{$0.01 \pm 0.001$} & \multicolumn{2}{|c|}{$0.24 \pm 0.02$} & \multicolumn{2}{|c|}{$22.1 \pm 1.6$} & $88 \pm 6$ \\
\hline $\mathrm{T} 201$ & \multicolumn{2}{|c|}{$0.01 \pm 0.001$} & \multicolumn{2}{|c|}{$0.23 \pm 0.02$} & \multicolumn{2}{|c|}{$21.3 \pm 1.5$} & $85 \pm 6$ \\
\hline T202 & \multicolumn{2}{|c|}{$0.01 \pm 0.000$} & \multicolumn{2}{|c|}{$0.23 \pm 0.01$} & \multicolumn{2}{|c|}{$21.3 \pm 0.7$} & $85 \pm 3$ \\
\hline $\mathrm{T} 203$ & \multicolumn{2}{|c|}{$0.01 \pm 0.001$} & \multicolumn{2}{|c|}{$0.22 \pm 0.02$} & \multicolumn{2}{|c|}{$20.4 \pm 1.9$} & $82 \pm 7$ \\
\hline
\end{tabular}


Table 4-2. 2003 Thermoluminescent Dosimeter Results. (cont)

\begin{tabular}{|c|c|c|c|c|c|c|c|}
\hline Location & Sample Period & EDP Code & mrem/hr & mrem/day & mrem/qtr & mrem/year & Days in Field \\
\hline \multirow[t]{10}{*}{ 100-F RA } & 1st Quarter '03 & T210 & 0.01 & 0.23 & 20.8 & 82 & 122 \\
\hline & & T211 & 0.01 & 0.23 & 20.8 & 82 & 122 \\
\hline & & T212 & 0.01 & 0.23 & 20.7 & 82 & 122 \\
\hline & & T213 & 0.01 & 0.22 & 20.2 & 80 & 122 \\
\hline & & $\mathrm{T} 214$ & 0.01 & 0.24 & 22 & 87 & 122 \\
\hline & 2nd Quarter '03 & $\mathrm{T} 210$ & 0.01 & 0.19 & 15.4 & 69 & 110 \\
\hline & & T211 & 0.01 & 0.19 & 15.4 & 69 & 110 \\
\hline & & T212 & 0.01 & 0.19 & 15.1 & 68 & 110 \\
\hline & & T213 & 0.01 & 0.18 & 14.8 & 67 & 110 \\
\hline & & $\mathrm{T} 214$ & 0.01 & 0.20 & 15.9 & 72 & 110 \\
\hline
\end{tabular}

100-F RA, Annual Average \pm 2 Standard Deviation (2SD)

EDP Code

T210

$\mathrm{T} 211$

$\mathrm{T} 212$

$\mathrm{T} 213$

$\mathrm{T} 214$ $\mathrm{mrem} / \mathrm{hr} \pm$ 2SD

$0.01 \pm 0.002$

$0.01 \pm 0.002$

$0.01 \pm 0.002$

$0.01 \pm 0.002$

$0.01 \pm 0.003$ mrem/day \pm 2SD

$0.21 \pm 0.05$

$0.21 \pm 0.05$

$0.21 \pm 0.06$

$0.20 \pm 0.05$

$0.22 \pm 0.06$ mrem/qtr \pm 2SD $19.1 \pm 4.6$

$19.1 \pm 4.6$

$18.9 \pm 5.0$

$18.5 \pm 4.7$

$20.0 \pm 5.4$ mrem/yr \pm 2SD

$76 \pm 18$

$76 \pm 18$

$76 \pm 20$

$74 \pm 19$

$80 \pm 22$ 
Table 4-2. 2003 Thermoluminescent Dosimeter Results. (cont)

\begin{tabular}{|c|c|c|c|c|c|c|c|}
\hline Location & Sample Period & EDP Code & mrem/hr & mrem/day & mrem/qtr & mrem/year & Days in Field \\
\hline \multirow[t]{33}{*}{ 100-K Area } & 1st Quarter '03 & T218 & 0.01 & 0.20 & 19.0 & 74 & 124 \\
\hline & & T219 & 0.01 & 0.22 & 20.6 & 80 & 124 \\
\hline & & T220 & 0.01 & 0.24 & 22.7 & 88 & 124 \\
\hline & & T221 & 0.01 & 0.28 & 26 & 101 & 124 \\
\hline & & T222 & 0.01 & 0.36 & 33.6 & 131 & 124 \\
\hline & & T223 & 0.01 & 0.21 & 19.7 & 76 & 124 \\
\hline & & T224 & 0.01 & 0.23 & 21.2 & 82 & 124 \\
\hline & & T225 & 0.06 & 1.44 & 135.4 & 526 & 124 \\
\hline & & T226 & 0.01 & 0.28 & 26.3 & 102 & 124 \\
\hline & & T227 & 0.02 & 0.40 & 37.3 & 145 & 124 \\
\hline & & Т228 & 0.01 & 0.23 & 21.8 & 85 & 124 \\
\hline & 2nd Quarter '03 & T218 & 0.01 & 0.21 & 18.8 & 76 & 119 \\
\hline & & T219 & 0.01 & 0.24 & 21.6 & 88 & 119 \\
\hline & & T220 & 0.01 & 0.27 & 23.8 & 97 & 119 \\
\hline & & T221 & 0.01 & 0.30 & 27.0 & 110 & 119 \\
\hline & & T222 & 0.06 & 1.54 & 138.2 & 561 & 119 \\
\hline & & T223 & 0.01 & 0.26 & 23.7 & 96 & 119 \\
\hline & & T224 & 0.01 & 0.24 & 21.7 & 88 & 119 \\
\hline & & T225 & 0.09 & 2.22 & 199.9 & 811 & 119 \\
\hline & & T226 & 0.01 & 0.31 & 28.1 & 114 & 119 \\
\hline & & T227 & 0.02 & 0.40 & 35.6 & 145 & 119 \\
\hline & & T228 & 0.01 & 0.24 & 21.4 & 87 & 119 \\
\hline & 3rd Quarter '03 & T218 & 0.01 & 0.21 & 20.5 & 77 & 127 \\
\hline & & T219 & 0.01 & 0.26 & 24.8 & 93 & 127 \\
\hline & & T220 & 0.01 & 0.25 & 24.2 & 91 & 127 \\
\hline & & T221 & 0.01 & 0.30 & 28.9 & 109 & 127 \\
\hline & & T222 & 0.14 & 3.43 & 332.3 & 1250 & 127 \\
\hline & & T223 & 0.02 & 0.36 & 35.1 & 132 & 127 \\
\hline & & T224 & 0.01 & 0.23 & 21.9 & 83 & 127 \\
\hline & & T225 & 0.02 & 0.48 & 46.2 & 174 & 127 \\
\hline & & T226 & 0.01 & 0.23 & 22.7 & 86 & 127 \\
\hline & & T227 & 0.01 & 0.35 & 34.3 & 129 & 127 \\
\hline & & T228 & 0.01 & 0.23 & 22.0 & 83 & 127 \\
\hline
\end{tabular}


Table 4-2. 2003 Thermoluminescent Dosimeter Results. (cont)

\begin{tabular}{cccccccc} 
Location & Sample Period & EDP Code & mrem/hr & mrem/day & mrem/qtr & mrem/year & Days in Field \\
\hline 100-K Area & 4th Quarter '03 & T218 & 0.01 & 0.21 & 19.1 & 77 & 120 \\
& & T219 & 0.01 & 0.21 & 18.9 & 77 & 120 \\
& & T220 & 0.01 & 0.25 & 22.1 & 90 & 120 \\
& T221 & 0.01 & 0.27 & 24.0 & 98 & 120 \\
& T222 & 0.01 & 0.31 & 28.0 & 113 & 120 \\
& & T223 & 0.01 & 0.22 & 20.1 & 82 & 120 \\
& & T224 & 0.01 & 0.23 & 20.7 & 84 & 120 \\
& & T225 & 0.01 & 0.30 & 27.4 & 111 & 120 \\
& & T226 & 0.01 & 0.23 & 21.0 & 85 & 120 \\
& & T227 & 0.01 & 0.36 & 32.0 & 130 & 120 \\
& & T228 & 0.01 & 0.24 & 21.4 & 87 & 120
\end{tabular}

100-K Area, Annual Average \pm 2 Standard Deviation (2SD)

\begin{tabular}{ccccc} 
EDP Code & $\mathbf{m r e m} / \mathbf{h r} \pm \mathbf{2 S D}$ & $\mathbf{m r e m} / \mathbf{d a y} \pm \mathbf{2 S D}$ & $\mathbf{m r e m} / \mathbf{q t r} \pm \mathbf{2 S D}$ & $\mathbf{m r e m} / \mathbf{y r} \pm \mathbf{2 S D}$ \\
\hline T218 & $0.01 \pm 0.000$ & $0.21 \pm 0.01$ & $19.0 \pm 0.8$ & $76 \pm 3$ \\
T219 & $0.01 \pm 0.002$ & $0.23 \pm 0.04$ & $21.1 \pm 3.8$ & $84 \pm 15$ \\
T220 & $0.01 \pm 0.001$ & $0.25 \pm 0.02$ & $22.8 \pm 1.9$ & $91 \pm 8$ \\
T221 & $0.01 \pm 0.001$ & $0.29 \pm 0.03$ & $26.1 \pm 3.0$ & $104 \pm 12$ \\
T222 & $0.06 \pm 0.122$ & $1.43 \pm 2.92$ & $131 \pm 266$ & $523 \pm 1060$ \\
T223 & $0.01 \pm 0.006$ & $0.27 \pm 0.14$ & $24.2 \pm 12.6$ & $97 \pm 50$ \\
T224 & $0.01 \pm 0.001$ & $0.23 \pm 0.01$ & $21.0 \pm 1.3$ & $84 \pm 5$ \\
T225 & $0.05 \pm 0.075$ & $1.10 \pm 1.79$ & $101 \pm 163$ & $402 \pm 653$ \\
T226 & $0.01 \pm 0.003$ & $0.26 \pm 0.08$ & $24.1 \pm 7.0$ & $97 \pm 28$ \\
T227 & $0.02 \pm 0.002$ & $0.38 \pm 0.05$ & $34.2 \pm 4.5$ & $137 \pm 18$ \\
T228 & $0.01 \pm 0.000$ & $0.23 \pm 0.01$ & $21.3 \pm 0.9$ & $85 \pm 4$
\end{tabular}


Table 4-2. 2003 Thermoluminescent Dosimeter Results. (cont)

\begin{tabular}{|c|c|c|c|c|c|c|c|}
\hline Location & Sample Period & EDP Code & mrem/hr & mrem/day & mrem/qtr & mrem/year & Days in Field \\
\hline \multirow[t]{16}{*}{ CVDF (100-K Area) } & \multirow[t]{4}{*}{ 1st Quarter '03 } & T347 & 0.01 & 0.22 & 20.9 & 81 & 124 \\
\hline & & T348 & 0.01 & 0.21 & 20.2 & 78 & 124 \\
\hline & & T349 & 0.01 & 0.20 & 18.9 & 74 & 124 \\
\hline & & T350 & 0.01 & 0.20 & 19.2 & 75 & 124 \\
\hline & \multirow[t]{4}{*}{ 2nd Quarter '03 } & Т347 & 0.01 & 0.23 & 20.5 & 83 & 119 \\
\hline & & T348 & 0.01 & 0.23 & 20.8 & 85 & 119 \\
\hline & & T349 & 0.01 & 0.22 & 19.5 & 79 & 119 \\
\hline & & T350 & 0.01 & 0.22 & 20.1 & 81 & 119 \\
\hline & \multirow[t]{4}{*}{ 3rd Quarter '03 } & Т347 & 0.01 & 0.23 & 22.6 & 85 & 127 \\
\hline & & T348 & 0.01 & 0.23 & 22 & 83 & 127 \\
\hline & & Т349 & 0.01 & 0.21 & 20.3 & 76 & 127 \\
\hline & & T350 & 0.01 & 0.23 & 22 & 83 & 127 \\
\hline & \multirow[t]{4}{*}{ 4th Quarter '03 } & T347 & 0.01 & 0.22 & 19.9 & 81 & 120 \\
\hline & & T348 & 0.01 & 0.22 & 19.4 & 79 & 120 \\
\hline & & Т349 & 0.01 & 0.21 & 18.8 & 76 & 120 \\
\hline & & T350 & 0.01 & 0.21 & 18.8 & 76 & 120 \\
\hline \multicolumn{8}{|c|}{ CVDF, Annual Average \pm 2 Standard Deviation (2SD) } \\
\hline EDP Code & \multicolumn{2}{|c|}{ mrem/hr $\pm 2 \mathrm{SD}$} & \multicolumn{2}{|c|}{ mrem/day \pm 2 SD } & \multicolumn{2}{|c|}{ mrem/qtr $\pm 2 S D$} & $\mathrm{mrem} / \mathrm{yr} \pm 2 \mathrm{SD}$ \\
\hline T347 & \multicolumn{2}{|c|}{$0.01 \pm 0.001$} & \multicolumn{2}{|c|}{$0.23 \pm 0.01$} & \multicolumn{2}{|c|}{$20.6 \pm 1.1$} & $82 \pm 4$ \\
\hline T348 & \multicolumn{2}{|c|}{$0.01 \pm 0.001$} & \multicolumn{2}{|c|}{$0.22 \pm 0.02$} & \multicolumn{2}{|c|}{$20.3 \pm 1.5$} & $81 \pm 6$ \\
\hline Т349 & \multicolumn{2}{|c|}{$0.01 \pm 0.001$} & \multicolumn{2}{|c|}{$0.21 \pm 0.01$} & 19.0 & 1.2 & $76 \pm 5$ \\
\hline T350 & 0.01 & 0.001 & 0.22 & 0.02 & 19.7 & 2.0 & $79 \pm 8$ \\
\hline
\end{tabular}


Table 4-2. 2003 Thermoluminescent Dosimeter Results. (cont)

\begin{tabular}{|c|c|c|c|c|c|c|c|}
\hline Location & Sample Period & EDP Code & $\mathrm{mrem} / \mathrm{hr}$ & mrem/day & mrem/qtr & mrem/year & Days in Field \\
\hline \multirow[t]{20}{*}{ 100-KR-1 RA } & 1st Quarter '03 & T364 & 0.01 & 0.25 & 22.9 & 90 & 123 \\
\hline & & T365 & 0.01 & 0.23 & 21.1 & 83 & 123 \\
\hline & & T366 & 0.01 & 0.26 & 24.2 & 95 & 123 \\
\hline & & T367 & 0.01 & 0.25 & 23.6 & 93 & 123 \\
\hline & & T368 & 0.01 & 0.27 & 24.7 & 97 & 123 \\
\hline & 2nd Quarter '03 & T364 & 0.01 & 0.25 & 22.7 & 92 & 119 \\
\hline & & T365 & 0.01 & 0.22 & 20.0 & 81 & 119 \\
\hline & & T366 & 0.01 & 0.28 & 25.0 & 101 & 119 \\
\hline & & T367 & 0.01 & 0.27 & 24.7 & 100 & 119 \\
\hline & & T368 & 0.01 & 0.28 & 25.5 & 104 & 119 \\
\hline & 3rd Quarter '03 & T364 & 0.01 & 0.26 & 25.5 & 96 & 127 \\
\hline & & T365 & 0.01 & 0.23 & 22.0 & 83 & 127 \\
\hline & & T366 & 0.01 & 0.27 & 26.4 & 99 & 127 \\
\hline & & T367 & 0.01 & 0.26 & 25.0 & 94 & 127 \\
\hline & & T368 & 0.01 & 0.28 & 26.9 & 101 & 127 \\
\hline & 4th Quarter '03 & T364 & 0.01 & 0.25 & 22.4 & 91 & 120 \\
\hline & & T365 & 0.01 & 0.23 & 20.9 & 85 & 120 \\
\hline & & T366 & 0.01 & 0.27 & 24.6 & 100 & 120 \\
\hline & & T367 & 0.01 & 0.28 & 24.8 & 101 & 120 \\
\hline & & T368 & 0.01 & 0.30 & 27.2 & 110 & 120 \\
\hline
\end{tabular}

100-KR-1, Annual Average \pm 2 Standard Deviation (2SD)

\begin{tabular}{crrrr} 
EDP Code & mrem/hr \pm 2SD & mrem/day \pm 2SD & mrem/qtr \pm 2SD & mrem/yr \pm 2SD \\
\hline T364 & $0.01 \pm 0.001$ & $0.25 \pm 0.01$ & $23.1 \pm 1.4$ & $92 \pm 5$ \\
T365 & $0.01 \pm 0.000$ & $0.23 \pm 0.01$ & $20.7 \pm 0.7$ & $83 \pm 3$ \\
T366 & $0.01 \pm 0.001$ & $0.27 \pm 0.02$ & $24.7 \pm 1.4$ & $99 \pm 5$ \\
T367 & $0.01 \pm 0.001$ & $0.27 \pm 0.02$ & $24.2 \pm 2.1$ & $97 \pm 8$ \\
T368 & $0.01 \pm 0.001$ & $0.28 \pm 0.03$ & $25.7 \pm 2.8$ & $103 \pm 11$
\end{tabular}


Table 4-2. 2003 Thermoluminescent Dosimeter Results. (cont)

\begin{tabular}{|c|c|c|c|c|c|c|c|}
\hline Location & Sample Period & EDP Code & mrem/hr & mrem/day & mrem/qtr & mrem/year & Days in Field \\
\hline \multirow[t]{42}{*}{ 100-N Area } & 1st Quarter '03 & T229 & 0.02 & 0.44 & 41.0 & 161 & 123 \\
\hline & & $\mathrm{T} 231$ & 0.02 & 0.45 & 41.7 & 164 & 123 \\
\hline & & $\mathrm{T} 234$ & 0.01 & 0.30 & 27.9 & 110 & 123 \\
\hline & & T235 & 0.02 & 0.57 & 53.4 & 210 & 123 \\
\hline & & $\mathrm{T} 240$ & 0.02 & 0.45 & 42.0 & 165 & 123 \\
\hline & & T243 & 0.01 & 0.22 & 20.1 & 79 & 123 \\
\hline & & $\mathrm{T} 245$ & 0.02 & 0.53 & 49.3 & 194 & 123 \\
\hline & & $\mathrm{T} 246$ & 0.01 & 0.28 & 25.7 & 101 & 123 \\
\hline & & $\mathrm{T} 248$ & 0.03 & 0.79 & 73.6 & 289 & 123 \\
\hline & & T249 & 0.11 & 2.67 & 248 & 973 & 123 \\
\hline & & $\mathrm{T} 250$ & 0.01 & 0.30 & 27.9 & 110 & 123 \\
\hline & & T251 & 0.01 & 0.29 & 27.1 & 107 & 123 \\
\hline & & T253 & 0.07 & 1.60 & 149.2 & 586 & 123 \\
\hline & & T254 & 0.04 & 0.91 & 84.6 & 332 & 123 \\
\hline & 2nd Quarter '03 & T229 & 0.02 & 0.51 & 46.8 & 188 & 120 \\
\hline & & T231 & 0.02 & 0.56 & 51.1 & 205 & 120 \\
\hline & & T234 & 0.01 & 0.31 & 28.5 & 114 & 120 \\
\hline & & T235 & 0.02 & 0.55 & 50.5 & 203 & 120 \\
\hline & & $\mathrm{T} 240$ & 0.02 & 0.44 & 39.9 & 160 & 120 \\
\hline & & T243 & 0.01 & 0.22 & 20.4 & 82 & 120 \\
\hline & & $\mathrm{T} 245$ & 0.03 & 0.67 & 61.0 & 245 & 120 \\
\hline & & T246 & 0.01 & 0.31 & 28.2 & 113 & 120 \\
\hline & & T248 & 0.03 & 0.80 & 72.8 & 292 & 120 \\
\hline & & T249 & 0.12 & 2.87 & 260.9 & 1047 & 120 \\
\hline & & T250 & 0.01 & 0.32 & 28.9 & 116 & 120 \\
\hline & & T251 & 0.01 & 0.31 & 28.5 & 114 & 120 \\
\hline & & T253 & 0.07 & 1.66 & 151.3 & 607 & 120 \\
\hline & & T254 & 0.04 & 0.92 & 83.6 & 335 & 120 \\
\hline & 3rd Quarter '03 & T229 & 0.02 & 0.53 & 50.4 & 192 & 126 \\
\hline & & T231 & 0.02 & 0.58 & 55.4 & 211 & 126 \\
\hline & & T234 & 0.01 & 0.34 & 32.6 & 124 & 126 \\
\hline & & T235 & 0.02 & 0.54 & 51.4 & 196 & 126 \\
\hline & & T240 & 0.02 & 0.44 & 42.4 & 161 & 126 \\
\hline & & $\mathrm{T} 243$ & 0.01 & 0.21 & 20.5 & 78 & 126 \\
\hline & & $\mathrm{T} 245$ & 0.03 & 0.67 & 64.8 & 246 & 126 \\
\hline & & T246 & 0.01 & 0.31 & 30.1 & 115 & 126 \\
\hline & & T248 & 0.03 & 0.77 & 74.2 & 282 & 126 \\
\hline & & T249 & 0.11 & 2.69 & 257.9 & 981 & 126 \\
\hline & & T250 & 0.01 & 0.32 & 30.4 & 116 & 126 \\
\hline & & T251 & 0.01 & 0.33 & 31.8 & 121 & 126 \\
\hline & & T253 & 0.07 & 1.68 & 161.5 & 614 & 126 \\
\hline & & T254 & 0.04 & 0.90 & 86.8 & 330 & 126 \\
\hline
\end{tabular}


Table 4-2. 2003 Thermoluminescent Dosimeter Results. (cont)

\begin{tabular}{|c|c|c|c|c|c|c|c|}
\hline Location & Sample Period & EDP Code & mrem/hr & mrem/day & mrem/qtr & mrem/year & Days in Field \\
\hline \multirow[t]{14}{*}{ 100-N Area } & \multirow[t]{14}{*}{ 4th Quarter '03 } & T229 & 0.02 & 0.58 & 52.4 & 210 & 121 \\
\hline & & $\mathrm{T} 231$ & 0.02 & 0.59 & 53.3 & 214 & 121 \\
\hline & & $\mathrm{T} 234$ & 0.01 & 0.30 & 27.3 & 110 & 121 \\
\hline & & $\mathrm{T} 235$ & 0.02 & 0.55 & 49.7 & 199 & 121 \\
\hline & & $\mathrm{T} 240$ & 0.02 & 0.43 & 38.9 & 156 & 121 \\
\hline & & $\mathrm{T} 243$ & 0.01 & 0.23 & 21.2 & 85 & 121 \\
\hline & & $\mathrm{T} 245$ & 0.03 & 0.67 & 61.4 & 246 & 121 \\
\hline & & T246 & 0.01 & 0.29 & 26.0 & 104 & 121 \\
\hline & & $\mathrm{T} 248$ & 0.03 & 0.73 & 66.3 & 266 & 121 \\
\hline & & T249 & 0.11 & 2.67 & 242.8 & 973 & 121 \\
\hline & & $\mathrm{T} 250$ & 0.01 & 0.30 & 27.6 & 111 & 121 \\
\hline & & $\mathrm{T} 251$ & 0.01 & 0.32 & 29.1 & 117 & 121 \\
\hline & & $\mathrm{T} 253$ & 0.04 & 0.96 & 86.9 & 349 & 121 \\
\hline & & $\mathrm{T} 254$ & 0.04 & 0.89 & 80.7 & 324 & 121 \\
\hline \multicolumn{8}{|c|}{ 100-N Area, Annual Average \pm 2 Standard Deviation (2SD) } \\
\hline EDP Code & \multicolumn{2}{|c|}{$\mathrm{mrem} / \mathrm{hr} \pm 2 \mathrm{SD}$} & \multicolumn{2}{|c|}{$\mathrm{mrem} / \mathrm{day} \pm 2 \mathrm{SD}$} & \multicolumn{2}{|c|}{$\mathrm{mrem} / \mathrm{qtr} \pm 2 \mathrm{SD}$} & mrem/yr $\pm 2 S D$ \\
\hline T229 & \multicolumn{2}{|c|}{$0.02 \pm 0.005$} & \multicolumn{2}{|c|}{$0.51 \pm 0.11$} & \multicolumn{2}{|c|}{$46.9 \pm 10.2$} & $187 \pm 41$ \\
\hline $\mathrm{T} 231$ & \multicolumn{2}{|c|}{$0.02 \pm 0.005$} & \multicolumn{2}{|c|}{$0.54 \pm 0.13$} & \multicolumn{2}{|c|}{$49.6 \pm 11.7$} & $198 \pm 47$ \\
\hline $\mathrm{T} 234$ & \multicolumn{2}{|c|}{$0.01 \pm 0.002$} & \multicolumn{2}{|c|}{$0.31 \pm 0.04$} & \multicolumn{2}{|c|}{$28.6 \pm 3.4$} & $114 \pm 13$ \\
\hline $\mathrm{T} 235$ & 0.02 & 0.001 & 0.55 & 0.03 & 50.4 & 3.0 & $202 \pm 12$ \\
\hline $\mathrm{T} 240$ & 0.02 & 0.001 & 0.44 & 0.02 & 40.1 & 1.8 & $161 \pm 7$ \\
\hline $\mathrm{T} 243$ & 0.01 & 0.001 & 0.22 & 0.02 & 20.2 & 1.5 & $81 \pm 6$ \\
\hline $\mathrm{T} 245$ & 0.03 & 0.006 & 0.64 & 0.14 & 58.2 & 13.1 & $233 \pm 52$ \\
\hline T246 & 0.01 & 0.002 & 0.30 & 0.04 & 27.1 & 3.4 & $108 \pm 13$ \\
\hline T248 & 0.03 & 0.003 & 0.77 & 0.06 & 70.6 & 5.8 & $282 \pm 23$ \\
\hline T249 & 0.11 & 0.008 & 2.72 & 0.20 & 248 & 17.9 & $993 \pm 71$ \\
\hline $\mathrm{T} 250$ & 0.01 & 0.001 & 0.31 & 0.02 & 28.3 & 1.7 & $113 \pm 7$ \\
\hline $\mathrm{T} 251$ & 0.01 & 0.001 & 0.31 & 0.03 & 28.7 & & $115 \pm 12$ \\
\hline T253 & 0.06 & 0.029 & 1.48 & 0.70 & 135 & 64 & $540 \pm 255$ \\
\hline $\mathrm{T} 254$ & 0.04 & 0.001 & 0.90 & 0.03 & 82.6 & & $330 \pm 10$ \\
\hline
\end{tabular}


Table 4-2. 2003 Thermoluminescent Dosimeter Results. (cont)

\begin{tabular}{|c|c|c|c|c|c|c|c|}
\hline Location & Sample Period & EDP Code & mrem/hr & mrem/day & mrem/qtr & mrem/year & Days in Field \\
\hline \multirow[t]{42}{*}{200 East Area } & 1st Quarter '03 & T259 & 0.01 & 0.24 & 21.7 & 87 & 121 \\
\hline & & T260 & 0.01 & 0.23 & 20.6 & 83 & 121 \\
\hline & & T261 & 0.01 & 0.23 & 21.3 & 84 & 122 \\
\hline & & T262 & 0.01 & 0.24 & 21.7 & 86 & 122 \\
\hline & & T263 & 0.01 & 0.22 & 20.5 & 81 & 122 \\
\hline & & T264 & 0.01 & 0.30 & 27.5 & 110 & 121 \\
\hline & & T265 & 0.01 & 0.30 & 26.9 & 108 & 121 \\
\hline & & T266 & 0.01 & 0.23 & 21.1 & 85 & 121 \\
\hline & & T267 & 0.01 & 0.25 & 22.6 & 91 & 121 \\
\hline & & T268 & 0.01 & 0.32 & 29.0 & 116 & 121 \\
\hline & & T269 & 0.01 & 0.24 & 21.9 & 88 & 121 \\
\hline & & $\mathrm{T} 270$ & 0.01 & 0.35 & 31.8 & 127 & 121 \\
\hline & & $\mathrm{T} 271$ & 0.01 & 0.26 & 23.4 & 94 & 121 \\
\hline & & T272 & 0.01 & 0.29 & 26.1 & 105 & 121 \\
\hline & & $\mathrm{T} 273$ & 0.01 & 0.22 & 20.2 & 81 & 121 \\
\hline & & T274 & 0.01 & 0.22 & 20.3 & 82 & 121 \\
\hline & & T275 & 0.01 & 0.24 & 21.7 & 87 & 121 \\
\hline & & T276 & 0.01 & 0.22 & 20.0 & 80 & 121 \\
\hline & & T277 & 0.01 & 0.25 & 22.7 & 91 & 121 \\
\hline & & T278 & 0.01 & 0.24 & 22.0 & 88 & 121 \\
\hline & & T279 & 0.01 & 0.23 & 20.6 & 83 & 121 \\
\hline & & $\mathrm{T} 280$ & 0.01 & 0.23 & 21.3 & 85 & 121 \\
\hline & & $\mathrm{T} 281$ & 0.01 & 0.23 & 21.3 & 85 & 121 \\
\hline & & $\mathrm{T} 282$ & 0.01 & 0.22 & 20.2 & 81 & 121 \\
\hline & & $\mathrm{T} 283$ & 0.01 & 0.23 & 20.6 & 83 & 121 \\
\hline & & $\mathrm{T} 284$ & 0.01 & 0.29 & 26.5 & 106 & 121 \\
\hline & & $\mathrm{T} 285$ & 0.02 & 0.56 & 51.8 & 205 & 122 \\
\hline & & T286 & 0.02 & 0.39 & 35.4 & 142 & 121 \\
\hline & & $\mathrm{T} 287$ & 0.02 & 0.38 & 34.7 & 138 & 122 \\
\hline & & T288 & 0.02 & 0.38 & 35.2 & 140 & 122 \\
\hline & & T289 & 0.02 & 0.40 & 36.6 & 147 & 121 \\
\hline & & Т290 & 0.01 & 0.30 & 27.6 & 110 & 122 \\
\hline & & Т291 & 0.02 & 0.46 & 41.9 & 166 & 122 \\
\hline & & T292 & 0.06 & 1.43 & 131.5 & 522 & 122 \\
\hline & & T293 & 0.02 & 0.36 & 33.3 & 132 & 122 \\
\hline & & T294 & 0.03 & 0.67 & 61.3 & 243 & 122 \\
\hline & & T295 & 0.02 & 0.54 & 49.6 & 197 & 122 \\
\hline & & T296 & 0.01 & 0.25 & 22.7 & 91 & 121 \\
\hline & & T297 & 0.01 & 0.24 & 22.0 & 88 & 121 \\
\hline & & T298 & 0.01 & 0.22 & 20.3 & 82 & 121 \\
\hline & & Т299 & 0.01 & 0.25 & 22.9 & 92 & 121 \\
\hline & & Т300 & 0.01 & 0.27 & 24.1 & 97 & 121 \\
\hline
\end{tabular}


Table 4-2. 2003 Thermoluminescent Dosimeter Results. (cont)

\begin{tabular}{|c|c|c|c|c|c|c|c|}
\hline Location & Sample Period & EDP Code & mrem/hr & mrem/day & mrem/qtr & mrem/year & Days in Field \\
\hline \multirow[t]{42}{*}{200 East Area } & 2nd Quarter '03 & T259 & 0.01 & 0.23 & 22.1 & 84 & 125 \\
\hline & & Т260 & 0.01 & 0.22 & 21.3 & 81 & 125 \\
\hline & & T261 & 0.01 & 0.23 & 21.8 & 84 & 124 \\
\hline & & T262 & 0.01 & 0.23 & 21.5 & 82 & 124 \\
\hline & & Т263 & 0.01 & 0.23 & 22.0 & 84 & 124 \\
\hline & & Т264 & 0.01 & 0.30 & 28.8 & 110 & 125 \\
\hline & & T265 & 0.01 & 0.29 & 27.6 & 105 & 125 \\
\hline & & T266 & 0.01 & 0.24 & 22.7 & 87 & 125 \\
\hline & & T267 & 0.01 & 0.25 & 23.6 & 90 & 125 \\
\hline & & T268 & 0.01 & 0.31 & 29.5 & 112 & 125 \\
\hline & & T269 & 0.01 & 0.24 & 23.1 & 88 & 125 \\
\hline & & $\mathrm{T} 270$ & 0.02 & 0.38 & 36.1 & 137 & 125 \\
\hline & & $\mathrm{T} 271$ & 0.01 & 0.25 & 24.2 & 92 & 125 \\
\hline & & $\mathrm{T} 272$ & 0.01 & 0.28 & 26.9 & 102 & 125 \\
\hline & & $\mathrm{T} 273$ & 0.01 & 0.23 & 22.0 & 84 & 125 \\
\hline & & $\mathrm{T} 274$ & 0.01 & 0.23 & 21.8 & 83 & 125 \\
\hline & & $\mathrm{T} 275$ & 0.01 & 0.22 & 21.4 & 81 & 125 \\
\hline & & $\mathrm{T} 276$ & 0.01 & 0.21 & 20.1 & 77 & 125 \\
\hline & & $\mathrm{T} 277$ & 0.01 & 0.25 & 23.6 & 90 & 125 \\
\hline & & $\mathrm{T} 278$ & 0.01 & 0.22 & 21.5 & 82 & 125 \\
\hline & & $\mathrm{T} 279$ & 0.01 & 0.21 & 20.4 & 77 & 125 \\
\hline & & $\mathrm{T} 280$ & 0.01 & 0.23 & 22.0 & 84 & 125 \\
\hline & & $\mathrm{T} 281$ & 0.01 & 0.23 & 22.1 & 84 & 125 \\
\hline & & T282 & 0.01 & 0.23 & 21.6 & 82 & 125 \\
\hline & & Т283 & 0.01 & 0.22 & 21.5 & 82 & 125 \\
\hline & & T284 & 0.01 & 0.28 & 26.8 & 102 & 125 \\
\hline & & T285 & 0.02 & 0.53 & 50.1 & 193 & 124 \\
\hline & & T286 & 0.02 & 0.42 & 40.0 & 152 & 125 \\
\hline & & Т287 & 0.02 & 0.44 & 41.8 & 161 & 124 \\
\hline & & $\mathrm{T} 288$ & 0.02 & 0.46 & 43.9 & 169 & 124 \\
\hline & & T289 & 0.02 & 0.36 & 34.9 & 133 & 125 \\
\hline & & T290 & 0.01 & 0.30 & 28.3 & 109 & 124 \\
\hline & & T291 & 0.02 & 0.45 & 42.8 & 165 & 124 \\
\hline & & T292 & 0.07 & 1.59 & 150.6 & 579 & 124 \\
\hline & & T293 & 0.01 & 0.35 & 33.3 & 128 & 124 \\
\hline & & T294 & 0.04 & 0.88 & 83.2 & 320 & 124 \\
\hline & & T295 & 0.01 & 0.23 & 21.9 & 84 & 124 \\
\hline & & Т296 & 0.01 & 0.25 & 23.5 & 90 & 125 \\
\hline & & T297 & 0.01 & 0.23 & 21.9 & 83 & 125 \\
\hline & & T298 & 0.01 & 0.22 & 21.1 & 80 & 125 \\
\hline & & T299 & 0.01 & 0.24 & 23.2 & 88 & 125 \\
\hline & & T300 & 0.01 & 0.24 & 23.4 & 89 & 125 \\
\hline
\end{tabular}


Table 4-2. 2003 Thermoluminescent Dosimeter Results. (cont)

\begin{tabular}{|c|c|c|c|c|c|c|c|}
\hline Location & Sample Period & EDP Code & mrem/hr & mrem/day & mrem/qtr & mrem/year & Days in Field \\
\hline \multirow{42}{*}{200 East Area } & 3rd Quarter '03 & T259 & 0.01 & 0.24 & 21.8 & 88 & 121 \\
\hline & & T260 & 0.01 & 0.23 & 20.5 & 82 & 121 \\
\hline & & T261 & 0.01 & 0.25 & 22.9 & 91 & 122 \\
\hline & & T262 & 0.01 & 0.24 & 21.7 & 86 & 122 \\
\hline & & $\mathrm{T} 263$ & 0.01 & 0.24 & 21.8 & 87 & 122 \\
\hline & & T264 & 0.01 & 0.31 & 28.7 & 114 & 122 \\
\hline & & T265 & 0.01 & 0.35 & 31.9 & 126 & 122 \\
\hline & & T266 & 0.01 & 0.24 & 22.5 & 89 & 122 \\
\hline & & $\mathrm{T} 267$ & 0.01 & 0.26 & 23.5 & 93 & 122 \\
\hline & & T268 & 0.01 & 0.33 & 30.4 & 121 & 122 \\
\hline & & T269 & 0.01 & 0.27 & 25.1 & 100 & 122 \\
\hline & & $\mathrm{T} 270$ & 0.02 & 0.40 & 37.1 & 147 & 122 \\
\hline & & $\mathrm{T} 271$ & 0.01 & 0.29 & 26.3 & 104 & 122 \\
\hline & & $\mathrm{T} 272$ & 0.01 & 0.30 & 27.6 & 109 & 122 \\
\hline & & T273 & 0.01 & 0.23 & 21.5 & 85 & 122 \\
\hline & & $\mathrm{T} 274$ & 0.01 & 0.25 & 22.6 & 90 & 122 \\
\hline & & $\mathrm{T} 275$ & 0.01 & 0.25 & 22.9 & 91 & 122 \\
\hline & & $\mathrm{T} 276$ & 0.01 & 0.23 & 20.9 & 83 & 122 \\
\hline & & T277 & 0.01 & 0.26 & 24.1 & 96 & 122 \\
\hline & & $\mathrm{T} 278$ & 0.01 & 0.23 & 20.9 & 83 & 122 \\
\hline & & T279 & 0.01 & 0.24 & 21.7 & 86 & 122 \\
\hline & & T280 & 0.01 & 0.24 & 22.3 & 88 & 122 \\
\hline & & T281 & 0.01 & 0.26 & 23.7 & 94 & 122 \\
\hline & & T282 & 0.01 & 0.23 & 21.0 & 83 & 122 \\
\hline & & T283 & 0.01 & 0.25 & 23.4 & 93 & 122 \\
\hline & & T284 & 0.01 & 0.30 & 28.0 & 111 & 122 \\
\hline & & T285 & 0.02 & 0.58 & 53.6 & 213 & 122 \\
\hline & & T286 & 0.02 & 0.37 & 33.8 & 134 & 122 \\
\hline & & T287 & 0.02 & 0.59 & 54.6 & 217 & 122 \\
\hline & & T288 & 0.02 & 0.59 & 54.2 & 215 & 122 \\
\hline & & T289 & 0.02 & 0.37 & 34.4 & 136 & 122 \\
\hline & & T290 & 0.01 & 0.33 & 30.6 & 121 & 122 \\
\hline & & T291 & 0.02 & 0.50 & 45.9 & 182 & 122 \\
\hline & & T292 & 0.06 & 1.38 & 126.9 & 504 & 122 \\
\hline & & T293 & 0.02 & 0.51 & 46.7 & 185 & 122 \\
\hline & & T294 & 0.03 & 0.62 & 56.7 & 225 & 122 \\
\hline & & T295 & 0.01 & 0.25 & 23.0 & 91 & 122 \\
\hline & & T296 & 0.01 & 0.26 & 24.0 & 95 & 122 \\
\hline & & T297 & 0.01 & 0.23 & 21.5 & 85 & 122 \\
\hline & & T298 & 0.01 & 0.22 & 19.9 & 79 & 122 \\
\hline & & T299 & 0.01 & 0.26 & 24.0 & 95 & 122 \\
\hline & & Т300 & 0.01 & 0.26 & 24.2 & 96 & 122 \\
\hline
\end{tabular}


Table 4-2. 2003 Thermoluminescent Dosimeter Results. (cont)

\begin{tabular}{|c|c|c|c|c|c|c|c|}
\hline Location & Sample Period & EDP Code & mrem/hr & mrem/day & mrem/qtr & mrem/year & Days in Field \\
\hline \multirow[t]{42}{*}{200 East Area } & 4th Quarter '03 & T259 & 0.01 & 0.24 & 21.7 & 87 & 121 \\
\hline & & Т260 & 0.01 & 0.22 & 19.8 & 80 & 121 \\
\hline & & T261 & 0.01 & 0.24 & 22.1 & 89 & 121 \\
\hline & & T262 & 0.01 & 0.22 & 20.2 & 81 & 121 \\
\hline & & Т263 & 0.01 & 0.23 & 21.0 & 84 & 121 \\
\hline & & Т264 & 0.01 & 0.29 & 26.5 & 108 & 120 \\
\hline & & T265 & 0.01 & 0.30 & 26.6 & 108 & 120 \\
\hline & & T266 & 0.01 & 0.23 & 21.1 & 86 & 120 \\
\hline & & T267 & 0.01 & 0.26 & 23.5 & 95 & 120 \\
\hline & & T268 & 0.01 & 0.32 & 28.8 & 117 & 120 \\
\hline & & T269 & 0.01 & 0.26 & 23.9 & 96 & 121 \\
\hline & & $\mathrm{T} 270$ & 0.02 & 0.38 & 34.4 & 139 & 120 \\
\hline & & $\mathrm{T} 271$ & 0.01 & 0.25 & 22.9 & 93 & 120 \\
\hline & & $\mathrm{T} 272$ & 0.01 & 0.28 & 25.4 & 102 & 121 \\
\hline & & $\mathrm{T} 273$ & 0.01 & 0.22 & 19.9 & 81 & 120 \\
\hline & & $\mathrm{T} 274$ & 0.01 & 0.23 & 20.6 & 84 & 120 \\
\hline & & $\mathrm{T} 275$ & 0.01 & 0.24 & 21.5 & 87 & 120 \\
\hline & & $\mathrm{T} 276$ & 0.01 & 0.23 & 20.8 & 84 & 120 \\
\hline & & $\mathrm{T} 277$ & 0.01 & 0.24 & 21.6 & 88 & 120 \\
\hline & & $\mathrm{T} 278$ & 0.01 & 0.23 & 20.7 & 83 & 121 \\
\hline & & $\mathrm{T} 279$ & 0.01 & 0.22 & 20.5 & 82 & 121 \\
\hline & & $\mathrm{T} 280$ & 0.01 & 0.23 & 20.8 & 83 & 121 \\
\hline & & $\mathrm{T} 281$ & 0.01 & 0.24 & 21.9 & 88 & 121 \\
\hline & & T282 & 0.01 & 0.24 & 22.0 & 88 & 121 \\
\hline & & Т283 & 0.01 & 0.26 & 23.4 & 94 & 121 \\
\hline & & T284 & 0.01 & 0.29 & 26.2 & 105 & 121 \\
\hline & & T285 & 0.02 & 0.55 & 49.7 & 199 & 121 \\
\hline & & T286 & 0.02 & 0.38 & 34.4 & 138 & 121 \\
\hline & & Т287 & 0.02 & 0.41 & 37.6 & 151 & 121 \\
\hline & & $\mathrm{T} 288$ & 0.03 & 0.78 & 71.1 & 285 & 121 \\
\hline & & T289 & 0.01 & 0.33 & 30.4 & 122 & 121 \\
\hline & & T290 & 0.02 & 0.40 & 36.8 & 148 & 121 \\
\hline & & T291 & 0.02 & 0.44 & 40.3 & 162 & 121 \\
\hline & & T292 & 0.04 & 0.87 & 79.6 & 319 & 121 \\
\hline & & T293 & 0.01 & 0.32 & 29.5 & 118 & 121 \\
\hline & & T294 & 0.02 & 0.50 & 45.5 & 182 & 121 \\
\hline & & T295 & 0.01 & 0.22 & 20.1 & 81 & 121 \\
\hline & & Т296 & 0.01 & 0.24 & 22.1 & 89 & 121 \\
\hline & & T297 & 0.01 & 0.23 & 20.8 & 83 & 121 \\
\hline & & T298 & 0.01 & 0.22 & 20.2 & 82 & 120 \\
\hline & & T299 & 0.01 & 0.23 & 20.3 & 82 & 120 \\
\hline & & T300 & 0.01 & 0.24 & 22.3 & 89 & 121 \\
\hline
\end{tabular}


Table 4-2. 2003 Thermoluminescent Dosimeter Results. (cont)

200 East Area, Annual Average \pm 2 Standard Deviation (2SD)

EDP Code

T259

$\mathrm{T} 260$

$\mathrm{T} 261$

T262

T263

T264

T265

T266

$\mathrm{T} 267$

T268

T269

$\mathrm{T} 270$

T271

T272

$\mathrm{T} 273$

T274

T275

T276

T277

T278

$\mathrm{T} 279$

T280

T281

T282

T283

T284

$\mathrm{T} 285$

T286

T287

T288

T289

T290

T291

T292

T293

T294

T295

T296

T297

T298

T299

Т300 $\mathrm{mrem} / \mathrm{hr} \pm$ 2SD

$0.01 \pm 0.000$

$0.01 \pm 0.000$

$0.01 \pm 0.001$

$0.01 \pm 0.001$

$0.01 \pm 0.001$

$0.01 \pm 0.001$

$0.01 \pm 0.002$

$0.01 \pm 0.000$

$0.01 \pm 0.001$

$0.01 \pm 0.001$

$0.01 \pm 0.001$

$0.02 \pm 0.002$

$0.01 \pm 0.001$

$0.01 \pm 0.001$

$0.01 \pm 0.001$

$0.01 \pm 0.001$

$0.01 \pm 0.001$

$0.01 \pm 0.001$

$0.01 \pm 0.001$

$0.01 \pm 0.001$

$0.01 \pm 0.001$

$0.01 \pm 0.001$

$0.01 \pm 0.001$

$0.01 \pm 0.001$

$0.01 \pm 0.001$

$0.01 \pm 0.001$

$0.02 \pm 0.002$

$0.02 \pm 0.002$

$0.02 \pm 0.008$

$0.02 \pm 0.015$

$0.02 \pm 0.002$

$0.01 \pm 0.004$

$0.02 \pm 0.002$

$0.06 \pm 0.026$

$0.02 \pm 0.007$

$0.03 \pm 0.013$

$0.01 \pm 0.013$

$0.01 \pm 0.001$

$0.01 \pm 0.001$

$0.01 \pm 0.000$

$0.01 \pm 0.001$

$0.01 \pm 0.001$ mrem/day \pm 2SD

$0.24 \pm 0.01$

$0.22 \pm 0.01$

$0.24 \pm 0.02$

$0.23 \pm 0.01$

$0.23 \pm 0.01$

$0.30 \pm 0.01$

$0.31 \pm 0.05$

$0.24 \pm 0.01$

$0.25 \pm 0.01$

$0.32 \pm 0.02$

$0.25 \pm 0.03$

$0.38 \pm 0.04$

$0.26 \pm 0.03$

$0.29 \pm 0.02$

$0.23 \pm 0.01$

$0.23 \pm 0.02$

$0.24 \pm 0.02$

$0.22 \pm 0.02$

$0.25 \pm 0.02$

$0.23 \pm 0.02$

$0.22 \pm 0.02$

$0.23 \pm 0.01$

$0.24 \pm 0.02$

$0.23 \pm 0.02$

$0.24 \pm 0.03$

$0.29 \pm 0.02$

$0.55 \pm 0.05$

$0.39 \pm 0.04$

$0.46 \pm 0.19$

$0.55 \pm 0.35$

$0.37 \pm 0.06$

$0.33 \pm 0.10$

$0.46 \pm 0.05$

$1.32 \pm 0.62$

$0.39 \pm 0.16$

$0.67 \pm 0.31$

$0.31 \pm 0.31$

$0.25 \pm 0.02$

$0.23 \pm 0.01$

$0.22 \pm 0.01$

$0.25 \pm 0.03$

$0.25 \pm 0.02$ mrem/qtr \pm 2SD

$21.6 \pm 0.8$

$20.3 \pm 0.7$

$21.7 \pm 1.7$

$21.0 \pm 1.3$

$21.0 \pm 1.1$

$27.6 \pm 1.3$

$27.9 \pm 4.9$

$21.6 \pm 1.0$

$23.0 \pm 1.2$

$29.1 \pm 1.7$

$23.2 \pm 3.0$

$34.4 \pm 4.1$

$23.9 \pm 2.9$

$26.2 \pm 1.7$

$20.7 \pm 1.1$

$21.1 \pm 1.8$

$21.6 \pm 1.9$

$20.2 \pm 1.7$

$22.8 \pm 1.6$

$21.0 \pm 1.5$

$20.5 \pm 1.8$

$21.3 \pm 1.2$

$21.9 \pm 2.2$

$20.9 \pm 1.6$

$21.9 \pm 3.2$

$26.5 \pm 1.9$

$50.6 \pm 4.3$

$35.4 \pm 3.8$

$41.6 \pm 17.4$

$50.4 \pm 31.7$

$33.6 \pm 5.2$

$30.4 \pm 9.0$

$42.2 \pm 4.6$

$121 \pm 56$

$35.2 \pm 15$

$60.8 \pm 28.7$

$28.3 \pm 27.9$

$22.8 \pm 1.4$

$21.2 \pm 1.2$

$20.2 \pm 0.6$

$22.4 \pm 2.8$

$23.2 \pm 2.1$ mrem/yr \pm 2SD

$86 \pm 3$

$81 \pm 3$

$87 \pm 7$

$84 \pm 5$

$84 \pm 4$

$110 \pm 5$

$112 \pm 20$

$86 \pm 4$

$92 \pm 5$

$116 \pm 7$

$93 \pm 12$

$138 \pm 16$

$96 \pm 12$

$105 \pm 7$

$83 \pm 4$

$84 \pm 7$

$86 \pm 8$

$81 \pm 7$

$91 \pm 6$

$84 \pm 6$

$82 \pm 7$

$85 \pm 5$

$88 \pm 9$

$84 \pm 6$

$88 \pm 13$

$106 \pm 8$

$202 \pm 17$

$142 \pm 15$

$166 \pm 69$

$202 \pm 127$

$134 \pm 21$

$122 \pm 36$

$169 \pm 18$

$482 \pm 225$

$141 \pm 60$

$243 \pm 115$

$113 \pm 112$

$91 \pm 6$

$85 \pm 5$

$81 \pm 2$

$89 \pm 11$

$93 \pm 8$ 
Table 4-2. 2003 Thermoluminescent Dosimeter Results. (cont)

\begin{tabular}{|c|c|c|c|c|c|c|c|}
\hline Location & Sample Period & EDP Code & mrem/hr & mrem/day & mrem/qtr & mrem/year & Days in Field \\
\hline \multirow[t]{48}{*}{200 West Area } & 1st Quarter '03 & T302 & 0.01 & 0.25 & 22.9 & 92 & 121 \\
\hline & & T303 & 0.01 & 0.31 & 28.6 & 115 & 121 \\
\hline & & T304 & 0.02 & 0.36 & 33.2 & 133 & 121 \\
\hline & & T305 & 0.01 & 0.24 & 22.1 & 89 & 121 \\
\hline & & T306 & 0.01 & 0.30 & 27.2 & 109 & 121 \\
\hline & & T307 & 0.01 & 0.30 & 27.0 & 109 & 121 \\
\hline & & T308 & 0.01 & 0.26 & 23.9 & 96 & 121 \\
\hline & & T309 & 0.01 & 0.24 & 21.4 & 86 & 121 \\
\hline & & T310 & 0.01 & 0.29 & 26.5 & 107 & 121 \\
\hline & & T311 & 0.01 & 0.26 & 23.6 & 95 & 121 \\
\hline & & T312 & 0.02 & 0.39 & 35.9 & 144 & 121 \\
\hline & & T313 & 0.02 & 0.56 & 50.9 & 204 & 121 \\
\hline & & T314 & 0.01 & 0.24 & 21.6 & 87 & 121 \\
\hline & & T315 & 0.01 & 0.24 & 21.9 & 88 & 121 \\
\hline & & T316 & 0.01 & 0.26 & 23.8 & 95 & 121 \\
\hline & & T317 & 0.01 & 0.25 & 22.7 & 91 & 121 \\
\hline & & T318 & 0.01 & 0.24 & 21.5 & 86 & 121 \\
\hline & & T319 & 0.01 & 0.24 & 21.8 & 88 & 121 \\
\hline & & T320 & 0.01 & 0.29 & 26.4 & 106 & 121 \\
\hline & & T321 & 0.01 & 0.29 & 26.1 & 105 & 121 \\
\hline & & T322 & 0.01 & 0.22 & 19.6 & 79 & 121 \\
\hline & & T323 & 0.01 & 0.24 & 22.2 & 89 & 121 \\
\hline & & T324 & 0.02 & 0.44 & 40.4 & 162 & 121 \\
\hline & & T325 & 0.01 & 0.33 & 30.4 & 122 & 121 \\
\hline & 2nd Quarter '03 & T302 & 0.01 & 0.25 & 23.6 & 90 & 125 \\
\hline & & Т303 & 0.01 & 0.30 & 29.0 & 110 & 125 \\
\hline & & T304 & 0.01 & 0.35 & 34.0 & 129 & 125 \\
\hline & & Т305 & 0.01 & 0.26 & 25.4 & 97 & 125 \\
\hline & & T306 & 0.01 & 0.27 & 25.9 & 98 & 125 \\
\hline & & T307 & 0.01 & 0.28 & 26.5 & 101 & 125 \\
\hline & & T308 & 0.01 & 0.26 & 24.8 & 94 & 125 \\
\hline & & Т309 & 0.01 & 0.23 & 22.4 & 85 & 125 \\
\hline & & T310 & 0.01 & 0.29 & 27.7 & 105 & 125 \\
\hline & & T311 & 0.01 & 0.25 & 23.6 & 90 & 125 \\
\hline & & T312 & 0.02 & 0.50 & 48.4 & 184 & 125 \\
\hline & & T313 & 0.02 & 0.50 & 48.5 & 184 & 125 \\
\hline & & T314 & 0.01 & 0.25 & 23.8 & 91 & 125 \\
\hline & & T315 & 0.01 & 0.24 & 22.7 & 86 & 125 \\
\hline & & T316 & 0.01 & 0.23 & 22.5 & 85 & 125 \\
\hline & & Т317 & 0.01 & 0.25 & 23.9 & 91 & 125 \\
\hline & & T318 & 0.01 & 0.23 & 21.9 & 83 & 125 \\
\hline & & T319 & 0.01 & 0.23 & 22.2 & 84 & 125 \\
\hline & & T320 & 0.01 & 0.28 & 26.6 & 101 & 125 \\
\hline & & T321 & 0.01 & 0.26 & 25.0 & 95 & 125 \\
\hline & & T322 & 0.01 & 0.21 & 20.0 & 76 & 125 \\
\hline & & T323 & 0.01 & 0.23 & 22.0 & 84 & 125 \\
\hline & & T324 & 0.01 & 0.27 & 26.1 & 99 & 125 \\
\hline & & T325 & 0.01 & 0.34 & 32.2 & 122 & 125 \\
\hline
\end{tabular}


Table 4-2. 2003 Thermoluminescent Dosimeter Results. (cont)

\begin{tabular}{|c|c|c|c|c|c|c|c|}
\hline Location & Sample Period & EDP Code & mrem/hr & mrem/day & mrem/qtr & mrem/year & Days in Field \\
\hline \multirow{48}{*}{200 West Area } & 3rd Quarter '03 & T302 & 0.01 & 0.26 & 23.5 & 94 & 121 \\
\hline & & Т303 & 0.01 & 0.34 & 31.4 & 126 & 121 \\
\hline & & Т304 & 0.02 & 0.36 & 33.0 & 132 & 121 \\
\hline & & Т305 & 0.01 & 0.25 & 23.1 & 93 & 121 \\
\hline & & Т306 & 0.01 & 0.31 & 28.4 & 114 & 121 \\
\hline & & Т307 & 0.01 & 0.31 & 28.3 & 113 & 121 \\
\hline & & Т308 & 0.01 & 0.29 & 26.5 & 106 & 121 \\
\hline & & Т309 & 0.01 & 0.25 & 22.7 & 91 & 121 \\
\hline & & Т310 & 0.01 & 0.31 & 28.1 & 113 & 121 \\
\hline & & T311 & 0.01 & 0.25 & 22.9 & 92 & 121 \\
\hline & & T312 & 0.02 & 0.52 & 47.4 & 190 & 121 \\
\hline & & T313 & 0.02 & 0.51 & 46.3 & 186 & 121 \\
\hline & & Т314 & 0.01 & 0.25 & 23.0 & 92 & 121 \\
\hline & & Т315 & 0.01 & 0.26 & 24.1 & 97 & 121 \\
\hline & & T316 & 0.01 & 0.26 & 23.3 & 93 & 121 \\
\hline & & Т317 & 0.01 & 0.26 & 23.3 & 93 & 121 \\
\hline & & T318 & 0.01 & 0.23 & 21.3 & 86 & 121 \\
\hline & & Т319 & 0.01 & 0.26 & 23.4 & 94 & 121 \\
\hline & & T320 & 0.01 & 0.31 & 28.5 & 114 & 121 \\
\hline & & T321 & 0.01 & 0.31 & 28.2 & 113 & 121 \\
\hline & & T322 & 0.01 & 0.23 & 20.9 & 84 & 121 \\
\hline & & Т323 & 0.01 & 0.25 & 23.1 & 93 & 121 \\
\hline & & Т324 & 0.01 & 0.33 & 29.9 & 120 & 121 \\
\hline & & T325 & 0.02 & 0.37 & 33.7 & 135 & 121 \\
\hline & 4th Quarter '03 & Т302 & 0.01 & 0.25 & 23.0 & 92 & 121 \\
\hline & & Т303 & 0.01 & 0.32 & 29.0 & 117 & 121 \\
\hline & & Т304 & 0.01 & 0.34 & 31.3 & 126 & 121 \\
\hline & & Т305 & 0.01 & 0.23 & 20.6 & 83 & 121 \\
\hline & & Т306 & 0.01 & 0.30 & 27.4 & 110 & 121 \\
\hline & & Т307 & 0.01 & 0.29 & 26.0 & 104 & 121 \\
\hline & & Т308 & 0.01 & 0.28 & 25.5 & 102 & 121 \\
\hline & & Т309 & 0.01 & 0.23 & 20.9 & 84 & 121 \\
\hline & & T310 & 0.01 & 0.29 & 26.3 & 105 & 121 \\
\hline & & Т311 & 0.01 & 0.24 & 22.1 & 89 & 121 \\
\hline & & Т312 & 0.02 & 0.42 & 38.3 & 153 & 121 \\
\hline & & Т313 & 0.02 & 0.50 & 45.1 & 181 & 121 \\
\hline & & Т314 & 0.01 & 0.24 & 21.7 & 87 & 121 \\
\hline & & T315 & 0.01 & 0.25 & 22.9 & 92 & 121 \\
\hline & & Т316 & 0.01 & 0.24 & 21.5 & 86 & 121 \\
\hline & & T317 & 0.01 & 0.25 & 22.7 & 91 & 121 \\
\hline & & Т318 & 0.01 & 0.22 & 19.9 & 80 & 121 \\
\hline & & Т319 & 0.01 & 0.23 & 20.6 & 82 & 121 \\
\hline & & T320 & 0.01 & 0.30 & 27.4 & 110 & 121 \\
\hline & & T321 & 0.01 & 0.29 & 26.0 & 104 & 121 \\
\hline & & T322 & 0.01 & 0.23 & 20.6 & 83 & 121 \\
\hline & & Т323 & 0.01 & 0.28 & 25.7 & 103 & 121 \\
\hline & & Т324 & 0.01 & 0.29 & 26.3 & 106 & 121 \\
\hline & & T325 & 0.01 & 0.33 & 30.4 & 122 & 121 \\
\hline
\end{tabular}


Table 4-2. 2003 Thermoluminescent Dosimeter Results. (cont)

\begin{tabular}{|c|c|c|c|c|}
\hline EDP Code & $\begin{array}{l}200 \text { West Area, } \\
\mathrm{mrem} / \mathrm{hr} \pm 2 \mathrm{SD}\end{array}$ & $\begin{array}{l}\text { l Average } \pm 2 \text { Stanc } \\
\mathrm{mrem} / \text { day } \pm 2 \mathrm{SD}\end{array}$ & $\begin{array}{l}\text { iation (2SD) } \\
\text { mrem/qtr } \pm \text { 2SD }\end{array}$ & $\mathrm{mrem} / \mathrm{yr} \pm 2 \mathrm{SD}$ \\
\hline T302 & $0.01 \pm 0.000$ & $0.25 \pm 0.01$ & $23.0 \pm 1.0$ & $92 \pm 4$ \\
\hline T303 & $0.01 \pm 0.002$ & $0.32 \pm 0.04$ & $29.2 \pm 3.3$ & $117 \pm 13$ \\
\hline T304 & $0.01 \pm 0.001$ & $0.36 \pm 0.02$ & $32.5 \pm 1.7$ & $130 \pm 7$ \\
\hline T305 & $0.01 \pm 0.001$ & $0.25 \pm 0.03$ & $22.5 \pm 3.0$ & $90 \pm 12$ \\
\hline T306 & $0.01 \pm 0.002$ & $0.30 \pm 0.04$ & $26.9 \pm 3.3$ & $108 \pm 13$ \\
\hline T307 & $0.01 \pm 0.001$ & $0.29 \pm 0.03$ & $26.7 \pm 2.7$ & $107 \pm 11$ \\
\hline Т308 & $0.01 \pm 0.001$ & $0.27 \pm 0.03$ & $24.9 \pm 2.8$ & $99 \pm 11$ \\
\hline Т309 & $0.01 \pm 0.001$ & $0.24 \pm 0.02$ & $21.6 \pm 1.6$ & $86 \pm 6$ \\
\hline T310 & $0.01 \pm 0.001$ & $0.29 \pm 0.02$ & $26.9 \pm 1.8$ & $107 \pm 7$ \\
\hline T311 & $0.01 \pm 0.001$ & $0.25 \pm 0.01$ & $22.8 \pm 1.3$ & $91 \pm 5$ \\
\hline T312 & $0.02 \pm 0.005$ & $0.46 \pm 0.12$ & $42.0 \pm 11.3$ & $168 \pm 45$ \\
\hline T313 & $0.02 \pm 0.002$ & $0.52 \pm 0.06$ & $47.2 \pm 5.2$ & $189 \pm 21$ \\
\hline T314 & $0.01 \pm 0.001$ & $0.24 \pm 0.01$ & $22.3 \pm 1.4$ & $89 \pm 5$ \\
\hline T315 & $0.01 \pm 0.001$ & $0.25 \pm 0.03$ & $22.7 \pm 2.3$ & $91 \pm 9$ \\
\hline T316 & $0.01 \pm 0.001$ & $0.25 \pm 0.03$ & $22.5 \pm 2.5$ & $90 \pm 10$ \\
\hline T317 & $0.01 \pm 0.000$ & $0.25 \pm 0.01$ & $22.9 \pm 0.6$ & $92 \pm 2$ \\
\hline T318 & $0.01 \pm 0.001$ & $0.23 \pm 0.02$ & $20.9 \pm 1.5$ & $84 \pm 6$ \\
\hline Т319 & $0.01 \pm 0.001$ & $0.24 \pm 0.03$ & $21.7 \pm 2.5$ & $87 \pm 10$ \\
\hline T320 & $0.01 \pm 0.001$ & $0.30 \pm 0.03$ & $26.9 \pm 2.8$ & $108 \pm 11$ \\
\hline T321 & $0.01 \pm 0.002$ & $0.29 \pm 0.04$ & $26.0 \pm 3.6$ & $104 \pm 14$ \\
\hline T322 & $0.01 \pm 0.001$ & $0.22 \pm 0.02$ & $20.1 \pm 1.9$ & $80 \pm 7$ \\
\hline T323 & $0.01 \pm 0.002$ & $0.25 \pm 0.04$ & $23.0 \pm 4.1$ & $92 \pm 16$ \\
\hline T324 & $0.01 \pm 0.006$ & $0.33 \pm 0.15$ & $30.3 \pm 14.1$ & $121 \pm 56$ \\
\hline T325 & $0.01 \pm 0.002$ & $0.34 \pm 0.04$ & $31.3 \pm 3.3$ & $125 \pm 13$ \\
\hline
\end{tabular}


Table 4-2. 2003 Thermoluminescent Dosimeter Results. (cont)

\begin{tabular}{|c|c|c|c|c|c|c|c|}
\hline Location & Sample Period & EDP Code & mrem/hr & mrem/day & mrem/qtr & mrem/year & Days in Field \\
\hline \multirow[t]{4}{*}{ 212-R (200 North) } & 1st Quarter '03 & T301 & 0.32 & 7.79 & 708.7 & 2843 & 121 \\
\hline & 2nd Quarter '03 & Т301 & 0.32 & 7.59 & 727.9 & 2769 & 125 \\
\hline & 3rd Quarter '03 & T301 & 0.39 & 9.29 & 845.8 & 3393 & 121 \\
\hline & 4th Quarter '03 & T301 & 0.36 & 8.57 & 788.8 & 3128 & 122 \\
\hline
\end{tabular}

212-R, Annual Average \pm 2 Standard Deviation (2SD)

EDP Code mrem/hr \pm 2SD mrem/day \pm 2SD

mrem/qtr \pm 2SD

mrem/yr \pm 2 SD $8.3 \pm 1.6$

$757 \pm 143$ $3030 \pm 571$ 
Table 4-2. 2003 Thermoluminescent Dosimeter Results. (cont)

\begin{tabular}{|c|c|c|c|c|c|c|c|}
\hline Location & Sample Period & EDP Code & mrem/hr & mrem/day & mrem/qtr & mrem/year & Days in Field \\
\hline \multirow[t]{32}{*}{300 Area } & 1st Quarter '03 & T332 & 0.01 & 0.24 & 22.3 & 86 & 125 \\
\hline & & T333 & 0.01 & 0.24 & 22.5 & 87 & 125 \\
\hline & & T334 & 0.01 & 0.23 & 21.7 & 83 & 125 \\
\hline & & T335 & 0.01 & 0.26 & 24.7 & 95 & 125 \\
\hline & & T336 & 0.01 & 0.22 & 21.0 & 81 & 125 \\
\hline & & T337 & 0.01 & 0.24 & 22.7 & 87 & 125 \\
\hline & & T338 & 0.01 & 0.31 & 29.2 & 112 & 125 \\
\hline & & T339 & 0.01 & 0.28 & 26.9 & 103 & 125 \\
\hline & 2nd Quarter '03 & T332 & 0.01 & 0.22 & 18.8 & 81 & 114 \\
\hline & & T333 & 0.01 & 0.23 & 20.0 & 86 & 114 \\
\hline & & T334 & 0.01 & 0.22 & 18.8 & 81 & 114 \\
\hline & & T335 & 0.01 & 0.25 & 21.3 & 92 & 114 \\
\hline & & T336 & 0.01 & 0.22 & 18.7 & 81 & 114 \\
\hline & & T337 & 0.01 & 0.25 & 21.1 & 91 & 114 \\
\hline & & T338 & 0.01 & 0.30 & 25.7 & 111 & 114 \\
\hline & & T339 & 0.01 & 0.32 & 27.2 & 117 & 114 \\
\hline & 3rd Quarter '03 & T332 & 0.01 & 0.22 & 22.0 & 81 & 130 \\
\hline & & Т333 & 0.01 & 0.25 & 25.0 & 91 & 130 \\
\hline & & T334 & 0.01 & 0.24 & 23.8 & 87 & 130 \\
\hline & & T335 & 0.01 & 0.25 & 24.6 & 90 & 130 \\
\hline & & T336 & 0.01 & 0.22 & 22.3 & 82 & 130 \\
\hline & & T337 & 0.01 & 0.24 & 23.5 & 86 & 130 \\
\hline & & Т338 & 0.01 & 0.30 & 30.3 & 111 & 130 \\
\hline & & T339 & 0.01 & 0.32 & 32.4 & 118 & 130 \\
\hline & 4th Quarter '03 & T332 & 0.01 & 0.22 & 20.2 & 82 & 120 \\
\hline & & Т333 & 0.01 & 0.24 & 21.6 & 87 & 120 \\
\hline & & T334 & 0.01 & 0.23 & 20.3 & 82 & 120 \\
\hline & & T335 & 0.01 & 0.25 & 22.5 & 91 & 120 \\
\hline & & T336 & 0.01 & 0.24 & 21.4 & 87 & 120 \\
\hline & & T337 & 0.01 & 0.24 & 21.6 & 88 & 120 \\
\hline & & Т338 & 0.01 & 0.31 & 27.9 & 113 & 120 \\
\hline & & Т339 & 0.01 & 0.28 & 25.4 & 103 & 120 \\
\hline
\end{tabular}

\begin{tabular}{|c|c|c|c|c|}
\hline EDP Code & $\mathrm{mrem} / \mathrm{hr} \pm$ 2SD & mrem/day \pm 2SD & mrem/qtr \pm 2SD & mrem/yr \pm 2SD \\
\hline T332 & $0.01 \pm 0.001$ & $0.23 \pm 0.01$ & $20.6 \pm 1.2$ & $82 \pm 5$ \\
\hline T333 & $0.01 \pm 0.001$ & $0.24 \pm 0.01$ & $22.0 \pm 1.2$ & $88 \pm 5$ \\
\hline T334 & $0.01 \pm 0.001$ & $0.23 \pm 0.01$ & $20.9 \pm 1.3$ & $83 \pm 5$ \\
\hline T335 & $0.01 \pm 0.001$ & $0.25 \pm 0.01$ & $22.9 \pm 1.1$ & $92 \pm 4$ \\
\hline T336 & $0.01 \pm 0.001$ & $0.23 \pm 0.02$ & $20.6 \pm 1.5$ & $82 \pm 6$ \\
\hline T337 & $0.01 \pm 0.001$ & $0.24 \pm 0.01$ & $22.0 \pm 1.0$ & $88 \pm 4$ \\
\hline Т338 & $0.01 \pm 0.000$ & $0.31 \pm 0.01$ & $27.9 \pm 0.6$ & $112 \pm 2$ \\
\hline Т339 & $0.01 \pm 0.002$ & $0.30 \pm 0.05$ & $27.6 \pm 4.2$ & $110 \pm 17$ \\
\hline
\end{tabular}


Table 4-2. 2003 Thermoluminescent Dosimeter Results. (cont)

\begin{tabular}{|c|c|c|c|c|c|c|c|}
\hline Location & Sample Period & EDP Code & mrem/hr & mrem/day & mrem/qtr & mrem/year & Days in Field \\
\hline \multirow[t]{24}{*}{300 TEDF } & \multirow[t]{6}{*}{ 1st Quarter '03 } & T326 & 0.01 & 0.23 & 21.4 & 82 & 125 \\
\hline & & T327 & 0.01 & 0.23 & 21.9 & 84 & 125 \\
\hline & & T328 & 0.01 & 0.24 & 23.0 & 88 & 125 \\
\hline & & T329 & 0.01 & 0.23 & 21.9 & 84 & 125 \\
\hline & & T330 & 0.01 & 0.21 & 20.2 & 78 & 125 \\
\hline & & T331 & 0.01 & 0.23 & 22.1 & 85 & 125 \\
\hline & \multirow[t]{6}{*}{ 2nd Quarter '03 } & T326 & 0.01 & 0.24 & 20.1 & 87 & 114 \\
\hline & & T327 & 0.01 & 0.23 & 19.2 & 83 & 114 \\
\hline & & T328 & 0.01 & 0.23 & 19.8 & 85 & 114 \\
\hline & & T329 & 0.01 & 0.23 & 19.7 & 85 & 114 \\
\hline & & T330 & 0.01 & 0.24 & 20.1 & 86 & 114 \\
\hline & & T331 & 0.01 & 0.23 & 19.9 & 86 & 114 \\
\hline & \multirow[t]{6}{*}{ 3rd Quarter '03 } & T326 & 0.01 & 0.23 & 23.4 & 85 & 130 \\
\hline & & T327 & 0.01 & 0.23 & 23.4 & 85 & 130 \\
\hline & & T328 & 0.01 & 0.27 & 26.9 & 98 & 130 \\
\hline & & T329 & 0.01 & 0.23 & 22.9 & 84 & 130 \\
\hline & & Т330 & 0.01 & 0.24 & 23.9 & 87 & 130 \\
\hline & & T331 & 0.01 & 0.23 & 23.4 & 85 & 130 \\
\hline & \multirow[t]{6}{*}{ 4th Quarter '03 } & T326 & 0.01 & 0.23 & 20.7 & 84 & 120 \\
\hline & & T327 & 0.01 & 0.23 & 20.7 & 84 & 120 \\
\hline & & T328 & 0.01 & 0.24 & 21.4 & 87 & 120 \\
\hline & & T329 & 0.01 & 0.23 & 20.9 & 85 & 120 \\
\hline & & T330 & 0.01 & 0.22 & 20.1 & 81 & 120 \\
\hline & & T331 & 0.01 & 0.25 & 22.8 & 93 & 120 \\
\hline \multicolumn{8}{|c|}{300 TEDF, Annual Average \pm 2 Standard Deviation (2SD) } \\
\hline EDP Code & \multicolumn{2}{|c|}{$\mathrm{mrem} / \mathrm{hr} \pm 2 \mathrm{SD}$} & \multicolumn{2}{|c|}{$\mathrm{mrem} / \mathrm{day} \pm$ 2SD } & \multicolumn{2}{|c|}{$\mathrm{mrem} / \mathrm{qtr} \pm 2 \mathrm{SD}$} & mrem/yr $\pm 2 S D$ \\
\hline T326 & \multicolumn{2}{|c|}{$0.01 \pm 0.000$} & \multicolumn{2}{|c|}{$0.23 \pm 0.01$} & \multicolumn{2}{|c|}{$21.1 \pm 0.9$} & $84 \pm 4$ \\
\hline T327 & \multicolumn{2}{|c|}{$0.01 \pm 0.000$} & \multicolumn{2}{|c|}{$0.23 \pm 0.01$} & \multicolumn{2}{|c|}{$21.0 \pm 0.6$} & $84 \pm 2$ \\
\hline T328 & \multicolumn{2}{|c|}{$0.01 \pm 0.001$} & \multicolumn{2}{|c|}{$0.25 \pm 0.03$} & \multicolumn{2}{|c|}{$22.5 \pm 2.9$} & $90 \pm 12$ \\
\hline T329 & \multicolumn{2}{|c|}{$0.01 \pm 0.000$} & \multicolumn{2}{|c|}{$0.23 \pm 0.00$} & \multicolumn{2}{|c|}{$21.1 \pm 0.3$} & $84 \pm 1$ \\
\hline T330 & \multicolumn{2}{|c|}{$0.01 \pm 0.001$} & \multicolumn{2}{|c|}{$0.23 \pm 0.02$} & \multicolumn{2}{|c|}{$20.8 \pm 2.3$} & $83 \pm 9$ \\
\hline T331 & \multicolumn{2}{|c|}{$0.01 \pm 0.001$} & \multicolumn{2}{|c|}{$\begin{array}{l}0.23 \pm 0.02 \\
0.24 \pm 0.02\end{array}$} & \multicolumn{2}{|c|}{$\begin{array}{l}20.0 \pm 2.3 \\
21.8 \pm 1.8\end{array}$} & $87 \pm 7$ \\
\hline
\end{tabular}


Table 4-2. 2003 Thermoluminescent Dosimeter Results. (cont)

\begin{tabular}{|c|c|c|c|c|c|c|c|}
\hline Location & Sample Period & EDP Code & mrem/hr & mrem/day & mrem/qtr & mrem/year & \multirow{2}{*}{ Days in Field } \\
\hline \multirow[t]{28}{*}{400 Area } & \multirow[t]{7}{*}{ 1st Quarter '03 } & T340 & 0.01 & 0.22 & 21.3 & 82 & \\
\hline & & Т341 & 0.01 & 0.21 & 20.1 & 77 & 125 \\
\hline & & Т342 & 0.01 & 0.22 & 20.7 & 80 & 125 \\
\hline & & Т343 & 0.01 & 0.22 & 20.8 & 80 & 125 \\
\hline & & T344 & 0.01 & 0.22 & 21.2 & 82 & 125 \\
\hline & & T345 & 0.01 & 0.22 & 21.0 & 81 & 125 \\
\hline & & T346 & 0.01 & 0.21 & 20.2 & 78 & 125 \\
\hline & \multirow[t]{7}{*}{ 2nd Quarter '03 } & T340 & 0.01 & 0.22 & 19.0 & 82 & 114 \\
\hline & & T341 & 0.01 & 0.22 & 18.7 & 80 & 114 \\
\hline & & Т342 & 0.01 & 0.23 & 19.2 & 83 & 114 \\
\hline & & T343 & 0.01 & 0.22 & 18.5 & 79 & 114 \\
\hline & & Т344 & 0.01 & 0.22 & 18.6 & 80 & 114 \\
\hline & & T345 & 0.01 & 0.21 & 17.8 & 77 & 114 \\
\hline & & T346 & 0.01 & 0.21 & 18.2 & 78 & 114 \\
\hline & \multirow[t]{7}{*}{ 3rd Quarter '03 } & T340 & 0.01 & 0.24 & 23.7 & 87 & 130 \\
\hline & & T341 & 0.01 & 0.21 & 21.4 & 78 & 130 \\
\hline & & T342 & 0.01 & 0.24 & 23.5 & 86 & 130 \\
\hline & & T343 & 0.01 & 0.22 & 21.8 & 80 & 130 \\
\hline & & T344 & 0.01 & 0.23 & 22.6 & 83 & 130 \\
\hline & & T345 & 0.01 & 0.22 & 22.0 & 80 & 130 \\
\hline & & T346 & 0.01 & 0.22 & 22.1 & 81 & 130 \\
\hline & 4th Quarter '03 & T340 & 0.01 & 0.24 & 21.9 & 89 & 120 \\
\hline & & T341 & 0.01 & 0.21 & 19.2 & 78 & 120 \\
\hline & & T342 & 0.01 & 0.23 & 21.1 & 86 & 120 \\
\hline & & T343 & 0.01 & 0.22 & 19.9 & 81 & 120 \\
\hline & & T344 & 0.01 & 0.22 & 20.0 & 81 & 120 \\
\hline & & T345 & 0.01 & 0.22 & 19.9 & 81 & 120 \\
\hline & & T346 & 0.01 & 0.21 & 19.1 & 78 & 120 \\
\hline & 400 & ea, Annual A & verage \pm 2 & andard Devi & ion (2SD) & & \\
\hline EDP Code & mrem/hr & 2SD & mrem/day & 2SD & mrem/qtr & 2SD & mrem/yr \pm 2SD \\
\hline T340 & 0.01 & 0.001 & 0.23 & 0.02 & 21.2 & 1.7 & $85 \pm 7$ \\
\hline Т341 & 0.01 & 0.000 & 0.21 & 0.01 & 19.6 & 0.7 & $78 \pm 3$ \\
\hline T342 & 0.01 & 0.001 & 0.23 & 0.02 & 20.9 & 1.5 & $83 \pm 6$ \\
\hline Т343 & 0.01 & 0.000 & 0.22 & 0.00 & 20.0 & 0.3 & $80 \pm 1$ \\
\hline T344 & 0.01 & 0.000 & 0.22 & 0.01 & 20.3 & 0.5 & $81 \pm 2$ \\
\hline T345 & 0.01 & 0.001 & 0.22 & 0.01 & 19.9 & 1.0 & $80 \pm 4$ \\
\hline T346 & 0.01 & 0.000 & 0.22 & 0.01 & 19.6 & 0.8 & $78 \pm 3$ \\
\hline
\end{tabular}


Table 4-2. 2003 Thermoluminescent Dosimeter Results. (cont)

\begin{tabular}{|c|c|c|c|c|c|c|c|}
\hline Location & Sample Period & EDP Code & mrem/hr & mrem/day & mrem/qtr & mrem/year & Days in Field \\
\hline \multirow[t]{12}{*}{ ERDF } & 1st Quarter '03 & T351 & 0.01 & 0.24 & 18.8 & 89 & 107 \\
\hline & & T352 & 0.01 & 0.29 & 22.6 & 107 & 107 \\
\hline & & T353 & 0.01 & 0.28 & 21.3 & 101 & 107 \\
\hline & 2nd Quarter '03 & T351 & 0.01 & 0.24 & 23.1 & 89 & 124 \\
\hline & & T352 & 0.01 & 0.27 & 25.2 & 97 & 124 \\
\hline & & T353 & 0.01 & 0.26 & 24.8 & 95 & 124 \\
\hline & 3rd Quarter '03 & T351 & 0.01 & 0.23 & 20.6 & 85 & 118 \\
\hline & & T352 & 0.01 & 0.27 & 23.5 & 98 & 118 \\
\hline & & T353 & 0.01 & 0.26 & 23.2 & 96 & 118 \\
\hline & 4th Quarter '03 & T351 & \multicolumn{5}{|c|}{ TLD not submitted for analysis } \\
\hline & & T352 & 0.01 & 0.26 & 23.1 & 96 & 118 \\
\hline & & T353 & 0.01 & 0.25 & 21.8 & 90 & 118 \\
\hline \multicolumn{8}{|c|}{ ERDF, Annual Average \pm 2 Standard Deviation (2SD) } \\
\hline EDP Code & \multicolumn{2}{|c|}{$\mathrm{mrem} / \mathrm{hr} \pm 2 \mathrm{SD}$} & \multicolumn{2}{|c|}{ mrem/day \pm 2 SD } & \multicolumn{2}{|c|}{ mrem/qtr \pm 2 SD } & $\mathrm{mrem} / \mathrm{yr} \pm 2 \mathrm{SD}$ \\
\hline T351 & \multicolumn{2}{|c|}{$0.01 \pm 0.001$} & \multicolumn{2}{|c|}{$0.24 \pm 0.01$} & \multicolumn{2}{|c|}{$21.9 \pm 1.1$} & $88 \pm 4$ \\
\hline T352 & \multicolumn{2}{|c|}{$0.01 \pm 0.001$} & \multicolumn{2}{|c|}{$0.27 \pm 0.03$} & \multicolumn{2}{|c|}{$24.8 \pm 2.7$} & $99 \pm 11$ \\
\hline T353 & \multicolumn{2}{|c|}{$0.01 \pm 0.001$} & \multicolumn{2}{|c|}{$0.26 \pm 0.02$} & \multicolumn{2}{|c|}{$23.9 \pm 2.2$} & $96 \pm 9$ \\
\hline
\end{tabular}




\subsection{0-N RIVERBANK SPRINGS MONITORING}

In 2003, water samples were taken only at the riverbank springs in the 100-N Area. All radiological analyses were performed onsite at the WSCF. Analyses for riverbank springs water included tritium, strontium-90, and gamma-emitting radionuclides. Sampling locations are illustrated in Figure 5-1.

Riverbank springs and/or shoreline seepage wells along the 100-N Area shoreline are sampled annually to verify that the reported radionuclide releases to the Columbia River are conservative (i.e., not underreported). In the past, radioactive effluent streams sent to the 1301-N and 1325-N LWDFs in the 100-N Area contributed to the release of radionuclides to the Columbia River through their migration with the groundwater. Radionuclides from these facilities enter the Columbia River along the riverbank region commonly called N Springs.

The amount of radionuclides entering the river at these springs is calculated based on analyses of samples routinely collected from monitoring well 199-N-46, located near the shoreline. To calculate these releases, conservatively high radionuclide activities in samples collected from well 199-N-46 are multiplied by the estimated groundwater discharged into the river. The estimated groundwater flow rate used to calculate 2003 releases from the springs was $42 \mathrm{~L} / \mathrm{min}$ (11 gal $/ \mathrm{min})$. The results of the annual riverbank spring samples can then be compared to the activities measured in well 199-N-46 to ensure that activities in the well reflect the highest activities of radionuclides in the groundwater. Additional discussion of the release calculations may be found in Environmental Releases for Calendar Year 2003, HNF-EP-0527-13 (Diediker 2004).

In October 2003, 10 samples were collected from the 13 shoreline wells. Three wells were dry and could not be sampled. The shoreline seepage well samples were collected using a bailer, carefully lowered into each well water column to avoid sediment suspension, and a 4-L (1-gal) sample was obtained. The sampling methods are discussed in more detail in DTS-OEM-001.

In 2003, strontium-90 was detected in eight of the ten riverbank springs samples. The highest concentrations were from wells Y302 (near well 199-N-46), and Y311 (downstream of well 199-N-46). Strontium-90 concentrations did not exceed the DOE DCG value at any well. Tritium and gamma-emitting radionuclide concentrations were below analytical detection limits in all ten samples collected in 2003. The 2003 data results from riverbank springs sampling are summarized in Table 5-1. Historical tritium and strontium-90 sampling results are provided in Tables 5-2 and 5-3. 


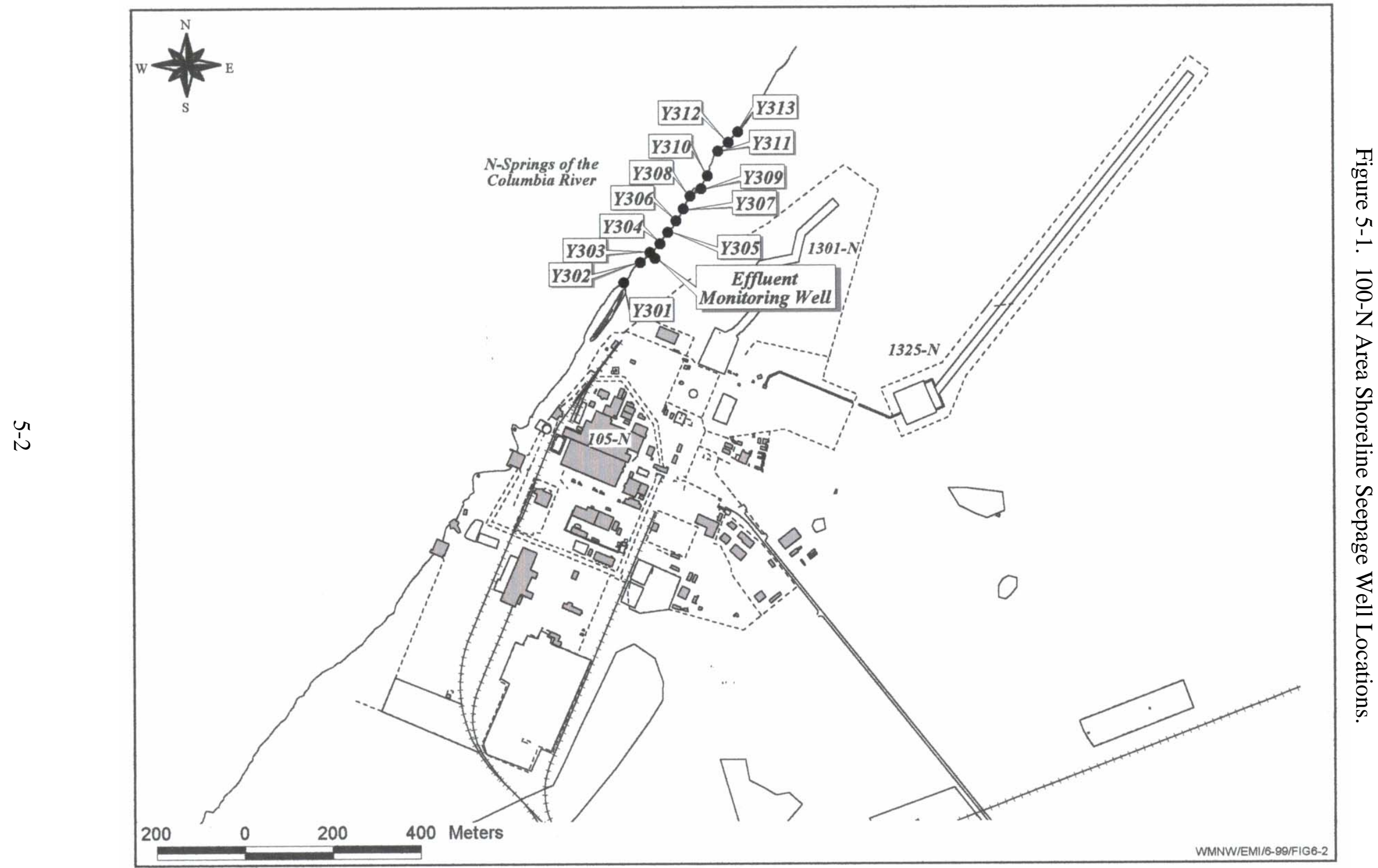


Table 5-1. 2003 Radiological Results for N-Springs Water Samples (pCi/L \pm total analytical uncertainty).

\begin{tabular}{|c|c|c|c|}
\hline Location & Isotope & Result \pm Uncertainty & $\mathbf{R Q}^{*}$ \\
\hline \multirow[t]{14}{*}{ Y302 } & ${ }^{144} \mathrm{Ce}$ & $-3.3 \mathrm{E}+00 \pm 3.3 \mathrm{E}+01$ & $\mathrm{U}$ \\
\hline & ${ }^{60} \mathrm{Co}$ & $4.2 \mathrm{E}+00 \pm 4.2 \mathrm{E}+00$ & $\mathrm{U}$ \\
\hline & ${ }^{134} \mathrm{Cs}$ & $-4.4 \mathrm{E}-01 \pm 4.3 \mathrm{E}+00$ & $\mathrm{U}$ \\
\hline & ${ }^{137} \mathrm{Cs}$ & $4.1 \mathrm{E}+00 \pm 6.3 \mathrm{E}+00$ & $\mathrm{U}$ \\
\hline & ${ }^{152} \mathrm{Eu}$ & $1.9 \mathrm{E}+00 \pm 1.3 \mathrm{E}+01$ & $\mathrm{U}$ \\
\hline & ${ }^{154} \mathrm{Eu}$ & $3.2 \mathrm{E}+00 \pm 1.1 \mathrm{E}+01$ & $\mathrm{U}$ \\
\hline & ${ }^{155} \mathrm{Eu}$ & $-7.0 \mathrm{E}+00 \pm 1.3 \mathrm{E}+01$ & $\mathrm{U}$ \\
\hline & ${ }^{3} \mathrm{H}$ & $2.4 \mathrm{E}+01 \pm 5.4 \mathrm{E}+01$ & $\mathrm{U}$ \\
\hline & ${ }^{103} \mathrm{Ru}$ & $-9.2 \mathrm{E}-01 \pm 4.5 \mathrm{E}+00$ & $\mathrm{U}$ \\
\hline & ${ }^{106} \mathrm{Ru}$ & $9.3 \mathrm{E}-01 \pm 9.3 \mathrm{E}+00$ & $\mathrm{U}$ \\
\hline & ${ }^{125} \mathrm{Sb}$ & $1.1 \mathrm{E}+01 \pm 1.3 \mathrm{E}+01$ & $\mathrm{U}$ \\
\hline & ${ }^{113} \mathrm{Sn}$ & $-4.4 \mathrm{E}+00 \pm 5.9 \mathrm{E}+00$ & $\mathrm{U}$ \\
\hline & ${ }^{90} \mathrm{Sr}$ & $2.3 \mathrm{E}+01 \pm 3.4 \mathrm{E}+00$ & \\
\hline & ${ }^{65} \mathrm{Zn}$ & $3.4 \mathrm{E}+00 \pm 9.5 \mathrm{E}+00$ & $\mathrm{U}$ \\
\hline \multirow[t]{14}{*}{ Y305 } & ${ }^{144} \mathrm{Ce}$ & $-1.4 \mathrm{E}+01 \pm 7.6 \mathrm{E}+01$ & $\mathrm{U}$ \\
\hline & ${ }^{60} \mathrm{Co}$ & $3.6 \mathrm{E}+00 \pm 5.3 \mathrm{E}+00$ & $\mathrm{U}$ \\
\hline & ${ }^{134} \mathrm{Cs}$ & $-2.5 \mathrm{E}+00 \pm 5.7 \mathrm{E}+00$ & $\mathrm{U}$ \\
\hline & ${ }^{137} \mathrm{Cs}$ & $1.0 \mathrm{E}+00 \pm 5.5 \mathrm{E}+00$ & $\mathrm{U}$ \\
\hline & ${ }^{152} \mathrm{Eu}$ & $-5.4 \mathrm{E}+00 \pm 2.1 \mathrm{E}+01$ & $\mathrm{U}$ \\
\hline & ${ }^{154} \mathrm{Eu}$ & $-4.5 \mathrm{E}+00 \pm 1.5 \mathrm{E}+01$ & $\mathrm{U}$ \\
\hline & ${ }^{155} \mathrm{Eu}$ & $-2.3 \mathrm{E}+00 \pm 2.0 \mathrm{E}+01$ & $\mathrm{U}$ \\
\hline & ${ }^{3} \mathrm{H}$ & $1.9 \mathrm{E}+01 \pm 4.9 \mathrm{E}+01$ & $\mathrm{U}$ \\
\hline & ${ }^{103} \mathrm{Ru}$ & $5.3 \mathrm{E}+00 \pm 5.9 \mathrm{E}+00$ & $\mathrm{U}$ \\
\hline & ${ }^{106} \mathrm{Ru}$ & $4.5 \mathrm{E}+01 \pm 4.9 \mathrm{E}+01$ & $\mathrm{U}$ \\
\hline & ${ }^{125} \mathrm{Sb}$ & $5.3 \mathrm{E}+00 \pm 1.6 \mathrm{E}+01$ & $\mathrm{U}$ \\
\hline & ${ }^{113} \mathrm{Sn}$ & $1.0 \mathrm{E}+00 \pm 7.3 \mathrm{E}+00$ & $\mathrm{U}$ \\
\hline & ${ }^{90} \mathrm{Sr}$ & $3.8 \mathrm{E}+00 \pm 1.3 \mathrm{E}+00$ & \\
\hline & ${ }^{65} \mathrm{Zn}$ & $-1.9 \mathrm{E}+01 \pm 1.9 \mathrm{E}+01$ & $\mathrm{U}$ \\
\hline \multirow[t]{14}{*}{ Y307 } & ${ }^{144} \mathrm{Ce}$ & $5.6 \mathrm{E}+01 \pm 7.2 \mathrm{E}+01$ & $\bar{U}$ \\
\hline & ${ }^{60} \mathrm{Co}$ & $-2.5 \mathrm{E}-01 \pm 2.5 \mathrm{E}+00$ & $\mathrm{U}$ \\
\hline & ${ }^{134} \mathrm{Cs}$ & $-4.3 \mathrm{E}-01 \pm 4.3 \mathrm{E}+00$ & $\mathrm{U}$ \\
\hline & ${ }^{137} \mathrm{Cs}$ & $1.4 \mathrm{E}+00 \pm 5.4 \mathrm{E}+00$ & $\mathrm{U}$ \\
\hline & ${ }^{152} \mathrm{Eu}$ & $3.6 \mathrm{E}+00 \pm 1.7 \mathrm{E}+01$ & $\mathrm{U}$ \\
\hline & ${ }^{154} \mathrm{Eu}$ & $-3.3 \mathrm{E}+00 \pm 1.4 \mathrm{E}+01$ & $\mathrm{U}$ \\
\hline & ${ }^{155} \mathrm{Eu}$ & $-3.6 \mathrm{E}+00 \pm 1.9 \mathrm{E}+01$ & $\mathrm{U}$ \\
\hline & ${ }^{3} \mathrm{H}$ & $4.5 \mathrm{E}-01 \pm 3.6 \mathrm{E}+00$ & $\mathrm{U}$ \\
\hline & ${ }^{103} \mathrm{Ru}$ & $-5.6 \mathrm{E}-01 \pm 5.4 \mathrm{E}+00$ & $\mathrm{U}$ \\
\hline & ${ }^{106} \mathrm{Ru}$ & $-4.0 \mathrm{E}+01 \pm 4.9 \mathrm{E}+01$ & $\mathrm{U}$ \\
\hline & ${ }^{125} \mathrm{Sb}$ & $4.1 \mathrm{E}-01 \pm 4.1 \mathrm{E}+00$ & $\mathrm{U}$ \\
\hline & ${ }^{113} \mathrm{Sn}$ & $-3.2 \mathrm{E}+00 \pm 6.8 \mathrm{E}+00$ & $\mathrm{U}$ \\
\hline & ${ }^{90} \mathrm{Sr}$ & $3.8 \mathrm{E}+00 \pm 9.5 \mathrm{E}-01$ & \\
\hline & ${ }^{65} \mathrm{Zn}$ & $3.8 \mathrm{E}-01 \pm 3.8 \mathrm{E}+00$ & $\mathrm{U}$ \\
\hline
\end{tabular}

\begin{tabular}{|c|c|c|c|}
\hline Location & Isotope & Result \pm Uncertainty & $\mathbf{R Q}^{*}$ \\
\hline \multirow[t]{14}{*}{ Y303 } & ${ }^{144} \mathrm{Ce}$ & $2.2 \mathrm{E}+01 \pm 5.8 \mathrm{E}+01$ & $\mathrm{U}$ \\
\hline & ${ }^{60} \mathrm{Co}$ & $-4.2 \mathrm{E}+00 \pm 5.2 \mathrm{E}+00$ & $\mathrm{U}$ \\
\hline & ${ }^{134} \mathrm{Cs}$ & $-2.3 \mathrm{E}+00 \pm 7.2 \mathrm{E}+00$ & $\mathrm{U}$ \\
\hline & ${ }^{137} \mathrm{Cs}$ & $8.1 \mathrm{E}+00 \pm 9.2 \mathrm{E}+00$ & $\mathrm{U}$ \\
\hline & ${ }^{152} \mathrm{Eu}$ & $-6.8 \mathrm{E}+00 \pm 1.5 \mathrm{E}+01$ & $\mathrm{U}$ \\
\hline & ${ }^{154} \mathrm{Eu}$ & $-4.5 \mathrm{E}+00 \pm 1.6 \mathrm{E}+01$ & $\mathrm{U}$ \\
\hline & ${ }^{155} \mathrm{Eu}$ & $-1.4 \mathrm{E}+00 \pm 1.4 \mathrm{E}+01$ & $\mathrm{U}$ \\
\hline & ${ }^{3} \mathrm{H}$ & $4.5 \mathrm{E}-01 \pm 2.4 \mathrm{E}+00$ & $\mathrm{U}$ \\
\hline & ${ }^{103} \mathrm{Ru}$ & $-8.5 \mathrm{E}-01 \pm 5.6 \mathrm{E}+00$ & $\mathrm{U}$ \\
\hline & ${ }^{106} \mathrm{Ru}$ & $2.1 \mathrm{E}+01 \pm 4.9 \mathrm{E}+01$ & $\mathrm{U}$ \\
\hline & ${ }^{125} \mathrm{Sb}$ & $1.5 \mathrm{E}+00 \pm 1.4 \mathrm{E}+01$ & $\mathrm{U}$ \\
\hline & ${ }^{113} \mathrm{Sn}$ & $-7.3 \mathrm{E}+00 \pm 1.1 \mathrm{E}+01$ & $\mathrm{U}$ \\
\hline & ${ }^{90} \mathrm{Sr}$ & $5.9 \mathrm{E}+00 \pm 1.5 \mathrm{E}+00$ & \\
\hline & ${ }^{65} \mathrm{Zn}$ & $4.3 \mathrm{E}+00 \pm 1.4 \mathrm{E}+01$ & $\mathrm{U}$ \\
\hline \multirow[t]{14}{*}{ Y306 } & ${ }^{144} \mathrm{Ce}$ & $1.2 \mathrm{E}+01 \pm 5.3 \mathrm{E}+01$ & $\mathrm{U}$ \\
\hline & ${ }^{60} \mathrm{Co}$ & $2.8 \mathrm{E}+00 \pm 4.9 \mathrm{E}+00$ & $\mathrm{U}$ \\
\hline & ${ }^{134} \mathrm{Cs}$ & $-2.6 \mathrm{E}+00 \pm 5.3 \mathrm{E}+00$ & $\mathrm{U}$ \\
\hline & ${ }^{137} \mathrm{Cs}$ & $-5.6 \mathrm{E}+00 \pm 5.6 \mathrm{E}+00$ & $\mathrm{U}$ \\
\hline & ${ }^{152} \mathrm{Eu}$ & $-8.9 \mathrm{E}+00 \pm 1.6 \mathrm{E}+01$ & $\mathrm{U}$ \\
\hline & ${ }^{154} \mathrm{Eu}$ & $2.5 \mathrm{E}+00 \pm 1.4 \mathrm{E}+01$ & $\mathrm{U}$ \\
\hline & ${ }^{155} \mathrm{Eu}$ & $-5.1 \mathrm{E}+00 \pm 1.4 \mathrm{E}+01$ & $\mathrm{U}$ \\
\hline & ${ }^{3} \mathrm{H}$ & $-7.5 \mathrm{E}+01 \pm 1.2 \mathrm{E}+02$ & $\mathrm{U}$ \\
\hline & ${ }^{103} \mathrm{Ru}$ & $7.0 \mathrm{E}+00 \pm 5.9 \mathrm{E}+00$ & $\mathrm{U}$ \\
\hline & ${ }^{106} \mathrm{Ru}$ & $1.3 \mathrm{E}+01 \pm 4.9 \mathrm{E}+01$ & $\mathrm{U}$ \\
\hline & ${ }^{125} \mathrm{Sb}$ & $-8.6 \mathrm{E}+00 \pm 1.3 \mathrm{E}+01$ & $\mathrm{U}$ \\
\hline & ${ }^{113} \mathrm{Sn}$ & $1.8 \mathrm{E}+00 \pm 6.1 \mathrm{E}+00$ & $\mathrm{U}$ \\
\hline & ${ }^{90} \mathrm{Sr}$ & $2.0 \mathrm{E}+00 \pm 7.0 \mathrm{E}-01$ & \\
\hline & ${ }^{65} \mathrm{Zn}$ & $7.1 \mathrm{E}-01 \pm 7.1 \mathrm{E}+00$ & $\mathrm{U}$ \\
\hline \multirow[t]{14}{*}{ Y308 } & ${ }^{144} \mathrm{Ce}$ & $6.9 \mathrm{E}+00 \pm 6.9 \mathrm{E}+01$ & $\mathrm{U}$ \\
\hline & ${ }^{60} \mathrm{Co}$ & $1.4 \mathrm{E}+00 \pm 5.1 \mathrm{E}+00$ & $\mathrm{U}$ \\
\hline & ${ }^{134} \mathrm{Cs}$ & $5.0 \mathrm{E}+00 \pm 5.7 \mathrm{E}+00$ & $\mathrm{U}$ \\
\hline & ${ }^{137} \mathrm{Cs}$ & $4.4 \mathrm{E}-01 \pm 4.3 \mathrm{E}+00$ & $\mathrm{U}$ \\
\hline & ${ }^{152} \mathrm{Eu}$ & $5.4 \mathrm{E}+00 \pm 1.5 \mathrm{E}+01$ & $\mathrm{U}$ \\
\hline & ${ }^{154} \mathrm{Eu}$ & $1.2 \mathrm{E}+01 \pm 1.5 \mathrm{E}+01$ & $\mathrm{U}$ \\
\hline & ${ }^{155} \mathrm{Eu}$ & $-9.4 \mathrm{E}+00 \pm 1.8 \mathrm{E}+01$ & $\mathrm{U}$ \\
\hline & ${ }^{3} \mathrm{H}$ & $-1.8 \mathrm{E}+00 \pm 7.7 \mathrm{E}+00$ & $\mathrm{U}$ \\
\hline & ${ }^{103} \mathrm{Ru}$ & $1.9 \mathrm{E}+00 \pm 5.1 \mathrm{E}+00$ & $\mathrm{U}$ \\
\hline & ${ }^{106} \mathrm{Ru}$ & $7.0 \mathrm{E}+01 \pm 7.7 \mathrm{E}+01$ & $\mathrm{U}$ \\
\hline & ${ }^{125} \mathrm{Sb}$ & $-1.6 \mathrm{E}+01 \pm 1.6 \mathrm{E}+01$ & $\mathrm{U}$ \\
\hline & ${ }^{113} \mathrm{Sn}$ & $3.4 \mathrm{E}+00 \pm 6.5 \mathrm{E}+00$ & $\mathrm{U}$ \\
\hline & ${ }^{90} \mathrm{Sr}$ & $8.4 \mathrm{E}+00 \pm 1.7 \mathrm{E}+00$ & \\
\hline & ${ }^{65} \mathrm{Zn}$ & $1.1 \mathrm{E}+00 \pm 1.1 \mathrm{E}+01$ & $\mathrm{U}$ \\
\hline
\end{tabular}

$\overline{\text { RQ* }^{*} \text { Result Qualifier. }} \mathrm{U}=$ The analyte was analyzed for but not detected. 
Table 5-1. 2003 Radiological Results for N-Springs Water Samples (pCi/L \pm total analytical uncertainty). (cont)

\begin{tabular}{|c|c|c|c|c|c|c|c|}
\hline Location & Isotope & Result \pm Uncertainty & $\mathbf{R Q}^{*}$ & Location & Isotope & Result \pm Uncertainty & RQ* \\
\hline \multirow[t]{14}{*}{ Y309 } & ${ }^{144} \mathrm{Ce}$ & $-6.5 \mathrm{E}+01 \pm 7.3 \mathrm{E}+01$ & $\bar{U}$ & Y311 & ${ }^{144} \mathrm{Ce}$ & $5.9 \mathrm{E}+01 \pm 5.6 \mathrm{E}+01$ & $\mathrm{U}$ \\
\hline & ${ }^{60} \mathrm{Co}$ & $2.1 \mathrm{E}+00 \pm 5.1 \mathrm{E}+00$ & $\mathrm{U}$ & & ${ }^{60} \mathrm{Co}$ & $-1.3 \mathrm{E}+00 \pm 4.1 \mathrm{E}+00$ & $\mathrm{U}$ \\
\hline & ${ }^{134} \mathrm{Cs}$ & $6.0 \mathrm{E}+00 \pm 5.7 \mathrm{E}+00$ & $\mathrm{U}$ & & ${ }^{134} \mathrm{Cs}$ & $-3.9 \mathrm{E}+00 \pm 4.3 \mathrm{E}+00$ & U \\
\hline & ${ }^{137} \mathrm{Cs}$ & $1.0 \mathrm{E}+00 \pm 5.6 \mathrm{E}+00$ & $\mathrm{U}$ & & ${ }^{137} \mathrm{Cs}$ & $7.9 \mathrm{E}-02 \pm 7.9 \mathrm{E}-01$ & $\mathrm{U}$ \\
\hline & ${ }^{152} \mathrm{Eu}$ & $-7.7 \mathrm{E}+00 \pm 1.9 \mathrm{E}+01$ & $\mathrm{U}$ & & ${ }^{152} \mathrm{Eu}$ & $1.1 \mathrm{E}+00 \pm 1.1 \mathrm{E}+01$ & $\mathrm{U}$ \\
\hline & ${ }^{154} \mathrm{Eu}$ & $-6.6 \mathrm{E}+00 \pm 1.4 \mathrm{E}+01$ & $\mathrm{U}$ & & ${ }^{154} \mathrm{Eu}$ & $1.9 \mathrm{E}+00 \pm 1.2 \mathrm{E}+01$ & $\mathrm{U}$ \\
\hline & ${ }^{155} \mathrm{Eu}$ & $-4.7 \mathrm{E}+00 \pm 2.0 \mathrm{E}+01$ & $\mathrm{U}$ & & ${ }^{155} \mathrm{Eu}$ & $2.2 \mathrm{E}+00 \pm 1.4 \mathrm{E}+01$ & $\mathrm{U}$ \\
\hline & ${ }^{3} \mathrm{H}$ & $-1.4 \mathrm{E}+02 \pm 1.7 \mathrm{E}+02$ & $\mathrm{U}$ & & ${ }^{3} \mathrm{H}$ & $1.9 \mathrm{E}+01 \pm 2.1 \mathrm{E}+01$ & $\mathrm{U}$ \\
\hline & ${ }^{103} \mathrm{Ru}$ & $-4.1 \mathrm{E}-01 \pm 4.1 \mathrm{E}+00$ & $\mathrm{U}$ & & ${ }^{103} \mathrm{Ru}$ & $8.8 \mathrm{E}-01 \pm 4.1 \mathrm{E}+00$ & $\mathrm{U}$ \\
\hline & ${ }^{106} \mathrm{Ru}$ & $-1.4 \mathrm{E}+01 \pm 4.9 \mathrm{E}+01$ & $\mathrm{U}$ & & ${ }^{106} \mathrm{Ru}$ & $1.0 \mathrm{E}+01 \pm 3.6 \mathrm{E}+01$ & $\mathrm{U}$ \\
\hline & ${ }^{125} \mathrm{Sb}$ & $-2.5 \mathrm{E}+00 \pm 1.5 \mathrm{E}+01$ & $\mathrm{U}$ & & ${ }^{125} \mathrm{Sb}$ & $6.2 \mathrm{E}-01 \pm 6.2 \mathrm{E}+00$ & $\mathrm{U}$ \\
\hline & ${ }^{113} \mathrm{Sn}$ & $4.8 \mathrm{E}+00 \pm 7.0 \mathrm{E}+00$ & $\mathrm{U}$ & & ${ }^{113} \mathrm{Sn}$ & $-1.8 \mathrm{E}+00 \pm 5.7 \mathrm{E}+00$ & $\mathrm{U}$ \\
\hline & ${ }^{90} \mathrm{Sr}$ & $1.7 \mathrm{E}+00 \pm 6.8 \mathrm{E}-01$ & $\mathrm{U}$ & & ${ }^{90} \mathrm{Sr}$ & $2.1 \mathrm{E}+01 \pm 3.1 \mathrm{E}+00$ & \\
\hline & ${ }^{65} \mathrm{Zn}$ & $2.0 \mathrm{E}+00 \pm 1.2 \mathrm{E}+01$ & $\mathrm{U}$ & & ${ }^{65} \mathrm{Zn}$ & $-1.3 \mathrm{E}+00 \pm 1.0 \mathrm{E}+01$ & $\mathrm{U}$ \\
\hline \multirow[t]{14}{*}{ Y312 } & ${ }^{144} \mathrm{Ce}$ & $-2.6 \mathrm{E}+00 \pm 2.6 \mathrm{E}+01$ & $\mathrm{U}$ & Y313 & ${ }^{144} \mathrm{Ce}$ & $9.6 \mathrm{E}+00 \pm 5.7 \mathrm{E}+01$ & $\mathrm{U}$ \\
\hline & ${ }^{60} \mathrm{Co}$ & $-7.1 \mathrm{E}+00 \pm 7.1 \mathrm{E}+00$ & $\mathrm{U}$ & & ${ }^{60} \mathrm{Co}$ & $-3.3 \mathrm{E}-01 \pm 3.3 \mathrm{E}+00$ & $\mathrm{U}$ \\
\hline & ${ }^{134} \mathrm{Cs}$ & $3.1 \mathrm{E}+00 \pm 5.6 \mathrm{E}+00$ & $\mathrm{U}$ & & ${ }^{134} \mathrm{Cs}$ & $1.0 \mathrm{E}+00 \pm 6.6 \mathrm{E}+00$ & $\mathrm{U}$ \\
\hline & ${ }^{137} \mathrm{Cs}$ & $-2.2 \mathrm{E}+00 \pm 5.8 \mathrm{E}+00$ & $\mathrm{U}$ & & ${ }^{137} \mathrm{Cs}$ & $-4.8 \mathrm{E}+00 \pm 5.5 \mathrm{E}+00$ & $\mathrm{U}$ \\
\hline & ${ }^{152} \mathrm{Eu}$ & $-6.4 \mathrm{E}+00 \pm 1.6 \mathrm{E}+01$ & $\mathrm{U}$ & & ${ }^{152} \mathrm{Eu}$ & $8.7 \mathrm{E}+00 \pm 1.5 \mathrm{E}+01$ & $\mathrm{U}$ \\
\hline & ${ }^{154} \mathrm{Eu}$ & $1.8 \mathrm{E}+00 \pm 1.6 \mathrm{E}+01$ & $\mathrm{U}$ & & ${ }^{154} \mathrm{Eu}$ & $8.8 \mathrm{E}+00 \pm 1.5 \mathrm{E}+01$ & $\mathrm{U}$ \\
\hline & ${ }^{155} \mathrm{Eu}$ & $-1.6 \mathrm{E}+01 \pm 2.1 \mathrm{E}+01$ & $\mathrm{U}$ & & ${ }^{155} \mathrm{Eu}$ & $3.9 \mathrm{E}+00 \pm 1.4 \mathrm{E}+01$ & $\mathrm{U}$ \\
\hline & ${ }^{3} \mathrm{H}$ & $-5.9 \mathrm{E}+01 \pm 5.9 \mathrm{E}+02$ & $\mathrm{U}$ & & ${ }^{3} \mathrm{H}$ & $-1.2 \mathrm{E}+02 \pm 1.3 \mathrm{E}+02$ & $\mathrm{U}$ \\
\hline & ${ }^{103} \mathrm{Ru}$ & $-2.3 \mathrm{E}-01 \pm 2.3 \mathrm{E}+00$ & $\mathrm{U}$ & & ${ }^{103} \mathrm{Ru}$ & $-4.3 \mathrm{E}-01 \pm 4.3 \mathrm{E}+00$ & $\mathrm{U}$ \\
\hline & ${ }^{106} \mathrm{Ru}$ & $1.9 \mathrm{E}+01 \pm 5.0 \mathrm{E}+01$ & $\mathrm{U}$ & & ${ }^{106} \mathrm{Ru}$ & $1.1 \mathrm{E}+01 \pm 4.9 \mathrm{E}+01$ & $\mathrm{U}$ \\
\hline & ${ }^{125} \mathrm{Sb}$ & $3.7 \mathrm{E}-01 \pm 3.7 \mathrm{E}+00$ & $\mathrm{U}$ & & ${ }^{125} \mathrm{Sb}$ & $-1.4 \mathrm{E}+00 \pm 1.3 \mathrm{E}+01$ & $\mathrm{U}$ \\
\hline & ${ }^{113} \mathrm{Sn}$ & $8.6 \mathrm{E}-02 \pm 8.6 \mathrm{E}-01$ & $\mathrm{U}$ & & ${ }^{113} \mathrm{Sn}$ & $-2.5 \mathrm{E}+00 \pm 5.9 \mathrm{E}+00$ & $\mathrm{U}$ \\
\hline & ${ }^{90} \mathrm{Sr}$ & $7.4 \mathrm{E}+00 \pm 1.5 \mathrm{E}+00$ & & & ${ }^{90} \mathrm{Sr}$ & $-1.0 \mathrm{E}-01 \pm 1.0 \mathrm{E}+00$ & $\mathrm{U}$ \\
\hline & ${ }^{65} \mathrm{Zn}$ & $-2.3 \mathrm{E}-01 \pm 2.3 \mathrm{E}+00$ & $\mathrm{U}$ & & ${ }^{65} \mathrm{Zn}$ & $-8.9 \mathrm{E}+00 \pm 1.2 \mathrm{E}+01$ & $\mathrm{U}$ \\
\hline
\end{tabular}

$\overline{\mathrm{RQ}^{*}=\text { Result Qualifier. }} \mathrm{U}=$ The analyte was analyzed for but not detected. 


\section{Table 5-2. Historical N-Springs Shoreline Tritium Concentrations}

(pCi/L \pm overall analytical uncertainty).

\begin{tabular}{|c|c|c|c|c|c|c|c|}
\hline Year & Y301 & Y302 & $\begin{array}{c}\text { Effluent } \\
\text { monitoring well }\end{array}$ & Y303 & Y304 & Y305 & Y306 \\
\hline 1987 & $6.8 \mathrm{E}+04$ & $7.6 \mathrm{E}+04$ & $9.5 \mathrm{E}+04$ & $9.2 \mathrm{E}+04$ & $9.4 \mathrm{E}+04$ & $8.8 \mathrm{E}+04$ & $7.9 \mathrm{E}+04$ \\
\hline 1988 & $5.7 \mathrm{E}+03$ & $2.8 \mathrm{E}+04$ & $7.5 \mathrm{E}+04$ & $6.9 \mathrm{E}+04$ & $7.4 \mathrm{E}+04$ & NS & NS \\
\hline 1989 & $2.5 \mathrm{E}+04$ & $2.8 \mathrm{E}+04$ & $3.9 \mathrm{E}+04$ & $3.6 \mathrm{E}+04$ & $5.0 \mathrm{E}+04$ & NS & $6.8 \mathrm{E}+04$ \\
\hline 1990 & $2.9 \mathrm{E}+04$ & $3.2 \mathrm{E}+04$ & $3.8 \mathrm{E}+04$ & $3.6 \mathrm{E}+04$ & NS & NS & $3.4 \mathrm{E}+03$ \\
\hline 1991 & $2.2 \mathrm{E}+02$ & $8.4 \mathrm{E}+01$ & $3.7 \mathrm{E}+04$ & $2.6 \mathrm{E}+03$ & $3.4 \mathrm{E}+04$ & NS & $4.0 \mathrm{E}+02$ \\
\hline 1992 & $7.2 \mathrm{E}+02$ & NS & $5.0 \mathrm{E}+04$ & 9.5E-01 & NS & NS & $1.5 \mathrm{E}+02$ \\
\hline 1993 & $2.8 \mathrm{E}+02$ & $1.3 \mathrm{E}+02$ & $2.7 \mathrm{E}+04 \pm 2.1 \mathrm{E}+03$ & $1.4 \mathrm{E}+02$ & $5.6 \mathrm{E}+02$ & $1.0 \mathrm{E}+02$ & $1.8 \mathrm{E}+02$ \\
\hline 1994 & NS & $4.0 \mathrm{E}+01 \pm 1.9 \mathrm{E}+02$ & $2.6 \mathrm{E}+04 \pm 2.1 \mathrm{E}+03$ & $4.0 \mathrm{E}+01$ & NS & NS & $1.2 \mathrm{E}+02 \pm 2.0 \mathrm{E}+02$ \\
\hline 1995 & NS & NS & $5.2 \mathrm{E}+03$ & $-1.2 \mathrm{E}+01 \pm 1.2 \mathrm{E}+02$ & NS & NS & $-4.4 \mathrm{E}+01 \pm 1.5 \mathrm{E}+02$ \\
\hline 1996 & $2.5 \mathrm{E}+02$ & $8.5 \mathrm{E}+02 \pm 2.5 \mathrm{E}+02$ & $2.0 \mathrm{E}+04 \pm 1.6 \mathrm{E}+03$ & $1.6 \mathrm{E}+04 \pm 1.3 \mathrm{E}+03$ & $4.2 \mathrm{E}+03 \pm 5.0 \mathrm{E}+02$ & $1.6 \mathrm{E}+02 \pm 2.2 \mathrm{E}+02$ & $2.2 \mathrm{E}+02 \pm 2.1 \mathrm{E}+02$ \\
\hline 1997 & $-4.3 \mathrm{E}+01 \pm 5.6 \mathrm{E}+01$ & $3.6 \mathrm{E}+01 \pm 3.2 \mathrm{E}+01$ & $1.6 \mathrm{E}+04 \pm 1.3 \mathrm{E}+03$ & $3.0 \mathrm{E}+03 \pm 6.0 \mathrm{E}+02$ & $-6.3 \mathrm{E}+02 \pm 1.1 \mathrm{E}+03$ & $-5.7 \mathrm{E}+01 \pm 9.1 \mathrm{E}+01$ & $-1.1 \mathrm{E}+02 \pm 5.4 \mathrm{E}+02$ \\
\hline 1998 & NS & $4.6 \mathrm{E}+02 \pm 2.1 \mathrm{E}+02$ & $1.6 \mathrm{E}+04 \pm 5.1 \mathrm{E}+03$ & $1.4 \mathrm{E}+02 \pm 3.6 \mathrm{E}+01$ & NS & $3.7 \mathrm{E}+02 \pm 2.6 \mathrm{E}+02$ & $4.3 \mathrm{E}+02 \pm 2.2 \mathrm{E}+02$ \\
\hline 1999 & $9.7 \mathrm{E}+01 \pm 7.4 \mathrm{E}+01$ & $1.0 \mathrm{E}+02 \pm 7.0 \mathrm{E}+01$ & $1.3 \mathrm{E}+02 \pm 7.9 \mathrm{E}+01$ & $1.9 \mathrm{E}+02 \pm 9.7 \mathrm{E}+01$ & NS & 4.7E+01 $\pm 4.7 \mathrm{E}+01$ & $3.6 \mathrm{E}+00 \pm 7.6 \mathrm{E}+00$ \\
\hline 2000 & $1.3 \mathrm{E}+03 \pm 3.3 \mathrm{E}+02$ & $2.8 \mathrm{E}+02 \pm 1.5 \mathrm{E}+02$ & $7.0 \mathrm{E}+03 \pm 3.1 \mathrm{E}+03$ & $2.8 \mathrm{E}+02 \pm 1.5 \mathrm{E}+02$ & $2.8 \mathrm{E}+02 \pm 1.4 \mathrm{E}+02$ & $2.0 \mathrm{E}+02 \pm 1.4 \mathrm{E}+02$ & $2.0 \mathrm{E}+02 \pm 1.4 \mathrm{E}+02$ \\
\hline 2001 & 9.9E-01 $\pm 8.9 \mathrm{E}-01$ & $9.9 \mathrm{E}-01 \pm 7.9 \mathrm{E}-01$ & $5.0 \mathrm{E}+03 \pm 5.1 \mathrm{E}+02$ & $9.6 \mathrm{E}+01 \pm 6.7 \mathrm{E}+01$ & NS & $5.9 \mathrm{E}+01 \pm 6.2 \mathrm{E}+01$ & $5.0 \mathrm{E}+01 \pm 6.5 \mathrm{E}+01$ \\
\hline 2002 & $-3.0 \mathrm{E}+02 \pm 4.8 \mathrm{E}+02$ & $-3.2 \mathrm{E}+02 \pm 5.1 \mathrm{E}+02$ & $2.9 \mathrm{E}+02 \pm 2.9 \mathrm{E}+01^{\mathrm{a}}$ & $-4.7 \mathrm{E}+02 \pm 8.0 \mathrm{E}+02$ & NS & $-4.9 \mathrm{E}+02 \pm 4.9 \mathrm{E}+02$ & $-3.6 \mathrm{E}+02 \pm 3.6 \mathrm{E}+02$ \\
\hline 2003 & NS & $2.4 \mathrm{E}+01 \pm 5.4 \mathrm{E}+01$ & $6.3 \mathrm{E}+02 \pm 6.7 \mathrm{E}+02^{\mathrm{a}}$ & $4.5 \mathrm{E}-01 \pm 2.4 \mathrm{E}+00$ & NS & $1.9 \mathrm{E}+01 \pm 4.9 \mathrm{E}+01$ & $-7.5 \mathrm{E}+01 \pm 1.2 \mathrm{E}+02$ \\
\hline Year & Y307 & Y308 & Y309 & Y310 & Y311 & Y312 & Y313 \\
\hline 1987 & $7.3 \mathrm{E}+04$ & $4.6 \mathrm{E}+04$ & $7.5 \mathrm{E}+04$ & $4.0 \mathrm{E}+03$ & $5.8 \mathrm{E}+04$ & $2.1 \mathrm{E}+04$ & $1.3 \mathrm{E}+03$ \\
\hline 1988 & $1.1 \mathrm{E}+04$ & $3.0 \mathrm{E}+04$ & $1.0 \mathrm{E}+04$ & NS & $2.9 \mathrm{E}+04$ & $1.9 \mathrm{E}+04$ & $3.0 \mathrm{E}+03$ \\
\hline 1989 & NS & $7.7 \mathrm{E}+04$ & $7.0 \mathrm{E}+04$ & $3.5 \mathrm{E}+04$ & $4.2 \mathrm{E}+04$ & NS & NS \\
\hline 1990 & NS & $1.4 \mathrm{E}+04$ & $3.5 \mathrm{E}+03$ & $9.7 \mathrm{E}+03$ & $3.8 \mathrm{E}+04$ & $2.0 \mathrm{E}+04$ & NS \\
\hline 1991 & $8.1 \mathrm{E}+02$ & $2.1 \mathrm{E}+03$ & $6.5 \mathrm{E}+03$ & $7.9 \mathrm{E}+02$ & $7.1 \mathrm{E}+02$ & $2.4 \mathrm{E}+03$ & $9.3 \mathrm{E}+00$ \\
\hline 1992 & NS & NS & $3.0 \mathrm{E}+02$ & $4.3 \mathrm{E}+02$ & $6.5 \mathrm{E}+02$ & $1.7 \mathrm{E}+02$ & NS \\
\hline 1993 & NS & NS & NS & NS & NS & NS & NS \\
\hline 1994 & $8.5 \mathrm{E}+01 \pm 1.9 \mathrm{E}+02$ & $1.3 \mathrm{E}+02 \pm 2.0 \mathrm{E}+02$ & $8.4 \mathrm{E}+01 \pm 1.9 \mathrm{E}+02$ & $4.0 \mathrm{E}+02 \pm 2.1 \mathrm{E}+02$ & $4.5 \mathrm{E}+02 \pm 2.1 \mathrm{E}+02$ & $2.9 \mathrm{E}+02 \pm 2.0 \mathrm{E}+02$ & NS \\
\hline 1995 & $-2.1 \mathrm{E}+00 \pm 2.1 \mathrm{E}+01$ & $-2.3 \mathrm{E}+01 \pm 1.4 \mathrm{E}+02$ & $-3.1 \mathrm{E}+01 \pm 1.4 \mathrm{E}+02$ & $-1.2 \mathrm{E}+01 \pm 1.2 \mathrm{E}+02$ & $3.2 \mathrm{E}+02 \pm 1.6 \mathrm{E}+02$ & $5.0 \mathrm{E}+02 \pm 1.8 \mathrm{E}+02$ & NS \\
\hline 1996 & $1.9 \mathrm{E}+02 \pm 2.1 \mathrm{E}+02$ & $2.4 \mathrm{E}+02 \pm 2.1 \mathrm{E}+02$ & NS & NS & $2.2 \mathrm{E}+02 \pm 2.1 \mathrm{E}+02$ & NS & NS \\
\hline 1997 & $-1.4 \mathrm{E}+02 \pm 1.4 \mathrm{E}+03$ & $-1.2 \mathrm{E}+02 \pm 8.2 \mathrm{E}+02$ & $-6.4 \mathrm{E}+01 \pm 1.0 \mathrm{E}+02$ & $-1.1 \mathrm{E}+02 \pm 5.4 \mathrm{E}+02$ & $2.6 \mathrm{E}+01 \pm 2.1 \mathrm{E}+01$ & NS & NS \\
\hline 1998 & $3.5 \mathrm{E}+02 \pm 2.6 \mathrm{E}+02$ & NS & $3.5 \mathrm{E}+02 \pm 2.7 \mathrm{E}+02$ & $3.0 \mathrm{E}+02 \pm 2.1 \mathrm{E}+02$ & $5.6 \mathrm{E}+02 \pm 2.5 \mathrm{E}+02$ & $6.2 \mathrm{E}+02 \pm 2.5 \mathrm{E}+02$ & $5.2 \mathrm{E}+02 \pm 2.6 \mathrm{E}+02$ \\
\hline 1999 & $2.7 \mathrm{E}+02 \pm 1.1 \mathrm{E}+02$ & $1.1 \mathrm{E}+02 \pm 8.8 \mathrm{E}+01$ & NS & $1.3 \mathrm{E}+02 \pm 8.5 \mathrm{E}+01$ & $1.8 \mathrm{E}+02 \pm 9.9 \mathrm{E}+01$ & $1.5 \mathrm{E}+02 \pm 9.8 \mathrm{E}+01$ & NS \\
\hline 2000 & $3.0 \mathrm{E}+02 \pm 1.5 \mathrm{E}+02$ & $2.4 \mathrm{E}+02 \pm 1.4 \mathrm{E}+02$ & $1.9 \mathrm{E}+02 \pm 1.3 \mathrm{E}+02$ & $2.4 \mathrm{E}+02 \pm 1.4 \mathrm{E}+02$ & $4.0 \mathrm{E}+02 \pm 1.6 \mathrm{E}+02$ & $3.7 \mathrm{E}+02 \pm 1.7 \mathrm{E}+02$ & $2.5 \mathrm{E}+02 \pm 1.4 \mathrm{E}+02$ \\
\hline 2001 & $9.7 \mathrm{E}+01 \pm 9.9 \mathrm{E}+01$ & NS & $1.9 \mathrm{E}+02 \pm 1.1 \mathrm{E}+02$ & $8.8 \mathrm{E}+01 \pm 9.7 \mathrm{E}+01$ & $5.3 \mathrm{E}+01 \pm 8.5 \mathrm{E}+01$ & $9.9 \mathrm{E}-01 \pm 2.4 \mathrm{E}+00$ & NS \\
\hline 2002 & $-4.3 \mathrm{E}+02 \pm 4.3 \mathrm{E}+02$ & $-3.2 \mathrm{E}+02 \pm 3.8 \mathrm{E}+02$ & $-3.5 \mathrm{E}+02 \pm 3.9 \mathrm{E}+02$ & $-3.4 \mathrm{E}+02 \pm 3.4 \mathrm{E}+02$ & $-2.6 \mathrm{E}+02 \pm 3.6 \mathrm{E}+02$ & $-2.3 \mathrm{E}+02 \pm 4.3 \mathrm{E}+02$ & NS \\
\hline 2003 & $4.5 \mathrm{E}-01 \pm 3.6 \mathrm{E}+00$ & $-1.8 \mathrm{E}+00 \pm 7.7 \mathrm{E}+00$ & $-1.4 \mathrm{E}+02 \pm 1.7 \mathrm{E}+02$ & NS & $1.9 \mathrm{E}+01 \pm 2.1 \mathrm{E}+01$ & $-5.9 \mathrm{E}+01 \pm 5.9 \mathrm{E}+02$ & $-1.2 \mathrm{E}+02 \pm 1.3 \mathrm{E}+02$ \\
\hline
\end{tabular}

NS - Not sampled.

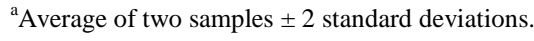




\section{Table 5-3. Historical N-Springs Shoreline Strontium-90 Concentrations}

(pCi/L \pm overall analytical uncertainty).

\begin{tabular}{|c|c|c|c|c|c|c|c|}
\hline Year & Y301 & Y302 & $\begin{array}{c}\text { Effluent } \\
\text { monitoring well }\end{array}$ & Y303 & Y304 & Y305 & Y306 \\
\hline 1987 & $1.7 \mathrm{E}+03$ & $2.7 \mathrm{E}+03$ & $6.1 \mathrm{E}+03$ & $8.3 E+03$ & $4.1 \mathrm{E}+03$ & $9.5 \mathrm{E}+02$ & $7.2 \mathrm{E}+02$ \\
\hline 1988 & $8.7 \mathrm{E}+02$ & $3.0 \mathrm{E}+03$ & $7.9 \mathrm{E}+03$ & $9.1 \mathrm{E}+03$ & $3.5 \mathrm{E}+03$ & NS & NS \\
\hline 1989 & $9.8 \mathrm{E}+02$ & $2.1 \mathrm{E}+03$ & $6.5 \mathrm{E}+03$ & $5.4 \mathrm{E}+03$ & $3.8 \mathrm{E}+03$ & NS & $8.9 E+02$ \\
\hline 1990 & $2.4 \mathrm{E}+03$ & $2.9 \mathrm{E}+03$ & $4.9 \mathrm{E}+03$ & $7.1 \mathrm{E}+03$ & NS & NS & $1.5 \mathrm{E}+02$ \\
\hline 1991 & $1.6 \mathrm{E}+01$ & $2.4 \mathrm{E}+01$ & $6.9 \mathrm{E}+03$ & $1.4 \mathrm{E}+03$ & $3.2 \mathrm{E}+03$ & NS & $8.6 \mathrm{E}+01$ \\
\hline 1992 & NS & NS & $6.3 \mathrm{E}+03$ & $1.5 \mathrm{E}+02$ & NS & NS & $9.6 \mathrm{E}+00$ \\
\hline 1993 & $1.2 \mathrm{E}+01$ & $8.3 \mathrm{E}+01$ & $7.4 \mathrm{E}+03 \pm 1.3 \mathrm{E}+03$ & $1.2 \mathrm{E}+02$ & $4.1 \mathrm{E}+03$ & $4.1 \mathrm{E}+01$ & $1.3 \mathrm{E}+01$ \\
\hline 1994 & NS & $1.1 \mathrm{E}+02 \pm 2.6 \mathrm{E}+01$ & $6.6 \mathrm{E}+03 \pm 1.4 \mathrm{E}+03$ & $1.2+\mathrm{E} 02$ & NS & NS & $6.4 \mathrm{E}+00 \pm 1.7 \mathrm{E}+00$ \\
\hline 1995 & NS & NS & $5.7 \mathrm{E}+03 \pm 1.4 \mathrm{E}+03$ & $3.0 \mathrm{E}+02 \pm 5.1 \mathrm{E}+01$ & NS & NS & $7.0 \mathrm{E}+00 \pm 1.4 \mathrm{E}+00$ \\
\hline 1996 & $5.8 \mathrm{E}+01$ & $2.6 \mathrm{E}+02 \pm 6.5 \mathrm{E}+01$ & $1.4 \mathrm{E}+04 \pm 4.1 \mathrm{E}+03$ & $5.8 \mathrm{E}+03 \pm 1.6 \mathrm{E}+03$ & $9.5 \mathrm{E}+02 \pm 2.6 \mathrm{E}+02$ & $3.7 \mathrm{E}+01 \pm 1.0 \mathrm{E}+01$ & $1.6 \mathrm{E}+01 \pm 4.2 \mathrm{E}+00$ \\
\hline 1997 & $3.1 \mathrm{E}+01 \pm 4.7 \mathrm{E}+00$ & $2.0 \mathrm{E}+02 \pm 2.8 \mathrm{E}+01$ & $1.0 \mathrm{E}+04 \pm 3.5 \mathrm{E}+03$ & $3.2 \mathrm{E}+03 \pm 3.8 \mathrm{E}+02$ & $1.7 \mathrm{E}+02 \pm 2.2 \mathrm{E}+01$ & $2.6 \mathrm{E}+01 \pm 4.7 \mathrm{E}+00$ & $3.1 \mathrm{E}+00 \pm 1.6 \mathrm{E}+00$ \\
\hline 1998 & NS & $1.1 \mathrm{E}+02 \pm 1.3 \mathrm{E}+01$ & $1.4 \mathrm{E}+04 \pm 2.1 \mathrm{E}+03$ & $1.9 \mathrm{E}+03 \pm 2.3 \mathrm{E}+02$ & NS & $1.7 \mathrm{E}+01 \pm 2.6 \mathrm{E}+00$ & $7.7 \mathrm{E}+00 \pm 1.5 \mathrm{E}+00$ \\
\hline 1999 & $7.1 \mathrm{E}+00 \pm 1.4 \mathrm{E}+00$ & $4.9 \mathrm{E}+01 \pm 7.4 \mathrm{E}+00$ & $3.2 \mathrm{E}+03 \pm 4.8 \mathrm{E}+02$ & $1.3 \mathrm{E}+03 \pm 2.0 \mathrm{E}+02$ & NS & $3.0 \mathrm{E}+01 \pm 4.5 \mathrm{E}+00$ & $8.1 \mathrm{E}+00 \pm 1.6 \mathrm{E}+00$ \\
\hline 2000 & $8.3 \mathrm{E}+00 \pm 1.7 \mathrm{E}+00$ & $1.1 \mathrm{E}+01 \pm 1.6 \mathrm{E}+00$ & $1.3 \mathrm{E}+04 \pm 4.0 \mathrm{E}+03$ & $1.3 \mathrm{E}+02 \pm 2.6 \mathrm{E}+01$ & $1.8 \mathrm{E}+02 \pm 2.7 \mathrm{E}+01$ & $7.1 \mathrm{E}+00 \pm 1.4 \mathrm{E}+00$ & $4.0 \mathrm{E}+00 \pm 1.0 \mathrm{E}+00$ \\
\hline 2001 & $4.3 \mathrm{E}+00 \pm 8.6 \mathrm{E}-01$ & $1.9 \mathrm{E}+01 \pm 2.8 \mathrm{E}+00$ & $9.7 \mathrm{E}+03 \pm 2.2 \mathrm{E}+03$ & $4.5 \mathrm{E}+01 \pm 6.8 \mathrm{E}+00$ & NS & $9.6 \mathrm{E}+00 \pm 1.9 \mathrm{E}+00$ & $3.3 \mathrm{E}+00 \pm 8.2 \mathrm{E}-01$ \\
\hline 2002 & $5.2 \mathrm{E}+00 \pm 1.0 \mathrm{E}+00$ & $2.2 \mathrm{E}+01 \pm 4.4 \mathrm{E}+00$ & $3.7 \mathrm{E}+03 \pm 3.7 \mathrm{E}+02^{\mathrm{a}}$ & $8.2 \mathrm{E}+01 \pm 1.6 \mathrm{E}+01$ & NS & $5.2 \mathrm{E}+00 \pm 1.0 \mathrm{E}+00$ & $1.6 \mathrm{E}+00 \pm 5.6 \mathrm{E}-01$ \\
\hline 2003 & NS & $2.3 \mathrm{E}+01 \pm 3.4 \mathrm{E}+00$ & $4.1 \mathrm{E}+03 \pm 2.0 \mathrm{E}+03^{\mathrm{a}}$ & $5.9 \mathrm{E}+00 \pm 1.5 \mathrm{E}+00$ & NS & $3.8 \mathrm{E}+00 \pm 1.3 \mathrm{E}+00$ & $2.0 \mathrm{E}+00 \pm 7.0 \mathrm{E}-01$ \\
\hline Year & Y307 & Y308 & Y309 & Y310 & Y311 & Y312 & Y313 \\
\hline 1987 & $1.3 \mathrm{E}+01$ & $4.2 \mathrm{E}+01$ & $2.4 \mathrm{E}+02$ & $5.7 \mathrm{E}+01$ & $6.6 \mathrm{E}+02$ & $5.8 \mathrm{E}+01$ & $5.0 \mathrm{E}+01$ \\
\hline 1988 & $1.5 \mathrm{E}+01$ & $3.2 \mathrm{E}+01$ & $4.1 \mathrm{E}+01$ & NS & $3.4 \mathrm{E}+02$ & $4.0 \mathrm{E}+01$ & $5.8 \mathrm{E}+01$ \\
\hline 1989 & NS & $7.8 \mathrm{E}+01$ & $2.9 \mathrm{E}+02$ & $1.6 \mathrm{E}+02$ & $9.5 \mathrm{E}+02$ & NS & NS \\
\hline 1990 & NS & $9.0 \mathrm{E}+01$ & $4.4 \mathrm{E}+01$ & $3.1 \mathrm{E}+01$ & $5.8 \mathrm{E}+02$ & $5.4 \mathrm{E}+01$ & NS \\
\hline 1991 & $1.4 \mathrm{E}+01$ & $2.8 \mathrm{E}+01$ & $1.0 \mathrm{E}+02$ & $1.5 \mathrm{E}+01$ & $4.0 \mathrm{E}+02$ & $8.9 \mathrm{E}+00$ & $8.1 \mathrm{E}+00$ \\
\hline 1992 & NS & NS & $8.1 \mathrm{E}+00$ & $6.7 \mathrm{E}+00$ & $1.1 \mathrm{E}+02$ & $7.1 \mathrm{E}+00$ & NS \\
\hline 1993 & NS & NS & NS & NS & NS & NS & NS \\
\hline 1994 & $3.8 \mathrm{E}+00 \pm 8.7 \mathrm{E}-01$ & $1.2 \mathrm{E}+01 \pm 2.5 \mathrm{E}+00$ & $3.4 \mathrm{E}+00 \pm 9.2 \mathrm{E}-01$ & $3.8 \mathrm{E}+00 \pm 1.0 \mathrm{E}+00$ & $5.1 \mathrm{E}+01 \pm 1.1 \mathrm{E}+01$ & $1.8 \mathrm{E}+01 \pm 4.3 \mathrm{E}+00$ & NS \\
\hline 1995 & $3.8 \mathrm{E}+00 \pm 8.0 \mathrm{E}-01$ & $1.4 \mathrm{E}+01 \pm 2.7 \mathrm{E}+00$ & $5.5 \mathrm{E}+00 \pm 1.2 \mathrm{E}+00$ & $7.0 \mathrm{E}+00 \pm 1.4 \mathrm{E}+00$ & $7.1 \mathrm{E}+01 \pm 1.3 \mathrm{E}+01$ & $1.9 \mathrm{E}+01 \pm 3.6 \mathrm{E}+00$ & NS \\
\hline 1996 & $6.5 \mathrm{E}+00 \pm 1.8 \mathrm{E}+00$ & $2.2 \mathrm{E}+01 \pm 5.7 \mathrm{E}+00$ & NS & NS & $1.7 \mathrm{E}+02 \pm 4.9 \mathrm{E}+01$ & NS & NS \\
\hline 1997 & $3.6 \mathrm{E}-01 \pm 1.9 \mathrm{E}+00$ & $1.1 \mathrm{E}+01 \pm 2.2 \mathrm{E}+00$ & $5.6 \mathrm{E}+00 \pm 1.7 \mathrm{E}+00$ & $7.6 \mathrm{E}-01 \pm 2.0 \mathrm{E}+00$ & $1.5 \mathrm{E}+02 \pm 2.0 \mathrm{E}+01$ & NS & NS \\
\hline 1998 & $1.5 \mathrm{E}+01 \pm 2.3 \mathrm{E}+00$ & NS & $5.1 \mathrm{E}+00 \pm 1.0 \mathrm{E}+00$ & $2.9 \mathrm{E}+00 \pm 8.7 \mathrm{E}-01$ & $1.1 \mathrm{E}+02 \pm 1.4 \mathrm{E}+01$ & $1.8 \mathrm{E}+01 \pm 2.3 \mathrm{E}+00$ & $3.6 \mathrm{E}+00 \pm 1.1 \mathrm{E}+00$ \\
\hline 1999 & $1.9 \mathrm{E}+00 \pm 7.6 \mathrm{E}-01$ & $2.5 \mathrm{E}+00 \pm 7.5 \mathrm{E}-01$ & NS & $4.0 \mathrm{E}+00 \pm 8.8 \mathrm{E}-01$ & $4.3 \mathrm{E}+01 \pm 6.5 \mathrm{E}+00$ & $9.2 \mathrm{E}+00 \pm 1.5 \mathrm{E}+00$ & NS \\
\hline 2000 & $3.3 \mathrm{E}+00 \pm 8.2 \mathrm{E}-01$ & $4.5 \mathrm{E}+00 \pm 1.1 \mathrm{E}+00$ & $2.2 \mathrm{E}+00 \pm 6.6 \mathrm{E}-01$ & $7.0 \mathrm{E}-01 \pm 5.6 \mathrm{E}-01$ & $4.8 \mathrm{E}+01 \pm 1.2 \mathrm{E}+01$ & $3.8 \mathrm{E}+00 \pm 7.6 \mathrm{E}-01$ & $2.0 \mathrm{E}-01 \pm 4.4 \mathrm{E}-01$ \\
\hline 2001 & $3.4 \mathrm{E}+00 \pm 8.5 \mathrm{E}-01$ & NS & $2.5 \mathrm{E}+00 \pm 7.5 \mathrm{E}-01$ & $2.8 \mathrm{E}+00 \pm 8.4 \mathrm{E}-01$ & $3.4 \mathrm{E}+01 \pm 5.1 \mathrm{E}+00$ & $6.0 \mathrm{E}+00 \pm 1.2 \mathrm{E}+00$ & NS \\
\hline 2002 & $6.0 \mathrm{E}-01 \pm 5.1 \mathrm{E}-01$ & $6.9 \mathrm{E}+00 \pm 1.4 \mathrm{E}+00$ & $1.9 \mathrm{E}+00 \pm 5.7 \mathrm{E}-01$ & $1.2 \mathrm{E}+00 \pm 6.6 \mathrm{E}-01$ & $3.1 \mathrm{E}+01 \pm 4.7 \mathrm{E}+00$ & $8.6 \mathrm{E}+00 \pm 1.7 \mathrm{E}+00$ & NS \\
\hline 2003 & $3.8 \mathrm{E}+00 \pm 9.5 \mathrm{E}-01$ & $8.4 \mathrm{E}+00 \pm 1.7 \mathrm{E}+00$ & $1.7 \mathrm{E}+00 \pm 6.8 \mathrm{E}-01$ & NS & $2.1 \mathrm{E}+01 \pm 3.1 \mathrm{E}+00$ & $7.4 \mathrm{E}+00 \pm 1.5 \mathrm{E}+00$ & $-1.0 \mathrm{E}-01 \pm 1.0 \mathrm{E}+00$ \\
\hline
\end{tabular}

NS - Not sampled.

average of two samples \pm 2 standard deviations. 


\subsection{RADIOLOGICAL SURVEYS}

In 2003, there were approximately 3,651 ha (9,022 acres) of posted outdoor contamination areas and 666 ha (1,646 acres) of posted underground radioactive materials areas at the Hanford Site. Survey locations are illustrated in Figures 6-1 through 6-10. These areas were typically associated with cribs, trenches, burial grounds, tank farms, and covered ponds and ditches.

The posted contamination areas vary in number and size between years because of an ongoing effort to clean, stabilize, and remediate areas of known contamination. Concurrently, new areas of contamination are also being identified. Approximately 2 ha (5 acres) in the 200 West Area were reclassified from underground radioactive materials areas to contamination/ soil contamination areas during 2003. Six, small contaminated sites in the 200 East Area and one site in the 200 West Area, all totaling less than 1 ha (2 acres), were stabilized and downposted to underground radioactive materials areas during the year. Several small contaminated areas totaling less than 0.5 ha ( 1 acre) were added to the $100-\mathrm{H}$ Area total.

Remedial actions in the 100 Areas are ongoing, with decommissioning of facilities and cleanup of inactive waste sites ahead of schedule. The amount of area already remediated is not fully reflected in the reporting of outdoor areas posted as soil contamination areas and/or underground radioactive material areas. These areas are in the various stages of sampling, analysis, regulatory approval, and the posting change status.

It was estimated that the external dose rate at $80 \%$ of the identified outdoor contamination areas was less than $1 \mathrm{mrem} / \mathrm{hr}$, although direct dose rate readings from isolated radioactive specks (a diameter less than $0.6 \mathrm{~cm}$ [0.25 in.]) could have been considerably higher. Contamination levels of this magnitude did not significantly add to dose rates for the public or Hanford Site workers in 2003. 


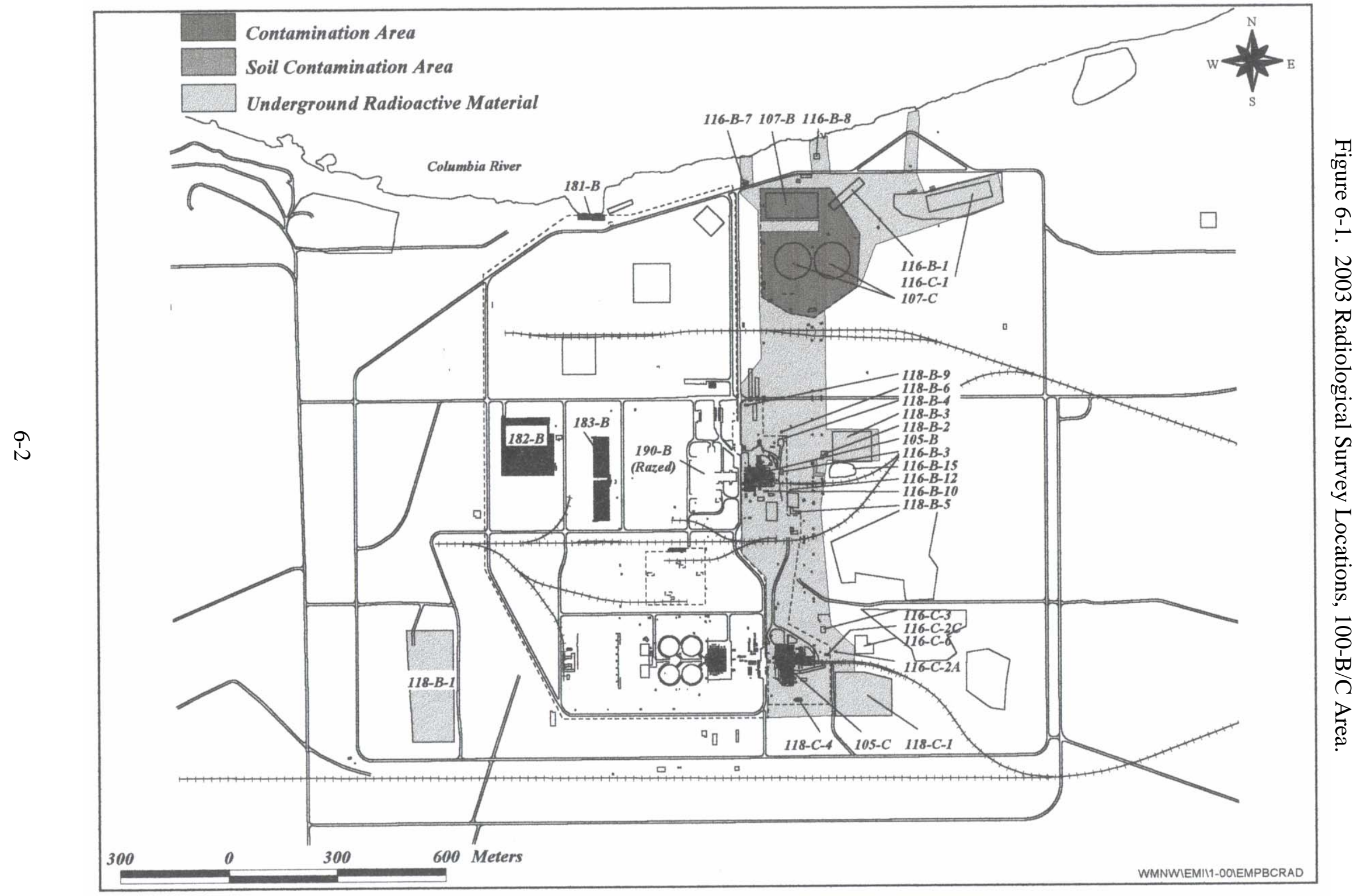




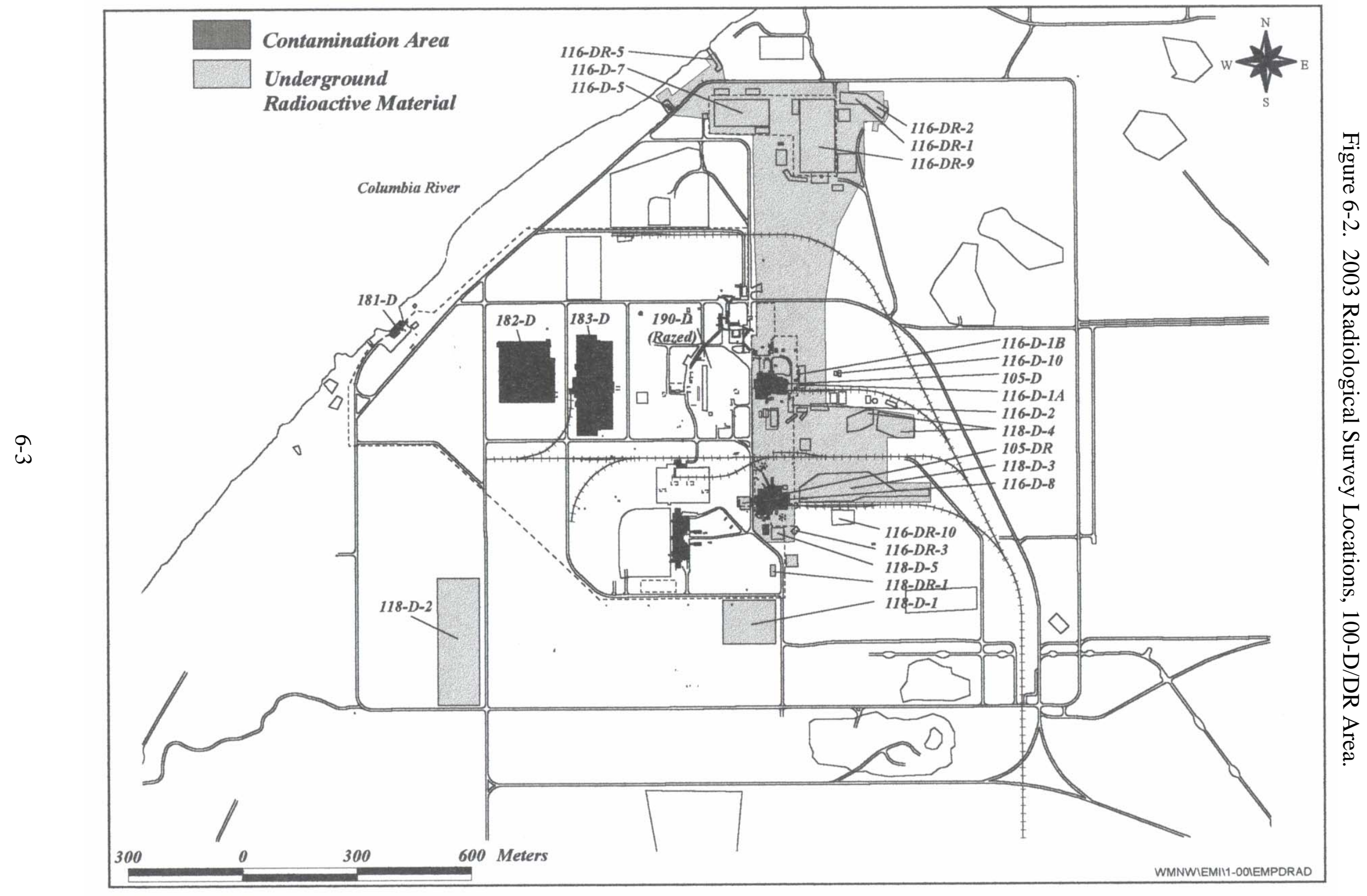




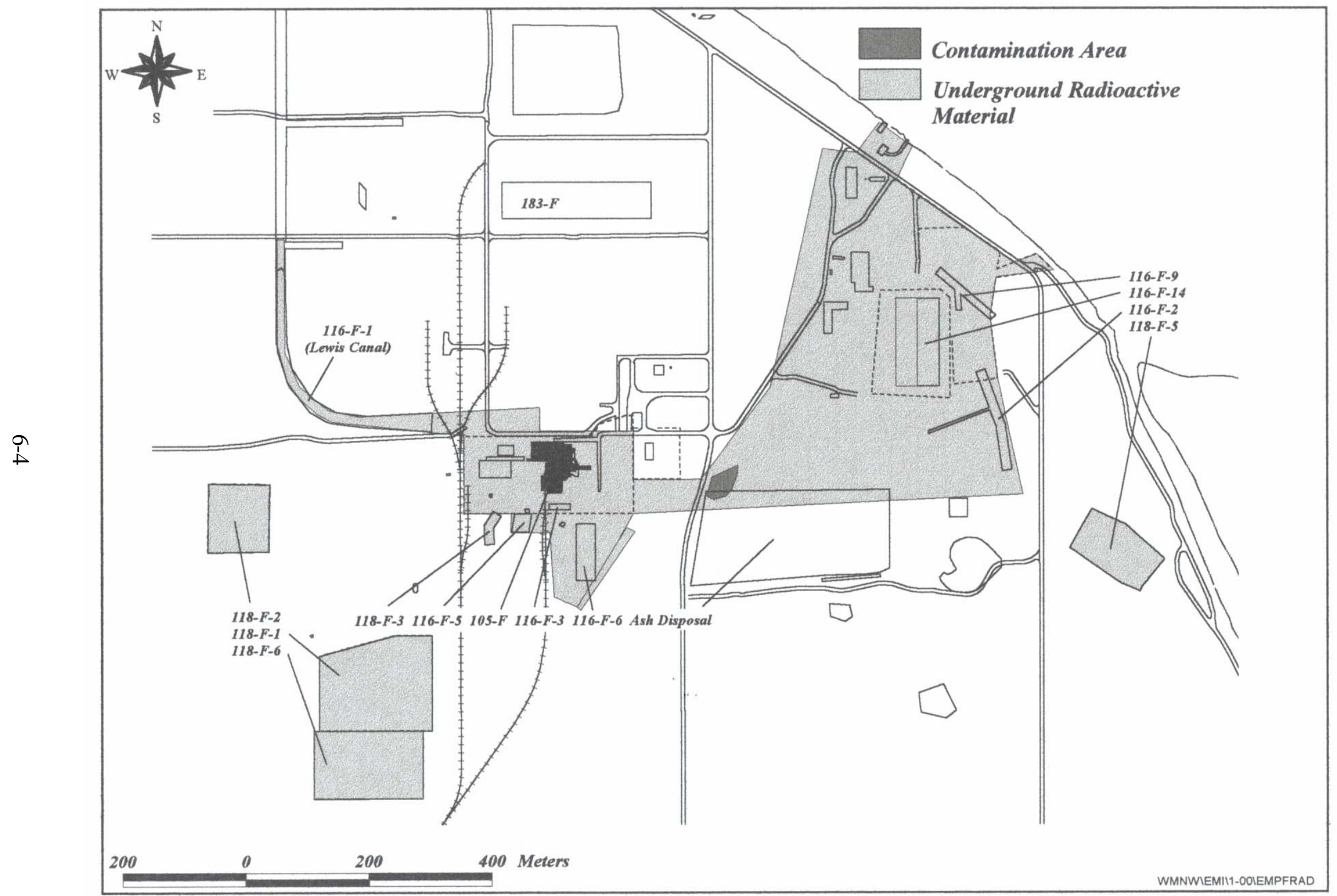

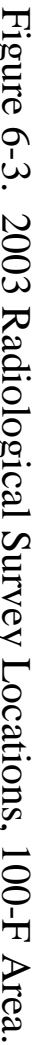




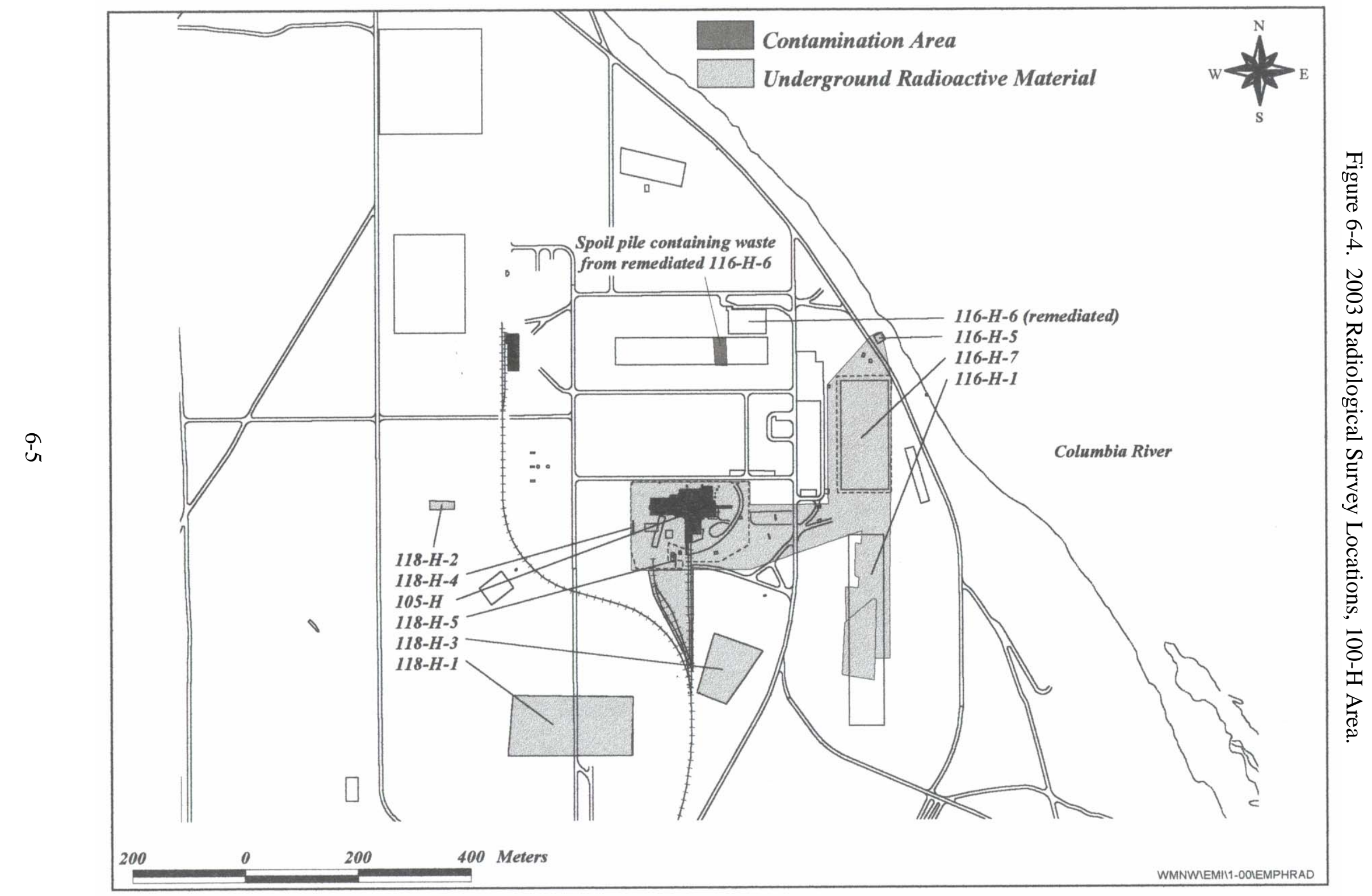




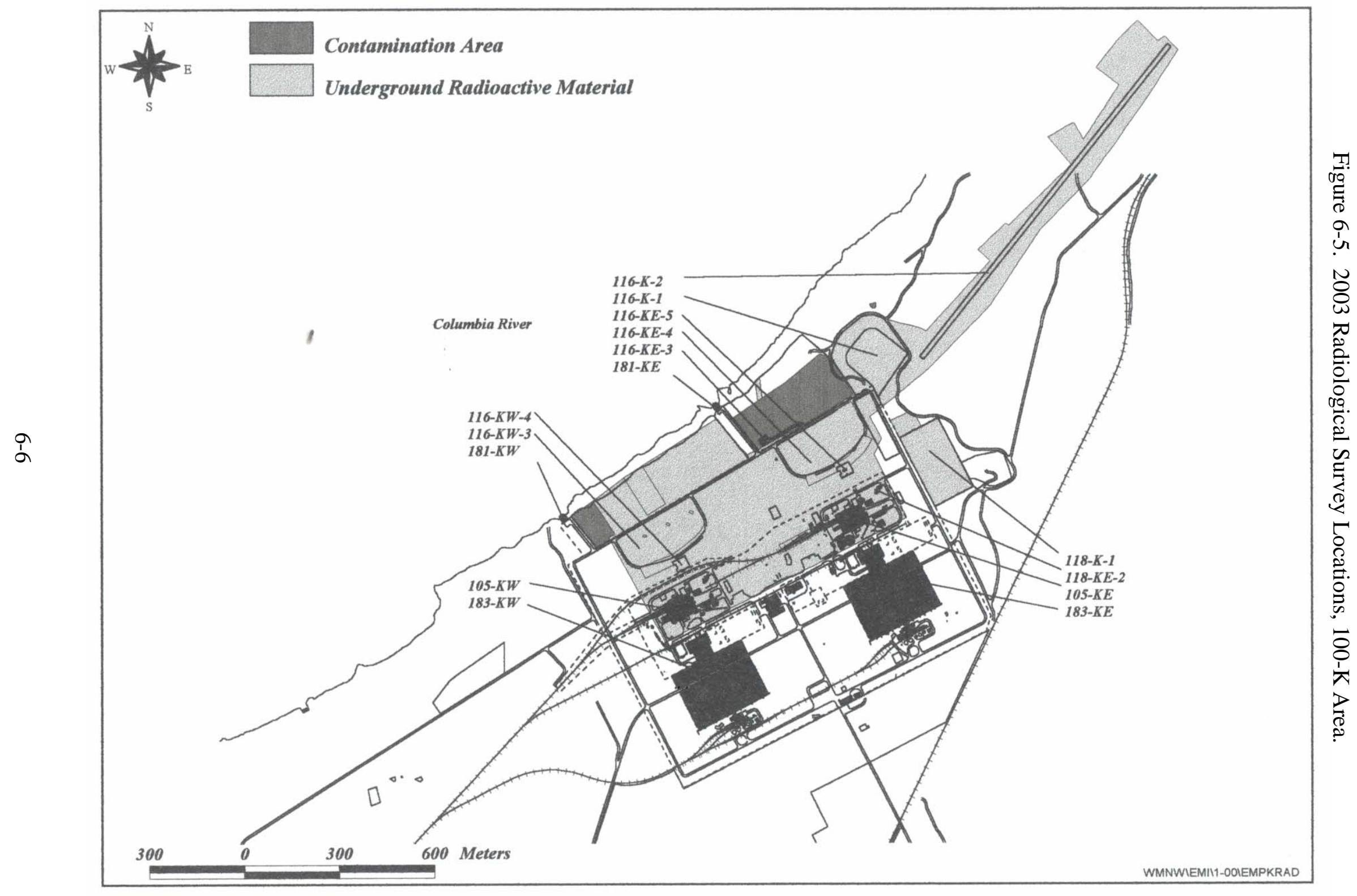




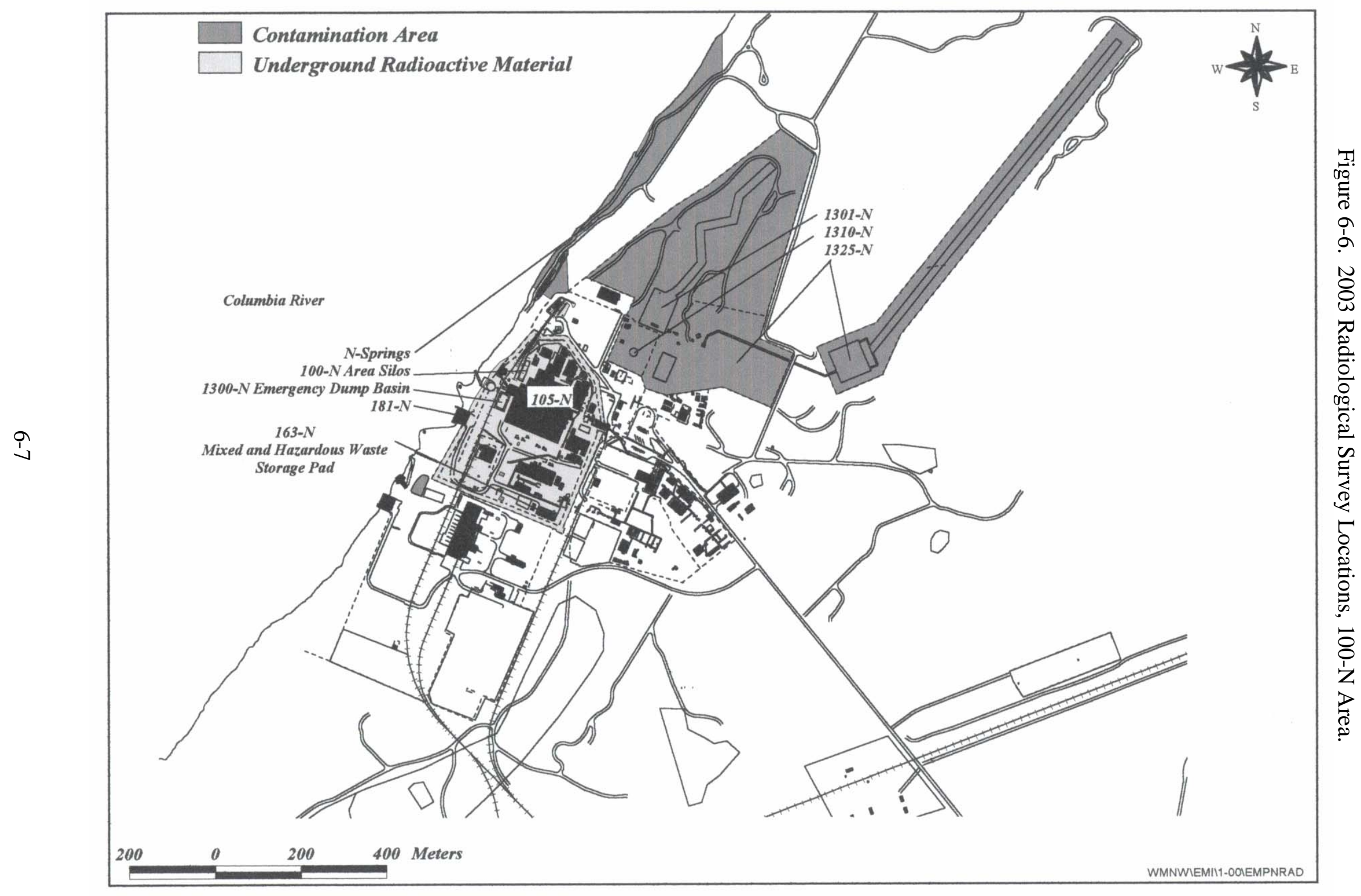




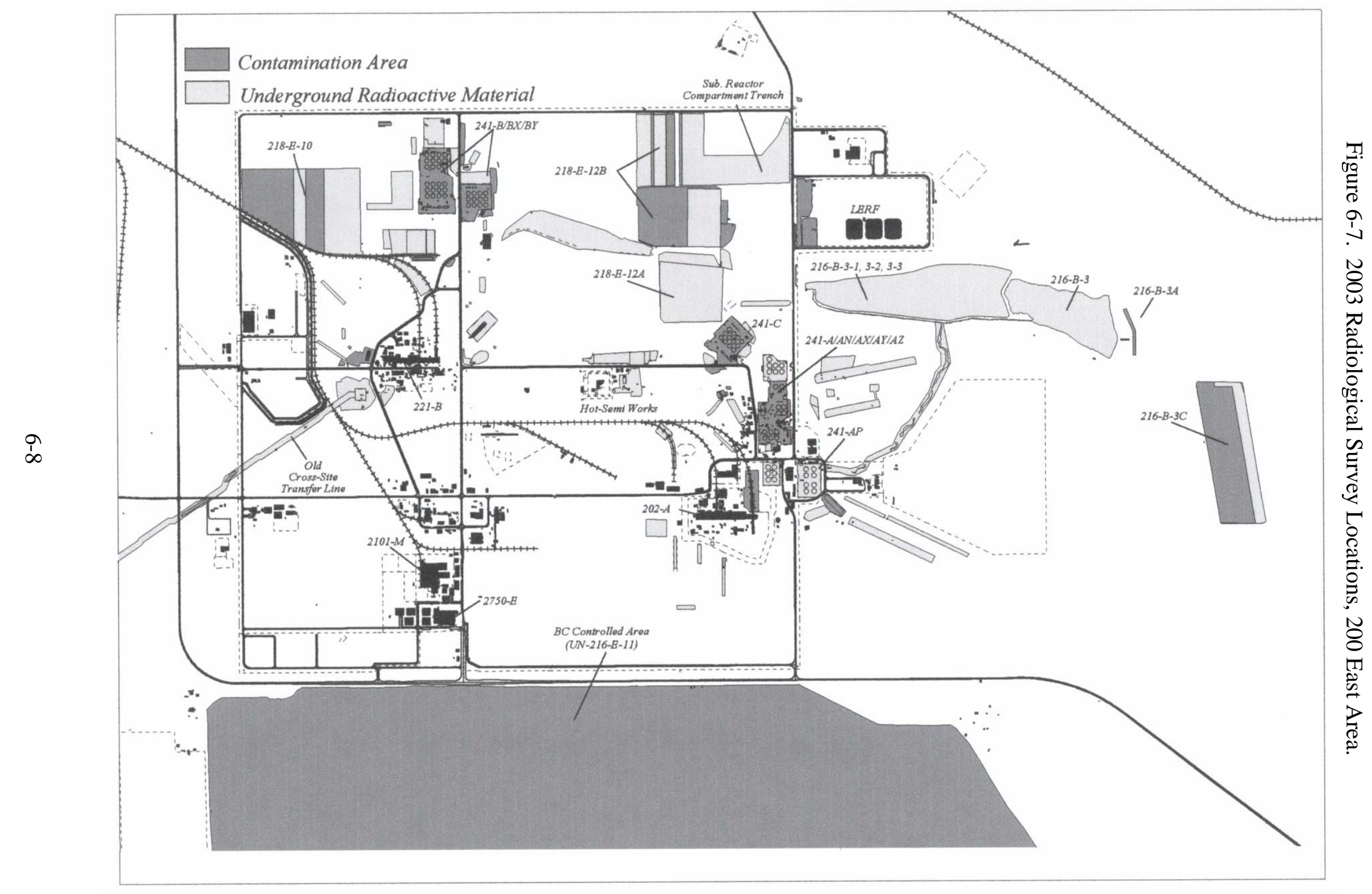




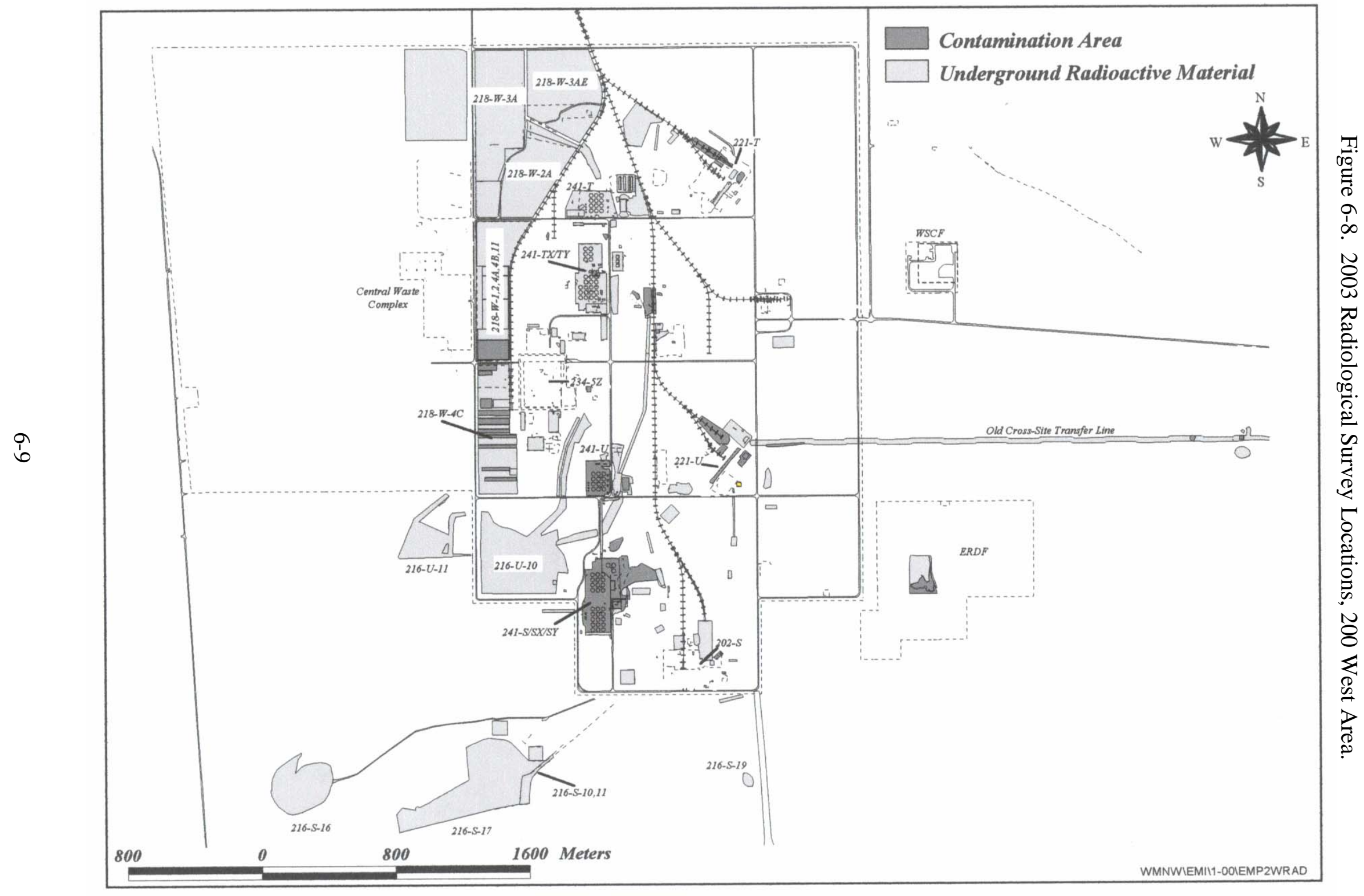




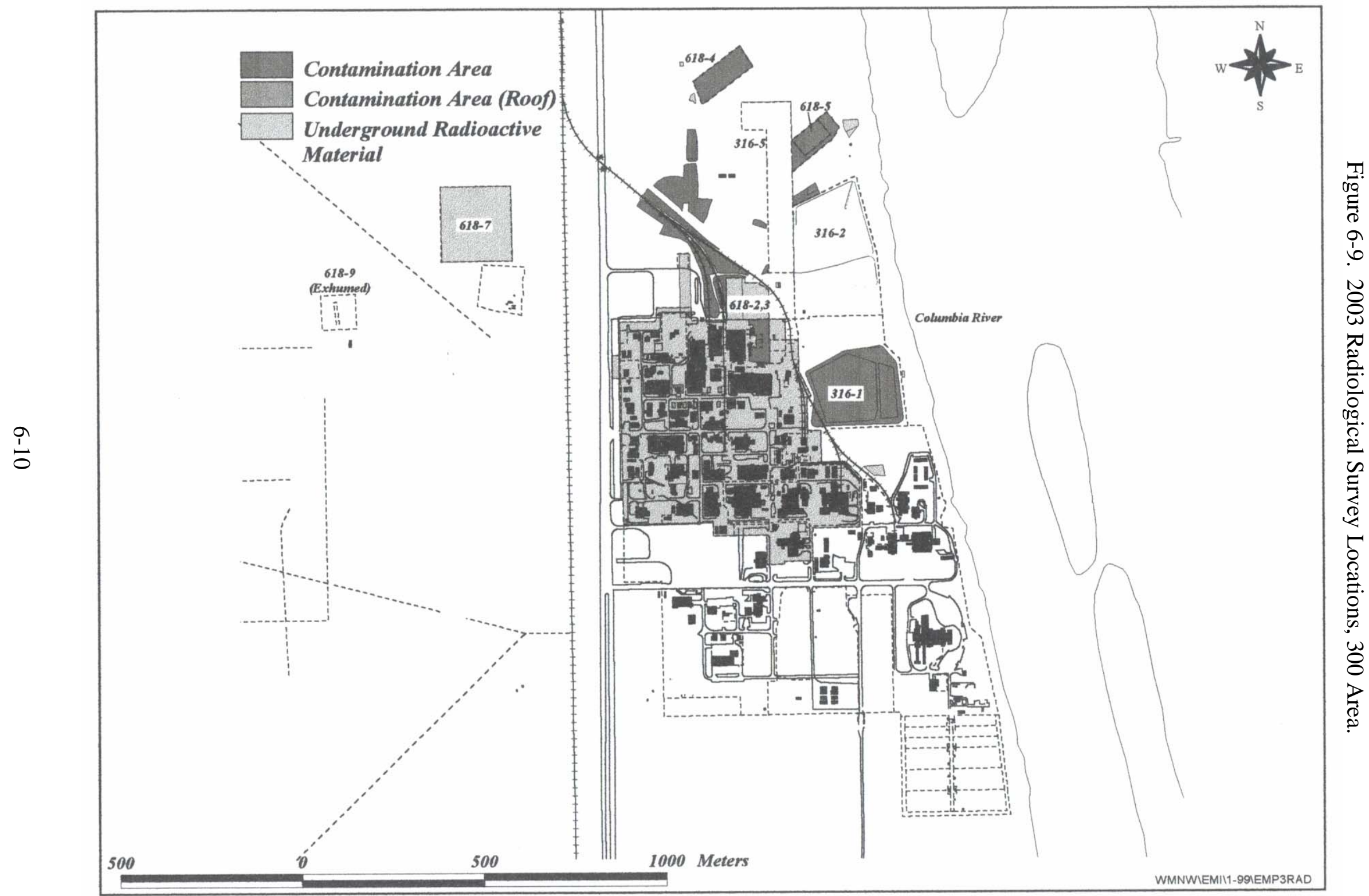




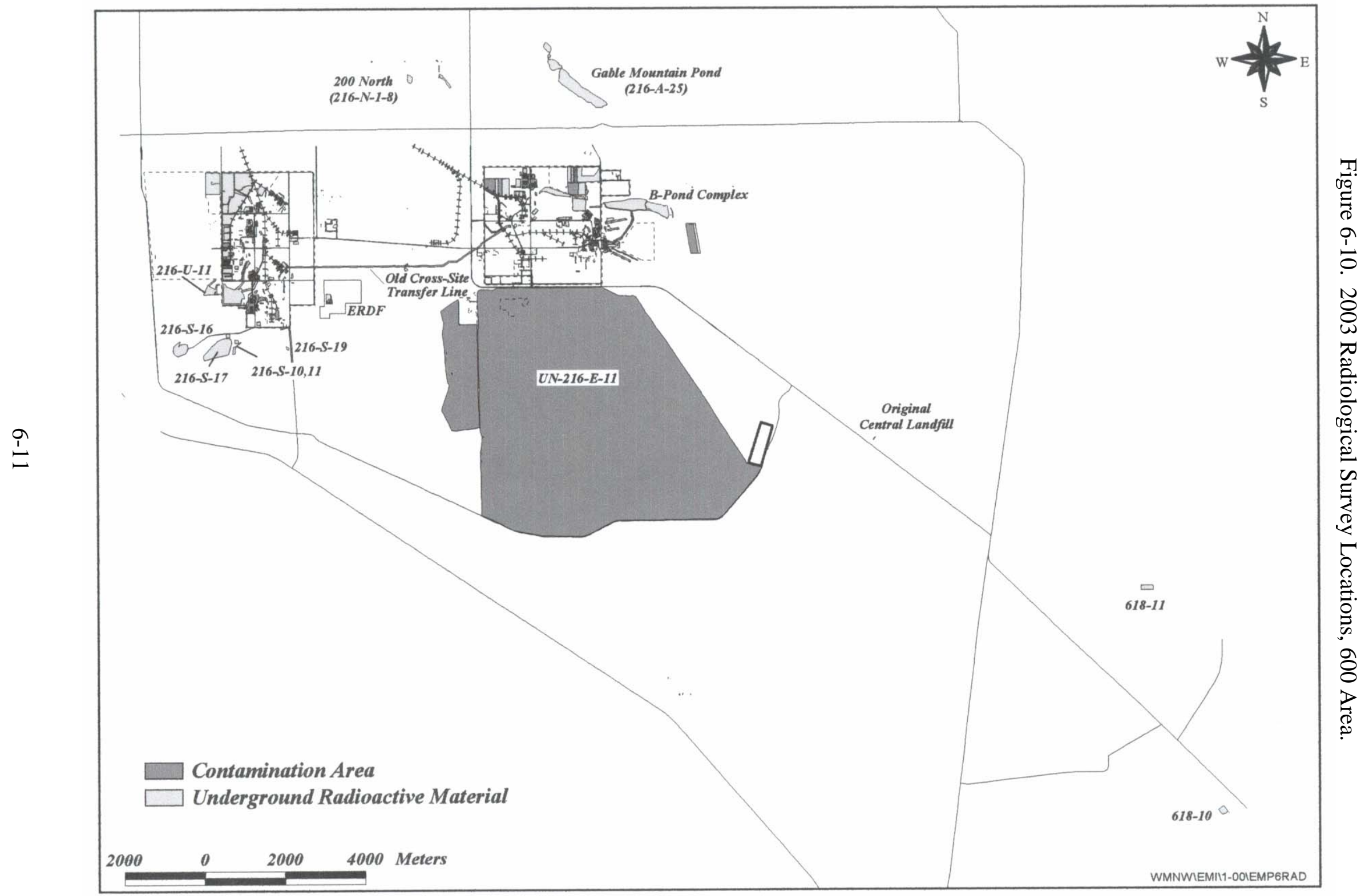


This page intentionally left blank.

6-12 


\subsection{INVESTIGATIVE SAMPLING}

Investigative samples were collected where known or suspected radioactive contamination was present, or to verify radiological conditions at project sites. Examples of investigative samples collected during 2003 included soil, vegetation, animals, and animal feces. Selected samples were analyzed for radionuclides at the 222-S Laboratory (200 West Area) and the results are provided in Table 7-1. Eighty-nine contaminated environmental samples were collected and disposed without isotopic analyses during clean-up operations during 2003. Field instrument readings were recorded for each and the results are provided in Table 7-2.

\subsection{SOIL}

During 2003, there were 30 instances of radiological contamination in which soil was identified as the carrier of contamination. One of these soil samples was submitted to the laboratory for radioisotopic analysis. Of the 30, 19 were identified only as specks or soil specks. Often, specks observed under high magnification are found to be small pieces of decomposed vegetation, most often tumbleweeds. External radioactivity levels ranged from slightly above background (approximately 2,500 disintegration's per minute $[\mathrm{dpm}] / 100 \mathrm{~cm}^{2}$ ) to $3,600,000 \mathrm{dpm} / 100 \mathrm{~cm}^{2}$. Contaminated areas were radiologically posted or cleaned up. The number of contamination incidents, the range of radiation dose rate levels, and radionuclide concentrations observed in 2003 were generally within historical ranges.

\subsection{VEGETATION}

In 2003, there were 32 instances in which vegetation was identified as the carrier of radiological contamination. None of these vegetation samples were submitted to the laboratory for radioisotopic analysis and the radioactivity levels were mostly within historical ranges. One contaminated vegetation instance had field readings in excess of one million $\mathrm{dpm} / 100 \mathrm{~cm}^{2}$.

The number of incidents in 2003 (32) is up from 16 in 2002, though still down from the high of 84 incidents in 1999. This overall decrease can be attributed to improvements in the deep-rooted weed prevention program. Nevertheless, contaminated tumbleweeds that grew in recent years continue to be identified by radiological surveys. It is expected that as contaminated vegetation from past years is identified and cleaned up, subsequent years will show the results of program improvements.

\subsection{ANIMALS}

Animals were collected either as part of an integrated pest management program or as a result of radiological surveys finding contaminated wildlife-related material (e.g., feces, nests, etc.). Animals were collected directly from or near facilities in an effort to monitor and track effectiveness of preventive measures designed to deter animal intrusion. For 2003, the number 
of animals or animal-related materials found to be contaminated with radioactivity, 32 instances, the radioactivity levels and the range of radionuclide concentrations were within historical ranges. Of the 32 instances of radiological contamination caused by animals, 19 were caused by mud dauber wasp activity in the 100-H (18) and 100-N (1) Areas. In each case, decommissioning activities exposed contaminated soil and made it available for mud dauber wasp nest building.

After the shut down of the 105-H reactor, the fuel storage basins were backfilled with soil. To prevent airborne contamination from the soil during decontamination and decommissioning activities, the backfill was kept wet. Mud dauber wasps then used the mud created during this activity to build nests at various places in the 100-H Area. Contamination levels found in the wasp nests ranged from a low of $32,500 \mathrm{dpm} / 100 \mathrm{~cm}^{2}$ beta/gamma to a high of $1,200,000 \mathrm{dpm} / 100 \mathrm{~cm}^{2}$ beta/gamma and 1,300 dpm/100 $\mathrm{cm}^{2}$ alpha. The contaminated areas created by the wasp nests were posted and will be cleaned up at a later date.

Similarly, in the 100-N Area, the contaminated mud dauber wasp nests came from the contaminated soil removal activities during the decommissioning of the $1325-\mathrm{N}$ trench.

In 2003, six animals, or animal-related contamination instances were identified and from these, nine samples were submitted to the laboratory for analysis.

- $\quad$ In November 2002, two separate instances of contaminated mice were found along the perimeter of the 241-BX/BY tank farm (200 East Area). The results are reported here as the analyses had not been completed in time to be included in the 2002 data report. Contaminants included strontium-89/90 and cesium-137.

- In February 2003, contaminated soil was found to the west of the 241-TX/TY tank farm (200 West Area) in an old construction debris site. Contaminants included strontium-89/90 and cesium-137.

- $\quad$ In June 2003, a contaminated mouse was found in the 105-KE radiological monitoring office. Contaminants included strontium-89/90 and cesium-137.

- $\quad$ In June 2003, a contaminated starling carcass was found in the 317 Building stair well. Contaminants included cobalt-60, strontium-89/90, and cesium-134/137.

- In August 2003, a contaminated house mouse was found at 105-KE reactor building. Contaminants included strontium-89/90 and cesium-137.

- $\quad$ In August 2003, a contaminated cottontail rabbit was found outside the 272-S paint shop east of the 241-S/SX/SY tank farm complex (200 West Area). The rabbit was divided into four parts, skin, bone, gastrointestinal tract, and muscle. Contaminants included strontium-89/90 and cesium-137 with the highest result in the muscle. 


\subsection{SPECIAL CHARACTERIZATION SAMPLING}

Special characterization projects were conducted in 2003 to ascertain the radiological status, and in some cases, the physical properties of site-specific operations and included the following:

A preoperational monitoring plan (Remote-Handled Immobilized Low-Activity Waste Disposal Facility Preoperational Monitoring Plan, RPP-6877 [Horton et al. 2000]) has been developed in support of the Waste Vitrification initiative. As part of this plan, an on-going environmental survey is being conducted on the proposed location for the Integrated Disposal Facility (IDF), formerly the Immobilized Low-Activity Waste Disposal (ILAW) Facility, in the 200 East Area. Tasks completed in 2003 included bulk soil sampling for geophysical properties. Following the completion of all the tasks outlined in the monitoring plan, the data collected will be published in a final report. The report is currently scheduled for publication in 2005.

Soil, vegetation and ground-dwelling invertebrate samples were collected at Gable Mountain Pond and B-Pond in October 2003 to identify potential exposure pathways and support remedial action decisions (Lane et al. 2003). 
Table 7-1. Investigative Sample Results, 2003.

\begin{tabular}{|c|c|c|c|c|c|c|}
\hline \multicolumn{5}{|c|}{ Sample } & \multirow[b]{2}{*}{ Isotope } & \multirow{2}{*}{$\begin{array}{r}\text { Result }^{\mathrm{a}} \quad \begin{array}{c}\text { Analytical } \\
(\mathrm{pCi} / \text { Sample })^{\mathrm{b}} \\
\pm\end{array} \text { Uncertainty } \\
\end{array}$} \\
\hline Number & Matrix & TSN & Location & Date & & \\
\hline \multirow[t]{10}{*}{6911} & Mouse & 180360 & 241-BX/BY Tank Farm & $11 / 13 / 02$ & ${ }^{60} \mathrm{Co}$ & $<6.8 \mathrm{E}+00$ \\
\hline & & & (200 East Area) & & ${ }^{89,90} \mathrm{Sr}$ & $3.7 \mathrm{E}+02 \pm 4.7 \mathrm{E}+00$ \\
\hline & & & & & ${ }^{134} \mathrm{Cs}$ & $<2.4 \mathrm{E}+01$ \\
\hline & & & & & ${ }^{137} \mathrm{Cs}$ & $1.4 \mathrm{E}+01 \pm 4.4 \mathrm{E}-01$ \\
\hline & & & & & ${ }^{152} \mathrm{Eu}$ & $<6.2 \mathrm{E}+01$ \\
\hline & & & & & ${ }^{154} \mathrm{Eu}$ & $<3.9 \mathrm{E}+01$ \\
\hline & & & & & ${ }^{155} \mathrm{Eu}$ & $<7.3 \mathrm{E}+01$ \\
\hline & & & & & ${ }^{\text {Total }} U^{c}$ & $<2.2 \mathrm{E}+04$ \\
\hline & & & & & ${ }^{238} \mathrm{Pu}$ & $<3.7 \mathrm{E}-01 \pm 1.0 \mathrm{E}+02$ \\
\hline & & & & & ${ }^{239,240} \mathrm{Pu}$ & $<2.1 \mathrm{E}-01 \pm 1.0 \mathrm{E}+02$ \\
\hline \multirow[t]{10}{*}{6912} & Mouse & 180360 & 241-BX/BY Tank Farm & $11 / 19 / 02$ & ${ }^{60} \mathrm{Co}$ & $<1.2 \mathrm{E}+00$ \\
\hline & & & (200 East Area) & & ${ }^{89,90} \mathrm{Sr}$ & $<7.0 \mathrm{E}+03 \pm 6.2 \mathrm{E}-01$ \\
\hline & & & & & ${ }^{134} \mathrm{Cs}$ & $<1.3 \mathrm{E}+01$ \\
\hline & & & & & ${ }^{137} \mathrm{Cs}$ & $<5.3 \mathrm{E}+04$ \\
\hline & & & & & ${ }^{152} \mathrm{Eu}$ & $<3.6 \mathrm{E}+01$ \\
\hline & & & & & ${ }^{154} \mathrm{Eu}$ & $<6.0 \mathrm{E}+00$ \\
\hline & & & & & ${ }^{155} \mathrm{Eu}$ & $<4.1 \mathrm{E}+01$ \\
\hline & & & & & ${ }^{\text {Total }} U^{\mathrm{c}}$ & $<1.3 \mathrm{E}=04$ \\
\hline & & & & & ${ }^{238} \mathrm{Pu}$ & $<3.3 \mathrm{E}-01 \pm 1.0 \mathrm{E}+02$ \\
\hline & & & & & ${ }^{239,240} \mathrm{Pu}$ & $<1.8 \mathrm{E}-01 \pm 1.0 \mathrm{E}+02$ \\
\hline \multirow[t]{10}{*}{6913} & Soil & 100 & 241-TX/YY Tank Farm & $02 / 13 / 03$ & ${ }^{60} \mathrm{Co}$ & $<1.8 \mathrm{E}-01$ \\
\hline & & & (200 West Area) & & ${ }^{89,90} \mathrm{Sr}$ & $<1.9 \mathrm{E}-01 \pm 1.4 \mathrm{E}+02$ \\
\hline & & & & & ${ }^{134} \mathrm{Cs}$ & $<1.4 \mathrm{E}-01$ \\
\hline & & & & & ${ }^{137} \mathrm{Cs}$ & $<3.0 \mathrm{E}+03 \pm 2.3 \mathrm{E}-01$ \\
\hline & & & & & ${ }^{152} \mathrm{Eu}$ & $<3.4 \mathrm{E}+00$ \\
\hline & & & & & ${ }^{154} \mathrm{Eu}$ & $<4.6 \mathrm{E}-01$ \\
\hline & & & & & ${ }^{155} \mathrm{Eu}$ & $<4.2 \mathrm{E}+00$ \\
\hline & & & & & ${ }^{\text {Total }} U^{c}$ & $<1.0 \mathrm{E}+03$ \\
\hline & & & & & ${ }^{238} \mathrm{Pu}$ & $<4.2 \mathrm{E}-01 \pm 1.0 \mathrm{E}+02$ \\
\hline & & & & & ${ }^{239,240} \mathrm{Pu}$ & $<5.9 \mathrm{E}-01 \pm 1.4 \mathrm{E}+02$ \\
\hline
\end{tabular}


Table 7-1. Investigative Sample Results, 2003. (cont)

\begin{tabular}{|c|c|c|c|c|c|c|}
\hline \multicolumn{5}{|c|}{ Sample } & \multirow[b]{2}{*}{ Isotope } & \multirow{2}{*}{ 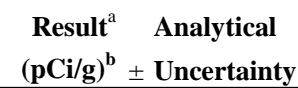 } \\
\hline Number & Matrix & TSN & Location & Date & & \\
\hline \multirow[t]{10}{*}{6914} & Mouse & 180360 & 105-KE RM Office & $06 / 13 / 03$ & ${ }^{60} \mathrm{Co}$ & $<1.1 \mathrm{E}+00$ \\
\hline & & & (100-K Area) & & ${ }^{89,90} \mathrm{Sr}$ & $1.00 \mathrm{E}+02 \pm 4.2 \mathrm{E}+00$ \\
\hline & & & & & ${ }^{134} \mathrm{Cs}$ & $<9.1 \mathrm{E}-01$ \\
\hline & & & & & ${ }^{137} \mathrm{Cs}$ & $3.70 \mathrm{E}+01 \pm 5.1 \mathrm{E}+00$ \\
\hline & & & & & ${ }^{152} \mathrm{Eu}$ & $<2.0 \mathrm{E}+00$ \\
\hline & & & & & ${ }^{154} \mathrm{Eu}$ & $<3.0 \mathrm{E}+00$ \\
\hline & & & & & ${ }^{155} \mathrm{Eu}$ & $<2.7 \mathrm{E}+00$ \\
\hline & & & & & ${ }^{\text {Total }} \mathrm{U}^{\mathrm{c}}$ & $<6.0 \mathrm{E}+02$ \\
\hline & & & & & ${ }^{238} \mathrm{Pu}$ & $<2.3 \mathrm{E}+00 \pm 9.6 \mathrm{E}+00$ \\
\hline & & & & & ${ }^{239,240} \mathrm{Pu}$ & $<2.1 \mathrm{E}+00 \pm 6.2 \mathrm{E}+00$ \\
\hline \multirow[t]{10}{*}{6915} & Starling Carcass & 179637 & 317 Building Stair Well & $06 / 13 / 03$ & ${ }^{60} \mathrm{Co}$ & $2.2 \mathrm{E}+00 \pm 9.2 \mathrm{E}+00$ \\
\hline & & & (300 Area) & & ${ }^{89,90} \mathrm{Sr}$ & $3.2 \mathrm{E}+01 \pm 7.3 \mathrm{E}+00$ \\
\hline & & & & & ${ }^{134} \mathrm{Cs}$ & $3.5 \mathrm{E}+00 \pm 7.8 \mathrm{E}+00$ \\
\hline & & & & & ${ }^{137} \mathrm{Cs}$ & $1.6 \mathrm{E}+03 \pm 2.5 \mathrm{E}-01$ \\
\hline & & & & & ${ }^{152} \mathrm{Eu}$ & $<1.6 \mathrm{E}+00$ \\
\hline & & & & & ${ }^{154} \mathrm{Eu}$ & $<5.8 \mathrm{E}-01$ \\
\hline & & & & & ${ }^{155} \mathrm{Eu}$ & $<2.0 \mathrm{E}+00$ \\
\hline & & & & & ${ }^{\text {Total }} \mathrm{U}^{\mathrm{c}}$ & $<5.2 \mathrm{E}+02$ \\
\hline & & & & & ${ }^{238} \mathrm{Pu}$ & $<9.7 \mathrm{E}-01 \pm 1.1 \mathrm{E}+01$ \\
\hline & & & & & ${ }^{239,240} \mathrm{Pu}$ & $<5.5 \mathrm{E}-01 \pm 1.4 \mathrm{E}+01$ \\
\hline \multirow[t]{10}{*}{6916} & House Mouse & 180360 & 105-KE & $08 / 05 / 03$ & ${ }^{60} \mathrm{Co}$ & $<1.0 \mathrm{E}+00$ \\
\hline & & & (100-K Area) & & ${ }^{89,90} \mathrm{Sr}$ & $7.6 \mathrm{E}+00 \pm 4.1 \mathrm{E}+00$ \\
\hline & & & & & ${ }^{134} \mathrm{Cs}$ & $<9.8 \mathrm{E}-01$ \\
\hline & & & & & ${ }^{137} \mathrm{Cs}$ & $2.0 \mathrm{E}+02 \pm 2.1 \mathrm{E}+01$ \\
\hline & & & & & ${ }^{152} \mathrm{Eu}$ & $<1.9 \mathrm{E}+00$ \\
\hline & & & & & ${ }^{154} \mathrm{Eu}$ & $<2.8 \mathrm{E}+00$ \\
\hline & & & & & ${ }^{155} \mathrm{Eu}$ & $<1.5 \mathrm{E}+00$ \\
\hline & & & & & ${ }^{\text {Total }} U^{c}$ & $<7.3 \mathrm{E}+02$ \\
\hline & & & & & ${ }^{238} \mathrm{Pu}$ & $<1.7 \mathrm{E}+00 \pm 1.0 \mathrm{E}+02$ \\
\hline & & & & & ${ }^{239,240} \mathrm{Pu}$ & $<1.3 \mathrm{E}+00 \pm 7.1 \mathrm{E}+00$ \\
\hline
\end{tabular}


Table 7-1. Investigative Sample Results, 2003. (cont)

\begin{tabular}{|c|c|c|c|c|c|c|}
\hline \multicolumn{5}{|c|}{ Sample } & \multirow[b]{2}{*}{ Isotope } & \multirow{2}{*}{ 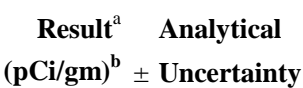 } \\
\hline Number & Matrix & TSN & Location & Date & & \\
\hline \multirow[t]{10}{*}{6917} & Cotton Tail Rabbit & 180126 & 272-S Paint Shop & $08 / 05 / 03$ & ${ }^{60} \mathrm{Co}$ & $<1.7 \mathrm{E}-01$ \\
\hline & Skin & & (200 West Area) & & ${ }^{89,90} \mathrm{Sr}$ & $2.3 \mathrm{E}+02 \pm 1.5 \mathrm{E}+00$ \\
\hline & & & & & ${ }^{134} \mathrm{Cs}$ & $<8.2 \mathrm{E}-01$ \\
\hline & & & & & ${ }^{137} \mathrm{Cs}$ & $1.3 \mathrm{E}+03 \pm 4.3 \mathrm{E}-01$ \\
\hline & & & & & ${ }^{152} \mathrm{Eu}$ & $<2.0 \mathrm{E}+00$ \\
\hline & & & & & ${ }^{154} \mathrm{Eu}$ & $<1.2 \mathrm{E}+00$ \\
\hline & & & & & ${ }^{155} \mathrm{Eu}$ & $<1.5 \mathrm{E}+00$ \\
\hline & & & & & ${ }^{\text {Total }} U^{c}$ & $<8.0 \mathrm{E}+02$ \\
\hline & & & & & ${ }^{238} \mathrm{Pu}$ & $<3.9 \mathrm{E}-01 \pm 1.0 \mathrm{E}+02$ \\
\hline & & & & & ${ }^{239,240} \mathrm{Pu}$ & $<2.0 \mathrm{E}-01 \pm 1.5 \mathrm{E}+01$ \\
\hline \multirow[t]{10}{*}{6918} & Cotton Tail Rabbit & 180126 & 272-S Paint Shop & $08 / 05 / 03$ & ${ }^{60} \mathrm{Co}$ & $<9.1 \mathrm{E}-01$ \\
\hline & Bone & & (200 West Area) & & ${ }^{89,90} \mathrm{Sr}$ & $3.1 \mathrm{E}+03 \pm 5.4 \mathrm{E}-01$ \\
\hline & & & & & ${ }^{134} \mathrm{Cs}$ & $<1.4 \mathrm{E}+00$ \\
\hline & & & & & ${ }^{137} \mathrm{Cs}$ & $1.4 \mathrm{E}+00 \pm 7.3 \mathrm{E}-01$ \\
\hline & & & & & ${ }^{152} \mathrm{Eu}$ & $<303 \mathrm{E}+00$ \\
\hline & & & & & ${ }^{154} \mathrm{Eu}$ & $<2.9 \mathrm{E}+00$ \\
\hline & & & & & ${ }^{155} \mathrm{Eu}$ & $<2.6 \mathrm{E}+00$ \\
\hline & & & & & ${ }^{\text {Total }} U^{c}$ & $<1.2 \mathrm{E}+03$ \\
\hline & & & & & ${ }^{238} \mathrm{Pu}$ & $<8.7 \mathrm{E}-01 \pm 1.0 \mathrm{E}+02$ \\
\hline & & & & & ${ }^{239,240} \mathrm{Pu}$ & $<4.4 \mathrm{E}-01 \pm 1.0 \mathrm{E}+02$ \\
\hline \multirow[t]{10}{*}{6919} & Cotton Tail Rabbit & 180126 & 272-S Paint Shop & $08 / 05 / 03$ & ${ }^{60} \mathrm{Co}$ & $<2.2 \mathrm{E}-01$ \\
\hline & GI Tract & & (200 West Area) & & ${ }^{89,90} \mathrm{Sr}$ & $2.7 \mathrm{E}+01 \pm 6.1 \mathrm{E}+00$ \\
\hline & & & & & ${ }^{134} \mathrm{Cs}$ & $<6.8 \mathrm{E}-01$ \\
\hline & & & & & ${ }^{137} \mathrm{Cs}$ & $1.2 \mathrm{E}+03 \pm 3.8 \mathrm{E}-01$ \\
\hline & & & & & ${ }^{152} \mathrm{Eu}$ & $<1.6 \mathrm{E}+00$ \\
\hline & & & & & ${ }^{154} \mathrm{Eu}$ & $<5.2 \mathrm{E}-01$ \\
\hline & & & & & ${ }^{155} \mathrm{Eu}$ & $<1.2 \mathrm{E}+00$ \\
\hline & & & & & ${ }^{\text {Total }} U^{c}$ & $<6.6 \mathrm{E}+02$ \\
\hline & & & & & ${ }^{238} \mathrm{Pu}$ & $<3.0 \mathrm{E}-01 \pm 1.0 \mathrm{E}+02$ \\
\hline & & & & & ${ }^{239,240} \mathrm{Pu}$ & $<1.7 \mathrm{E}-01 \pm 1.0 \mathrm{E}+02$ \\
\hline
\end{tabular}


Table 7-1. Investigative Sample Results, 2003. (cont)

\begin{tabular}{|c|c|c|c|c|c|c|}
\hline \multicolumn{5}{|c|}{ Sample } & \multirow[b]{2}{*}{ Isotope } & \multirow{2}{*}{$\begin{array}{c}\text { Result }^{\mathrm{a}} \quad \text { Analytical } \\
(\mathbf{p C i} / \mathrm{L})^{\mathrm{d}} \pm \text { Uncertainty }\end{array}$} \\
\hline Number & Matrix & TSN & Location & Date & & \\
\hline \multirow[t]{10}{*}{6920} & Cotton Tail Rabbit & 180126 & 272-S Paint Shop & $08 / 05 / 03$ & ${ }^{60} \mathrm{Co}$ & $<2.2 \mathrm{E}-01$ \\
\hline & Muscle & & (200 West Area) & & ${ }^{89,90} \mathrm{Sr}$ & $6.3 \mathrm{E}+01 \pm 1.9 \mathrm{E}+00$ \\
\hline & & & & & ${ }^{134} \mathrm{Cs}$ & $<9.4 \mathrm{E}-01$ \\
\hline & & & & & ${ }^{137} \mathrm{Cs}$ & $2.2 \mathrm{E}+03 \pm 2.9 \mathrm{E}-01$ \\
\hline & & & & & ${ }^{152} \mathrm{Eu}$ & $<2.3 \mathrm{E}+00$ \\
\hline & & & & & ${ }^{154} \mathrm{Eu}$ & $<5.9 \mathrm{E}-01$ \\
\hline & & & & & ${ }^{155} \mathrm{Eu}$ & $<1.6 \mathrm{E}+00$ \\
\hline & & & & & ${ }^{\text {Total }} \mathrm{U}^{\mathrm{C}}$ & $<9.1 \mathrm{E}+02$ \\
\hline & & & & & ${ }^{238} \mathrm{Pu}$ & $<4.0 \mathrm{E}-01 \pm 1.0 \mathrm{E}+02$ \\
\hline & & & & & ${ }^{239,240} \mathrm{Pu}$ & $<2.6 \mathrm{E}-01 \pm 1.0 \mathrm{E}+02$ \\
\hline
\end{tabular}

\footnotetext{
${ }^{\mathrm{a} A ~ "<" ~ s y m b o l ~ i n d i c a t e s ~ t h a t ~ t h e ~ a n a l y t e ~ w a s ~ a n a l y z e d ~ f o r ~ b u t ~ n o t ~ d e t e c t e d . ~ U n c e r t a i n t y ~ v a l u e s ~ w e r e ~ n o t ~ r e p o r t e d ~ b y ~ t h e ~}$ laboratory for all results.

${ }^{\mathrm{b}}$ To convert to international metric system units (SI), multiply pCi/g by 0.03704 to obtain Bq/g.

${ }^{\mathrm{c}}$ Total uranium concentrations are reported by the laboratory in units of ug/g. These results have been converted to $\mathrm{pCi} / \mathrm{g}$ using a specific activity of $9.6 \mathrm{E}+05 \mathrm{pCi} / \mathrm{g}$ for total uranium.

${ }^{\mathrm{d}}$ To convert to international metric system units (SI), multiply pCi/L by 0.03704 to obtain $\mathrm{Bq} / \mathrm{L}$.
} 
Table 7-2. Investigative Samples Not Analyzed, 2003.

\begin{tabular}{|c|c|c|c|}
\hline Date & Sample matrix & Location & $\begin{array}{c}\text { Field reading } \\
\text { (Beta/Gamma) }\end{array}$ \\
\hline $02 / 10 / 03$ & Speck in soil & West of 241-U tank farm perimeter & $199,000 \mathrm{dpm} / 100 \mathrm{~cm}^{2}$ \\
\hline $02 / 19 / 03$ & Soil & 200-W-92 West of 241-TX/TY & $750,000 \mathrm{dpm} / 100 \mathrm{~cm}^{2}$ \\
\hline $02 / 21 / 03$ & Spot on ground & RBA South of 221-B Plant & $11,000 \mathrm{dpm} / 100 \mathrm{~cm}^{2}$ \\
\hline 03/03/03 & Soil between RR Tracks & 200-W-83 & $200,000 \mathrm{dpm} / 100 \mathrm{~cm}^{2}$ \\
\hline 03/06/03 & Soil & 216-B-3A Pond SCA/CA Area & $2,500 \mathrm{dpm} / 100 \mathrm{~cm}^{2}$ \\
\hline 03/07/03 & Rabbit Feces & $200-W-54$ & $10,000 \mathrm{dpm} / 100 \mathrm{~cm}^{2}$ \\
\hline 03/10/03 & Speck in soil & West of 241-C Tank Farm & $60,000 \mathrm{dpm} / 100 \mathrm{~cm}^{2}$ \\
\hline 03/18/03 & Choker & 6290 Crane \& Rigging Facility & $30,000 \mathrm{dpm} / 100 \mathrm{~cm}^{2}$ \\
\hline $03 / 24 / 03$ & Speck & 241-C Tank Farm Perimeter & $400,000 \mathrm{dpm} / 100 \mathrm{~cm}^{2}$ \\
\hline $03 / 25 / 03$ & Speck & 241-AX Tank Farm Perimeter & $80,000 \mathrm{dpm} / 100 \mathrm{~cm}^{2}$ \\
\hline 03/31/03 & 8-Tumbleweeds & 216-A-34 Crib & $36,000 \mathrm{dpm} / 100 \mathrm{~cm}^{2}$ \\
\hline $04 / 08 / 03$ & Scaffold Knuckle & 300 Area Laydown Yard & $10,000 \mathrm{dpm} / 100 \mathrm{~cm}^{2}$ \\
\hline $04 / 16 / 03$ & Concrete Pad & 219-S TSD & $440,000 \mathrm{dpm} / 100 \mathrm{~cm}^{2}$ \\
\hline $04 / 17 / 03$ & Tumbleweed & 241-B Tank Farm Perimeter & $990,000 \mathrm{dpm} / 100 \mathrm{~cm}^{2}$ \\
\hline $04 / 21 / 03$ & Electrical Wire & 2101-M Laydown Yard & 1,204dpm/100 $\mathrm{cm}^{2}$ Alpha \\
\hline $04 / 29 / 03$ & Soil Speck & NW Corner 241-TX/TY Tank Farm Fence & $199,000 \mathrm{dpm} / 100 \mathrm{~cm}^{2}$ \\
\hline $05 / 06 / 03$ & 8-Tumbleweeds & 216-B-3 Pond & $24,000 \mathrm{dpm} / 100 \mathrm{~cm}^{2}$ \\
\hline 05/13/03 & Soil & Outside of the posted 200-E-121UPR & $14,000 \mathrm{dpm} / 100 \mathrm{~cm}^{2}$ \\
\hline $05 / 14 / 03$ & Soil & Outside the posted 200-W-091 & $30,000 \mathrm{dpm} / 100 \mathrm{~cm}^{2}$ \\
\hline $05 / 21 / 03$ & Tumbleweeds/Grass & 218-E-12A Burial Ground & $114,000 \mathrm{dpm} / 100 \mathrm{~cm}^{2}$ \\
\hline $05 / 28 / 03$ & Speck & South East Corner 241-A Tank Farm & $11,000 \mathrm{dpm} / 100 \mathrm{~cm}^{2}$ \\
\hline 06/13/03 & Specks & West side of the 241-B Tank Farm & $90,000 \mathrm{dpm} / 100 \mathrm{~cm}^{2}$ \\
\hline $06 / 26 / 03$ & Mud Dauber Wasp Nest & Counting Booth at the 105-H Reactor Building & $1,200,000 \mathrm{dpm} / 100 \mathrm{~cm}^{2}$ \\
\hline 07/08/03 & Rocks/Soil & 100-H Fuel Storage Basin & $2,000,000 \mathrm{dpm} / 100 \mathrm{~cm}^{2}$ \\
\hline 07/09/03 & Soil Specks & 200-E-122 & $200,000 \mathrm{dpm} / 100 \mathrm{~cm}^{2}$ \\
\hline 07/14/03 & Mud Dauber Wasp Nest & Counting Booth at the 105-H Reactor Building & $440,000 \mathrm{dpm} / 100 \mathrm{~cm}^{2}$ \\
\hline 07/14/03 & Mud Dauber Wasp Nest & Counting Booth at the 105-H Reactor Building & $1,300 \mathrm{dpm} / 100 \mathrm{~cm}^{2}$ alpha \\
\hline 07/16/03 & Specks & West side of 241-B Tank Farm & $100,000 \mathrm{dpm} / 100 \mathrm{~cm}^{2}$ \\
\hline 07/23/03 & Specks & North side of 241-S Tank Farm & $300,000 \mathrm{dpm} / 100 \mathrm{~cm}^{2}$ \\
\hline 07/23/03 & Specks & Near the 272-S Maintenance Shop & $400,000 \mathrm{dpm} / 100 \mathrm{~cm}^{2}$ \\
\hline $08 / 05 / 03$ & Mud Dauber Wasp Nest & Inside Air Monitoring Dog House @ 1325-N & $>2,000 \mathrm{dpm} / 100 \mathrm{~cm}^{2}$ \\
\hline $08 / 08 / 03$ & Rabbit Feces & 272-S RMA & $100,000 \mathrm{dpm} / 100 \mathrm{~cm}^{2}$ \\
\hline 08/08/03 & Base of Rabbit Brush/Soil & 272-S RMA & $240,000 \mathrm{dpm} / 100 \mathrm{~cm}^{2}$ \\
\hline 08/08/03 & Speck & Near the 241-SX Tank Farm & $90,000 \mathrm{dpm} / 100 \mathrm{~cm}^{2}$ \\
\hline 08/08/03 & Mud Dauber Wasp Nests & 105-H Drinking Water Station & $200,000 \mathrm{dpm} / 100 \mathrm{~cm}^{2}$ \\
\hline 08/11/03 & Speck & 241-ER-151Perimeter & $350,000 \mathrm{dpm} / 100 \mathrm{~cm}^{2}$ \\
\hline 08/11/03 & Mud Dauber Wasp Nests & 105-H Power Poles \& Chemical Storage Cabinet & $270,000 \mathrm{dpm} / 100 \mathrm{~cm}^{2}$ \\
\hline $08 / 12 / 03$ & Mud Dauber Wasp Nests & 1143 Maintenance Build. Generator & $260,000 \mathrm{dpm} / 100 \mathrm{~cm}^{2}$ \\
\hline $08 / 13 / 03$ & Mud Dauber Wasp Nests & Outside the $105-\mathrm{H}$ D\&D Perimeter Fence & $800,000 \mathrm{dpm} / 100 \mathrm{~cm}^{2}$ \\
\hline 08/13/03 & Bird Feces & Outside the $105-\mathrm{H}$ D\&D Perimeter Fence & $800,000 \mathrm{dpm} / 100 \mathrm{~cm}^{2}$ \\
\hline 08/13/03 & Mud Dauber Wasp Nests & 1713-H Warehouse Building & $560,000 \mathrm{dpm} / 100 \mathrm{~cm}^{2}$ \\
\hline 08/18/03 & Mud Dauber Wasp Nests & 126-H-2 (105-H Clear Well) & $1,200,000 \mathrm{dpm} / 100 \mathrm{~cm}^{2}$ \\
\hline 08/18/03 & Tumbleweed Fragments & inside posted CA @ 200-E-121 & $900,000 \mathrm{dpm} / 100 \mathrm{~cm}^{2}$ \\
\hline 08/19/03 & Mud Dauber Wasp Nests & 1713-H Warehouse Parking Area RR Ties & $399,000 \mathrm{dpm} / 100 \mathrm{~cm}^{2}$ \\
\hline $08 / 20 / 03$ & Owl Pellet & Outside the 105-H Fuel Storage Basins & $57,900 \mathrm{dpm} / 100 \mathrm{~cm}^{2}$ \\
\hline $08 / 20 / 03$ & Tumbleweed & Outside the 241-B Tank Farm & $1,200,000 \mathrm{dpm} / 100 \mathrm{~cm}^{2}$ \\
\hline $08 / 21 / 03$ & Soil \& Tumbleweed Fragments & 200-E-132 outside 241-BX/BY & $3,600,000 \mathrm{dpm} / 100 \mathrm{~cm}^{2}$ \\
\hline $08 / 21 / 03$ & Mud Dauber Wasp Nests & Power poles approx. $1 / 2 \mathrm{~m}$. west of $1713-\mathrm{H}$ & $106,000 \mathrm{dpm} / 100 \mathrm{~cm}^{2}$ \\
\hline
\end{tabular}


Table 7-2. Investigative Samples Not Analyzed, 2003. (cont)

\begin{tabular}{|c|c|c|c|}
\hline Date & Sample Matrix & Location & $\begin{array}{c}\text { Field reading } \\
\text { (Beta/Gamma) }\end{array}$ \\
\hline $08 / 28 / 03$ & Speck & East Perimeter of 241-S & $800,000 \mathrm{dpm} / 100 \mathrm{~cm}^{2}$ \\
\hline 08/28/03 & Mud Dauber Wasp Nest & Power Poles east of $1713-\mathrm{H}$ & $114,000 \mathrm{dpm} / 100 \mathrm{~cm}^{2}$ \\
\hline 09/02/03 & Mud Dauber Nest & Power pole $\# 130$ west of $1713-\mathrm{H}$ & $32,500 \mathrm{dpm} / 100 \mathrm{~cm}^{2}$ \\
\hline 09/02/03 & Speck & 241-ER-151Perimeter & $450,000 \mathrm{dpm} / 100 \mathrm{~cm}^{2}$ \\
\hline 09/03/03 & Specks in soil & North stairs of the $1713-\mathrm{H}$ Warehouse Building & $199,000 \mathrm{dpm} / 100 \mathrm{~cm}^{2}$ \\
\hline 09/03/03 & Soil & Around perimeter of UPR-200-E-78 & $600,000 \mathrm{dpm} / 100 \mathrm{~cm}^{2}$ \\
\hline 09/03/03 & 3-Soil Specks & 241-BX-155 Diversion Box & $>1,000,000 \mathrm{dpm} / 100 \mathrm{~cm}^{2}$ \\
\hline 09/04/03 & Tumbleweed & South of the 241-B Diversion Box & $60,000 \mathrm{dpm} / 100 \mathrm{~cm}^{2}$ \\
\hline 09/05/03 & Fence Post (wasp nests) & West of the $1713-\mathrm{H}$ Warehouse & $200,000 \mathrm{dpm} / 100 \mathrm{~cm}^{2}$ \\
\hline 09/10/03 & Tumbleweed & Northeast of 241-U (UN-216-W-35) & $120,000 \mathrm{dpm} / 100 \mathrm{~cm}^{2}$ \\
\hline 09/11/03 & Stainless Steel Canister & 126-B-3 Coal Pit & $100,000 \mathrm{dpm} / 100 \mathrm{~cm}^{2}$ \\
\hline 09/16/03 & Tumbleweed Fragment & North Fenceline of 241-SY Tank Farm & $70,000 \mathrm{dpm} / 100 \mathrm{~cm}^{2}$ \\
\hline 09/17/03 & Tumbleweed & Northeast of 241-C (UN-216-E-115) & $6,000 \mathrm{dpm} / 100 \mathrm{~cm}^{2}$ \\
\hline 09/22/03 & Speck & inside 244-A lift station perimeter & $400,000 \mathrm{dpm} / 100 \mathrm{~cm}^{2}$ \\
\hline 09/22/03 & (6) Mud Dauber Nests & $105-\mathrm{H}$ south of $116-\mathrm{H}-1$ & $280,000 \mathrm{dpm} / 100 \mathrm{~cm}^{2}$ \\
\hline $10 / 03 / 03$ & (21) Mud Dauber Nests & $105-\mathrm{H}$ south of $116-\mathrm{H}-1$ & $540,000 \mathrm{dpm} / 100 \mathrm{~cm}^{2}$ \\
\hline $10 / 03 / 03$ & Tumbleweeds & 216-U-10 Pond & $60,000 \mathrm{dpm} / 100 \mathrm{~cm}^{2}$ \\
\hline $10 / 14 / 03$ & Tumbleweed Fragments & 6290 Riggers Loft & $220,000 \mathrm{dpm} / 100 \mathrm{~cm}^{2}$ \\
\hline $10 / 15 / 03$ & Starling Carcass & B-Cell inside of 327 Building & $100,000 \mathrm{dpm} / 100 \mathrm{~cm}^{2}$ \\
\hline $10 / 21 / 03$ & Tumbleweeds & TC-4 Rail Road Spur UPR-200-E-43 & $30,000 \mathrm{dpm} / 100 \mathrm{~cm}^{2}$ \\
\hline $10 / 21 / 03$ & Tumbleweed Fragment & Old 200-E Burn Pit 200-E-53 & $60,000 \mathrm{dpm} / 100 \mathrm{~cm}^{2}$ \\
\hline $10 / 28 / 03$ & Rabbit Brush/Tumbleweeds & 200-E-139 (CA north of 241-C) & $18,000 \mathrm{dpm} / 100 \mathrm{~cm}^{2}$ \\
\hline $10 / 29 / 03$ & Specks & 241-C Tank Farm Perimeter & $400,000 \mathrm{dpm} / 100 \mathrm{~cm}^{2}$ \\
\hline $10 / 29 / 03$ & Choker & MO-235 Crane \& Rigging Facility & $240,000 \mathrm{dpm} / 100 \mathrm{~cm}^{2}$ \\
\hline $10 / 30 / 03$ & Tumbleweeds & 216-U-10 Pond & $60,000 \mathrm{dpm} / 100 \mathrm{~cm}^{2}$ \\
\hline $10 / 31 / 03$ & Tumbleweeds & 216-U-10 Pond & $96,000 \mathrm{dpm} / 100 \mathrm{~cm}^{2}$ \\
\hline $11 / 03 / 03$ & Tumbleweeds & East Side of 200-E-121 & $85,000 \mathrm{dpm} / 100 \mathrm{~cm}^{2}$ \\
\hline $11 / 03 / 03$ & Tumbleweeds & 216-U-10 Pond & $96,000 \mathrm{dpm} / 100 \mathrm{~cm}^{2}$ \\
\hline $11 / 04 / 03$ & Tumbleweeds & 216-U-10 Pond & $60,000 \mathrm{dpm} / 100 \mathrm{~cm}^{2}$ \\
\hline $11 / 04 / 03$ & Mud Dauber Nests & Well 199-H3-2A, 105-H area & $349,000 \mathrm{dpm} / 100 \mathrm{~cm}^{2}$ \\
\hline $11 / 05 / 03$ & Tumbleweeds & 216-U-10 Pond & $60,000 \mathrm{dpm} / 100 \mathrm{~cm}^{2}$ \\
\hline $11 / 11 / 03$ & Tumbleweeds & 216-T-21 Trench & $240,000 \mathrm{dpm} / 100 \mathrm{~cm}^{2}$ \\
\hline $11 / 11 / 03$ & Tumbleweeds & 241-A Tank Farm Perimeter & $21,500 \mathrm{dpm} / 100 \mathrm{~cm}^{2}$ \\
\hline $11 / 11 / 03$ & Tumbleweeds & 241-BYTank Farm Perimeter & $60,000 \mathrm{dpm} / 100 \mathrm{~cm}^{2}$ \\
\hline $11 / 12 / 03$ & Tumbleweeds & 216-Z-5 Trench & $42,000 \mathrm{dpm} / 100 \mathrm{~cm}^{2}$ \\
\hline $11 / 12 / 03$ & Mud Dauber Wasp Nest & Well 1-N-125 south of the 1304-N Dump Tank & $156,000 \mathrm{dpm} / \mathrm{PA}$ \\
\hline $11 / 17 / 03$ & Tumbleweeds & North East perimeter of 218-E-12B & $800,000 \mathrm{dpm} / 100 \mathrm{~cm} 2$ \\
\hline $11 / 19 / 03$ & Tumbleweed Fragments & Eastern Fenceline 241-SX/SY & $99,000 \mathrm{dpm} / 100 \mathrm{~cm} 2$ \\
\hline $11 / 19 / 03$ & Tumbleweeds & 216-U-10 Pond & $96,000 \mathrm{dpm} / 100 \mathrm{~cm} 2$ \\
\hline $11 / 20 / 03$ & Tumbleweeds & UPR-600-20 Cross Site Transfer Line & $24,000 \mathrm{dpm} / 100 \mathrm{~cm} 2$ \\
\hline $12 / 04 / 03$ & Tumbleweeds & East of 241-A \& West of 216-A-8 Crib & $30,000 \mathrm{dpm} / 100 \mathrm{~cm} 2$ \\
\hline $12 / 16 / 03$ & Soil & North of 241-SX/SY by 242-S & $80,000 \mathrm{dpm} / 100 \mathrm{~cm} 2$ \\
\hline
\end{tabular}


This page intentionally left blank. 


\subsection{NOXIOUS WEED CONTROL PROGRAM}

Ten plant species are on a high priority list for control at the Hanford Site. These species are listed below, with a summary of the 2003 control activities. Major populations of noxious weeds on the Hanford Site are illustrated in Figure 8-1.

Yellow Starthistle (Centaurea solstitialis). Yellow starthistle represents the most rapidly expanding weed infestation in the Western United States. Hanford is at a critical point in the infestation cycle. Over 2,023 ha (5,000 acres) have been infested, and a seed bank has been established in the soil. Many additional acres have scattered starthistle infestation. Applications of aerial herbicides in 1998 and 1999 have been effective, with minimal germination in 2002. In 2003, significant germination was observed and plants were again controlled by aerial herbicide applications. Biological control organisms, primarily the hairy weevil (Eustenopus villosus) and the bud weevil (Bangasternus orientalis) were commonly found in starthistle during 2003. It was observed that buds flowering early through mid season were heavily infested with weevils. However, buds flowering late in the season showed reduced infestation of the flowering heads.

Rush Skeletonweed (Chondrilla juncea). Rush skeletonweed is widely scattered over large areas on the Hanford Site. Although areas of dense infestation have largely been eliminated, a considerable population remains as scattered individuals. Populations of skeletonweed have increased on some areas burned in the 24 Command fire (June 2000).

In 2003, control of rush skeletonweed concentrated on the area north of HAMMER and the Hanford Patrol Training Academy. Herbicides were aerially applied to approximately 1,200 acres with a relatively heavy rush skeletonweed population. The effectiveness of the application will be evaluated during the spring of 2004.

As in most years, some populations were highly affected by the bio-controls, and flowering was eliminated. Other populations were less affected, and some were not significantly impacted by the bio-control agent.

Medusahead (Taeniatherum asperum). Hand pulling was once again used to control the small population of Medusahead on the 200 Area plateau. Plants were pulled before seeds were mature. Monitoring and eradication efforts will continue in 2004 as the plants mature to the point where they can be distinguished from neighboring grass species.

Babysbreath (Gypsophila paniculata). Efforts to control babysbreath in 2003 concentrated on the main infestation at the Hanford Townsite. Although babysbreath is resistant to control by herbicides, effective herbicides exist that kill the upper portions of the plant. Controlling the top of the plant prevents flowering and additional seed production as well as depleting energy reserves in the roots until the plant succumbs. The invasion on the Hanford Site is relatively small, and control by attrition is the practical alternative. 
Dalmatian Toadflax (Linaria genistifolia ssp. Dalmatica). In 2003, control of dalmatian toadflax focused on a small population at 100-B/C Area. The species at Hanford has yielded to past control efforts. Seedlings of the long-lived perennial plant will be eliminated as they are identified.

Spotted Knapweed (Centaurea maculosa). Most populations of spotted knapweed on the Hanford Site have been reduced to scattered individuals, or seedlings germinating from the longlived seeds. Cooperative work with neighboring landowners continues to eliminate spotted knapweed near the Hanford Site.

Diffuse Knapweed (Centaurea diffusa). Aerial applications for control of diffuse knapweed have been effective in the past. Spot treatment of scattered individuals continued in 2003. Herbicide control of populations of diffuse knapweed near the high water mark of the Columbia River has not been actively pursued due to the biological sensitivity of the area. Biological controls have been established and their effectiveness continues to be monitored.

Russian Knapweed (Acroptilon repens). Biological controls for Russian knapweed are limited, and their success in the arid climate of Hanford has been poor. Chemicals and techniques are being developed that may prove effective with this difficult to control species.

Saltcedar (Tamarix spp.). Several individual plants of saltcedar are found on the Hanford Site. Most are remaining from ornamental plantings near homes in the early part of the previous century. A few populations are the result of natural seed dispersal. Most individuals south and west of the Columbia River have been eliminated. Those remaining continue to be treated with herbicide and will be monitored until they no longer show signs of life.

Purple Loosestrife (Lythrum salicaria). Purple loosestrife has established only sparse populations along the south and west bank of the Columbia River. Portions of the riverbank and slews are monitored for purple loosestrife and identified individuals are controlled. 
Figure 8-1. Major Populations of Noxious Weeds, 2003.

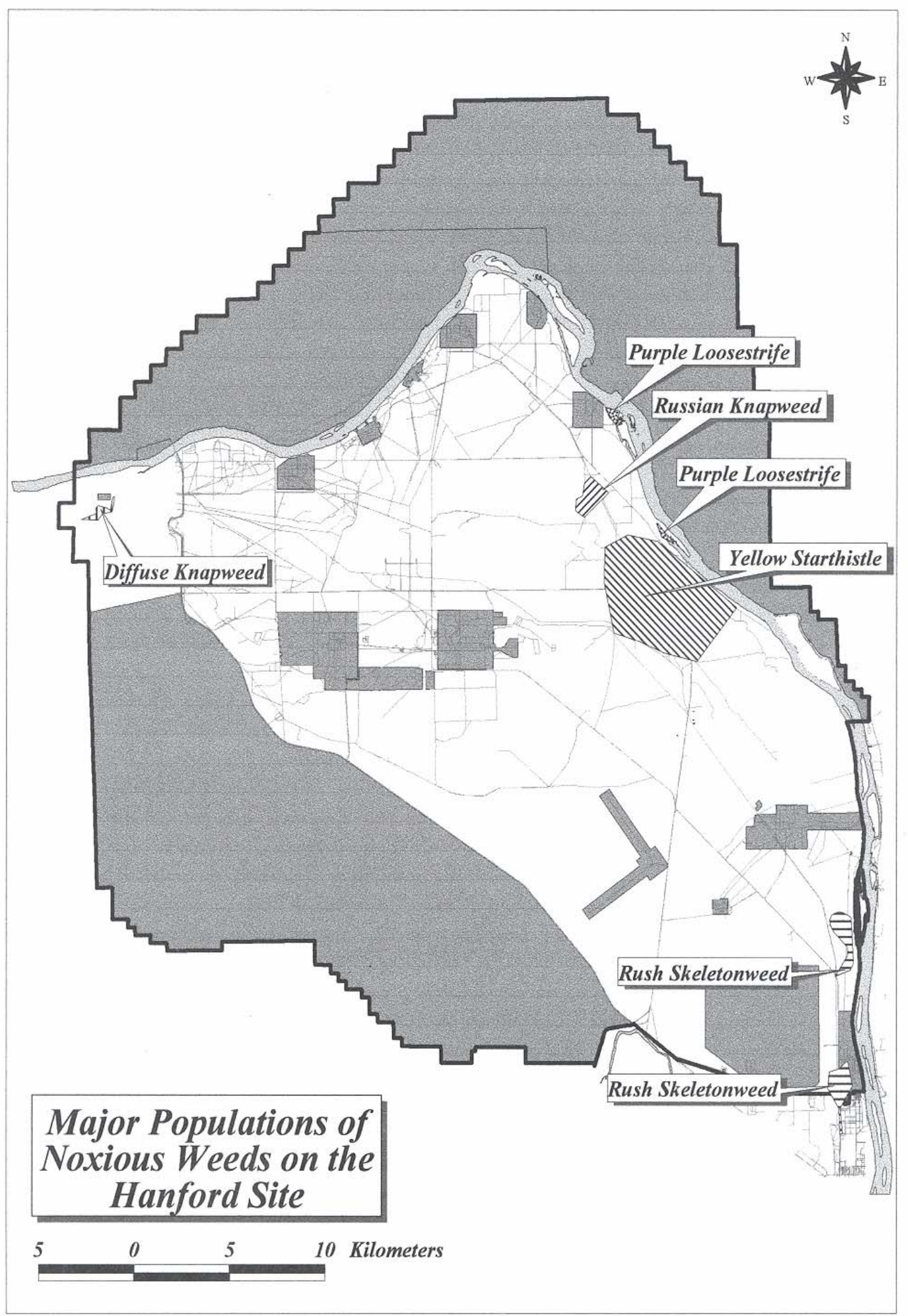


This page intentionally left blank. 


\subsection{QUALITY ASSURANCE}

QA may be defined as the actions necessary to provide confidence that an item, process, or program meets or exceeds that user's requirements and expectations. The near-facility environmental monitoring QA program consists of procedures and guides to demonstrate that environmental monitoring techniques and analyses are performed within established limits of acceptance. This is documented in the Near-Facility Environmental Monitoring Quality Assurance Project Plan (McKinney 2003).

Written operating procedures are an integral part of near-facility environmental monitoring QA. Procedures for field operations are provided in internal manual DTS-OEM-001. This section briefly describes the essential components of the near-facility environmental monitoring QA program.

\subsection{DOCUMENTATION}

Record keeping is a vital part of any environmental monitoring program. Maintenance of environmental data is important from a QA standpoint, from a regulatory standpoint, and for trend analyses and optimization of environmental monitoring procedures. Each phase of near-facility environmental monitoring is documented. This documentation includes environmental sample logbooks, quarterly reports, annual reports, and occurrence reports.

\subsection{SAMPLE REPLICATION}

Replicate sampling and subsequent analyses are the primary means of assessing sample variability. Duplicate samples of air, water, soil, sediment, and vegetation are collected.

\subsection{DATA ANALYSIS}

Environmental data are reviewed to determine compliance with applicable federal and company guides. The data are analyzed both graphically and by standard statistical tests to determine trends and impacts on the environment. Newly acquired data are compared with historical data and natural background levels. Routine environmental data are stored on both magnetic media (i.e., in a computer environment) and hard-copy printouts.

\subsection{TRAINING}

To ensure quality and consistency in sample collection and handling, all personnel performing such work received formal training. All radiological control technicians are required to complete a certification program. In addition, those radiological control technicians assigned to environmental monitoring receive special classroom orientation and on-the-job training by 
experienced personnel. Duratek Technical Services Environmental Monitoring and Investigations personnel, in addition to their formal training received while obtaining professional degrees, have received training in courses taught through Washington State University, the Harvard School of Public Health, and various other institutions.

\subsection{SAMPLE FREQUENCY}

1. Ambient air sample filters are collected biweekly.

2. Radiological surveys of roads are performed quarterly, bimonthly, or annually.

3. The TLDs are exchanged quarterly.

4. Radiological surveys of waste sites are performed quarterly, semiannually, or annually depending on the operating status, condition, and history of the site.

5. Soil, vegetation, and surface water samples are collected annually.

\subsection{ANALYTICAL PROCEDURES}

Three laboratories provided routine analytical support to the near-facility environmental monitoring: PNNL, the WSCF, and the 222-S Analytical Laboratory. Samples are analyzed in accordance with prescribed procedures and quality control guides that are described briefly in the following paragraphs.

\subsubsection{Pacific Northwest National Laboratory Radiation Standards and Engineering}

9.6.1.1 Thermoluminescent Dosimeters. External radiation levels are measured using TLDs. The Hanford Site uses the Harshaw 8807 dosimeter and the Harshaw 8800 reader. The TLDs are calibrated, packaged, and read by the PNNL Radiation Calibration Laboratory, Radiation Standards and Engineering Department. All TLD work is performed in accordance with formal, written procedures.

\subsubsection{2-S and Waste Sampling and Characterization Facility Analytical Laboratories}

The 222-S and WSCF laboratories also provide analytical support to near-facility environmental monitoring. Formal, written laboratory procedures are used in analyzing samples. The 222-S Laboratory is normally used for samples containing higher than normal environmental levels of radioactivity. The WSCF is used for the samples containing typical environmental 
levels of radioactivity. The WSCF also participates in an annual Quality Assurance Task Force (QATF) intercomparison program coordinated by the Radiation Protection division of the WDOH. 
This page intentionally left blank. 


\subsection{GLOSSARY}

Accessible Soils: Hanford soils that are not behind security fences must meet a $10 \mathrm{mrem} / \mathrm{yr}$ effective dose equivalent (EDE) limit from Hanford Site operations to the most exposed member of the public.

As Low As Reasonably Achievable (ALARA): The implementation of ALARA is described in HNF-PRO-1620, ALARA Program Scope. This concept applies to maintaining releases at or below prescribed regulatory limits.

Average Soil Contamination: Contamination generally dispersed through the soil. Numerically, the radioactivity content averaged over a suitable mass of soil.

Background Radiation: Refers to regional levels of radioactivity produced by sources other than those of specific interest (e.g., the nuclear activities at the Hanford Site).

Becquerel (Bq): The standard international unit of radioactivity. One Becquerel is one disintegration per second or: $\mathrm{Bq}=2.7 \mathrm{E}-11 \mathrm{Ci}$

Biological Transport: Means of biological transport may include one or more of the following processes:

- Movement of subsurface radioactivity to the surface by physiological vegetative processes.

- $\quad$ Dispersion of such vegetation by the wind.

- $\quad$ Contaminated urine and feces deposited by animals that have gained access to and ingested radioactive materials.

- $\quad$ Contaminated animals themselves that have ingested radioactive materials directly or ingested other contaminated animals or plants.

- $\quad$ Physical displacement of radioactive materials by burrowing animals.

- $\quad$ Nests built using contaminated materials.

Biota: The plant and animal life of a specific region.

Burial Ground: A land area specifically designated to receive contaminated solid or solidified liquid waste packages and equipment. The contaminated articles are usually placed in trenches and covered with overburden. 
Byproduct: A material that is not one of the primary products of a production process and is not solely or separately produced by the production process. Examples are process residues such as slag or distillation column bottoms. The term does not include a coproduct that is produced for the general public's use and is ordinarily used in the form in which it is produced by the process.

Calibration: Determining the deviation of an instrument from a standard traceable to the National Bureau of Standards or other recognized agency and reporting the deviations and/or eliminating them by adjustment.

Chemical Processing: Chemical treatment of material to separate desired components selectively. At the Hanford Site, plutonium, uranium, and fission products were chemically separated from irradiated fuels.

Committed Dose Equivalent: The predicted total dose equivalent to a tissue or organ over a 50-year period after a known intake of a radionuclide into the body. It does not include contributions from external dose. Committed dose equivalent is expressed in units of rem (or sievert).

Committed Effective Dose Equivalent: The sum of the committed dose equivalents to various tissues in the body, each multiplied by the appropriate weighing factor. Committed effective dose equivalent is expressed in units of rem (or sievert).

Composite Sample: A number of random samples initially collected from a waste and combined into a single sample; this sample is analyzed for the contaminants of concern.

\section{Comprehensive Environmental Response, Compensation, and Liability Act of 1980}

(CERCLA): Commonly known as "Superfund," CERCLA was enacted to respond to uncontrolled releases of hazardous substances to the environment, primarily at inactive sites that were not adequately addressed by the Resource Conservation and Recovery Act of 1976 (RCRA). CERCLA also applies to actively managed facilities and any onshore or offshore facility.

Controlled Area: An area where access is controlled to protect individuals from exposure to radiation and/or radioactive materials.

Contamination Area: Any area where contamination levels are greater than the values specified in Chapter 2, Table 2-2, PHMC Radiological Control Manual, HNF-5173, but less than or equal to 100 times those values.

Crib: An underground structure designed to receive liquid waste that percolates into the soil directly or percolates into the soil after having traveled through a connected tile field.

Decommissioning: Actions taken to reduce the potential health and safety impacts of DOE-controlled contaminated facilities. Actions could include stabilizing, reducing, or removing radioactivity or demolishing the contaminated facilities. 
Decontamination: The removal of radioactive or hazardous contamination from facilities, equipment, or soils by washing, heating, chemical or electrochemical treating, mechanical cleaning, or other techniques.

Derived Concentration Guide for Public Exposure (DCG-Public): The concentration of a radionuclide in air or water that, under conditions of continuous exposure for one year by one exposure mode (e.g., ingestion of water, submersion in air, or inhalation of air), would result in an EDE equal to the annual dose limit applicable to the group exposed. For exposure of the public, the DCG is the radionuclide concentration in air or water that would result in an EDE of $100 \mathrm{mrem}(1 \mathrm{mSv})$ to a person having the characteristics of the reference manual.

Diffuse Source: A source or sources of radioactive or chemical contaminants released into the environment that do not have a defined point or origin of release (a nonpoint source). Such sources are also known as area sources.

Disposal Facility: Any facility or part of a facility where hazardous and/or radioactive waste is intentionally placed or where any land or water wastes will remain after closure.

Ditch: An open surface site for transport of liquid wastes to a pond or trench structure designed for percolation.

Ecology: The Washington State Department of Ecology.

Effective Dose Equivalent: The summation of the products of the dose equivalent received by specified tissues of the body and a tissue-specific weighing factor. This sum is a risk-equivalent value and can be used to estimate the health-effects risk of the exposed individual. The tissuespecific weighing factor represents the fraction of the total health risk resulting from uniform whole-body irradiation that would be contributed by that particular tissue. The EDE includes the committed EDE from internal deposition of radionuclides and the EDE caused by penetrating radiation from sources outside the body. EDE is expressed in units of rem (or sievert).

Effluent: An airborne or liquid discharge from a facility after all engineered waste treatment and effluent controls have been performed. The term includes onsite discharges to the atmosphere, lagoons, ponds, cribs, injection wells, French drains, or ditches. The term does not include solid waste stored or removed for disposal or waste that is contained in retention basins or tanks before treatment and/or disposal.

Emissions Unit: Regarding air pollutant emissions, any part of a stationary source that emits or would have the potential to emit any pollutant subject to regulation.

Environmental Monitoring Plan: A two-part document prepared for each site, facility, or process that uses, generates, releases, or manages significant pollutants or hazardous materials.

Environmental Sites Database (ESD): A database of environmental sites that is administered by the ERC. 
External Radiation: Radiation originating from a source outside the body.

Facility: A processing plant, tank farm, shop, laboratory, powerhouse, or laundry. Including all contiguous land and structures, other appurtenances, and improvements on land used for recycling, reusing, reclaiming, transferring, storing, and treating of dangerous waste (including treatment, storage, and disposal sites as well as groundwater wells). (40 CFR 264, "Standards for Owners and Operators of Hazardous Waste Treatment Storage and Disposal Facilities," and WAC 173-303-040.)

Facility-Specific Environmental Monitoring: Routine environmental monitoring of all environmental media (air, biota, etc.) around facility parameters.

Field Blank: Aliquots of analyte-free water or solvents brought to the field in sealed containers and transported to the laboratory with the sample container. Field blanks include trip blanks and equipment blanks.

Field Duplicate: Field duplicates are collected at specified frequencies and are used to document precision. The field duplicate precision depends on the variance of waste composition, sampling techniques, and analytical technique.

Fugitive Emissions: Material that is generated incidental to an operation, process, or activity and that is released or dispersed into the open air. Fugitive emissions occur via pathways that do not allow routine measurement at the point of release.

Grab Sample: A single sample removed from a sample medium over a short time interval.

Groundwater: Water that exists below the water table, also referred to as the zone of saturation. However, the capillary fringe directly above can be completely saturated if the sediment is fine enough. To avoid this ambiguous term, the use of phreatic water, which is water that enters freely into wells under both confined and unconfined conditions is suggested. Phreatic water is a term originally applied only to water that occurs in the upper part of the zone of saturation under water table conditions (unconfined groundwater or well water), but has come to be applied to all water in the zone of saturation, thus making it an exact synonym of groundwater. Above the water table is the vadose zone, where water pressures are less than atmospheric pressure. This zone still contains water, but the water is held to the soil particles or other groundwater material by capillary force. Thus, while this water still can move within the vadose zone, it cannot move out of the zone into a well or other place is exposed to atmospheric pressure. The dividing line between water in the vadose zone and phreatic water is the atmospheric pressure between the two, with the pressure of vadose water being below atmospheric pressure and that of phreatic water (i.e., groundwater) above atmospheric pressure.

High-Efficiency Particulate Air (HEPA) Filter: To qualify as a HEPA filter, a filter must achieve an efficiency of $99.97 \%$ under laboratory conditions and $99.95 \%$ after installation for the removal of airborne particulates greater than 3 E-05 cm (0.3 microns). 
High-Level Nuclear Waste: Spent nuclear fuel or radioactive waste resulting directly from the dissolution and reprocessing of spent nuclear fuel. Secondary waste streams resulting from the dissolution and reprocessing of spent nuclear fuel are not considered high-level waste.

Immobile Radionuclides: All those radionuclides that are sorbed onto Hanford Site soils and usually would not migrate through the vadose zone or the groundwater below the future control zone.

Inaccessible Soils: Areas from which the general public is excluded (by fences, posting, patrols, or distance), but that are still subject to meteorological effects, are subject to a $10 \mathrm{mrem} / \mathrm{yr}$ operational EDE limit.

Inactive Crib: A crib that has been designated as permanently out of service.

Inactive Radioactive Waste Site: Any waste site that is no longer needed for current operational programs and that is not currently an active waste disposal site.

Inactive Waste Sites: Inactive waste sites include units such as burial grounds, unplanned release sites, cribs, ditches, ponds, trenches, and basins, abandoned storage areas, drains, singleshell tank piping, transfer pits, and jumper boxes.

Less Than Detectable: An analytical term for a concentration in a sample that is lower than the minimum detection capabilities of that analytical equipment or process.

Low-Level Waste: Any gaseous, liquid, or solid radioactive waste not classified as high-level waste, transuranic waste, or spent nuclear fuel, as defined by DOE Order 435.1, Radioactive Waste Management.

Maximum Contaminant Level (MCL): The drinking water standards specified in 40 CFR 141, "National Primary Drinking Water Regulations." See Appendix C, "Maximum Contaminant Levels."

Mean: Average value of a series of measurements.

Minimum Detection Limit: Smallest amount or concentration of a radionuclide or nonradioactive element that can be reliably detected in a sample.

Mixed Waste: Dangerous waste that also contains enough radioactivity to be classified as radioactive waste.

Monitoring System: Instrumentation that provides measurement of an airborne or liquid waste stream parameters. The system includes a detector and associated readout components. A continuous monitoring system measures the stream parameters on a near-real-time basis or as specified in applicable Environmental Protection Agency regulations, 40 CFR 52, "Approval and Promulgation of Implementation Plans," Appendix E; 40 CFR 51, "Requirements for Preparation, Adoption, and Submittal of Implementation Plans," Appendix P, or as defined in 
applicable American National Standards Institute standards. A radiation monitoring system is a system in which radiation or radioactivity is the measured parameter. An integrating monitoring system totals the instantaneously measured parameter over some time period. A sampling system does not measure or read out an instantaneous stream parameter.

Near Facility Environmental Monitoring: The collection and analysis of samples of air, water, soil, biota, and other media near nuclear facilities on DOE sites and their environs and the measurement of external radiation to demonstrate compliance with applicable standards and assess radiation exposures to employees and members of the public, and the near-field environment.

Nonroutine Activities: Any actions on a large-scale ( $>5$ acres), including stabilization, soil removal, fixative or sealant application, other surface treatments, or other activities that could affect future remediation activities in an inactive waste site.

Not Detected: A reporting term which describes any or all of the following: the overall analytical error was greater than the radionuclide concentration itself; or, after allowing for the subtraction of the background level of the radionuclide, the resulting concentration was less than zero; or, no radio analytical peak was detected during the analysis.

Operations: In this report, this term loosely refers to Fluor Project Hanford activities including chemical processing, waste management, and decommissioning.

Pesticide: As defined in 40 CFR 162 ("State Registration of Pesticide Products"), the term pesticide covers all pest-control chemicals such as herbicides, rodenticides, and insecticides.

Plutonium Processing and Handling Facility: Any facility constructed primarily to process plutonium (including plutonium-238) and that handles in-process plutonium.

Plutonium Storage Facility: Any facility constructed to store strategic (category I) quantities of plutonium.

Point Source: A single defined point (origin) of an airborne release, such as a vent or stack.

Pond: A surface impoundment used to contain or percolate low-level liquid radioactive waste, mixed waste, or hazardous waste.

Quality Assurance: A process designed to maintain the quality of the results of a program within established limits of acceptance.

Radiation Survey: Evaluation of an area or object with portable instruments to identify radioactive materials and radiation fields present. 
Radioactive Byproduct: Any radioactive material (except special nuclear material) yielded in or made radioactive by exposure to the radiation incident to the process of producing or using special nuclear material. The nonradioactive hazardous component of the waste material will be subject to regulation under the RCRA.

Radioactive Liquid Effluent: A liquid effluent that has a reasonable potential for containing radioactive materials in quantities such that the annual average concentration is equal to or greater than the MCL.

Radiological Control Area: An area where access is controlled to protect individuals from exposure to radiation and/or radioactive materials. Radiological control areas include, but are not limited to, areas posted as radiation areas, surface contamination, and underground radioactive materials, to describe the radiological condition of the area within.

Radiological Posting: Information in the form of signs and barriers to inform people of radiological conditions that warrant avoidance or special precautions for entry.

Representative Sample: The average stream parameter being measured occurs in the sample in the same average proportion that it occurs in the environmental discharge.

Retired Waste Site: A waste site that is isolated and no longer available to receive waste in any form.

Routine Activities: Any actions on a small-scale ( $<5$ acres), including radioactive hot-spot removal, vegetation removal, fencing, posting, herbicide spraying, stabilization, or immediate spill response) in an inactive waste site. In general, these routine actions shall not interfere with RCRA/CERCLA response or site investigations.

Sampling System: Instrumentation and equipment that remove a part of a liquid or airborne waste stream for subsequent quantitative determination of stream parameters. The system generally employs such devices as filters, other sample collection media, or effluent traps of some kind. A continuous sampling system removes a part of the stream continuously except during sample change, maintenance, repair, or other necessary outages. A grab sampling system removes an instantaneous part of the stream or removes a part of the stream over a time period.

Sediment Column: The sediment beneath a crib. It can mean either all the sediment beneath the bottom of the crib extending to the water table or all sediment beneath a crib contaminated by radioactive materials.

Site: The location of a significant event, a prehistoric or historic occupation or activity, or a building or structure (whether standing, ruined, or vanished) where the location itself maintains historical or archeological value, regardless of the value of any existing structure.

Soil at depth: Soil below $91 \mathrm{~cm}$ (36 in.).

Soil Contamination: Contaminated soil not releasable in accordance with DOE Order 5400.5. 
Solid Waste: Any discarded material that is not excluded by WAC 173-303-017(2) or that is not excluded by a variance granted under WAC 173-303-017(5). Materials are solid waste if they are: (1) abandoned by being disposed of, burned, or incinerated, or (2) accumulated, stored, or treated (but not recycled) before (or in lieu of) being abandoned by being disposed of, burned, or incinerated. In addition, a solid waste includes any material considered to be inherently wastelike.

Speck Contamination: Single grains of soil, rust particles, feces, or pieces of vegetation.

Spot Contamination: A spot or quantity of contamination less than $1 \mathrm{~cm}^{3}$ in volume, or areal contamination less than $15 \mathrm{~cm}^{2}$ in area.

Stabilization: The process of covering surface contaminated areas with clean backfill or topsoil.

Standard: A specified set of rules or conditions concerned with the classification of components; delineation of procedures; definition of terms; designation of materials, performance, design, or operations; or measurements of quality in describing materials, products, systems, services, or practices. A standard is more general than a procedure or specification and more specific than a criterion.

Standard Deviation: A measure of the range of values about the mean.

Standard Error of the Mean: A measure of the uncertainty in the estimated mean of averaged values.

Surface Soil: Soil from $0 \mathrm{~cm}$ (0 in.) to $5 \mathrm{~cm}$ (2 in.) deep.

Surplus Facilities: Surplus facilities include all facilities that have been accepted into a decommissioning program.

Survey: A method to detect the release, disposal, or presence of radioactive materials or hazardous substances under a specific set of conditions to determine actual or potential hazards. Such an evaluation may include, but is not limited to, tests, physical examinations, and measurements of radiation or concentrations of materials.

Suspect Waste Site: A site, believed to have been previously unknown or undocumented, that, because of characteristics present at the site or historical information about the site, is suspected of containing waste (i.e., non-dangerous, hazardous, dangerous, mixed, and radioactive).

Tank Farm: An area of large underground tanks designed to store up to 1 Mgal each of high-level liquid waste. 
Thermoluminescent Dosimeter: A chip or series of chips used for measuring external gamma radiation. It consists of a material capable of absorbing energy imparted by ionizing radiation, then emitting light as a result of thermal stimulation. A measure of that light is proportional to the radioactivity absorbed.

Topsoil: The soil used as a plant growth medium at the surface to a depth of $30 \mathrm{~cm}$ as measured at the restabilization site. Topsoil is added soil to support the stabilization of a retired disposal facility with the objective of controlling erosion, establishing the growth of perennial grasses, and preventing the growth of deep-rooted vegetation.

Total Analytical Uncertainty: All analytical measurements include some degree of uncertainty as a consequence of a series of unavoidable and unintentional inaccuracies related to the collection and analysis of samples. Examples of these inaccuracies can include errors associated with reading and recording results, sample handling and processing, instrument calibrations, numerical rounding, and randomness of radioactive decay. The total analytical uncertainty value implies that approximately $95 \%$ of the time a recount or reanalysis of the sample would give a value somewhere in the range between the initial reported value plus or minus the total analytical uncertainty.

Transuranic (TRU) Radionuclide: Any radionuclide having an atomic number greater than 92 (DOE Order 435.1).

Transuranic Waste: Without regard to source or form, radioactive waste that at the end of institutional control periods is contaminated with alpha-emitting transuranium radionuclides with half-lives greater than 20 years and concentrations greater than $100 \mathrm{nCi} / \mathrm{g}(3700 \mathrm{~Bq} / \mathrm{g})$. The Waste Isolation Pilot Plant, high-level waste, and spent nuclear fuel as defined by DOE Order 435.1 are specifically excluded from this definition.

Trip Blank: A type of field blank used to accompany sample containers to and from the field and to detect contamination or cross-contamination that occurs during sample handling and transportation.

Uncontaminated Soil: A soil or a land area that requires no controls or restrictions in any way for radiation protection purposes and/or meets the contamination limit specifications.

Underground Radioactive Material: A radiological posting status where subsurface radioactivity is present but where surface contamination does not exceed the soil standards.

Unity Rule: If more than one radionuclide is present, the sum of the fractions represented by each radionuclide concentration divided by its respective limiting concentration (administrative control value) shall not exceed unity. This rule could also apply to parameters other than radionuclide concentration.

Unplanned Release Site: An area that was contaminated by an unplanned release of radioactive contamination, making it a radiological control area. 
Unrestricted Release: Values below which unrestricted release of soils will occur will be defined in an applicable record of decision.

U.S. Environmental Protection Agency: The federal agency chartered with carrying out and monitoring the environmental regulations.

Waste Information Data System: A database that identifies waste management units on the Hanford Site. It is a subset of the ESD.

Waste Management: The activity involved with storing, disposing of, shipping, handling, and monitoring all radioactive waste.

Waste Sites: Any facility used for the planned disposal of hazardous, radioactive, toxic, or nonradioactive/nontoxic waste.

Water Table: The upper boundary of an unconfined aquifer below which saturated groundwater occurs. 
Table 10-1. Radionuclide Nomenclature.

\begin{tabular}{|c|c|c|c|c|c|}
\hline Radionuclide & Symbol & Half-Life & Radionuclide & Symbol & Half-Life \\
\hline Tritium & ${ }^{3} \mathrm{H}$ & $12.3 \mathrm{yr}$ & Cesium-134 & ${ }^{134} \mathrm{Cs}$ & $2.1 \mathrm{yr}$ \\
\hline Beryllium-7 & ${ }^{7} \mathrm{Be}$ & $53.28 \mathrm{~d}$ & Cesium-137 & ${ }^{137} \mathrm{Cs}$ & $30.3 \mathrm{yr}$ \\
\hline Carbon-14 & ${ }^{14} \mathrm{C}$ & $5.72 \mathrm{E}+03 \mathrm{yr}$ & Cerium-141 & ${ }^{141} \mathrm{Ce}$ & $32.5 \mathrm{~d}$ \\
\hline Sodium-22 & ${ }^{22} \mathrm{Na}$ & $2.6 \mathrm{yr}$ & Cerium-144 & ${ }^{144} \mathrm{Ce}$ & $284.6 \mathrm{~d}$ \\
\hline Potassium-40 & ${ }^{40} \mathrm{~K}$ & $1.26 \mathrm{E}+09 \mathrm{yr}$ & Promethium-147 & ${ }^{147} \mathrm{Pm}$ & $13.4 \mathrm{~min}$ \\
\hline Argon-41 & ${ }^{41} \mathrm{Ar}$ & $1.8 \mathrm{~h}$ & Europium-152 & ${ }^{152} \mathrm{Eu}$ & $13.5 \mathrm{yr}$ \\
\hline Chromium-51 & ${ }^{51} \mathrm{Cr}$ & $27.7 \mathrm{~d}$ & Europium-154 & ${ }^{154} \mathrm{Eu}$ & $8.6 \mathrm{yr}$ \\
\hline Manganese-54 & ${ }^{54} \mathrm{Mn}$ & $312 \mathrm{~d}$ & Europium-155 & ${ }^{155} \mathrm{Eu}$ & $4.7 \mathrm{yr}$ \\
\hline Cobalt-58 & ${ }^{58} \mathrm{Co}$ & $71 \mathrm{~d}$ & Thallium-208 & ${ }^{208} \mathrm{Tl}$ & $3.1 \mathrm{~min}$ \\
\hline Iron-59 & ${ }^{59} \mathrm{Fe}$ & $45 \mathrm{~d}$ & Bismuth-212 & ${ }^{212} \mathrm{Bi}$ & $60.6 \mathrm{~min}$ \\
\hline Cobalt-60 & ${ }^{60} \mathrm{Co}$ & $5.3 \mathrm{yr}$ & Lead-212 & ${ }^{212} \mathrm{~Pb}$ & $10.6 \mathrm{~h}$ \\
\hline Nickel-63 & ${ }^{63} \mathrm{Ni}$ & $100 \mathrm{yr}$ & Polonium-212 & ${ }^{212} \mathrm{Po}$ & $0.3 \times 10^{-6} \mathrm{~s}$ \\
\hline Zinc-65 & ${ }^{65} \mathrm{Zn}$ & $243.8 \mathrm{~d}$ & Polonium-216 & ${ }^{216} \mathrm{Po}$ & $0.15 \mathrm{~s}$ \\
\hline Krypton-85 & ${ }^{85} \mathrm{Kr}$ & $10.7 \mathrm{yr}$ & Radon-220 & ${ }^{220} \mathrm{Rn}$ & $55.6 \mathrm{~s}$ \\
\hline Strontium-89 & ${ }^{89} \mathrm{Sr}$ & $50.5 \mathrm{~d}$ & Radium-226 & ${ }^{226} \mathrm{Ra}$ & $1.60 \mathrm{E}+03 \mathrm{yr}$ \\
\hline Strontium-90 & ${ }^{90} \mathrm{Sr}$ & $29.1 \mathrm{yr}$ & Radium-228 & ${ }^{228} \mathrm{Ra}$ & $5.75 \mathrm{yr}$ \\
\hline Niobium-95 & ${ }^{95} \mathrm{Nb}$ & $35.0 \mathrm{~d}$ & Thorium-232 & ${ }^{232} \mathrm{Th}$ & $1.40 \mathrm{E}+10 \mathrm{yr}$ \\
\hline Zirconium-95 & ${ }^{95} \mathrm{Zr}$ & $64.0 \mathrm{~d}$ & Uranium Total & $\begin{array}{c}\text { U or } \\
\text { Uranium }\end{array}$ & $4.50 \mathrm{E}+09 \mathrm{yr}$ \\
\hline Technetium-99 & ${ }^{99} \mathrm{Tc}$ & $2.12 \mathrm{E}+05 \mathrm{yr}$ & Uranium-234 & ${ }^{234} \mathrm{U}$ & $2.40 \mathrm{E}+05 \mathrm{yr}$ \\
\hline Ruthenium-103 & ${ }^{103} \mathrm{Ru}$ & $39.4 \mathrm{~d}$ & Uranium-235 & ${ }^{235} \mathrm{U}$ & $7.00 \mathrm{E}+08 \mathrm{yr}$ \\
\hline Ruthenium-106 & ${ }^{106} \mathrm{Ru}$ & $1.0 \mathrm{yr}$ & Uranium-236 & ${ }^{236} \mathrm{U}$ & $2.30 \mathrm{E}+07 \mathrm{yr}$ \\
\hline Tin-113 & ${ }^{113} \mathrm{Sn}$ & $115 \mathrm{~d}$ & Uranium-238 & ${ }^{238} \mathrm{U}$ & $4.50 \mathrm{E}+09 \mathrm{yr}$ \\
\hline Antimony-124 & ${ }^{124} \mathrm{Sb}$ & $60 \mathrm{~d}$ & Plutonium-238 & ${ }^{238} \mathrm{Pu}$ & $87.7 \mathrm{yr}$ \\
\hline Antimony-125 & ${ }^{125} \mathrm{Sb}$ & $2.7 \mathrm{yr}$ & Plutonium-239/240 & ${ }^{239,240} \mathrm{Pu}$ & $2.40 \mathrm{E}+04 \mathrm{yr}$ \\
\hline Iodine-129 & ${ }^{129} \mathrm{I}$ & $1.7 \mathrm{E}+07 \mathrm{yr}$ & Plutonium-241 & ${ }^{241} \mathrm{Pu}$ & $14.4 \mathrm{yr}$ \\
\hline Iodine-131 & ${ }^{131} \mathrm{I}$ & $8.0 \mathrm{~d}$ & Americium-241 & ${ }^{241} \mathrm{Am}$ & $433 \mathrm{yr}$ \\
\hline Barium-133 & ${ }^{133} \mathrm{Ba}$ & $10.53 \mathrm{yr}$ & & & \\
\hline
\end{tabular}


This page intentionally left blank. 


\subsection{STANDARDS}

Table 11-1. U.S. Department of Energy Derived Concentration Guides. ${ }^{\mathrm{a}}$

\begin{tabular}{|c|c|c|c|c|c|}
\hline \multirow[b]{3}{*}{ Radionuclide } & \multicolumn{2}{|c|}{ DCG } & \multirow[b]{3}{*}{ Radionuclide } & \multicolumn{2}{|c|}{ DCG } \\
\hline & Air & Liquid & & Air & Liquid \\
\hline & $\left(\mathrm{pCi} / \mathrm{m}^{3}\right)$ & $(\mathrm{pCi} / \mathrm{L})$ & & $\left(\mathrm{pCi} / \mathrm{m}^{3}\right)$ & $(\mathrm{pCi} / \mathrm{L})$ \\
\hline${ }^{3} \mathrm{H}$ & $1.0 \mathrm{E}+05$ & $2.0 \mathrm{E}+06$ & ${ }^{147} \mathrm{Pm}$ & $3.0 \mathrm{E}+02$ & $1.0 \mathrm{E}+05$ \\
\hline${ }^{14} \mathrm{C}$ & $6.0 \mathrm{E}+03$ & $7.0 \mathrm{E}+04$ & ${ }^{152} \mathrm{Eu}$ & $5.0 \mathrm{E}+01$ & $2.0 \mathrm{E}+04$ \\
\hline${ }^{40} \mathrm{~K}$ & $9.0 \mathrm{E}+02$ & $7.0 \mathrm{E}+03$ & ${ }^{154} \mathrm{Eu}$ & $5.0 \mathrm{E}+01$ & $2.0 \mathrm{E}+04$ \\
\hline${ }^{41} \mathrm{Ar}$ & $1.0 \mathrm{E}+04$ & $0.0 \mathrm{E}+00$ & ${ }^{155} \mathrm{Eu}$ & $3.0 \mathrm{E}+02$ & $1.0 \mathrm{E}+05$ \\
\hline${ }^{51} \mathrm{Cr}$ & $6.0 \mathrm{E}+04$ & $1.0 \mathrm{E}+06$ & ${ }^{208} \mathrm{Tl}$ & $5.0 \mathrm{E}+03$ & $0.0 \mathrm{E}+00$ \\
\hline${ }^{54} \mathrm{Mn}$ & $2.0 \mathrm{E}+03$ & $5.0 \mathrm{E}+04$ & ${ }^{212} \mathrm{Bi}$ & $6.0 \mathrm{E}+02$ & $1.0 \mathrm{E}+05$ \\
\hline${ }^{59} \mathrm{Fe}$ & $8.0 \mathrm{E}+02$ & $2.0 \mathrm{E}+04$ & ${ }^{214} \mathrm{Bi}$ & $2.0 \mathrm{E}+03$ & $6.0 \mathrm{E}+05$ \\
\hline 58 Co & $2.0 \mathrm{E}+03$ & $4.0 \mathrm{E}+04$ & ${ }^{212} \mathrm{~Pb}$ & $8.0 \mathrm{E}+01$ & $3.0 \mathrm{E}+03$ \\
\hline${ }^{60} \mathrm{Co}$ & $8.0 \mathrm{E}+01$ & $5.0 \mathrm{E}+03$ & ${ }^{214} \mathrm{~Pb}$ & $2.0 \mathrm{E}+03$ & $2.0 \mathrm{E}+05$ \\
\hline${ }^{65} \mathrm{Zn}$ & $6.0 \mathrm{E}+02$ & $9.0 \mathrm{E}+03$ & ${ }^{212} \mathrm{Po}$ & $1.0 \mathrm{E}+00$ & $8.0 \mathrm{E}+01$ \\
\hline${ }^{85} \mathrm{Kr}$ & $3.0 \mathrm{E}+06$ & $0.0 \mathrm{E}+00$ & ${ }^{216} \mathrm{Po}$ & $1.0 \mathrm{E}+00$ & $8.0 \mathrm{E}+01$ \\
\hline${ }^{89} \mathrm{Sr}$ & $3.0 \mathrm{E}+02$ & $2.0 \mathrm{E}+04$ & ${ }^{220} \mathrm{Rn}$ & $3.0 \mathrm{E}+03$ & $0.0 \mathrm{E}+00$ \\
\hline${ }^{90} \mathrm{Sr}$ & $9.0 \mathrm{E}+00$ & $1.0 \mathrm{E}+03$ & ${ }^{224} \mathrm{Ra}$ & $4.0 \mathrm{E}+00$ & $4.0 \mathrm{E}+02$ \\
\hline${ }^{95} \mathrm{Zr}$ & $6.0 \mathrm{E}+02$ & $4.0 \mathrm{E}+04$ & ${ }^{226} \mathrm{Ra}$ & $1.0 \mathrm{E}+00$ & $1.0 \mathrm{E}+02$ \\
\hline${ }^{95} \mathrm{Nb}$ & $3.0 \mathrm{E}+03$ & $6.0 \mathrm{E}+04$ & ${ }^{228} \mathrm{Ac}$ & $4.0 \mathrm{E}+01$ & $6.0 \mathrm{E}+04$ \\
\hline${ }^{99} \mathrm{Tc}$ & $2.0 \mathrm{E}+03$ & $1.0 \mathrm{E}+05$ & ${ }^{232} \mathrm{Th}$ & $7.0 \mathrm{E}-03$ & $5.0 \mathrm{E}+01$ \\
\hline${ }^{103} \mathrm{Ru}$ & $2.0 \mathrm{E}+03$ & $5.0 \mathrm{E}+04$ & ${ }^{\text {Total }} \mathrm{U}$ & $1.0 \mathrm{E}-01$ & $6.0 \mathrm{E}+02$ \\
\hline${ }^{106} \mathrm{Ru}$ & $3.0 \mathrm{E}+01$ & $6.0 \mathrm{E}+03$ & ${ }^{234} \mathrm{U}$ & $9.0 \mathrm{E}-02$ & $5.0 \mathrm{E}+02$ \\
\hline${ }^{113} \mathrm{Sn}$ & $1.0 \mathrm{E}+03$ & $5.0 \mathrm{E}+04$ & ${ }^{235} \mathrm{U}$ & $1.0 \mathrm{E}-01$ & $6.0 \mathrm{E}+02$ \\
\hline${ }^{124} \mathrm{Sb}$ & $6.0 \mathrm{E}+02$ & $1.0 \mathrm{E}+04$ & ${ }^{236} \mathrm{U}$ & $1.0 \mathrm{E}-01$ & $5.0 \mathrm{E}+02$ \\
\hline${ }^{125} \mathrm{Sb}$ & $1.0 \mathrm{E}+03$ & $5.0 \mathrm{E}+04$ & ${ }^{238} \mathrm{U}$ & $1.0 \mathrm{E}-01$ & $6.0 \mathrm{E}+02$ \\
\hline${ }^{129} \mathrm{I}$ & $7.0 \mathrm{E}+01$ & $5.0 \mathrm{E}+02$ & ${ }^{238} \mathrm{Pu}$ & $3.0 \mathrm{E}-02$ & $4.0 \mathrm{E}+01$ \\
\hline${ }^{131} \mathrm{I}$ & $4.0 \mathrm{E}+02$ & $3.0 \mathrm{E}+03$ & ${ }^{239,240} \mathrm{Pu}$ & $2.0 \mathrm{E}-02$ & $3.0 \mathrm{E}+01$ \\
\hline${ }^{134} \mathrm{Cs}$ & $2.0 \mathrm{E}+02$ & $2.0 \mathrm{E}+03$ & ${ }^{241} \mathrm{Pu}$ & $1.0 \mathrm{E}+00$ & $2.0 \mathrm{E}+03$ \\
\hline${ }^{137} \mathrm{Cs}$ & $4.0 \mathrm{E}+02$ & $3.0 \mathrm{E}+03$ & ${ }^{241} \mathrm{Am}$ & $2.0 \mathrm{E}-02$ & $3.0 \mathrm{E}+01$ \\
\hline${ }^{141} \mathrm{Ce}$ & $1.0 \mathrm{E}+03$ & $5.0 \mathrm{E}+04$ & Total Alpha & $2.0 \mathrm{E}-02$ & $3.0 \mathrm{E}+01$ \\
\hline${ }^{144} \mathrm{Ce}$ & $3.0 \mathrm{E}+01$ & $7.0 \mathrm{E}+03$ & Total Beta & $9.0 \mathrm{E}+00$ & $1.0 \mathrm{E}+03$ \\
\hline
\end{tabular}

${ }^{\mathrm{a}}$ From DOE Order 5400.5. 
Table 11-2. EPA Concentration Levels for Environmental Compliance. ${ }^{\mathrm{a}}$ (Radionuclide Concentrations $\left[\mathrm{pCi} / \mathrm{m}^{3}\right]$ in Air)

\begin{tabular}{cc} 
Radionuclide & Concentration \\
\hline${ }^{3} \mathrm{H}$ & $1.5 \mathrm{E}+03$ \\
${ }^{14} \mathrm{C}$ & $1.0 \mathrm{E}+01$ \\
${ }^{40} \mathrm{~K}$ & $2.7 \mathrm{E}-02$ \\
${ }^{41} \mathrm{Ar}$ & $1.7 \mathrm{E}+03$ \\
${ }^{51} \mathrm{Cr}$ & $3.1 \mathrm{E}+01$ \\
${ }^{54} \mathrm{Mn}$ & $2.8 \mathrm{E}-01$ \\
${ }^{59} \mathrm{Fe}$ & $6.7 \mathrm{E}-01$ \\
${ }^{58} \mathrm{Co}$ & $6.7 \mathrm{E}-01$ \\
${ }^{60} \mathrm{Co}$ & $1.7 \mathrm{E}-02$ \\
${ }^{65} \mathrm{Zn}$ & $9.1 \mathrm{E}-02$ \\
${ }^{85} \mathrm{Kr}$ & $1.0 \mathrm{E}+06$ \\
${ }^{89} \mathrm{Sr}$ & $1.8 \mathrm{E}+00$ \\
${ }^{90} \mathrm{Sr}$ & $1.9 \mathrm{E}-02$ \\
${ }^{95} \mathrm{Zr}$ & $6.7 \mathrm{E}-01$ \\
${ }^{95} \mathrm{Nb}$ & $2.2 \mathrm{E}+00$ \\
${ }^{99} \mathrm{Tc}$ & $1.4 \mathrm{E}-01$ \\
${ }^{103} \mathrm{Ru}$ & $2.6 \mathrm{E}+00$ \\
${ }^{106} \mathrm{Ru}$ & $3.4 \mathrm{E}-01$ \\
${ }^{113} \mathrm{Sn}$ & $1.4 \mathrm{E}+00$ \\
${ }^{124} \mathrm{Sb}$ & $5.3 \mathrm{E}-01$ \\
${ }^{134} \mathrm{I}$ & $1.6 \mathrm{E}-01$ \\
${ }^{129} \mathrm{Sb}$ & $9.1 \mathrm{E}-03$ \\
${ }^{125} \mathrm{Cs}$ & $2.1 \mathrm{E}-01$ \\
$2.7 \mathrm{E}-02$ \\
\end{tabular}

\begin{tabular}{cc} 
Radionuclide & Concentration \\
\hline${ }^{137} \mathrm{Cs}$ & $1.9 \mathrm{E}-02$ \\
${ }^{141} \mathrm{Ce}$ & $6.3 \mathrm{E}+00$ \\
${ }^{144} \mathrm{Ce}$ & $6.2 \mathrm{E}-01$ \\
${ }^{147} \mathrm{Pm}$ & $1.1 \mathrm{E}+01$ \\
${ }^{152} \mathrm{Eu}$ & $2.0 \mathrm{E}-02$ \\
${ }^{154} \mathrm{Eu}$ & $2.3 \mathrm{E}-02$ \\
${ }^{155} \mathrm{Eu}$ & $5.9 \mathrm{E}-01$ \\
${ }^{212} \mathrm{Bi}$ & $5.6 \mathrm{E}+01$ \\
${ }^{214} \mathrm{Bi}$ & $1.4 \mathrm{E}+02$ \\
${ }^{212} \mathrm{~Pb}$ & $6.3 \mathrm{E}+00$ \\
${ }^{214} \mathrm{~Pb}$ & $1.2 \mathrm{E}+02$ \\
${ }^{224} \mathrm{Ra}$ & $1.5 \mathrm{E}-01$ \\
${ }^{226} \mathrm{Ra}$ & $3.3 \mathrm{E}-03$ \\
${ }^{228} \mathrm{Ac}$ & $3.7 \mathrm{E}+00$ \\
${ }^{232} \mathrm{Th}$ & $6.2 \mathrm{E}-04$ \\
${ }^{234} \mathrm{U}$ & $7.7 \mathrm{E}-03$ \\
${ }^{235} \mathrm{U}$ & $7.1 \mathrm{E}-03$ \\
${ }^{236} \mathrm{U}$ & $7.7 \mathrm{E}-03$ \\
${ }^{238} \mathrm{U}$ & $8.3 \mathrm{E}-03$ \\
${ }^{238} \mathrm{Pu}$ & $2.1 \mathrm{E}-03$ \\
${ }^{239 / 240} \mathrm{Pu}$ & $2.0 \mathrm{E}-03$ \\
${ }^{241} \mathrm{Pu}$ & $1.0 \mathrm{E}-01$ \\
& $1.9 \mathrm{E}-03$ \\
\end{tabular}

$\overline{\text { a - from } 40 \text { CFR } 61}$, Subpart I, Appendix E, Table 2 
Table 11-3. Inaccessible Soil Concentrations (pCi/g).

\begin{tabular}{|c|c|c|c|c|c|c|}
\hline Radionuclide & 100 BDKN & $100 \mathrm{~F}, \mathrm{H}$ & 200 West Area & 200 East Area & 300 Area & 400 Area \\
\hline${ }^{3} \mathrm{H}$ & $1.4 \mathrm{E}+08$ & $7.4 \mathrm{E}+07$ & $3.7 \mathrm{E}+08$ & $2.0 \mathrm{E}+08$ & $9.5 \mathrm{E}+06$ & $1.4 \mathrm{E}+07$ \\
\hline${ }^{14} \mathrm{C}$ & $6.2 \mathrm{E}+05$ & $6.2 \mathrm{E}+05$ & $6.2 \mathrm{E}+05$ & $6.2 \mathrm{E}+05$ & $6.2 \mathrm{E}+05$ & $6.2 \mathrm{E}+05$ \\
\hline${ }^{55} \mathrm{Fe}$ & $9.7 \mathrm{E}+06$ & $9.7 \mathrm{E}+06$ & $3.6 \mathrm{E}+10$ & $1.9 \mathrm{E}+10$ & $1.0 \mathrm{E}+07$ & $1.4 \mathrm{E}+09$ \\
\hline${ }^{58} \mathrm{Co}$ & $9.8 E+06$ & $9.8 \mathrm{E}+06$ & $8.1 \mathrm{E}+09$ & $4.3 \mathrm{E}+09$ & $1.2 \mathrm{E}+07$ & $3.1 \mathrm{E}+08$ \\
\hline${ }^{60} \mathrm{Co}$ & $9.9 \mathrm{E}+05$ & $9.9 \mathrm{E}+05$ & $5.7 \mathrm{E}+08$ & $3.0 \mathrm{E}+08$ & $1.0 \mathrm{E}+06$ & $9.9 \mathrm{E}+06$ \\
\hline${ }^{63} \mathrm{Ni}$ & $1.5 \mathrm{E}+08$ & $1.5 \mathrm{E}+08$ & $6.9 \mathrm{E}+09$ & $6.9 \mathrm{E}+09$ & $1.5 \mathrm{E}+08$ & $2.2 \mathrm{E}+08$ \\
\hline${ }^{90} \mathrm{Sr}^{*}$ & $8.3 E+05$ & $8.3 E+05$ & $2.2 \mathrm{E}+08$ & $1.2 \mathrm{E}+08$ & $8.3 \mathrm{E}+05$ & $8.4 \mathrm{E}+06$ \\
\hline${ }^{99} \mathrm{Tc}$ & $1.3 \mathrm{E}+07$ & $1.3 \mathrm{E}+07$ & $1.3 \mathrm{E}+07$ & $1.3 \mathrm{E}+07$ & $1.3 \mathrm{E}+07$ & $1.3 \mathrm{E}+07$ \\
\hline${ }^{106} \mathrm{Ru}^{*}$ & $2.0 \mathrm{E}+07$ & $2.0 \mathrm{E}+07$ & $5.7 \mathrm{E}+08$ & $3.0 \mathrm{E}+08$ & $1.5 \mathrm{E}+07$ & $2.2 \mathrm{E}+07$ \\
\hline${ }^{125} \mathrm{Sb}^{*}$ & $9.1 \mathrm{E}+06$ & $9.1 \mathrm{E}+06$ & $5.7 \mathrm{E}+09$ & $3.0 \mathrm{E}+09$ & $9.2 \mathrm{E}+06$ & $1.1 \mathrm{E}+08$ \\
\hline${ }^{129} \mathrm{I}$ & $2.8 \mathrm{E}+05$ & $2.8 \mathrm{E}+05$ & $2.8 \mathrm{E}+05$ & $2.8 \mathrm{E}+05$ & $2.2 \mathrm{E}+05$ & $2.8 \mathrm{E}+05$ \\
\hline${ }^{134} \mathrm{Cs}$ & $1.7 \mathrm{E}+04$ & $1.7 \mathrm{E}+04$ & $2.5 \mathrm{E}+08$ & $1.4 \mathrm{E}+08$ & $2.4 \mathrm{E}+04$ & $9.7 \mathrm{E}+06$ \\
\hline${ }^{137} \mathrm{Ce}^{*}$ & $1.7 \mathrm{E}+04$ & $1.7 \mathrm{E}+04$ & $3.5 \mathrm{E}+08$ & $1.8 \mathrm{E}+08$ & $1.7 \mathrm{E}+04$ & $1.3 \mathrm{E}+07$ \\
\hline${ }^{144} \mathrm{Cs}^{*}$ & $1.4 \mathrm{E}+06$ & $1.4 \mathrm{E}+06$ & $7.4 \mathrm{E}+08$ & $4.0 \mathrm{E}+08$ & $1.9 \mathrm{E}+06$ & $2.8 \mathrm{E}+07$ \\
\hline${ }^{147} \mathrm{Pm}$ & $3.4 \mathrm{E}+07$ & $3.4 \mathrm{E}+07$ & $7.4 \mathrm{E}+09$ & $4.0 \mathrm{E}+09$ & $3.5 \mathrm{E}+07$ & $2.8 \mathrm{E}+08$ \\
\hline${ }^{152} \mathrm{Eu}$ & $4.5 \mathrm{E}+06$ & $4.5 \mathrm{E}+06$ & $1.2 \mathrm{E}+09$ & $6.2 \mathrm{E}+08$ & $4.6 \mathrm{E}+06$ & $4.5 \mathrm{E}+07$ \\
\hline${ }^{154} \mathrm{Eu}$ & $3.3 \mathrm{E}+06$ & $3.3 \mathrm{E}+06$ & $8.8 \mathrm{E}+08$ & $4.7 \mathrm{E}+08$ & $3.3 \mathrm{E}+06$ & $3.4 \mathrm{E}+07$ \\
\hline${ }^{155} \mathrm{Eu}$ & $2.3 \mathrm{E}+07$ & $2.3 \mathrm{E}+07$ & $6.9 \mathrm{E}+09$ & $3.7 \mathrm{E}+09$ & $2.4 \mathrm{E}+07$ & $2.6 \mathrm{E}+08$ \\
\hline${ }^{226} \mathrm{Ra}^{*}$ & $1.3 \mathrm{E}+05$ & $1.3 \mathrm{E}+05$ & $2.1 \mathrm{E}+05$ & $2.1 \mathrm{E}+05$ & $1.3 \mathrm{E}+05$ & $1.4 \mathrm{E}+05$ \\
\hline${ }^{227}$ Ac* & $2.4 \mathrm{E}+03$ & $2.4 \mathrm{E}+03$ & $5.4 \mathrm{E}+04$ & $2.9 \mathrm{E}+04$ & $1.4 \mathrm{E}+03$ & $2.1 \mathrm{E}+03$ \\
\hline${ }^{232} \mathrm{Th}^{*}$ & $2.0 \mathrm{E}+04$ & $2.0 \mathrm{E}+04$ & $2.0 \mathrm{E}+04$ & $2.0 \mathrm{E}+04$ & $4.7 \mathrm{E}+03$ & $7.1 \mathrm{E}+03$ \\
\hline${ }^{232} \mathrm{U}^{*}$ & $5.5 \mathrm{E}+04$ & $5.5 \mathrm{E}+04$ & $1.4 \mathrm{E}+05$ & $1.4 \mathrm{E}+05$ & $9.9 \mathrm{E}+03$ & $1.5 \mathrm{E}+04$ \\
\hline${ }^{233} \mathrm{U}$ & $4.5 \mathrm{E}+05$ & $4.5 \mathrm{E}+05$ & $4.5 \mathrm{E}+05$ & $4.5 \mathrm{E}+05$ & $6.7 \mathrm{E}+04$ & $1.0 \mathrm{E}+05$ \\
\hline${ }^{234} \mathrm{U}$ & $4.6 \mathrm{E}+05$ & $4.6 \mathrm{E}+05$ & $4.6 \mathrm{E}+05$ & $4.6 \mathrm{E}+05$ & $6.9 \mathrm{E}+04$ & $1.0 \mathrm{E}+05$ \\
\hline${ }^{235} \mathrm{U}^{*}$ & $4.9 \mathrm{E}+05$ & $4.9 \mathrm{E}+05$ & $4.9 \mathrm{E}+05$ & $4.9 \mathrm{E}+05$ & $7.3 \mathrm{E}+04$ & $1.1 \mathrm{E}+05$ \\
\hline${ }^{236} \mathrm{U}$ & $4.9 \mathrm{E}+05$ & $4.9 \mathrm{E}+05$ & $4.9 \mathrm{E}+05$ & $4.9 \mathrm{E}+05$ & $7.1 \mathrm{E}+04$ & $1.1 \mathrm{E}+05$ \\
\hline${ }^{238} \mathrm{U}^{*}$ & $4.7 \mathrm{E}+05$ & $4.7 \mathrm{E}+05$ & $4.7 \mathrm{E}+05$ & $4.7 \mathrm{E}+05$ & $7.7 \mathrm{E}+04$ & $1.2 \mathrm{E}+05$ \\
\hline${ }^{237} \mathrm{~Np}^{*}$ & $8.9 \mathrm{E}+02$ & $8.9 \mathrm{E}+02$ & $8.9 \mathrm{E}+02$ & $8.9 \mathrm{E}+02$ & $8.9 \mathrm{E}+02$ & $8.9 \mathrm{E}+02$ \\
\hline${ }^{238} \mathrm{Pu}$ & $1.3 \mathrm{E}+04$ & $1.3 \mathrm{E}+04$ & $8.8 \mathrm{E}+05$ & $4.7 \mathrm{E}+05$ & $1.3 \mathrm{E}+04$ & $3.4 \mathrm{E}+04$ \\
\hline${ }^{239} \mathrm{Pu}$ & $1.2 \mathrm{E}+04$ & $1.2 \mathrm{E}+04$ & $1.2 \mathrm{E}+04$ & $1.2 \mathrm{E}+04$ & $1.2 \mathrm{E}+04$ & $1.2 \mathrm{E}+04$ \\
\hline${ }^{240} \mathrm{Pu}$ & $1.2 \mathrm{E}+04$ & $1.2 \mathrm{E}+04$ & $1.4 \mathrm{E}+04$ & $1.4 \mathrm{E}+04$ & $1.2 \mathrm{E}+04$ & $1.2 \mathrm{E}+04$ \\
\hline${ }^{241} \mathrm{Pu}$ & $6.1 \mathrm{E}+05$ & $6.1 \mathrm{E}+05$ & $4.2 \mathrm{E}+07$ & $2.2 \mathrm{E}+07$ & $6.1 \mathrm{E}+05$ & $1.2 \mathrm{E}+06$ \\
\hline${ }^{241} \mathrm{Am}$ & $2.5 \mathrm{E}+04$ & $2.5 \mathrm{E}+04$ & $7.4 \mathrm{E}+05$ & $4.0 \mathrm{E}+05$ & $1.9 \mathrm{E}+04$ & $2.8 \mathrm{E}+04$ \\
\hline
\end{tabular}

Note: Asterisks mark nuclides with progeny that are assumed to be present in equilibrium amounts. However, ${ }^{234} \mathrm{U}$ was not included in the ${ }^{238} \mathrm{U}$ limits. For supporting references see WHC-SD-EN-TI-070. 
Table 11-4. Accessible Soil Concentrations (pCi/g).

\begin{tabular}{|c|c|c|c|c|c|c|}
\hline Radionuclide & 100 BDKN & $100 \mathrm{~F}, \mathrm{H}$ & 200 West Area & 200 East Area & 300 Area & 400 Area \\
\hline${ }^{3} \mathrm{H}$ & $1.4 \mathrm{E}+08$ & $7.4 \mathrm{E}+07$ & $3.7 \mathrm{E}+08$ & $2.0 \mathrm{E}+08$ & $9.5 \mathrm{E}+06$ & $1.4 \mathrm{E}+07$ \\
\hline${ }^{14} \mathrm{C}$ & $6.2 \mathrm{E}+05$ & $6.2 \mathrm{E}+05$ & $6.2 \mathrm{E}+05$ & $6.2 \mathrm{E}+05$ & $6.2 \mathrm{E}+05$ & $6.2 \mathrm{E}+05$ \\
\hline${ }^{55} \mathrm{Fe}$ & $5.3 \mathrm{E}+05$ & $5.3 \mathrm{E}+05$ & $5.3 \mathrm{E}+05$ & $5.3 \mathrm{E}+05$ & $5.3 \mathrm{E}+05$ & $5.3 \mathrm{E}+05$ \\
\hline${ }^{58} \mathrm{Co}$ & $1.8 \mathrm{E}+01$ & $1.8 \mathrm{E}+01$ & $1.8 \mathrm{E}+01$ & $1.8 \mathrm{E}+01$ & $1.8 \mathrm{E}+01$ & $1.8 \mathrm{E}+01$ \\
\hline${ }^{60} \mathrm{Co}$ & $7.1 \mathrm{E}+00$ & $7.1 \mathrm{E}+00$ & $7.1 \mathrm{E}+00$ & $7.1 \mathrm{E}+00$ & $7.1 \mathrm{E}+00$ & $7.1 \mathrm{E}+00$ \\
\hline${ }^{63} \mathrm{Ni}$ & $2.5 \mathrm{E}+07$ & $2.5 \mathrm{E}+07$ & $2.5 \mathrm{E}+07$ & $2.5 \mathrm{E}+07$ & $2.5 \mathrm{E}+07$ & $2.5 \mathrm{E}+07$ \\
\hline${ }^{90} \mathrm{Sr}^{*}$ & $2.8 \mathrm{E}+03$ & $2.8 \mathrm{E}+03$ & $2.8 \mathrm{E}+03$ & $2.8 \mathrm{E}+03$ & $2.8 \mathrm{E}+03$ & $2.8 \mathrm{E}+03$ \\
\hline${ }^{99} \mathrm{Tc}$ & $1.0 \mathrm{E}+06$ & $1.0 \mathrm{E}+06$ & $1.0 \mathrm{E}+06$ & $1.0 \mathrm{E}+06$ & $1.0 \mathrm{E}+06$ & $1.0 \mathrm{E}+06$ \\
\hline${ }^{106} \mathrm{Ru}^{*}$ & $7.7 \mathrm{E}+01$ & $7.7 \mathrm{E}+01$ & $7.7 \mathrm{E}+01$ & $7.7 \mathrm{E}+01$ & $7.7 \mathrm{E}+01$ & $7.7 \mathrm{E}+01$ \\
\hline${ }^{125} \mathrm{Sb}^{*}$ & $3.7 \mathrm{E}+01$ & $3.7 \mathrm{E}+01$ & $3.7 \mathrm{E}+01$ & $3.7 \mathrm{E}+01$ & $3.7 \mathrm{E}+01$ & $3.7 \mathrm{E}+01$ \\
\hline${ }^{129} \mathrm{I}$ & $1.0 \mathrm{E}+04$ & $1.0 \mathrm{E}+04$ & $1.0 \mathrm{E}+04$ & $1.0 \mathrm{E}+04$ & $1.0 \mathrm{E}+04$ & $1.0 \mathrm{E}+04$ \\
\hline${ }^{134} \mathrm{Cs}$ & $1.0 \mathrm{E}+01$ & $1.0 \mathrm{E}+01$ & $1.0 \mathrm{E}+01$ & $1.0 \mathrm{E}+01$ & $1.0 \mathrm{E}+01$ & $1.0 \mathrm{E}+01$ \\
\hline${ }^{137}$ Cs* & $3.0 \mathrm{E}+01$ & $3.0 \mathrm{E}+01$ & $3.0 \mathrm{E}+01$ & $3.0 \mathrm{E}+01$ & $3.0 \mathrm{E}+01$ & $3.0 \mathrm{E}+01$ \\
\hline${ }^{144} \mathrm{Ce}^{*}$ & $3.3 \mathrm{E}+02$ & $3.3 \mathrm{E}+02$ & $3.3 \mathrm{E}+02$ & $3.3 \mathrm{E}+02$ & $3.3 \mathrm{E}+02$ & $3.3 \mathrm{E}+02$ \\
\hline${ }^{147} \mathrm{Pm}$ & $1.1 \mathrm{E}+06$ & $1.1 \mathrm{E}+06$ & $1.1 \mathrm{E}+06$ & $1.1 \mathrm{E}+06$ & $1.1 \mathrm{E}+06$ & $1.1 \mathrm{E}+06$ \\
\hline${ }^{152} \mathrm{Eu}$ & $1.5 \mathrm{E}+01$ & $1.5 \mathrm{E}+01$ & $1.5 \mathrm{E}+01$ & $1.5 \mathrm{E}+01$ & $1.5 \mathrm{E}+01$ & $1.5 \mathrm{E}+01$ \\
\hline${ }^{154} \mathrm{Eu}$ & $1.4 \mathrm{E}+01$ & $1.4 \mathrm{E}+01$ & $1.4 \mathrm{E}+01$ & $1.4 \mathrm{E}+01$ & $1.4 \mathrm{E}+01$ & $1.4 \mathrm{E}+01$ \\
\hline${ }^{155} \mathrm{Eu}$ & $6.3 \mathrm{E}+02$ & $6.3 \mathrm{E}+02$ & $6.3 \mathrm{E}+02$ & $6.3 \mathrm{E}+02$ & $6.3 \mathrm{E}+02$ & $6.3 \mathrm{E}+02$ \\
\hline${ }^{226} \mathrm{Ra}^{*}$ & $1.0 \mathrm{E}+01$ & $1.0 \mathrm{E}+01$ & $1.0 \mathrm{E}+01$ & $1.0 \mathrm{E}+01$ & $1.0 \mathrm{E}+01$ & $1.0 \mathrm{E}+01$ \\
\hline${ }^{227} \mathrm{Ac}^{*}$ & $1.0 \mathrm{E}+01$ & $1.0 \mathrm{E}+01$ & $1.0 \mathrm{E}+01$ & $1.0 \mathrm{E}+01$ & $1.0 \mathrm{E}+01$ & $1.0 \mathrm{E}+01$ \\
\hline${ }^{232} \mathrm{Th}^{*}$ & $5.9 \mathrm{E}+00$ & $5.9 \mathrm{E}+00$ & $5.9 \mathrm{E}+00$ & $5.9 \mathrm{E}+00$ & $5.9 \mathrm{E}+00$ & $5.9 \mathrm{E}+00$ \\
\hline${ }^{232} \mathrm{U}^{*}$ & $1.0 \mathrm{E}+01$ & $1.0 \mathrm{E}+01$ & $1.0 \mathrm{E}+01$ & $1.0 \mathrm{E}+01$ & $1.0 \mathrm{E}+01$ & $1.0 \mathrm{E}+01$ \\
\hline${ }^{233} \mathrm{U}$ & $6.3 \mathrm{E}+02$ & $6.3 \mathrm{E}+02$ & $6.3 \mathrm{E}+02$ & $6.3 \mathrm{E}+02$ & $6.3 \mathrm{E}+02$ & $6.3 \mathrm{E}+02$ \\
\hline${ }^{234} \mathrm{U}$ & $6.3 \mathrm{E}+02$ & $6.3 \mathrm{E}+02$ & $6.3 \mathrm{E}+02$ & $6.3 \mathrm{E}+02$ & $6.3 \mathrm{E}+02$ & $6.3 \mathrm{E}+02$ \\
\hline${ }^{235} \mathrm{U} *$ & $1.7 \mathrm{E}+02$ & $1.7 \mathrm{E}+02$ & $1.7 \mathrm{E}+02$ & $1.7 \mathrm{E}+02$ & $1.7 \mathrm{E}+02$ & $1.7 \mathrm{E}+02$ \\
\hline${ }^{236} \mathrm{U}$ & $6.7 \mathrm{E}+02$ & $6.7 \mathrm{E}+02$ & $6.7 \mathrm{E}+02$ & $6.7 \mathrm{E}+02$ & $6.7 \mathrm{E}+02$ & $6.7 \mathrm{E}+02$ \\
\hline${ }^{238} \mathrm{U}^{*}$ & $3.7 \mathrm{E}+02$ & $3.7 \mathrm{E}+02$ & $3.7 \mathrm{E}+02$ & $3.7 \mathrm{E}+02$ & $3.7 \mathrm{E}+02$ & $3.7 \mathrm{E}+02$ \\
\hline${ }^{237} \mathrm{~Np}^{*}$ & $4.8 \mathrm{E}+01$ & $4.8 \mathrm{E}+01$ & $4.8 \mathrm{E}+01$ & $4.8 \mathrm{E}+01$ & $4.8 \mathrm{E}+01$ & $4.8 \mathrm{E}+01$ \\
\hline${ }^{238} \mathrm{Pu}$ & $2.1 \mathrm{E}+02$ & $2.1 \mathrm{E}+02$ & $2.1 \mathrm{E}+02$ & $2.1 \mathrm{E}+02$ & $2.1 \mathrm{E}+02$ & $2.1 \mathrm{E}+02$ \\
\hline${ }^{239} \mathrm{Pu}$ & $1.9 \mathrm{E}+02$ & $1.9 \mathrm{E}+02$ & $1.9 \mathrm{E}+02$ & $1.9 \mathrm{E}+02$ & $1.9 \mathrm{E}+02$ & $1.9 \mathrm{E}+02$ \\
\hline${ }^{240} \mathrm{Pu}$ & $1.9 \mathrm{E}+02$ & $1.9 \mathrm{E}+02$ & $1.9 \mathrm{E}+02$ & $1.9 \mathrm{E}+02$ & $1.9 \mathrm{E}+02$ & $1.9 \mathrm{E}+02$ \\
\hline${ }^{241} \mathrm{Pu}$ & $1.0 \mathrm{E}+04$ & $1.0 \mathrm{E}+04$ & $1.0 \mathrm{E}+04$ & $1.0 \mathrm{E}+04$ & $1.0 \mathrm{E}+04$ & $1.0 \mathrm{E}+04$ \\
\hline${ }^{241} \mathrm{Am}$ & $1.8 \mathrm{E}+02$ & $1.8 \mathrm{E}+02$ & $1.8 \mathrm{E}+02$ & $1.8 \mathrm{E}+02$ & $1.8 \mathrm{E}+02$ & $1.8 \mathrm{E}+02$ \\
\hline
\end{tabular}

Note: Asterisks mark nuclides with progeny that are assumed to be present in equilibrium amounts. However, ${ }^{234} \mathrm{U}$ was not included in the ${ }^{238} \mathrm{U}$ limits. For supporting references see WHC-SD-EN-TI-070. 


\subsection{DATA SUMMARY METHODS}

Measuring any physical quantity has some degree of inherent uncertainty. This uncertainty results from the combination of all possible inaccuracies in the measurements process, including such factors as the reading of the result, the calibration of the measuring device, and numerical rounding errors.

In this report, individual radioactive measurements are accompanied by a plus or minus $( \pm)$ value, which represents the total propagated analytical uncertainty (or 2-sigma counting error). The two-sigma counting error gives information on what the measurement might be if the same sample were counted again under identical conditions. The two-sigma counting error implies that approximately $95 \%$ of the time, a recount of the same sample would give a value within plus or minus the two-sigma counting error at the value reported.

Values in the tables that are less than the minimum detectable activity indicate that the reported result might have come from a sample with no radioactivity. Such values are considered below the detection limits of the measuring instrument. Also note that each radioactive measurement must have the random background radioactivity of the measuring instrument subtracted; therefore, negative results are possible, especially when the sample has very little radioactivity.

Reported averages also are accompanied by a plus or minus $( \pm)$ value, which represents two standard deviations from the mean. If the data fluctuate randomly, this is a measure of the uncertainty in the estimated average of the data because of this randomness.

Where averages of averages are reported, the plus or minus $( \pm)$ value represents two standard errors of the mean.

The mean, $\mathrm{X}$, is computed as:

$$
X=\frac{1}{n} \sum_{i=1}^{n} X_{i}
$$

where $\mathrm{X}_{\mathrm{i}}$ is the ith measurement and $\mathrm{n}$ is the number of measurements.

The standard error of the mean was computed as:

$$
S E=\sqrt{\frac{S^{2}}{n}}
$$


where $\mathrm{S}^{2}$, the variance of the $\mathrm{n}$ measurements, was computed as:

$$
S_{M}^{2}=\frac{1}{n-1} \sum_{i=1}^{n}\left(X_{i}-X\right)^{2}
$$

This estimator, $\mathrm{S}^{2}$, includes the variance among the samples and the counting variance. The estimated $S^{2}$ occasionally may be less than the average counting variance. 


\subsection{REFERENCES}

10 CFR 835, “Occupational Radiation Protection,” Code of Federal Regulations, as amended.

40 CFR 51, "Requirements for Preparation, Adoption, and Submittal of Implementation Plans," Code of Federal Regulations, as amended.

40 CFR 52, “Approval and Promulgation of Implementation Plans," Code of Federal Regulations, as amended.

40 CFR 61, Subpart H, "National Emissions Standards for Hazardous Air Pollutants," Code of Federal Regulations, as amended.

40 CFR 141, "National Primary Drinking Water Regulations," Code of Federal Regulations, as amended.

40 CFR 162, "State Registration of Pesticide Products," Code of Federal Regulations, as amended.

40 CFR 264, "Standards for Owners and Operators of Hazardous Waste Treatment Storage and Disposal Facilities," Code of Federal Regulations, as amended.

Comprehensive Environmental Response, Compensation, and Liability Act of 1980, 42 USC 9601, et seq.

Dyekman, D. L., 2004, Environmental Releases for Calendar Year 2003, HNF-EP-0527-13, Fluor Daniel Hanford, Inc., Richland, Washington.

DTS-OEM-001, Operational Environmental Monitoring, Duratek Technical Services, Richland, Washington.

DOE, 2004, Environment, Safety, and Health Reporting Manual, DOE Manual 231.1-1A,

U.S. Department of Energy, March 19, 2004.

DOE, 2003, Environmental Protection Program, DOE Order 450.1, U.S. Department of Energy, Washington, D.C.

DOE, 2001, Radioactive Waste Management, DOE Order 435.1, U.S. Department of Energy, Washington, D.C.

DOE 1993, Radiation Protection of the Public and the Environment, DOE Order 5400.5, U.S. Department of Energy, Washington, D.C. 
Hartman, M. J., L. F. Morasch, and W. D. Webber, 2004, Hanford Site Groundwater Monitoring for Fiscal Year 2003, PNNL-14548, Pacific Northwest National Laboratory, Richland, Washington.

HNF-PRO-1620, ALARA Program Scope, Fluor Hanford, Richland, Washington.

HNF-5173, PHMC Radiological Control Manual, Fluor Hanford, Richland, Washington.

Horton, D. G., S. P. Reidel, Yi-Ju Chien, and R. M. Mitchell, 2000, Remote-Handled Immobilized Low-Activity Waste Disposal Facility Preoperational Monitoring Plan, RPP-6877, Pacific Northwest National Laboratory and Waste Management Federal Services, Inc., Northwest Operations for CH2MHill Hanford Group, Inc., Richland, Washington.

Lane, N. K., J. K. Linville, R. M. Mitchell and R. S. Zack, 2003, Sampling and Analysis Instruction for Soil, Vegetation, and Soil Invertebrate Sampling at Gable Mountain Pond, B-Pond, and a 200 West Reference Location, DRAFT, Fluor Hanford, Richland Washington.

McKinney, S. M., 2003, Near-Facility Environmental Monitoring QA Project Plan, HNF-EP-0538-8, Duratek Technical Services, Inc., Richland, Washington.

PNNL, 2004, Hanford Site Environmental Report for Calendar Year 2003, PNNL-14687, Pacific Northwest National Laboratory, Richland, Washington.

Resource Conservation and Recovery Act of 1976, 42 USC 6901, et seq.

WAC 173-303, “Dangerous Waste Regulations,” Washington Administrative Code, as amended.

WAC 246-247, “Radiation Protection-Air Emissions,” Washington Administrative Code, as amended. 


\section{Distribution}

No. of

Copies

\section{OFFSITE}

P L. Block

U.S. Department of Interior

Fish and Wildlife Service

Fish and Wildlife Enhancement

Moses Lake, Field Office

P.O. Box 1157

Moses Lake, WA 98837

P D. Goeke

U.S. Department of Interior

Fish and Wildlife Service

Columbia National Wildlife Refuge

P.O. Box Drawer F

Othello, WA 99344

P R. Jim

Yakima Indian Nation

Environmental Restoration/Waste Management Program

P. O. Box 151

Toppenish, WA 98948

P J. E. McDonald

Energy Northwest

P.O. Box 968, MD 1020

Richland, WA 99352

P A. Palmer

US Ecology, Inc.

P.O. Box 638

Richland, WA 99352

P Mr. Patrick Sobotta

Nez Perce Tribe

Environmental Restoration and Waste Management

P.O. Box 365

Lapwai, ID 83540
No. of

Copies

2P U.S. Environmental Protection Agency, Region 10

1200 Sixth Avenue

Seattle, WA 98081

ATTN: J. M. Leitch

OAQ-107

R. W. Poeton

OAQ-107

4CD Washington State Department of Health

Air Emissions and Defense Waste

Division of Radiation Protection

7171 Cleanwater Lane

Building 5

Olympia, WA 98504-7837

ATTN: L. Albin
A. W. Conklin
J. L. Erickson
D. McBaugh

8CD Washington State Department of Health Air Emissions and Defense Waste

Division of Radiation Protection

309 Bradley Boulevard, Suite 201

Richland, WA 99352

ATTN: R. S. Acselrod

J. C. Berkey

A. T. Cooper

R. A. Danielson

R. E. Jaquish

P. J. Martell

T. M. Priddy

J. W. Schmidt

P J. P. Wilkinson

Confederated Tribes of the Umatilla

Indian Reservation

P.O. Box 638

Pendleton, OR 97801 
No. of

Copies

\section{ONSITE}

E. J. Antonio (CD)

J. G. April (CD)

L. E. Borneman (CD)

O. L. Bostic (CD)

E. M. Bowers (P/CD)

M. W. Bowman (CD)

H. C. Boynton (P)

R. C. Brunke (CD)

S. L. Bump (CD)

L. L. Cadwell (CD)

C. J. Chou (P)

R. T. Coffman (CD)

B. L. Curn (CD)

E. B. Dagan (CD)

A. K. Dasgupta (CD)

L. P. Diediker (8P/10CD)

R. L. Dirkes (P)

J. N. Diven (CD)

B. J. Dixon (CD)

J. W. Donnelly (P)

J. J. Dorian (P/CD)

P. F. Dunigan, Jr. (P)

D. L. Dyekman (CD)

R. G. Egge (CD)

R. E. Elder (CD)

R. H. Engelmann (CD)

B. G. Erlandson (CD)

J. D. Fancher (P)

K. R. Fecht (CD)

B. G. Fritz (CD)

K. A. Gano (CD)

R. H. Gurske (CD)

J. B. Hall (P)

R. W. Hanf (P)

L. M. Hay (P)

W. M. Hayward (P)

R. P. Henckel (CD)

R. D. Hildebrand (CD)

J. S. Hill (CD)

N. A. Homan (CD)

D. G. Horton (CD)

A. R. Johnson (5P)

D. L. Johnson (CD)
No. of

\section{Copies}

V. G. Johnson (CD) E6-35

R. D. Julian (P)

T4-04

R1-51

A4-70

H9-03

H6-03

K6-96

A3-03

H1-11

H1-11

H1-11

X5-50

H9-03

S2-42

K6-75

H1-11

H8-40

T1-27

K6-75

T6-12

L1-04

K6-81

K3-54

S2-55

A5-15

H8-13

L4-19

S5-08

T4-04

K8-03

H8-13

B5-18

S6-72

K3-75

A2-17

X3-79

H0-23

H9-03

N2-02

B1-07

H6-08

H0-09

H2-53

P8-55 